Robert Weber

\title{
Kulturlandschaftswandel in Zentralsulawesi
}

Historisch-geographische Analyse einer indonesischen Bergregenwaldregion

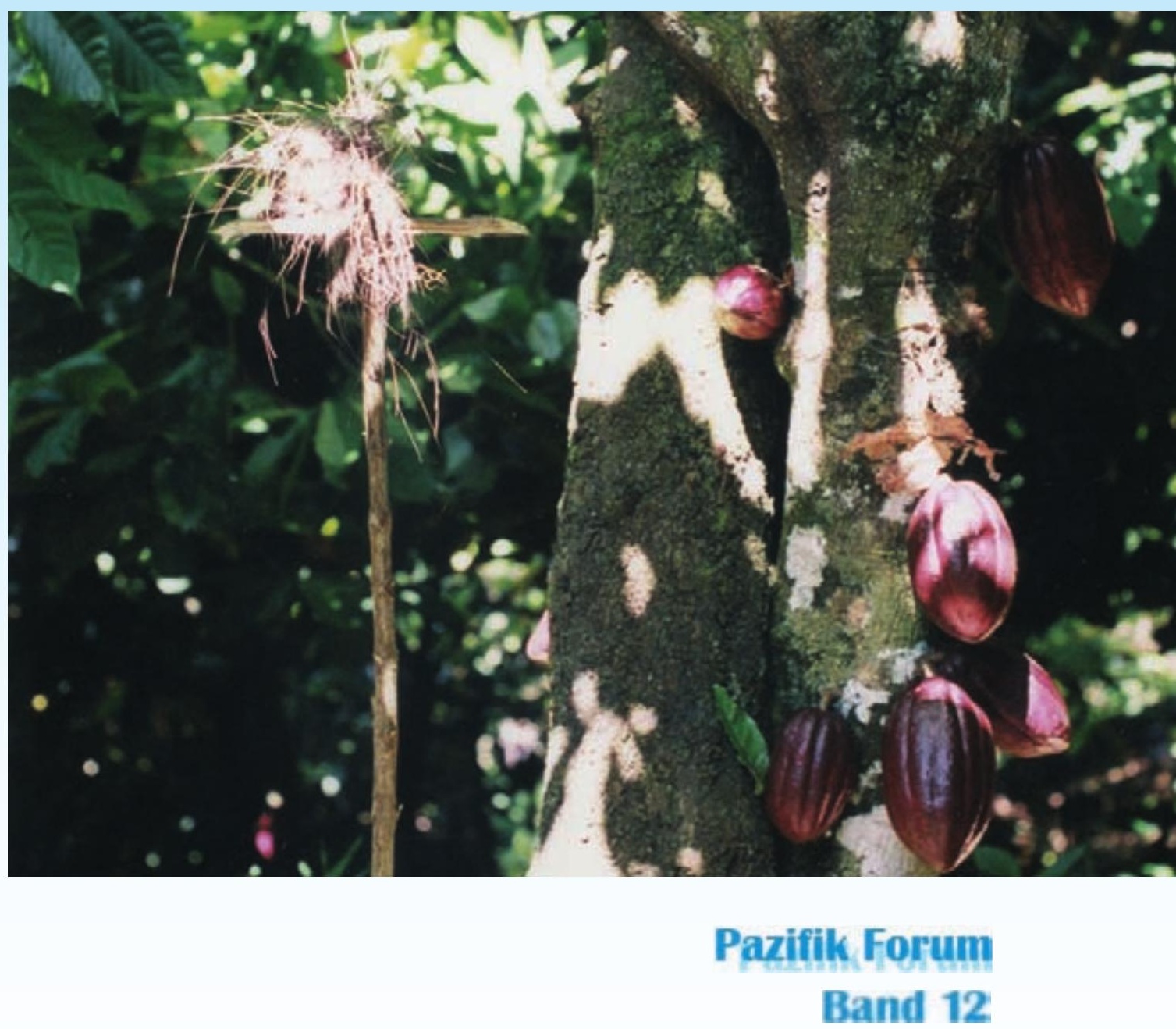



Robert Weber

Kulturlandschaftswandel in Zentralsulawesi

This work is licensed under the Creative Commons License 3.0 "by-nd", allowing you to download, distribute and print the document in a few copies for private or educational use, given that the document stays unchanged and the creator is mentioned.

You are not allowed to sell copies of the free version. 
erschienen im Universitätsverlag Göttingen 2006 
Robert Weber

Kulturlandschaftswandel in

Zentralsulawesi

Historisch-geographische

Analyse einer indonesischen

Bergregenwaldregion

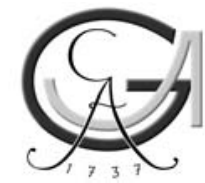

Universitätsverlag Göttingen

2006 


\section{Bibliographische Information der Deutschen Nationalbibliothek}

Die Deutsche Nationalbibliothek verzeichnet diese Publikation in der Deutschen Nationalbibliographie; detaillierte bibliographische Daten sind im Internet über $<$ http://dnb.ddb.de $>$ abrufbar.

Dieses Buch ist auch als freie Onlineversion über die Homepage des Verlags sowie über den OPAC der Niedersächsischen Staats- und Universitätsbibliothek (http://www.sub.uni-goettingen.de) erreichbar und darf gelesen, heruntergeladen sowie als Privatkopie ausgedruckt werden. Es gelten die Lizenzbestimmungen der Onlineversion. Es ist nicht gestattet, Kopien oder gedruckte Fassungen der freien Onlineversion zu veräußern.

Umschlagabbildung:

Das aus Kokosfasern gefertigte, animistische Ornament an der Spitze des Holzkreuzes weist auf Kontinuitäten traditioneller Geisteshaltungen der lokalen Bevölkerung hin. Das Kreuz selbst steht als christliches Symbol beispielhaft für den religiösen Wandel, während die Kakaopflanze eine Facette des landwirtschaftlichen Wandels in der Lore-Lindu-Region darstellt.

(C) Robert Weber 2001.

Satz und Layout: Margo Bargheer

Umschlaggestaltung: Margo Bargheer, Kilian Klapp

\section{(C) 2006 Universitätsverlag Göttingen}

ISBN-10: 3-938616-52-0

ISBN-13: 978-3-938616-52-9 
Arbeitsgemeinschaft für Pazifische Studien

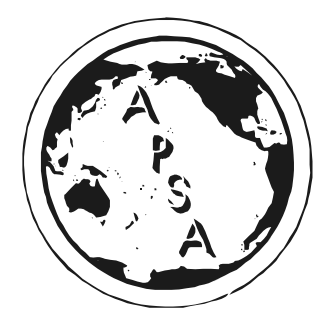

ROBERT WEBER

\section{Kulturlandschaftswandel in Zentralsulawesi}

Historisch-geographische Analyse einer indonesischen Bergregenwaldregion

Hrsg.: Heiko Faust

Werner Kreisel

Michael Waibel 



\section{Vorwort}

Dieser Band enthält eine überarbeitete Fassung meiner Dissertation, welche im Jahr 2005 unter dem Titel „Kulturlandschaftswandel während des 20. Jh. in Zentralsulawesi - eine historisch-geographische Analyse der Lore-Lindu-Bergregenwaldregion“ bei der Niedersächsischen Staats- und Universitätsbibliothek Göttingen digital publiziert wurde. Die Dissertation entstand im Rahmen des Sonderforschungsbereichs SFB 552 - STORMA „Stability of Rainforest Margins in Indonesia“ der Deutschen Forschungsgemeinschaft (DFG). Ich bedanke mich beim Vorstand der Arbeitsgemeinschaft für Pazifische Studien e.V. für sein Interesse an einer Veröffentlichung dieser Arbeit in der Reihe Pazifik-Forum.

Die erfolgreiche Fertigstellung dieser Arbeit ist der freundlichen Unterstützung durch eine Viezahl von Personen zu verdanken. Ein ganz besonderer Dank gebührt den Einwohnern meiner Untersuchungsdörfer für ihre freundliche Aufnahme in ihrer Mitte und ihre Unterstützung meiner Feldforschung, insbesondere den Dorfbürgermeistern, meinen Gastfamilien und allen Interviewpartnern. Ohne ihre Bereitschaft wäre die empirische Datengrundlage für diese Arbeit nicht zustande gekommen. Sie haben meinen Aufenthalt in der Lore-Lindu-Region zudem zu einem unvergesslichen Erlebnis gemacht. Ich bedanke mich bei Akib, meinem Forschungsassistenten, der mir nicht nur unermüdlich bei der Datenerhebung half, sondern mich auch in zahlreiche Aspekte der lokalen Kultur einwies und mir zum Freund wurde. Ebenso bedanke ich mich bei Eka und Sri, die mit unglaublicher Energie die zahlreichen Tonbandaufzeichnungen transkribierten.

Mein herzlicher Dank gilt Herrn Prof. Dr. Werner Kreisel, der mir die Gelegenheit gab, diese Dissertation zu erstellen, und der mir mit seinem Rat zur Seite stand. Herrn Prof. Dr. Gerhard Gerold danke ich dafür, die Korreferenz für diese Doktorarbeit übernommen zu haben. Ebenso danke ich Herrn PD Dr. Heiko Faust ganz herzlich für seine ermutigende Beratung während der gesamten Zeit. Ferner möchte ich, neben den bereits genannten Personen, Frau Prof. Dr. Brigitta HauserSchäublin, Herrn Prof. Dr. Manfred Zeller und Herrn Prof. Dr. Karl-Heinz Pörtge dafür danken, meine Arbeit im Rahmen der Disputation kritisch unter die Lupe genommen zu haben.

Danken möchte ich auch Prof. Dr. Sulaiman Mamar und Dr. Endriatmo Sutarto, unseren Forschungspartnern (Supervisors) in Palu und Bogor, sowie den indonesischen Doktorandenkollegen unseres Teilprojekts, Melani Abdulkadir-Sunito, Moh. Shohibuddin und Ivanovich Agusta, für ihre Unterstützung, den anregenden fachlichen Austausch und ihre Gastfreundschaft. 
Darüber hinaus möchte ich mich auch bei allen STORMA-Kolleginnen und -Kollegen bedanken, die mir während meines Aufenthalts in Indonesien in vielfältiger Weise behilflich waren, sei es durch anregende und konstruktive Diskussionen oder durch unvergessliche Karaokeabende im Dewi und Teratai („Buka pintu-u-u“). Besonders erwähnen möchte ich hierbei Sylvia Ebersberger, Christian H. Schulze, Michael Hoppe, Regina Birner, Stefan Schwarze, Günter Burkard und Teunis van Rheenen. Georg Dechert danke ich für die breit gefächerten Gesprächs- und Diskussionsthemen und den Spaß, den wir gemeinsam als Oberbrandinspektoren oder Entdeckungsreisende hatten. Sehr herzlich möchte ich mich auch bei Gesa Milferstädt, Miet Maertens und Kerstin Bohmann, meinen Mitbewohnerinnen in Palu, bedanken, mit denen ich eine meiner bisher angenehmsten Wohngemeinschaften bildete. Ich danke den Teams von Z4 in Göttingen, Bogor und Palu, insbesondere Rina, Arman und allen Fahrern des Forschungsprojekts. Ferner gilt mein Dank Greg Acciaioli, Lorraine Aragon, Stephanie Haberer, David Henley, Dinah Loerke und Tobias Reeh für die fruchtbaren Fachgespräche und die aufbauende Unterstützung.

Mein größter Dank gilt jedoch meiner Freundin Stella für die Kraft und Liebe, die sie mir gab, und für das tapfere Ertragen meiner psychischen Anspannung (v.a. während der letzten Monate der Fertigstellung) sowie meiner Familie, die mich stets großzügig unterstützt hat und immer von dem Gelingen meines Vorhabens überzeugt war.

Göttingen, im Mai 2006

Robert Weber

„Knowledge is never pure but is always situated in the complex and sometimes contradictory social locations of its producers and audience."

(Women and Geography Study Group 1997: 14) 
Für Gertraud, Fritz, Andrea und Stella 



\section{Inhaltsverzeichnis}

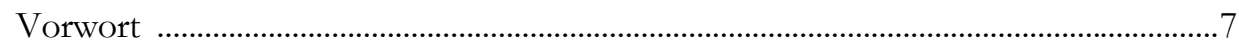

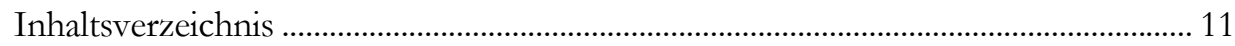

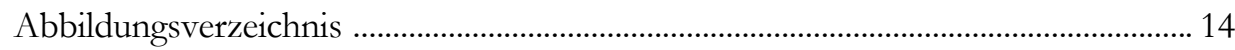

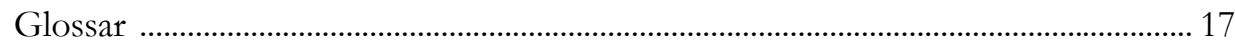

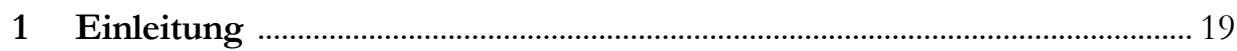

1.1 Problemstellung, Zielsetzung ……………………………………………..... 19

1.2 Die Kulturlandschaft - nur ein soziokulturelles Konstrukt? ....................... 23

1.3 Forschungsbedarf und Hypothesen ……………………………………...... 29

1.4 Forschungsverlauf und -methodik ................................................................ 33

1.5 Aufbau der Arbeit ...................................................................................... 40

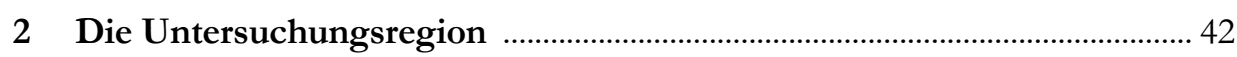

2.1 Einführung in das Untersuchungsgebiet ........................................................ 42

2.2 Naturgeographische Grundlagen der Lore-Lindu-Region ........................... 45

2.3 Historische Entwicklung der Untersuchungsregion und deren Einbindung in die Geschichte Indonesiens ................................................... 49

2.3.1 Die präkoloniale Phase (bis 1904) ………………………………...... 50

2.3.2 Die ersten Europäer in Zentralsulawesi .............................................. 53

2.3.3 Die niederländische und japanische Kolonialherrschaft

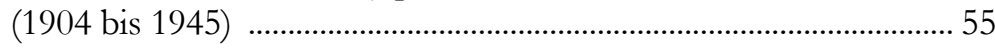

2.3.4 Die Phase der Unabhängigkeit Indonesiens (ab 1945) .................... 59

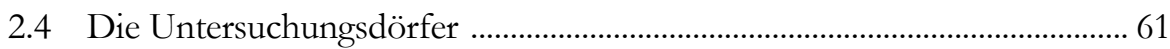

3 Veränderungen der Bevölkerungsstrukturen in der

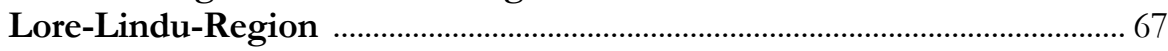

3.1 Bevölkerung und Bevölkerungsdynamik ...................................................... 67

3.1.1 Charakterisierung der lokalen Bevölkerungsgruppen ......................... 69

3.1.2 Quantifizierung der Bevölkerung zu Beginn des 20. Jh. ................... 72

3.1.3 Bevölkerungsdynamik in der Lore-Lindu-Region ............................. 74

3.2 Migrationsprozesse in der Lore-Lindu-Region ............................................ 75 
3.3 Allgemeine Aspekte der Zu- und Abwanderung im

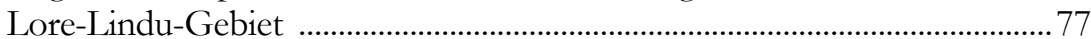

3.4 Staatlich geplante Umsiedlungen ...................................................................... 81

3.4.1 Staatlich geplante, lokale Umsiedlungen .............................................. 82

3.4.2 Staatlich geplante, nationale Umsiedlungen Das Transmigrasi-Programm .................................................................. 89

3.5 Halbfreiwillige Umsiedlung am Beispiel des Dorfes Lawe .......................... 94

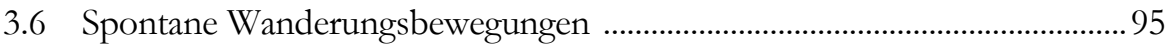

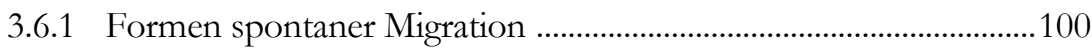

3.6.2 Typisierung von Wanderungsmotiven ...............................................103

3.6.3 Fallbeispiel: Spontane Zuwanderung in das Palolo-Tal Das Beispiel Berdikari ........................................................................105

3.7 Zu- und Abwanderung als Auswirkung regionaler Konflikte .................... 108

3.7.1 Die Rebellionen von DI/TII und Permesta und ihre Auswirkungen auf die Lore-Lindu-Region 108

3.7.2 Der Poso-Konflikt und die Fluchtmigration nach Lore Utara

4 Veränderungen der Siedlungsstrukturen ............................................................ 110

4.1 Die historische Siedlungsentwicklung in der Lore-Lindu-Region .............112

4.1.1 Allgemeine Bemerkungen zur Rekonstruktion der Siedlungsentwicklung ....................................................................113

4.1.2 Präkoloniale Besiedlung der Lore-Lindu-Region ............................114

4.1.3 Wandel der Siedlungsformen unter dem kolonialen Einfluss

4.1.4 Regionale Unterschiede der Siedlungsentwicklung ab 1945

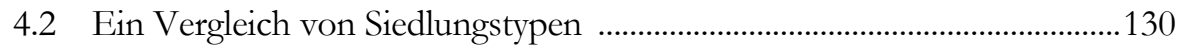

4.2.1 Fallbeispiel Maranatha ........................................................................131

4.2.2 Fallbeispiele Lempelero und Bolapapu ..............................................132

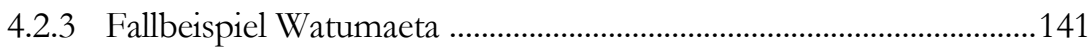

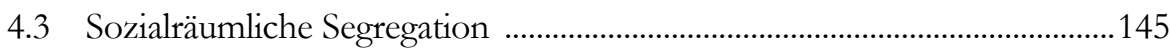

4.3.1 Allgemeine Überlegungen zum Verhaltensmuster sozialer Abgrenzung

4.3.2 Zur Entwicklung multi-ethnischer Dorfgemeinschaften in der Lore-Lindu-Region

4.3.3 Segregationsmuster im Vergleich 149 
5 Der Wandel von Landnutzung und Landbesitz ....................................... 157

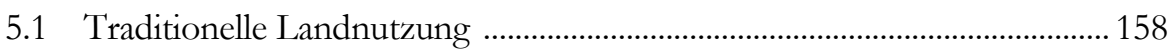

5.2 Von der Subsistenzwirtschaft in die Weltmarktproduktion ....................... 164

5.2.1 Vom Wasserbüffel zum Handtraktor - Ausbau und Technisierung des Nassreisanbaus

5.2.2 Der Kakaoboom - Wohlstandsmotor auf tönernen Füßen

5.2.3 Vom nachbarschaftlichen Arbeitskräftetausch zur Lohnarbeit

5.3 Landkonflikte - ein Problem der Zuwanderung?

6 Politische Einflüsse auf die Veränderungen der Kulturlandschaft 184

6.1 Die Ethische Politik der Niederländer 184

6.1.1 Der kolonialpolitische Entstehungsprozess der Ethischen Politik

6.1.2 Die Bedeutung der Ethischen Politik für die Lore-Lindu-Region

6.2 Bildungspolitik und die Bedeutung von Bildung für die ökonomische Entwicklung

6.3 Kontinuitäten und Brüche traditioneller Herrschaftsstrukturen 196

6.4 Das Spannungsfeld Nationalpark 198

6.4.1 Historische Entwicklung der Mensch-Wald-Interaktion in der Untersuchungsregion 198

6.4.2 Der Dongi-Dongi-Konflikt und seine Folgen 203

6.4.3 Räumliche Differenzierung von Wahrnehmung und Handeln in bezug auf den Lore-Lindu-Nationalpark

Quellen- und Literaturverzeichnis 215

Anhang 233

Abstract 234 


\section{Abbildungsverzeichnis}

Abb. 1 Der Biodiversitäts-Hotspot Wallacea

Abb. 2 Primärwaldbestand und Ratio Primärwaldfläche zur gesamten

Landfläche südostasiatischer Staaten im Jahr 2000

Abb. 3 Jährliche Abnahme des Waldbestandes südostasiatischer Staaten 1990 - 2000 im Verhältnis zum Gesamtbestand im Jahr 2000

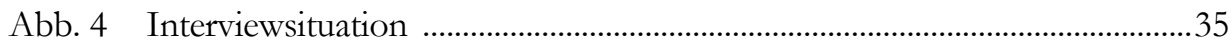

Abb. 5 Die Untersuchungsregion mit Lokalisierung der Untersuchungsdörfer

Abb. 6 Karte der kolonialzeitlichen Verwaltungseinheiten im westlichen Zentralsulawesi

Abb. 7 Reparaturarbeiten an der Verbindungsstrasse zwischen Palu- und Kulawi-Tal nach einem Erdrutsch

Abb. 8 Ausbauarbeiten am Fuß- und Pferdepfad nach Lawe

Abb. 9 Prozentanteile der Bevölkerung verschiedener Teilregionen an der Gesamtbevölkerung Indonesiens im Jahr 2000

Abb. 10 Bevölkerungsdichte verschiedener Teilregionen Indonesiens im

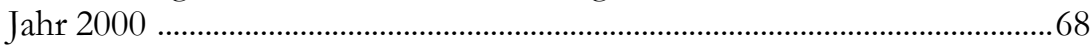

Abb. 11 Einwohnerzahlen und Bevölkerungsdichte nach Kecamatan 2001 ...........68

Abb. 12 Verteilung der ethnischen Gruppen des westlichen Zentralsulawesi in den 1930er Jahren

Abb. 13 Bevölkerungsentwicklung in der Lore-Lindu-Region nach Kecamatan 1938 - 2001 .79

Abb. 14 Bevölkerungszuwachsraten in der Lore-Lindu-Region nach Kecamatan 1938 - 2001 80

Abb. 15 Planskizze von Maranatha . .87

Abb. 16 Lage der Transmigrationssiedlungen im Untersuchungsgebiet . .91

Abb. 17 Besiedlung des Palu-Tals und des nördlichen Kulawi .96

Abb. 18 Besiedlung des südlichen Kulawi von Rampi und über das Bada-Tal .97

Abb. 19 Besiedlung von Napu- und Besoa-Tal . .98

Abb. 20 Stadt- und Landbevölkerung in Indonesien 1950 - 2030 (ab 2005 prognostizierte Werte)

\section{Pazifik Forum Bd. 12}


Abb. 21 Entwicklung der Bevölkerungsverteilung zwischen Stadt und Land bzw. agrarisch und nicht-agrarisch in Indonesien $1961-2001$

Abb. 23 Der Dorfwall von Lamba, Napu-Tal 115

Abb. 24 Karte des Napu-Tals von 1908 116

Abb. 25 Karte des Napu-Tals von 1905

Abb. 26 Siedlungsgebiete im westlichen Zentralsulawesi Anfang der 1920er Jahre

Abb. 27 Dorfgründungen in der Lore-Lindu-Region 1906 - 2001

Abb. 29 Räumliche Verteilung der einzelnen Dorfteile von Lempelero sowie der Dörfer Tompi Tua und Tompi Bangka

Abb. 30 Das Dorf Bolapapu und die räumliche Verteilung seiner Dorfteile 2001

Abb. 31 Soziopolitische Stratifikation der Gesellschaft im präkolonialen Kulawi

Abb. 32 Soziopolitische Stratifikation der Gesellschaft im kolonialen Kulawi 138

Abb. 33 Kartenskizze von Laone und seiner Besiedlung 140

Abb. 34 Raum-zeitliche Ausdehnung der Wohnareale in Watumaeta 142

Abb. 35 Ein alter lesung batu in Powanuanga als Hinweis auf die ehemalige anthropogene Nutzung dieses Gebiets 144

Abb. 36 Räumliche Segregation der Bevölkerung von Maranatha nach Herkunftsorten

Abb. 37 Sozial-räumliche Segregation in Sintuwu 154

Abb. 38 Anteile der 2001 in der Lore-Lindu-Region angebauten

Feldfrüchte und ihre Relevanz für das Haushalteinkommen der Landwirte

Abb. 40 Brachefeld in der Nähe von Lawe 159

Abb. 41 Nassreisflächen im Palu-Tal 1938 166

Abb. 42 Pflugbewirtschaftung eines Nassreisfeldes im Palu-Tal 168

Abb. 43 Kakaoproduktion von Kleinbauern in Indonesien 1984 - 1994 173

Abb. 44 Anzahl der Kakao produzierenden Kleinbauern im Ländervergleich 1999

Abb. 45 Durchschnittlicher Jahresweltmarktpreis für Kakao 1960 - 2003 174

Abb. 46 Karikatur über den politischen Kampf zwischen der Stuw-Gruppe und dem Reichseinheitsblock: ,Zieht doch! Und vor allem nicht umschauen." 
Abb. 47 Blick von Westen aus auf den Ort Lemo und die entwaldeten Hänge des heutigen Lore-Lindu Nationalparks 1911 200

Abb. 48 Blick vom Regierungs-Gästehaus (ehem. Lemo) von Bolapapu aus nach Osten auf die Hänge des Lore-Lindu Nationalparks 201

Abb. 49 Rodung des Waldes in Sintuwu entlang der sog. Jalan Jepang ......................206

Abb. 50 Hangerosion in Sintuwu .207

\section{Tabellenverzeichnis}

Tab. 1 Administrative Verteilung der Untersuchungsdörfer auf das Untersuchungsgebiet

Tab. 2 Distanzen der Untersuchungsdörfer zum Hauptort des jeweiligen Kecamatan 


\section{Glossar}

${ }^{\circ} \mathrm{C}$

a

a. a. $\mathbf{O}$.

Abb.

A. D.

Adat

Anm. d. Verf.

BK

BPS

bspw.

Bupati

bzgl.

Camat

DepSos

Desa

DFG

d.h.

Dusun

ebd.

ehem.

FAO

ha

i.d.R.

insbes.

IPB

$\mathrm{HH}$

Kab.

Kabupaten

Kap.
Grad Celsius

Ar

am angegebenen Ort

Abbildung

Anno Domini

Gewohnheitsrecht

Anmerkung des Verfassers dieser Arbeit

Bala Keselamatan (Heilsarmee)

Badan Pusat Statistik (Zentrale Statistikbehörde)

beispielsweise

Chef eines Kabupaten

bezüglich

Chef eines Kecamatan

Departmen Sosial (Behörde für soziale Angelegenheiten)

Dorf

Deutsche Forschungsgemeinschaft

das heißt

Dorfteil, Dorfviertel

ebenda

ehemals

Food and Agricultural Organisation

Hektar

in der Regel

insbesondere

Institut Pertanian Bogor

Haushalt

Kabupaten

Distrikt (in etwa vergleichbar mit einem deutschen

Regierungsbezirk)

Kapitel 
Kec.

Kecamatan

km

LLNP

m

NGO

o.A.

o.g.

o. J.

o.O.

Pak

s.

s.o.

s.u.

sawah

SFB

sog.

STORMA

Tab.

TNLL

u.

u.a.

ü. NN.

UNTAD

vgl.

v.u.Z.

z.B.

z.T.
Kecamatan

Sub-Distrikt (in etwa vergleichbar mit einem deutschen Landkreis)

Kilometer

Lore-Lindu Nationalpark

Meter

Non-Government Organization (Nichtregierungsorganisation)

ohne Autorenangabe

oben genannt

ohne Jahreszahl

ohne Ortsangabe

von: Bapak (wörtlich übersetzt: „Vater“; im Allgemeinen als Anrede „Herr" verwendet)

siehe

siehe oben

siehe unten

Nassreis

Sonderforschungsbereich

so genannt(-e/-er/-es)

Stability of Rainforest Margins in Indonesia

Tabelle

Taman Nasional Lore Lindu (Lore-Lindu Nationalpark)

und

unter anderem / und andere

über Normalnull

Universitas Tadulako

vergleiche

vor unserer Zeitrechnung

zum Beispiel

zum Teil 


\section{$1 \quad$ Einleitung}

\subsection{Problemstellung, Zielsetzung}

Die Lore-Lindu-Region in Zentralsulawesi stellt als Teil des Biodiversitäts-Hotspots Wallacea (vgl. Abb. 1) ein - unter Biodiversitätsaspekten betrachtet - einmaliges Gebiet Indonesiens und eines der weltweit bedeutendsten Zentren endemischer Arten dar (Waltert et al. 2004; Schulze et al. 2004). Als eines der beiden einzigen Schutzreservate Zentralsulawesis nimmt der Lore-Lindu Nationalpark eine Schlüsselrolle für den Erhalt der einzigartigen Flora und Fauna der Insel Sulawesi ein (vgl. Coates et al. 1997; Myers et al. 2000; Stattersfield et al. 1998). 228 bisher entdeckte verschiedene Vogelarten und eine beachtliche Anzahl wichtiger endemischer Landsäuger sind dort beheimatet (Waltert et al. 2004: 328 u. 331). Den Kern des Parks bildet einer der wenigen noch existierenden artenreichen Primärwälder des Archipels.

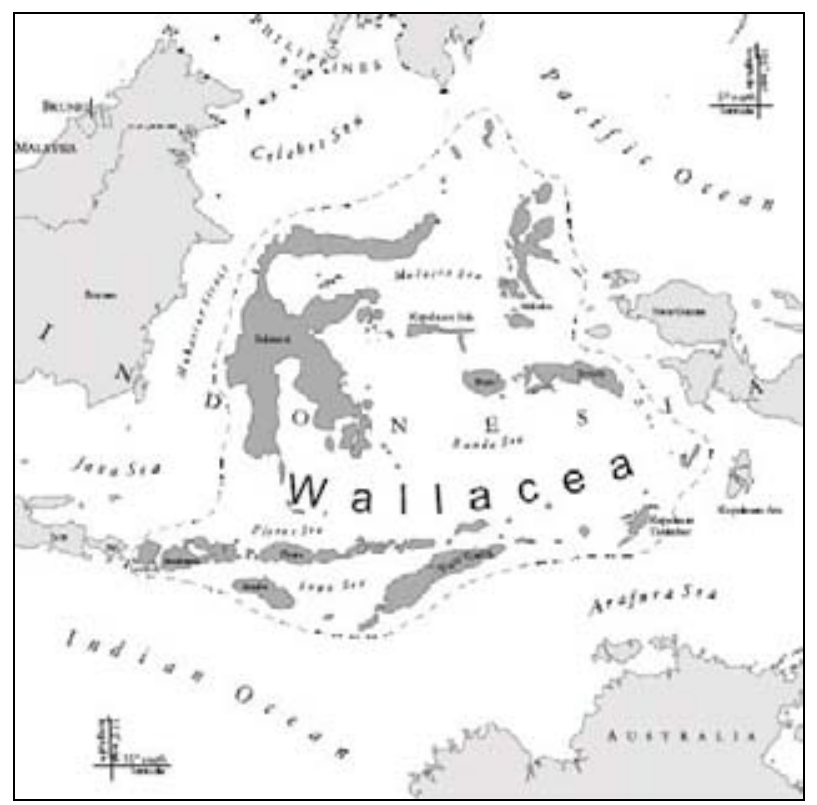

Abb. 1 Der Biodiversitäts-Hotspot Wallacea

(Quelle: Conservation International 2002) 
Im südostasiatischen Vergleich verfügte Indonesien im Jahr 2000 über den größten Bestand an Primärwald. Mit über 95.000 ha weist der Inselstaat knapp dreimal soviel Primärwald auf wie Myanmar, welches den zweiten Rang einnimmt (vgl. Abb. 2).

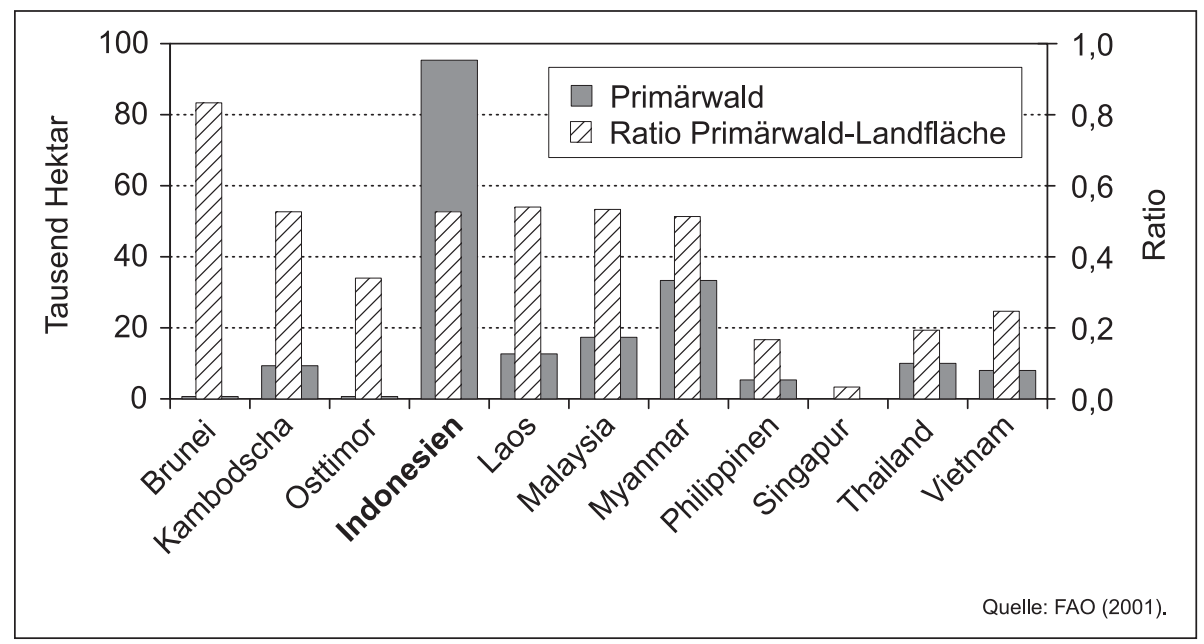

Abb. 2 Primärwaldbestand und Ratio Primärwaldfläche zur gesamten Landfläche südostasiatischer Staaten im Jahr 2000

Selbst wenn man den Umstand berücksichtigt, dass es sich bei Indonesien um den flächengrößten Staat Südostasiens handelt, und die Verhältniswerte von Primärwaldfläche der Staaten zu der jeweiligen gesamten Landfläche betrachtet, positioniert sich Indonesien mit $53 \%$ immer noch unter den vorderen Rängen.

Abgesehen von Vietnam nimmt der Waldbestand in allen verglichenen Ländern Südostasiens ab. Indonesien offenbart sich dabei als eines der Länder mit den höchsten Entwaldungsraten in der regionalen Synopse. Zwischen 1990 und 2000 wurde der Waldbestand jährlich um 1,2 \% bzw. 1.300 ha dezimiert, ${ }^{1}$ ein Wert, der nur von Myanmar und den Philippinien überschritten wird, welche eine jährliche Entwaldungsrate von 1,4\% während der letzten Dekade des 20. Jh. aufweisen (vgl. Abb. 3). In der Lore-Lindu-Region nahm der Bestand an Naturwald zwischen 1971 und 2002 um rund $18 \%$ ab, bei einer durchschnittlichen jährlichen Entwaldungsrate von 0,6\% (Erasmi et. al. 2004: 294). ${ }^{2}$

1 Während des Zeitraums 1982-1990 belief sich die Entwaldung in Indonesien auf 937.000 ha pro Jahr (Olive 1998: 2)

2 Die große Diskrepanz zwischend den Entwaldungsraten der Lore-Lindu-Region einerseits und Gesamt-Indonesiens andererseits kann durch die unterschiedlichen Zeitintervalle (1971-2002 bzw. 1990-2000) und Datenquellen erklärt werden (Erasmi et. al. 2004: 295f). 


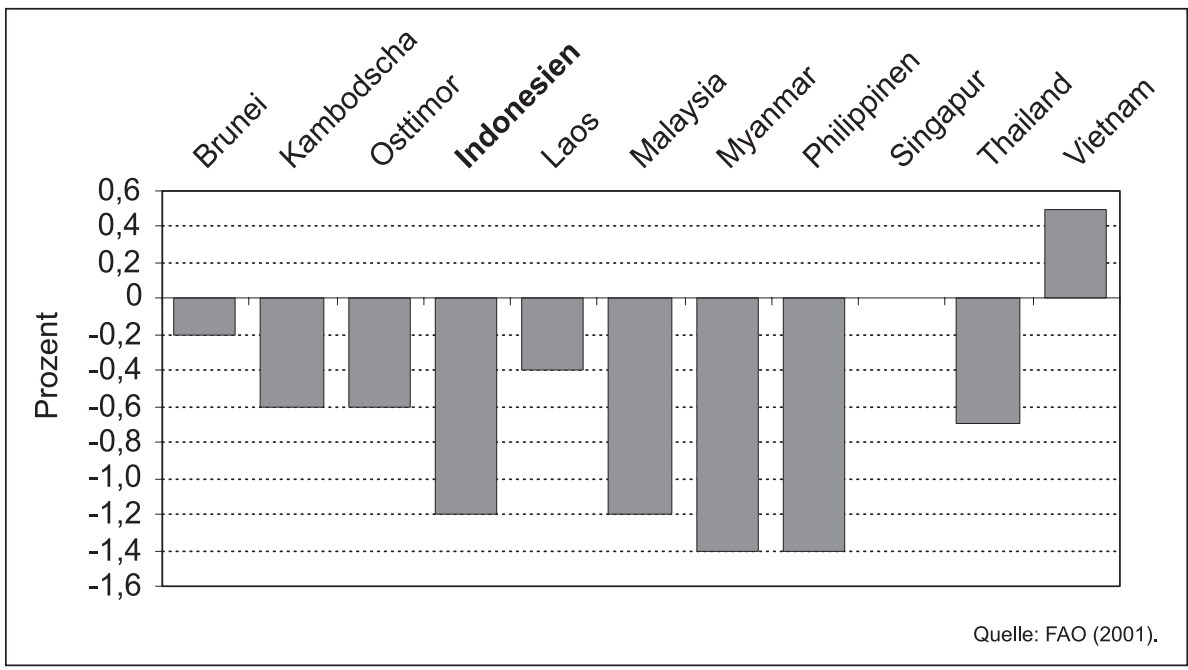

Abb. 3 Jährliche Abnahme des Waldbestandes südostasiatischer Staaten 1990 - 2000 im Verhältnis zum Gesamtbestand im Jahr 2000

Mit dem Rückgang der Waldreserven ist auch eine Dezimierung der in Interaktion mit dem Wald lebenden Tier- und Pflanzenpopulationen verbunden. Besonders für Naturwissenschaftler ${ }^{3}$, allen voran Biologen, ist die Untersuchung von Biodiversitäts-Hotspots wie der Lore-Lindu-Region daher von großer Bedeutung. Bestandsaufnahmen bisher noch nicht umfassend inventarisierter Pflanzen- und Tierarten liefern dabei neben der Erforschung von Tier-Pflanzen-Interaktionen wichtige wissenschaftliche Erkenntnisse im Bereich der Grundlagenforschung zu tropischen Regenwaldregionen. Ebenso sind Untersuchungen zu den Luft- und Wasserregimen sowie zur Bodenbeschaffenheit einer solchen Region von besonderer Wichtigkeit. Diese Forschungsthemen erzeugen bei flüchtiger Betrachtung den Anschein, man bewege sich ausschließlich inmitten der Anökumene, und es drängt sich die Frage nach dem Menschen in diesem Gefüge auf. Immerhin bevölkern zum Ende des 20. Jh. über 120.000 Menschen die Lore-Lindu-Region, welche einen bedeutenden Einfluss auf ihre Umwelt haben.

Aus diesem Grund bietet die Lore-Lindu-Region nicht nur aus naturwissenschaftlicher Perspektive ein äußerst interessantes Forschungsfeld. Der dynamische

3 Im Folgenden wird bzgl. der Verwendung weiblicher und männlicher Formen im Singular das Geschlecht unterschieden. Bei Pluralformen wird dagegen die männliche Schreibweise verwendet, welche jedoch die weibliche Form automatisch mit einschließt. Handelt sich im Plural ausschließlich um männliche oder weibliche Subjekte im grammatikalischen Sinn, wird die Form wiederum unterschieden. 
sozioökonomische und kulturelle Wandel in diesem Gebiet eröffnet ein ebenso weites, wie für eine Einschätzung naturwissenschaftlicher Erkenntnisse wichtiges Untersuchungsfeld. So stellt die Gründung des Lore-Lindu-Nationalparks selbst eine große Herausforderung für die Vereinbarung von Naturschutzinteressen auf der einen Seite und den Belangen der im Randbereich des Parks lebenden Menschen andererseits dar. Für die Generierung umfassender Lösungsansätze in diesem, wie auch in anderen aktuellen Problembereichen sind die Kenntnisse über die Entwicklung der Kulturlandschaft und ihre beeinflussenden Faktoren von großer Bedeutung. Neben naturgeographischen Gegebenheiten ist der Mensch durch sein Handeln der größte prägende Faktor für die Veränderungsprozesse einer bestimmten Region der Erde. Heute lebt eine Vielzahl unterschiedlicher Gruppen von Menschen in der Lore-Lindu-Region, welche ihre Umgebung prägen und tagtäglich verändern. Dies passiert auf individueller Ebene eines jeden Bewohners der Region ebenso wie auf der Ebene unterschiedlicher Gruppen. Veränderungen der Kulturlandschaft können sowohl durch interne, als auch externe Faktoren und Prozesse hervorgerufen werden.

Der zeitliche Untersuchungsrahmen dieser Arbeit erstreckt sich über das gesamte 20. Jahrhundert. Durch diesen Zeitrahmen sind die drei wichtigsten geschichtlichen Epochen abgedeckt: die letzten Jahre der vorkolonialen Periode bis 1904, die Kolonialzeit bis 1945 und die daran anschließende Ära der Unabhängigkeit Indonesiens. Somit ist gewährleistet, dass nicht nur aktuelle Veränderungsprozesse der Kulturlandschaft erfasst werden, sondern auch geschichtliche Voraussetzungen und Entwicklungen in die Analyse mit einfließen können. Ausgehend von der Annahme, dass beispielsweise zahlreiche Komponenten postkolonialer Gesellschaften in vielen Bereichen auf den Fundamenten kolonialzeitlicher Einflussnahme basieren (Kößler 1994: 85ff), aber auch, dass traditionelle, vorkoloniale Aspekte in Form von Kontinuitäten fortbestehen, ist historisches Wissen über eine Region für die Ausformung nachfolgender Prozesse unentbehrlich (vgl. Behrendt 1965). Der zeitliche Rahmen kann nicht immer strikt eingehalten werden. So werden die zeitlichen Eckpunkte, die Jahre 1900 und 2000, an manchen Stellen dieser Arbeit bei Bedarf auch überschritten.

Erste entscheidende Veränderungen der Kulturlandschaft ereigneten sich bereits Anfang des 20. Jh. mit der kolonialen Eroberung durch die Holländer. Nach der Unabhängigkeit Indonesiens setzte sich der Wandel fort und vollzog sich vor allem in den letzten zehn Jahren besonders rapide. Die Einrichtung des Lore-Lindu Nationalparks Anfang der 1990er Jahre mit seinem geschützten Waldreservat und die damit verbundene Verknappung von Landressourcen ließ diese Region zu einem äußerst sensiblen Kulturlandschaftsgebilde wandeln. Besonders die Migration auf regionalem und lokalem Maßstab führte in der Lore-Lindu-Region zu einem Mosaik unterschiedlichster Kulturlandschaftseinheiten auf relativ kleinem Raum.

Ziel dieser Arbeit ist es, die Veränderungen der Kulturlandschaft dieser Region seit Beginn der holländischen Unterwerfung zu analysieren. Es wird dabei unter-

\section{Pazifik Forum Bd. 12}


sucht, in welchen räumlichen und funktionalen Bereichen welche Faktoren auf Makro- (global und national), Meso- (regional), und Mikroebene (lokal) die Kulturlandschaft der Lore-Lindu-Region geprägt und zu einer Differenzierung des Kulturlandschaftsgefüges beigetragen haben. Hierzu werden die Bereiche Bevölkerung, Siedlungswesen, Landbesitz und -nutzung sowie Politik beleuchtet. Fallbeispiele auf regionaler und lokaler Ebene sollen einen tieferen Einblick in die Veränderungsprozesse geben und der vermuteten Komplexität der Wandlungsprozesse Rechnung tragen.

Die vorliegende Arbeit entstand im Rahmen des Sonderforschungsbereichs 552 - STORMA. Die Lore-Lindu-Region wurde daher nicht vom Verfasser dieser Arbeit persönlich ausgewählt, sondern war durch den regionalen Forschungsrahmen des SFB vorgegeben. Bereits während der Vorbereitungsphase dieses interdisziplinären, wissenschaftlichen Projekts hatte der Verfasser im Rahmen seiner Diplomarbeit den westlichen Teil Zentralsulawesis als Forschungsraum ausgewählt und die Auswirkungen der Kolonialzeit auf die Kulturlandschaft dieses Gebietes anhand von unveröffentlichten Archivquellen, sowie zeitgenössischer und aktueller Literatur untersucht (vgl. Weber 2000). Im Zuge der Beantragung des Sonderforschungsbereichs wurde von der DFG die besondere Bedeutung einer geographischen Untersuchung zur Kulturlandschaft der gesamten Lore-Lindu-Region als Grundlagenarbeit zur Interpretation der Forschungsergebnisse anderer sozialwissenschaftlicher, aber v.a. auch naturwissenschaftlicher Disziplinen innerhalb des SFB erkannt.

\subsection{Die Kulturlandschaft - nur ein soziokulturelles Konstrukt?}

Verbunden mit der Frage nach der soziokulturellen Konstruktion einer Kulturlandschaft ist unweigerlich auch die Frage, auf welcher Grundlage Begriffe wie „Ethnie“ und „Ethnizität“ basieren. Bezeichnete das Wort „Ethnie“" in der griechischen Antike alle nicht-hellenischen „Völker“, so wurde ab dem 18. Jh. mit der Einführung des Wissenschaftsbegriffs „Ethnologie“ unter Ethnien zunächst staatenlose, relativ kleine Gesellschaften außerhalb Europas verstanden. Mittlerweile gibt es eine Vielzahl an Definitionen zu den Begriffen „Ethnie“ und „Ethnizität“ (vgl. u.a. Balibar \& Wallerstein 1992; Banks 1996; Barth 1969; Le Vine 1997; Tilley 1997; Welz 1994; Wsevolod 1981).

Eine klare Aussage darüber zu treffen, was unter dem Begriff „Ethnie“ zu verstehen ist, fällt nicht leicht. Sie hängt stark von dem eigenen Standpunkt der definierenden Person ab. Zu einer wohl am weitesten verbreiteten Definition von „Ethnie“ gehört jene, die einer solchen Gruppe von Menschen bestimmte Charakteristika nachsagt, welche von den Gruppenmitgliedern geteilt werden. Dies können die Abstammung, die Geschichte, die Religion, die Sprache und eine oft nicht klarer bestimmte Kultur sein. Hierbei handelt es sich in der Regel um Fremdzuschreibun- 
gen, wie sie in ihrer extremsten Form unter Einbeziehung negativer, biologistischer Stereotype im Rassismus und Antisemitismus verwendet werden. Barth (1969) kritisiert die Annahme, dass Merkmale einer Ethnie rein objektiv bestimmt werden könnten. Vielmehr sei die Selbstzuschreibung der Gruppenmitglieder ausschlaggebend für die Definition einer Gruppe von Menschen als „Ethnie“.

Da der Begriff „Ethnizität“ als eine kollektive Identität bestimmt werden kann, welche von den Gruppenmitgliedern geteilt wird, lässt sich schließlich definieren, dass die „Ethnizität“ eine „Ethnie“ bestimmt. Hierbei ist es völlig unwichtig, ob sich alle Mitglieder dieser Gruppe objektiv durch eines der oben genannten Charakteristika auszeichnen. Entscheidend ist, dass alle Mitglieder daran glauben, dass ihre Gruppe das eine oder andere gemeinsame Charakteristikum verbindet. Allerdings kann die Selbstzuschreibung, welche oft auch in Abgrenzung zu anderen Gruppen erfolgen kann, nie von der Fremdzuschreibung getrennt werden. Kahn (1993: 180) beschreibt, dass der für eine Gruppe in Westsumatra mittlerweile feststehende Begriff „Minangkabau“ im 19. Jh. noch keine abgegrenzte, kulturelle Einheit darstellte. Dies erfolgte erst unter dem Einfluss der niederländischen Kolonialpräsenz (vgl. auch Kahn 1999). ${ }^{4}$ Ein Beispiel aus dem regionalen Kontext dieser Arbeit liefert die Gruppe der Toraja in Südsulawesi. Bevor diese Bergbevölkerung den Namen Toraja für sich selbst angenommen hatte, wurde sie von den Bewohnern des küstennahen Tieflandes abschätzig mit diesem nach Vrocklage als ,rückständiger Mensch“ (1936: 227) zu übersetzenden Begriff betitelt. ${ }^{5}$ Die Selbstwahrnehmung und -bezeichnung dieser Hochlandbevölkerung als Toraja begann ähnlich wie bei den Minangkabau mit der Einflussnahme des Kolonialismus. Hinzu kam in diesem Fall noch die christliche Missionierung, die den Toraja einen religiösen Sonderstatus im überwiegend muslimischen Südsulawesi einbrachte (vgl. Velthoen 2002). ${ }^{6}$

Generell ist „Ethnizität“ und damit die Bestimmung einer „Ethnie“ von einem ständigen Prozess wechselseitiger Selbst- und Fremdbestimmungen geprägt (Kohl 1998: 272). Da ein Individuum automatisch einer Vielzahl kultureller Gruppen angehört und sich meist nur schlecht in klare Merkmalsstrukturen pressen lässt, kann die Ethnie als generell konstruiert betrachtet werden. Dies bedeutet jedoch nicht, dass sich eine solche Ethnie nicht auch real verankern kann. Kohl merkt hierzu an:

„Auch wenn sie auf nichts anderem als erfundenen Traditionen beruben sollten, werden ethnische Gruppen, sobald sie sich erst einmal als solche ausgebildet baben, zu realen Größen, zu geschichtswirksamen Faktoren."(1998: 284)

4 Zur Konstruktion von Ethnien und Ethnizität vgl. u.a. Banks (1996) und Kaschuba (1999).

5 Mit dieser Übersetzung des Begriffs „Toraja“ übernahm Vrocklage gleichermaßen die negative Konnotation, wie sie von den Tieflandbewohnern produziert worden war.

6 Für weitere Erläuterungen zum Terminus „Toraja“vgl. Fußnote 31. 
Ethnien bilden sich jedoch nicht nur zu geschichtswirksamen, sondern auch zu raumwirksamen Größen aus, da der Definition einer Ethnie meist auch eine räumliche Begrenzung zugrunde gelegt wird. Dieser räumliche Bezug kann in administrativen Grenzen verankert sein (z.B. bei der Definition eines Staatsvolkes), aber auch in fiktiven Grenzen, welche zwischen den Mitgliedern einer Ethnie je nach ihrer jeweiligen mental map variieren können. In Bezug auf die Nation bringt Anderson den fiktiven, konstruierten Charakter solcher Einheiten folgendermaßen auf den Punkt:

„It [die Nation; Anm. d. Verf.] is imagined because the members of even the smallest nations will never know most of their fellow-members, meet them, or even hear of them, yet in minds of each lives the image of their communion. "(1983: 6).

Wie eine Ethnie kann auch eine Kulturlandschaft als ein Konstrukt verstanden werden. Dennoch wäre es falsch davon zu sprechen, dass Kulturlandschaft nur noch als ein virtueller Raum gesehen werden darf. Vielmehr muss man zwischen einer physisch-realen Kulturlandschaft und einer soziokulturell konstruierten Kulturlandschaft unterscheiden. Erstere stellt die im klassischen Sinne durch den Menschen umgewandelte Naturlandschaft dar (Sauer 1963: 341). Dies können zum Beispiel Siedlungen, Strassen, Agrarflächen oder anthropogen angelegte Wälder sein, kurz: „das Werk des Menschen im Rahmen dessen, was die Natur erlaubt." (Schwind 1951: 19). Bei der Umformung von Naturlandschaft in Kulturlandschaft sei hierbei zu beachten, dass:

„[...] the cultural landscape is fashioned from a natural landscape by a culture group. Culture is the agent, the natural area is the medium, and the cultural landscape the result. The natural landscape is very important for it supplies the materials out of which the cultural landscape is formed. The shaping force, however, lies in the culture itself" (Sauer 1963: 343)

Kultur nimmt in der von Sauer an die Kulturlandschaftsidee Schlüters (1906, 1928) angelehnten Konzeption allerdings den Charakter eines superorganischen Akteurs an.

Während sich Geographen wie Zelinsky (1973) oder Ethnologen wie Steward (1955) mit seiner Theorie der Cultural Ecology noch bis weit in die zweite Hälfte des 20. Jh. auf das Konzept Carl Sauers bezogen, vollzog sich ab den 1980er Jahren eine Wende in der Betrachtung von Kultur und Kulturlandschaft. Kultur wurde nun als Produkt der sozialen Zusammenhänge einer Gesellschaft und seiner Individuen gesehen (Cosgrove et al. 1987: 95), ,socially produced through myriad struggles over and in space, scales and landscapes" (Mitchell 2000: xvi). Im Gegensatz zu klassischen Ansätzen zur Kulturlandschaft, welche die physischen Gegebenheiten als Container für die Gesellschaft sehen, erkennt Jackson (1989) die Landschaft als eine Struktur, die von der Gesellschaft selbst erschaffen wird. Begründet wird dies damit, dass sich menschliche Ideen in Verhaltensweisen äußern, welche anschließend die Kulturlandschaft entstehen lassen. Im Umkehrschluss reflektiert die Kulturlandschaft soziale Beziehungen und Institutionen und formt diese Beziehungen, weshalb Jackson (1989) von einem sozialräumlich dialektischen Modell spricht. Ein Beispiel für diese 
neue Wechselbeziehung stellen Cosgroves (1984) Untersuchungen zu der Entwicklung klassischer Architekturformen und Landschaftsparks als Ausdruck der Veränderungen des kapitalistischen Systems und seiner sozialen Beziehungen dar. Erweitert wurde dieses Modell durch Werlens (1993) „Theorie der Alltagskultur“, welche die Vielzahl unterschiedlicher kultureller Welten und Lebensstile auf kleinem Raum betont (Werlen et al. 1995). Weitere aktuelle Arbeiten im Bereich der Kulturgeographie, wie jene von Crang (1998), Mitchell (2000), Norton (2000), Peet (1998) und Schurmer-Smith (2002), weisen deutlich den Weg dieser Neubetrachtung von Kultur und Kulturlandschaft im Zuge des sog. Cultural Turn. ${ }^{7}$

Wie bereits angedeutet, ist eine solche Kulturlandschaft nicht starr, sondern einem ständigen Wandel durch das Handeln des Menschen unterworfen (Kreisel et al. 2004: 40; vgl. auch Ewald 1996: 99 und Konold 1996: 121ff; Scherer-Hall 1996: 24; Terkenli 2005: 167). Analog zu der Erklärung des Begriffs „Kultur“ als „a dynamic mix of symbols, beliefs, languages and practices that people create, not a fixed thing or entity governing humans" (Anderson et al. 1992: 3) ist die Kulturlandschaft daher als ein vom Menschen geschaffenes, dynamisches Kontinuum zu verstehen, „always in the nature of ,work in progess" (Ingold 1993: 162).

Die Untersuchung des Kulturlandschaftswandels ist auch mit der Frage verbunden, in wieweit sich Akkulturations- oder sogar Assimilationsprozesse abzeichnen. Akkulturation kann nach Herskovits (1958: 14) entstehen, wenn eine Gruppe von Menschen dem längeren Einfluss einer anderen Gruppe ausgesetzt ist, wobei sich die beiden Gruppen in bestimmten kulturellen Prägungselementen unterscheiden. Dabei lässt Herskovits (1967: 180) sowohl die Möglichkeit eines Wandels unter Druck, als auch die eines Akkulturationsprozesses auf freiwilliger Basis gelten, während Naylor (1996: 50f) nur den unfreiwilligen Wandel als Akkulturation definiert. Akkulturationsprozesse bedeuten nicht zwingend eine Annäherung einer Minderheitengruppe an die Kultur einer Bevölkerungsmajorität. Es kann ebenso ein Akkulturationsprozess in die entgegengesetzte Richtung erfolgen. Die durch Akkulturation hervorgebrachten Veränderungen der Kulturlandschaft können sich sowohl in ihrer physisch-realen, als auch in ihrer soziokulturell konstruierten Version niederschlagen.

Die Kulturlandschaft ist zum einen also die im Raum verortete Veränderung der Naturlandschaft durch das Handeln des Menschen. Sie ist etwas reales, etwas sichtbares. Darüber hinaus definiert der Mensch seine Umgebung aber auch anhand seiner eigenen soziokulturellen Prägungen, die den im Bourdieuschen Sinne durch

7 Ein guter Überblick über die Theorieentwicklung innerhalb der Geographie und den Cultural Turn ist u.a. bei Hubbard et al. (2002) zusammengestellt (vgl. u.a. auch Barnett (1998), Bodman

(1991, 1995) und Cosgrove \& Jackson (1987)) 
die Sozialisation erworbenen „Habitus“ (1976: 165) ausmachen. ${ }^{8}$ Somit entsteht eine zweite, eine konstruierte Kulturlandschaft. ${ }^{9}$ Diese konstruierte Kulturlandschaft ist, im Gegensatz zur physisch-realen, das Konglomerat der zugrunde liegenden kulturellen Prägungen jener Menschen, welche die reale Kulturlandschaft bewohnen. Unter kultureller Prägung ist dabei all das zu subsumieren, was das einzelne Individuum und sein Handeln ausmacht, seien es bestimmte „Traditionen“ 10, Ethnizität, soziale, politische oder ökonomische Werte und Fähigkeiten und vieles mehr. Greider \& Garkovich sehen soziokulturell konstruierte Landschaften als Produkte einer Selbstreflexion:

„Cultural groups socially construct landscapes as reflections of themselves. In the process, the social, cultural and natural environments are meshed and become part of the shared symbols and believes of members of the groups. Thus, the natural environment and changes in it take on different meanings depending on the social and cultural symbols affiliated with it." (1994: 8).

Wie Head (2000: 49) richtig anmerkt, werden in den Humanwissenschaften Kernbegriffe wie Kultur oder Landschaft mittlerweile nicht mehr als gegebene Realitäten angesehen, sondern als durch soziale Prozesse konstruierte Kategorien mit vielfältigen und wechselnden Bedeutungen" ${ }^{11}$. Somit kann "landscape as cultural product" (Robertson \& Richards 2003: 2) und ,landscape as cultural process" (ebd.: 6) verstanden werden. Die konstruierte Kulturlandschaft findet sich als verortetes Resultat in der physisch-realen Kulturlandschaft wieder. Ein Beispiel hierfür wäre die Errichtung von Handelsplätzen durch Menschen, deren konstruierte Kulturlandschaft von einer Geschäftstüchtigkeit und den nötigen Marktkenntnissen geprägt ist. Auch der Bau von religiösen Versammlungsstätten (im konkreten Fall der Lore-Lindu-Region etwa Kirchen und Moscheen) manifestiert die jeweilige religiös geprägte, mentale Kulturlandschaft als für jeden sichtbares Zeichen. Die oben genannte Neuausrichtung in der Kulturgeographie ist auch in Hinsicht auf die Untersuchung konstruierter Kulturlandschaften wegweisend.

Anhand der genannten Beispiele offenbart sich zudem, dass beide Kulturlandschaften, die konstruierte wie die reale, räumlich nicht klar abgegrenzt sind, weder voneinander, noch in sich selbst. Durch endogene oder exogene Einflüsse kann aus

8 Zu einer kritischen Betrachtung des Habitus-Begriffs bei Bourdieu in bezug auf Kultur vgl. Acciaioli (1981: 36f).

9 Vgl. dazu auch Norton (1989), der zwischen „visible landscapes“ und „symbolic landscapes“ (ebd.: 4) unterscheidet.

10 Der Traditionsbegriff ist schwierig zu fassen und daher in Anführungszeichen gesetzt. Zu verstehen ist darunter an dieser Stelle ein gewonnenes Bewusstsein über individuelle oder gruppenspezifische Regelmäßigkeiten im menschlichen Handeln. Oft ist den betreffenden Personen die Entstehung mancher ihrer Traditionen nicht bekannt. Durch Erklärungen wie: „Das ist bei uns so Tradition." wird diesen jedoch ein quasi-gesetzmäßiger Charakter verliehen.

11 Vgl. auch die bisherigen Ausführungen zur realen Kulturlandschaft in diesem Kapitel. 
einer realen Kulturlandschaft des Reisanbaus die des Kakaoanbaus werden, deren räumliche Grenzen sich hierdurch verschieben können. Konnte man vor Beginn dieser Einflüsse etwa eine Region A, geprägt von dem kulturlandschaftlichen Element Reisanbau, von einer durch den Kakaoanbau geprägten Region B unterscheiden, wird danach die Region A - zumindest was das Element Landnutzung anbelangt - räumlich mit der Region B verbunden. Die räumlichen Grenzen einer realen Kulturlandschaft haben sich somit verschoben. Ebenso muss eine bestimmte konstruierte Kulturlandschaft nicht in den Köpfen aller Mitglieder einer Gruppe von Menschen dieselbe interne oder externe Abgrenzung implizieren. Nimmt man hierzu das Beispiel der konstruierten Kulturlandschaft in bezug auf die Bewohner Bayerns, ergeben sich eine Vielzahl von Ausprägungen dieses Konstrukts. Für den einen mag mit Bayern eine nostalgische Rückschau auf die ehemalige Monarchie unter Ludwig II. verbunden sein. Für die andere mögen Begriffe wie Gemütlichkeit, Katholizismus und die liberalitas Bavariae die prägende Basis sein. Für einen dritten setzt sich diese konstruierte Kulturlandschaft aus Elementen wie Amigo-System, Provinzialität und Konservatismus zusammen. Wie bereits erwähnt stellen auch diese drei Beispiele konstruierter Kulturlandschaft keine Konstanten dar, sondern können - sie müssen es allerdings nicht zwingend - einen dynamischen Charakter annehmen.

Die Frage im Titel dieses Kapitels nach der rein sozialen Konstruktion von Kulturlandschaft lässt sich folglich mit einem Nein beantworten. Allerdings, so haben es die o.g. Ausführungen gezeigt, spielt die sozial konstruierte Kulturlandschaft eine entscheidende Rolle bei der Ausprägung der physisch-realen Kulturlandschaft. Beide lassen sich, im Gegensatz zu den Andeutungen von Muir (1998), nicht voneinander trennen. Die physisch-reale Kulturlandschaft stellt nicht nur ein Spiegelbild soziokulturell geprägter, konstruierter Kulturlandschaften dar, sondern ,needs to be understood as enmeshed within the processes which shape how the world is organized, experienced and understood, rather than read as its end product" (Seymour 2000: 214). Jones et al. (1997: 280) beschreiben die Bedeutung der Kulturlandschaft in einer gerade für die in dieser Arbeit untersuchten Region im Randbereich eines Nationalparks sehr treffenden Weise:

„,[...] the cultural landscape provides an arena in which different interest groups struggle to influence the formation of our physical surroundings, exemplified in the conflicts that often arise between the production of economic goods and the production of environmental goods."

Die in diesem Zitat genannten Konflikte um wirtschaftliche und Umweltgüter nehmen in der Lore-Lindu-Region einen besonderen Stellenwert ein, in dessen Mittelpunkt der Lore-Lindu Nationalpark als ein durch seine Grenzziehung entstandenes Kulturlandschaftselement steht. Um ihn gruppieren sich die räumlich und inhaltlich unterschiedlich ausgeformten physisch-realen und soziokulturell konstruierten Kulturlandschaften. Diese werden in ihrer Komplexität und Vielfältigkeit erst sichtbar, wenn man sie auf Ebenen unterschiedlicher Betrachtungsdichte in räumlicher und zeitlicher Perspektive analysiert. Zwar liegt der Schwerpunkt dieser 
Arbeit auf der Untersuchung des Wandels der physisch-realen Kulturlandschaft(en), angesichts der oben genannten Erklärungen lässt sich dieser Wandel jedoch nicht völlig losgelöst von den Wahrnehmungen der Umwelt durch die, sowie von der soziokulturellen Bedeutung für die dort lebenden Menschen analysieren.

\subsection{Forschungsbedarf und Hypothesen}

Die vorliegende Arbeit ist im Bereich der Grundlagenforschung anzusiedeln, da eine umfassende, kulturgeographische Erfassung des Wandels in der Lore-Lindu-Region für den gesamten Zeitraum des 20. Jh. bislang noch nicht existiert. Zu Zentralsulawesi gibt es mittlerweile zahlreiche Arbeiten, welche neben dem empirischen Datenmaterial als Informationsgrundlage herangezogen werden können.

Zur Kolonialzeit in dieser Provinz liegen diverse ethnographische Beschreibungen zeitgenössischer Autoren vor. Besonders hervorzuheben sind hier die beiden niederländischen Missionare Albert Christiaan Kruyt und Nicolaus Adriani. Kruyt wurde im Jahr 1891 als erster Missionar von dem damaligen Assistent-Resident von Gorontalo zur Mündung des Poso-Flusses begleitet, um dort mit seiner Missionstätigkeit zu beginnen. Enttäuscht von der mangelnden Bereitschaft der lokalen Bevölkerung, sich bekehren zu lassen, begrüßte er die spätere Erschließung des Gebietes durch die niederländische Kolonialverwaltung (Coté 1979:36). Der an Sprachen interessierte Adriani wurde 1895 von der Niederländischen Bibel-Gesellschaft nach Poso geschickt, um eine Ausgabe der Bibel in der lokalen Bare'e-Sprache zu verfassen. Im Gegensatz zu Kruyt war er jedoch kein großer Freund der niederländischen Kolonialpolitik. Die landschaftskundlichen und ethnographischen Ergebnisse ihrer Reisen im östlichen und westlichen Zentralsulawesi schrieben die beiden Missionare in zahlreichen Veröffentlichungen nieder, welche eine unschätzbare Informationsquelle für Nachforschungen in diesen Regionen darstellen (vgl. u.a. Adriani 1913, 1915; Adriani \& Kruyt 1898, 1912-1914; Hissink 1912; Kruyt 1903a, 1903b, 1906, 1908, 1909, 1926, 1929, 1935, 1938; Kruyt \& Kruyt 1921; o.A. 1912; Schuyt 1911). Die Autoren, welche Zentralsulawesi bereits weit vor der niederländischen Kolonisierung der Lore-Lindu-Region (ab 1904) besuchten, hielten sich nur in Palu auf und erwähnen darüber hinaus höchstens noch einen kurzen Eindruck vom nördlichen Palu-Tal (vgl. Bastian 1889; Hart 1853; Valentijn 1724-1726; Wichmann 1890). Zeitgenössische Monographien und Aufsätze zum kulturlandschaftlichen Status quo in der Untersuchungsregion während der niederländischen Kolonialzeit wurden von Grubauer (1913, 1923), Kaudern (1925a, 1925b, 1941), Kornrumpf (1935) und Vrocklage (1936) verfasst.

Umfangreiche Informationen über die kolonialzeitliche Entwicklung der Untersuchungsregion sind auch in den sog. Memories van Overgave enthalten, welche vom Verfasser dieser Arbeit bereits für den Westteil der Lore-Lindu-Region ausgewertet wurden (vgl. Weber 2000a, 2000b; Weber et al. 2003). In der zweiten Hälfte des 19. Jh. mussten die Kolonialstellen in den Niederlanden feststellen, dass sie immer 
schlechter über die Situation und die Entwicklungen in den Kolonialgebieten des indonesischen Archipels informiert wurden (Boomgard 1991: 24). Dies konnte nicht an der technischen Seite der Kommunikation zwischen Mutterland und Kolonie gelegen haben, da die Entwicklung der Seefahrt (z.B. Dampfschiffe, Öffnung des Suez-Kanals 1869) eher eine Beschleunigung des Austausches mit sich brachte. Vielmehr war das Problem bei der Kolonialregierung in Batavia zu suchen, die dazu überging, ohne Berücksichtigung der Interessen der Niederlande zu regieren (vgl. Cribb 1994). Die Kolonisatoren dachten nur noch an die Wahrung ihrer eigenen finanziellen Interessen. Um dieser Entwicklung einer Verselbständigung Niederländisch-Indiens entgegenzuwirken, erließ das Kolonialministerium 1867 eine Anweisung, wonach von den Kolonialverwaltern regelmäßig Protokolle über den Stand der Dinge in der Kolonie nach Den Haag zu schicken waren. ${ }^{12}$ Zwei Jahre später wurde dieses neue Regierungsreglement umgesetzt. Es wurden sog. Mailrapporten von Batavia nach Den Haag geschickt, welche in ein wichtiges Medium der Quellenforschung eingingen. Dieses Medium waren die Memories van Overgave, die Übergabeprotokolle der aus ihrem Amt scheidenden Gouverneure, Residenten und Kontrolleure der einzelnen Verwaltungsgebiete des Archipels. Mit Hilfe dieser Memories wurde Den Haag über Zustand und Veränderungen u.a. in den Bereichen Infrastruktur, Siedlungswesen, Bevölkerung, Wirtschaft, Handel und Missionierung in den entsprechenden Regionen auf dem laufenden gehalten. Heute befinden sich die Memories van Overgave im Nationaalarchief in Den Haag. Sie erstrecken sich für den gesamten indonesischen Archipel über die Zeit von 1849 bis 1962. Dabei wird jedoch nicht für alle Teilregionen der komplette Zeitraum abgedeckt. Im Fall des Untersuchungsgebiets der vorliegenden Arbeit ist dies in bezug auf den Beginn der Erfassungsperiode nachvollziehbar, da die Niederländer dort erst ab 1904 verwalterisch tätig wurden. In manchen Fällen ist die Schuld aber bei den Verfassern der Protokolle selbst zu suchen. In einigen der ausgewerteten Memories wird im Vorwort auf die unzureichende Umsetzung der Protokollvorschriften durch die Verfasser der Vorgänger-Protokolle hingewiesen (vgl. Geuns 1906; Logeman 1922). Andere erklären, dass ihr Amtsvorgänger vorzeitig abberufen wurde und/oder kein Memorie hinterlassen hatte (vgl. Van Rhijn 1941; Vorstman 1935). Zudem sind einige der in den Protokollen erwähnten Vorgängerprotokolle nicht im Nationaalarchief vorhanden. ${ }^{13}$

12 Soweit nicht anders belegt, beziehen sich die folgenden Informationen auf das Einleitungskapitel zum Findbuch der Memories van Overgave im Nationaalarchief in Den Haag.

13 Das Nationaalarchief in Den Haag hat die Memories van Overgave nach folgenden Kriterien geordnet: Es besteht eine sog. MMK-Sammlung sowie eine sog. KIT-Sammlung. MMK steht für das Ministerie van Kolonien in Den Haag, KIT für das Koninklijke Institunt voor de Tropen in Amsterdam. Dies gibt die Orte an, an welchen die Memories während der Kolonialzeit gesammelt wurden. Die Protokolle für das Kolonialministerium wurden von den Gouverneuren bzw. Residenten als höchsten Amtsinhabern der einzelnen Verwaltungsgebiete verfaßt. Im KIT wurden v.a. ergänzende Protokolle gesammelt, die von Kontrolleuren oder militärischen Befehlshabern der Afdeelingen, Onderafdeelingen 
Neben diesen zeitgenössischen Quellen finden sich aber auch aktuellere Arbeiten die sich, zumindest in Teilen, mit der Kolonialzeit in Zentralsulawesi befassen. Allerdings behandeln diese Arbeiten nur Teilaspekte der Kulturlandschaftsentwicklung und decken dabei meist nur Teilregionen des Untersuchungsgebietes ab. Der Ethnologe Acciaioli (1987, 1989) gibt zwar einen Überblick der kolonialen Einflussnahme in Zentralsulawesi wieder, im Kern konzentrieren sich seine Werke jedoch auf die Region um den Lindu See. Aragon fasst die Ergebnisse ihres Feldaufenthalts in Tobaku (Pipikoro-Region, Kulawi) zusammen und beschäftigt sich vor allem mit religiösen Aspekten $(1992,2000 \mathrm{~b})$ und dem niederländischen Einfluss auf die lokalen Tauschbeziehungen (1996d). Mit ihrem Aufsatz über die kurze japanische Kolonialherrschaft über Zentralsulawesi (1996a) leistet Aragon zudem einen sehr hilfreichen Beitrag zur Aufarbeitung jenes für die Untersuchungsregion kaum dokumentierten Zeitraums. Kotilainen (1992) widmet sich in ihrer Arbeit insbesondere der materiellen Kultur. Eine sehr detaillierte Recherche zur Demographie Nord- und Zentralsulawesis ist bei dem Geographen Henley (2004) zu finden. Allerdings nehmen die Kapitel zu Zentralsulawesi, insbesondere zur Lore-Lindu-Region, in seiner Monographie nur einen geringen Raum ein. Diese Arbeit dient jedoch als ein guter Beleg für die vergleichsweise unbefriedigende Quellenlage bzgl. der in der vorliegenden Arbeit behandelten Region. Gestützt wird dieser Beleg auch von der Aufarbeitung der demographischen Geschichte Indonesiens bis 1942 durch Gooszen (1999), der für Zentralsulawesi nur teilweise Daten vorlegen kann, welche an mancher Stelle auch nur Schätzwerte darstellen. Interessante Informationen zur sog. Ethischen Politik der Niederländer ab 1901 (vgl. auch Kap. 2.3.3 und 6.1) enthalten die Schriften von Coté (1979, 1996). Regional liegt dessen Schwerpunkt aber in der Region der Pamona, welche sich östlich des Untersuchungsgebiets befindet. Auf die gleiche Region konzentriert sich die Arbeit des Ethnologen Schrauwers (2000a).

Auch die Mehrzahl der aktuelleren Arbeiten von Autoren mit sozialwissenschaftlichem Disziplinhintergrund, welche sich nicht bzw. nicht nur mit der Kolonialzeit in Zentralsulawesi beschäftigen, haben nicht die gesamte Lore-Lindu-Region als Untersuchungsbasis. Schrauwers (1998, 1999, 2000a, 2000b) bezieht sich in seinen Untersuchungen weiterhin auf die Poso-Region. Acciaioli (1985, 1987, 1989, 1999, 2000, 2001) bleibt ebenfalls hauptsächlich auf seine Feldforschungsregion des Lindu Sees fixiert, während Aragon die Religion und traditionelle Kulturelemente behandelt (1996b, 1996c, 2003). Ferner hat sie sich einer Analyse des mit religiöser Symbolik aufgeladenen Poso-Konflikts angenommen (vgl. Aragon 2000a, 2001, 2002). Die Arbeiten von Li (1999, 2001a, 2001b, 2002a, 2002b, 2002c) behandeln unterschiedliche, v.a. ethnologisch basierte Aspekte mit Bezug zu Zentralsulawesi.

oder Landschappen (von den Niederländern eingeführte Verwaltungseinheiten im Kolonialgebiet) dieser Regionen verfaßt worden waren. 
Im Rahmen des SFB 552 - STORMA entstanden Arbeiten, die sich dezidiert mit der Lore-Lindu-Region befassen, doch auch hier decken die meisten Werke nur Teilräume des Untersuchungsgebiets ab (vgl. Abdulkadir-Sunito 2004; Burkard 2002a, 2002b; Faust \& Chairil 2002; Fremerey 2002; Hoppe 2003; Hoppe \& Faust 2004; Keil et al. 2003; Savitri 2004; Sitorus 2002a, 2002b; Sunito 2004; Sutarto 2003; Weber 2000a, 2000b, 2003; Weber et al. 2003). Auch unter den Veröffentlichungen, die auf der Basis der Forschungsarbeiten im SFB 552 zustande kamen, behandeln bislang nur einige die gesamte Lore-Lindu-Region und dies vorwiegend unter ökonomischen Gesichtspunkten (vgl. Alene et al. 2005; Birner et al. 2002; Faust et al. 2003; Maertens 2003; Maertens et al. 2002, 2004; Mappatoba 2004; Schwarze 2004; Schwarze et al. 2005; Van Rheenen et al. 2004). Bezogen auf die historische Kulturlandschaftsentwicklung dieses Gebietes existiert derzeit nur der Aufsatz von Kreisel et al. (2004), welcher einige vorläufige Ergebnisse der Feldforschung des Verfassers der vorliegenden Arbeit zusammenfasst.

Der aktuelle Stand der Literatur zeigt, dass eine kulturgeographische Arbeit, welche nicht nur den derzeitigen Status quo der Kulturlandschaftsentwicklung widerspiegelt, sondern auch deren Veränderungsprozesse während des gesamten 20. Jh. beleuchtet, bislang noch aussteht. Die vorliegende Arbeit hat nicht nur den Anspruch, allgemeine Aussagen über die gesamte Untersuchungsregion zu machen oder sich in der detaillierten Behandlung räumlich begrenzter Fallbeispiele zu verlieren. Vielmehr sollen beide Ebenen miteinander „,verschmolzen“ werden. Dadurch wird vermieden, dass - um eine Redewendung zu bemühen - man weder den Wald vor lauter Bäumen aus dem Blick verliert, noch den einzelnen Baum inmitten des dichten Waldes übersieht.

Die vorliegende Arbeit geht von zwei Arbeitshypothesen aus. Erstens wird angenommen, dass die Kulturlandschaft der Lore-Lindu-Region durch ein mehrdimensionales Geflecht von Veränderungsprozessen geprägt ist. Dieses Geflecht zeichnet sich durch eine vertikale sowie eine horizontale Ebene aus. Vertikal sind die einflussnehmenden Faktoren der Makro- (global und national), Meso- (regional) und Mikroebene (lokal) zu unterscheiden und in ihrer Bedeutung für die Veränderungen in der Kulturlandschaft miteinander zu vergleichen. Horizontal gilt das Augenmerk dieser Untersuchung den räumlichen Unterschieden und Gemeinsamkeiten, aber auch der Bedeutung der verschiedenen Bevölkerungsgruppen als wirkungsaktive Größen.

Darauf aufbauend wird zweitens behauptet, dass keine über die Gesamtregion homogene Ausprägung der Kulturlandschaft abgeleitet werden kann. Zwar sind möglicherweise einige herausragende Phänomene in der gesamten Untersuchungsregion gleichermaßen zu beobachten. Diese könnten jedoch zu voreiligen Schlussfolgerungen verleiten, welche der angenommenen Heterogenität des untersuchten Raumes nicht gerecht würden. 


\subsection{Forschungsverlauf und -methodik}

Um die in den Arbeitshypothesen vermutete Heterogenität der Kulturlandschaftsentwicklung in der Lore-Lindu-Region analysieren zu können, ist eine rein quantitativ angelegte Datenerhebung nicht ausreichend. Die Grundlage der vorliegenden Arbeit bildet daher das qualitativ erhobene Datenmaterial aus einer vom Verfasser durchgeführten Feldforschung in der Lore-Lindu-Region Zentralsulawesis. Diese Feldforschung war integrierter Bestandteil des Sonderforschungsbereichs 552 - STORMA „Stability of Rainforest Margins in Indonesia“. Der SFB 552 ist ein wissenschaftliches Kooperationsprojekt zwischen zwei indonesischen und zwei deutschen Universitäten. Von deutscher Seite aus sind die Georg-August-Universität Göttingen und die Universität Gesamthochschule Kassel beteiligt. Das Institut Pertanian Bogor und die Universitas Tadulako in Palu nehmen als indonesische Partnerinstitutionen an diesem Sonderforschungsbereich teil.

Im Rahmen der ersten Förderungsphase des SFB (Juli 2000 - Juni 2003) hat der Verfasser als Mitarbeiter des von Prof. Dr. Werner Kreisel und PD Dr. Heiko Faust (Geographisches Institut, Abteilung Kultur- und Sozialgeographie, Georg-AugustUniversität Göttingen) geleiteten Teilprojekts A1 „Regionalanalyse der Kulturlandschaft und ihrer Veränderungsprozesse" qualitative und quantitative Daten in der oben genannten Region gesammelt. Hierfür besuchte der Verfasser die indonesische Provinz Zentralsulawesi zwischen November 2000 und Oktober 2002 für insgesamt 17 Monate. Ein weiterer sechswöchiger Aufenthalt im Jahr 2003 diente der Komplettierung des Datenmaterials, sowie der Präsentation erster Forschungsergebnisse in den Untersuchungsdörfern. Zwei zusätzliche Forschungsaufenthalte (insgesamt fünf Monate) im Jahr 2004 nutzte der Verfasser für weitere Validierungen der Analyseergebnisse.

Der Schwerpunkt der Feldforschung lag auf einer qualitativen Erhebung in neun ausgewählten Dörfern. Als Grundlage für die Fallstudien dieser neun Dörfern diente das sog. STORMA village sample (vgl. Zeller et al. 2002). Die 12 Dörfer dieses Samples wurden anhand dreier Kriterien, nämlich Bevölkerungsdichte, ethnische Komposition und Nähe zum Lore-Lindu Nationalpark, durch eine geschichtete Zufallsauswahl aus der Gesamtzahl der vorhanden Dörfer der Untersuchungsregion ermittelt (vgl. auch Schwarze 2004: 21f). Die Dörfer befinden sich in vier verschiedenen Kecamatan ${ }^{14}$, welche wiederum zwei Kabupaten ${ }^{15}$ der Provinz Sulawesi Tengah (Zentralsulawesi) zugeordnet sind. Die genaue administrative Einordnung der Un-

14 Der indonesische Begriff „Kecamatan“ kann in etwa mit der deutschen Verwaltungseinheit des Landkreises verglichen werden. Repräsentant eines Kecamatan ist der Camat, vergleichbar mit einem Landrat in Deutschland.

15 Analog zum Begriff des Kecamatan ist ein indonesisches Kabupaten mit einem deutschen Regierungsbezirk zu vergleichen. Einem Kabupaten steht der Bupati vor. 
tersuchungsdörfer ist in Tab. 1 in Kap. 2.1 aufgeführt (vgl. auch Abb. 5). Eine erste grobe Charakterisierung dieser Dörfer erfolgt in Kap. 2.4.

Die Datenerhebung war mit einem mehrwöchigen Aufenthalt in jedem der neun Dörfer verbunden. Die durchgängigen Aufenthalte im Dorf betrugen eine bis zwei Wochen bei einer maximalen Aufenthaltsdauer von bis zu sechs Wochen pro Dorf. Der Verfasser wohnte während der Aufenthalte im Dorf bei Gastfamilien, entweder bei der des Dorfbürgermeisters (kepala desa) oder bei einer von diesem vermittelten Familie. Die längeren Verweildauern und die Unterbringung bei örtlichen Familien erlaubten zum einen, die Distanz zwischen Forscher und Bevölkerung zu minimieren und das für die Untersuchung benötigte Vertrauen der Menschen in den Dörfern zu gewinnen. Zum anderen konnten zusätzliche Informationen im Rahmen einer teilnehmenden Beobachtung zusammengetragen werden. ${ }^{16}$ Es zeigte sich, dass derartige, informell erhaltene Daten ein wichtiges Korrektiv für die aus formellen Interviews gewonnenen Erkenntnisse darstellt. Generell sollte nicht unerwähnt bleiben, dass Mitglieder unterschiedlicher Gesellschaften die Beziehungen zwischen Mensch und Mensch, sowie zwischen Mensch und Umwelt unter verschiedenen konzeptionellen Betrachtungsweisen wahrnehmen (vgl. Cronon 1996; Evernden 1992; Merchant 1980; Simmons 1993). Hierbei sei darauf hingewiesen, dass die partizipative, informelle Datenerhebung, wie auch die Informationen aus formellen Interviews, selbstverständlich keinen Anspruch auf absolute Korrektheit zulassen. Sie sind besonders davon abhängig, wie der Wissenschaftler und sein Forschungsvorhaben von den Informanten wahrgenommen werden. ${ }^{17}$

Die qualitative Datenerhebung wurde mittels halbstrukturierter Leitfadeninterviews durchgeführt (vgl. Abb. 4), welche im Fall ,gewöhnlicher" Haushalte biographisch angelegt waren und zudem auf die Erfassung der Wahrnehmung der Befragten im Hinblick auf die kulturlandschaftlichen Veränderungen im jeweiligen Dorf sowie in der entsprechenden Region (z.B. dem Kecamatan) abzielten. Handelte es sich um Haushalte, deren Familienvorstand zugleich ein Amtsträger im Dorf ist (z.B. Bürgermeister oder Dorfteilrepräsentant), wurden zusätzlich Daten

16 Zur Methodik der teilnehmenden Beobachten vgl. u.a. Jorgensen (1989). Die teilnehmende Beobachtung im Rahmen dieser Forschungsarbeit beinhaltete die Teilnahme an diversen Zeremonien, an Teilen des Alltagsleben der Gastfamilien, sowie an geselligen Treffen der Dorfbevölkerung. Die für eine qualitative Untersuchung relativ kurze Verweildauer in den jeweiligen Dörfern erlaubte allerdings keine umfassende teilnehmende Beobachtung, wie sie in der klassischen Ethnologie Verwendung findet.

17 Wird beispielsweise trotz einer entsprechenden Klarstellung angenommen, dass der Wissenschaftler anhand der erhaltenen Informationen über die Landnutzung die Regierung zu Düngemittelspenden veranlassen wird, kann sich der Dateninhalt deutlich unterscheiden von jenem, der unter der Annahme erzielt wird, der Wissenschaftler arbeite - entgegen allen Beteuerungen - mit der Steuerbehörde zusammen. Dennoch zeigt die Erfahrung, dass gerade informelle Gespräche Hintergrundinformationen liefern können, welche die formell erhobenen Daten in einen völlig anderen Kontext setzen. 
erfragt, die sich anhand der entsprechenden Funktion des Respondenten im Dorf versprechen ließen (vgl. Interviewleitfäden im Anhang).

Diese Methode erweist sich in zweierlei Hinsicht als vorteilhaft. Zum einen ist es dadurch möglich, die übergeordneten Themen der Untersuchung in allen Interviews anzusprechen. Im Gegensatz zum rein narrativen, freien Interview besteht somit nicht die Gefahr, dass wichtige Rahmenthemen während einer Befragung unbeachtet bleiben. Zum anderen erlaubt die Methode im Vergleich zu standardisierten Fragebogeninterviews, den Interviewten interessante Themen und Aspekte in das Gespräch einfließen zu lassen (vgl. Yin 1984; Oswald \& Schöpfle 1987). Dadurch können nicht nur neue Gesichtspunkte für die weiteren Untersuchungen aufgegriffen werden, der Befragte wird so auch zu einem gewissen Grad vom Objekt zum Subjekt erhoben. Gerade dieser letzte Effekt ist für die Mensch-MenschInteraktion bei sozialwissenschaftlichen Forschungen äußerst entscheidend für die Qualität der gewonnenen Daten. Das Bewusstsein des Befragten, einen Sachverhalt ausführlich darlegen und, mehr noch, eigene Themen und Gesichtspunkte in das Gespräch mit einbringen zu dürfen, begünstigt die gesamte Interviewsituation durch die Verminderung des formalen Befragungscharakters.

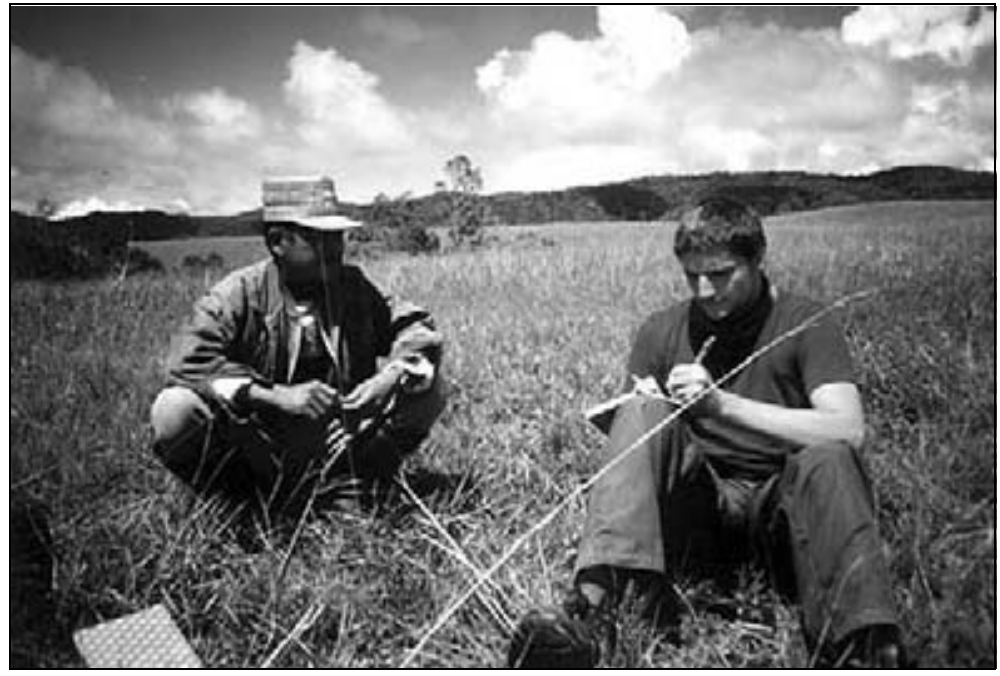

Abb. 4 Interviewsituation

(Quelle: Aufnahme von Syliva Ebersberger, 2002)

Da die eigenen Indonesischkenntnisse zu Beginn der Feldforschung für Tiefeninterviews noch nicht ausreichten, wurden die Befragungen zusammen mit einem indonesischen Assistenten durchgeführt. Dieser stellte nicht nur das sprachliche Bindeglied zwischen Interviewer und Interviewten dar, sondern wurde auch in die Bewertung der Befragungsergebnisse eingebunden. Es soll nicht verschwiegen werden, dass eine Übersetzung gewisse Reibungsverluste mit sich bringt. Durch die im 
Laufe der Feldforschung verbesserten Indonesischkenntnisse des Verfassers konnten diese zu einem guten Teil vermindert werden. ${ }^{18}$ Bei den gleichzeitig auf Tonband aufgezeichneten Interviews liegen die Aussagen der Befragten in Form von Transkripten in nahezu unverfälschtem Original vor. ${ }^{19}$

Der indonesische Assistent war auch als Informationsquelle bzgl. lokaler Gepflogenheiten eine wertvolle Hilfe. Zudem achtete er mit darauf, ob die Gesprächspartner durch Mimik oder Gestik ein abnehmendes Interesse an der weiteren Befragung erkennen ließen, welches eine Klärung mit der interviewten Person über die Beendigung bzw. Vertagung des Interviews erforderlich machte. Bis auf wenige Ausnahmen fanden alle Interviews im Haus der jeweils befragten Person statt. Die dadurch erhaltene vertraute Umgebung der Gesprächspartner sollte helfen, den formellen Charakter der Befragung zu vermindern. Einerseits wurde damit die Chance vergrößert, dass sich auch die Ehepartner am Interview beteiligen können. Andererseits erhöhte diese Wahl der Örtlichkeit jedoch die Gefahr, dass Nachbarn, Freunde und Bekannte dem Interview beiwohnen und Aussagen der Interviewpartner aktiv oder passiv beeinflussen. In einigen Fällen konnte eine solche Beeinflussung festgestellt und die Aussagen entsprechend gewertet werden.

Abhängig von der Anzahl der einzelnen Dorfviertel (dusun), welche in manchen Dörfern zum Teil eigenständige Charakteristika aufweisen, und von der Komplexität der verschiedenen Thematiken wurden pro Dorf zwischen 17 und 30 Interviews mit Vertretern der Dorfinstitutionen, ausgewählten Haushalten und weiteren Schlüsselpersonen geführt. Die Gesamtzahl der Interviews beträgt 196, wovon der größte Teil auf Tonband aufgezeichnet und anschließend transkribiert wurde. Grundsätzlich wurden die Interviewpartner um deren Einwilligung in die Aufzeichnung des Gesprächs gebeten. Dabei wurde besonders darauf hingewiesen, dass die gewonnenen, personenbezogenen Daten nicht an staatliche Stellen, wie etwa der Steuerbehörde, weitergegeben werden. Bei Verweigerung der Aufnahmegenehmigung bzw. im Falle starker Nebengeräusche wurde auf eine Tonbandaufzeichnung verzichtet. Neben den Tonbandmitschnitten wurden für jedes Interview Feldnotizen angefertigt, um einerseits einen direkten Überblick über die Hauptinformationen des Interviews zu haben und andererseits den kompletten Datenverlust im Falle technischer Probleme bei der Tonbandaufnahme zu verhindern.

18 In einigen wenigen Fällen sprachen die Gesprächspartner nur ihre Lokalsprache. Teilweise war dann eine Übersetzung in zwei Schritten erforderlich, wobei ein Familienmitglied um Unterstützung bei der Übersetzung von der Lokalsprache ins Indonesische gebeten wurde. Handelte es sich in solchen Fällen um eine dem Forschungsassistenten vertraute Lokalsprache, wurden die Interviews direkt in der Lokalsprache geführt.

19 Da die Transkripte der aufgezeichneten Interviews nicht eigenständig angefertigt wurden, ist ein Reibungsverlust auch hier nicht hundertprozentig auszuschließen. Allerdings zeigen eigene praktische Erfahrungen mit dem Transkribieren von Interviews, dass es dabei selbst bei konzentrierter Bearbeitung zu Übertragungsfehlern kommen kann. 
Die Dauer der Interviews variiert zwischen 20 Minuten und drei Stunden, bei einem Durchschnitt von etwa 80 Minuten. Spätestens nach 90 Minuten wurde mit dem Interviewpartner bzw. der Interviewpartnerin abgeklärt, ob das Gespräch fortgesetzt werden kann, oder ob es auf einen späteren Zeitpunkt vertagt werden soll. Die glücklicherweise nur selten vorkommende geringe Interviewdauer von unter einer halben Stunde ist auf Verunsicherungen seitens der befragten Personen zurückzuführen. In diesen Fällen gelang es dem Interviewer und dessen Assistenten nicht, das nötige Vertrauen des bzw. der Interviewten zu gewinnen. In der Regel wurde das Familienoberhaupt eines Haushalts befragt, welches in der überwiegenden Mehrzahl männlichen Geschlechts war. Nur wenn das Familienoberhaupt nicht in der Lage war, das Interview zu führen (z.B. aufgrund alters- oder krankheitsbedingter Behinderungen), oder wenn es nicht angetroffen werden konnte, wurde ein anderes Mitglied der Familie, meist die Ehefrau, befragt.

Im Vergleich zu qualitativen Befragungen in Deutschland zeigte sich bei der Feldforschung in der Lore-Lindu-Region eine enorm hohe Bereitschaft der Menschen zu einem Interview. Nur in wenigen Fällen wurde dem Verfasser ein Interview verweigert. Meistens waren bereits von anderen Forschern des SFB durchgeführte Befragungen der Grund für die Ablehnung. Als Ursache der großen Interviewbereitschaft der Bevölkerung konnten drei Hauptmotive isoliert werden. Erstens wurde die Befragung als Pflicht angesehen, ähnlich der Verpflichtung, sich für eine Befragung durch Mitarbeiter staatlicher Behörden zur Verfügung zu stellen. Zweitens erhofften sich die Befragten einen materiellen Nutzen von der Befragung. ${ }^{20}$ Drittens wollten sie dem Verfasser bei seinem persönlichen Anliegen, der Verfassung einer Doktorarbeit, behilflich sein. Die ersten beiden Motive spielten für die meisten der Befragten die größte Rolle. Vor allem der Pflichtgedanke erwies sich als ein Erschwernisfaktor bei den Interviews. Gepaart mit der Überzeugung der Befragten, keine hilfreichen Informationen liefern zu können, war es in zahlreichen Fällen nur schwer möglich, den Interviews einen narrativen Charakter zu verleihen. So konnten viele Informationspakete, z.B. Biographien, nur durch vielfaches Nachfragen zusammengesetzt werden, was einen erheblichen Zeitaufwand bedeutete.

Der Verfasser ist sich bewusst, dass angesichts der vorwiegend männlichen Interviewpartner einige wichtige genderspezifische Themen nicht hinreichend erfasst werden konnten. Die Felderfahrungen zeigten jedoch auch die Schwierigkeiten, als Mann Interviews mit Frauen führen zu können. Beispielhaft hierfür ist eine erlebte

20 Die berechtigte Frage nach dem Nutzen der Befragungen für die Bevölkerung ist ein weitverbreitetes und immer wiederkehrendes Thema aller Forschungsaktivitäten des SFB. Oft werden diese wissenschaftlichen Untersuchungen mit den Aktivitäten von NGOs in der Region in Verbindung gebracht. Diese Tatsache bewirkte, dass zahlreiche Respondenten trotz entsprechender Aufklärung oft ungefragt materielle Mängel in ihrem Dorf thematiserten, in der Hoffnung, dass dem eine entsprechende Unterstützung des Dorfes folgt. 
Interviewsituation: Die Befragung wurde aufgrund der Abwesenheit des männlichen Haushaltsvorstands mit dessen Ehefrau begonnen. Diese zögerte anfangs, sich für das Interview zur Verfügung zu stellen, mit dem Verweis, dass ihr Mann nicht zugegen sei. Bei der Mehrzahl der gestellten Fragen verwies die Frau auf das Wissensmonopol ihres Mannes. Als dieser auftauchte, übergab sie ihm sofort die Position des Interviewpartners und zog sich zurück. Generell wurde versucht, bei den Befragungen beide Eheleute zum Interview einzuladen. Nur in sehr wenigen Fällen beteiligte sich die Frau an dem Interview. Selbst der Versuch, die Ehefrau aktiv in das Gespräch einzubeziehen, indem Fragen explizit an sie gerichtet wurden, führte in der Regel nicht zu dem erhofften Ergebnis. Die ursprünglich geplante Vorgehensweise, zusammen mit einer indonesischen Doktorandin die Feldforschung in den selben Dörfern durchzuführen, konnte leider nicht umgesetzt werden. ${ }^{21}$

Das aus den Befragungen gewonnene, qualitative Datenmaterial wurde durch Experteninterviews mit Vertretern staatlicher Behörden (z.B. des u.a. für lokale Umsiedlungsprogramme zuständigen Departmen Sosial) in der Provinzhauptstadt Palu ergänzt. Ferner wurde das verfügbare statistische und Kartenmaterial ausgewertet. Sowohl in bezug auf die Expertengespräche als auch auf das statistische Material konnten nicht alle potentiellen Informationsquellen für das Kabupaten Poso ausgeschöpft werden, da die Bezirkshauptstadt Poso aufgrund der bürgerkriegsähnlichen Lage in jener Region nicht besucht werden konnte.

Im Zusammenhang mit der Auswertung empirischer Daten zur Bildung neuer Theorien bzw. Theorieansätzen stellt sich immer wieder die Frage, ob ein deduktives oder ein induktives Vorgehen sinnvoll ist. Lamnek merkt dazu an:

\section{„Das Vorgehen der Theoriegewinnung ist in der quantitativen Position deduktiv, in dem quali- tativen Paradigma induktiv. Basis für die Theorieentwicklung ist in der qualitativen Sozialfor- schung immer die soziale Realität des zu untersuchenden Feldes, weshalb die so produzierten Theorien , realistischer' sind. "(1993: 129).}

Ebenso sieht Steinke (1999: 20) den induktiven Ansatz strikt mit der qualitativen Forschungsmethode verbunden. Ausschlaggebend für diese enge Verbindung in der empirischen Sozialforschung ist die von Glaser und Strauss ins Leben gerufene sog. Grounded Theory (vgl. Glaser \& Strauss 1967) im Zuge der zunehmenden Kritik an der in den 1960er Jahren noch starken Position der deduktiv vorgehenden quantitativen Forschungsmethode. Diese postulierte, dass allein durch mathematisch-statistische Berechnungen quantitativer Daten eine wissenschaftlichen Analyse von Hypothesen gewährleistet ist, wobei die allgemeine Annahme anhand des empirischen Materials überprüft wird. Glaser \& Strauss (1967: 35ff) hingegen sehen in den

21 Der Verfasser möchte an dieser Stelle insbesondere den Forschungskolleginnen Melani Abdulkadir-Sunito, Sylvia Ebersberger und Laksmi Adriani Savitri für deren interessante Informationen danken. 
erhobenen Daten den Ausgangspunkt der Theoriebildung. Dieser soll sich ohne vorgegebene Theorien und Kategorisierung und ohne Kenntnis der entsprechenden Fachliteratur dem Untersuchungsfeld angenährt werden. Diese Vorgehensweise steht im klaren Widerspruch zu derjenigen, die eine bestimmte Fragestellung voraussetzt, anhand derer die empirischen Daten untersucht werden können (vgl. Popper 1984: 375). Im Zuge der Kritik an der Grounded Theory weicht auch Strauss (1987) von der ursprünglich strikten Ablehnung der a priori-Position von Hypothesen ab. Auch Kelle (1994) und Kelle \& Kluge (1999) gehen davon aus, dass eine rein induktive Herangehensweise bei der Analyse empirischer Daten und der anschlieBenden Generierung von Theorien nicht möglich ist. Die Auswertung qualitativ erhobener Daten birgt generell eine gewisse Disposition zur induktiven Forschungsmethode. Besonders durch das semi-strukturierte Interview, welches es zulässt, Themen zu erfassen, die über die vorab entworfenen Forschungsfragen hinausgehen, eröffnet sich ein Raum, der ein induktives Vorgehen anbietet. Dabei werden Erkenntnisse gewonnen, mit deren Hilfe nicht nur aufgestellte Hypothesen überprüft werden können, sondern auch die Vertiefung und Erweiterung einer Fragestellung ermöglicht wird. Solche neuen, zusätzlichen Informationen dienen dann als Grundlage, als Hypothese, welche anhand des empirischen Datenmaterials überprüft und verfeinert werden kann.

In der vorliegenden Arbeit wurden die quantitativen und qualitativen Daten in zweierlei Hinsicht bearbeitet. Zum einen dienten sie der deskriptiven Auswertung, um ein Bild der Kulturlandschaft(en) der Lore-Lindu-Region und dessen Wandel im Verlauf des 20. Jh. zu zeichnen. Zum anderen wurden die Daten, insbesondere jene qualitativer Art, dahin gehend untersucht, Ursachen und Einflussfaktoren für Veränderungsprozesse der Kulturlandschaft(en) zu filtern. Dabei wurde besonderes Augenmerk auf die raum-zeitlichen Veränderungen gelegt, um herausfinden zu können, wann, wie und warum sich kulturlandschaftliche Veränderungen in der gesamten Untersuchungsregion oder nur in bestimmten Teilgebieten vollzogen haben. Insbesondere die qualitativen Interviews dienten zusätzlich der Analyse von kleinräumigen Besonderheiten kulturlandschaftlicher Ausprägung und der Erfassung gruppen- und individuenspezifischer Entscheidungsfacetten. Mit Hilfe dieses Auswertungsspektrums sollte eine Annäherung an die Komplexität des Kulturlandschaftswandels in der Lore-Lindu-Region erreicht werden.

Die Auswertung qualitativer Daten zielt nicht darauf ab, durchgehend repräsentative Ergebnisse zu liefern. Vielmehr dient sie der Erfassung dessen, was durch das Raster quantitativer Untersuchungen fällt. Es werden dabei Hinweise geliefert, in welcher Form das menschliche Handeln und der dadurch bewirkte Wandel einer Kulturlandschaft sich im Detail verhält und wie das repräsentative Bild von kleinräumigen Prozessen überlagert oder durchbrochen wird.

An dieser Stelle seien noch einige Anmerkungen zum Umgang mit direkten Zitaten gemacht. Kurze Zitate aus niederländischen Quellen werden im Text im Original wiedergegeben, sofern davon ausgegangen werden kann, dass sie auch für 
Leserinnen und Leser ohne entsprechende Sprachkenntnisse zu verstehen sind. Handelt es sich um längere und/oder schwer verständliche niederländische Zitate, so erscheint im Text die deutsche Übersetzung, wobei das Original in der zugehörigen Fußnote wiedergegeben wird. Beim Zitieren indonesischer Quellen, insbesondere der während des Feldaufenthalts befragten Personen, wird im Text nur die deutsche Übersetzung benutzt. Die Originaltexte liegen dem Verfasser als Tonbandaufzeichnungen und Interviewtranskripte vor. Für die Interviews, bei welchen aus den genannten Gründen keine Tonbandmitschnitte angefertigt wurden, können Zitate nur sinngemäß anhand der Feldnotizen wiedergegeben werden.

\subsection{Aufbau der Arbeit}

Nach dem Einleitungskapitel wird im zweiten Hauptkapitel die Untersuchungsregion unter naturgeographischen und historischen Gesichtspunkten dargestellt. Nach einer Einführung in das Untersuchungsgebiet (Kap. 2.1) und einer Darstellung der naturgeographischen Grundlagen (Kap. 2.2) werden die für die untersuchte Region bedeutenden geschichtlichen Entwicklungen während des 20. Jh. anhand verschiedener zeitlicher Querschnitte beschrieben (Kap. 2.3). Diese sollen als Grundlage für die nachfolgende Analyse der Prozesse auf Meso- (Region/Sub-Region) und Mikroebene (Dorf/Haushalt/Individuum) dienen. Daran anschließend werden die Untersuchungsdörfer dargestellt (Kap. 2.4).

In den Kapiteln 3 bis 6 wird der Kulturlandschaftswandel anhand der übergeordneten Themen Bevölkerungsentwicklung, Siedlungswesen, Landnutzung und politische Aspekte auf der Basis des empirischen Datenmaterials herausgearbeitet. Als erstes dieser Oberthemen werden in Kapitel 3 die Veränderungen der Bevölkerungsstrukturen in der Lore-Lindu-Region sowie die dafür entscheidenden Migrationsprozessen einer ausführlichen Analyse unterzogen. Hierdurch soll gewährleistet werden, dass die Migration als ein bedeutender Faktor für die Veränderung der Kulturlandschaft bei der Betrachtung der nachfolgenden Themenblöcke (Siedlungsstruktur etc.) bereits als Grundlagenwissen zur Verfügung steht. Zahlreiche Aspekte dieser übrigen Themengebiete beziehen sich auf die Migrationsprozesse in der Untersuchungsregion. Ein logisches Aufeinanderaufbauen der Einzelthemen kann somit ebenso erreicht werden wie die Vermeidung allzu häufiger Redundanzen. Als Basis für dieses Hauptkapitel wird in Kap. 3.1 die Bevölkerung der Untersuchungsregion charakterisiert und für den Beginn des 20. Jh. quantifiziert. Ebenso wird in diesem Kapitel die Bevölkerungsdynamik in der Lore-Lindu-Region beleuchtet. Mit einer Einordnung des in dieser Arbeit verwendeten Migrationsbegriffs wird der umfassenden Themenbereich der Migrationsprozesse in der Untersuchungsregion (Kap. 3.2 bis 3.7) eingeleitet. Nach einem Überblick über allgemeine Aspekte der Migration in der Lore-Lindu-Region (Kap. 3.3) untergliedert sich das Kapitel 3 in die Betrachtung staatlich geplanter und habfreiwilliger Umsiedlungen (Kap. 3.4 und 3.5), spontaner Migration (Kap. 3.6), sowie der Fluchtmigration (Kap. 3.7). Anhand

\section{Pazifik Forum Bd. 12}


der spontanen Wanderungsbewegungen werden verschiedene Formen dieser Migration aufgezeigt (Kap. 3.6.1) und eine Typisierung von Wanderungsmotiven vorgenommen (Kap. 3.6.2).

Das vierte Kapitel widmet sich der Herausarbeitung bedeutender Veränderungsprozesse im Siedlungswesen. Zuerst wird die historische Siedlungsentwicklung von der vorkolonialen Besiedlung bis zum gegewärtigen Status quo nachgezeichnet (Kap. 4.1). Anschließend werden unterschiedliche Siedlungsformen in der Lore-Lindu-Region anhand von Fallbeispielen verschiedener Dörfer dargestellt (Kap. 4.2). Den Abschluß des Hauptkapitels zum Siedlungswesen bildet eine Erörterung sozialräumlicher Segregationsmuster als Folge der Herausbildung multiethnischer Dorfgemeinschaften (Kap. 4.3). Dabei werden, nach einigen allgemeinen Überlegungen zum Verhaltensmuster der sozialen Ausgrenzung, die gemeinsamen sowie lokal unterschiedlichen Einflussfaktoren für das Entsehen sozialräumlicher Segragation anhand der einzelnen Untersuchungsdörfer herausgearbeitet.

Da der Wandel in der Landnutzung und dessen Ursachen für die ländlich geprägte Untersuchungsregion von großer Bedeutung sind und ein generell wichtiges Kriterium der Entwicklung von Kulturlandschaften im ländlichen Raum darstellen, wird diesem Aspekt ein eigenes Kapitel (5) gewidmet. Ausgehend von den Grundlagen der traditionellen Landnutzung (Kap. 5.1) werden in Kap. 5.2 die Einflüsse der Moderne auf die Landwirtschaft in der Region untersucht. Besonderes Augenmerk wird dabei der v.a. auf dem Reisanbau basierenden landwirtschaftliche Subsistenzwirtschaft einerseits und der Produktion von cash crops (z.B. Kakao) geschenkt. Zudem wird der Übergang von der landwirtschaftlichen Nachbarschaftshilfe zur Ausbreitung der Lohnarbeitsverhältnisse untersucht. Nach einer allgemeinen Analyse der sozioökonomischen Auswirkungen des Wandels in der Landnutzung (Kap. 5.3), soll die Behandlung des Themas Landkonflikte mit der Frage nach seiner Ursächlichkeit (Kap. 5.4) in der Zuwanderung einen weiteren Bogen zur Migration spannen.

Um der Bedeutung politischer Prozesse, insbesondere ihrer individuellen Ausformungen auf Dorfebene, für die Veränderungen der Kulturlandschaft und deren räumlich differenzierte Ausprägung gerecht zu werden, wird in Kapitel 6 der politische Einfluss anhand von Fallbeispielen auf Makro-, Meso- und Mikroebene untersucht. Neben den Auswirkungen der sog. Ethischen Politik der Niederländer (Kap. 6.1) wird der Frage nachgegangen, in welcher Form die Region von soziopolitischen Kontinuitäten und Brüchen geprägt ist und wie der Kulturlandschaftswandel davon betroffen ist (Kap. 6.2). Zudem wird die Bedeutung von Bildungspolitik untersucht (Kap. 6.3). Das letzte Unterkapitel befasst sich mit dem Spannungsfeld Nationalpark (Kap. 6.4), in dem sich eine Vielzahl von Aspekten aus den Kap. 3 bis 5 bündeln.

Abschließend werden in Kapitel 7 die aus den Ergebnissen der Feldforschung gewonnen Erkenntnisse zusammengefasst. Anhand der analysebefunde werden dabei die in der Einleitung aufgestellten Hypothesen überprüft. 
Es gliedert sich in die zwei Kabupaten Donggala und Poso, welche ihrerseits in mehrere Kecamatan unterteilt werden können (vgl. Tab. 1). Die in dieser Arbeit untersuchte Region umfasst die Kecamatan Sigi-Biromaru, Palolo und Kulawi (alle im Kabupaten Donggala; das Territorium von Palolo ging im Jahr 1997 als eigenständiges Kecamatan aus dem Kec. Sigi-Biromaru hervor). Ferner umfasst die untersuchte Region auf dem Gebiet des Kab. Poso das Kecamatan Lore Utara. ${ }^{22}$

Tab. 1 Administrative Verteilung der Untersuchungsdörfer auf das Untersuchungsgebiet

\begin{tabular}{ccc}
\hline Kabupaten & Kecamatan & Desa (Dorf) \\
\hline \multirow{3}{*}{ Donggala } & Sigi-Biromaru & Maranatha \\
& & Pandere \\
\cline { 2 - 3 } & Kulawi & Bolapapu \\
& Palolo & Lempelero \\
& & Lawe \\
\hline \multirow{2}{*}{ Poso } & Lordikari \\
& \multirow{2}{*}{ Lo Utara } & Sintuwu \\
\hline
\end{tabular}

Während der Feldforschung wurde der südliche Teil des Kec. Lore Utara zu dem neuen Kec. Lore Tengah erhoben. In gleicher Weise wurde das Pipikoro-Gebiet im Mai 2002 als eigenständiges Kecamatan vom Territorium des Kec. Kulawi getrennt. Hierdurch würde die Untersuchungsregion mittlerweile sieben Kecamatan umfassen. Die vorliegende Arbeit bezieht sich jedoch generell auf die ehemals fünf Kecamatan.

Im Jahr 1910, noch zu Beginn der niederländischen Kolonialherrschaft über das Lore-Lindu-Gebiet, war Zentralsulawesi (damals Midden-Celebes genannt) in vier sog. Onderafdeelingen (Unterabteilungen) unterteilt. Für das Untersuchungsgebiet entscheidend sind davon die Onderafdeelingen Palu und Poso. ${ }^{23}$ Für die Onderafdeeling Palu sind in bezug auf das Untersuchungsgebiet dieser Arbeit die damaligen Landschappen (Landschaften; Untereinheiten einer Onderafdeeling) Biromaru, Sigi, Dolo, Kulawi, Lindu und Tobaku von Bedeutung.

22 Das Kecamtan Lore Selatan ist zwar eine Teilregion des STORMA-Untersuchungsgebiets, in der vorliegenden Arbeit wird es aber nur am Rande betrachtet, da das der Arbeit zugrunde liegende Interviewmaterial nur in Dörfern der übrigen vier Kecamatan gesammelt wurde. 


\section{Die Untersuchungsregion}

\subsection{Einführung in das Untersuchungsgebiet}

Das in dieser Arbeit als Lore-Lindu-Region bezeichnete Untersuchungsgebiet umfasst einen Teil der Provinz Zentralsulawesi in Indonesien (vgl. Abb. 5).

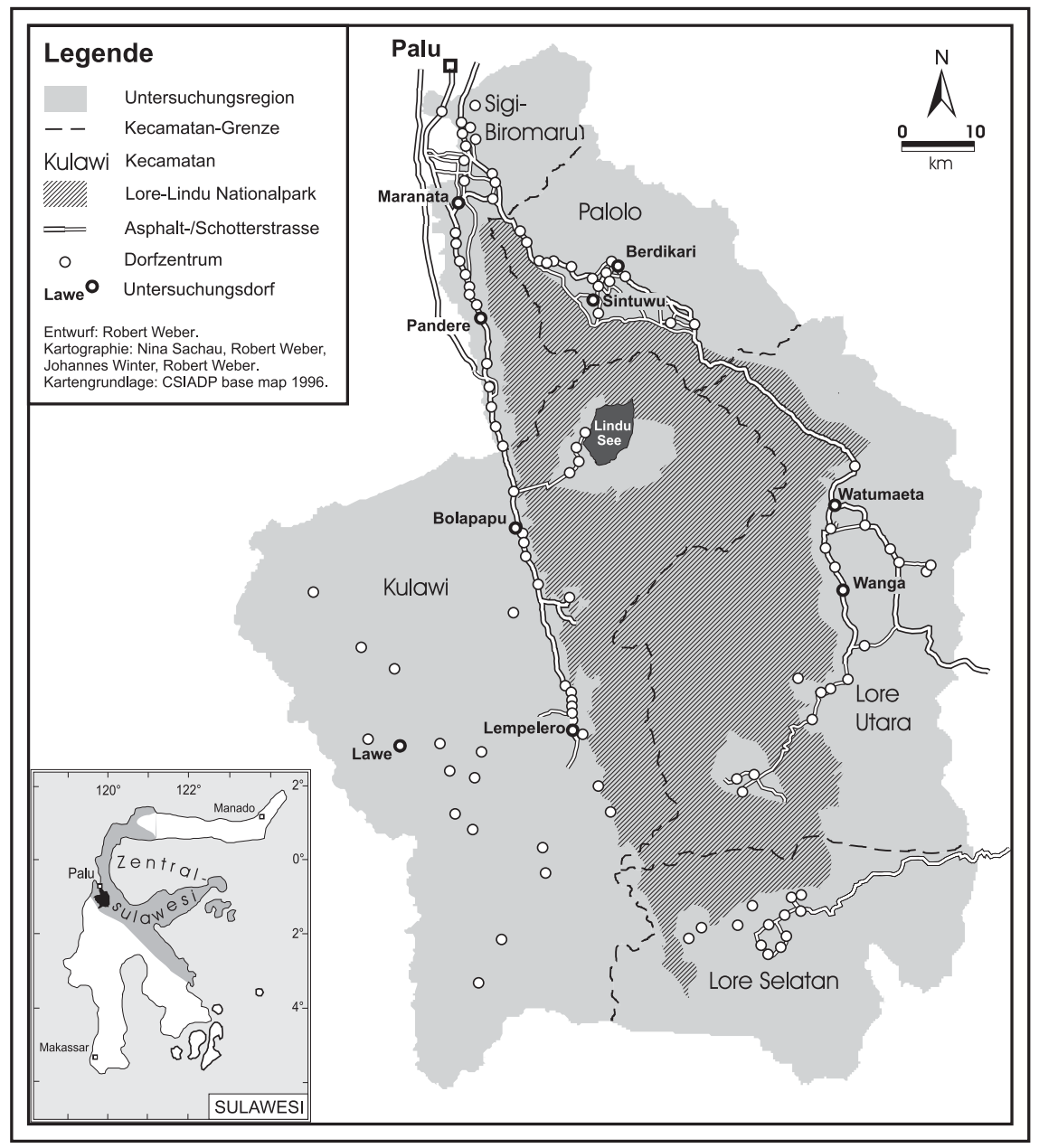

Abb. 5 Die Untersuchungsregion mit Lokalisierung der Untersuchungsdörfer 


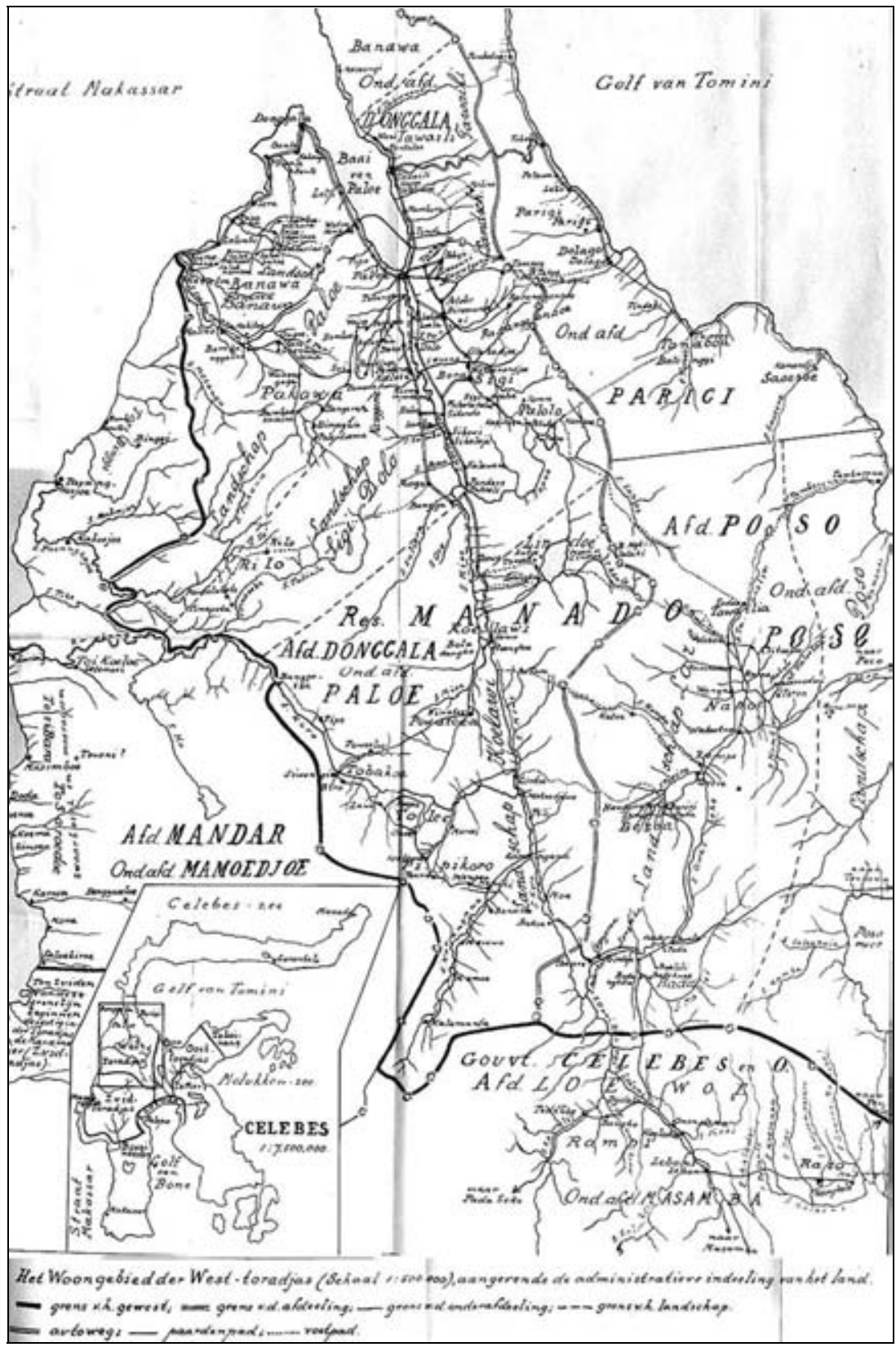

Abb. 6 Karte der kolonialzeitlichen Verwaltungseinheiten im westlichen Zentralsulawesi (Quelle: Kruyt 1938, Anhang) 
Für Poso sind dies die Landschappen Tawaeli, Napu, Besoa und Bada (Van Hengel 1930: 12f), welche ab 1916 als Landschap Lore zusammengefasst wurden (Koloniaal Verslag 1917: 35). Im Jahr 1922 bestand die Onderafdeeling Palu nur noch aus den vier Landschappen Palu, Dolo, dem zusammengeführten Sigi-Biromaru und Kulawi (Logeman 1922: 103). Gegen Ende der niederländischen Kolonialzeit wurden SigiBiromaru und Dolo schließlich zur Landschap Sigi-Dolo zusammengefasst, so dass vor dem Übergang zur Unabhängigkeit Indonesiens in der Untersuchungsregion die Landschappen Sigi-Dolo, Kulawi und Lore als administrative Einheiten existierten (vgl. Abb. 6).

Die neun im Rahmen dieser Forschungsarbeit genauer untersuchten Dörfer verteilen sich auf die Lore-Lindu-Region wie in Tab. 1 und Abb. 5 dargestellt. Im Zentrum der Untersuchungsregion, einige Kilometer südöstlich der Provinzhauptstadt Palu befindet sich der ca. 220.000 Hektar große Lore-Lindu Nationalpark, welcher aus tropischem Bergregenwald besteht (Anzdec 1997: 10). Zum Zeitpunkt der Feldforschung zählte die Lore-Lindu-Region 119 Dörfer. Das Untersuchungsgebiet untergliedert sich in einzelne Täler: das Palu-Tal, das einen Großteil des Kecamatan Sigi-Biromaru ausmacht, das Palolo-Tal im gleichnamigen Kecamatan, das SedoaTal und das Napu-Tal im Kecamatan Lore Utara, sowie das Kulawi-Tal und Gimpu-Tal im nördlichen bzw. südlichen Kecamatan Kulawi. ${ }^{24}$ Diese Täler werden von mehr oder weniger stark ausgeprägten Gebirgen begrenzt. Hinzu kommt noch die Pipikoro-Region, ein sehr gebirgiges und schwer zugängliches Gebiet im westlichen Teil des Kecamatan Kulawi. Die o.g. Täler sind von Palu aus über asphaltierte Strassen erreichbar, deren Passieren v.a. in den gebirgigen Übergängen zwischen den Tälern nach intensiven Niederschlägen durch Erdrutsche erschwert werden kann (vgl. Abb. 7).

\subsection{Naturgeographische Grundlagen der Lore-Lindu- Region $^{25}$}

„Mittel-Celebes ist in vielerlei Hinsicht ein merkwürdiges Land. Erstens wegen seiner Form: nach Süden, Südosten, Norden und Osten streckt es seine Arme aus, wodurch große Golfe oder Buchten entstehen, die den Weg bestimmen, wie man von vier Seiten her in das Land eindringen kann. Große Flüsse münden in die Meerbusen, welche [die Flüsse; Anm. d. Verf.] kaum

24 Das Bada-Tal in Lore Selatan zählt zwar zur Forschungsregion des SFB 552, jedoch nicht mehr zum Kerngebiet der Untersuchungen dieser Arbeit. Es wird daher nur am Rande behandelt.

25 Sofern nicht anders belegt, beziehen sich die nachfolgenden Angaben auf Koninklijk Nederlandsch Aardrijkskundig Genootschap (1938), Whitten et al. (1988) und Kruyt (1938, Bd. 1). 
befahrbar sind, welche jedoch den Menschen, die auf diese Insel kamen, um sich dort niederzulassen, den Weg gewiesen haben, um in das unbekannte Landesinnere zu gelangen. "26

beschreibt Kruyt (1935: 585) Zentralsulawesi, wohl wissend, dass neben anderen Aspekten auch die naturräumlichen Gegebenheiten für die Besiedlung und die Ausbildung der jeweiligen Kulturlandschaft eines Raumes ausschlaggebend sind.

Sulawesi ist eine von etwa 13.000 Inseln im indonesischen Archipel. Sie erstreckt sich etwa zwischen $3^{\circ}$ n.Br. und $6^{\circ}$ s.Br. sowie zwischen $118^{\circ}$ ö.L. und $125^{\circ}$ ö.L. und liegt zwischen Borneo im Westen und den Molukken im Osten. Etwas nördlich der zentralsulawesischen Provinzhauptstadt Palu schneidet die Insel den Äquator. Mit einer Fläche von $189.200 \mathrm{~km}^{2}$ ist Sulawesi die viertgrößte Insel Indonesiens nach Borneo, Sumatra und Papua (ehem. Irian Jaya). In der erdumspannenden Tethys befand sich auch die Insel Sulawesi. In diesem Meer wurden seit dem Paläozoikum im Bereich des heutigen Sulawesi u.a. Kalk- und Sandsteinsedimente auf Gneisen und anderen Gesteinen abgelagert. Die Orogenese Sulawesis erfolgte zur Zeit der alpidischen Gebirgsfaltung im Tertiär. Dabei wurden die verfestigten Sedimente in mehreren Phasen aufgefaltet und aus dem Meer gehoben. Im Eozän begann die vulkanische Tätigkeit und setzte sich im Miozän verstärkt fort. Ein schwaches vulkanisches Epizentrum befindet sich heute nordwestlich des Lindu Sees. Vermutlich kollidierte Westsulawesi im späten Pliozän mit Borneo und trennte sich während des Quartärs wieder.

Das Untersuchungsgebiet ist hauptsächlich von kristallinem und metamorphem Gestein bestimmt. Im Quartär lagerten sich weitere Sedimente ab und verfestigten sich. Im Westen Zentralsulawesis bestehen im nördlichen Teil des Palu-Tals Alluvialböden, welche durch frühere oder rezente Überschwemmungen geprägt sind. Die Palu-Bucht und das Palu-Tal sollen einmal ein kleines Meer dargestellt haben, welches am heutigen Nordende der Bucht durch ein Randgebirge abgeschlossen war. Dieses Randgebirge war vermutlich aus den gleichen basischen Gesteinen aufgebaut, die noch im nördlichen Teil der Bucht an den Rändern zu finden sind. Nach dem Durchbruch dieses Randgebirges spülte das abfließende Wasser das sedimentierte Material weg und legte die Palu-Ebene frei. Den südlichen Teil des Tals nehmen Regosole ein. Die Gebirgsböden bilden einen Bodenkomplex, der bislang noch wenig erforscht war. Erste Untersuchungen im Rahmen des STORMA-Projekts stellten in den Tälern der Untersuchungsregion fluviale Kambiosole, Fluviosole und Gleyosole fest. Die unteren Hangbereiche sind mit kolluvialem Material bedeckt,

26 Zitat im Original: „Midden-Celebes is in vele opzichten een merkwaardig land. In de eerste plaats al om zijn vorm: naar het Zuiden, Zuidoosten, Noorden en Oosten steekt het zijn armen uit, waardoor groote golven of bochten ontstaan, die den weg aanwijzen, hoe men van vier kanten het land binnen kan dringen. Groote rivieren monden in die golven uit, die veelal weinig bevaarbaar zijn, maar die toch aan de menschen, die op dit eiland een woonplaats kwamen zoeken, den weg hebben gewezen om het onbekende binnenland in te gaan." 
während die Talböden junge kolluviale, alluviale und lacustre Sedimente aufweisen. Kambiosole und Leptiosole bedecken die oberen Hanglagen sowie das Hochland (Dechert 2003: 10). Die Gebirgsrücken erstrecken sich in Zentralsulawesi in der Regel in Nord-Süd-Richtung, nur auf der nord-östlichen Halbinsel weicht diese Richtung nach West-Ost ab. Durch die starke Faltung bildete sich ein sehr ausgeprägtes Relief heraus, dessen Gipfel bis in eine Höhe von $2.500 \mathrm{~m}$ reichen.

Sulawesi, das reich an Mineralien (Nickel, Kupfer, Silber, weniger Gold) ist, kann in drei große geologische „Provinzen“ aufgeteilt werden, eine West-, eine Ostund eine Banggai-Sula-Provinz, welche sich hinter Luwu auf der NO-Halbinsel befindet. Zwischen der West- und der Ost-Provinz verläuft eine tektonische Verwerfung, welche sich von Palu aus in südöstlicher Richtung verlaufend bis zum Golf von Bone durchzieht. Diese tektonische Störung, an welcher das Palu-Tal entlang läuft, wird Palu-Koro-Verwerfung oder, nach ihren Entdeckern benannt, SarasinLinie genannt (vgl. Sarasin \& Sarasin 1901, 1905).

Mit seiner Lage um den Äquator ist Sulawesi in die Warmtropen eingebunden und durch Monsun-Klima geprägt. In höheren Lagen der Gebirge wechselt das Klima in den Kalttropenbereich. Die Windrichtung dreht, über das Jahr verteilt, von NW bis W (Januar) auf O bis NO (April), SO bzw. SW (Juli) und W bis NW (November). Gemäß seiner Äquatoriallage ändert sich die Tagestemperatur über das Jahr hin nur mäßig und bewegt sich zwischen $25^{\circ}$ und $31^{\circ} \mathrm{C}$. Mit der Höhe nimmt die Temperatur ab und kann somit in den Hochebenen oft nur $16^{\circ}$ bis $22^{\circ} \mathrm{C}$ erreichen. Die Jahreszeiten sind durch die Regenzeit geprägt, die etwa im April beginnt und bis Oktober reicht. Durch die Faltung der Gebirge liegen die meisten Täler in Nord-Süd-Richtung, wodurch sie sich fast das ganze Jahr im Regenschatten befinden. Das Palu-Tal, welches zu den trockensten Gebieten Indonesiens gehört, weist meist Jahresniederschlagsmengen unter $600 \mathrm{~mm}$ auf. Braun gedörrte Grasflächen gegen Ende der Trockenzeit sowie das Vorkommen von Kakteenarten v.a. im nördlichen Teil des Tals geben einen deutlichen Hinweis für dieses, gemessen an seiner nahen Äquatorlage, klimatisch ungewöhnliche Gebiet. Mit der Höhe wechseln die Klimazonen von 5-6 humiden bei maximal drei ariden Monaten bis zu 1012 humiden bei maximal zwei ariden Monaten pro Jahr. Maximalwerte erreicht der Niederschlag im westlichen Zentralsulawesi in den Gebirgen, wo zwischen 2000 und $3000 \mathrm{~mm}$ Niederschlag gemessen werden. Eine Folge der hohen Niederschläge während der Regenzeit sind häufige Erdrutsche, welche die Verkehrsinfrastruktur der region beeinflussen können (vgl. Abb. 7). Der nicht verdunstete Niederschlag wird über die relativ kurzen Flüsse ins Meer abtransportiert. Der Lariang, welcher sich im Untersuchungsgebiet befindet, ist mit $225 \mathrm{~km}$ der mit großem Abstand längste Fluss der Insel. Als stehende Gewässer findet man in Zentralsulawesi den Lindu See östlich des Palu- und des Kulawi-Tals, den Rano See im Napu-Tal sowie den Poso See südlich der Stadt Poso.

Sulawesi nimmt, bezogen auf Flora und Fauna, eine gewisse Sonderrolle ein. Die Insel war während ihrer geologischen Vergangenheit weder mit dem asiatischen 
Festland, noch mit dem australischen Kontinent verbunden. Es existierte lediglich eine Landbrücke zu den Philippinen. Der britische Naturforscher A. R. Wallace (1823-1923) postulierte eine imaginäre Linie, die sog. Wallace-Linie, die sich zwischen Bali und Lombok hindurch nach Norden westlich an den Philippinen vorbeizieht und dabei Borneo und Sulawesi trennt. Durch diese Linie werden die verschiedenen Faunen und Floren, die zu unterschiedlichen Landmassen der letzten Eiszeit gehören, voneinander abgegrenzt. Im Osten trennt die sog. Weber-Linie Sulawesis Tier- und Pflanzenwelt von jener der Molukken, Neuguineas und Australiens. Diese Lage ermöglichte die Entwicklung zahlreicher endemischer Tier- und Pflanzenarten im Biodiversitäts-Hotspot Wallacea, wie sie etwa im Lore-Lindu-Nationalpark in Zentralsulawesi vorkommen (vgl. u.a. Waltert et al. 2004; Schulze et al. 2004).

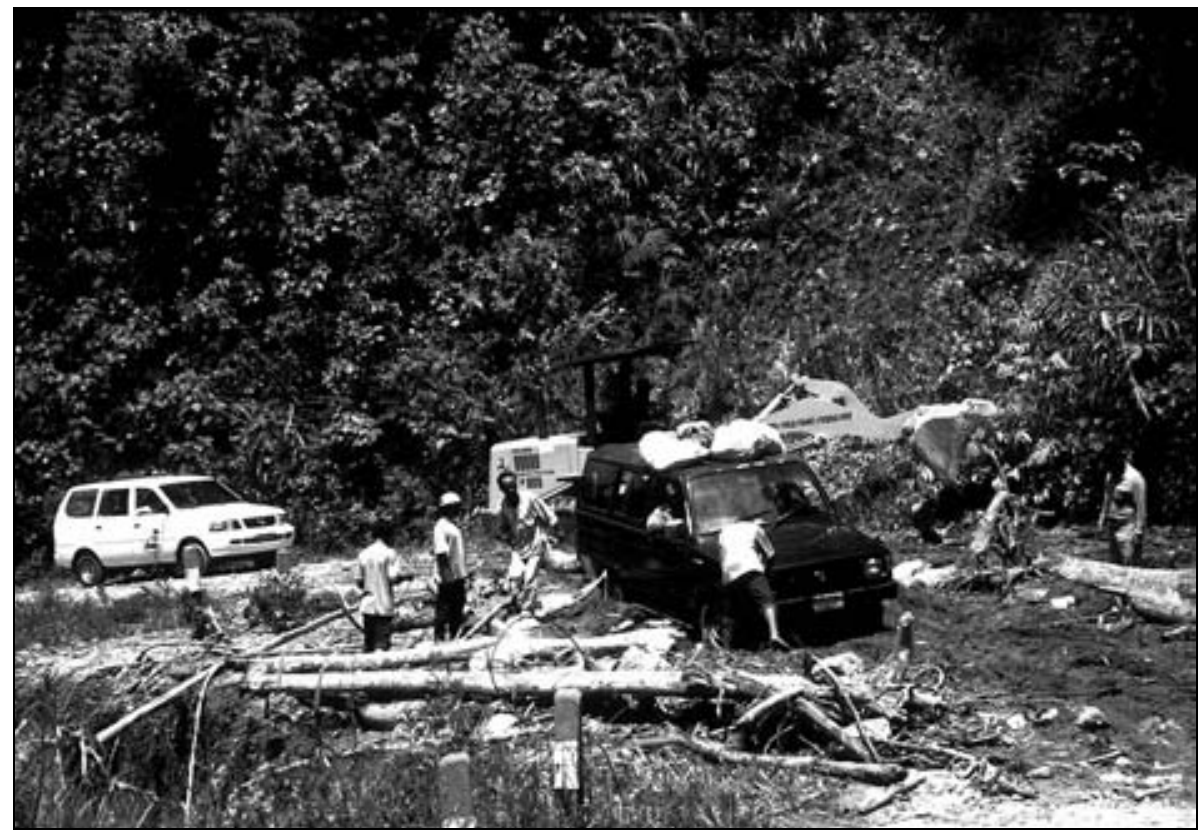

Abb. 7 Reparaturarbeiten an der Verbindungsstrasse zwischen Palu- und Kulawi-Tal nach einem Erdrutsch

(Quelle: Eigene Aufnahme, 2000)

Im Palu-Tal ist die natürliche Vegetation dem trockenen Klima angepasst. Um das Tal herum geht die Vegetation in Regenwald über, der auf Sulawesi durch seinen hohen Palmen-Anteil auffällt. War im frühen 18. Jh. das Palu-Tal mit Reisfeldern übersät, so präsentierte es sich 200 Jahre später sehr karg. Kriegerische Auseinandersetzungen einerseits und die Verhinderung einer funktionierenden Bewässerung wegen großflächiger Materialabtragungen von den umliegenden Bergen in Folge 
massiver Entwaldung (im Süden und Südosten des Tals waren die Berge 1896 bis in eine Höhe von $1700 \mathrm{~m}$ baumlos) andererseits führten zur Auflassung der Reisfelder. Das ausgeprägte Relief Zentralsulawesis und der dichte Bewuchs mit Regenwald ermöglichen kaum großflächigeren landwirtschaftlichen Anbau. Neben der PaluEbene bieten sich im Untersuchungsgebiet noch Flussniederungen in Kulawi, Palolo und Napu (Lore Utara) oder südlich des Lindu Sees für eine ausgedehntere agrarische Nutzung an.

\subsection{Historische Entwicklung der Untersuchungsregion und deren Einbindung in die Geschichte Indonesiens}

Die vorliegende Arbeit befasst sich mit Ereignissen, welche sich über den Zeitraum eines Jahrhunderts verteilen. Zur besseren Nachvollziehbarkeit ist daher ein Überblick über die geschichtliche Entwicklung der untersuchten Region unabdingbar. Bereits während der niederländischen Kolonialherrschaft wurden in Zentralsulawesi entscheidende Maßnahmen ergriffen, welche die Basis für aktuelle Veränderungsprozesse in der Lore-Lindu-Region darstellen.

Mittels zeitlicher Querschnitte wird die historische Darstellung im Folgenden zunächst die präkolonialen Phase vor Beginn der effektiven ${ }^{27}$ Kolonialherrschaft der Niederländer in der Untersuchungsregion behandeln. Danach werden die ersten Europäer in Zentralsulawesi im Allgemeinen und der Lore-Lindu-Region im Besonderen vorgestellt. Einige dieser Personen besuchten die Untersuchungsregion bereits in der vorkolonialen Zeit, wodurch dieser Abschnitt geeignet erscheint, um von der präkolonialen zur Kolonialzeit überzuleiten. Daran anschließend erfolgt eine Abhandlung der wichtigsten Ereignisse während der niederländischen und japanischen Kolonialherrschaft, welche für die Lore-Lindu-Region ab 1904 bzw. 1942 begonnen hat. Abschließend erfolgt ein Überblick der im bezug auf die Untersuchungsregion relevanten historischen Entwicklung seit der Proklamierung der Unabhängigkeit Indonesiens im Jahr 1945. Da einige der historischen Aspekte in späteren Kapiteln noch genauer abgehandelt werden, werden diese bei der geschichtlichen Übersicht dieses Kapitels nur grob skizziert.

27 Unter effektiver Kolonialherrschaft ist an dieser Stelle jener Zeitraum zu verstehen, in dem die Niederländer aktiv in das Geschehen der Region eingreifen. Dieser Zeitraum beginnt ab 1904 mit der vertraglichen Unterwerfung der lokalen Herrscher unter die niederländische Krone. Vor diesem Wendepunkt zählte Zentralsulawesi zwar auch schon zu dem Kolonialterritorium der Niederländer. Es wurden allerdings nur einige Küstenort (z.B. Palu) als Umschlagplatz für Kolonialwaren genutzt (vgl. Weber 2000a, 2000b; Weber et al. 2003). 


\subsubsection{Die präkoloniale Phase (bis 1904)}

Zentralsulawesi befand sich Jahrhunderte lang abgeschieden von äußeren Einflüssen. „In contrast to Java, Bali, and, to some degree coastal South Sulawesi, Central Sulawesi was little affected by Indianization during and after the first millennium A.D.“ (Aragon 1992: 105). Nur die Küstenregionen waren schon frühzeitig vom Einfluss islamischer Händler geprägt. In den tief eingeschnittenen Tälern Zentralsulawesis - das ausgeprägte Relief stellt bis in die heutige Zeit ein Problem für den Ausbau der Infrastruktur dar - haben die verschiedenen Gruppen eigene Sprachen herausgebildet und lange Zeit relativ isoliert voneinander gelebt (Kruyt 1935: 586f). Die in Zentralsulawesi lebende Bevölkerung kommuniziert nach archäologischen und linguistischen Forschungen in austronesischen Sprachen, welche vermutlich um 2000 v.u.Z. von den Philippinen und aus Taiwan eingeführt wurden. Parallelen bestehen im Bezug auf die sog. barkcloth-Technologie und -Terminologie zwischen Polynesien und Zentralsulawesi. ${ }^{28}$ Die vorherrschende Sprache der Untersuchungsregion ist Kaili, doch haben sich viele unterschiedliche Sprachen durch die Isolation der einzelnen Gruppen herausgebildet (vgl. Aragon 1992: 106ff, 112). ${ }^{29}$ Auch zur Zeit der großen Königreiche und der Fürstentümer Südsulawesis war der Außenkontakt der Bevölkerung von Zentralsulawesi noch sehr gering.

Nach der Herrschaft der hinduistischen Königreiche Sriwijaya (mit Zentrum auf Sumatra) und Majapahit (Ostjava) entwickelten sich im 13. Jh. die Bugis-Königreiche von Gowa, Bone, Sopeng, Wajo etc. Handelskontakte bestanden in jener Zeit bereits mit den Molukken und der kleinen Sundainsel Sumbawa. Sogar aus China kamen bereits Güter nach Sulawesi. Seit dieser Zeit haftete Sulawesi das Stigma eines Piratengebietes an. Da viele Boote aus Malakka von Bugis ausgeraubt wurden,

28 Unter barkcloth, (Baum-)Rindenkleidung, versteht man Kleidungsstücke, welche aus der Rinde einer bestimmten Baumart hergestellt wird. In der Lore-Lindu-Region war die Verwendung dieser Kleidungsstücke für den alltäglichen Gebrauch bis in die erste Hälfte des 20. Jahrhundert weit verbreitet. Nachdem im Laufe der Jahrzehnte zunehmend Baumwoll- und andere Textilkleidungen angeboten wurden, gibt es in der Region mittlerweile nur noch wenige Frauen, die dieses Handwerk beherrschen. Gewänder aus bearbeiteter Baumrinde werden meist nur noch für besondere Adat-Zeremonien (Hochzeit, Beerdigung) verwendet. Bei der Herstellung eines Rindenstoffs wird die vom Baum abgetrennte Rinde (indones.: kulit kayu) mit geriffelten Eisenklöppeln solange bearbeitet, bis sich die Rinde auffasert. Danach werden die Rindenstücke, ähnlich der Papyrusherstellung, quer übereinander gelegt und mit zunehmend feiner geriffelten Klöppeln behauen, bis sich die Fasern der einzelnen Rindenlagen verbunden haben. Der so entstandene Stoff gilt als besonders strapazierfähig.

29 Die Sprache Kaili lässt sich in über 30 Dialekte, wie etwa Ledo, Da'a, Tara etc., untergliedern. Der Kaili-Sprachraum erstreckt sich über das Gebiet der Palu-Bucht, des Palu-Tals und Palolos.

Daneben finden sich auch noch Sprachen aus der Kulawi-Region (z.B. Moma und Uma), sowie aus dem Napu-, Behoa- (Besoa-) und Bada-Gebiet. Teilweise werden die Kulawisprachen ebenfalls der Kaili-Sprachgruppe eingegliedert. Diese Gliederung ist auf die Herrschaft des vorkolonialen SigiReiches über das Kulawi-Reich zurückzuführen, welches sein Zentrum in Bora, im Palu-Tal hatte. 
verfestigte sich dieses Vorurteil und diente den Niederländern später als Legitimation zur Unterdrückung.

Im 15. Jh. existierten in Südsulawesi im Königreich Luwu knapp 50 Fürstentümer, meist winzige Stadtstaaten. 1540 führten portugiesische Priester dort den Katholizismus ein. Die Missionierung schlug jedoch fehl, woraufhin die Fürsten Koranlehrer aus Malaya anforderten, um den Islam in Sulawesi zu verbreiten. In der Minahassa $^{30}$ (Nordsulawesi) hatte der Katholizismus der Portugiesen mehr Erfolg. Das Fürstentum Gowa, das seine Macht in vielfachen Kriegen über Ost-Kalimantan, Lombok, Sumbawa, Flores, Timor und auch Toli-Toli und Palu (Zentral- Sulawesi) ausdehnte, wurde 1605 islamisiert. Engere Beziehungen mit den in den Bergregionen lebenden Bevölkerungsgruppen (von den Europäern wie Bewohnern des Tieflands von Sulawesi wurde für sie der pauschalisierende Begriff „Toraja“31 verwendet) wollten die Bugis ${ }^{32}$ aus Angst vor der Kopfjagd nicht eingehen.

Im Jahr 1607, zu einer Zeit, als an Zentralsulawesi noch kein europäisches Interesse bestand, errichteten die Niederländer in Makassar bereits einen Handelsposten. 1613 erkannten die Engländer die Bedeutung dieses Handelsplatzes, fünf Jahre spä-

30 Nach Leirissa (1996: 266) setzt sich „Minahassa“ aus den Wörtern „mina“ und „esa“ zusammen und bedeutet in etwa ,,vereinigt sein“. Der Begriff soll von niederländischen, protestantischen Missionaren Anfang des 19. Jahrhunderts eingeführt worden sein und grenzt damit die dort lebende, christlich missionierte Gemeinde von muslimischen Regionen wie Gorontalo, Bolaang Mongondow oder den christlich geprägten Sangihe- und Talaud-Inseln ab. Es handelt sich also um eine ethnische Fremdbezeichnung, die später auch von der niederländischen Kolonialverwaltung übernommen wurde.

31 Nach Vroklage (1936: 227) setzt sich das Wort „Toraja“ aus „To“, der Bezeichnung für „Mensch“, und dem buginesisch geprägten „raja“ zusammen, wodurch sich eine Übersetzung von „Toraja“ als „Oberländer“ bzw. ,,rückständiger Mensch“ ergibt. Nooy-Palm (1975: 54) führt den Begriff „Toraja“ auf den Term „to ri aja“ (Bewohner des Hochlandes) aus der Sprache der Bevölkerung von Luwu, einer Küstenregion in Südsulawesi, zurück. In den 1930er Jahren haben die Bewohner des heutigen Tanah Toraja in Südsulawesi den Terminus „Toraja“ als Selbstbezeichnung übernommen (vgl. Bigalke 1981: 13ff). Das Wort „raja“ entspricht dem malayischen „dajak“, welches analog für die Bergbevölkerung der Dayak verwendet wurde. Coté (1979) hat vermutlich als erster Nicht-Indonesier diese ungenaue Bezeichnung durch die einzelnen Sprachgruppen-Namen dieses Gebietes ersetzt. Die Bewohner Zentralsulawesis hatten nach Kotilainen (1992: 16) nie eine einheitliche Bezeichnung für alle Gruppen der Region verwendet. In den Kolonialquellen und selbst noch in der jüngeren Literatur (vgl. Lebar 1972; Holmgren \& Spertus 1989) wird der Universalbegriff „Toraja“ für alle Hochlandbewohner verwendet. Wie indonesische (z.B. Mattulada 1991) oder westliche Wissenschaftler (z.B. Aragon 2000b) wird auch in der vorliegenden Arbeit der Sammelbegriff „Toraja" nicht verwendet, da er der Komplexität der verschiedenen ethischen Gruppen Zentralsulawesis nicht gerecht wird.

32 Die Bugis stellen in Südsulawesi eine der größten ethnischen Gruppen dar. Dank ihrer Unternehmensfreudigkeit, ihrer hohen Mobilität, ihrer Kenntnisse im Schiffsbau und in der Seefahrt sowie ihrer Risikobereitschaft, fremde Gebiete mit neuen Nutzungsmehtoden zu kultivieren, waren Bugis bereits im 17. Jh. in zahlreichen Küstenregionen des Archipels vertreten (Uhlig 1984: 84 u. 94; Hardjono 1977: 84). 
Kapitel 2 - Die Untersuchungsregion

ter die Dänen. Händler aus Arabien, Indien, China, von den Philippinen etc. gingen dort ihren Handelsgeschäften nach. Im 16. Jh. wurde Makassar (auch bekannt unter dem Namen Ujung Pandang) Hauptstadt von Gowa und zugleich kosmopolitisches Handelszentrum. Im 17. Jh. spezialisierte sich das aufblühende Makassar-Reich, unterstützt von den Portugiesen, auf den Gewürznelken- und Muskathandel und zog somit den Zorn der Niederländer auf sich (Emmer et al. 1988: 216; Ricklefs 1981: 61f). In Nordsulawesi errichtete die VOC (Verenigde Oostindische Compagnie) 1608 einen weiteren Umschlagplatz in Manado. 1658 wurde dort den Spaniern, welche bereits Ende des 16. Jh. versuchten, sich im Norden der Insel zu behaupten, und dem Einfluss von Ternate (Molukken) mit der Errichtung einer Festung begegnet. Bei Parigi (Zentralsulawesi) richteten die Spanier Mitte des 17. Jh. einen Handelsposten ein, der unter dem Druck Ternates wieder eingestellt wurde. (Kotilainen 1992: 46; Ricklefs 1981: 62). Als die Niederländer 1660 portugiesische Schiffe im Hafen von Makassar angegriffen, das Fort besetzt und den Sultan von Gowa zu Gesprächen gedrängt hatten, übernahmen sie nach der Gewinnung eines BugisPrinzen als Alliierten und kriegerischen Auseinandersetzungen 1667 schließlich die Macht. Viele Bugis flüchteten nach Kalimantan oder Malaya, manche versuchten ihr Glück auch an den Küsten von Zentralsulawesi (Ricklefs 1981: 61f; Emmer et al. 1988: 216), während die Niederländer weiterhin mit ihrem Verbündeten Bone in die Auseinandersetzungen zwischen dem Makassar- und dem Bugisstaat involviert waren und ihre eigene Stellung in Makassar zu sichern suchten (Ricklefs 1981: 64).

Wie über Ostsulawesi herrschte auch über Zentralsulawesi vor dem Einmarsch der Niederländer das Sultanat von Ternate. So schreibt Wichmann:
„Die Landschaft Kajeli (Kaïli) gehört zu den wenigen bekannten Gegenden von Celebes. [...] Nur soviel mag bier bemerkt werden, dass dieselbe in früherer Zeit dem Sultan von Ternate an- gehörte, demselben aber von dem Raja von Gowa abgenommen und erst in Folge des Vertrags von Bungaya (1667) dem ursprünglichen Eigentümer zurückgegeben wurde." (1890: 984).

Bereits im 17. Jh. wurde von Palu als wichtigem Hafen im Seehandelsverkehr zwischen den Königreichen von Aceh, Bantan und Melaka (heute: Malakka) berichtet (Aragon 1992: 136). Im Jahr 1684 fiel das Gebiet an die VOC, verblieb dem Sultan von Ternate aber als Lehen. 1710 wurde dieses Gebiet dem Gouvernement von Makassar unterstellt (Wichmann 1890: 984). Nach 1840 stand der nördliche Teil Sulawesis unter der Rechtsprechung von Ambon (Molukken). Die Palu-Region wurde zuerst unter Manado verwaltet, kam 1824 aber unter die Jurisdiktion von Makassar (Südsulawesi), ,so as to unite the island politically. "(Aragon 1992: 137).

$\mathrm{Zu}$ dieser Zeit ,the interior highland groups were already involved in trade and tribute relationships with lowland kingdoms, primarily Sigi, and islamicized Kaili polity that was vassal to the kingdom of Bone in South Sulawesi. "(Aragon 1992: 111). Doch schon im neunten oder zehnten Jahrhundert sollen Bugis-Beziehungen mit der Kaili-Bevölkerung des PaluTals unternommen worden sein (ebd.). Acciaioli (1989: 65) stellt hingegen fest, dass ,[...] the Palu Valley and its surrounding mountains have long constituted a frontier for Bugis 
movement outside their homeland of the southwestern peninsula." Die Lösung ist mit Kotilainen vermutlich darin $\mathrm{zu}$ finden, dass

„,...] a clear distinction has to be made between the coastal area, i.e. Palu V alley and Tomini Gulf, and the interior mountain region. The inhabitants of Palu V alley and of Tomini Gulf had for centuries been in contact with foreign merchants and sailors, while the people living in the central highlands had less contact with people from outside their own area and had preserved more of their traditional religion and way of life." (1992: 49).

Die Bewohner dieser Berggebiete blieben nach o. A. (1912: 6ff) während der Einwanderung der sog. parigisch-kajelischen (parigisch-kailischen) Gruppe, welche vom Miu-Fluss in das Palu-Tal gekommen sein soll, in den fruchtbaren Tiefebenen der Bergregionen südlich des Palu-Tals zurück. Im Laufe der Zeit wurden sie jedoch durch Einfluss von außen in die Berge gedrängt. ${ }^{33}$

Die Lore-Lindu-Region war vor der Unterwerfung durch die Niederländer von mehreren Königreichen beherrscht. Auf der Westseite hatte das Reich von Sigi mit seinem Hauptsitz in Bora, im heutigen Kecamatan Sigi-Biromaru, den größten Einfluss, welchem auch das Herrschaftsgebiet der To Kulawi ${ }^{34}$ unterstand (Kruyt 1938, Bd. 1:27). Im Osten der Untersuchungsregion bildeten die To Pekurehua (Fremdbezeichnung: To Napu) ein großes Einflußgebiet, die, wie auch das kleine Reich der To Tawaeli (heute: Sedoa) nördlich des Napu-Tals, gegenüber dem Herrscher von Sigi einen Vasallenstatus einnahmen (Kruyt 1908: 1272 u. 1331)

\subsubsection{Die ersten Europäer in Zentralsulawesi}

In der Mitte des 19. Jh. hatten das Militär und die Zivilregierung der Niederländer viele Fragen bzgl. des Gebiets um den Poso-Fluss aufgeworfen, aber erst in den 1890ern wurde die Region systematisch studiert (Kaudern 1925b: 29). Der niederländische Missionar Albert Christiaan Kruyt (1869-1949) wurde 1891 als erster vom Assistent-Resident von Gorontalo zur Mündung des Poso-Flusses begleitet, um dort mit seiner Missionsarbeit für die niederländische Missionsgesellschaft (Nederlandsch Zendinggenootschap) zu beginnen. Enttäuscht über die mäßige Bereitschaft der Bevölkerung, sich bekehren zu lassen, begrüßte er später die Erschließung des Gebietes durch die niederländische Kolonialverwaltung (Coté 1979: 36).

Der zweite bedeutende Missionar in dieser Region war der an Sprachen interessierte Nicolaus Adriani (1965-1926), welcher 1895 von der Niederländischen BibelGesellschaft (Nederlandsch Bijbel Genootschap) nach Poso geschickt wurde, um eine

33 Wer oder was mit diesem Einfluss gemeint ist, wird leider nicht erwähnt. Es könnte sich um Bugis aus dem Süden oder um Menschen um das Fürstentum Sigi in der Palu-Ebene gehandelt haben bzw. um Auseinandersetzungen der beiden im Gebiet dieser Bergregion. „To“ bedeutet Mensch. „To Kulawi“ kann somit als „Menschen von Kulawi“ übersetzt werden. 
Kapitel 2 - Die Untersuchungsregion

Ausgabe der Bibel in der lokalen Bare'e-Sprache zu erarbeiten. Er war im Gegensatz zu Kruyt kein großer Freund der Kolonisierungspolitik, wie sie von der niederländischen Kolonialregierung betrieben wurde (Kotilainen 1992: 21). Mit ihren Werken wie „De Bare'e-sprekende Toradjas van Midden-Celebes“ von 1912-14 sammelten jedoch beide die Grundlagen, mit deren Hilfe die Kolonialregierung ihre Kolonisation vorantrieb. Den beiden Missionaren Adriani \& Kruyt folgten nach einigen Jahren die Brüder Sarasin, zwei Schweizer, die vor allem geologische Untersuchungen in Zentralsulawesi zu ihrer Aufgabe machten (ebd.; Sarasin \& Sarasin 1901). Die weitere Entwicklung der Kolonisierung bringt Kaudern folgendermaßen auf den Punkt: „Later, development proceeded so quickly that at present very little is left of the origin culture. [...] Villages and heathentemples have been levelled with the ground, and new villages have been built after modern principles. "(1925b: 29f).

Im westlichen Teil des Untersuchungsgebiets, der viel schwerer zugänglich ist als der östliche Teil, lebten die Menschen lange Zeit ungestört in ihren Bergregionen. Vor der Erschließung des Westens von Zentralsulawesi 1905 waren schon verschiedene Forschungsreisende in Palu gewesen. Mehr als über diesen Hafenort und einen Blick in das Palu-Tal wussten sie aber meist noch nicht zu berichten. So schreibt Hart über das Palu-Tal: „Das Land scheint sehr fruchtbar zu sein, und wenn der Boden bearbeitet wird, sollte hier dann ein großer Vorrat an allerlei (Erd-)früchten, wie es tropischen Gebieten eigen ist, geerntet werden können. ${ }^{35}$ (1853: 264). Die Einheimischen, so Harts abschätzige Beurteilung, seien aber faul und träge und täten nur das, was für den täglichen Lebensunterhalt von Nöten wäre. Auf die Fruchtbarkeit des Tals und den „Charakter` der dort lebenden Menschen geht auch Wichmann ein:
„Da uns in Makassar gesagt worden war, dass es hier Reis in Ueberflusse gäbe, - und das ganze Strandgebiet ist in der That sehr fruchtbar [...]. Durchaus zutreffend sagte schon der biedere $V$ alentijn: „, het is een gezegnet land“; wenn er jedoch unmittelbar darauffolgen lässt „,dog van le- ven en vervloekt Sodom" (Oud- en Nieun Oost-Indien I, 1727, p.75), so thut er damit der Be- völkerung bitteres Unrecht an." (1890: 984f).

Bastian (1889) geht in seinen Reiseaufzeichnungen ebenfalls nur kurz auf Palos alias Palu ein: „Der Fürst von Kajeli residiert (als Maradika) in Palos [...]. Von der Bucht in Palos (bei Kajeli) findet sich ein Handelsweg durch Celebes nach den Bergen von Tomini. " (1889: 46). Die bereits erwähnten Brüder Paul und Fritz Sarasin kritisieren in ihren geographisch-geologischen Beschreibungen (1901) die Verwendung des Namens Palos für den Ort Palu, wie es auch Bastian (1889) getan hatte, und erklären: „Wir vermuten, daß dies Versehen den Spaniern zur Last fällt, welche durch den Namen Palu an den andalusischen Hafen Palos erinnert wurden, von welchem Columbus seine erste Ausfahrt genommen hat."

35 Zitat im Original: „Het land vertoont zich zeer vruchtbaar en wanneer de grond goed bewerkt wird, zoude hij ruimer voorraad, dan nu, van allerlei aardgewassen en vruchten kunnen voortbrengen, aan tropische gewesten eigen." 
(Sarasin \& Sarasin 1901: 163) ${ }^{36}$. Auf ihrer Reise von Palu im Norden nach Palopo im Süden konnten die Sarasins die Wasserläufe entlang der sog. Sarasin-Linie kartieren und die Richtung der Gebirgszüge festhalten. Als erste Europäer besuchten sie die Gebiete von Gimpu, Bada und Leboni (Kaudern 1925b: 30). Um die Einheimischen von Napu zu besuchen, welche als die gefürchtetsten Kopfjäger ganz Zentralsulawesis bezeichnet wurden, gingen bereits zwei Jahre vorher die Missionare Adriani \& Kruyt 1898 zum raja ${ }^{37}$ von Sigi im Palu-Tal, um ihn um seine Erlaubnis zu bitten, da er nominell der Herrscher von Napu war. Ihnen wurde erlaubt, über Lindu nach Napu zu gehen. Sie kamen sicher nach Kulawi und später auch nach Lindu, konnten aber, nach der Angst der Leute von Lindu vor den To Napu zu urteilen, nicht nach Napu weiter und mussten umkehren (ebd.).

Nach der „Befriedung“ von Zentralsulawesi unternahmen eine Reihe von Missionaren, Armeeangehörigen, Naturwissenschaftlern und Ethnologen Reisen in dieses Gebiet. Neben den Missionaren Schuijt und Ten Kate studierte der ab 1918 in Kantewu stationierte Heilsarmee-Offizier Woodward die lokalen Kulturen und Sprachen. Loois, ebenfalls Heilsarmist, erkundete die Sprachen der Kulawi-Region. Unter den niederländischen Offizieren erforschten Kiliaan, Hissink und Captain Boonstra van Heerdt Zentralsulawesi. Hissink studierte dabei die Gebiete des PaluTals und jene südlich des Palu-Flusses bis hin zum Koro-Fluss (Lariang-Fluss). Van Heerdt erwarb sich Meriten mit der Erforschung der Quellen von Palu- und KoroFluss. Der Geologe Abendanon untersuchte Zentralsulawesi in den Jahren 1909 und 1910. Ein Jahr später durchquerte Grubauer das Gebiet, indem er, von Poso kommend, in einem Bogen über Gimpu nach Palu gelangte. Nach ihm machte der Amerikaner Raven zoologische Sammlungen u.a. am Lindu See, im Kulawi- und im Gimpu-Tal (Kaudern 1925b: 31f).

\subsubsection{Die niederländische und japanische Kolonialherrschaft} (1904 bis 1945)

Bereits Anfang des 18. Jh. erbat der magau von Palu eine Intervention der VOC gegen die seeräuberisch tätigen Mandar. Nach Acciailoi (1989: 76) hatten Mandaresen seit 1730 die Palu-Region so sehr verwüstet, dass nicht mehr genug Kokosöl für die Tributzahlungen an das Gouvernement in Makassar produziert werden konnte.

36 Die Brüder Sarasin zitieren ihrerseits noch Bleeker (1856). Dieser habe geschrieben: „Central-Celebes ist uns ebensowenig bekannt, als unsere Autorität gering ist. Die Wünschbarkeit einer wissenschaftlichen Expedition dahin ist nicht zweifelhaft" (ebd.). Diese Worte galten nach Sarasin \& Sarasin (1901: 170) ,,also noch jetzt für den bier ins Auge gefassten Theil der Insel“"

37 Höchste Position in der traditionellen soziopolitischen Gesellschaftsstratifikation. Entspricht in etwa einem/einer Fürsten/Fürstin oder König/Königin. 
Kapitel 2 - Die Untersuchungsregion

Das westliche Zentralsulawesi kam ab 1904 unter faktische niederländische Verwaltung. Zwar war bspw. Palu bereits 1864 offiziell den Niederlanden untergeben (Atlas Van Tropisch Nederland 1938, Blatt 10a) ${ }^{38}$, doch der direkte Eingriff in die Verwaltung dieser Region wurde erst im 20. Jh. im Rahmen der Expansions- und „Entwicklungshilfe“-Politik unter „ethischem“ Vorzeichen - die sog. Ethische Politik - vollzogen (vgl. Kap. 6.1). ${ }^{39} \mathrm{Im}$ Jahr 1888 wurde Zentralsulawesi gegen die Engländer gesichert. 1894 wurde in Poso, der späteren Verwaltungshauptstadt des östlichen Zentralsulawesi, ein Kontrolleur eingesetzt (Coté 1996: 93). In der Region Palu-Bucht wurde 1891 in Donggala ein Posthouder eingesetzt und 1893 die Afdeeling Paloebaai besiegelt. Im Jahr 1895 wurde der Posthouder durch einen Civiel Gezaghebber ersetzt. 1905 wurde schließlich die Afdeeling Midden-Celebes gegründet, wobei Donggala und Tawaeli unter die Aufsicht eines Controleurs in Donggala kamen, während die Gebiete von Palu nach Süden (Palu, Sigi-Biromaru, Dolo und Kulawi) dem Controleur von Palu unterstanden (Acciaioli 1989: 73f).

Mit der „Befriedung“ der Außenbesitzungen und der Einführung der sog. Korte Verklaringen ${ }^{40}$ wichen die Niederländer von der bis dahin herrschenden Enthaltungspolitik ${ }^{41}$ ab. C. Snouck Hurgronje, ${ }^{42}$ ein einflussreicher Arabist, und J. B.

38 Die Residenz Manado als nördlicher Teil Sulawesis wurde 1824 als Teil des Gouvernements der Molukken geschaffen und 1865 nach Aufgabe der Monopolstellung im Gewürzhandel in den nördlichen Provinzen zu einer unabhängigen Verwaltungseinheit (Leirissa 1996: 267).

39 Mit der verstärkten Aufarbeitung der Kolonialgeschichte des eigenen Landes in den 1980er und 1990er Jahren wurden darin v.a. sozioökonomische und historische Themenkomplexe der niederländischen Kolonialherrschaft in Indonesien analysiert. Die Aufarbeitung der niederländischen Kolonialgeschichte wurde seinerzeit durch eine Imperialismus-Debatte angeregt, die der Frage nachging, ob die Expansion der Kolonialherrschaft auf die sog. Äußeren Inseln des indonesischen Archipels als moderner Imperialismus zu werten sei oder nicht. An der Debatte beteiligten sich z.B. Locher-Scholten (1994), Kuitenbrouwer (1991), Coté (1996), Cribb (1994), Prince (1995). Bezugnehmend auf die seit etwa 1901 propagierte Ethische Politik, wurde auch eine Auseinandersetzung über den ethischen Gehalt der niederländischen Kolonialpolitik in Südostasien geführt. Aufbauend auf dem Standardwerk von Locher-Scholten (1981), griffen Veröffentlichungen u.a. von Otterspeer (1989), Boomgaard (1986, 1993), Houben (1994), Coté (1996) und Wedema (1998) dieses Thema auf.

40 Vor der Einführung der Kurzdeklarationen (Korte Verklaringen) gab es auch noch Verträge in längerer Fassung (vgl. Modell-Fassung von 1875 in Spit (1911: Beiblatt II)), die für die Unterwerfung der direkt verwalteten Gebiete eingesetzt wurden.

41 Diese Politik begnügte sich mit der Ausbeutung der Kolonie, ohne sich um Belange der einheimischen Bevölkerung zu kümmern. Ein Beispiel aus den Koloniale Verslagen von 1900 bzgl. der Region Djambi (Jambi) auf Sumatra macht diesen Politikwandel deutlich: „In Djambi heerschte een staat van anarchie als sinds lang niet voorgekommen was. De sultan, een oud en afgelefd man, bemoeide zich bijna niet meer met het bestuur des landes. Dij de Indische regeering zijn thans maatregelen in overweging om aan den toestand van regeerinsloosheid en einde te maken. "(zitiert nach Loze 1929: 13).

42 Snouck Hurgronje verfolgte Mitte des 19. Jahrhunderts eine Teile-und-herrsche-Politik. Er vertrat den Standpunkt, dass die Niederlande einen größeren Gewinn aus einer pro-islamischen Politik ziehen würden als aus einer Konvertierung der Bevölkerung zum Christentum (Kipp 1990: 32f). 
van Heutzs, der Hauptverantwortliche für die brutale Niederschlagung der Acehrevolten 1878-1908, gaben den Anstoß zu dieser neuen Politikform (Loze 1929: 11). Ab 1907 wurde mit dem Beginsel-Programma Van Heutsz die vorherige NichtEinmischungspolitik aufgegeben und auch in den sog. zelfbesturende landschappen (selbstverwaltete „Landschaften“) die Wohlfahrt und „Entwicklung“ von Land und Bevölkerung als Pflicht der Kolonialregierung festgelegt. Verwaltung und Rechtsprechung sollten unter Vermeidung eines gewalttätigen Auftretens ebenso verbessert werden wie ,den oeconomischen en socialen toestand der inheemsche bevolking" (Loze 1929: Beilage 1). Dies müsste unterstützt werden durch eine Entwicklung ,van de natuurlijke bronnen van welvaart des landes" (ebd.). Mit den zitierten Punkten aus dem Programm von Van Heutsz wird die Vermutung gestärkt, dass es im Untersuchungsgebiet zu umfangreichen und tiefgreifenden Einmischungen in das traditionelle Gefüge der Kulturlandschaft seitens der niederländischen Kolonialmacht gekommen sein muss.

Mit der Unterzeichnung der Korte Verklaringen wurden die zelfbesturende landschap$p e n^{43}$ eingerichtet, d.h. Gebiete, die im Gegensatz zu direkt verwalteten Regionen ${ }^{44}$ weiterhin über die lokalen traditionellen Fürsten verwaltet wurden. Die Korte Verklaring war eine uniforme Erklärung, die aus drei Artikeln bestand. Mit der Zustimmung zu Artikel 1 erkannte die betreffende landschap die Oberherrschaft der Niederlande an. Artikel 2 forderte das Versprechen ein, sich mit keinen fremden Mächten auf staatlicher Ebene anzunähern. Durch Artikel 3 wurde versprochen, dass die Bevölkerung des betreffenden Gebietes allen Befehlen etc. der Königin der Niederlande, des Generalgouverneurs und dessen Stellvertretern nachkommen würde. Durch die Unterzeichnung dieses Schriftstücks erhielten die Kolonialbeamten eine uneingeschränkte Regelungsbefugnis, auch wenn Spit (1911: 132) behauptet, dass ein Freibrief für jedes Unrecht nicht vorgelegen hätte. Loze (1929: 14) erwähnt, dass die Verwaltungsbeamten von dieser Befugnis durchaus großzügig

43 Unter den verschiedenen Vertretern der Kolonialpolitik bzw. entsprechender Akademiker herrschte durchaus Uneinigkeit in bezug auf Sinn und Zweck von Selbstverwaltungen. Loze (1929: 23f) führt hierzu drei Positionen an: Abdul Moeis zeigte sich mit der Regierungspolitik unzufrieden. Nach ihm hätte eine Modernisierung der Selbstverwaltung, Schulung der Selbstverwalter und das Herausheben der Bevölkerung aus dem Elend mit Nachdruck angepackt werden müssen, und nicht, wie in vielen Gegenden (z.B. Midden-Celebes), nur wegen der Bequemlichkeit und des leichteren Regierens. Die Binnlandsch Bestuur (Inländische Verwaltung) antwortete darauf, dass Modernisierung ein Kostenfaktor und gerade in Midden-Celebes eine entsprechende Entwicklung nicht der Mühe wert wäre. Van Hinloopen Laberton wandte sich 1920 wiederum mit folgender Begründung gegen die Selbstverwaltungspolitik, welche Loze (1929: 24) folgendermaßen zusammenfaßt: „Die Selbstverwalter, u.a. in Celebes, sind ihm zufolge, willenlose Automaten ' in den Händen einer absolut autokratischen Verwaltung. Er nennt die Kurydeklaration einen ,Scheinvertrag: “(Zitat im Original: „De Zelfbesturen o.a. in Celebes zijn volgens hem, willoze automaten' in de handen van een absoluut autocratisch bestuur. Hij noemt de Korte Verklaring een ,contract van schijn'“').

Auf Sulawesi waren z. B. die meisten Landschappen Südsulawesis direkt verwaltet worden. 
Kapitel 2 - Die Untersuchungsregion

Gebrauch gemacht hatten, obwohl es nach seinen Angaben - ganz im ,ethischen“ Sinne - in der Anfangszeit vornehmlich darum gegangen wäre, Ordnung in das Chaos zu bringen, welches die Nicht-Einmischungspolitik hervorgerufen hätte. Das Fürstentum Sigi, dessen politisches Machtzentrum in dem Dorf Bora (im heutigen Kecamatan Sigi-Biromaru) lag, unterzeichnete im Jahr 1904 als erstes der vorkolonialen Herrschaftsgebiete der Lore-Lindu-Region die Korte Verklaring (Hissink 1909: 95 u. 127; Hirschmann 1934: 27). Erst zwei Jahre später wurde das KulawiGebiet unter koloniale Vorherrschaft gebracht. Die für die Kulawi-Krieger erfolgreiche Schlacht gegen die unter niederländischem Befehl stehenden Soldaten am Berg Momi im Jahr 1905 ist vor allem unter der älteren Bevölkerung Kulawis bis heute ein wichtiger Baustein ihrer Ethnizität. Nachdem der Versuch der Niederländer, über diesen Berg, der Kulawi nach Norden hin gegenüber dem Kec. Sigi-Biromaru abgrenzt, in die Region vorzudringen, gescheitert war, setzten die Niederländer unter Androhung eines Massakers die Einwohner des Ortes Tuwa unter Druck, woraufhin diese einen Alternativweg nach Kulawi entlang des Flusses Miu preisgaben. Inowaa Tomatorengke, seinerzeit raja von Kulawi, wurde schließlich von den Niederländern aufgefordert, alle Krieger aus den Bergen zurückzuziehen, da ansonsten das gesamte Kulawi-Gebiet niedergebrannt würde. Am 30. November 1908 wurde mit der Unterzeichnung einer Korte Verklaring den Niederländern die offizielle Hoheit über Kulawi zugesprochen (Departmen Pendidikan Dan Kebudayaan 1996/1997: 115f; Pamei 1997: 6ff). Die Unterzeichnung der Korte Verklaring für die Region Napu erfolgte im Jahr 1908. Der Unterwerfung der lokalen Bevölkerung unter die niederländische Krone ging eine militärische Auseinandersetzung im Jahr 1907 voraus, die unter der Bezeichnung Peore-Krieg (perang Peore) bekannt ist (Kruyt 1908: 1276).

Die europäische Verwaltung der Residentie Manado wurde in die Hände dreier Assistent-Residenten gelegt. Diese hatten Afdeeling-, Onderafdeeling-Chefs, Kontrolleure, Befehlshaber, Verwaltungsbeamte und Hilfskräfte unter sich (Logeman 1922: 96). Die Residenz Manado wurde in Afdeelingen eingeteilt, welche ihrerseits in Onderafdeelingen separiert wurden. Die Afdeelingen unterstanden einem niederländischen Assistent-Resident, während die Onderafdeelingen durch einen Controleur überwacht wurden. Die Basis des Verwaltungssystems bildeten die lokalen Dörfer, welche in Distrikte zusammengefasst waren. Die Afdeeling basierte auf der ethnischen Einteilung der Bevölkerung. Die Distrikte waren an die Sprachgrenzen angepasst (Leirissa 1996: 267). Die Niederländer erschlossen weite Teile der Lore-Lindu-Region mit einem Strassen- und Wegenetz, führten Umsiedlungen der lokalen Bevölkerung und Neustrukturierungen des Siedlungswesens durch, bereiteten den Weg für die christlichen Missionierung und bewirkten eine Monetarisierung und zunehmende Marktausrichtung der Wirtschaft. Eine detailliertere Untersuchung dieser Eingriffe in das Kulturlandschaftsgefüge der Region wird in den nachfolgenden Kapiteln vorgenommen (vgl. Kap. 3 bis 6). 
Im Jahr 1942 besetzte Japan den indonesischen Archipel. In der Lore-Lindu-Region führten die Japaner viele der von den Niederländern begonnen Maßnahmen fort. Die dreijährige Präsenz der Japaner stand unter dem Einfluss des 2. Weltkriegs, wodurch v.a. der Handel zum Erliegen kam und die landwirtschaftliche Produktion stärker als während der niederländischen Herrschaft auf die Interessen der Kolonialherren fokussiert wurde. Die Anwesenheit der Japaner, deren Einflüsse auf die Lore-Lindu-Region bislang kaum dokumentiert sind ${ }^{45}$ (vgl. Aragon 1996a; Weber et al. 2003), wird von lokalen Zeitzeugen als unterdrückender als die niederländische Kolonialpräsenz erinnert. Während die Niederländer unter dem Einfuss ihrer Ethischen Politik (vgl. Kap. 6.1) z.T. durchaus versuchten, die Interessen der lokalen Bevölkerung zu berücksichtigen, setzten die Japaner ihren Willen grundsätzlich mit Gewalt durch. Das Verhältnis zwischen Kolonialmacht und Lokalbevölkerung war, anders als unter den Niederländern, von permanenter Angst geprägt. Wurde den Anordnungen der japanischen Besatzer nicht entsprochen, setzten diese physische Gewalt gegen die betreffenden Personen ein.

In der Gegenüberstellung der niederländischen und der japanischen Phase der Kolonialherrschaft haben alle Befragten übereinstimmend die niederländische Hegemonie als positiver dargestellt. Allerdings ist hierbei, und auch bei der Interpretation des Interviewmaterials, zu berücksichtigen, dass die sehr negativen Erfahrungen der nachfolgenden japanischen Herrschaft die Wahrnehmungen über die vorangegangene Einflussnahme der Niederländer beeinflusst haben.

\subsubsection{Die Phase der Unabhängigkeit Indonesiens (ab 1945)}

Die beiden großen politischen Phasen Indonesiens ab 1945 bringen Cribb \& Brown folgendermaßen auf den Punkt: „In 1945 the task at hand had been defending independence against the Dutch. In 1966 the task defined by the Suharto government was development, pembangunan." (1995:115).46 Die unter der niederländischen Herrschaft bestandenen selbstverwalteten Gebiete wurden in den 1950 er Jahren in ein dreigliedriges Verwaltungssystem überführt, das von da an aus Provinzen, Kabupaten und Dörfern bestand. Die Zentralregierung jener Zeit sah es aufgrund der Größe und Diversität Indonesiens als notwendig an, einen Teil der politischen Entscheidungsprozesse in die Verantwortung der Provinzen zu

45 Eine Quellenrecherche in japanischen Archiven konnte aus Sprachgründen vom Verfasser nicht vorgenommen werden. Bei dem Vergleich der Inhalte niederländischer Archivquellen mit den Interviewaussagen von Zeitzeugen hat sich eine sehr hohe Übereinstimmung ergeben. Daher erscheint es angesichts der relativ kurzen Periode der japanischen Kolonialherrschaft in der Untersuchungsregion gerechtfertigt, diese Zeit „nur“ anhand des gewonnenen Interviewmaterials zu untersuchen. 
Kapitel 2 - Die Untersuchungsregion

überstellen, da nur so eine effektive Regierung funktionsfähig war (Cribb \& Brown 1995: 36f). Gegenüber der Aufteilung in zwei Residentie unter der niederländischen Kolonialherrschaft bestand die Insel Sulawesi im Jahr 1950 nur noch aus einer Provinz. Diese wurde von Makassar aus regiert.

Im Laufe der 1950er Jahre bildeten sich auf der Insel verschiedene politische Einflusssphären aus, welche von bewaffneten Konflikten ausgelöst wurden. Der bedeutendste dieser Konflikte wurde zwischen Anhängern der Darul IslamBewegung $^{47}$ (DI/TII) ${ }^{48}$ und der Staatsarmee ausgetragen. Herausragender Anführer der Bewegung war Kahar Muzzakar, der in seiner Heimat Südsulawesi eine Abspaltung von der Zentralregierung durchsetzen und eine autonome islamisch fundierte Region gründen wollte. Dieser Konflikt blieb allerdings nicht nur auf den Süden der Insel beschränkt. Er löste eine Flucht vor den gewalttätigen Auseinandersetzungen aus, die eine Reihe von Menschen nach Zentralsulawesi und in die Lore-Lindu-Region führte. Ein weiterer Konflikt entstand durch die PermestaBewegung (1957-1961), welche in Nordsulawesi einen bewaffneten Kampf führte. Diese Bewegung wurde 1957 in Makassar proklamiert und hatte die Erlangung größerer Autonomie von der Zentralregierung in Jakarta zum Ziel. Ende der 1950er Jahre bildete sich in Zentralsulawesi die Gerakan Pemuda Sulawesi Tengah (GPST; Jugendbewegung Zentralsulawesi), welche an der Seite der staatlichen Armee die Permesta bekämpfte (Velthoen 2002).

Die 1950er und 1960er Jahre brachten der Lore-Lindu-Region daneben kaum nennenswerte Entwicklungen. Erst mit dem militärischen Staatsstreich Suhartos, dem die blutige Niederschlagung eines inszenierten Putschversuchs angeblich kommunistischer Kräfte im Jahr 1966 vorausging, durchlief die Region nachhaltige Veränderungen. Mit Hilfe enormer Steigerungen der Exporteinnahmen aus dem Erdölverkauf, die von 1970 bis 1981 um 45,5\% zunahmen und 1981 82\% aller Exporterlöse ausmachten (Vatikiotis 1993: 35), konnten die von der Regierung geplanten infrastrukturellen Entwicklungprogramme umgesetzt werden. Diese in Form von Fünf-Jahres-Plänen (repelita) strukturierten Programme förderten die landwirtschaftliche Technisierung und Intensivierung, welche v.a. auch die sog. Äußeren Inseln des Archipels einen wirtschaftlichen Aufschwung erfahren lassen sollten. Zudem wurden die lokalen Umsiedlungsprogramme aus den Zeiten der niederländischen Kolonialherrschaft forciert, um die sog. primitive Bergbevölkerung aus ihrer wirtschaftlichen und infrastrukturellen Isolation herauszuholen.

47 Darul Islam (wörtlich übersetzt: „Haus des Islam“) ist der Name für eine Rebellion, die sich 1948 in Westjava entwicklete und bis in die 1960er Jahre hinein aktiv war. Sie hatte ihren Ursprung in der Weigerung islamischer Rebellen, die zentralstaatliche Autorität Indonesiens nach Erlangung der Unabhängigkeit des Staates anzuerkenen. Von Java aus breitete sich diese Rebellion während der 1950er Jahre nach Sumatra und Südsulawesi aus (Liefer 1996: 93f).

48 DI/TII = Darul Islam / Tentara Islam Indonesia (wörtlich übersetzt: „Haus des Islam/Soldaten des indonesischen Islam“). 
Mit der Verabschiedung des Regionalen Verwaltungsgesetzes (Gesetz Nr. 1/1957) wurden sämtliche autonomen Verwaltungseinheiten abgeschafft, welche bis zu diesem Zeitpunkt noch von Sultanen oder traditionellen Führern regiert worden waren. Die Suharto-Regierung verfolgte einen streng hierarchischen Aufbau der Verwaltung, mittels dessen die politischen Interessen und Ideologien der Zentralregierung in Jakarta bis auf die Dorfebene transportiert wurden. Eine entsprechend hohe Autorität genossen die lokalen politischen Repräsentanten gegenüber der Bevölkerung. Das Handeln des Dorfchefs wurde als das der Regierung identifiziert und Kritik an diesem Handeln wurde als Kritik an der Zentralregierung wahrgenommen, welche über einen ausgedehnten Repressionsapparat verfügte.

Erst mit dem Sturz Suhartos, der Verabschiedung des Gesetze zur Regionalen Autonomie und politischen Dezentralisierung und den damit verfolgten Demokratisierungsbestrebungen wurde das autoritär-repressive System der sog. Neuen Ordnung (Orde Baru) teilweise aufgebrochen. Der rasche Übergang von der jahrzehntelangen, streng autoritären Politikführung löste eine politische Unsicherheit der gesamten Gesellschaft darüber aus, in welcher Weise die neu gewonnenen Freiheiten ausgelegt werden können. Eine der daraus resultierenden Folgen war das offene Austragen der während der Suharto-Ära bereits latent vorhandenen, aber vom Militär niedergehaltenen regionalen Konflikte (vgl. Weber 2004). Entweder handelte es sich dabei um Separationsbestrebungen (z.B. in Osttimor) oder um Auseinandersetzungen zwischen alteingesessener Bevölkerung einer Region und Migranten, wobei die lokale Bevölkerung versuchte, die an die Migranten zum Teil verlorene politische und wirtschaftliche Hegemonie zurückzugewinnen (z.B. in der an das Untersuchungsgebiet angrenzenden Poso-Region).

\subsection{Die Untersuchungsdörfer}

In Tab. 1 wurden die im Rahmen dieser Arbeit untersuchten neun Dörfer bereits benannt und ihre Zuordnung zu den unterschiedlichen Verwaltungseinheiten der Region vorgenommen. Die räumliche Verortung der Dörfer kann der Übersichtskarte in Abb. 5 entnommen werden. Die Distanz zum Hauptort des jeweiligen Kecamatan ist in Tab. 2 dargestellt. Dieses Kapitel dient nun einer überblicksartigen Skizzierung dieser Dörfer. Zwar wird auf die einzelnen Dörfer im Verlauf der folgenden Kapitel immer wieder Bezug genommen werden, doch erscheint die Vorwegnahme einer Gesamtschau für eine bessere Orientierung hilfreich.

Die neun Untersuchungsdörfer sind über die gesamte Forschungsregion des SFB 552 - STORMA, mit Ausnahme des Kecamatan Lore Selatan, verteilt. Der Ausschluss des Kecamatan Lore Selatan ergibt sich aus dem sog. STORMA village sample (vgl. Kap. 1.4). Zwei der Dörfer, Maranatha und Pandere, befinden sich im Palu-Tal (Kec. Sigi-Biromaru) und liegen mit ca. 20 bzw. 44 km Entfernung mit 
deutlichem Abstand am nächsten an der Provinzhauptstadt, dem politischen und wirtschaftlichen Hauptbezugsort für alle untersuchten Dörfer.

Maranatha wurde 1969 im Rahmen einer staatlichen Maßnahme zur Umsiedlung der Bevölkerung aus verschiedenen Orten der westlichen und östlichen Randgebirge des Palu-Tals aufgebaut. Bis zu seiner offiziellen Anerkennung als eigenständiges Dorf im Jahr 1976 trug die Siedlung den Namen Rarantikala. Die übergroße Bevölkerungsmehrheit des rund 2.400 Einwohner ${ }^{49}$ zählenden Dorfes bilden Kaili aus den Randgebirgen des Palu-Tals.

Tab. 2 Distanzen der Untersuchungsdörfer zum Hauptort des jeweiligen Kecamatan

\begin{tabular}{|c|c|c|c|}
\hline \multirow[b]{2}{*}{ Dorfname } & \multirow[b]{2}{*}{$\begin{array}{l}\text { Hauptort des } \\
\text { Kecamatan }\end{array}$} & \multicolumn{2}{|c|}{ Entfernung zum Hauptort (in km) } \\
\hline & & $\begin{array}{l}\text { per PKW befahr- } \\
\text { barer Strecken- } \\
\text { teil }\end{array}$ & $\begin{array}{c}\text { nicht per PKW } \\
\text { befahrbarer Stre } \\
\text { ckenteil }\end{array}$ \\
\hline Maranatha & Mpanau & 16 & - \\
\hline Pandere & Mpanau & 40 & - \\
\hline Bolapapu & Bolapapu & 0 & - \\
\hline Lempelero & Bolapapu & 32 & - \\
\hline Lawe & Bolapapu & 47 & 30 \\
\hline Berdikari & Makmur & 6 & - \\
\hline Sintuwu & Makmur & $4(20)^{50}$ & - \\
\hline Watumaeta & Wuasa & 3 & - \\
\hline Wanga & Wuasa & 10 & - \\
\hline
\end{tabular}

Das im südlichen Teil des Palu-Tals gelegene Pandere (2.180 Einwohner) wurde bereits 1927 als Zielort einer lokalen Zwangsumsiedlung durch die niederländische Kolonialmacht gegründet. Im Gegensatz zu Maranatha befindet sich ein großer Teil des Dorfterritoriums innerhalb des Lore-Lindu-Nationalparks. Während die Dusun 1

49 Die Einwohnerzahlen der Untersuchungsdörfer beruhen auf den statistischen Erhebungen der Dorfadministrationen von 2001 bzw. 2002.

50 Die direkte Verbindung nach Makmur verlief bis zum Beginn des 21. Jh. entlang einer Schotterstrasse, welche gelegentlich nicht passierbar war. Der in Klammern gesetzte Wert gibt die ungefähre Entfernung über die Alternativstrecke entlang der sog. Jalan Jepang (Japanische Strasse) auf der Südseite des Palolo-Tals an. Seit 2004 hat sich die Erreichbarkeit Sintuwus durch strassenbauliche Maßnahmen verbessert. 
und 3 mehrheitlich von der lokalen Kaili-Bevölkerung bewohnt sind, leben in Dusun 2 v.a. regionale Migranten aus Manado (Nordsulawesi). Den größten Teil der landwirtschaftlich genutzten Fläche nehmen Nassreisfelder ein. Etwa 20 \% der Einwohner bauen Kaffee an, 50 \% Kakao. Daneben bestehen auch Kokoskulturen.

Im Kecamatan Kulawi liegen drei weitere der Untersuchungsdörfer: Bolapapu, Lempelero und Lawe. Das Dorf Bolapapu setzt sich aus mehreren, während der Kolonialzeit noch eigenständigen Dörfern zusammen und erstreckt sich etwa acht Kilometer entlang der Hauptstrasse Palu-Kulawi, wobei die bebauten Areale der einzelnen Dorfteile nur im Zentrum des Dorfes (Dusun 1 und 2) direkt ineinander übergehen. Das Dorf, welches den Hauptort des Kec. Kulawi repräsentiert, grenzt nach Osten hin direkt an den Nationalpark. Auf etwa 700 m ü. N.N. werden neben Nassreis (Dusun 1 bis 4) vor allem Kaffee und Kakao angebaut. Im Dusun 5 weicht der Nassreisanbau aufgrund der Verschmälerung des Tals nach Norden hin hinter den Trockenreisanbau zurück. Der Anteil der zugewanderten Bevölkerung des 4.027 Einwohner zählenden Ortes ist angesichts der infrastrukturellen Rahmenbedingungen relativ gering. In allen Dorfteilen halten die Kulawi die absolute Bevölkerungsmehrheit. Vor allem in Dusun 1 bilden Bugis neben Chinesen, Javanern und Toraja, welche alle die ökonomische Elite des Dorfes vertreten, die größte Migrantengruppe. In Dusun 2 bilden Migranten aus Napu und Besoa sowie aus Manado und Java die größten nicht-einheimischen Bevölkerungsgruppen. Nennenswert ist ferner eine kleinere Anzahl von Bugis-Familien in Dusun 4 und v.a. Dusun 5.

Lempelero (1.044 Einwohner) befindet sich im Gimpu-Tal im Süden Kulawis, am Ende der asphaltierten Verbindungsstrasse nach Palu. Das Dorf wurde im Jahr 1972 gegründet, nachdem die Bewohner ihr vorheriges Dorf Tompi Bangka nach einem verwüstenden Erdrutsch verlassen mussten. Heute ist das Dorf auf drei Siedlungsgebiete verteilt welche bis zu zehn Kilometer voneinander entfernt liegen. Diese Verteilung beruht darauf, dass sich nicht alle der ehemaligen Bewohner von Tompi Bangka in der Hauptsiedlung Lempeleros niedergelassen haben. Alle heutigen Siedlungsteile des Dorfes befinden sich auf dem traditionellen Land der Vorfahren ihrer Bewohner. Neben lokalen Migranten, v.a. aus der Pipikoro-Region westlich von Lempelero, haben sich hauptsächlich Bugis aus Südsulawesi angesiedelt, welche als Händler im Hauptort des Dorfes direkt an der Asphaltstrasse wohnen.

Lawe nimmt unter den neun Untersuchungsdörfern eine gewisse Sonderrolle ein. Das Dorf befindet sich im Pipikoro-Gebiet im westlichen Kulawi, der verkehrstechnisch rückständigsten Region des Forschungsgebiets. Die Distanz zur Hauptstrasse Palu-Kulawi beträgt ca. 40 km, welche größtenteils nur zu Fuß oder per Pferd zurückgelegt werden kann. Im Jahr 1980 war die Bevölkerung des Dorfes, welches bereits in vorkolonialer Zeit existierte, aufgrund der unzureichenden Verfügbarkeit von Wasser gezwungen, ihre Siedlung einige Kilometer vom ursprünglichen Dorf entfernt neu zu errichten. Lawe, das mit knapp 400 Einwohnern das kleinste Untersuchungsdorf ist, hebt sich von den übrigen Untersuchungsdörfern auch dadurch ab, dass der Reis noch heute auf steilen Trockenfeldern im Rotations- 
system angebaut wird. Daneben wird jedoch auch Kaffee und seit den 1980er Jahren auch Kakao angebaut. Die schlechte Verkehrsanbindung (vgl. Abb. 8) an die Absatzmärkte der cash crops stellt allerdings eine deutliche Entwicklungsbremse für das Dorf dar. Dementsprechend ist Lawe v.a. als Abwanderungsdorf zu klassifizieren.

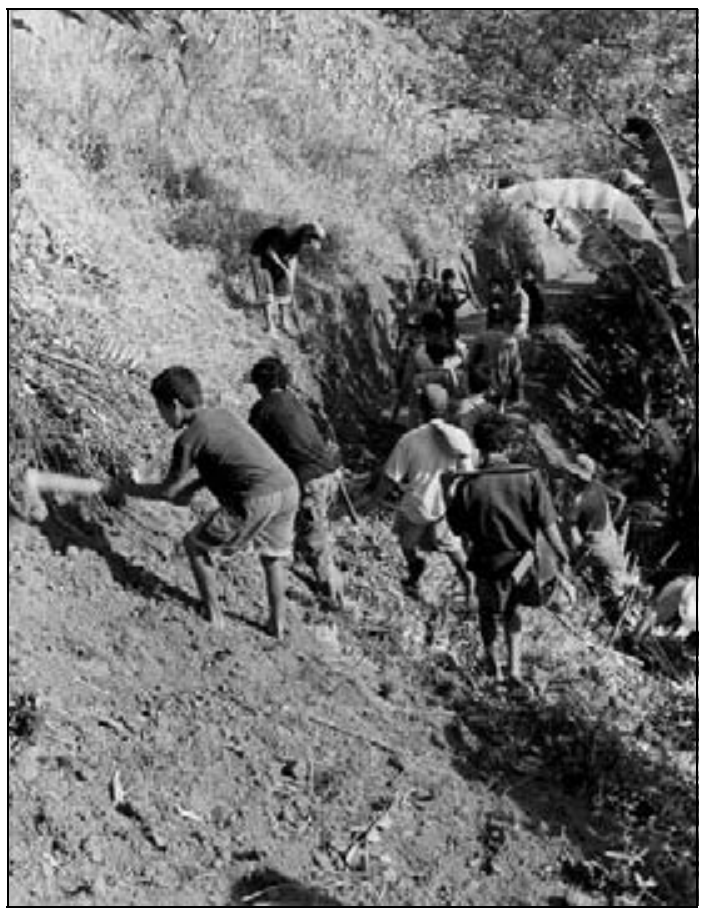

Abb. 8 Ausbauarbeiten am Fuß- und Pferdepfad nach Lawe (Quelle: Eigene Aufnahme, 2001)

Im Kecamatan Palolo befinden sich die beiden Untersuchungsdörfer Berdikari und Sintuwu. Berdikari, das heute etwa 1.200 Einwohner zählt, wurde 1954 von sieben Kulawi-Familien aus dem Pipikoro-Gebiet gegründet. Heute besteht das Dorf aus drei Dusun, wobei Dusun 2 und 3 seit 1965 durch die Transmigrationssiedlung Bahagia getrennt sind, welche von umgesiedelten Javanern bewohnt wird. Das Dorf zieht sich entlang der asphaltierten Hauptstrasse, die das Palolo-Tal mit Palu verbindet. Während Kulawi-Familien die übergroße Mehrheit in den Dusun 1 und 2 ausmachen, stellen in Dusun 3, welches am spätesten besiedelt wurde, Toraja und Bugis die Bevölkerungsmehrheit. Neben Nassreis war die Gewürznelke in den 1980er Jahren noch eine bedeutende Anbaufrucht der Dorfbewohner. Seitdem ist sie aber, wie auch in anderen Regionen, aufgrund des Verfalls der Gewürznelkenpreise durch Kakao abgelöst worden, welcher aufgrund der günstigen klimatischen 
Bedingungen spätestens seit Mitte der 1990er Jahre neben dem Nassreis das Landschaftsbild des gesamten Palolo-Tals prägt.

Das 1.400 Einwohner zählende Dorf Sintuwu ist 1961 im Rahmen staatlich geplanter, lokaler Umsiedlungen der im Übergangsbereich zwischen Palu- und PaloloTal lebenden Kaili-Bevölkerung gegründet worden. Nach Lawe ist es das am zweitschlechtesten infrastrukturell ausgebaute der neun Untersuchungsdörfer. Es ist ausschließlich über Schotterstrassen zu erreichen und musste einen Teil des Dorfterritoriums an den Lore-Lindu-Nationalpark abtreten. Zwar stellt die Kaili-Bevölkerung die Dorfmehrheit, doch die ab den 1980er Jahren in großer Zahl zugewanderten Bugis stellen mit ca. $30 \%$ eine relativ große und daneben ökonomisch sehr bedeutsame Migrantengruppe dar. Mit etwa $10 \%$ der Dorfbevölkerung bilden die Kulawi die zweitgrößte zugewanderte Gruppe. Die drei ethnischen Hauptgruppen haben größtenteils exklusive Wohnbezirke im Dorf. War Sintuwu anfangs v.a. durch den Nassreisanbau geprägt, so wurden ab den 1980er Jahren viele sawah-Flächen in Kakaokulturen umgewandelt.

Die verbleibenden beiden Untersuchungsdörfer, Watumaeta und Wanga, befinden sich im Kecamatan Lore Utara. Watumaeta, das zu Beginn des 21. Jh. 1.125 Einwohner zählte, wurde ab 1929 von Kulawi-Familien und lokalen Familien besiedelt. Im Jahr 1938 wurde das Dorf Watumaeta gegründet. Während sich bereits während der 1930er Jahre einige Bugis und Toraja in dem Ort aufhielten, ereignete sich - abgesehen von lokaler Heiratsmigration - erst wieder gegen Ende der 1980er Jahre, nach dem Ausbau der Verbindungsstrasse zwischen Palolo und Lore Utara, eine nennenswerte Zuwanderung. Diese betraf v.a. Bugis aus Südsulawesi und verstärkte sich mit dem Boom des Kakaopreises ab Mitte der 1990er Jahre beträchtlich. Während die lokale Bevölkerung Watumaetas sich hauptsächlich im Zentrum des Ortes aufhält, haben sich die Migranten v.a. entlang der Strasse nach Alitupu, später auch westlich der Strasse nach Wuasa im Randbereich des Lore-Lindu-Nationalparks niedergelassen. Neben Nassreis zählen Kakao und Gemüse (v.a. bei Javanern und Bugis) inzwischen zu den Hauptanbauprodukten des Ortes.

Wanga (436 Einwohner) wurde 1923 von einem Teil der damals noch in Watutau residierenden Herrscherfamilie von Lore besiedelt und 1925 von den Niederländern zum Dorf erklärt. Die größte Migrantengruppe im Dorf bilden Kulawi-Familien, die in den 1980er Jahren von dem als lokales Umsiedlungsprojekt entstandenen Dorf Kamarora (Kec. Palolo) nach Wanga umzogen und die ersten Wohnhäuser im heutigen Dusun 2 errichteten. Daneben ließ sich 1999 eine Gruppe von Familien und Einzelpersonen aus Flores in Wanga nieder, die ihr Herkunftsgebiet nach einem schweren Erdbeben verlassen mussten. Abgesehen von einigen wenigen Bugis- und Toraja-Familien, bilden die Migranten eine ökonomisch relativ schwache Gruppe. Während Dusun 1 fast ausschließlich von der lokalen Bevölkerung bewohnt ist, befinden sich die Wohnkomplexe der Migranten vornehmlich in Dusun 2. Hauptanbauprodukte des Dorfes sind Nassreis und Mais. Letzterer wird zum größten Teil 
nach Kalimantan exportiert. Daneben bietet der nahegelegene Rano See eine Einkommensquelle aus dem Fischfang. 


\section{Veränderungen der Bevölkerungsstrukturen in der Lore-Lindu-Region}

\subsection{Bevölkerung und Bevölkerungsdynamik}

Bevor im Folgenden die Migrationsprozesse in der Untersuchungsregion eine eingehende Betrachtung erfahren, werden zunächst einige generelle Aussagen zur Bevölkerung und ihrer Dynamik im Lore-Lindu-Gebiet vorweggenommen. Mit 7,2 \% ist Sulawesi nach Java und Sumatra die drittbevölkerungsreichste Insel des indonesischen Archipels (Abb. 9), während sie flächenmäßig erst an vierter Stelle nach Kalimantan steht.

Allerdings zählt die Insel zu den im gesamtindonesischen Vergleich relativ gering bevölkerten Regionen. Weit abgeschlagen hinter den dicht bevölkerten Inseln Java und Bali nimmt sie im regionalen Vergleich (vgl. Abb. 10) hinsichtlich der Bevölkerungsdichte nur den fünften Platz ein.

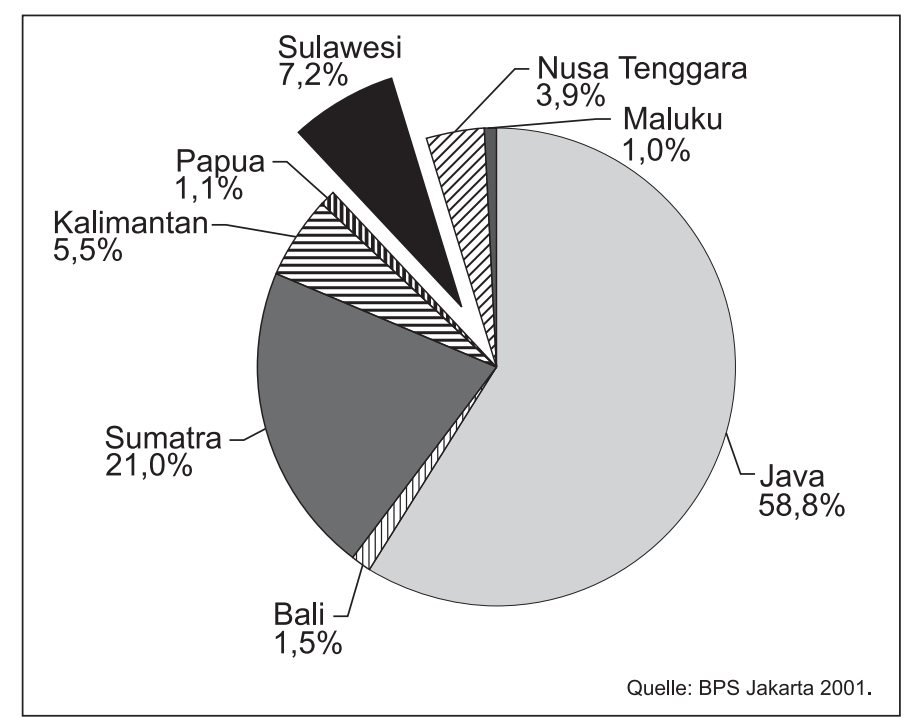

Abb. 9 Prozentanteile der Bevölkerung verschiedener Teilregionen an der Gesamtbevölkerung Indonesiens im Jahr 2000 


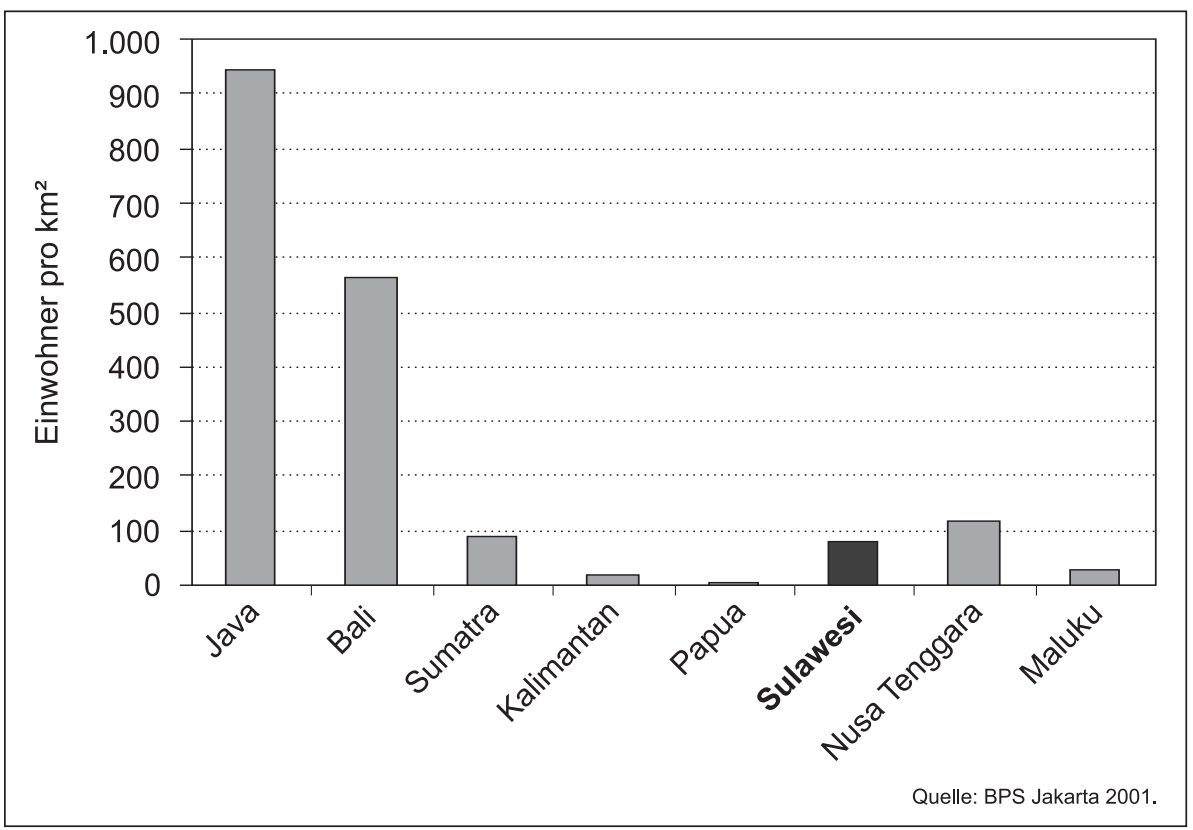

Abb. 10 Bevölkerungsdichte verschiedener Teilregionen Indonesiens im Jahr 2000

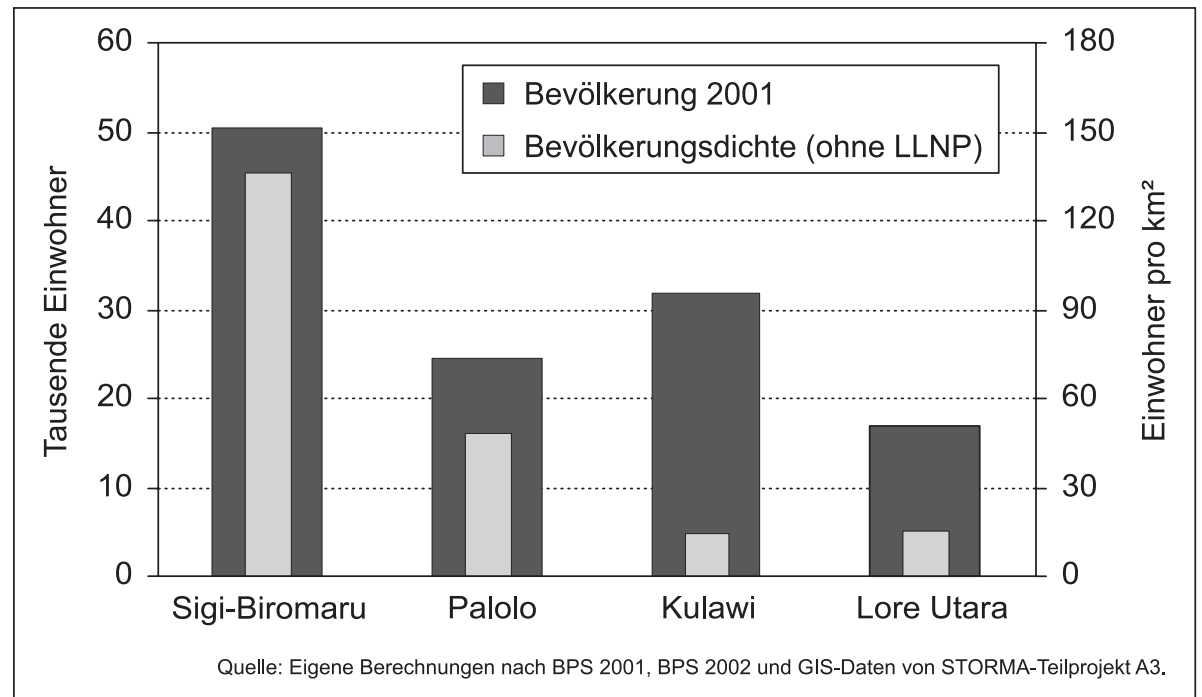

Abb. 11 Einwohnerzahlen und Bevölkerungsdichte nach Kecamatan 2001

In der Untersuchungsregion lebten im Jahr 2001 ca. 123.000 Menschen (vgl. Abb. 11). Bezogen auf die vier Kecamatan unterscheiden sich die Einwohnerzahlen deut- 
lich. Während in Sigi-Biromaru mit etwas über 50.000 die meisten Menschen leben, folgt an zweiter Stelle das flächenmäßig größte Kecamatan Kulawi mit knapp 32.000 Einwohnern. Lore Utara nimmt den letzten Rang ein, obwohl die Fläche dieses Kecamatan mehr als sechsmal Mal so groß ist wie die von Sigi-Biromaru und über viermal so groß wie die Fläche Palolos (jeweils bereits abzüglich des vom LoreLindu Nationalpark eingenommenen Flächenanteils). Entsprechend weisen Kulawi und Lore Utara die geringsten Bevölkerungsdichten auf. Während Sigi-Biromaru, Palolo und Kulawi ungefähr die gleichen Flächenanteile innerhalb des Lore-Lindu Nationalparks aufweisen, und diese für die Besiedlung irrelevanten Flächen bzgl. des Vergleichs der Bevölkerungsdichte des jeweiligen Kecamatan kaum ins Gewicht fallen, muss im Fall Lore Utara in Betracht gezogen werden, dass dort der Anteil der Nationalparkfläche an der Gesamtfläche des Kecamatan deutlich höher liegt. Dadurch erklärt sich die dritthöchste Bevölkerungsdichte für Lore Utara und die Schlussposition Kulawis.

\subsubsection{Charakterisierung der lokalen Bevölkerungsgruppen}

Die lokale Bevölkerung der Lore-Lindu-Region kann in drei ethnische Hauptgruppen unterteilt werden: Kaili, Kulawi und Lore. Anhand ihrer vorkolonialen Siedlungsgebiete lässt sich die räumliche Verteilung dieser Gruppen am deutlichsten darstellen, da die verschiedenen Ethnien zu jener Zeit noch relativ isoliert voneinander lebten. Die Kaili-Bevölkerung besiedelte die heutigen Kecamatan Sigi-Biromaru und Palolo, während die Kulawi-Menschen im heutigen Kulawi und die LoreBevölkerung in den Kec. Lore Utara und Lore Selatan beheimatet war. Das Verbreitungsgebiet der Kaili ging über die beiden genannten Verwaltungseinheiten des Untersuchungsgebiet hinaus und schloss sowohl die Bergregionen westlich und östlich des Palu-Tals als auch die Gebiete der Palu-Bucht mit ein.

Das bedeutendste Unterscheidungsmerkmal der drei Hauptgruppen ist ihre Sprache, welche jeweils weitere Untergruppen herausbildete (vgl. Abb. 12). So zählen zur Sprachgruppe der Kaili u.a. die Dialekte Kaili-Ledo (Untersuchungsregion und östliche Palu Bucht), Kaili-Da'a (westliches Randgebirge des Palu-Tals und westliche Palu-Bucht), Kaili-Ado (Pandere, Pakuli, Sibalaya) und Kaili-Tea (westliches Hügelland des heutigen Palolo-Tals). Die Kulawigruppe ist unterteilt in die Dialekte Moma (nördliches Kulawi und Kulawibecken um den Hauptort Bolapapu), Uma (südliches Kulawi/Gimpuebene und Pipikoro) und Tado (Lindu See). Im Lore-Gebiet unterscheiden sich Sedoa (Sedoa-Tal), Napu (ursprünglich Pekurehua ${ }^{51}$;

51 Vgl. Fußnote 58. 
Kapitel 3 - Veränderungen der Bevölkerungsstrukturen

verbreitet im Napu-Tal) und Behoa/Besoa ${ }^{52}$ (Besoa-Tal). Die drei ethnischen Gruppen Kaili, Kulawi und Lore decken sich mit den drei einflussreichsten Königreichen (kerajaan), welche sich in vorkolonialer Zeit herausgebildet hatten: Sigi (Kaili-Bevölkerung), Kulawi und Lore (vgl. Abb. 12). ${ }^{53}$

Nach Informationen aus den jeweiligen Regionen stellen diese Königreiche Zusammenschlüsse vormals kleinerer Einheiten dar. Demnach hatte es in Kulawi auch einmal die Kerajaan Pipikoro und Gimpu gegeben. Während der niederländischen Kolonialzeit wurden diese der Rechtsprechung von Kulawi unterstellt (Aragon 2000b: 54f). Auch für die Ostseite der Untersuchungsregion werden die unterschiedlichen Einheiten Sedoa/Tawaelia, Pekurehua und Besoa/Behoa genannt. Nach Departmen Pendidikan Dan Kebudayaan (1996/1997: 29) gab es in frühester Zeit auf dem Territorium des späteren Sigi u.a. auch die Kerajaan Raranggonau, Dolo, Palu und Sidondo. In der Folge kriegerischer Auseinandersetzungen entwickelte sich Sigi zum einflussreichsten Königreich der Region.

52 Die ursprüngliche Bezeichnung der Sprache und des Tals lautet Behoa. Erst durch die Niederländer kam es zu einer Umwandlung in Besoa, jenen Begriff, welcher sich bis heute durchgesetzt hat. Nach Vermutungen von Zeitzeugen hatte die Namensänderung ihre Ursache in der einfacheren Aussprache für die Niederländer. Eine definitive Erklärung dieses Lautwandels konnte jedoch nicht gefunden werden. Aus Befragungen ergab sich ferner, dass manche heutige Ortsbezeichnungen auf einem Missverständnis zwischen der lokalen Bevölkerung und den Niederländern beruhen.

53 Über die Aufteilung der Bevölkerung von Zentralsulawesi gab es schon früh unterschiedliche Ansichten. Generell ist die Rede von den sog. Toraja (vgl. Fußnote 31). Nach Coté (1979: 42) war es Albert C. Kruyt, der diesen Terminus erstmals 1897 auf die Gruppen des nördlichen Zentralsulawesi angewendet hatte. Zusammen mit Adriani nannte er die Bewohner des östlichen Zentralsulawesi „Posso’sch-Todjo'sche groep, „Oost-Toradja groep“ oder „Bare'e-Toradja“, jene des westlichen Teils „Parigi'sch-Kaili’sche groep“ oder „West-Toradja groep“. Dazu kam noch die „Sadanggroep“ (heute „Sa'dan Toraja“ genannt), die sich im zentralen Hochland Südsulawesis aufhielt (Adriani\& Kruyt 1912-14, Bd. 1: 3). Kaudern (1925, Bd. 1: 26f) unterschied zwischen südlichen und nördlichen Toraja und teilte diese folgendermaßen weiter auf (Übernahme der Schreibweise, wie sie in der Quelle erfolgt): A) Nord-Toradja, A.1: Poso-Toradja, A.2: Paloe-Toradja (Stämme am Golf von Tomini, Stämme in der Paloe Bay und im Paloe-Tal,, Stämme in den Bergdistrikten um die Flüsse Goembasa und Mioe, Zuflüsse des Paloe-Flusses), A.3: Koro-Toradja (Stämme im Distrikt um den Koro-Fluss, allgemein genannt Pipikoro, Stämme im Distrikt am oberen Teil des Koro, wo er Belanta und Tawaeli genannt wird, Stämme im Distrikt der südlichen Zuflüsse des Koro: dem Rampi und dem Leboni, am Kalaena-Fluss leben einige Stämme, die wahrscheinlich zu dieser Gruppe gehören und gleichzeitig Transitionen zu den Poso-Toradja sind), B) Süd-Toradja: Sadang-Toradja (Stämme an der Quelle des Karama-Flusses, Stämme am Rokong-Fluss, Stämme am Sadang-Fluss). 


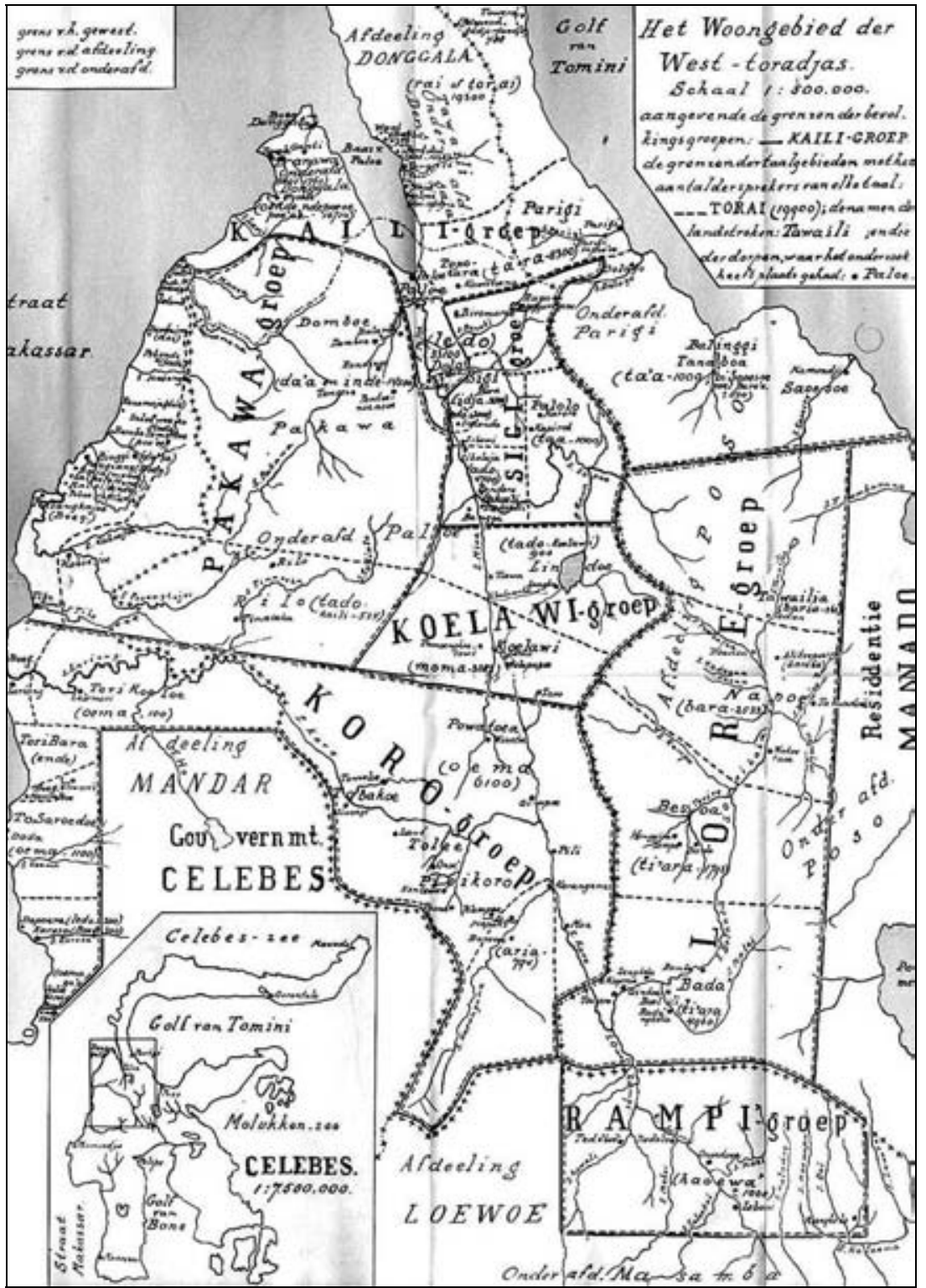

Abb. 12 Verteilung der ethnischen Gruppen des westlichen Zentralsulawesi in den 1930er Jahren

(Quelle: Kruyt 1938, Anhang)

Exkurs: Sulawesi - eine „nationale Einheit“‘?

Aus den verwendeten Quellen geht hervor, dass es von kolonialer Seite her die Vorstellung gab, die Insel Sulawesi könnte als eine „nationale Einheit‘ gesehen werden. Die De- 
konstruktion dieser Idee wurde allerdings selbst von Beteiligten des Kolonialapparates betrieben. Die Beschreibung der „Völker' in Manado wäre sehr schwierig, merkt z.B. Tideman (1926: 12) in seinem Memorie van Overgave an, da die Unterscheidung in Art, Entwicklung und Sprache so groß sei. Zu jener Zeit gab es in Manado 34 Sprachen. Man sollte laut Tideman (ebd.) dabei zu der Überzeugung kommen, dass von einer nationalen Einheit Celebes, die man sich von gewisser Seite so gerne zu organisieren wünschte, eigentlich keine Rede sein könnte. Wenn man von Stammesverband spräche, so Tideman (ebd.: 13) weiter, müßte man sich erst einmal fragen, was unter Stamm zu verstehen sei, da nicht jede Gruppe von Personen, die sich als von einem einzelnen Stammvater abstammend betrachtete, einen Stamm bildete. Henley (1989: 2) geht davon aus, dass das Konzept Sulawesi als kulturelle und politische Kategorie weitestgehend ein Produkt der klassifikatorischen Phantasien westlicher Beobachter war. Denn von Alters her war die Insel Heimat unterschiedlichster Sprachgruppen und Ethnien, die - gerade in gebirgigen Regionen - über lange Zeit isoliert lebten. Auch die Fremdherrschaften, seien es die Königreiche von Gowa, Bone oder Luwu im Süden der Insel, oder die Herrschaft der Spanier, Philippinen oder der Häupter von Ternate, wie sie im 16. und 17. Jh. vorherrschten, zeigen auf, dass vor der Ausbreitung der niederländischen Kolonialverwaltung Anfang des 20. Jh. in Zentralsulawesi die Insel Sulawesi nie als Einheit betrachtet wurde. Im August 1923 wurde in Manado unter der Leitung eines Herrn Tjoroaminoto der sog. Nationaal Celebes Congres ins Leben gerufen. Es wurde ein Komitee gebildet, um Vorbereitungen für einen nationalen Verband Celebes zu treffen. Seitdem wurde von diesem Komitee nichts mehr vernommen, was von Resident Tideman (1926: 93) als logisch bezeichnet wurde, da nun einmal so etwas wie eine Nation Celebes nicht bestünde und auch nicht bestehen könnte.

\subsubsection{Quantifizierung der Bevölkerung zu Beginn des 20. Jh.}

Die Quantifizierung der Bevölkerung, welche nach Reid (1988-1993, Bd. 2: 11) das größte Problem für die Geschichtsrekonstruktion im vorkolonialen Südostasien ausmacht, stellt auch für die Lore-Lindu-Region eine besondere Schwierigkeit dar. Genaue Aussagen über die Zahl der Einwohner vor dem Einmarsch der Niederländer sind aufgrund der dürftigen Quellenlage in der Tat nur schwer zu treffen. ${ }^{54}$ Zwar besuchten Adriani \& Kruyt (1898) Kulawi bereits im Jahr 1897, auch die SarasinBrüder gelangten während ihrer geologischen Erkundungen schon 1902 in die entlegeneren Gebiete südlich des Palu-Tals (Sarasin \& Sarasin 1905). Aufgrund der Tatsache, dass ein Großteil der lokalen Bevölkerung zu dieser Zeit noch verstreut in den Bergregionen lebte (vgl. Kap. 4.1), ist bei den wenigen vorhandenen Größenangaben aus jener Zeit nicht davon auszugehen, dass alle Einwohner einer Region darin eingeschlossen sind. In der Regel beruhen die Größenangaben nur auf groben

54 Selbst für die ersten Jahrzehnte des 20. Jh. erlaubt die sehr dürftige, vorhandene Datendichte für viele Regionen der sog. Äußeren Inseln des Archipels, darunter insbesondere auch Zentralsulawesi, nur grobe Abschätzungen. Zur Bevölkerungsquantifizierung außerhalb Javas um die Wende zum 20. Jahrhundert vgl. u.a. Gooszen (1999), Hugo (1980), McDonald (1980) und Wander (1965); speziell zu Zentralsulawesi vgl. Goozen (1999) und Henley (2004). 
Schätzungen, teilweise auf Überlieferungen aus dritter Hand. Selbst während des Auftretens der Niederländer in der Lore-Lindu-Region sind die meisten Angaben über die Bevölkerungszahlen geschätzt. Nach Henley (2004: 57) war ein weit verbreitetes Vorgehen zur Schätzung der Bevölkerung einer Region, die Anzahl der Dörfer eines Gebietes erst mit der durchschnittlichen Zahl der Häuser pro Dorf und danach mit der vermuteten Anzahl der Bewohner pro Haus zu multiplizieren. Neben der dabei zu erwartenden Ungenauigkeit der Ergebnisse muss in Hinblick auf die Verlässlichkeit der Daten aus jener Zeit auch die mehr oder weniger differenzierte Erfassung der Siedlungen berücksichtigt werden. Manche Europäer erkannten, dass die Dörfer (in den zeitgenössischen Quellen meist dorpen oder kampung genannt) auch als Distrikte betrachtet werden könnten, da sie eine Vielzahl kleiner, schwerer auffindbarer Siedlungen umfassten. Die meisten übersahen jedoch die Tatsache dieser verstreut lebenden Bevölkerung (Henley 2004: 57).

Der nördliche Teil des Palu-Tals wird von Adriani \& Kruyt (1898: 438), die wegen ihrer intensiven Reisen in der Untersuchungsregion eher zur ersten Gruppe der eben unterschiedenen Europäer gezählt werden können, ${ }^{55}$ als dicht besiedeltes Gebiet beschrieben. Kruyt hebt die hohe Bevölkerungszahl dieses Teilgebiets im Vergleich zum generell nur dünn bevölkerten Zentralsulawesi besonders hervor: „Die Bevölkerung von Mittel-Celebes ist sehr gering [...]. Nur eine Strecke in dem genannten Teil von Mittel-Celebes ist etwas stärker bevölkert, nämlich das Palu-Tal, aber von Überbevölkerung ist bier noch keine Rede [...]. " (1903b: 191). ${ }^{56}$ Er schreibt diesen Umstand dem bereits praktizierten Nassreisanbau im Palu-Tal zu. Zudem lässt sich die höhere Bevölkerungsdichte dieses Gebietes auf die Nähe zum Zentrum des mächtigen Sigi-Reiches mit Hauptsitz in dem Dorf Bora zurückführen. Dieses Fürstentum, dessen Einfluss sich von Parigi über Palu bis in das nördliche Kulawi erstreckte, soll 1869 nach Aussagen Dritter über 13.000 Bewohner gezählt haben (Adriani 1913b: 1617). Aus der gleichen Quelle stammen die Schätzungen der Bevölkerung der Hochebene um den Lindu See auf 1.300 Menschen, sowie der des Kulawi-Tals ${ }^{57}$ auf etwa 1.000 Personen. Eine Schätzung von Adriani \& Kruyt (1898: 501) geht für das Kulawi-Tal von ca. 2.200 Einwohnern im Jahr 1897 aus. Über die Anzahl der Bewohner der Pipi-

55 Kruyt (1908: 1309) kritisiert z.B. die Bevölkerungszählung im Napu-Tal durch das niederländische Militär kurz nach dessen Sieg im sog. Peore-Krieg. Diese basiere nur auf der Anzahl der wehrfähigen Männer. Ferner wäre es zu jener Zeit aufgrund der verstreuten Siedlungsstruktur nicht möglich gewesen alle Frauen und Kinder zu zählen.

56 Zitat im Original: „De bevolking van Midden-Celebes is dus zeer schaarsch [...]. Slechts in het genoemde gedeelte van Midden-Celebes is wat sterker bevolkt, namlijk het Paloedal, maar van overbevolking is hier nog geen sprake [...].“

57 Da die Region um den Lindu See extra aufgeführt ist, kann davon ausgegangen werden, dass es sich hierbei nur um das Tal handelt, dessen Hauptort heute Bolapapu darstellt. Sowohl das GimpuTal im Süden, als auch die Pipikoro-Region dürften in der Schätzung von 1.000 Menschen nicht enthalten sein. 
Kapitel 3 - Veränderungen der Bevölkerungsstrukturen

koro-Region im westlichen Kulawi gibt es aus jener Zeit keine genauen Angaben. Für die To Napu ${ }^{58}$ gibt Kruyt (1908: 1309) eine Größenordnung von 1.486 Einwohnern kurz nach der Unterwerfung unter die niederländische Krone an. Zur selben Zeit sollen sich im Besoa-Tal, das bis Anfang des 21. Jh. zusammen mit dem NapuTal das Kecamatan Lore Utara bildete, 1.413 Menschen aufgehalten haben (ebd.: 1337). Allerdings legt der Verfasser der Quelle die Vermutung nahe, dass beide Angaben zu niedrig seien. Das Bada-Tal, das heutige Lore Selatan, umfasste nach Kruyt (1909: 351) rund 4.000 Einwohner.

\subsubsection{Bevölkerungsdynamik in der Lore-Lindu-Region}

Auch wenn v.a. das Palu-Tal bereits zu vorkolonialen Zeiten eine vergleichsweise stark bevölkerten Region darstellte, sind es erst die Migrationsbewegungen nach 1945, die zu einer deutlichen, über das natürliche Wachstum hinausgehenden Zunahme der Bevölkerungszahlen geführt haben. Hierbei lassen sich jedoch unterschiedliche zeitliche und räumliche Entwicklungen ausmachen (vgl. Abb. 13 und Abb. 14 in Kap. 3.3).

Grundsätzlich lässt sich sagen, dass der westliche Teil der Untersuchungsregion (Kec. Sigi-Biromaru und Kec. Kulawi) von einem kontinuierlicheren Bevölkerungszuwachs geprägt ist als der östliche Teil (Kec. Palolo und Kec. Lore Utara). Dort nehmen die Bevölkerungszuwächse ab den 1980er bzw. 1990er Jahren eine exponentielle Dynamik an, wobei sich Palolo bereits während der 1960er bis 1980er Jahre durch einen vergleichsweise extrem hohen Zuwachs der Einwohnerzahlen auszeichnet. Da die gesamten Bevölkerungsdynamiken insbesondere auf die Zuund Abwanderung in der Lore-Lindu-Region zurückzuführen sind, unterbleibt an dieser Stelle vorerst eine weitergehende, detailliertere Betrachtung der regionalen

58 To Napu (Napu-Menschen) bezeichnet die ethnische Bevölkerungsgruppe, welche das Napu-Tal bewohnt. Allerdings handelt es sich hierbei um eine Fremdbezeichnung durch die Bewohner des Poso-Pamona-Gebietes östlich des Napu-Tals. Ursprünglich nannten sich die Bewohner des Tals To Pekurehua, eine Bezeichnung, die sich nach Aussagen der Bevölkerung auf einen Vogel zurückführen lässt, welcher in dem Gebiet weit verbreitet war und dessen Ruf wie „kureu-kureu“ klang. Auch Kruyt (1908: 1296) erwähnt diese ursprüngliche Selbstbezeichnung der Menschen des NapuTals. Napu soll sich nach lokalen Überlieferungen aus der Abkürzung des Begriffs nasaya pura aus der Poso-Sprache gebildet haben, welcher ,totschlagen“ "bedeutet. Der Hintergrund dieser Fremdbezeichung der To Pekurebua als To Napu (Totschläger) liegt in den gewalttätigen Raubzügen der Bewohner des Hochlandes bei ihren Tieflandnachbarn des Poso-Pamona-Gebiets. Als die Niederländer später die Tieflandbewohner fragten, wer sich denn in dem Gebiet jenseits der westlichen Bergkette befände, erhielten sie als Antwort, dass dort die to napu, die „Totschläger“"wohnten. Ein weiterer lokaler Informant leitet die Fremdbezeichnung napu von dem Namen einer Bambusart ab, welche bei Hautkontakt einen Juckreiz auslöst. Gemeint ist damit ein metaphorischer Bezug zu der leidvollen Auswirkung der Übergriffe der To Pekurehua auf die Bewohner der Poso-Ebene. 
Bevölkerungsveränderungen. Diese werden im Folgenden in direkter Beziehung zu den Migrationsprozessen untersucht.

\subsection{Migrationsprozesse in der Lore-Lindu-Region}

Für die Analyse der Migrationsprozesse in der Lore-Lindu-Region muss zunächst grundsätzlich festgelegt werden, unter welchen Bedingungen eine räumliche Mobilität als Migration bezeichnet wird. Für die vorliegende Untersuchung wurde als Migrantin oder Migrant definiert, wer seinen Herkunftsort mit der festen Absicht verlassen hat, am Zielort ihren/seinen neuen Lebensmittelpunkt zu haben. Diese Definition umfasst darüber hinaus auch Personen, welche für eine längere Zeit permanent an einen anderen $\mathrm{Ort}^{59}$ wechseln, um dort eine Arbeit aufzunehmen, eine Ausbildung (z.B. Schul- oder Universitätsbesuch) zu absolvieren, etc., und in diesem Ort für die Dauer ihres Aufenthalts wohnen. Nicht eingeschlossen ist hierbei ein kurzzeitiger Wohnortwechsel von weniger als einem halben Jahr, nach welchem die Person wieder in den Ausgangsort zurückkehrt. Hingegen wird für Migrationsbiographien, die auch kurzzeitige Aufenthalte an verschiedenen Orten beinhalten, definiert, dass es sich bei der betreffenden Person in den einzelnen Zwischenstationen der Migration um eine Migrantin bzw. einen Migranten handelt.

Wohnt eine Person von Geburt an in einem bestimmten Ort, so wird sie in dieser Arbeit als Lokale bzw. Lokaler dieses Ortes bezeichnet. Entsprechend werden die Begriffe lokale Gruppe, lokale Bevölkerung, etc. verwendet. Ist eine Person in einem bestimmten Ort nicht geboren, so wird sie als Migrantin/Migrant dieses Ortes definiert. Analog verhält es sich mit entsprechenden Gruppenbezeichnungen. Wie sich später noch zeigen wird, befinden sich in der Untersuchungsregion auch Orte, deren erstangesiedelte Bevölkerung nicht aus der näheren Umgebung des Ortes stammt. In diesen Fällen wird die Bevölkerungsgruppe des Ortes, deren ethnische Zugehörigkeit sich mit jener der Erstansiedler deckt, mit dem Terminus „lokal“" benannt. Gegebenenfalls wird hierbei allerdings zusätzlich angegeben, dass es sich bei den Betreffenden um lokale Migranten (s. auch unten) handelt. Ausgenommen von der Regelung, Erstansiedler eines Ortes trotz ihrer Migrationsgeschichte als lokale Bevölkerungsgruppe zu bezeichnen, sind jedoch sämtliche Personen, die im Rahmen interinsularer Umsiedlungsprogramme in einem neu gegründeten Dorf angesiedelt werden. Sie werden auch dann als Migranten bezeichnet, wenn sie die Erstansiedler eines Ortes stellen. Gerade die während der Untersuchungen zutage getretenen subjektiv unterschiedlichen Selbstzuschreibungen zu einer lokalen oder zugewanderten Bevölkerungsgruppe machen diese objektive Definition erforderlich.

59 Mit dem Begriff „Ort“ ist in dieser Arbeit in der Regel ein ganzes Dorf bzw. eine ganze Stadt, nicht jedoch ein Dorf- oder Stadtteil gemeint. 
Sollte in Einzelfällen von den hier bestimmten Begrifflichkeiten abgewichen werden, wird dies an den entsprechenden Stellen benannt und erklärt werden.

Nach dieser zeitlichen und funktionalen Differenzierung des Migrationsbegriffs wird der Terminus nun für seine Anwendung in der vorliegenden Untersuchung räumlich definiert. Hierbei ist zwischen lokaler, regionaler und nationaler Migration zu unterscheiden. Als lokale Migration sind im Rahmen dieser Arbeit all jene Wanderungsbewegungen zu verstehen, welche ihren Ursprungs- und Zielort innerhalb des Untersuchungsgebietes haben. Unter regionaler Migration hingegen werden die Wanderungen von anderen Gebieten der Insel Sulawesi in die Untersuchungsregion und umgekehrt zusammengefasst. Nationale Migration bedeutet schließlich eine Wanderungsbewegung zwischen Untersuchungsgebiet und anderen Inseln des indonesischen Archipels. Zudem unterscheiden sich Stadt-Stadt-, Land-Land-, StadtLand- und Land-Stadt-Migration, welche sich auch in Form von Etappenmigration miteinander verbinden lassen. Die Etappenmigration ist eine in der Untersuchungsregion sehr häufig vorkommende Art der räumlichen Mobilität.

Die Erfassung der Migration für ein Gebiet wie die Lore-Lindu-Region stellt eine Forscherin oder einen Forscher vor gewisse Schwierigkeiten in bezug auf die verfügbare Datengrundlage. Die Probleme vergrößern sich sowohl in räumlicher als auch in zeitlicher Perspektive. Räumlich gesehen muss generell zwischen der Untersuchung von Migration in einer in globaler Sichtweise ,zentralen“ Region und jener in einer „peripheren“ Region bezüglich einer Industriestaaten-Trikont-Dichotomie unterschieden werden. Erstere weisen in der Regel eine höhere Dichte an statistischem Datenmaterial mit einer scheinbar oder tatsächlich höheren Genauigkeit der enthaltenen Informationen auf. Dennoch lassen sich z.B. großräumige Migrationsbewegungen, wie etwa die zwischen Kontinenten, Ländern oder auch Großregionen eines Landes mittlerweile relativ einfach erschließen. Möchte man allerdings eine vergleichsweise kleine Raumeinheit untersuchen, ist man, bei vorerst reiner Berücksichtigung bereits vorhandener statistischer Daten auf die Grenzen der Datenerfassung für die entsprechende Region angewiesen. Im konkreten Fall der Lore-LinduRegion erleichtert die Übereinstimmung der Forschungsregion mit den politischadministrativen Raumeinheiten (z.B. Kecamatan) die Verwendung existierender statistischer Daten. Doch bereits auf dieser Ebene lassen sich Migrationsbewegungen nur bedingt quantifizieren, da in den offiziellen Veröffentlichungen zwar Zahlen zur staatlichen Transmigration (transmigrasi) (vgl. Kap. 3.4.2) erfasst werden, andere Migrationsformen (z.B. spontane Migration) jedoch unberücksichtigt bleiben. Vielmehr muss die Gesamtmigration aus den statistischen Vorlagen zur räumlichen Bevölkerungsverteilung abgeleitet werden. Spätestens an dieser Stelle sind qualitative Zusatzinformationen für eine wirklichkeitsnahe Einschätzung von Migrationsbewegungen unabdingbar. Da das verfügbare Sekundärmaterial nur sehr eingeschränkt Aussagen über die Bevölkerungsentwicklung einzelner Gebietsteile eines Kecamatan oder sogar einzelner Dörfer zulässt, lassen sich daraus entsprechend unzureichend bis gar keine Informationen über die Zu- und Abwanderung bzgl. einzelner Ge-

\section{Pazifik Forum Bd. 12}


bietsteile bzw. Dörfer generieren. Hier ermöglichen nur detaillierte Fallstudien eine Annäherung an die realen Migrationsprozesse. Besonders auch die räumliche Mobilität zwischen einzelnen Teilregionen des Untersuchungsgebiets ist allein über die Auswertung bestehender Statistiken kaum fassbar.

Daneben ist die Zeitkomponente zu berücksichtigen. Die vorliegende Arbeit umfasst den Zeitraum eines Jahrhunderts. Kolonialzeitliches Datenmaterial, hauptsächlich in den Kolonialberichten und zeitgenössischen Veröffentlichungen enthalten, beruht größtenteils auf Schätzwerten für einige zeitliche Fixpunkte. Zeitreihendatensätze zur Bevölkerung der Untersuchungsregion existieren nicht. Statistiken der staatlichen statistischen Behörden (BPS) bieten erst ab dem Zensus von 1970 Daten, welche zumindest Aussagen auf der Kecamatan-Ebene erlauben. Allerdings sind auch in diesem Fall Zeitreihenkalkulationen nur bedingt möglich, da die Statistiken der verschiedenen Jahre nicht alle benötigten Informationen in gleicher Detaildichte enthalten. Auch hier dienen Primärdatenerhebungen auf Meso- und Mikroebene dazu, Migrationsprozesse in ihrem zeitlichen Umfang besser erfassen zu können.

Darüber hinaus gibt das veröffentlichte Datenmaterial kaum Aufschlüsse über die Motivation von Migranten. Ebenso wenig lassen sich daraus Migrationsbiographien ablesen, die - sofern überhaupt erfasst - über die drei Komponenten Herkunftsort/-region, Zeitpunkt der Migration und Zielort/-region hinausreichen. Wie bereits für die vorliegende Arbeit im Ganzen erwähnt, sei auch im Bezug auf diesen Themenkomplex ausdrücklich betont, dass die im Rahmen der Forschungsarbeit erhobenen Daten die Bevölkerungsdynamik und die Migrationsprozesse in der Lore-Lindu-Region nicht umfassend darstellen und analysieren können. Vielmehr sollen die groben Bevölkerungs- und Migrationsstrukturen verfeinert und anhand von Fallbeispielen die Komplexität dieser Strukturen untersucht werden. Zudem soll herausgestellt werden, welche unterschiedlichen Faktoren die Migrationsprozesse beeinflussen.

\subsection{Allgemeine Aspekte der Zu- und Abwanderung im Lore-Lindu-Gebiet}

Migration spielt in der Lore-Lindu-Region eine maßgebliche Rolle bei der Veränderung der Kulturlandschaft. Gerade die Jahrhunderte lange, weitgehende Isolation Zentralsulawesis im Allgemeinen und großer Teile der Lore-Lindu-Region im Besonderen ließ das Gebiet im Laufe der Jahrzehnte eine wachsende Zuwanderung bei insgesamt relativ geringer Abwanderung erfahren. Daneben setzte ab der niederländischen Kolonialzeit eine beachtliche Binnenmigration ein, wobei als eine erste grobe Annäherung die Westseite der Lore-Lindu-Region als Binnenabwanderungsgebiet, die Ostseite als Binnenzuwanderungsgebiet bezeichnet werden kann. Wie 
Kapitel 3 - Veränderungen der Bevölkerungsstrukturen

sich die Migrationsprozesse während des 20. Jh. im Detail darstellen, wird in den nachfolgenden Unterkapiteln erläutert.

Die Zu- und Abwanderung ist räumlich, zeitlich und in ihrer Art zu unterscheiden. Räumlich gesehen ist die Migration im Untersuchungsgebiet erstens in einen Bezug auf die Zuwanderung in die bzw. die Abwanderung aus der Untersuchungsregion zu differenzieren. Da die Zuwanderung in die Region bedeutend größer ist als die Abwanderung, welche auch nur einen sehr geringen Einfluss auf den Kulturlandschaftswandel hat, bleibt die Abwanderung in dieser Arbeit weitgehend unberücksichtigt. Zweitens sind $\mathrm{Zu}-$ und Abwanderung innerhalb der Lore-Lindu-Region zu unterscheiden.

Über die Bevölkerungsentwicklung während der ersten 70 Jahre des 20. Jh. lassen sich bezogen auf die heute existierenden administrativen Einheiten (insbesondere die Kecamatan-Ebene) nur grobe Aussagen treffen. Zum einen ist die Datengrundlage zur Bevölkerung insgesamt während dieses Zeitraums sehr lückenhaft, zum anderen ist ein exakter Vergleich zur jüngeren Entwicklung durch Unterschiede in der räumlichen Aufteilung der statistischen Erfassung erschwert (vgl. Kap. 3.1). Gooszen (1999: 96) zählt Zentralsulawesi mit einer Anzahl von 14.600 Zugewanderten im Jahr 1930 während der ersten Jahrzehnte des 20. Jh. zu den „,non-migration regions" (ebd.: 114) des Archipels. Die Wachstumsraten der Bevölkerung Zentralsulawesis werden von Gooszen (1999: 225) auf 0 bis 1,0 für den Zeitraum 1900-1920 und auf 1,25 bis 1,75 für den Zeitraum 1920-1930 geschätzt, Werte, die mit denjenigen von Nordwestsumatra und Zentralborneo gleichgestellt werden. Damit bewohnen im Jahr 1930 knapp über 800.000 Menschen die Region Zentralsulawesi.

Ein Blick auf die Verteilung der Bevölkerungsentwicklung in der Lore-LinduRegion nach Kecamatan im Laufe der letzten 40 Jahre (Abb. 13) offenbart enorme räumliche und zeitliche Unterschiede, die sich nur durch den Einfluss von Migrationsprozessen erklären lassen. Zwar nimmt die Bevölkerung in allen fünf Kecama$\tan \mathrm{zu}$, doch unterscheiden sich die Zuwachsraten erheblich, wie aus der Abb. 14 abzulesen ist.

Das Palu-Tal, in welchem sich das heutige Kecamatan Sigi-Biromaru befindet, erfuhr bereits zu vorkolonialen ${ }^{60}$ Zeiten einen größeren Bevölkerungszuwachs. Dieser ist durch die Ansiedlung von Bugis aus Südsulawesi begründet (vgl. Lineton 1975). Gleichzeitig fanden mit dem Damar-Boom ${ }^{61}$ gegen Ende des 19. Jh. erste Formen der Arbeitsmigration statt. Belege hierzu finden sich insbesondere in bezug auf die Regionen Kulawi und Bada (vgl. u.a. Adriani \& Kruyt 1898: 501; Kruyt 1908: 1337; Kruyt 1909: 349; o. A. 1912: 25). Während der Kolonialherrschaft führten

60 Der Begriff ,,vorkolonial“ bezieht sich hier auf die Zeit vor der effektifen niederländischen Unterwerfung des genannten Gebiets ab 1904.

61 Damar ist ein helles, gelbliches Harz, welches bei der Herstellung von Lacken, Fackeln und Batiken eingesetzt werden kann. 
Zwangsumsiedlungen aus den westlichen und östlichen Gebirgszügen zu einer weiteren Verdichtung der Bevölkerungszahl im Palu-Tal. Die Hauptgründe für die genannten Umsiedlungsmaßnahmen waren zum einen der Erosionsschutz durch die Unterbindung der Wanderfeld-Brandrodung ${ }^{62}$ an den Berghängen, zum anderen die erleichterte Kontrollierbarkeit der einheimischen Bevölkerung und die effizientere Steuereintreibung (s. auch Kap. 3.4).

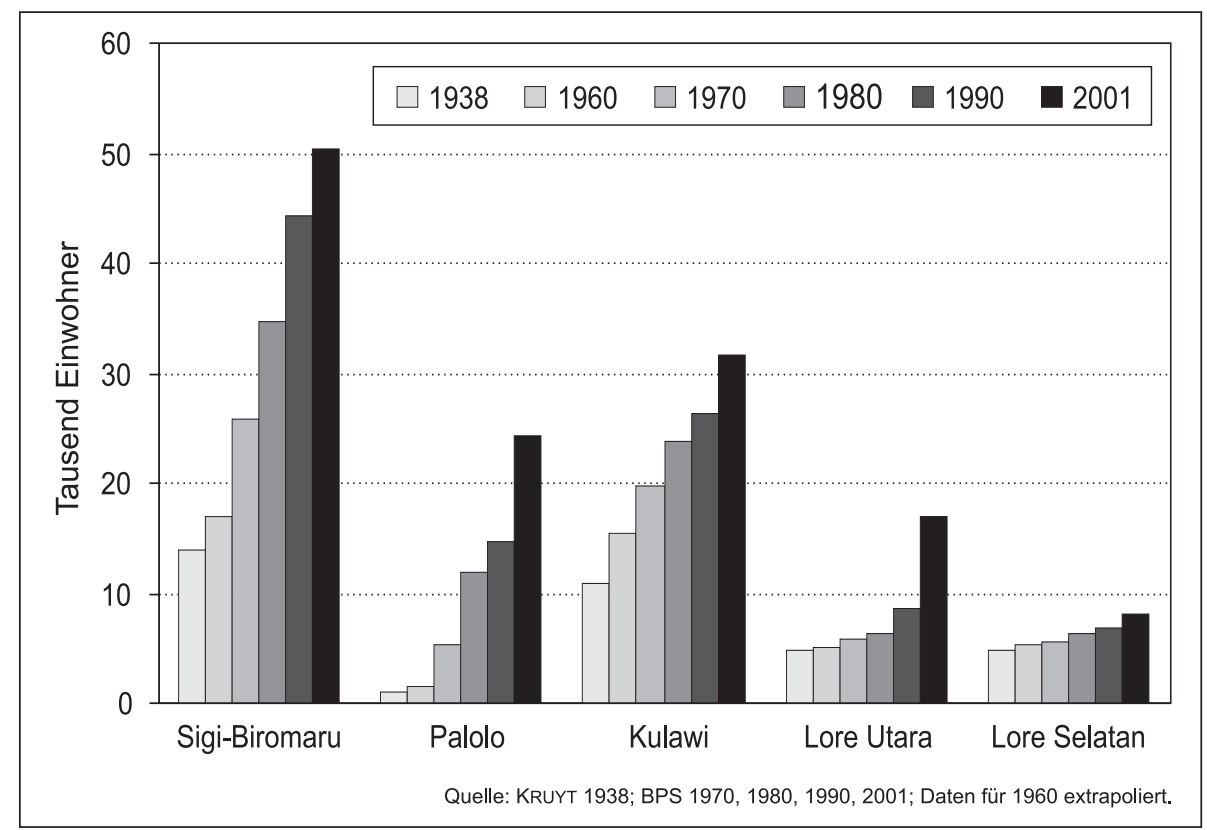

Abb. 13 Bevölkerungsentwicklung in der Lore-Lindu-Region nach Kecamatan $1938-2001$

In Palolo bewegen sich die Dekadenzuwächse der Bevölkerungsanzahl zwischen 1960 und 1980 in einem Bereich von über 100 Prozent, im Zeitraum von 1960 bis 1970 nimmt in diesem Kecamatan die Bevölkerung sogar um über 250 Prozent zu. Ursache hierfür ist, dass der größte Teil des Palolo-Tals bis in die 1950er Jahre noch nicht in Kultur genommen war. Wälder und Grasflächen beherrschten das Landschaftsbild, insbesondere der Osthälfte des Tals. Erst Ende der 1950er Jahre

62 Wanderfeldbau stellt ein Landnutzungssystem dar, bei welchem in räumlicher und zeitlicher Rotation unter dem Einsatz von Feuer Flächen für den Ackerbau gerodet werden

(Brown \& Schreckenberg 1998: 2). Je nach Bevölkerungsdichte wird dabei ein längerer oder kürzerer Rotationsrhythmus eingehalten, wodurch die Brachzeiten bereits bewirtschafteter Felder und damit deren Bodenfruchtbarkeit zu- oder abnehmen. 
wurde die Erschließung des Gebietes vorangetrieben. Zum einen wurden KailiFamilien, die bis dato in den bereits existierenden Dörfern am westlichen Ende des Palolo-Tals siedelten, von der Regierung dazu genötigt, Neuland weiter im Osten zu erschließen. Zum anderen wanderten ab den 1950er Jahren zahlreiche Familien aus der gebirgigen Pipikoro-Region Kulawis nach Palolo. Bedingt durch die Verbesserung der Verkehrsinfrastruktur nach und in Palolo sowie durch die Landverknappung in Südsulawesi und den einsetzenden Boom des Kakaopreises siedelten sich vor allem ab 1990 zahlreiche Bugis-Familien aus Südsulawesi in den noch relativ jungen, neuen Dörfern des Palolo-Tals an.

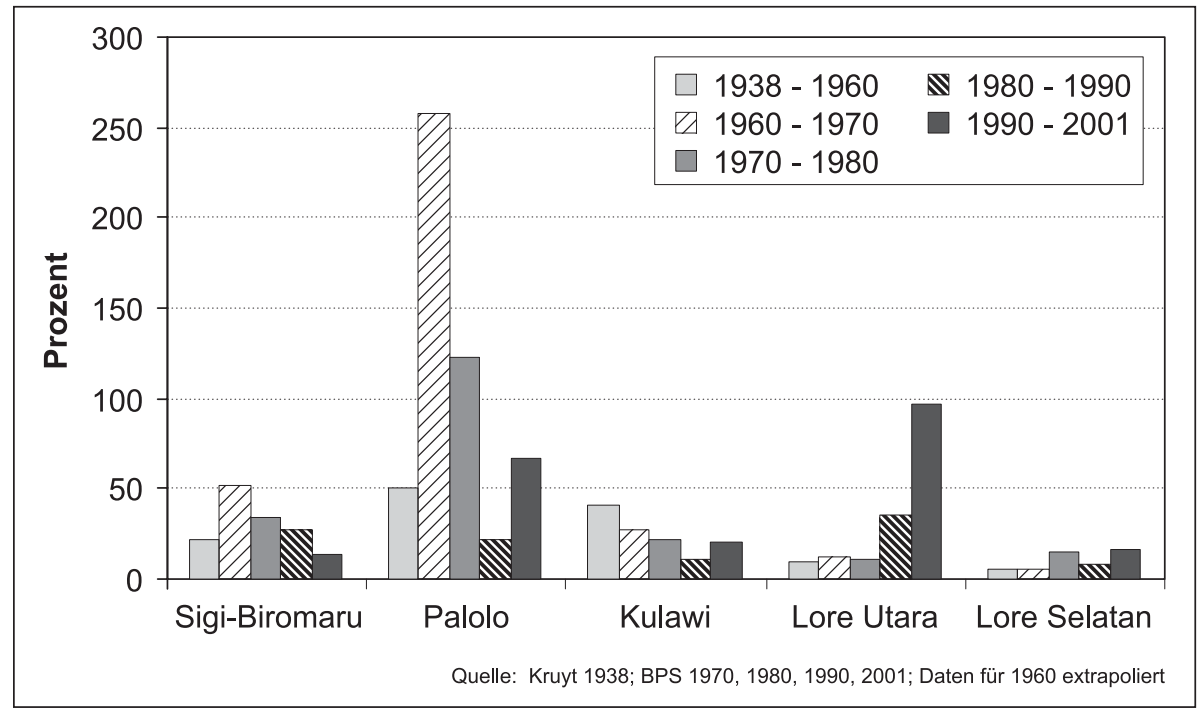

Abb. 14 Bevölkerungszuwachsraten in der Lore-Lindu-Region nach Kecamatan 1938 - 2001

Neben Palolo weist auch das Kec. Lore Utara extreme Bevölkerungsveränderungen auf (vgl. Abb. 14) Auf Grund seiner bis in die 1970er Jahre verkehrsinfrastrukturellen Isolation blieb das Kecamatan Lore Utara trotz seines weitläufigen Talbodens relativ gering besiedelt. Mit dem Ausbau der Straßenverbindung Palu - Lore Utara im Jahr 1982 und der dadurch verbesserten Marktanbindung entwickelte sich das Gebiet in den 1980er Jahren zum zweiten großen Zuwanderungsgebiet neben dem Palolo-Tal. ${ }^{63}$ Vor allem der Boom des Kakaopreises und die Landverknappung in

63 Kruyt (1908: 1328) bezeichnet den Verbindungspfad vom Sedoa-Tal (im Norden von Lore Utara) in das Palolo-Tal als einen der ältesten Wege in Zentralsulawesi. 
Südsulawesi führten in Lore Utara, wie bereits im Palolo-Tal zu einer verstärkten Ansiedlung von Bugis-Migranten.

Mit der steigenden Bedeutung von landwirtschaftlichen Exportprodukten, allen voran dem Kakao, kam es neben der Zuwanderung in das Lore-Lindu-Gebiet auch zu Wanderungsbewegungen innerhalb dieser Region. Als Ausgleichsentwicklung zu der auf Grund der Nähe zur Küste bereits früh erreichten dichten Besiedlung des Kecamatan Sigi-Biromaru, begann ein Teil dessen Bewohner damit, nach Palolo und Lore Utara umzusiedeln. Betroffen war hiervon vor allem die jüngere Generation. Die traditionelle Erbteilung des Landes hatte bei anwachsender Bevölkerungszahl dazu geführt, dass nur noch kleine Landparzellen an die nachfolgende Generation weitergegeben werden konnte. Einen Ausweg aus dieser Situation bot schließlich für viele der Betroffenen der Umzug in die zu jener Zeit noch weniger dicht besiedelten Regionen im östlichen Lore-Lindu-Gebiet an.

Abgesehen von dem ökonomischen Faktor ist der Hauptgrund für die Migration innerhalb des Untersuchungsgebiets die Heirat. Dieser Migrationsprozess führte dazu, dass sich die zu vorkolonialer Zeit relativ isoliert lebenden ethnischen Gruppen über die Lore-Lindu-Region verbreiteten und dadurch zu einer ethnischen Diversifizierung der einzelnen Dorfgemeinschaften beitrugen. Neben diesen spontanen intraregionalen Migrationsbewegungen, hatten staatlich geplante Umsiedlungen einen entscheidenden Einfluss auf den Wandel der Kulturlandschaft. Angefangen unter der niederländischen Kolonialherrschaft wurde diese planvolle Migration innerhalb der Lore-Lindu-Region sowie die intra-insulare Umsiedlung auch nach der Unabhängigkeit Indonesiens fortgesetzt. Mit einer genaueren Betrachtung dieser staatlich geplanten Migration beginnt die nachfolgende Detailuntersuchung der soeben kurz umrissenen, für das Untersuchungsgebiet bedeutenden Migrationsprozesse.

\subsection{Staatlich geplante Umsiedlungen}

Staatlich geplante Umsiedlungsmaßnahmen fanden in der Lore-Lindu-Region während des gesamten Untersuchungszeitraums statt. Hierbei lassen sich regionale bzw. lokale Umsiedlungen (vgl. Kap. 3.4.1) von nationalen bzw. zwischen einzelnen Inseln des Archipels stattfindenden Umsiedlungen (vgl. Kap. 3.4.2) unterscheiden.

Wie bereits die niederländische Kolonialregierung ab Beginn des 20. Jh., machte es sich nach Erreichen der Unabhängigkeit Indonesiens insbesondere die Regierung unter Präsident Suharto zur Aufgabe, mittels ihrer Fünf-Jahres-Pläne die Entwicklung besonders der peripheren Regionen des indonesischen Archipels voranzutreiben. Sowohl während der Kolonialzeit, als auch während der Suharto-Ära wurde dieses Vorhaben mit staatlich geplanten Umsiedlungsmaßnahmen verbunden. 


\subsubsection{Staatlich geplante, lokale Umsiedlungen}

Eine Vielzahl der heute vorhandenen Siedlungen in der Lore-Lindu-Region sind auf die lokalen Umsiedlungsmaßnahmen der niederländischen Kolonialverwaltung zurückzuführen. Im Rahmen der durch die Kolonialverwaltung durchgeführten lokalen Umsiedlungsmaßnahmen der Bergbevölkerung kam es zu einer Konzentration der vormals bestandenen Streusiedlungen. ${ }^{64}$

Neben der Verringerung der Straßenbaukosten durch die Einrichtung solcher Sammelsiedlungen spielte auch die bessere Kontrolle der dort lebenden Bevölkerung eine wichtige Rolle in den Überlegungen der Niederländer. Deren Hauptinteresse lag nämlich darin, dass die Menschen von den Erträgen ihrer Felder ihre Steuern bezahlen konnten (Kruyt 1926: 543). Die Konzentration der Bevölkerung auf die leichter zugänglichen Talböden erleichterte den Kolonialherren sowohl das Eintreiben der Steuern als auch die Rekrutierung zu Arbeitsdiensten, insbesondere im Straßenbau. Ferner sollte durch diese Umsiedlungen und der damit verbundenen besseren Kontrolle auch die Einhaltung der Grenzen von Waldschutzreservaten gewährleistet werden. Letzteres spielte vor allem für die Umsiedlung in das Palu-Tal eine Rolle, wird von Zeitzeugen aber auch für das Palolo-Tal berichtet. Das Ziel der Niederländer, den Nassreisanbau in den Tälern auszudehnen bzw. ehemals genutzte Felder wieder zu bewirtschaften, war durch eine zu geringe Wasserführung der Flüsse und Bäche aus den Bergen gefährdet. Dieses Problem wurde der durch den Brandrodungswanderfeldbau hervorgerufenen Entwaldung der Berghänge zugeschrieben. Die kolonialen Umsiedlungsmaßnahmen aus den Bergregionen führten zu erheblichen Einschnitten in die Lebensweise der Bevölkerung. Das Leben in permanenten Siedlungen war für die Menschen ebenso neu (Kruyt 1938: 501), wie die klimatischen Bedingungen in den Tälern ungewohnt waren. Insbesondere die höheren Temperaturen waren für viele eine große Umstellung. Vorstman (1935: 13) stellte fest, dass die neue Umgebung zu ,een algemeen gebrek an levenshust en energie" der umgesiedelten Menschen führte. Die Geburtenraten sanken, während sich die Zahl der Sterbefälle deutlich erhöhte, ein Umstand, den Kruyt (1926: 542) u.a. mit den Folgen einer Ruhrepidemie und einer allgemeinen Geschwächtheit der ehemaligen Bergbevölkerung in Verbindung bringt. Es ist daher nicht verwunderlich, dass zahlreiche dieser Menschen wieder in ihre Herkunftsgebiete zurückkehrten. Anfangs wurde noch versucht, die Umgesiedelten durch die Präsenz von kolonialen Verwaltungsbeamten in den Dörfern zu halten und sie zum Anbau von Nassreis zu zwingen. Das Eintreiben der Steuern und andere Aufgaben band mit der Zeit allerdings immer mehr Kräfte, so dass die Rückwanderungen in die Bergregionen mit Abnahme der täglichen Kontrolle durch die Kolonialbeamten stetig zunahmen (Vorstman 1935: 13). Beeinflusst von der Ethischen Politik (vgl. Kap. 6.1) vermerkt

64 Auf die Veränderungen im Siedlungswesen wird im Kapitel 4 noch ausführlicher eingegangen.

\section{Pazifik Forum Bd. 12}


Vorstman (ebd.) schließlich, dass man diese Menschen doch in ihre Herkunftsgebiete zurückkehren lassen sollte, bestünde nicht die Gefahr weiterer Walddegradation durch die Brandrodungslandwirtschaft und somit einer Bedrohung der ausreichenden Wasserversorgung für die Nassreisfelder. Zudem betont Vorstman (ebd.), dass die Belange der zehnmal so großen Talbevölkerung über jene der Bergbewohner gingen. Als Kompromisslösung wurde den Menschen erlaubt, sich auch wieder an den Hängen, jedoch außerhalb der von den Niederländern angelegten Waldschutzreservate anzusiedeln (Kruyt 1926: 543).

Obwohl die lokalen Umsiedlungen also nicht in vollem Umfang permanent umgesetzt worden waren, resultierten diese Maßnahmen doch in einer ersten massiven Umgestaltung der Kulturlandschaft der Lore-Lindu-Region. Der Hauptteil der lokalen Bevölkerung siedelte nun in den Tälern, konzentriert in zahlreichen neu geschaffenen Dörfern. Die Agrarlandschaft stellte sich zunehmend in permanent bewirtschafteten Feldern mit vorwiegender Nassreisbewirtschaftung dar, auch wenn es noch weit bis in die Dekaden der Unabhängigkeit Indonesiens dauern sollte, bis der Wanderfeldbau dauerhaft minimiert wurde.

Die Suharto-Regierung griff das System staatlich geplanter lokaler Umsiedlungen wieder auf. Zentralsulawesi wies nach Babcock \& Cummings (1984: 19f) noch in den 1970er Jahren die größte Konzentration der sog. masyarakat terasing in ganz Indonesien auf. Mit dem Begriff masyarakat terasing wird im Behördenjargon jene Bevölkerung umschrieben, welche sich zumeist in unzugänglichen Bergregionen mit einer geringen Infrastruktur (Verkehrswege, Zugang zu Märkten und Bildung, etc.) aufhält. Von Mitarbeitern der für lokale Umsiedlungen verantwortliche Behörden wird in bezug auf masyarakat terasing auch von „primitiven“ Menschen gesprochen, das sich an den urspünglich verwendeten Begriff suku terasing anlehnt. ${ }^{65}$ Weitere, im Zusammenhang mit lokalen Umsiedlungsprogrammen verwendete Termini, deren voneinander abgrenzende Definierung selbst den entsprechenden Behörden (DepSos, PMD, etc.) nicht in eindeutiger Form gelingt, sind masyarakat terpencil bzw. masyarakat terisolir. Trotz ihrer im Vergleich zu den inter-insularen Transmigranten (vgl. Kap. 3.4.2) viel geringeren Anzahl, spielen diese Bevölkerungsgruppen nach Tsing (1993: 28) eine bedeutende ideologische Rolle. Sie werden nicht als gefährlich betrachtet, wodurch ihnen ein negativer, aber dennoch in gewisser Weise aufwertender Status beigemessen werden könnte. Die Zuschreibung der Hochlandbewohner Zentralsulawesis als „Kopfjäger“ (vgl. Grubauer 1913) führte z.B. bei Außen-

65 Es kommt auch vor, dass Dorfautoritäten die Bergregionen des Dorfterritoriums als leer in bezug auf die dörfliche Bevölkerungsverteilung beschreiben. Li (2000: 12) machte dabei die Erfahrung, dass auf insistierende Nachfrage hin die Bergbewohner zwar erwähnt werden, diesen aber zugleich Attribute wie „primitiv“ oder ",rückständig“" zugeschrieben. „They sometimes refer to the mountain dwellers as orang dayak, a term they have picked up through media exposure to the apparently wild and primitive people of the Kalimantan interior, and now use to label and characterize their own backwoods." (ebd.). 
stehenden einen gewissen, von Angst gespeisten Respekt herbei, der lange Zeit einen Kontakt mit dieser Bevölkerung verhindert hatte. Ähnlich verhält es sich mit der Fremdzuschreibung der Gruppe der To Pekurehua im heutigen Lore Utara als To Napu, als „Totschläger", welche in Fußnote 58 bereits detailliert beschrieben wurde. ${ }^{66}$ Tsing (1993: 28) zufolge werden der masyarakat terasing von staatlichen Behörden statt dessen Prädikate der Unordnung, Schwäche etc. zugesprochen, und die Betonung ihrer marginalen gesellschaftlichen Lage kann dazu dienen, dass sich die schon weiter „entwickelte“, ländliche, arme Bevölkerung mit diesem Negativbeispiel vor Augen räumlich zentraler positioniert. Dass das Bild von den ,primitiven“ Menschen von unterschiedlichen Institutionen und für divergierende Zielinteressen verwendet wird, beschreibt Tsing an Beispielen aus Borneo:

\section{„Ecological activists argue for the conservation of Bornean rainforests based on images of nature- loving primitive tribes. Such images of primitive conservatism are also used by developers to prove the necessity for progress in the form of forced resettlement and export-oriented resource appropria- tion." (1993: 32).}

Das in den 1970er Jahren von der Staatsregierung ins Leben gerufene IDT-Programm (Instruksi presiden Desa Tertinggal; wörtlich übersetzt: Instruktionen des Präsidenten für zurückgebliebene Dörfer) soll dazu dienen, eben jene Gemeinden dem nationalen Entwicklungsstand anzugleichen. Neben direkten materiellen, staatlichen Zuwendungen, welche die Ergebnisse anderer Inpres-Programme ${ }^{67}$ darstellen, spielen sich die lokalen Umsiedlungsprogramme vorwiegend im IDT-Rahmen ab. Die Einschätzung von Akita \& Szeto (2000: 170), es handle sich bei den IDT-Programmen um eine "political and economic devolution to regional governments" besonders auf fiskalischer Ebene, erscheint angesichts der Einschätzung in den betreffenden Behörden auf Provinzebene allerdings zu hoch gegriffen. Von Vertretern dieser regionalen Verwaltungseinrichtungen wird deutlich herausgestellt, dass sie selbst nach Ende der Suharto-Ära nur ausführende Organe der Anweisungen aus Jakarta sind. Manche der in ihren Augen notwendige Umsiedlungsprojekte scheiterten allein an der positiven Bestätigung des Innenministeriums und der damit verbundenen Verweigerung der benötigten finanziellen Mittel.

Lokale Umsiedlungsprogramme werden in erster Linie von der Behörde für Gemeindeentwicklung, PMD (Pembangunan Masyarakat Desa), in Palu durchgeführt (Lagarens 2002). ${ }^{68}$ Vom Innenministerium in Jakarta erthält die Behörde die Planvorgaben für durchzuführende lokale Umsiedlungen. Aus Vertretern einer

66 Vgl. auch Hefner (1990) zu der Beziehung zwischen Hoch- und Tieflandbewohnern auf Java in früherer Zeit, welche von Furcht vor und gleichzeitiger Bewunderung für erstere durch die Küstenbewohner geprägt war.

67 Inpres $=$ Instruksi presiden (Instruktionen des Präsidenten).

68 Seit 1999 führt auch die Transmigrationsbehörde lokale Umsiedlungsprogramme durch, da dort nach dem Ende der Transmigrationsprogramme Ressourcen frei geworden sind. 
Reihe anderer staatlicher Verwaltungseinrichtungen, wie der Landwirtschaftsbehörde (Departmen Pertanian), der Forstbehörde (Departmen Kehutanan) der Gesundheitsbehörde (Departmen Kesehatan) und der Behörde für soziale Angelegenheiten (Departmen Sosial), wird anschließend ein Untersuchungsteam zusammengestellt. Dieses vom Departmen Sosial geführte Team erarbeitet einen Bericht über die infrastrukturellen und naturräumlichen Gegebenheiten der Ursprungsregion der umzusiedelnden Bevölkerung. Darin werden u.a. Erosionsgefährdungen, unzureichende Gesundheitsversorgung, der Zugang zu natürlichen Ressourcen wie Wasser und zu Bildungsmöglichkeiten, sowie die generelle verkehrstechnische Erschließung des Gebietes festgehalten. Anschließend werden potentielle Zielregionen der Umsieldung untersucht, wobei insbesondere die Faktoren der Bodenfruchtbarkeit und Verfügbarkeit von Landressourcen berücksichtigt werden.

Im Rahmen staatlich geplanter, lokaler Umsiedlungsprogramme sollten mit der Abwanderung der lokalen Bergbevölkerung mögliche Erosionsgefahren minimiert werden. Zum anderen bot die Besiedlung der bis dato noch ungenutzten Gebiete in den Talböden die Möglichkeit zur Steigerung der landwirtschaftlichen Bewirtschaftung, insbesondere des Nassreisanbaus, der die Selbstversorgung mit Grundnahrungsmitteln gewährleisten sollte. Ist die Zielregion ausgewählt, werden die Bewohner der Ursprungsgemeinde darüber informiert, dass sie umgesiedelt werden sollen. Dabei wird ihnen laut Darstellung von PMD eine Verbesserung ihrer Einkommenssituation in Aussicht gestellt. Ferner weisen die Behördenvertreter gegenüber den betroffenen Familien auf den positiven Effekt hin, nicht mehr der Brandrodungslandwirtschaft nachgehen zu müssen. In den jeweiligen Zielgebieten werden den Migranten Landgrundstücke zur Verfügung gestellt. Ferner erhalten die neuen Bewohner Nahrungsbeihilfen, um die Monate zwischen Ankunft und der ersten eigenen Ernte zu überbrücken. Es werden Schulen, Gesundheitsstationen und religiöse Einrichtungen errichtet sowie Pflanzensaat, Vieh und Werkzeuge für die Landwirtschaft bereitgestellt. Während der ersten fünf Jahre ${ }^{69}$ wohnen auch drei bis fünf Beamte der beteiligten Behörden (v.a. des Departmen Sosial) in den Dörfern, um die Entwicklungen des Aufbaus zu überwachen.

Hauptzielregion staatlich geplanter, lokaler Umsiedlungen im Untersuchungsgebiet ist das Kecamatan Palolo, wo allein in die beiden Dörfer Rahmat und Kamarora Ende der 1970er Jahre 1.450 Haushalte umgesiedelt worden sind. Auch fand dort eines der bislang letzten von PMD in der Lore-Lindu-Region durchgeführten Umsiedlungsprojekte in den Jahren 1984 bis 1986 nach Lembangtongoa statt. Eine bedeutende Herkunftsregion innerhalb des Lore-Lindu-Gebiets ist das Kecamatan Kulawi.

69 Seit dem Jahr 2000 wurde diese Zeitspanne auf drei Jahre verkürzt. 
Anhand des Untersuchungsdorfes Maranatha im Kecamatan Sigi-Biromaru werden solche lokalen Umsiedlungsprojekte im Folgenden am konkreten Beispiel veranschaulicht. Es handelt sich hierbei um das erste Projekt lokaler Umsiedlung in Zentralsulawesi seit der Unabhängigkeit (Nirboyo 1990: 71). Im Falle des Dorfes Maranatha wurde die Bevölkerung mehrerer Gemeinden umgesiedelt. Die Herkunftsorte befinden sich in den Hanglagen der das Palu-Tal säumenden Gebirgsketten. Der Zielort befindet sich im Palu-Tal östlich des Palu-Flusses, etwa 16 km südlich der Provinzhauptstadt Palu. Die Umsielung begann im Jahr 1969. Die Hauptphase der staatlich gelenkten Migration endete zwei Jahre später. Das Departmen Sosial in Palu organisierte zwar die Umsiedlungsmaßnahmen. Die Behörde bediente sich allerdings der Heilsarmee für die Überzeugungsarbeit in den einzelnen Bergsiedlungen. So berichtet ein Major der Heilsarmee in Maranatha, wie er 1968 im Auftrag des Departmen Sosial regelmäßig die entsprechenden Dörfer besuchte, um die Bevölkerung von den positiven Auswirkungen einer Umsiedlung zu überzeugen. Gleichzeitig wies er auch auf die Folgen einer Verweigerung seitens der Bevölkerung hin, in dem er die Drohung der Behörde in Palu weitergab, dass in solch einem Fall die Häuser und Felder der Menschen niedergebrannt würden. Diese Androhung wird auch von anderen Bewohnern Maranathas bestätigt. Noch drastischere Maßnahmen erinnert eine der umgesiedelten Personen und erzählt, dass die Regierung angedroht hätte, die Menschen bei Verweigerung der Umsiedlung zu erschießen. Anhand des Einsetzens der Heilsarmee treten die Kontinuitäten dieses postkolonialen Umsiedlungsprojekts sehr deutlich zu Tage, waren doch schon während der niederländischen Kolonialherrschaft Missionare mit der Kommunikation mit der lokalen Bevölkerung betraut worden. Den Menschen wurde jedoch nicht nur gedroht, sondern es wurden ihnen auch die Vorteile einer Umsiedlung nahe gebracht. Die Aussagen der befragten Dorfbewohner hierzu decken sich mit den aus den Interviews mit Vertretern von DepSos und PMD gewonnenen Informationen.

Das Zielgebiet der Umsiedlung bestand beim Eintreffen der ersten Migranten aus einer Waldfläche. Die Neuankömmlinge, bei denen es sich anfangs nur um die Familienväter oder alleinstehende Männer handelte, begannen damit, den Wald für die späteren Grundstücke zu roden. Während dieser Zeit wurden sie in zwei großen Holzbaracken untergebracht, die von der Regierung bereitgestellt worden waren. Die Baracken waren mit einem Raum pro Familie und einer Gemeinschaftsküche ausgestattet. Die gefällten Bäume wurden zu Brettern für die zukünftigen Häuser verarbeitet. Den Standort der Häuser bestimmte die Verwaltung anhand eines Bebauungsplans (vgl. Abb. 15). Deutlich zu erkennen ist der planmäßige Aufbau der Siedlung. Die Befragungen ergaben, dass die Bevölkerung den jeweiligen Herkunftsorten entsprechend auf unterschiedliche Bereiche des Bebauungsgebiets verteilt wurden (vgl. auch Abb. 36.) Jede Familie erhielt ein Hausgrundstück von 50 mal 50 Metern Fläche, auf dem vier mal sechs Meter große Häuser erbaut wurden. Als landwirtschaftliche Nutzfläche wurden zwar zwei Hektar pro Familie versprochen, letztendlich aber nur ein Hektar zur Verfügung gestellt. Manchen, die erst 
später nach Maranatha zogen, wurde nur noch ein halber Hektar Land zugesprochen. Die Regierung versorgte die Bevölkerung mit Zinkblech für die Hausdächer und mit Grundnahrungsmitteln für die ersten Monate des Aufenthalts, bis die Familien sich von der Ernte ihrer eigenen Felder versorgen konnten. Als der Bau der Dorfstraßen durch Maranatha begann, wurden diejenigen Hauhalte, deren Häuser sich nicht entlang der Straße befanden, aufgefordert, ihre Häuser an der Straße wieder aufzubauen.

Bereits zwei Wochen nach ihrer Ankunft im Jahr 1969 kehrten 50 der insgesamt 60 ersten Umsiedler wieder in die Berge zurück, da sie aufgrund der zu jener Zeit herrschenden Trockenheit im Palu-Tal keine landwirtschaftliche Verbesserung für sich und ihre Familien sahen. Nachdem die in Maranatha Verbliebenen jedoch ihre erste ausreichende Ernte erzielen konnten, siedelten sich auch die anderen wieder in der Ebene an. Im Jahr 1972, als das Palu-Tal von einer lang anhaltenden Dürreperiode heimgesucht wurde, entschieden sich zahlreiche Familien im damals noch Rarantikala genannten Maranatha für eine Rückkehr in ihre Herkunftsdörfer.

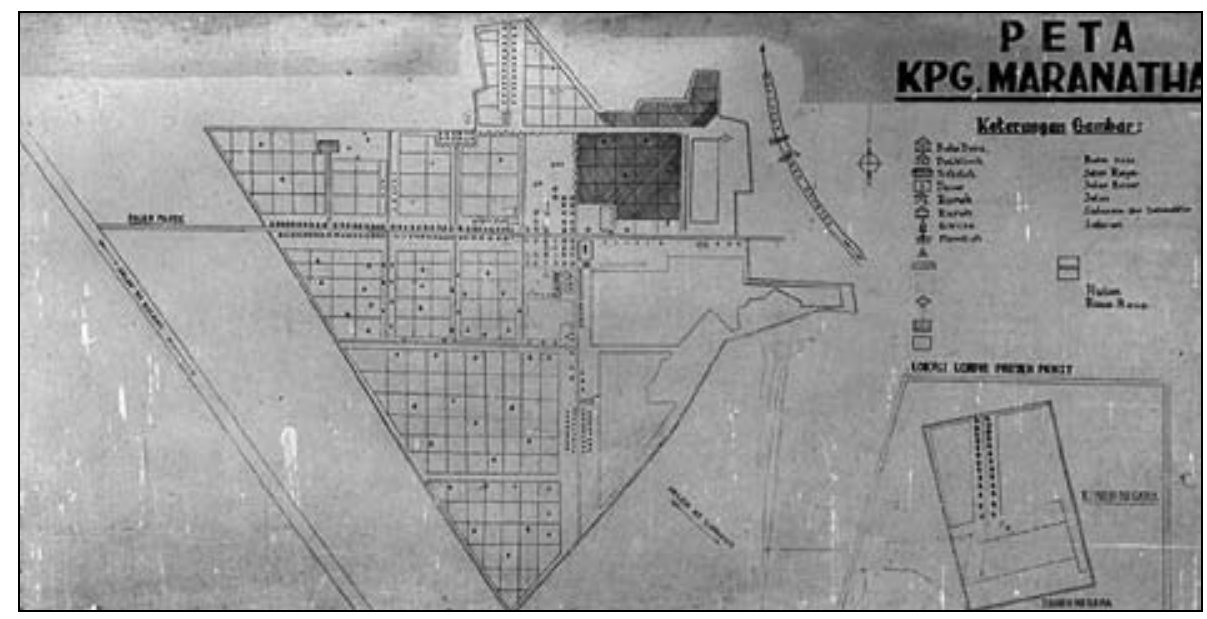

Abb. 15 Planskizze von Maranatha

(Quelle: Eigene Aufnahme, 2001)

$\mathrm{Zu}$ diesem Zeitpunkt existierte noch keine ausreichende Bewässerungsanlage ${ }^{70}$ für die Nassreisbewirtschaftung und die ausbleibenden Niederschläge jener Zeit reichten vielerorts nicht aus, um die Grundnahrungsversorgung sicher zu stellen. Viele Familien sahen sich daher genötigt, in ihre regenreicheren Ursprungsgebiete zurück-

70 Der Ausbau der Gumbasa-Bewässerungsanlage wurde 1976 abgeschlossen. Auch aus Maranatha waren zahlreiche Leute als Tagelöhner an den Arbeiten beteiligt. 
zukehren. Die Mehrzahl dieser Familien kehrte nach dem Ende der Dürreperiode wieder nach Maranatha zurück, einige verblieben jedoch in ihren Herkunftsdörfern. Auch hier zeigen sich Parallelen zur kolonialen Umsiedlungspolitik. Bereits die Kolonialverwaltung verzichtete, geleitet von der sog. Ethischen Politik der Niederlande in bezug auf das Verhalten gegenüber der einheimischen Bevölkerung in den Kolonialbesitzungen, auf eine gewaltsame, wiederholte Umsiedlung aus den Berggebieten in die neu gegründeten Dörfer im Tal. Eine hundertprozentige Rückkehrquote nach Maranatha war allein schon durch die spezielle Landvergabepolitik der Regierungsbehörden illusorisch. Diese sah nämlich vor, dass das Land von abgewanderten Hauhalten nach spätestens einem Jahr anderen Familien zur Verfügung gestellt wurde. Ohne das Land sahen die betroffenen Haushalte schließlich keinen Grund, nach Maranatha zurückzukehren.

Im Jahr 1976 wurde das Umsiedlungsprogramm offiziell beendet. Der ursprüngliche Dorfname Rarantikala (übersetzt: im dornigen Gras) sollte einem neuen Namen weichen, der die Identifikation der Bevölkerung mit dem Dorf stärkt und weitere Rückwanderungen in die Herkunftsgebiete nach Möglichkeit verhindert. Der Name Maranatha schien dafür geeignet zu sein. Er bedeutet „Warten auf den Messias" und gibt den christlich missionierten Dorfbewohnern, die heute die überwiegende Mehrheit in der Gemeinde ausmacht, zu verstehen, dass sie solange an diesem Ort bleiben soll, bis ihnen Jesus erscheint. In der Tat kam es in den Folgejahren zu keinen nennenswerten Rückwanderungen in die Herkunftsdörfer mehr. Auch bereut mittlerweile kaum jemand, seinerzeit umgesiedelt worden zu sein. Positiv wird angemerkt, dass Maranatha eine Straßenanbindung hat, während manche der Ursprungsdörfer teilweise mehrere Stunden von der nächsten per Auto befahrbaren Strasse entfernt lagen. Auch der Nassreisanbau wurde gegenüber dem Trockenfeldanbau schnell als Verbesserung angesehen. Zudem gehört die bessere Erreichbarkeit höherer Schulen zu den meistgenannten Aufwertungen des Zielortes der Umsiedlung.

Nicht alle Bewohner der Ursprungsdörfer wurden nach Maranatha umgesiedelt. Manche schlossen sich gegen Ende der 1970er Jahre anderen lokalen Umsiedlungsprojekten, v.a. in die Dörfer Banpres ${ }^{71}$ und Kamarora im Palolo-Tal an. Nur der ehemalige Herkunftsort Lampio, dessen Bevölkerung sich in Dusun 6 von Maranatha angesiedelt hatte, existiert heute nicht mehr. In den anderen Dörfern stieg die Einwohnerzahl im Laufe der Jahre wieder an. Die Anzahl der aktuell dort lebenden Familien bewegt sich je nach Dorf zwischen etwa 30 und 200. Ein Besuch des Herkunftsdorfes Balumpeva bestätigte die genannten Größenordungen exemplarisch. Ein Informant in Balumpeva erklärte, dass sich der Großteil der Dorfbevölkerung in den unwegsamen Gebieten hangaufwärts aufhält. Es handelt sich, wie in den

71 Banpres ist die Abkürzung von bantuan presiden (Hilfe des Präsidenten). 
meisten Fällen staatlich geplanter, lokaler Umsiedlungsmaßnahmen in der Untersuchungsregion, also nicht um Totalumsiedlungen bei anschließendem Wüstfallen der Herkunftsorte.

\subsubsection{Staatlich geplante, nationale Umsiedlungen - Das Transmigrasi- Programm}

Nach Boomgard et al. (1991: 55) ist die Migration von Javanern auf die äußeren Inseln nicht allein Erscheinung des 20. Jh., insbesondere der Transmigrasi-Politik des unabhängigen Indonesien, sondern:

„In addition to the migration of labourers, transmigration (or ,colonisation" as it was originally called) formed an important element of the mass migrations from Java to the Outer Islands. The idea had already been considered in the $19^{\text {th }}$ century of permanently resettling Javanese in other parts of the Archipelago with the stated aim of improving the exploitation of the ,natural riches" there." (ebd.).

Eine Entlastungsmigration von der Hauptinsel Java fand also bereits während der niederländischen Kolonialherrschaft statt. Dabei standen politische und wirtschaftliche Aspekte an oberster Stelle, wie im Folgenden noch belegt werden soll. Die durch finanzielle Anreize geförderte Migration von Javanern nach Sumatra, Kalimantan und Sulawesi hatte bis 1941 einen Umfang von ca. 80.000 Personen (Cribb 1993: 237).

In der Untersuchungsregion gab es während der Kolonialzeit nur einen einzigen Transmigrationsversuch. Im Jahr 1906 wurden christliche Javaner in dem Dorf Kalawara-naputih (heute: Kalawara) im Palu-Tal angesiedelt (Van Hengel 1910: 21). Unter der Leitung der Heilsarmee sollten die Migranten v.a. die Ausweitung des Nassreisanbaus befördern, der lokalen Kaili-Bevölkerung die entsprechenden Anbaumethoden vorführen und die Entwicklung der zu jener Zeit noch dünn besiedelten Palu-Ebene befördern (Encyclopaedie 1921; Bd. 2: 257). Im Jahr 1922 lebten 69 Javaner in Kalawara, 1926 waren es 148, zwei Jahre später bereits 208 (Logeman 1922: 43; Tideman 1926: 91; Metzner 1981: 51). Das Transmigrationsprojekt wurde letztendlich nicht als Erfolg gewertet. ${ }^{72}$ Vielmehr stellte Kalawara eine Enklave dar. Allein die religiöse Zuordnung der Javaner führte zu Konflikten in dem bereits frühzeitig islamisch beeinflussten Palu-Tal. Nach Hirschmann (1934: 28f) wurde das Ziel der Niederländer, eine stärkere Verbindung von Javanern und Kaili durch Heirat zu erreichen, nicht in dem erwünschten Umfang und Tempo umgesetzt. Zudem

72 Metzner (1981: 51) merkt interessanterweise an, dass es sich bei Kalawara um ein ermutigendes Beispiel gehandelt habe und dass für 1939 eine weitere Ansiedlung von Javanaern in der Region geplant gewesen sei. Die Einlassungen der damaligen Kolonialverwalter deckt sich mit dieser Aussage jedoch in keiner Weise. 
widersprachen sich die Interessen der Kolonialregierung und der Mission der Heilsarmee. Letztere stellte den Migranten nur kleine Felder schlechter Qualität für den Nassreisanbau zur Verfügung, während Kokospalmen- und Kaffeefelder im Besitz der Missionare verblieben. Die Javaner mussten zweimal pro Woche unentgeltlich auf deren Feldern arbeiten. Hierdurch sollte gewährleistet werden, dass sich die Kolonie Kalawara nicht nur selbst unterhält, sondern auch die Kosten der Heilsarmee für ihre Tätigkeit in der gesamten Palu-Region getragen werden (Hirschmann 1934: 31). Ein weiterer Grund für das Misslingen des Transmigrationsprojekts findet sich im Fehlen eines Leiters der Siedlung, der selbst mit den Anbaumethoden vertraut ist, die javanische Sprache beherrscht und Handelsgeist besitzt (Hirschmann 1934: 29). Die Unzufriedenheit der Javaner mit ihrer neuen Umgebung führte in der Folge zu zahlreichen spontanen Abwanderungen in andere Dörfer des Palu-Tals (z.B. Sibalaya und Sibowi) oder nach Palu, wo sich viele als Hausangestellte verdingten (Hirschmann 1934: 28). Kalawara war zusammenfassend das erste und letzte Transmigrationsprojekt in der Lore-Lindu-Region während der niederländischen Kolonialzeit.

Unter der Präsidentschaft Suhartos erfuhr das Prinzip der Umsieldung von den dicht besiedelten Inseln Java und Bali auf die viel geringer bevölkerten sog. Äußeren Inseln des Archipels eine Revitalisierung. Das ab 1969 unter dem Namen transmigrasi firmierende Programm, mit dessen Hilfe zwischen 1969 und 1994 etwa 6,8 Mio. Menschen umsiedelt wurden, ist hinsichtlich seiner politischen Grundlagen, Bedeutung und Auswirkungen für Indonesien insgesamt bereits vielfältig dokumentiert (vgl. u.a. Guiness 1977; Hardjono 1977; Kebschull 1984, 1986, 1987; Kebschull \& Fasbender 1987; Fasbender \& Erbe 1990; Scholz 1992; Fearnside 1997). Sie sollen daher in der vorliegenden Arbeit nicht eingehend resümiert werden. Erst seit kurzem jedoch gibt es auch Arbeiten, die sich mit der Transmigration speziell in die Lore-Lindu-Region befassen (vgl. Hoppe 2003; Hoppe \& Faust 2003) und die Auswirkung der Bildung von Transmigrationssiedlungen mit Fallbeispielen aus anderen tropischen Agrarkolonisationsräumen vergleichen (vgl. Faust im Druck). Die Transmigrationsprojekte im Kec. Lore Utara, welche in den oben genannten Schriften behandelt werden, werden anhand eigenen Interviewmaterials im Folgenden durch ein weiteres im Kec. Palolo ergänzt. Berücksichtigt man die Beschreibungen zur Bevölkerungsentwicklung und zu den allgemeinen Aspekten der Migration im Lore-Lindu-Gebiet (vgl. Kap. 3.1 und 3.3), erscheint es nicht verwunderlich, dass ausschließlich der östliche Teil der Untersuchungsregion ein Zielgebiet für staatlich geplante nationale Umsiedlungsmaßnahmen wurde. Die relativ späte verkehrs- und siedlungsmäßige Erschließung (letzteres v.a. in Palolo) dieser Landstriche war bei der Wahl der Zielregion von maßgeblicher Bedeutung. Zwar würde zumindest der Faktor Verkehrsinfrastruktur auch auf Regionen wie das entlegene Pipikoro im Kec. Kulawi zutreffen. Hier träfe aber eine weitere entscheidende Einflussgröße nicht zu, nämlich die große Verfügbarkeit von landwirtschaftlich nutzbaren Landressourcen für den bei Javanern und Balinesen bevorzugten Nassreisanbau. 
Insgesamt handelt es sich in der Lore-Lindu-Region um vier Transmigrationsprojekte. Drei der Projekte fanden in Lore Utara statt und führten zur Gründung der Siedlungen UPT Tamadue (Mekarsari), UPT Wanga (Siliwanga) und KaduwaaTrans. Die vierte Transmigrationssiedlung, welche in Palolo gegründet wurde, trägt den Namen Bahagia (vgl. Abb. 16).

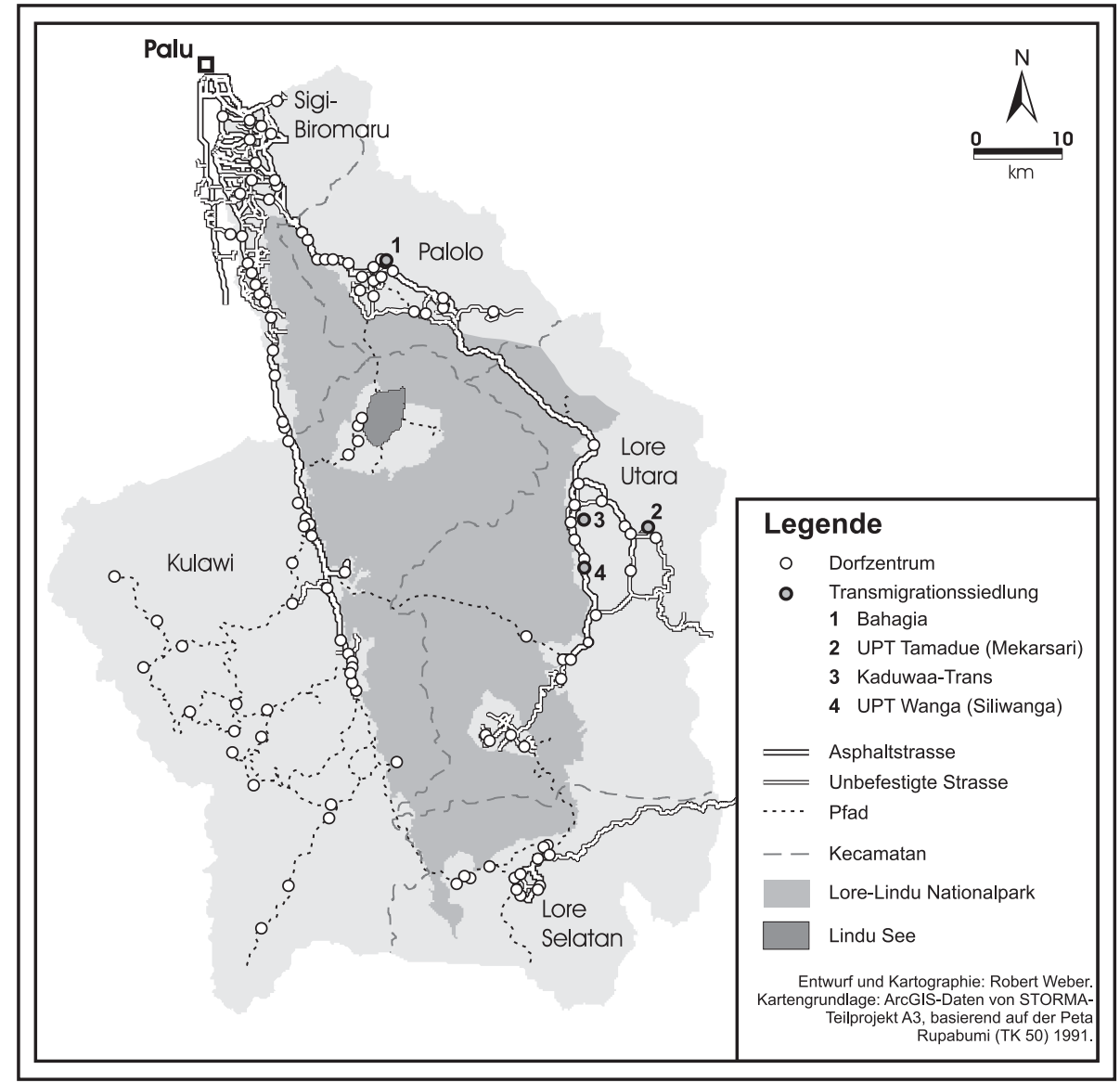

Abb. 16 Lage der Transmigrationssiedlungen im Untersuchungsgebiet

Entsprechend ihrer räumlichen Unterteilung lässt sich auch die zeitliche Einordnung der Entstehung dieser Siedlungen trennen. Während Bahagia mit dem Jahr 1965 noch in die Endphase der Regierungszeit Sukarnos fällt, datiert die Gründung der anderen drei Siedlungen auf die Jahre 1991 und 1993 zurück. Nach Hoppe \& Faust (2004: 7) plante die Transmigrationsbehörde, in Lore Utara insgesamt 600 Haushalte anzusiedeln. Die Transmigrasi-Projekte sollten der Kultivierung von Grass- bzw. Waldgebieten dienen, welche vor Beginn der Umsiedlungen von der lokalen 
Bevölkerung als Weideflächen bzw. Rattan- und Holzsammelgebiete genutzt wurde. Zehn Jahre nach Beginn der drei Umsiedlungsprojekte kommen Hoppe \& Faust (2004: 9ff) zu dem Ergebnis, dass alle drei Projekte nicht erfolgreich im Sinne der Transmigrationspolitik waren. In Siliwanga, dezimierte sich die Zahl der Transmigranten-Haushalte innerhalb einer Dekade auf gerade noch $29 \%$ der ursprünglich anvisierten 300 Haushalte. Eine ähnliche Entwicklung vollzogen die anderen beiden Siedlungen. In der Regel verblieben nur noch die Haushalte einer Herkunftsregion im Dorf, während die meisten Mitglieder anderer ethnischer Gruppen aus Java, Bali oder Nusa Tenggara Barat nahezu geschlossen in ihre Herkunftsgebiete oder in andere Regionen Zentralsulawesis abwanderten. Ursächlich für die Abwanderung sind ungünstige landwirtschaftliche Bodenbedingungen, klimatische Differenzen zwischen Herkunfts- und Zielregion, unzureichende infrastrukturelle Unterstützung von Seiten der Regierungsbehörden, Landkonflikte sowie die insgesamt ungewohnte, neue Situation im Zielgebiet.

In Siliwanga, wo von Anfang an Balinesen die Mehrheit der Transmigranten ausmachten, konnte keine Nassreisbewirtschaftung im angestrebten Umfang durchgeführt werden, da, entgegen den Versprechungen vor der Umsiedlung, keine ausreichenden Bewässerungsanlagen zur Verfügung gestellt wurden. Zudem offenbarten sich Konflikte über das von der Transmigrationsbehörde vorgesehene Land und den Ansprüchen der Nachbargemeinde Wanga. Ein Teil der Einwohner Wangas wurde von der Regierung ebenfalls aufgefordert, sich in Siliwanga anzusiedeln. Der Hintergedanke, dadurch eine bessere Integration der Transmigranten in die lokale Bevölkerung zu erreichen, erbrachte jedoch nicht den erhofften Erfolg. Vielmehr offenbarten sich Doppelvergaben von Landgrundstücken an Transmigranten und aus Wanga zugewanderte Personen, welche zu entsprechenden Landkonflikten führten und dem Integrationsgedanken entgegenwirkten. Ähnliche Konflikte brachen auch im Fall Kaduwaa-Trans und UPT Tamadue auf. Auch in bezug auf die klimatischen Verhältnisse in Lore Utara wurden die Betroffenen im Vorfeld nur unzureichend informiert. So wurde den umgesiedelten Familien erst nach ihrer Ankunft bewusst, dass sich das Napu-Tal auf einer Höhe von etwa 1.100 m ü. N.N. befindet. Erhebliche gesundheitliche Probleme waren die Folge. Die positivste Entwicklung durchlief letztendlich die Transmigrationssiedlung UPT Tamadue. Hoppe \& Faust (2004: 22) führen dies auf den vergleichsweise hohen Integrationswillen der lokalen Bevölkerung von Tamadue zurück, der auch eine Schlichtung von Landkonflikten zuließ.

Das Transmigrasi-Projekt nach Bahagia im Kec. Palolo startete im Jahr 1965. Ursprünglich war allerdings nicht Bahagia das geplante Ziel dieser Umsiedlung von Java. Anfangs sollten die Migranten sich in Kalimantan ansiedeln. Statt dessen wurden sie nach Palu gebracht, von wo aus sie nach Parigi in der Tomini Bucht transportiert werden sollten. Auch diese Planung wurde kurzfristig geändert und entschieden, dass die Javaner in dem damals noch dünn bevölkerten Palolo-Tal angesiedelt werden sollen. Der zu jenem Zeitpunkt in Betracht gezogene Zielort Rahmat 
auf der Südseite des Tals kam letztendlich jedoch auch nicht für die Umsieldung in Frage, da die Brücke dorthin noch nicht existierte. Die isolierte Lage Rahmats hätte verhindert, dass die Multiplikatoreffekte durch das Vorexerzieren von Anbaumethoden in der Nassreisbewirtschaftung durch die Javaner, einem der Gründe für die Durchführung solcher Transmigrationsprogramme, nicht allzu wirksam geworden wären. Zudem befanden sich in dem Gebiet große Sumpfflächen, eine nur suboptimale Voraussetzung für den Nassreisanbau. Schließlich entschloss sich die Transmigrationsbehörde für eine Ansiedlung der Javaner auf dem Territorium des Dorfes Berdikari auf der Nordseite des Tals. Im Anfangsjahr der Umsiedlung erreichten 81 Familien Berdikari. Das Gebiet war noch nicht von den Einwohnern Berdikaris in Kultur genommen worden und bot daher ausreichend freie Landressourcen für die Transmigranten. 1976 lebten in dem Berdikari 2 benannten Gebiet bereits rund 400 Haushalte. Zwei Jahre später wurde Berdikari 2 ein eigenständiges Dorf und auf den Wunsch seiner Bewohner in Bahagia (übersetzt: glücklich) umbenannt. Bahagia steht für ein relativ erfolgreiches Transmigrationsprojekt, was sich auch aus der Namenswahl des Dorfes ableiten lässt.

Zusammenfassend lässt sich sagen, dass die in der Lore-Lindu-Region stattgefundenen interinsularen Umsiedlungsprojekte tendenziell nur von mäßigem Erfolg waren. Maßgeblich ausschlaggebend hierfür war die unzureichende Kommunikation zwischen staatlichen Behörden auf der einen sowie den Transmigranten und der lokalen Bevölkerung der Zielregionen auf der anderen Seite. Die positive Entwicklung im Fall Bahagia dürfte hauptsächlich dem Zufall geschuldet sein, dass sich das Palolo-Tal zu jener Zeit in einer Phase enormen infrastrukturellen Inputs befand und dass die Bewohner des Dorfes Berdikari selbst Zugewanderte aus Kulawi waren und sich dadurch keine Landkonflikte ergaben, bei welchen sich die „Lokalbevölkerung ${ }^{673}$ auf ihr Ahnenland beziehen konnten.

73 Mit der Verwendung der Anführungszeichen soll dem Umstand Rechnung getragen werden, dass es sich bei den ersten Einwohnern Berdikaris auch um Migranten handelt. Die Befragungen in dem Dorf haben ergeben, dass sich dessen Bewohner in der Regel selbst heute noch als orang Kulawi (Kulawi-Menschen) sehen und nicht etwa als orang Palolo. Selbst unter der jüngeren Generation, die in Berdikari geboren ist, überwiegt die ethnische Beziehung auf die Herkunftsregion ihrer Eltern. Die darin involvierten Aspekte der Ethnizität spielen bei der Frage der Segregation eine im gesamten Untersuchungsgebiet herausragende Rolle und werden in Kap. 4.3 noch genauer beleuchtet. Prinzipiell gilt aber für diese Untersuchung - dies wurde in dieser Arbeit bereits definiert (s.o.) -, dass unter der lokalen Bevölkerung eines Dorfes die zuerst dort angesiedelten Menschen zu verstehen sind. 


\subsection{Halbfreiwillige Umsiedlung am Beispiel des Dorfes Lawe}

Neben staatlich organisierten, lokalen Umsiedlungsprojekten lassen sich in der Untersuchungsregion auch durch solche Migrationsprogramme beeinflusste halbfreiwillige Wanderungsbewegungen ausmachen. Beispielhaft wird eine solche Form der Migration im Folgenden an dem Dorf Lawe im südwestlichen Distrikt Kulawi beschrieben.

Wie bereits im vorangehenden Kapitel erwähnt, waren vor allem jene Berggebiete von lokalen Umsiedlungsmaßnahmen betroffen, welche über eine unzureichende Verkehrsinfrastruktur verfügten und/oder ein relativ hohes Potenzial einer Hangerosion aufwiesen. Die Pipikoro-Region Kulawis, in der sich das Dorf Lawe befindet, zählt zu den infrastrukturell bislang am schlechtesten erschlossenen Gegenden der Lore-Lindu-Region. Ein stark zerklüftetes Relief, welches durch den Fluss Lariang ${ }^{74}$ und dessen Nebenarme geschaffen wurde, prägt das gesamte Gebiet. Die vielfach auf den bis zu 750 m ü. NN. erreichenden Bergrücken verorteten Dörfer sind durch unbefestigte Wege miteinander verbunden, die während der niederländischen Kolonialzeit in Zwangsarbeit angelegt worden waren. Bis heute ist die Region für PKW oder gar LKW unzugänglich. Waren und Güter werden immer noch entweder zu Fuß oder per Pferd transportiert. ${ }^{75}$

Das Dorf Lawe befindet sich etwa 47 Wegkilometer von der asphaltierten Hauptstraße Palu-Lempelero entfernt und kann in ein bis zwei Tagen nach Überwindung mehrerer Bergkämme erreicht werden. Die knapp 400 Einwohner bewohnen überwiegend Häuser aus Holz oder pitate (Bambusmaterial) und bauen noch heute Mais und Trockenreis im Wanderfeldbau an, der seit den 1950er Jahren durch Kaffee, seit den 1970er Jahren durch Gewürznelken und seit den 1980er Jahren durch Kakao ergänzt wird. Das gegebene Relief erfordert einen Anbau an bis zu 80 Grad geneigten Hängen, die aufgrund der Feldrotation durchaus mehrere Kilometer vom Dorfzentrum entfernt liegen können. Neben der Feldwirtschaft gibt es noch die für christlich geprägte Gebiete typische Schweinehaltung, welche jedoch nur im geringen Umfang betrieben wird.

Bis 1980 befand sich das Dorf Lawe noch rund zwei Kilometer nördlich seines heutigen Standorts. Im Folgenden soll daher zwischen Alt-Lawe und Lawe unterschieden werden. Bereits im Jahr 1969 sollte nach dem Wunsch der Provinzregierung Zentralsulawesis eine Umsiedlung der Bevölkerung Lawes an den Lindu See durchgeführt werden (vgl. Abb. 5). Diese Migrationsmaßnahme wurde allerdings

74 In der Lokalsprache Uma bedeutet koro „Fluss“. Pipi koro heisst übersetzt „entlang des Flusses“. Gemeint ist damit der Fluss Lariang, welcher sich durch das Pipikoro-Gebiet zieht.

75 Seit kurzem können einige Dörfer Pipikoros auch mit dem Motorrad erreicht werden. Die schmalen Wege entlang steiler Berghänge bergen jedoch, besonders bei Regen, ein hohes Unfallrisiko. 
ebenso wenig umgesetzt wie die Umsiedlungspläne im Jahr 1971 nach Pili Makujawa, 1973 in das einige Kilometer entfernte Dorf Onu, wo die Migranten aus Lawe Nassreisfelder anlegen sollten, oder nach Panggaiba im Folgejahr (Kerjasama Badan Perencanaan Pembangunan Nasional Yayasan Agro Ekonomika 1995: 19). Ende der 1970er Jahre machten Vertreter der Distriktregierung, die Lawe besuchten, einen letzten Vorschlag, in das Umsiedlungs-Projektdorf Kamarora im Distrikt Palolo umzuziehen, um das Problem einer mangelhaften Wasserversorgung in Alt-Lawe zu beheben. Obwohl sich der damalige Dorfbürgermeister selbst am liebsten der kleinen Gruppe migrationswilliger Familien im Dorf angeschlossen und somit für eine Umsiedlung der gesamten Dorfbevölkerung nach Kamarora plädiert hätte, sah er sich dem Willen der Mehrheit verpflichtet. Folglich informierte er die Regierungsbeamten, dass sein Dorf dieser planmäßigen Umsiedlung nicht zustimmt. Während fünf Familien Alt-Lawe verließen und nach Kamarora abwanderten, räumte die Distriktregierung dem Dorfoberhaupt eine Frist von einem Jahr ein, um einen geeigneten Standort für ein neues Dorf in der lokalen Umgebung zu suchen. Sollte diese Frist ohne ein sinnvolles Ergebnis verstreichen, würde die Bevölkerung AltLawes unter Zwang nach Kamarora umgesiedelt werden.

\subsection{Spontane Wanderungsbewegungen}

Spontane Migration kam in der Lore-Lindu-Region nach den Informationen Einheimischer bereits zu vorkolonialer Zeit vor (vgl. auch Departemen Pendidikan Dan Kebudayaan 1996/1997). Demnach soll z.B. die Bevölkerung Kulawis teilweise ursprünglich in der Region Bada (Lore Selatan) beheimatet gewesen sein. Allerdings kollidiert diese Aussage mit dem in Kulawi verbreiteten Glauben an eine Abstammung der lokalen Bevölkerung von mythischen Wurzeln, die keine Migrationselemente enthalten.

Kaudern (1925a: 19ff) geht jedoch davon aus, dass die ersten Siedler des nördlichen Kulawi-Tals von Osten her zugewandert waren und auf dem Bolapapu-Hügel (Bulu Bolapapu) die Orte Bolapapu und Lemo gegründet hatten. Von da aus sollen sie sich erst in der Kulawi-Ebene und später weiter nach Norden und Süden ausgebreitet haben (vgl. Abb. 17). Die Bewohner des südlichen Kulawi (Gimpu-Tal) und der Pipikoro-Region stammt nach Kaudern (1925a: 193) größtenteils aus den Regionen Bada und Rampi (vgl. Abb. 18). Die ersten Menschen des Napu-Tals migrierten dieser Quelle zufolge aus Bada und dem Poso-Tiefland in die Hochebene von Napu (Kaudern 1925a: 88ff; vgl. Abb. 19). Für die anfängliche Besiedlung des Palu-Tals und seiner Randgebirge nennt Kaudern (1925a: 45ff) mehrere Wanderungsbewegungen verschiedener Gruppen, wobei die Bewohner des nördlichen Palu-Tals von der Tomini Bucht im Osten (nordwestlich von Poso) zugewandert waren. Die südlichen Gebiete des heutigen Sigi-Biromaru (in etwa 
Sidondo bis Pakuli) wurden seinen Untersuchungen zufolge vom Napu-Tal ausgehend über den Lindu See erreicht (vgl. Abb. 17).

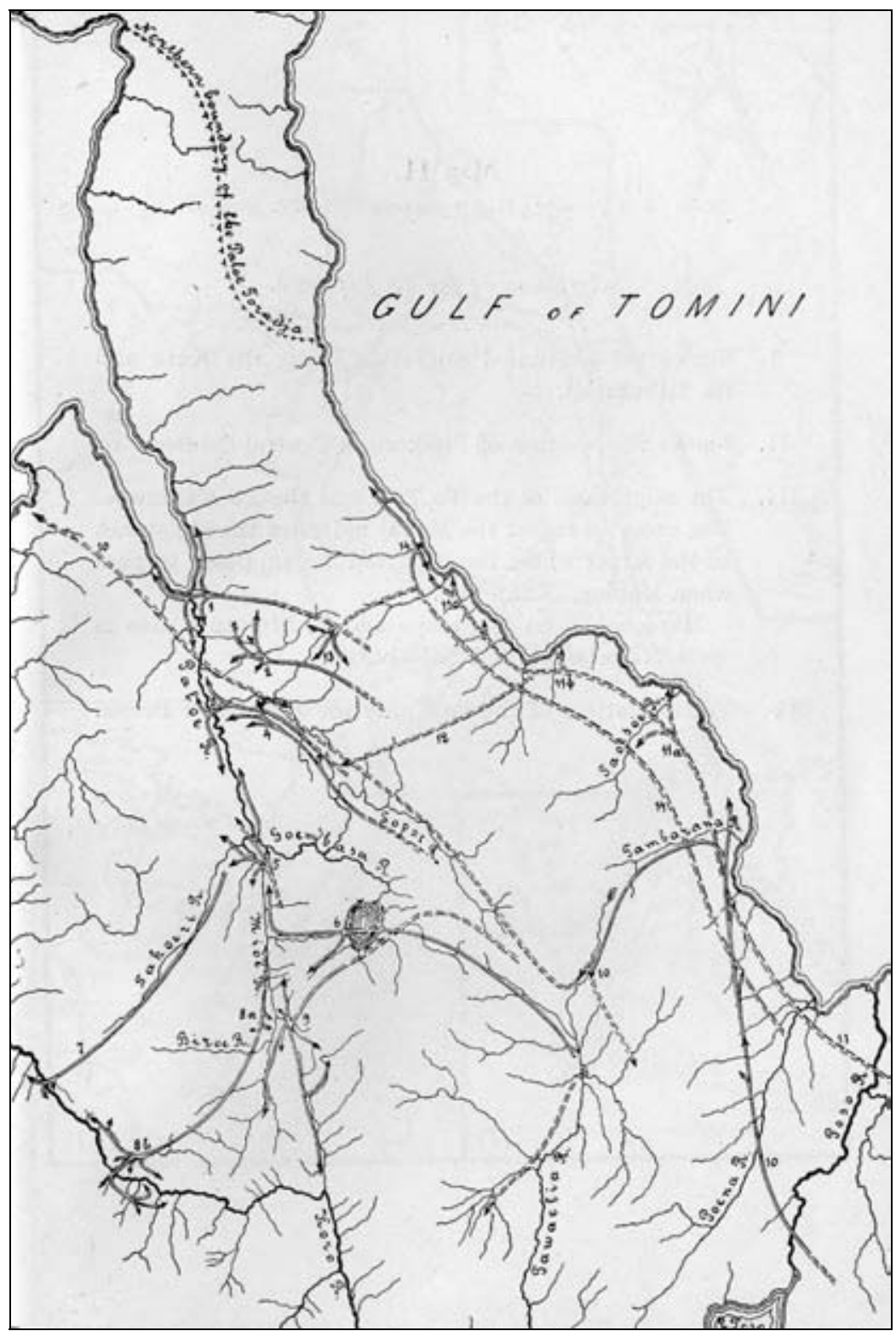

Abb. 17 Besiedlung des Palu-Tals und des nördlichen Kulawi (Quelle: Kaudern 1925a: 183) 


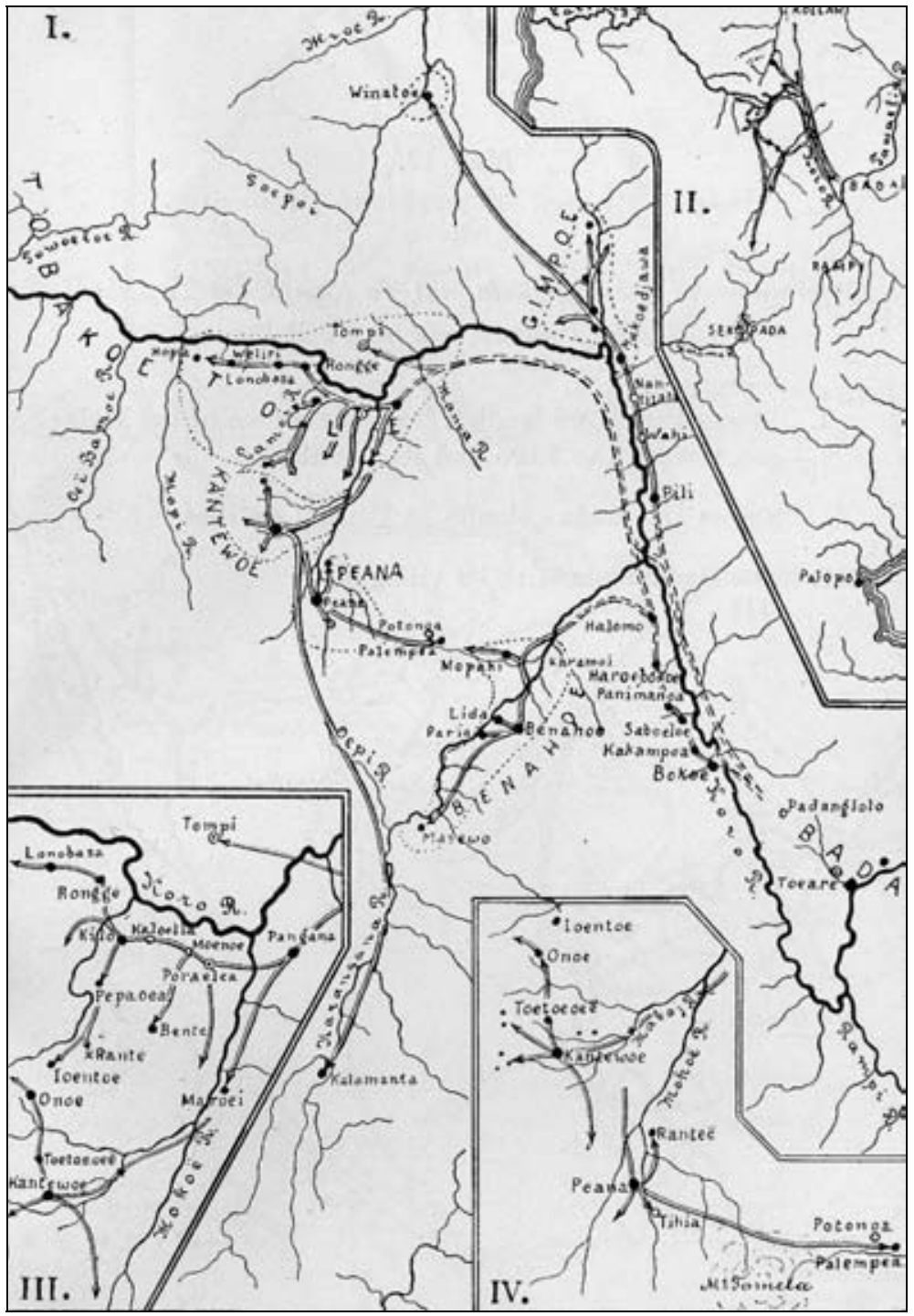

Abb. 18 Besiedlung des südlichen Kulawi von Rampi und über das Bada-Tal (Quelle: Kaudern 1925a: 1985) 


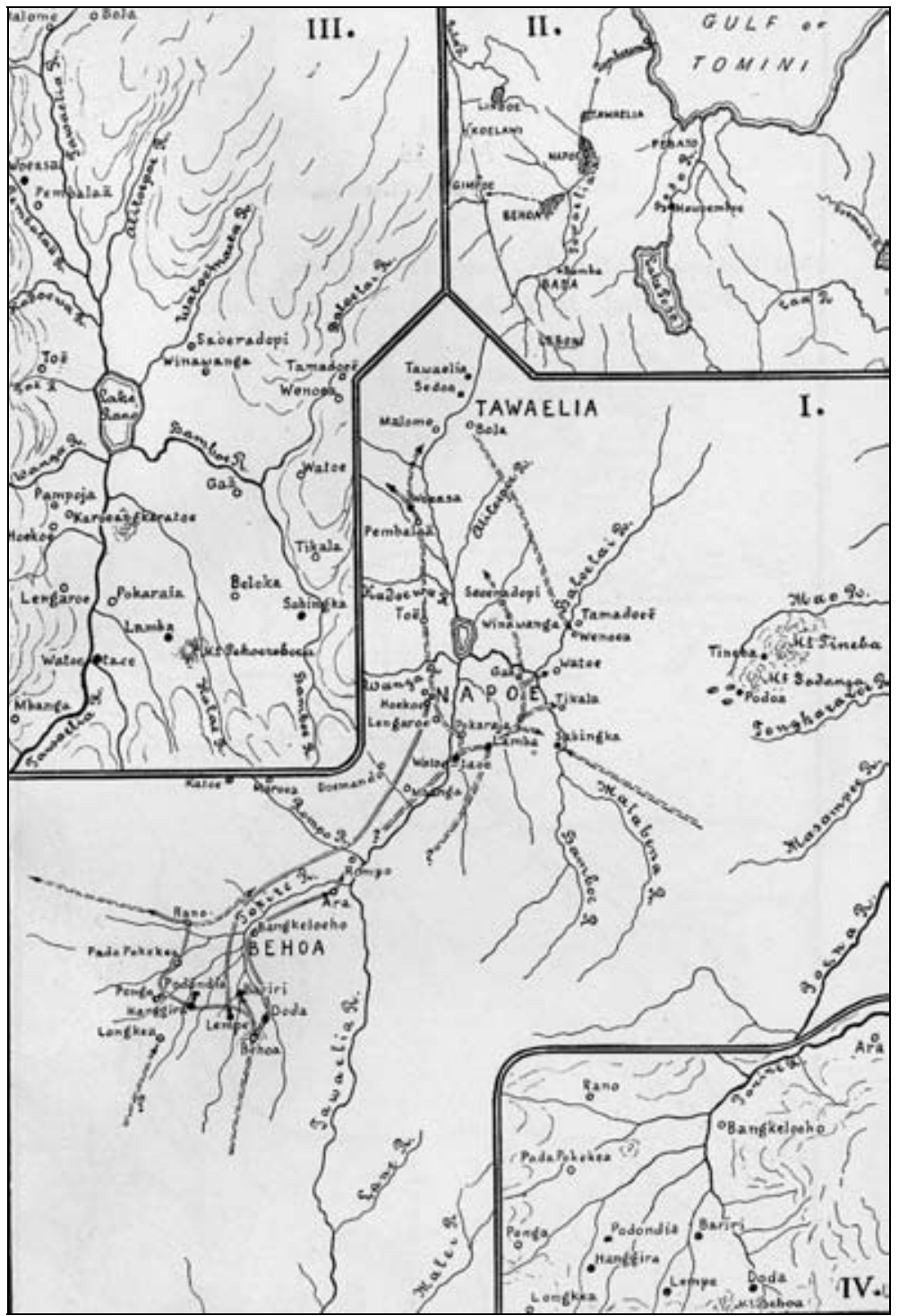

Abb. 19 Besiedlung von Napu- und Besoa-Tal (Quelle: Kaudern 1925a: 189)

Die präkolonialen Tauschbeziehungen, etwa zwischen den Königreichen Kulawi und Sigi (vgl. Aragon 1996d), welche mit dem Einfluss der Niederländer zunehmend einer Geldwirtschaft weichte und sich zunehmend intensivierte, führten zu einer stärkeren Annäherung der Bevölkerungen aus dem Hoch- und Tiefland. Hierdurch entwickelten sich Wanderungsbewegungen hauptsächlich auf der Basis von 
Heiratsbeziehungen. Auch innerhalb der einzelnen ethnischen Gruppen fand spontane Migration zwischen den Siedlungsgebieten der Familienclans zur Gewährleistung der Exogamie statt. Heirat lag auch der post-kolonialen spontanen Migration innerhalb der Lore-Lindu-Region als Hauptmotivation zugrunde und legte den Grundstein für die heute vorfindbaren, räumlich weit verzweigten Verwandtschaftsbeziehungen in der Untersuchungsregion.

Besonders ab der Mitte des 20. Jh. erhalten spontane Migrationsbewegungen einen immer bedeutender werdenden Stellenwert in der Lore-Lindu-Region. War die Kolonialzeit vor allem von erzwungenen Umsiedlungen (vgl. Kap. 3.4) und die ersten Jahre des neugegründeten indonesischen Staates von einer insgesamt niedrigen räumlichen Mobilität geprägt, so setzten ab Mitte der 1950er Jahre vermehrt spontane Wanderungen ein. Anfangs waren es, wie bereits erwähnt, hauptsächlich lokale Zu- und Abwanderungen innerhalb eines Kecamatan oder zwischen den einzelnen Kecamatan des Untersuchungsgebiets. Die Zuwanderung in das Palolo-Tal aus den infrastrukturell gering erschlossenen Bergregionen des Pipikoro-Gebiets im westlichen Kulawi während der 1950er bis 1980er Jahre (vgl. Abb. 14) kann hierbei als eines der deutlichsten Beispiele für die erste Zunahme spontaner Migration herangezogen werden (vgl. Kap. 3.6.1). Während das Kecamatan Sigi-Biromaru dank seiner Nähe zur Provinzhauptstadt Palu eine relativ kontinuierliche spontane Zuwanderung verzeichnet, erfahren entlegenere Gebiete wie das Kecamatan Lore Utara erst ab den 1980er Jahren bemerkenswerte Zunahme der Immigration. Es handelt sich dabei vornehmlich um die Ansiedlung von Bugis-Migranten aus Südsulawesi, ,searching for good fortune" (Acciaioli 1989). Push-Faktoren wie Landverknappung in den Herkunftsregionen sowie Pull-Faktoren, z.B. die Verfügbarkeit von landwirtschaftlich nutzbarem Land und/oder bereits existierende soziale, ethnische Netzwerke in der Zielregion, haben die Mehrzahl dieser Personen zu einem entsprechenden Wohnortwechsel bewogen. Seit einem Boom der Kakaopreise Mitte der 1990er Jahre (vgl. Kap. 5.2.2) intensivieren sich die spontanen Wanderungsbewegungen in der gesamten Untersuchungsregion. Gepackt vom „Kakao-Fieber“ nehmen die Einwohnerzahlen in jenen Regionen am deutlichsten zu, welche über die geeigneten Boden- und Klimavoraussetzungen für einen effektiven Anbau des Exportprodukts verfügen. Hierzu können alle Gebiete der Lore-Lindu-Region außerhalb des Palu-Tals gezählt werden. Allerdings ist die regionale Ausprägung dieser letztgenannten Migrationserscheinung noch anderen Faktoren als allein dem Kakaoboom geschuldet. Dieser kurze Überblick deutet bereits auf die räumliche Heterogenität spontaner Wanderungsbewegungen in dem Untersuchungsgebiet hin. Im Folgenden werden daher die spontane $\mathrm{Zu}$ - und Abwanderung für die einzelnen Kecamatan der Lore-Lindu-Region ausführlich untersucht. 


\subsubsection{Formen spontaner Migration}

Eine sehr weit verbreitete Form spontaner räumlicher Mobilität ist die Kettenmigration. Diese ist nicht nur bei interregionaler (regionaler bzw. nationaler) Migration zu finden, sondern auch bei intraregionalen (lokalen) Wanderungsbewegungen. Hierbei unterscheiden sich zwei Verlaufsmuster. Im ersten Fall gibt es eine Person oder Familie, welche in der Pionierphase in einen Zielort migriert. Dabei können die Motive für die jeweilige Migration durch verschiedene Push- und Pullfaktoren begründet sein (vgl. Kap. 3.6.2). Die nachfolgende Kettenmigration erfolgt im Anschluss an einen Informationsaustausch zwischen Pionieren und Folgemigranten über die bestehenden sozialen Netzwerke. In der Regel handelt es sich um Netzwerke innerhalb einer Verwandtschaftsgruppe oder, größer gefasst, innerhalb einer ethnischen Gruppe. Im zweiten Fall beteiligt sich eine Gruppe von mehreren Einzelpersonen oder Familien an der Pionierphase. Dabei setzt sich die Gruppe meist aus Mitgliedern eines Verwandtschaftsverbandes in einem Dorf zusammen. In selteneren Fällen sind es Freundschaftsbeziehungen, welche die Zusammensetzung der Migrationsgruppe bestimmen. Die anschließende Kettenmigration wird wiederum durch den Informationsaustausch innerhalb der sozialen Netzwerke ausgelöst.

Der Pioniermigration muss nicht grundsätzlich eine Kettenmigration folgen. In den Untersuchungsdörfern gibt es durchaus auch Beispiele für Einzelmigrationen. Diese ereignen sich mehrheitlich, wenn auch nicht ausschließlich, unter der jeweiligen Lokalbevölkerung einer Region. Klassische Beispiele hierfür sind der Wohnortwechsel in Folge von Heirat zweier in unterschiedlichen Dörfern lebender Menschen oder als Ergebnis eines Dienstortwechsels von staatlichen Angestellten. Daneben können Beschäftigungsangebote in der privaten Wirtschaft Einzelmigrationen verursachen, welche in vielen Fällen, ähnlich der Migration zu Ausbildungszwecken (z.B. Studium an der Universität in Palu), jedoch keinen permanenten Wohnortwechsel darstellen. Besonders jene Wanderungsbewegungen, welche sich an der Nachfrage nach Arbeitskräften orientieren, stellen oft Zwischenetappen der individuellen Migrationsbiographie dar. Diese Form der Etappen-Migration wird von der Bevölkerung i.d.R. als merantau (übersetzt: in die Fremde gehen) bezeichnet (vgl. dazu Kap. 3.6.2).

Um einen Eindruck von den unterschiedlichen Migrationsformen der Bewohner in der Lore-Lindu-Region zu erhalten, werden im Folgenden drei Migrationsbiographien wiedergegeben:

Biographisches Beispiel 1: Bodin ${ }^{76}$ wurde 1939 in Petapa (8 km nördlich von Parigi (zwischen Palu und Poso) geboren. Sein Vater war dort Koksnusspflücker auf Tagelohnbasis. Als sein Vater 1946 starb, nahm Bodins Schwester ihren Bruder mit nach Lasoani (heute Teil von Palu), wo seine Großeltern lebten. Dort ab-

76 Die Personennamen in allen drei Beispielen wurden vom Verfasser geändert. 
solvierte er 1944 die Grundschule. Bodins Eltern waren ursprünglich aus Lasoani und zogen später nach Parigi, als dort Kokospflücker gesucht wurden. Nach der Schulausbildung arbeitete Bodin in Lasoani als Ziegenhirte und heiratete 1955. Nach der Heirat sammelte er Damar in den Wäldern zwischen Palu und Parigi. Im Jahr 1972 zog er über Salua nach Saluki, wo er Rattan sammelte. Daneben bot er sich mit seiner Kuh bei den Dorfbewohnern zum Feld pflügen an. Mit dem verdienten Lohn erwarb er ein Stück Land, auf dem er versuchte, Kokospalmen anzupflanzen. Nachdem die Pflanzen jedoch von Schweinen zerstört worden waren und seine Schwiegereltern in Lasoani verstorben waren, verkaufte Bodin sein Land in Saluki und kehrte 1977 nach Lasoani zurück. Der Preis für Damar war inzwischen stark gesunken und so verdingte sich Bodin in einem Steinbruch. Mit dieser Lohnarbeitsbeschäftigung war Bodin nur mäßig zufrieden, da er eigentlich Bauer sein wollte. Das Stück Land, das seine Frau und er von ihren Schwiegereltern geerbt hatten, war jedoch für eine Bewirtschaftung zu trocken. Im Jahr 1978 zog Bodin daher nach Kapiroe (Palolo), wo er mit seiner Frau bei Verwandten wohnte. Der Bürgermeister von Kapiroe war zu jener Zeit der Chef eines RattanUnternehmens und stellte Bodin als Rattansammler ein. Neben dieser Tätigkeit bewirtschaftete er während seines dreijährigen Aufenthalts in Kapiroe auch ein Trockenfeld (Reis, Gemüse) auf bagi basil-Basis. ${ }^{77}$ Von Verwandten nach Sintuwu gerufen verließ Bodin Kapiroe im Jahr 1980. Der Bürgermeister von Sintuwu teilte Bodin ein Viertel Hektar Land zu, auf dem er ein Haus bauen konnte. Als Bodin nach Sintuwu zog, wohnten bereits neun weitere Familien in diesem Dorfabschnitt von Sintuwu. Alle stammten aus Lasoani und waren über Kapiroe nach Sintuwu migriert. Wie Bodin erhielten auch die neun anderen Familien nur ein Viertel Hektar Land, und der Bürgermeister untersagte ihnen, weiteres Land zu kultivieren. Neben der Feldarbeit arbeiteten die Menschen aus Lasoani auch auf den Maisfeldern von Kaili-Familien (bagi hasil-System), transportierten Fisch vom Lindu See nach Sintuwu und/oder sammelten Rattan, das sie an einen Händler im Ort verkauften. Nachdem Bodins Tochter einen Mandar-Mann (aus Südsulawesi) geheiratet hatte, ging Bodin 1982 nach Südsulawesi um Kakaosamen zu erwerben, da sich in Sintuwu durch den Zuzug von Bugis die guten Erträge des Kakaoanbaus herumgesprochen hatten. Vor Ort in Südsulawesi konnte sich Bodin persönlich von den Vorteilen des Kakaoanbaus überzeugen. Bereits 1985 konnte Bodin die erste Ernte der auf seinem 0,25 ha großen Grundstück gepflanzten Kakaobäume einfahren.

Biographisches Beispiel 2: Ibrahim wurde 1956 als Sohn von sawah-Bauern ohne Schulausbildung in einem Dorf in Südsulawesi geboren. Seine Familie verließ Südsulawesi im Jahr 1960 aufgrund der DI/TII-Unruhen. Ibrahims Onkel rief die Familie nach Palu, wo dieser schon seit einiger Zeit lebte. Als seine Eltern erfuhren, dass der Dorfchef von Sidondo I (Kec. Sigi-Biromaru) kostenlos Land für Migranten zur Verfügung stellte, siedelte sich seine Familie dort an, wo er die ersten beiden Klassen der Grundschule besuchte. In Sidondo I rodeten Ibrahims Eltern ein Stück Wald und pflanzten Mais und Reis an. Nachdem jedoch Schweine ihre Ernte vernichtet hatten, verließen sie das Dorf und zog zusammen mit etwa zehn weiteren Bugis-Familien nach Pandere. Sie erhielten vom Dorfchef Land in einem sumpfigen Gebiet in Dusun 1 in der Nähe des Palu-Flusses. Aller-

77 Bagi hasil bedeutet, dass der Besitzer eines Feldes dieses einer anderen Person zur Bewirtschaftung gibt. Beide Personen erhalten einen Teil der Ernte. 
dings wurde ihnen vom Dorfchef erklärt, dass sie ab der siebenten Ernte einen Teil des Ertrags an das Dorf abgeben müssten. Die Bugis-Familien lebten für insgesamt 10 Jahre in diesem Gebiet in kleinen Hütten auf ihren Feldern. Einige der lokalen Familien gaben ihnen Waldland zur Kultivierung, für welches diese ebenfalls nach der sechsten Ernte einen Teil des Ertrags verlangten. Ibrahims Eltern, die auf dem zusätzlichen Land Kokspalmen pflanzten, kauften während dieser Zeit ein kleines Stück Land entlang der Hauptstrasse und ließen sich dort schließlich im Jahr 1971 nieder. Auch die anderen Bugis-Familien erwarben nach und nach Land, das sich zum größten Teil an der Strasse befand, und errichteten dort Anfang der 1970er Jahre ihre neuen Häuser. Heute kultiviert Ibrahim weiterhin Nassreis und Kokospalmen. In Pandere besitzt er kein Kakaofeld. Dafür hatte er 1980 ein Feld in Pantai Timur erworben, auf dem er Gewürznelken anbaut. Zudem gehört ihm seit 1998 ein Stück Land in Alitupu (Lore Utara), das mit Mais, roten Bohnen und einigen wenigen Kakaobäumen bestellt ist.

Biographisches Beispiel 3: Kornelius wurde 1956 in dem Dorf Siwongi im Pipikoro-Gebiet Kulawis geboren. Seine Eltern hatten dort im Schnitt etwa 2 ha an steilen Berghängen gelegenes Land auf der Basis von Wanderfeldbau, auf dem sie Trockenfeldreis, Mais und Cassava im Wechsel anbauten. Nach einer Rotationsdauer von etwa sechs Jahren kehrten sie an das Ausgangsfeld zurück. Daneben unterhielten sie zwei Kaffeefelder. Kornelius verließ Siwongi im Jahr 1969, nachdem er ab der 5. Grundschulklasse keine Lust mehr hatte, weiter die Schule zu besuchen. Er zog nach Onu (ebenfalls im Pipikoro-Gebiet), wo er für zwei Jahre bei seinem Onkel lebte und diesem bei der Bestellung des Nassreisfeldes behilflich war. Im Jahr 1973 folgte Kornelius seinem Bruder nach Salua, wo zu jener Zeit schon einige Verwandte (Brüder, Cousins) von Kornelius wohnten. In Salua lebte er zusammen mit seinen Verwandten in einer Hütte und verdingte sich wie die anderen als Fischträger vom Lindu See nach Salua oder als Tagelöhner auf dem Feld. Zwei Jahre später zog Kornelius allein weiter nach Pandere. Der Grund für diesen Wohnortswechsel bestand in dem Angebot eines Bugis aus Pandere, den er während eines Marktbesuchs in Pandere kennenlernte und der ihm eine Lohntätigkeit auf dessen Kokospalmenfeld in Aussicht stellte. In Pandere wohnte er bei seinem Arbeitgeber und schnitt das Gras zwischen den Palmen auf dessen Feld. Noch im gleichen Jahr verließ Kornelius jedoch Pandere und migrierte nach Banpres (Kec. Palolo), da er von der dort geplanten Errichtung einer lokalen Transmigrationssiedlung erfuhr, wofür Arbeiter zum Roden des Waldes gesucht wurden. Kornelius entschied sich für diesen Wechsel, da er in Pandere für seine Arbeit auf Tagesbasis bezahlt wurde, in Banpres jedoch einen monatlichen Lohn erhalten würde. Nachdem Kornelius sechs Monate zusammen mit etwa 300 anderen Kontraktarbeitern in Banpres gearbeitet hatte, folgte er den anderen Arbeitern nach Palu Barat, um dort in einem Wiederaufforstungsprojekt in der Nähe der Dörfer Doda, Kalora und Paneki zu arbeiten. Obwohl das Projekt noch nicht abgeschlossen war, wanderte Kornelius zusammen mit drei Freunden Ende 1975 nach Berdikari, wo sich inzwischen einer seiner Onkel aus seinem Heimatdorf Siwongi im Rahmen der spontanen Kettenmigration von Pipikoro-Familien nach Berdikari (vgl. Kap. 3.6.3) niedergelassen hatte. Nach seiner Heirat im Jahr 1979 arbeitete Kornelius zuerst auf dem Feld seiner Schwiegereltern, bei welchen er zusammen mit seiner Ehefrau wohnte. 1982 erhielt er vom Dorfbürgermeister einen Hektar Land, das er anfangs mit Mais und Trockenreis, später mit Kakao bestellte. Zusätzlich erhielt er von seinem Onkel 0,75 ha Nassreisfeld. 


\subsubsection{Typisierung von Wanderungsmotiven}

Befragt man spontane Migranten in der Lore-Lindu-Region nach ihrer Motivation, den Wohnort zu wechseln, erhält man in der überwiegenden Anzahl der Fälle die lapidare Antwort: „cari hidup yang lebih baik" („ein besseres Leben suchen“). Bei genauerer Nachfrage enthüllt diese sehr allgemein gehaltene Antwort eine Reihe unterschiedlicher Wanderungsmotive spontaner Migranten. Vergleicht man die wichtigsten Wanderungsmotive, so nahm deren Vielfalt im Verlauf des 20. Jh. zu. Während der niederländischen Kolonialzeit waren v.a. Heirat, Handelsinteressen, sowie klimatische und medizinische Verhältnisse die Hauptursachen für einen Wohnortwechsel.

Heirat, welche in hohem Maße die soziale Integration der Migrantin bzw. des Migranten im Zielort befördert, war zu Beginn des 20. Jh. insbesondere für die lokale Bevölkerung ein häufiges Wanderungsmotiv. Vor allem auf der Ebene der traditionellen „Noblen“ (raja, maradika, etc.) kam es hierbei auch zu Wanderungen in andere soziopolitische Herrschaftsgebiete (z.B. zwischen Kulawi und Sigi). Die gewöhnliche Bevölkerung bewegte sich hingegen weitgehend innerhalb der lokalen Herrschaftsgebiete. Die Handelsinteressen betrafen v.a Bugis, Chinesen und Araber, die im Gefolge der niederländischen Unterwerfung der Lore-Lindu-Region von den Küstengebieten um Palu oder Poso in das Palu-Tal und nach Kulawi bzw. Napu (Lore Utara) vordrangen, um dort ihre Handelsaktivitäten zu entfalten. Anfangs waren sie noch als mobile Händler in diesen Gebieten tätig. Manche ließen sich jedoch in den jeweiligen Zentren (z.B. Bolapapu in Kulawi) permanent nieder. Die klimatischen und medizinischen Verhältnisse waren besonders für die von den Niederländern zwangsumgesiedelte Bergbevölkerung die Ursachen für eine Migration. Wie bereits erwähnt, konnten sich viele dieser Menschen nur schwer an die klimatischen Bedingungen im Tal gewöhnen oder wurden von Krankheiten, z.B. Malaria, heimgesucht. Sie kehrten daher wieder in ihre Ursprungsgebiete in den Bergen zurück.

Nach der Unabhängigkeit Indonesiens kam das Motiv der Suche nach verfügbarem Land hinzu. Mit der Verknappung der Landressourcen in der Herkunftsregion, welche durch das natürliche Bevölkerungswachstum, durch Zuwanderung, die infrastrukturellen bzw. naturgeographischen Bedingungen und/oder die staatliche Einschränkung der Nutzung bestimmter Waldareale hervorgerufen wurde, gewann dieses Wanderungsmotiv an Bedeutung. Neben der Verbesserung der Verkehrsinfrastruktur (v.a. in Palolo und nach Lore Utara) führte das zunehmende Interesse am Anbau von cash crops wie Kaffee, Gewürznelken oder Kakao dazu, dass zum einen Migranten aus Südsulawesi in großer Anzahl in die neu erschlossenen, ressourcenreichen und klimatisch günstigen Gebiete auf der Ostseite der Lore-LinduRegion zuwanderten. Besonders unter den Bugis-Migranten, die ab den 1980er Jahren aus Südsulawesi zugewandert sind, ist das in der Lore-Lindu-Region verfügbare Land ein wichtiger Pull-Faktor. Viele von ihnen besaßen in ihrer Herkunftsregion zwar über die finanziellen Mittel, Land zu erwerben. Für den Anbau von Kakao auf 
Grund der natürlichen Gegebenheiten geeignetes Land war in den 1980er Jahren in Südsulawesi allerdings nur noch in verkehrsinfrastrukturell schlecht erschlossenen Regionen verfügbar. Die Verbesserung der Infrastruktur v.a. in Palolo und Lore Utara und die Bereitschaft der lokalen Bevölkerung dieser Gebiete zum Landverkauf zog daher viele finanzkräftige Migranten aus dem Süden der Insel an. Für weniger wohlhabende, regionale Migranten aus Südsulawesi und anderen Provinzen der Insel waren es insbesondere die sozialen, ethnischen Netzwerke, welche sich in den Zielregionen etablierten. Zum anderen nahm dieses Motiv auch für die lokale Bevölkerung im Palu-Tal oder in Kulawi einen in den vergangenen zwei Dekaden stetig wachsenden Stellenwert ein. Ursächlich hierfür ist z.B. die traditionelle Regelung der Erbteilung, gepaart mit Landverkäufen und dem beschränkten Zugriff auf den familiären Landbesitz durch die Einrichtung des Lore-Lindu Nationalparks. Reicht das eigene, geerbte oder als Mitgift erhaltene Land als Versorgungsgrundlage für die Familie nicht aus, migriert diese - sofern sich keine Beschäftigungsmöglichkeiten als Lohnarbeiter ${ }^{78}$ auf den Feldern anderer Dorfbewohner bzw. außerhalb der Landwirtschaft bieten oder angestrebt werden - in ein anderes Dorf der Untersuchungsregion. Auch hier spielen in vielen Fällen die sozialen Netzwerke der eigenen ethnischen Gruppe eine wichtige Rolle. Die Suche nach Land kann daher als das insgesamt bedeutendste Migrationsmotiv seit der Unabhängigkeit Indonesiens gewertet werden.

Die Zunahme des produzierenden und Dienstleistungsgewerbes, v.a. in Palu, und die wachsende Bedeutung von Lohnarbeitsbeschäftigung im agraren und nichtagraren Bereich eröffnete ein weiteres Motiv für einen Wohnortwechsel. Ebenso wurde mit dem Ausbau des Bildungssystems (v.a. der höheren Schulen) während der Regierungszeit von Präsident Suharto eine neue Motivgrundlage geschaffen. Dies führte zu einer, meist zeitlich begrenzten Wanderung in die Hauptorte des jeweiligen Kecamatan oder nach Palu (v.a. für Hochschulbesuche).

Auf die Migrationsentscheidungen wirkt sich nicht nur die Verkehrsinfrastruktur im Zielgebiet beeinflussend aus, sondern auch die des Herkunftsgebiets. Beispielhaft hierfür ist die Pipikoro-Region im westlichen Kulawi. Mit zunehmender Bedeutung der Märkte entschlossen sich ab den 1970er und 1980er Jahren zahlreiche Familien, das gebirgige und größtenteils nur mit Fußwegen erschlossene Pipikoro-Gebiet zu verlassen, um sich in der Gimpu-Ebene niederzulassen. Die hohen Transportkosten, um die produzierten landwirtschaftlichen Güter zu den Märkten und Händlern zu bringen, ergaben für sie eine zu schlechte Kosten-Nutzen-Bilanz. Andere verließen die Region aber auch, weil ihnen die Bewirtschaftung der an den bis zu $80 \mathrm{Grad}$ steilen Berghängen gelegenen Felder zu mühsam wurde. Zudem darf

78 Lohnarbeit wird generell als makan gaji bezeichnet. Daneben gibt es z.B. auch die Möglichkeit des bagi hasil, ein System, bei welchem anstelle eines Geldlohns die produzierte Ernte geteilt wird. Auch dies stellt eine alternative Unterhaltsquelle im genannten Fall dar. 
der Einfluss des Mediums Fernsehen nicht übersehen werden. Das Fernsehprogramm führt den Menschen mit seinen in den javanischen Metropolen produzierten Seifenopern oder den US-amerikanischen Serien und Spielfilmen tagtäglich ein Leben vor, das in extremem Kontrast zu dem Lebensstandard der Fernsehzuschauer in den Dörfern Pipikoros steht. Zwar sind sich die Menschen i.d.R. darüber im Klaren, dass sie diesen hohen Lebensstandard nicht werden erreichen können. Manche von ihnen erhoffen sich aber, durch eine Migration in besser erschlossene Gebiete der Lore-Lindu-Region dem Lebensstandard der Protagonisten aus dem Fernsehen zumindest ein Stück weit näher kommen zu können.

Ein spezieller, bei den Befragungen häufig genannter Migrationsgrund ist merantau. Übersetzt bedeutet das Wort: „,in die Fremde gehen“. Es sind in der Regel Männer, die in jüngeren Jahren ihr Elternhaus verlassen, um sich in anderen Orten und Regionen eine Lohnbeschäftigung zu suchen. Diese Personengruppe weist meist eine sehr bewegte Migrationsbiographie auf. Sie bleiben nur so lange an einem Ort, wie es das Arbeitsangebot zulässt. Ergibt sich nach dem Ablauf der Vertragslaufzeit keine Möglichkeit auf eine andere lukrative Tätigkeit am selben Ort, ziehen sie weiter. Den Abschluss solcher Migrationsbiographien bilden meist die Heirat und die permanente Niederlassung an einem Ort (vgl. auch Kap. 3.6.1).

In der Auflistung der verschiedenen Wanderungsmotive tauchten bereits wiederholt die ethnischen und sozialen Netzwerke als migrationsbeeinflussende Größen auf. In der Tat ist diesem Faktor, vielleicht abgesehen von der Migration zu Ausbildungszwecken, ein enormer Stellenwert bei der Entscheidung zur Migration beizumessen. Wie bereits in Kap. 3.6.1 erwähnt, bilden diese Netzwerke die Grundlage von Kettenmigrationen in der Untersuchungsregion. Nur in seltenen Fällen kommt es vor, dass soziale Kontakte keine Rolle beim Entscheidungsprozess spielen. Dabei dienen diese Netzwerke nicht nur dem Informationsaustausch über einen potentiellen Zielort. Die durch vorangegangene Wanderungen erzeugten, räumlich weitverzweigten familiären Netzwerke führen dazu, dass in einer Vielzahl von Orten der Region Verwandte wohnen, welche als erste Anlaufstelle nach vollzogener Migration zur Verfügung stehen. Wie die im vorangegangenen Kapitel dargestellten Migrationsbiographien zeigen, sind es oft der Onkel oder andere Verwandte, welche den Migranten bzw. die Migrantin während der ersten Zeit im Zielort der Migration bei sich aufnehmen.

\subsubsection{Fallbeispiel: Spontane Zuwanderung in das Palolo-Tal - Das Beispiel Berdikari}

Bis zu Beginn der 1950er Jahre war die Ebene des Palolo-Tals noch weitgehend unbewohnt. Nur am westlichen Ende der Region, in den bergigen Übergangsbereichen zum Palu-Tal siedelte die lokale Bevölkerung, welche während der niederländischen Kolonialzeit, wie in anderen Gebieten der Lore-Lindu-Region, mit Hilfe von Zwangsumsiedlungen in einigen Dörfern konzentriert worden war. Das Tal selbst 
bestand noch überwiegend aus Waldgebieten und vereinzelten Gras- und Sumpfflächen. Allein im westlichen Teil begann sich die Bevölkerung im Zuge der von den Niederländern verordneten Ausweitung der Nassreisproduktion auch im Talbereich anzusiedeln. Ende der 1940er Jahre reichte das Wegenetz bis zum Dorf Menusi, aus welchem später die Siedlungen Ampera und Makmur hervorgingen. Makmur ist der heutige Hauptort des Kecamatan Palolo, dessen Territorium 1997 von dem des Kecamatan Sigi-Biromaru abgetrennt wurde und seitdem eine eigene Verwaltungseinheit bildet. Die Wälder im Tal dienten der lokalen Bevölkerung in der Mitte des 20. Jh. ausschließlich der Entnahme von Brenn- oder Bauholz, Heilpflanzen und anderern Forstprodukten. Die Grassflächen wurden zu jener Zeit als Weidegrund für die damals noch zahlreichen Wasserbüffel genutzt. Manche Gebiete wurden aufgrund überlieferter Mythen mit dämonischen Bezügen gemieden. Hierzu zählt z.B. das Gebiet, in welchem das Dorf Berdikari gegründet wurde. Der Legende aus dem Mythenschatz der alteingesessenen Bevölkerung von Sigi-Biromaru zufolge soll sich dort ein übernatürliches Wesen mit dem Namen Mperengge befunden haben. Jongo, ein schwarzer Hund, und Wurika, ein tigerartiger Hund, bewachten dieses menschenartige Wesen. Wenn sich ein Mensch diesem Gebiet näherte, soll er krank geworden und schließlich gestorben sein. Diese Legende kann u.a. als Erklärung dafür herangezogen werden, warum es keine Familien aus dem Palu-Tal waren, die sich als erste im heutigen Berdikari niedergelassen haben, sondern Familien aus Kulawi, die keinen Bezug zu dieser Mythe haben. Lokale Legenden sind in der Untersuchungsregion bis heute verbreitet. In der Schule wird die Lokalgeschichte, deren Bestandteil diese Legenden sind, nicht behandelt, sondern hauptsächlich Nationalepen und deren Heldenfiguren thematisiert. Dennoch haben sich zahlreiche lokale Mythen bis heute gehalten. Besonders die schwarze und weiße Magie spielt selbst bei der jungen Generation noch immer eine große Rolle. Das oben genannte Beispiel zeigt, dass dieser Themenbereich nicht nur aus rein ethnologischer Perspektive interessant ist, sondern auch für die Erforschung von Migrations- und Siedlungsprozessen unentbehrliche Erklärungsansätze bietet.

In den 1950er Jahren siedelten sich die ersten Familien aus der Pipikoro-Region Kulawis in Menusi an, da sie sich dort bessere landwirtschaftliche Bedingungen erhofften. Unter der Führung des späteren ersten Bürgermeisters von Berdikari begannen im Jahr 1954 sieben miteinander verwandte Kulawi-Familien damit, sich im Grasland nordöstlich von Menusi anzusiedeln und dieses in Nassreisfelder umzuwandeln. Zwölf Jahr später zählte die neue Siedlung Karawa Maluo ${ }^{79}$ bereits 40 Familien, welche nach und nach aus den Herkunftsdörfern der ersten sieben Familien zu Fuß (vier Tage) oder per Pferd (ein Tag) ebenfalls in das Palolo-Tal migriert waren. Die Familien kamen v.a. aus den Dörfern Kantewu, Siwongi und Towulu.

79 Der Siedlungsname bedeutet: ,weites Grasland“. 
Im Jahr 1964 reiste der 1935 geborene und ab 1959 in Berdikari angesiedelte Set Saito $^{80}$ nach Kantewu, dem Herkunftsort seiner Familie. Er berichtete den Dorfbewohnern von den guten Lebensbedingungen im Palolo-Tal und betonte insbesondere die besseren Möglichkeiten schulischer Ausbildung. Ebenso teilte er dem Camat von Kulawi mit, dass er die Bewohner Kantewus nach Berdikari rufen möchte. Bereits vor 1964 war von der Regierung vorgesehen, die Bevölkerung Kantewus an den Lindu See umzusiedeln. Da dieser Plan jedoch nicht umgesetzt worden war, gab der Camat von Kulawi den Bewohnern die Erlaubnis, auf Wunsch nach Berdikari auszuwandern. In den ersten Jahren folgten nur wenige der Initiative von Set Saito, da die meisten kein Interesse hatten, alles aufzugeben, um in Berdikari wieder „bei Null“ zu beginnen. Doch schon bald erwägten mehr Familien die Migration in das Palolo-Tal. Ab 1965 wanderten auch Familien aus Towulu und Siwongi nach Berdikari, nachdem sich die Informationen über das Siedlungsgebiet auf der Ostseite der Lore-Lindu-Region im Pipikoro-Gebiet verbreitet hatten. Mit dem Beginn der 1970 er Jahre siedelten sich neben Kulawi-Familien auch Bugis in Berdikari an, die sich neben der Landwirtschaft auch als Kioskbesitzer, Schreiner, Steinhauer etc. verdingten.

Zwar nimmt Berdikari für die Migration von Kulawi-Familien aus der PipikoroRegion einen besonderen Stellenwert ein, da es das einzige Dorf in Palolo ist, in dem diese Migrantengruppe die ersten Siedler des Dorfes repräsentiert. Für Menschen aus der Pipikoro-Region wurden aber auch andere Dörfer Palolos (z.B. Sintuwu, Kamarora) zum Migrationsziel. Daneben waren es v.a. die Landverknappung und der Kakaoboom der 1990er Jahre, die als Push-Faktoren für lokale Migranten aus dem Palu-Tal (Kec. Sigi-Biromaru) nach Palolo wirkten und sich dort mit den Pullfaktoren der Verfügbarkeit von Landressourcen und den für den Anbau von Kakao günstigen klimatischen und bodenspezifischen Gegebenheiten zu migrationswirksamen Einflussfaktoren verbanden. Die lokale Zuwanderung aus Kulawi und Sigi-Biromaru, sowie die regionale Zuwanderung aus Nord- und Südsulawesi (vgl. Kap. 5.2.2) führte zu einem von Dorf zu Dorf unterschiedlich ausgeprägten Siedlungsgefüge und sozialräumlichen Segregationsmuster, welche in Kap. 4.1 im Detail untersucht werden.

80 Set Saito ist heute für die Öffentlichkeitsarbeit in Berdikari verantwortlich. 


\subsection{Zu- und Abwanderung als Auswirkung regionaler Kon- flikte}

\subsubsection{Die Rebellionen von DI/TII und Permesta und ihre Auswirkungen auf die Lore-Lindu-Region}

Darul Islam (wörtlich übersetzt: „Haus des Islam“) ist der Name für eine Rebellion, die sich 1948 in Westjava entwicklete und bis in die 1960er Jahre hinein wirkte. Sie hatte ihren Ursprung in der Weigerung islamischer Rebellen, die zentralstaatliche Autorität Indonesiens nach Erlangung der Unabhängigkeit des Staates anzuerkennen. Von Java aus breitete sich diese Rebellion während der 1950er Jahre nach Sumatra und Südsulawesi aus (Liefer 1996: 93f). In Südsulawesi entstand so die DI/TII (Darul Islam/Tentara Islam Indonesia)-Bewegung. Die Rebellen dieser Gruppe, welche in den 1950er Jahren unter der Führung von Kahar Muzzakar in Makassar entstand und die Gründung einer von der Zentralregierung autonomen Region streng islamischer Ausprägung verfolgte, verschanzten sich vor den Soldaten der Staatsarmee in den Bergregionen des nördlichen Südsulawesi und des südlichen Zentralsulawesi (z.B. in der Region Rampi). Teile der dortigen Bevölkerung flüchteten vor den Rebellen zum Teil nach Zentralsulawesi. In Gimpu (Kec. Kulawi) wurde eine Vielzahl dieser Flüchtlinge aufgenommen und schließlich auf mehrere Dörfer, darunter das Dorf Toro, verteilt.

Die Permesta-Bewegung wurde von dem regionalen Militärkommandeur für Ostindonesien, Sumual, im Jahr 1957 ins Leben gerufen. Die Bewegung zielte nicht auf eine Abspaltung von der Republik Indonesien, forderte aber finanzielle Regionalautonomie, einen höheren Anteil am Entwicklungsbudget des Landes und den von Japan zu leistenden Kriegsreparationszahlungen. Die Nationalversammlung Indonesiens sollte in Form eines Oberhauses die Interessen der einzelnen Regionen des Archipels wahren (Cribb \& Brown 1995: 78). Die Permesta-Rebellion, gegen die sich in Zentralsulawesi in den frühen 1960er Jahren eine Gegenbewegung, die Gerakan Pemuda Sulawesi Tengah (GPST; Jugendbewegung Zentralsulawesi) auf Seiten der statlichen Armee gegründet hatte, war neben Palolo v.a in Lore Utara aktiv. Bewohner von Watumaeta berichten, dass sich lokale Sympathisanten der Permesta-Bewegung im Zuge der Verfolgung der Rebellen durch die staatliche Armee zum Lindu See flüchteten, wo sich bereits zuvor Verwandte von ihnen angesiedelt hatten.

\subsubsection{Der Poso-Konflikt und die Fluchtmigration nach Lore Utara}

Zwar fallen die Auswirkungen des Poso-Konflikts bereits in das 21. Jh., dennoch sollen sie hier eine kurze Erwähnung finden. Bei dem Poso-Konflikt handelt es sich um einen ursprünglich sozioökonomisch und politisch begründeten und bereits während der Suharto-Ära schwelenden Konflikt zwischen der lokalen Bevölkerung und den aus Südsulawesi zugewanderten Bugis. Diese regionalen Migranten konnten 
sich nicht nur im wirtschaftlichen Sektor etablieren, sondern waren auch in hohen politischen Ämtern vertreten. Es bildete sich daraus ein Spannungsverhältnis, das von Neid, Missgunst und Gier nach Einfluss geprägt war. Ausgelöst von einer Schlägerei unter alkoholisierten Jugendlichen beider Gruppen entlud sich ab 2000 schließlich ein blutiger Konflikt, der mehrere hundert Menschenleben forderte (vgl. Aragon 2000a, 2001, 2002).

Die Konstellation aus einer christlich missionierten lokalen Bevölkerung einerseits und muslimischen Migranten andererseits führte bald zu einer Aufladung des Konflikts mit religiösen Symbolen. Neben Wohnhäusern wurden dadurch auch Kirchen bzw. Moscheen zu Angriffszielen der beiden rivalisierenden Gruppen. Tausende Menschen flüchteten aus der Poso-Region. Während ein Groteil der muslimischen Flüchtlinge in Palu Aufnahme fand, wanderten mehrere Hundert christliche Familien nach Lore Utara, wo sie v.a. in den Dörfern des nördlichen Teils des Napu-Tals aufgenommen wurden. 


\section{$4 \quad$ Veränderungen der Siedlungsstrukturen}

Indonesien ist noch heute ein sehr stark rural geprägtes Land. Knapp über 120 Millionen Einwohner des Archipels zählen noch immer zur ländlichen Bevölkerung, während etwa 100 Millionen Menschen in Städten wohnen (vgl. Abb. 20). Eine Umkehr dieser Disparität prognostiziert die FAO (2003) erst für das Jahr 2010.

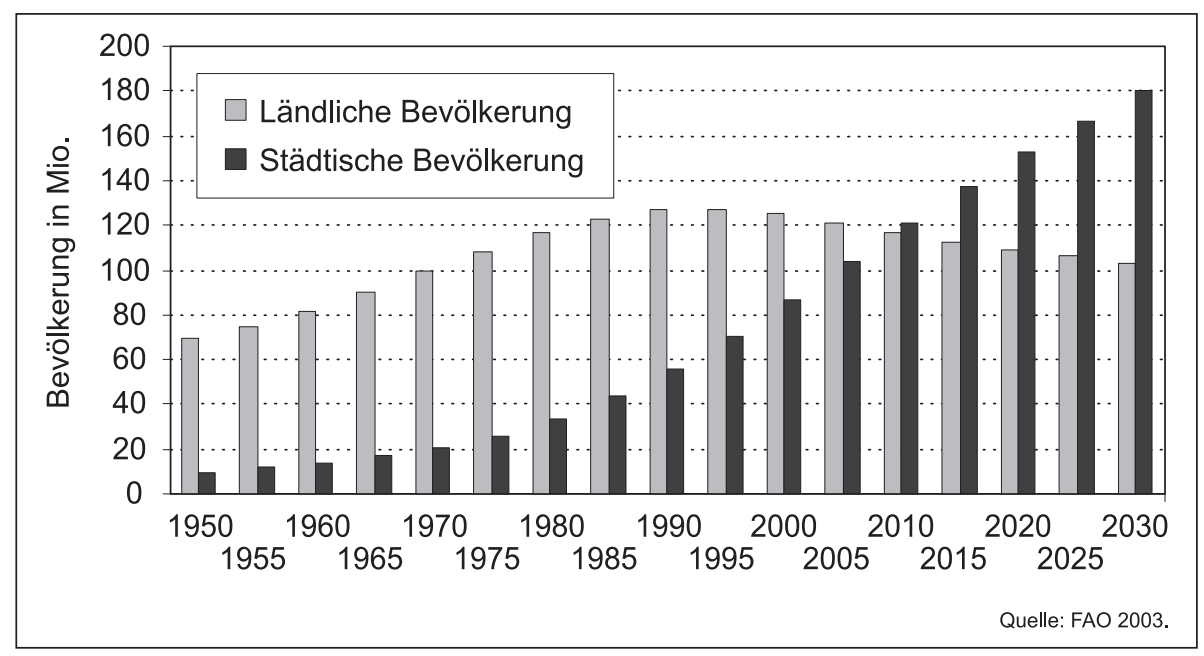

Abb. 20 Stadt- und Landbevölkerung in Indonesien 1950 - 2030

(ab 2005 prognostizierte Werte)

Vergleicht man hingegen die Bevölkerungsentwicklung Indonesiens im landwirtschaftlichen bzw. nicht- landwirtschaftlichen Bereich (vgl. Abb. 21), zeichnet sich ein gegenläufiger Trend bereits in den 1990er Jahren ab. ${ }^{81}$ Während das Verhältnis von Land- zu Stadtbevölkerung im Jahr 1991 noch bei 2,70 lag, näherte sich das Verhältnis zwischen Agrar- und Nicht-Agrarbevölkerung mit einem Wert von 1,08 bereits dem Equilibrium an. Fünf Jahre später sank dieser Wert auf 0,88 und kehrte das Verhältnis von landwirtschaftlich geprägter zu nicht-landwirtschaftlich geprägter

81 Schon in den 1980er Jahren hielt der Agrarsektor in Indonesien nur noch einen Anteil von ca. 25 Prozent am BIP des Landes, während er im Jahr 1970 noch bei ca. 50 \% lag (Thorbecke \& van de Pluijm 1993: 5). Allerdings ist dieser Rückgang weniger auf die Abnahme landwirtschaftlicher Produktion zurückzuführen, als vielmehr auf den Boom der Erdölexporte gegen Ende der 1970er Jahre. 
Bevölkerung um. Für die Untersuchungsregion kann diese Entwicklung nicht bestätigt werden. Noch heute ist das Gebiet sehr von der Landwirtschaft geprägt. Selbst wenn man die Provinzhauptstadt in die Überlegungen mit einbezieht, muss weiterhin von einer überwiegend ländlichen Bevölkerung gesprochen werden. Ebenfalls ist die Region noch weit von der Wende von einer Agrar- zu einer NichtAgrargesellschaft entfernt. Die Erklärung hierfür lässt sich in der peripheren Lage der Untersuchungsregion und in seiner naturräumlichen Ausstattung 82 (vgl. auch Kap. 2) finden.

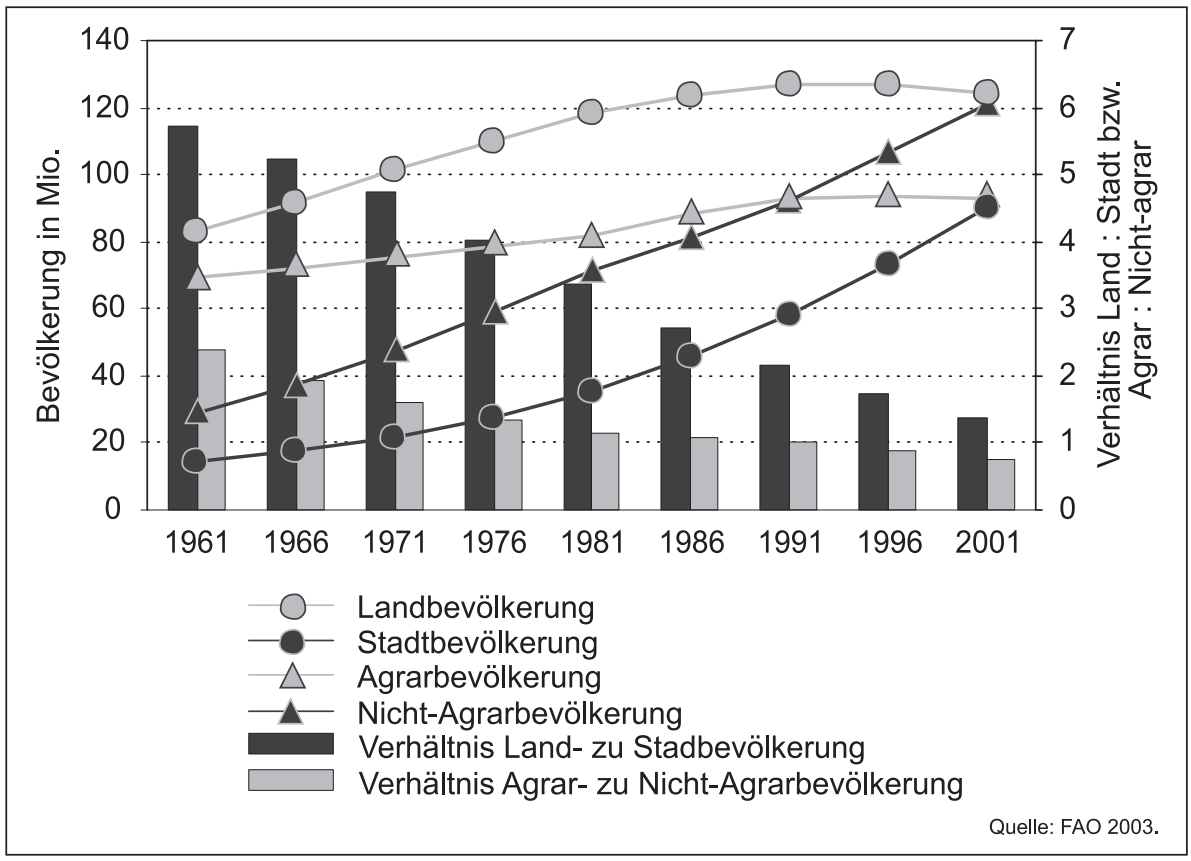

Abb. 21 Entwicklung der Bevölkerungsverteilung zwischen Stadt und Land bzw. agrarisch und nicht-agrarisch in Indonesien 1961 - 2001

Zwar hat sich mittlerweile in zahlreichen der 119 Dörfer der Lore-Lindu-Region ein tertiärer Sektor etabliert, welcher sich durch die Errichtung kleiner Gemischt-

82 Hierbei ist anzumerken, dass nicht die naturräumliche Ausstattung an sich ausschlaggebend für die Siedlungsentwicklung ist. Vielmehr sind auch die politisch-ökonomischen Rahmenbedingungen zu berücksichtigen, welche beispielsweise den Ausbau der Infrastruktur behindern. Das Gegenbeispiel der Stadt Salt Lake City (Utah, USA) belegt, dass natürliche Ungunsträume unter den entsprechenden wirtschaftlichen Gegebenheiten siedlungstechnisch durchaus qualitativ hochrangig in Wert gesetzt werden können. 
warenläden, Gaststätten, Werkstätten etc. oder durch den Betrieb von Beförderungsunternehmen des öffentlichen Nahverkehrs und anderem darstellt. Die Zahl derjenigen Bevölkerung, welche mittels dieser Einrichtungen ihr Einkommen erwirtschaftet, nimmt sich jedoch im Vergleich zum primären Sektor immer noch gering aus (vgl. Schwarze 2004: 52). Die Landwirtschaft ist weiterhin einer der wichtigsten prägenden Faktoren des Siedlungsbildes in der Untersuchungsregion.

\subsection{Die historische Siedlungsentwicklung in der Lore- Lindu-Region}

Die gegenwärtige Verteilung der Siedlungen über die Lore-Lindu-Region zeigt eine deutliche Ausrichtung auf das vorhandene Verkehrswegenetz (vgl. Abb. 22). Die meisten Dörfer befinden sich entlang der beiden Hauptverkehrsachsen Palu-Kulawi im Westen und Palu-Lore Utara im Osten. Allein in der stark reliefierten PipikoroRegion Kulawis befindet sich eine Reihe von Siedlungen, die fernab der Hauptverkehrsachse liegen und deren Verteilung einen stärkeren Streuungscharakter annimmt.

Ermittelt man die Siedlungsdichte (Dörfer $/ 100 \mathrm{~km}^{2}$ ) für die einzelnen Kecamatan ergibt sich eine deutlich Differenzierung. Während das Kec. Sigi-Biromaru einen Wert von 6,8 erreicht, ist die Siedlungsdichte in Palolo um etwa ein Drittel geringer $(4,2)$. Kulawi und Lore Utara erreichen hingegen nur einen Wert von 1,7 bzw. 1,9. ${ }^{83}$ In bezug auf Kulawi ist zu beachten, dass dieses Kecamatan mit der Pipikoro-Region ein relativ großes, gebirgiges Gebiet beinhaltet. Die beiden nördlichen Kecamatan Sigi-Biromaru und Palolo weisen dennoch die mit Abstand höchsten Siedlungsdichten auf. Allerdings hat bereits die in Kap. 3 vorgenommene Untersuchung der Migrationsprozesse gezeigt, dass sich in den beiden Kecamatan eine historisch unterschiedliche Entwicklung der Besiedlung vollzogen hatte.

Die Deckung der Siedlungsverteilung mit der Verkehrsinfrastruktur der Untersuchungsregion ist einleuchtend. Der beispielhafte Vergleich von Sigi-Biromaru und Palolo lässt jedoch die Bedeutung einer Beantwortung der Frage erkennen, wie sich die gegenwärtige Verteilung historisch entwickelt hat und welche regionalen und lokalen Besonderheiten dabei festzustellen sind. Zudem gilt zu untersuchen, welche Faktoren für die Entwicklung der Siedlungsstrukturen in der Gesamtregion, wie in ihren Teilgebieten ausschlaggebend waren.

83 Die Werte wurden anhand der von STORMA-Teilprojekt A3 ermittelten Flächengrößen der einzelnen Kecamatan berechnet. Von den Flächen sind bereits die Anteile innerhalb des Lore-Lindu Nationalparks abgezogen. 


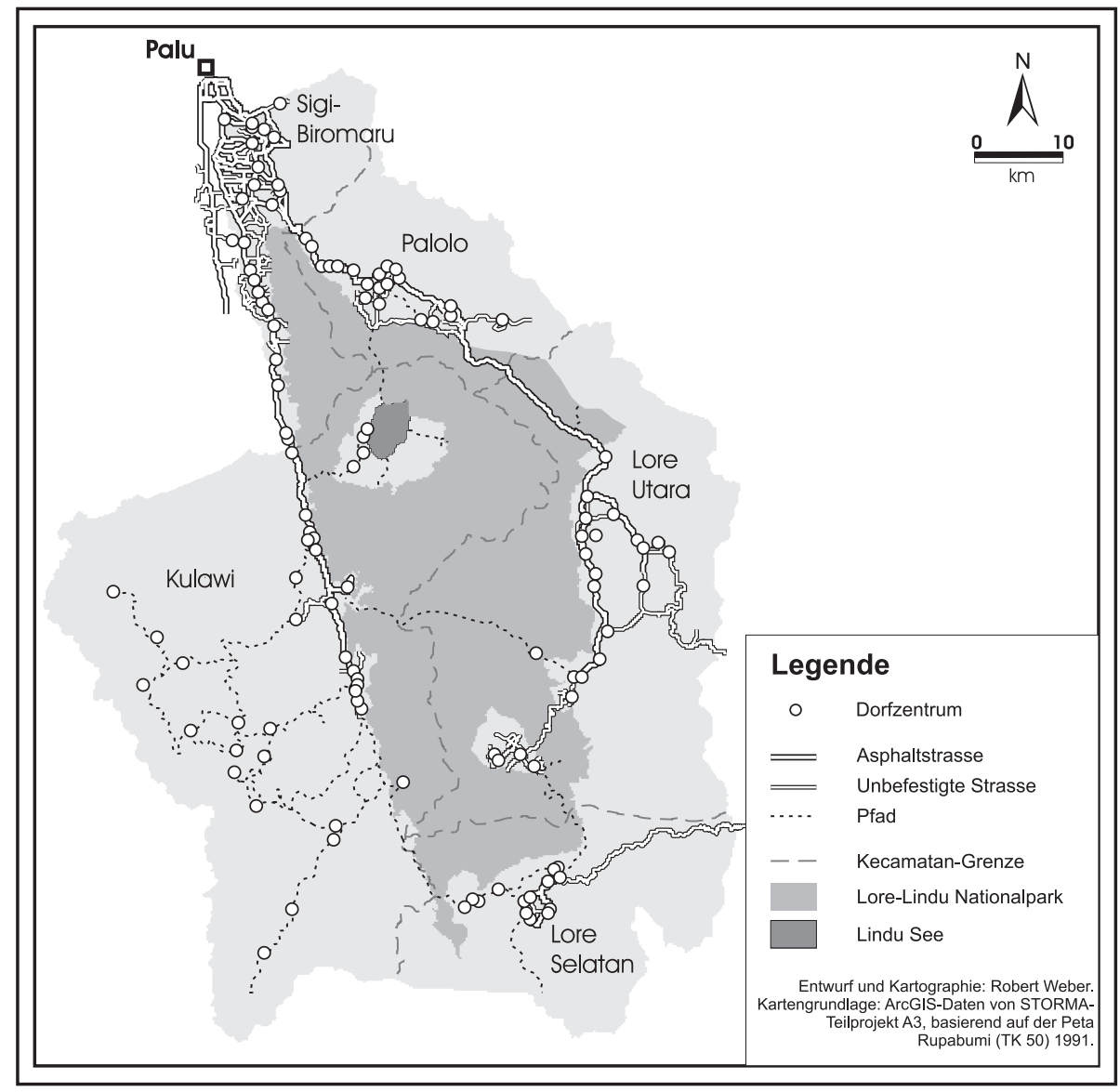

Abb. 22 Lage der Dörfer in der Lore-Lindu-Region in bezug zum Verkehrswegenetz

\subsubsection{Allgemeine Bemerkungen zur Rekonstruktion der Siedlungsentwicklung}

Die Rekonstruktion der historischen Siedlungsgenese in einer Region wie Zentralsulawesi kann im Gegensatz zu Forschungen dieser Art in Deutschland nicht hauptsächlich anhand von Kartenmaterial, Katastern und anderen Registern sowie Fotographien erfolgen. Der Grund dafür liegt in der geringen Anzahl bzw. dem völligen Fehlen entsprechender Quellen. Der überwiegende Teil des Landbesitzes ist bis heute nicht offiziell registriert, geschweige denn in Form von Ortskatastern verfügbar. In bezug auf Karten gibt es zwar einige kolonialzeitliche Quellen (vgl. Kaudern 1925a, 1925b; Kruyt 1908; Kruyt 1938). Diese geben jedoch nur die Siedlungsbereiche in der Region bzw. die (teilweise noch sehr ungenau kartographierte) Lage der 
einzelnen Siedlungen wieder. Kleinräumigere Karten aus der niederländischen Kolonialzeit existieren vorwiegend für größere Städte (z.B. Batavia; heute: Jakarta) oder bedeutende Küstenfestungen (z.B. auf Ambon; Molukken). Siedlungen des ländlichen Raumes wurden statt dessen auf Grund ihrer geringeren Bedeutung nicht detailgenau kartographisch erfasst. In jüngerer Zeit wurden topographische Karten anhand von Satellitenaufnahmen erstellt. Aber auch diese erfassen weder Dorfgrenzen, noch eine detaillierte Darstellung der Verteilung von Wohnbereichen und landwirtschaftlichen Nutzflächen. In der Lore-Lindu-Region waren es v.a. NGOs (z.B. TNC), die ab den 1990er Jahren die Dorfgemeinden zur Anfertigung von Dorfkarten ermunterten oder selbst Kartierungen einiger Dörfer vornahmen. Allerdings variieren die von der Dorfadministration selbst angefertigten Karten sehr stark in bezug auf Maßstabsgenauigkeit und Detaildichte der enthaltenen Informationen.

Trotz Würdigung dieser relativ dürftigen Quellen besteht weiterhin das Problem, einen chronologischen Besiedlungsverlauf nicht nachzeichnen zu können. Einen Ausweg aus diesem Dilemma bietet die Sammlung qualitativer Informationen zur Siedlungsgenese in ausgewählten Dörfern. Dem Verfasser ist bewusst, dass noch weitere Methoden (z.B. umfassende Transekte) herangezogen werden müssten, um ein noch detailgetreueres Bild der Siedlungsentwicklung zu zeichnen. Dies konnte jedoch nur ansatzweise umgesetzt werden, da es den zeitlichen Rahmen der Forschung überschritten hätte. ${ }^{84}$

\subsubsection{Präkoloniale Besiedlung der Lore-Lindu-Region}

Vor Beginn der aktiven niederländischen Kolonialherrschaft in Zentralsulawesi besiedelte die Bevölkerung der Lore-Lindu-Region vorwiegend die Berghänge. Es bestanden noch keine Dorfstrukturen in Form eines kompakten Dorfes mit Funktionsteilung. Vielmehr lebten die Menschen in ihren Familienverbänden in einfachen Hütten und verlagerten ihren Wohnort mit dem durch den Wanderfeldbau bedingten Wechsel ihrer landwirtschaftlichen Felder. Daneben gab es einige wenige befes-

84 Die Durchführung umfassender Transekte ist v.a. durch die Größe der Dorfterritorien und die Siedlungsstrukturen in der Lore-Lindu-Region ein mühsames Unterfangen. Manche der Dörfer bestehen aus verschiedenen Einzelsiedlungen (meist als eigene Dorfteile ausgewiesen). Für jedes dieser einzelnen Siedlungsgebiete wären mehrere Transekte über mehrere Kilometer Länge erforderlich gewesen, um eine detailgenaue Kartierung durchführen zu können. Damit wäre jedoch erst der gegenwärtige Zustand erfasst worden. Um die historische Entwicklung zu untersuchen, wäre ein enormer zusätzlicher Zeitaufwand durch die Befragung von Schlüsselinformanten während der Transektbegehungen erforderlich gewesen. Da die Sielungsentwicklung nur einen Teilaspekt der Gesamtuntersuchung dieser Arbeit bildet, wurde „,nur“ die Interviewbefragung als Hauptmethode verwendet. Zwar mangelt es den daraus gewonnen Ergebnissen an Detailgenauigkeit, dafür konnte aber zumindest eine Vergleichbarkeit mehrerer Siedlungen in unterschiedlichen Regionen des Untersuchungsgebiets gewährleistet werden. 
tigte Dorfanlagen (vgl. Abb. 23), die hauptsächlich von den ,aristokratischen“ Familien bewohnt wurden (vgl. u.a. Adriani 1915: 469ff; Adriani \& Kruyt 1912-1914, Bd. 1: 149; Kruyt 1908: 1308f).

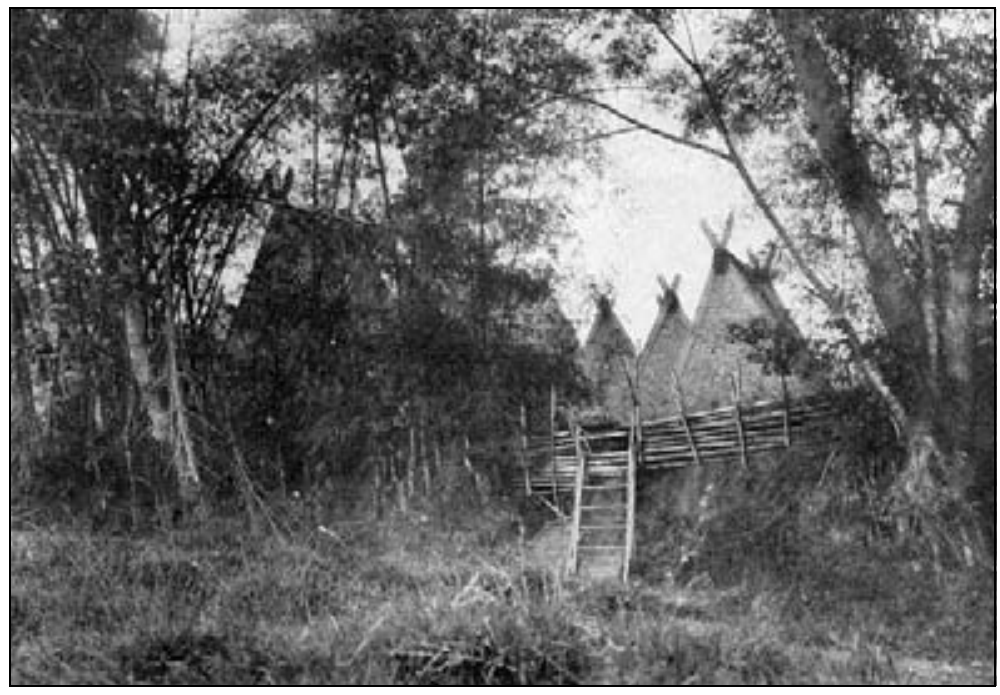

Abb. 23 Der Dorfwall von Lamba, Napu-Tal (Quelle: Grubauer 1913: 473)

Unter solchen „Dorffestungen“ sind jedoch meist relativ kleine Siedlungen mit nur wenigen Häusern zu verstehen. „Sabinka ist ein kleines Dorf von drei Häusern.", 85 beschreibt Kruyt (1908: 1289) kurz nach der niederländischen Eroberung eine der Siedlungen im Napu-Tal und weist damit auf die teilweise geringe Bevölkerungsdichte dessen hin, was Europäer jener Zeit bereits als Dorf definierten. Das Dorf Gimpu, heute der Hauptort des gleichnamigen Tals bestand im Jahr 1911 nur „aus 7 oder 8 Häusern und einem Lobo" (Grubauer 1923: 145). Die Siedlungsverbände stellten keine völlig geschlossene, endogame Einheit dar. Verbindungen mit anderen Siedlungen durch Heirat kamen nach Angaben eines Respondenten aus Bolapapu durchaus vor.

Teilweise existieren in den Teilregionen des Untersuchungsgebiets relativ detaillierte Legenden über die vorkoloniale Besiedlung der Talebenen. Zwar nehmen diese Legenden zum Teil mythenhaften Charakter an. Viele Elemente in bezug auf die geschaffenen Siedlungsstrukturen decken sich jedoch mit den Aufzeichnungen europäischer Forschungsreisender, welche die Regionen zu Beginn der effektiven

85 Zitat im Original: „Sabinka is een klein dorp van drie huizen.“ 
niederländischen Kolonialherrschaft über das jeweilige Gebiet besucht hatten. Am Beispiel des Napu-Tals wird in dem nachfolgenden Exkurs eine der Besiedlungslegenden wiedergegeben (vgl. hierzu Abb. 24).

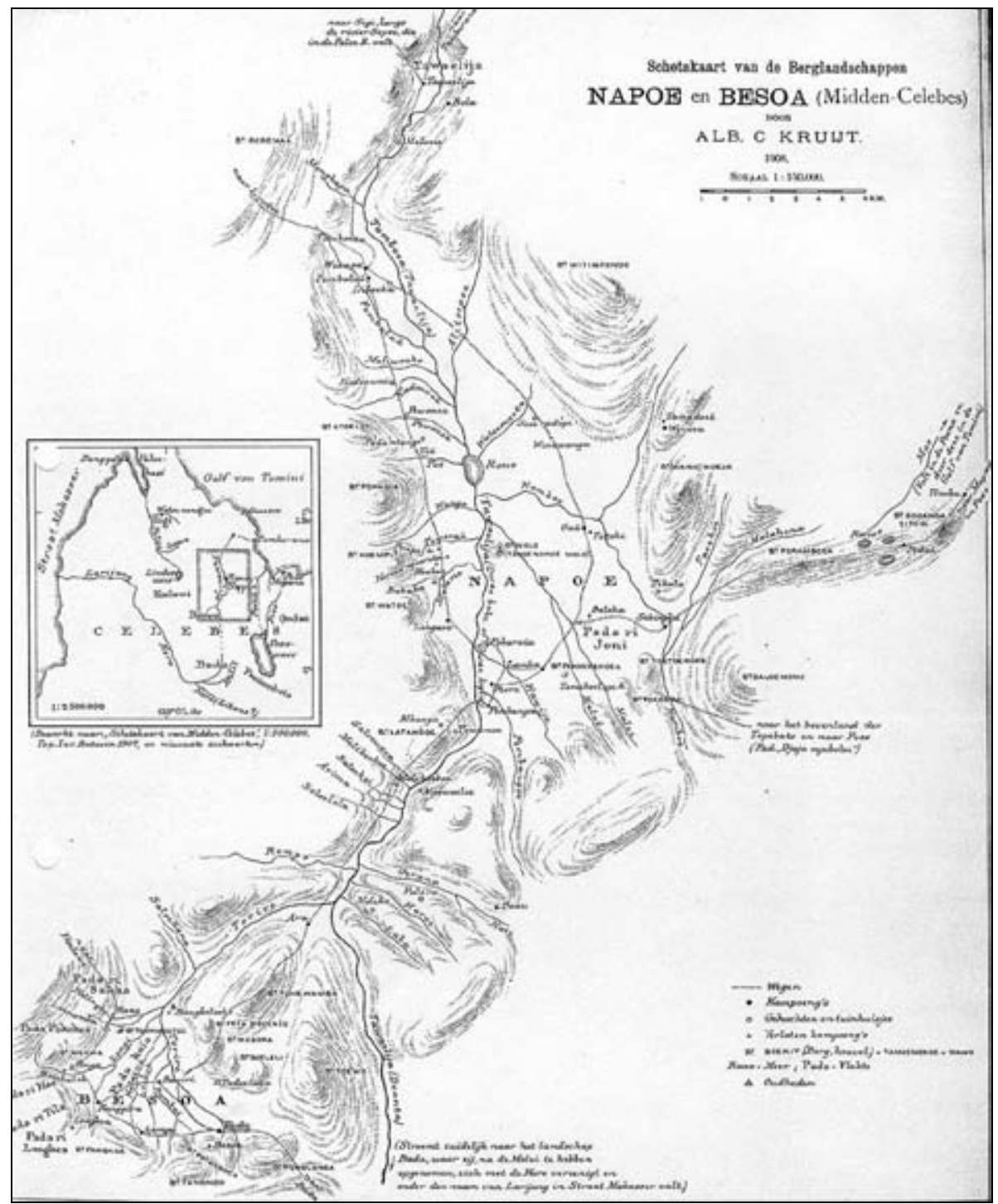

Abb. 24 Karte des Napu-Tals von 1908

(Quelle: Kruyt 1908: 1345) 


\section{Exkurs: Legende von der vorkolonialen Besiedlung des Napu-Tals}

Im Napu-Tal kursiert die folgende Legende über das Tal und seine Besiedlung: ${ }^{86}$ Während der sog. Raba-Zeit, vor Tausenden von Jahren, war das Napu-Tal ein See (Raba See). Auf den Hügeln um den See gab es zu jener Zeit Siedlungsgebiete verschiedener Stämme, die sich nur im Rahmen von Adat-Zeremonien trafen. Die beiden größten Gruppen waren To Winoa im Osten (oberhalb des heutigen Tamadue) und To Huku im Westen (oberhalb von Wuasa). Daneben gab es noch weitere kleinere Gruppen: To Uana, To Beau, To Malibubu, To Wawownla, To Kapa, To Beloka und To Petingkea. Jeder dieser Gruppen stand ein tuana (vergleichbar mit maradika in Kulawi) vor. ${ }^{87}$ In ihrer animistischen Glaubensvorstellung spielten die Towalia eine bedeutende Rolle als Mediatoren zwischen den Menschen und dem übernatürlichen Wesen, welches die Bäume und Steine beseelte. In jeder der genannten Gruppen übte eine Person die Funktion dieses Mediators aus. Eines Tages beschlossen die Menschen, dass der See austrocknen soll, um sich dort ansiedeln zu können. Daraufhin trafen sich alle Towalia an einem Ort, um das übernatürliche Wesen zu bitten, den See trocken zu legen. Das Wesen teilte ihnen mit, dass sie sieben Mal um den See laufen und in jeder Siedlung modondi (ein Vorläufer des heute v.a. in der Region Poso weit verbreiteten (mo-)dero-Tanzes) tanzen müssten. Nachdem die Towalia den See sieben Mal umrundet hatten, wählten sie am südlichen Ende des Tals eine Stelle aus, an der sie mit Hilfe des pehungki, einer goldenen Schaufel, einen Entwässerungskanal gruben. Hundert Jahre später war der See schließlich verschwunden. Der Rano See, nordwestlich von Wanga, stellt das letzte Relikt des alten Sees dar. Der nördliche Teil des ausgetrockneten Tals war nach der Entwässerung mit Wald bestanden, während sich im südlichen Teil Grassland erstreckte. Die Menschen wanderten danach von Winoa aus nach Kalide, von Huku aus nach Lengaro, Pembangu und Peore. Die Mitglieder der anderen Gruppen verteilten sich auf Kalide und Lengaro. Zu jener Zeit aßen die Menschen nur Wild und Waldfrüchte, da ihnen die Landwirtschaft noch völlig unbekannt war. Etwa im 10. Jh. kam Tomanuru vom Himmel und heiratete eine Frau in Kalide. Er stellte Werkzeuge für die Landwirtschaft her und lehrte die Menschen den Ackerbau, woraufhin diese mit der Nassreisbewirtschaftung begannen. Tomanuru zeugte sieben Kinder, sechs Buben und ein Mädchen. Nachdem seine Kinder das Erwachsenenalter erreicht hatten, verschwand Tomanuru. Tindarura, alias Gumang Kuana, blieb im Napu-Tal. Die anderen sechs Kinder verließen das Tal: Madusila ging nach Gowa, Ralinu zum Lindu See oder nach Parigi, Madika Mpudu alias Kompailo nach Wentira (Reich der Geister), Pua nach Manado, Rabuho (die Tochter) nach Palopo und Rampaili nach Bada. Tinadura war es nach dieser Legende auch, der Pekurebua als Gebietsbezeichnung für das Napu-Tal einführte, ${ }^{88}$ nachdem er während der Jagd die

86 Über die Entstehung des Napu- sowie des Besoa-Tals hat Grubauer die folgende Legende notiert: „'Vorzeitlich bestand auch das Territorium der heutigen Hochebene aus hohem Berglande, wie alles Gebiet von Meer zu Meer. Da kam aus dem Hochgebirge herab ein riesiger Büffel, um in den Napu-Bergen Weide zu suchen. Er fand hier so zarte Äsung, daß er sich vor Wohlbehagen niederlegte und im Grase wälzte. Zweimal drehte er sich dabei um sich selbst, und die hierdurch entstandenen Flächen stellen die durch einen vorspringenden Hügelzug geteilte Hochebene von Napu dar. Auf seiner Streife weiterziehend, kam der Büffel hierauf ins Besoa-Gebirge. Auch hier wälzte er sich einmal, und die entstandene tiefe Mulde ist das heutige Hochtal von Besoa."“ (1923: 133). Vgl. den Exkurs in Kap. 4.2.2 zur soziopolitischen Gesellschaftsstratifikation in Kulawi.

88 Das ursprüngliche Gebiet von Pekurehua befand sich im Süden des Napu-Tals. In der Karte von Kruyt (1908: 1345; vgl. Abb. 24) ist ein Hügel in der Nähe des Dorfes Lamba mit der Bezeichnung Bukit Pekurehua vermerkt. Heute sind es allerdings auch Bewohner der Dörfer im Norden des 
Stimme eines Vogels hörte. Er fragte die Menschen, um welchen Vogel es sich dabei handelte, und erhielt als Antwort, dass es ein Kureu-Vogel wäre. Schließlich nannte er das Gebiet, in dem er die Stimme gehört hatte Pekurehua. Nachdem das Tal diese Bezeichnung erhalten hatte, gründeten die Menschen südlich von Kalide eine Siedlung, die sie Habingka nannten, und legten sawah-Felder an. Als die Bevölkerung des Ortes zu groß geworden war, zogen einige der Bewohner weiter, um das Dorf Gaa zu gründen. Daneben wurden auch noch die Siedlungen Mamboli und Korowosi errichtet. Mit der Zeit entstanden vier einflussreiche „Fürstentümer“ in Habingka, Gaa, Lengaro und Watutau $^{89}$ (dem früheren Pembangu), die jeweils von einem sog. bangsawan geführt wurden. Tindarura wurde schließlich von den vier bangsawan zu ihrem tadulako (Kriegsheld) ernannt, der neben den landwirtschaftlichen Werkzeugen auch Waffen herstellte. Er trug nun den Namen Gumang Kuana. In dem Ort Lamba wurde eine baruga (Versammlungshaus) ${ }^{90}$ als Treffpunkt aller bangsawan errichtet. Im Laufe der Zeit siedelten sich dort Familien aus den anderen Dörfern an. Bevor Gumang Kuana verstarb, teilte er den Menschen mit, sie sollten ihn nach seinem Ableben in einem Sarg unter der baruga beisetzen, aber nicht begraben. Ferner erklärte er, dass die Menschen ihn im Fall einer großen Gefahr wiedererwecken könnten, indem sie ein Ei gegen seinen Sarg werfen. Gegen Ende des 12. Jh. wurde die Bevölkerung des Napu-Tals von den To Mene $e^{91}$ attackiert. Alle Menschen im Norden des Tals wurden dabei getötet. Daraufhin wurde Gumang Kuana wiedererweckt, der die Feinde schließlich zwischen dem heutigen Watumaeta und Sedoa köpfte. Bevor er in die Schlacht zog, forderte er die bangsawan auf, in der Zwischenzeit seinen Sarg zu zerstören. Als Gumang Kuana zur baruga zurückkehrte, sah er, dass der Sarg unversehrt war. Er erklärte den Menschen, dass er ihnen von nun an nicht mehr helfen könne, da sie ihm nicht geholfen, seinen Befehl nicht ausgeführt hätten. Er legte sich in den Sarg und verstarb für immer. Kurze Zeit darauf wurde die baruga von einem Feuer zerstört und die Asche begrub den Sarg von Gumang Kuana.

Kruyt fasst den vorkolonialen Zustand der Besiedlung Zentralsulawesis in einem im Juli 1902 verfassten Aufsatz folgendermaßen zusammen:

„Die Dörfer sind alle klein und liegen weit voneinander entfernt. Viele Landstriche sind durch große Urwälder voneinander getrennt, die man in nicht weniger als zwei bis drei Tagen durchwandern kann. Diese ausgedehnten Wälder findet man zwischen den Talgebieten aller größeren Flüsse, während sich die Torajas, wie so viele andere Volksstämme des Indischen Archipels immer in der Nähe eines Hauptstroms sammeln. So benötigt man drei Tage um das wüste, mit dichtem Wald begrünte Bergland zu durchstreifen, das die Posso-Ebene, wo die meisten Stämme der Barée-Sprecher wohnen, von dem Tawaelia-Tal teilt, wo die Stämme der Tobesoa, Tobanahoe [vermutlich sind damit die To Napu gemeint; Anm. d. Verf.], Tobada angesiedelt sind. $V$ on bier muss man weiter durch ein Berggebiet von etlichen Tagesreisen, um in das Stromgebiet des Palu-Flusses bei Tolindoe, Tokoelawi und den Stämmen im Palu-Tal zu kommen. [...] Die Bevölkerung von Mittel-Celebes ist sehr gering [...]. Nur eine Strecke in dem genannten Teil von

Tals, die Pekurehua als ehemalige Bezeichnung ihrer Umgebung definieren und somit das gesamte Napu-Tal unter diesem Terminus zusammenfassen.

89 Watutau bedeutet übersetzt: ,steinerner Mann“.

90 Frühere Bezeichnung: dubunga. In Kulawi werden diese Versammlunghäuser lobo genannt.

91 Nach Kruyt (1908: 1318) handelt es sich bei den To Mene um Mandaresen. 
Mittel-Celebes ist etwas stärker bevölkert, nämlich das Palu-Tal, aber von Überbevölkerung ist bier noch keine Rede [...]. "(Kruyt 1903b: 190f). ${ }^{92}$

Bis zur Ankunft der Niederländer befanden sich in der Lore-Lindu-Region ausschließlich kleine Pfade, meist Trampelpfade, welche die vorwiegend in den Bergregionen lebende Bevölkerung miteinander verband. Ein Verkehrswegenetz wurde allerdings erst nach den Eingriffen der effektiven Kolonialherrschaft in dieser Region installiert. So wird in einem Aufsatz aus dem Jahre 1912 über den Zustand in den Bergen noch folgendes vermerkt: „V $V$ an wegen kann men in de Berglandschappen niet spreken."(o.A. 1912: 3).

Wie bereits erwähnt, lebte die Bevölkerung der Lore-Lindu-Region zu Beginn des 20. Jh. in Familienverbänden bzw. Clans zusammen. Diese waren miteinander durch die Vorstellung verbunden, von ein und demselben Urahnen abzustammen. Durch die verschiedenen existierenden Königreiche/Fürstentümer (kerajaan) wurde eine Clan übergreifende Identitätsstruktur aufgebaut. Diese hatte jedoch keinen Einfluss auf die Siedlungsstruktur. Die Identität äußerte sich hauptsächlich in einem Zusammengehörigkeitsgefühl im Falle von kriegerischen Auseinandersetzungen mit anderen Fürstentümern der Region (Mattulada 1990: 3). Für die Tobaku-Region (Pipikoro-Gebiet, westliches Kulawi) erklärt Aragon (2000: 57), dass die heutigen offiziellen Dörfer ursprünglich Zeremonieplätze waren.

„The precolonial ,ceremonial village' thus consisted of a large temple (lobo, Uma), a small temple (sou eo), which doubled as an inn, and a few other multibearth dwellings that would each periodically shelter several families who shared a common horticultural settlement for that year. "(ebd.)

Diese Dörfer führten also zu einer kurzfristigen Veränderung der Wohnstruktur. Während die Familien die meiste Zeit des Jahres dezentralisiert siedelte, wohnten sie aus Anlass bestimmter Zeremonien gemeinsam an einem Ort. Dieses Verhalten war auch nach Einführung der christlichen Religion zu beobachten. Bis in die zweite Hälfte des 20. Jh. hinein wohnte der Großteil der Einwohner eines Dorfes in kleinen Hütten auf ihren Feldern und kam nur am Wochenende zum Besuch des Got-

92 Zitat im Original: „De dorpen zijn alle klein en liggen ver van elkaar af. Vele landstreken zijn door droote oerwouden van elkaar gescheiden, die men in niet minder dan twee à drie dagen kan doortrekken. Deze uitgestrekte wouden vindt men tusschen de dalgebieden van alle groote rivieren, want als zoovele andere volksstammen van den Indischen Archipel trekken de Toradja's zich steeds samen in de nabijheid van een hoofdstroom. Zoo heeft men drie dagen nodig om het woeste bergland met zwaar bosch begroeid door te trekken, dat de Posso-depressie, alwaar de meeste stammen der Barée-sprekers wonen scheidt van het Tawaelia-dal, alwaar de stammen der Tobesoa, Tobanahoe, Tobada gevestigt zijn. Van hier moet men weer door een bergland van ettlijke dagreizen om in het stroomgebied te komen van de Paloerivier bij Tolindoe, Tokoelawi en de stammetjes in het Paloedal. [...] De bevolking van Midden-Celebes is dus zeer schaarsch [...]. Slechts in het genoemde gedeelte van Midden-Celebes is wat sterker bevolkt, namlijk het Paloedal, maar van overbevolking is hier nog geen sprake [...].“ 
tesdienstes ins Dorf (vgl. Kap 4.1.3). Anfang des 20. Jh. wurden zahlreiche brachliegende Nassreisfelder in den Tallagen, z.B. des Palu-Tals (Hissink 1909: 62f) oder des Napu-Tals (Kruyt \& Kruyt 1921: 407), vorgefunden, was auf eine ehemals dichtere Besiedlung der jeweiligen Tallagen schließen lässt. Kriegerische Auseinandersetzung zwischen den einzelnen Fürstentümern könnten allerdings der Grund dafür sein, dass sich die Bevölkerung zunehmend in die strategisch vorteilhafteren, höheren Berglagen zurückgezogen hatten (Henley 2004: 131).

\section{Exkurs: Zur Attraktivität der Bergregionen für die lokale Bevölkerung}

In diesem Exkurs soll der interessanten Frage nachgehen werden, warum das Hochland in vorkolonialer Zeit überhaupt eine anscheinend so hohe Attraktivität für die Bevölkerung dargestellt hatte. Reid (1997) geht in seinem Aufsatz zu Umsiedlungen auf Sumatra während der Kolonialzeit auch auf Zentralsulawesi ein und stellt dabei die Frage:

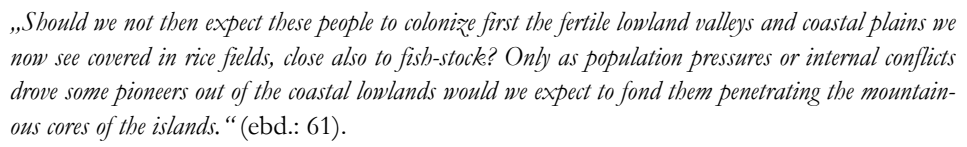

Eine Ursache liegt vermutlich in den Herkunftsmythen der einzelnen Gesellschaften, die nicht von der Küste als Ausgangspunkt sprechen, ,but of a kind of ethnogenesis in the mountains. " (ebd.). Für das Poso-Gebiet Zentralsulawesis bestätigt auch Coté (1979) eine solche Mythenbasis. Reid weist darauf hin, dass:
„During the rapid population growth of the twentieth century, the most significant migrations in Indo- nesia have not been of lowlanders into the sparsely settled highlands, as has been a marked feature in China and Vietnam [...], but the reverse."(1997: 62).

In Sulawesi muss man jedoch noch zwischen Bugis, Makassar und Mandar im Südwesten der Insel einerseits und den meisten übrigen Inselbewohnern andererseits unterscheiden (ebd.: 63). So hält Reid für Zentralsulawesi fest:
„Other highland lakes [als Lake Tondano; d. Verf.] such as Lake Poso and Lake Lindu were im- portant centers of sedentarization, although the main centre of the mysterious megalith remains of Cen- tral Sulawesi is in an upland valley mid-way between these two lakes. The Toraja of the central massif also developed a complex stateless civilization at elevations above 1,000 metres. "(ebd.: 63f).

Damit ist allerdings erst eine Zustandsbeschreibung vollzogen worden. Wichtig ist jedoch noch die Frage, welche Faktoren diese Entwicklung bewirkt hatten. Auch hier gibt Reid (1997: 78) einen Lösungsvorschlag. Seiner Meinung nach spielten die Landwirtschaft, die Gesundheit, Staatenlosigkeit, Sicherheitsfragen und die Kultur eine Rolle. Auf einige dieser Faktoren soll nun genauer eingegangen werden. Der landwirtschaftliche Anbau im Tiefland war der Gefahr von Überflutungen ausgesetzt. Diese vernichteten die Ernte, brachten das Vieh in Gefahr und schränkten die Frischwasserversorgung ein. Die Ebenen waren nur mit großflächiger Bewässerung und Drainage, welche die Wassermassen kontrollierte, zu bewirtschaften. Zudem benötigten die Nassreisfelder des Tieflandes einen hohen Arbeitskräfteaufwand und brachten eine komplette Veränderung der natürlichen Umwelt mit sich. „With relatively simple technology and small family units of labour, bighland valleys with small but permanent streams of water are far easier to manage." (ebd.). Weniger die Malariagefahr war es, die den gesundheitlichen Aspekt der Ansiedlung im Hochland ausmachte, denn malariafreie Gebiete kamen auch in manchen Tiefebenen vor. Vielmehr waren es die Krankheiten, welche durch unsauberes Wasser verbreitet wurden, wie beispielsweise Typhus oder Cholera. „Coastal and river-mouth cities were the worst death-traps of 
all." (Reid 1997: 79). Für die Inlandregionen einiger der östlichen Inseln des Archipels stellt Reid (1988: 18) durchaus komplexe vorkoloniale wirtschaftliche Systeme fest, welche beispielsweise von Hochland-Tiefland-Tauschbeziehungen geprägt waren (Reid 1988: 28; vgl. auch Aragon (1996d) für Zentralsulawesi). Zu dem Faktor der Staatenlosigkeit resümiert Reid:

,With some exceptions in Java and Bali, all states in the archipelago have been based at river mouths or strategic coastal locations. They absorbed some of the interior people and established loose relations of tribute with others, but most highland populations defend their autonomies by a mixture of guerilla warfare, diplomatic flexibility, and deliberate exaggeration of myths about their savagery. "(1997: 82).

Letzteres taten die Hochlandbewohner etwa, indem sie selbst ihren angeblichen Kannibalismus übertrieben nach außen trugen, um somit die Europäer abzuschrecken. Für das Untersuchungsgebiet könnte das z.B. die Kopfjagd (vgl. Grubauers (1913) Buchtitel „Unter Kopfjägern in Central-Celebes“) gewesen sein. Bezüglich kultureller Ursachen für die Präferenz von höher gelegenen Regionen als Siedlungszentren spricht Reid das Bewusstsein der Hochlandbevölkerung an, im Falle einer Migration von der muslimischen Bevölkerung im Tiefland solange verächtlich betrachtet zu werden, wie sie nicht deren Kleidung, Sprache, Gebräuche etc. angenommen hätten. Die Bergregionen können daher auch als ein passiv-strategisches Rückzugsgebiet betrachtet werden, welches Schutz vor entsprechenden soziokulturellen Veränderungen gewährte. Die Konversion der Hochlandbewohner zu Islam oder Christentum markierte daher , a crucial breakdown of this cultural barrier in the long term, even if in the short term it sometimes strengthened it. "(Reid 1997: 82). Reids Faktoren, welche die Bergbevölkerung von einer Ansiedlung in der Tiefebene fernhielten, scheinen plausibel zu sein und entsprechen zudem dem Bild in der Palu-Region, wo die Ebene anfangs mit buginesischen Immigranten durchzogen war, welche sich im Laufe der Zeit Handel betreibend in die Bergregionen des Hinterlandes vorgewagt hatten. Neben den von Reid angeführten Erklärungen ist auch der verteidigungspolitische Aspekt für die Präferenz der Bergregionen als Siedlungsgebiet zu berücksichtigen. Die Höhenlage bot angesichts der häufigen kriegerischen Auseinandersetzungen einen großen verteidigungsstrategischen Vorteil.

Aus siedlungsgeographisch-kartographischer Sicht interessant ist eine Karte der östlichen Lore-Lindu-Region (Lore Utara, Lore Selatan) aus niederländischen Archivbeständen zur Kolonialzeit (vgl. Abb. 25). Die Karte stammt aus dem Inventaris van de verzamling Politieke Verslagen en Berichten wit de Buitengewesten, 1898-1940, welches im Nationaalarchief in Den Haag archiviert ist. Die falsch genordete Karte (der Nordpfeil zeigt auf der Karte nach Osten) stellt das Napu-Tal, die Besoa-Enklave, sowie Verbindungswege zu den Regionen Kulawi, Bada und Poso dar. Die Karte, welche auf das Jahr 1905 datiert ist, wurde im Rahmen einer Geheimmission des Resident van Menado, des obersten niederländischen Verwalters des nördlichen Teils von Sulawesi, erstellt. Nach Angaben des niederländischen Missionars Kruyt (1908: 1275) besuchte der niederländische Leutnant H. J. Voskuil im August 1905 als erster Europäer die Napu-Ebene. Möglicherweise entstand die vorliegende Karte unter seiner Federführung. Die große kartographische Ungenauigkeit der Karte lässt vermuten, dass die meisten Inhalte mehr auf den Informationen Dritter beruhen, als dass sie von den Niederländern nach selbständiger Kartierung eingefügt wurden. Obwohl eine Karte von Kruyt (1908: 1345; vgl. Abb. 24) das Gebiet viel detailierter 
und realitätsnäher wiedergibt, ist die vorliegende Karte dadurch interessanter, dass sie bereits vor der Unterwerfung des Napu-Tals durch die Holländer im Jahr 1907 angefertigt wurde und interessante siedlungsgeographische Hinweise liefert.

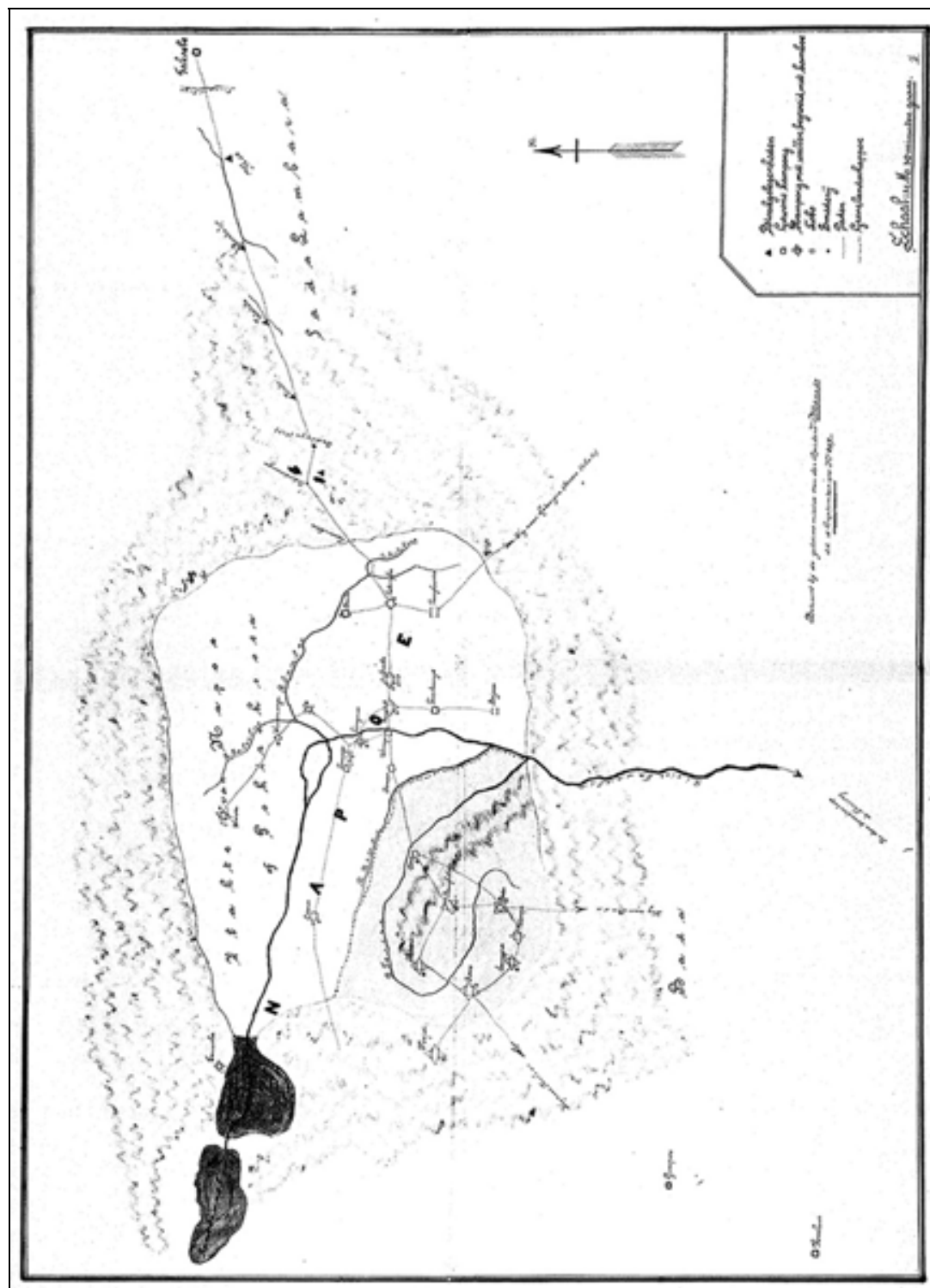

Quelle: Ministerie van Kolonien, Pol. Versl. Buitengewesten, 1898-1940, Menado, Karten \& Tekeningen, Fiche: 722A, Algemeen Nationaalarchief, Den Haag.

Abb. 25 Karte des Napu-Tals von 1905 
Im Napu-Tal bestand nach Aussage dieser Karte bereits zu Beginn des 20. Jh. eine gewisse Anzahl von Siedlungen. Die Signatur „Kampong met wallen begroeid, met bamboe", markiert die Hauptorte der Region. Die Feststellung Einheimischer heute, dass die Orte Winoa, Lengaru, Peore ${ }^{93}$ und Lamba vor Beginn der niederländischen Okkupation politische Zentren des Napu-Tals waren, decken sich nicht nur mit den Aussagen zeitgenössischer Berichte (vgl. Kruyt 1908), sondern auch mit den Angaben in dieser Karte. Die verstärkte Konzentration von lobos (Versamlungshäuser, religiöse Kultstätten) in den genannten Orten bestätigt auch das Ergebnis aus der oral history, dass die politische Funktion dieser Siedlungen auch mit einer religiösen Bedeutung verknüpft war. Außer Wuasa und Tawailia (das spätere Sedoa) konzentrieren sich die Siedlungen auf den südlichen Teil des NapuTals, der im Vergleich zum damals noch stark bewaldeten Norden schon früh durch ausgedehnte Grassflächen gekennzeichnet war. Die Lage der Verdichtungsräume auf der Karte decken sich auch mit der Verortung der heute noch vorhandenen Relikte einer alter Megalithkultur. ${ }^{94}$

\subsubsection{Wandel der Siedlungsformen unter dem kolonialen Einfluss}

Erst mit der von der Kolonialmacht durchgeführten massiven lokalen Umsiedlung der Bergbevölkerung kam es, vor allem in den Tälern, zu dem Aufbau deutlich strukturierter Dörfer. Den Niederländern war insbesondere daran gelegen, die verstreut in den Bergen siedelnden Familien in fest verorteten Dörfern zu konzentrieren (vgl. u.a. Kaudern 1925b: 39; Kruyt 1908: 1308; Kruyt 1926: 542; Vorstman 1935: 13). Dies erfüllte zum einen den Zweck, die Menschen besser verwalten zu können. So wurde beispielsweise das Eintreiben der Abgaben für das von den Kolonialherren eingeführte Steuersystem (Van Hengel 1910: 3; Logeman 1922: 131f) erleichtert. Denn gerade in den Bergregionen wurde das Erheben der Steuern bei der verstreut lebenden Bevölkerung erschwert (Voorn 1925: 15) Zum anderen konnte schneller auf die zum Strassen- und Wegebau herangezogene männliche Bevölkerung zugegriffen werden (Logeman 1992: 128). Die große Bedeutung für die Niederländer, die Siedlungsstruktur in der Region zu verändern, sowie die Vorgehensweise fasst Adriani folgendermaßen zusammen:

„Eine zweite, sehr wichtige Maßregel war das Neuanlegen von Dörfern. Innerhalb des Stammesgebiets mussten alle Dörfer von den Hügelspitzen herunterkommen und wurden an flachen, gut

93 Peore war das Zentrum der entscheidenden Schlacht zwischen den einheimischen Kriegern und den niederländischen Soldaten im Jahr 1907, welche bis heute als Peore-Krieg bekannt ist.

94 Das bedeutendste Zentrum der Megalithkultur stellt das Bada-Tal dar. Bislang konnten nur wenige Fragen über die Megalithen, ihren zeitlichen Ursprung und ihre gesellschaftlichen Bezüge beantwortet werden. 
zugänglichen Orten in die Nähe von Wasser neu aufgebaut, und zwar so, dass der große Weg, der durch das Stammesgebiet angelegt wird, keine großen Bögen machen muss, um alle Dörfer des Stammes zu durchschneiden. Alle Durchsetzungskraft des Herrn Mazee und alle Überredungskunst der Mission war nötig, um dafür zu sorgen, dass das klappte. "(1915: 469)..$^{95}$

Die neu geschaffene Siedlungsstruktur ist, wie auch das Zitat belegt, eng mit dem Ausbau der Verkehrsinfrastruktur verbunden. Von Palu aus wurde jeweils eine Strasse $^{96}$ westlich und östlich des Palu-Flusses nach Süden vorangetrieben. Auf der östlichen Seite entwickelte sich ein stärker verzweigtes Straßennetz. Ab dem Dorf Pandere führte dann eine Strasse weiter, welche zum Ende der Kolonialzeit bis nach Bolapapu, dem heutigen Hauptort des Kecamatan Kulawi reichte. Von dort aus wurde ein Weg weiter nach Süden errichtet, der sich im westlichen Kulawi bis in das gebirgige Pipikoro-Gebiet hinein verzweigte. Ein weiterer Weg wurde aus dem PaluTal heraus nach Osten bis zum Beginn des Palolo-Tals gebaut. Allein das Napu-Tal (Lore Utara) war ausschließlich über die östlich von Palu an der Tomini Bucht gelegene Hafenstadt Poso durch einen neu errichteten Weg verbunden, welcher sich im Napu-Tal verzweigte. Zwar waren das Palolo- und das Napu-Tal mit einem Trampelpfad verbunden, eine ausgebaute Verbindung bestand jedoch bis in die frühen 1980er Jahre nicht. Im Napu-Tal wählten die Niederländer fünf Gebiete aus, in welchen sich die lokale Bevölkerung in Dörfern ansiedeln mussten. Dies waren die Siedlungen Peore-Pembangu ${ }^{97}$, Gaa-Totohi, Winoa-Tamadue, Sabinka und WuasaPembalaa $^{98}$ (Kruyt 1908: 1309). Die Doppelnamen ergaben sich aus dem Umstand heraus, dass eine Siedlung (z.B. Gaa) bereits vor dem Einmarsch der Niederländer existierte, dieses Wehrdorf jedoch durch einen umgebenden Erdwall räumlich begrenzt war (vgl. Abb. 23). Der zwangsumgesiedelten Bevölkerung wurde schließlich ein Gebiet außerhalb des Walls zugewiesen, welches einen eigenen Namen erhielt (ebd.: 1308).

Kaudern (1925b: 38) verdeutlicht die durch die niederländische Siedlungspolitik beeinflusste Umstrukturierung anhand eines zeitlichen Vergleichs. Für die Region, welche das heutige Kecamatan Kulawi umfasst, untersucht er die Differenzen zwi-

95 Zitat im Original: „Een tweede, zeer belangrijke maatregel was het stichten van nieuwe dorpen. Binnen het grondgebied van de stam moesten alle dorpen van hunne heuveltoppen alkomen en herbouwd worden op vlakke, althans toegankelijke plaatsen, in de nabijheid van water en zóó, dat de groote weg, die door het gebied van den stam wird aangelegd, geen groote bochten behoefde te maken om alle dorpen van den stam te doorsnijden. Alle doorzettingskracht van den heer Mazee en alle overredingskracht der Zendelingen is noodig geweest, om te zorgen dat dit gebeurde.“

96 In bezug auf die Kolonialzeit ist unter dem Begriff „Strasse“ immer eine mit dem Auto befahrbare Strasse zu verstehen, während Wege höchstens per Pferd passierbar waren.

97 Peore-Pembangu wurde später in den noch heute existierenden Dorfnamen Watutau umbenannt.

98 Wuasa stellte nach Kruyt (1908: 1321f) bereits zu vorkolonialer Zeit ein sehr großes Dorf dar. Er belegt dies u.a. mit der Tatsache, dass seine Bewohner in früherer Zeit die Angriffe von Kriegern des raja von Sigi mehrere Male erfolgreich schlagen konnten, bevor diese mit Hilfe einer Wasservergiftung das nördliche Napu-Tal für das Sigi-Reich unterwerfen konnten. 
schen der Häuserzählung von Boonstra van Herdt aus dem Jahre 1911 und seinen eigenen Zählungen im Jahr 1918. Dabei fällt ihm auf, dass die Siedlungen der schwerer zugänglichen Berggebiete 1918 fast immer eine geringere Anzahl von Häusern aufweisen als noch 1911. Kaudern sieht den Hintegrund in ,the endeavour of the Dutch Government to make the natives leave the highlands, and come to live in more accessible districts." (1925b: 39).

Nachdem die neuen Dörfer entlang der Strassen und Wege errichtet worden waren, siedelten sich die Menschen in den Dörfern zunehmend entlang der Strassen an. Noch heute ist diese Entwicklung anhand der nahezu im gesamten Untersuchungsgebiet vorherrschenden Siedlungsform des Straßendorfes erkennbar. Zwar zweigen innerhalb der Dörfer kleinere Gassen (sog. lorong) von den Hauptstrassen $\mathrm{ab}$, in den wenigsten Dörfern bildete sich aber ein klassischer Dorfkern, vergleichbar etwa mit dem eines Angerdorfes, heraus. Die von den Niederländern eingeführte, geordnete Siedlungsform zeigt Grubauer am Beispiel des Dorfes Watutau auf: „Brände haben das Dorf vernichtet, und die in neuerer Zeit entstandenen Baulichkeiten haben wohl den alten Typ nach Art des Häuptlingshauses beibehalten, sind aber in breiten regelmäßigen Reihen angelegt und mit gut gepflegten Gärten versehen."(1923: 133). Am Beispiel des Neuaufbaus des Dorfes Lemo (Teil des heutigen Bolapapu, Kecamatan Kulawi) verweist auch Kaudern auf den kolonialen Einfluss auf die Siedlungsformen:

„,...] or an earthquake has in exceptional cases destroyed a village as was the case in 1909 with the village of Lemo in Koelawi. The new village that was built instead of the old one, has obviously been influenced by modernism, and does not look like a genuine Koelawi village [...]" (1925b: 39).

Eine Karte von Walter Kaudern (1925b: 33) zeigt, auf welche Regionen sich die Siedlungsgebiete im westlichen Zentralsulawesi Anfang der 1920er Jahre beschränkten (vgl. Abb. 26). Das größte Siedlungsgebiet befindet sich darin im PaluTal. Gut zu erkennen sind auch die Bergregionen Pekawa und Raraggonau westlich und östlich des Tals, aus welchen große Teile der Bevölkerung von den Niederländern zwangsumgesiedelt wurden (vgl. Kap. 3.4.1). Der größte Bereich des PaloloTals war zu jener Zeit noch unbesiedelt. Nur die Bergregion im Übergang zum Palu-Tal ist in der Karte als bevölkertes Gebiet vermerkt. Im nördlichen Kulawi befanden sich nur kleinere Siedlungsbereiche. Neben dem Kulawi-Tal und dem Gimpu-Tal zählten v.a. die Region um den Lindu See, Toro und Tangkulowi (in der Karte: Tamoengkolowi) dazu. Auffallen groß sind die bevölkerten Gebiete der Pipikoro-Region im westlichen Kulawi. Im Osten des Untersuchungsgebiets ist das Napu-Tal als größtes Siedlungsareal vermerkt. Der zu vorkolonialer Zeit noch stark bewaldete nördliche Teil des Napu-Tals ist in der Karte von Kaudern (1925b: 33; vgl. Abb. 26) bereits als bevölkerte Region ausgewiesen, was sich durch den Einfluss der Niederländer auf das Siedlungswesen erklären lässt. Die kompakte Form der Siedlungsgebiete auf der Karte verdeutlicht die zu jener Zeit durchgeführten lokalen Umsiedlungsmaßnahmen von den Bergen in die Talbereiche, sowie die Konzentration der Siedlungsgebiete in der Pipikoro-Region. 


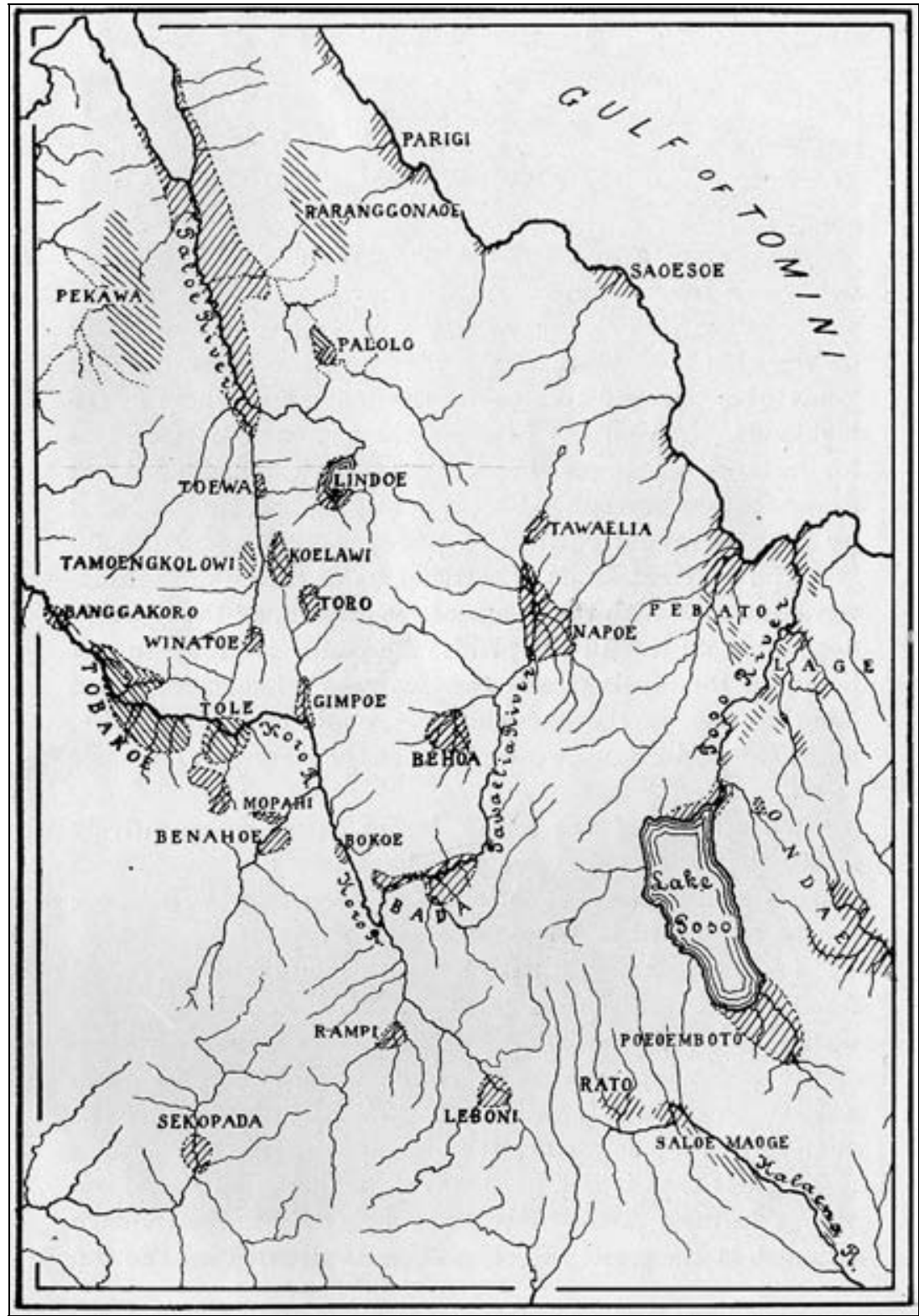

Abb. 26 Siedlungsgebiete im westlichen Zentralsulawesi Anfang der 1920er Jahre (Quelle: Kaudern 1925b: 33)

Die siedlungspolitischen Maßnahmen der Niederländer führten zwar dazu, dass feste Siedlungsstrukturen in Form permanenter Dörfer angelegt wurden. Dies darf jedoch nicht darüber hinweg täuschen, dass bis in die 1970er Jahre hinein nur ein 
kleiner Teil der Bevölkerung eines Dorfes auch tatsächlich permanent in den Dörfern wohnte. Man kann sogar soweit gehen, dass es v.a. der christlichen Missionierung zu verdanken ist, dass die Dorfbewohner regelmäßig in ihren Dorfbehausungen anzutreffen waren. In der Regel war nur die Dorfadministration (also die Führungsschicht der Dorfgesellschaft), in einigen Dörfern auch chinesische, arabische oder buginesische Händler, dauerhaft in den Dörfern anwesend. Die restliche Bevölkerung wohnte weiterhin die Woche über in ihren einfachen Hütten auf den Feldern und hielt sich nur zum Gottesdienst am Sonntag im Dorf auf. Befragte Missionare der Heilsarmee bestätigen dieses Verhalten. Sie fanden v.a. in Regionen, in welchen (noch) keine Nassreisfelder angelegt waren, unter der Woche fast ausgestorbene Siedlungen vor. Selbst in denjenigen Dörfern, deren Bevölkerung unter dem Einfluss der Niederländer Nassreis bewirtschaftete, befanden sich die Wohnhäuser im Dorf entgegen der Vorstellungen der Niederländer anfangs noch nicht generell entlang der neu gebauten Strassen, sondern abseits in der Nähe der Felder. Erst mit dem Ausbau der Strassen und der zunehmenden Bedeutung der Anbindung an Absatzmärkte verlagerten die Menschen allmählich ihre Häuser an die Strasse.

\subsubsection{Regionale Unterschiede der Siedlungsentwicklung ab 1945}

Die Abb. 27 zeigt die Entwicklung der Dorfgründungen in der Untersuchungsregion für drei Zeitintervalle an. ${ }^{99}$ Die Anzahl und Verteilung der neuen Dörfer in der linken Karte der Abbildung weisen zwei Besonderheiten auf. Erstens verdeutlichen sie noch einmal den hohen Grad der Dorfneugründungen während der Kolonialzeit, wie er bereits im vorangegangenen Kapitel erläutert wurde. Zweitens erkennt man daraus, dass während jener Periode in nahezu der gesamten Lore-Lindu-Region neue Siedlungen errichtet wurden. Auffallend ist ferner der weiterhin unbesiedelte Ostteil des Palolo-Tals ${ }^{100}$. Auf der mittleren Karte (1946-1975) erkennt man insbesondere im Palolo-Tal eine starke Intensität an Dorfneugründungen. Diese hängt mit der Migrationsentwicklung Palolos in jener Zeit zusammen (vgl. Kap. 3, insbes. Kap. 3.6.3). Zudem lässt sich die Verbesserung der Verkehrsinfrastruktur im Berggebiet zwischen Palu- und Kulawi-Tal sowie am Ende des Gimpu-Tals im südlichen Kulawi anhand der linearen Struktur der Dorfgründungen in diesen Gebieten ablesen. Ab 1976 (vgl. rechte Karte in Abb. 27) wurden deutlich weniger neue Dörfer errichtet als noch in den beiden vorangegangenen Zeitintervallen. Die größten

99 In der Abbildung sind nur 80 der insgesamt 119 Dörfer erfasst. Dennoch können daraus die wichtigsten Entwicklungen der Siedlungsverteilung abgelesen werden.

100 Die in der linken Karte der Abb. 27 siedlungsfreie Fläche im Nordwesten Kulawis ist, bedingt durch das Relief, bis heute unbesiedelt. 
Aktivitäten fanden diesbezüglich in der östlichen Hälfte des Palolo-Tals statt. Verantwortlich hierfür zeichnet vor allem die Verbindung des Tals mit dem südöstlich angrenzenden Napu-Tal (Lore Utara) durch eine per Auto befahrbare Strasse, die im Jahr 1982 fertiggestellt worden war.

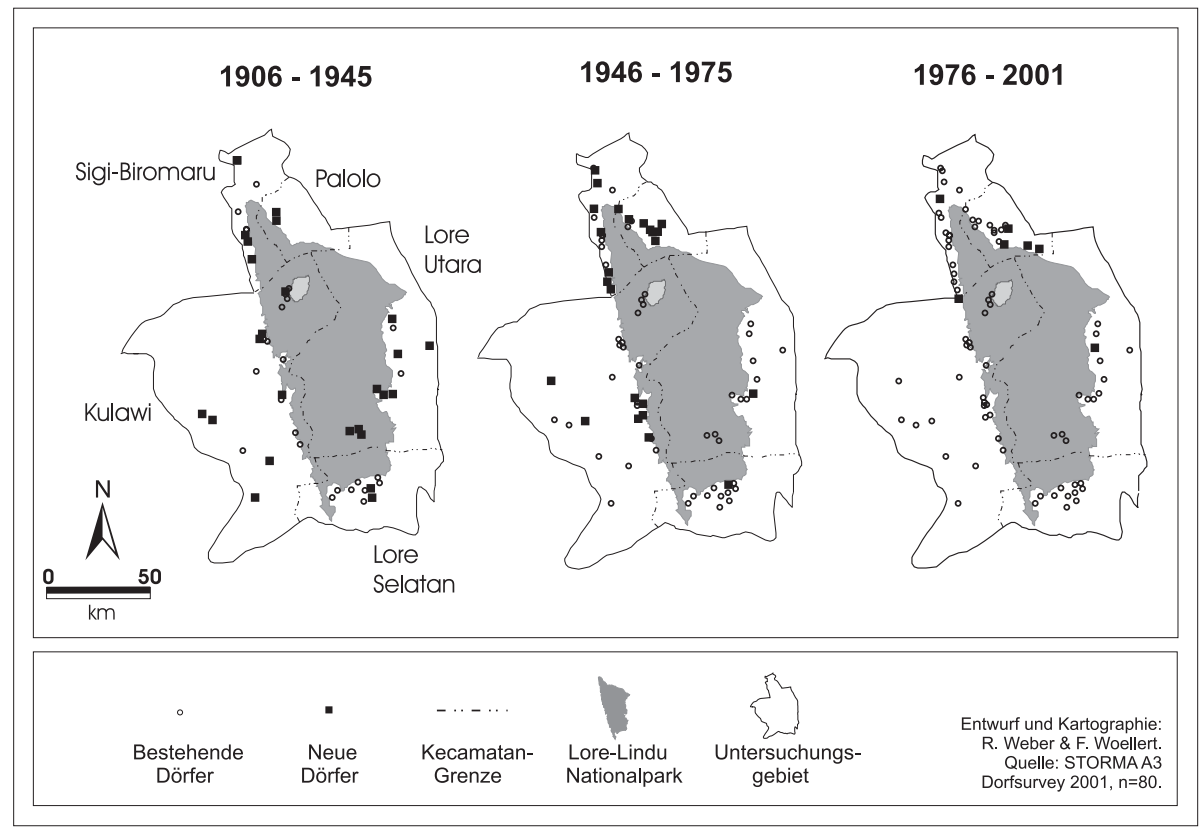

Abb. 27 Dorfgründungen in der Lore-Lindu-Region 1906 - 2001

Da gerade in den letzten zwei Dekaden des 20. Jh. die Zuwanderung in die LoreLindu-Region, bedingt durch den Boom des Kakaopreises einerseits (vgl. Kap. 5.2.2) sowie durch Transmigrationsprojekte andererseits (vgl. Kap. 3.4.2), erheblich zunahm, mag die geringe Anzahl der Dorfneugründungen ab 1976 auf den ersten Blick verwundern. Die Zunahme der Bevölkerung führt jedoch nicht automatisch zu einer Vermehrung der Dörfer. Entscheidend ist, auf welche Regionen sich die Bevölkerungszuwächse konzentrieren und welche Einwohnerdichte die Siedlungen dieser Gebiete bislang aufweisen. Wie in Kapitel 3 bereits aufgezeigt wurde, waren besonders Palolo und Lore Utara von der enormen Zuwanderung betroffen. Palolo bestand in den 1980er Jahren aus vielen noch sehr jungen Dörfern. Mit der Reform der administrativen Gemeindeverwaltung Indonesiens durch das Gesetz Nr. 5/1979 wurde das javanische Prinzip des territorial basierten Dorfes für alle Dörfer des Archipels übernommen. Dieser Dorftyp ist durch seine festen Grenzen bestimmt. Ferner sind alle Menschen, die innerhalb dieser Grenzen leben automatisch Mitglieder der Dorfgemeinde. Mit der landesweiten Anwendung dieses Prinzips wurde der außerhalb Javas verbreitete Clan-basierte Dorftyp unterminiert. 
Das Gesetz von 1979 legte auch fest, dass jedes Dorf in Dorfteile, sog. dusun zergliedert wird (Soemardjan \& Breazeale 1993: 12f). Solange die Dörfer noch verfügbare Landressourcen aufwiesen, konnte deren Einwohnerdichte durch natürlichen, aber auch durch migrationsbedingten Bevölkerungszuwachs erhöht werden, ohne dass neue Dörfer gegründet werden mussten. Das neu erschlossene Land des Dorfterritoriums wurde in der Folge nach und nach mit neuen Dusun-Nummern versehen. Bestanden zu Beginn der Umsetzung des Gesetzes Nr. 5/1979 die meisten Dörfer nur aus ein oder zwei Dusun, zählen manche Gemeinden mittlerweile bis zu fünf oder sechs Dorfteile. Neu zugewanderte Familien konnten sich in Palolo folglich auf eine große Anzahl von Dörfern mit noch geringer Einwohnerzahl verteilen. Das Kecamatan Lore Utara erlaubte durch seine relativ große Talausdehnung mit einem noch hohen Bestand an nicht bewirtschafteten Flächen ebenfalls, die bereits bestehenden Dörfer dichter zu besiedeln. Dabei wurde in der Regel die bebaute Fläche zu den Dorfgrenzen hin ausgedehnt. Besonders seit Beginn des 21. Jh. kommt es in der Lore-Lindu-Region zu Dorfteilungen, wobei ein Teil der Dusunin der Regel jene mit den höchsten Ordnungsnummern - zu der Verwaltungseinheit eines eigenständigen Dorfes zusammengefasst werden. Der Grund hierfür liegt in der zu hohen Einwohnerzahl des jeweiligen Dorfes und/oder in der Distanz dieser Dorfteile zum politischen Zentrum des Dorfes, das sich generell im Dusun mit der Ordnungsnummer 1 befindet.

In diesem Zusammenhang ist die Unterschiedlichkeit der Dörfer in ihrem Aufbau zu beachten. Die Residenzbereiche der meisten Dörfer der Lore-Lindu-Region erstrecken sich entlang der Hauptstrassen. Maßgeblich für diese Struktur ist, wie bereits erwähnt, die kolonialzeitliche Siedlungsentwicklung einerseits und deren Fortsetzung ab 1945 andererseits. Die Wohngebiete dieser Dörfer dehnten sich entlang der Hauptstrasse zu den Dorfgrenzen hin aus. Abhängig von den naturgeographischen Voraussetzungen, sowie der Landvergabepolitik der einzelnen Dorfregierungen entstanden zudem Wohnbereiche, die sich von der Hauptstrasse weg zu den Seiten entwickelten. In manchen Fällen wurden mehrere in sich geschlossene Siedlungen zu einem Dorf zusammengeschlossen. Beispielhaft steht hierfür das Dorf Bolapapu, der Hauptort des Kecamatan Kulawi. Nach der kolonialen Umsiedlung der Bergbevölkerung hin zur Hauptstrasse von Palu nach Kulawi entstandene, noch eigenständige Dörfer wurden später zum Dorf Bolapapu zusammengefasst. Von dem Dusun Salua, welches 1985 von Bolapapu abgetrennt und zu einem eigenständigen Dorf wurde, bis zum Dusun 1, dem politischen Zentrum Bolapapus, betrug die Distanz ca. zwölf Kilometer. Abgesehen von den Dorfteilen 1 und 2, die eine durchgehende Bebauung entlang der Strasse aufweisen, sind die Wohnbezirke der einzelnen Dusun teils mehrere Kilometer voneinander entfernt und vermitteln dem Laien den Eindruck, es handle sich jeweils um ein eigenes Dorf (vgl. Abb. 30). 


\subsection{Ein Vergleich von Siedlungstypen}

Bisher wurden die großräumigen Veränderungen der Siedlungsformen, des sog. Siedlungsmusters im Untersuchungsgebiet diskutiert. Daneben lassen sich aber auch in kleinerem Maßstab Wandlungen der Siedlungsformen aufzeigen. Anhand des Siedlungsganges verschiedener ausgewählter Dörfer sollen nun Unterschiede und Gemeinsamkeiten der Strukturierung von Siedlungen in der Lore-Lindu-Region herausgearbeitet werden.

Zwar handelt es sich bei den meisten Dörfern des Untersuchungsgebiets um Straßensiedlungen, doch lassen sich eine Reihe verschiedener Formen der Anordnung der einzelnen Dusun eines Dorfes ausmachen. Die Mehrzahl der Dörfer in der Untersuchungsregion weisen ein relativ kompaktes Gefüge der Verteilung ihrer Wohnbezirke auf. Bei Straßendörfern verlaufen die meisten Dusun-Grenzen entlang der Hauptstrasse. Darüber können ein oder zwei Dorfteile ohne direkten Anschluss an diese Strasse bestehen. Es gibt jedoch auch Ausnahmen, die zudem belegen, dass das Clan-basierte Prinzip der Gemeindegrenzziehung in der Lore-Lindu-Region nach 1979 nicht komplett ersetzt wurde. Ein Beispiel hierfür stellt das in Kap. 4.2.2 beschriebene Dorf Lempelero im Kecamatan Kulawi dar.

Die funktionale Siedlungsstruktur ist in der Lore-Lindu-Region kaum von der Tierhaltung beeinflusst. Das Dorf Lonebasa im Pipikoro-Gebiet (Kec. Kulawi) stellt hierbei eine besondere Ausnahme dar. Das gesamte bebaute Siedlungsareal (Wohnbezirke, landwirtschaftliche Felder) ist eingezäunt. Der Zugangsweg zu diesem Bereich ist durch ein Tor begrenzt. Schweine und Ziegen dürfen sich nur außerhalb des umzäunten Gebiets aufhalten. Zum einen ist dadurch ein Schutz der angebauten Feldpflanzen vor Verbiss und Vertritt gegeben, zum anderen können die Bewohner die Verbreitung von Krankheiten und Seuchen reduzieren. Diese Form der Umgrenzung der Wohnsiedlung geht v.a. auf den Einfluss von NGOs oder der Gesundheitsbehörde zurück. Daneben gab es traditionell aber schon mit Schutzbefestigungen versehene Dörfer, welche sich in erster Linie aus verteidigungsstrategischen Beweggründen entwickelten. Das in der Abb. 23 dargestellte Dorf Lamba im Napu-Tal (Kec. Lore Utara) zeigt eine solche Dorfumwallung zu Beginn des 20. Jh.

In den nachfolgenden Kapiteln (4.2.1 bis 4.2.3) werden drei unterschiedliche Aspekte der Ausgestaltung von Siedlungen näher beleuchtet. Das Fallbeispiel des Dorfes Maranatha im Kecamatan Sigi-Biromaru steht für staatlich geplante Siedlungen im Rahmen der vom DepSos und anderen Behörden durchgeführten lokalen Umsiedlungsprogrammen ab den 1960er Jahren. Am Beispiel der Dörfer Lempelero im Süden Kulawis und Bolapapu im Norden Kulawis wird aufgezeigt, dass manche Dörfer in der Lore-Lindu-Region eine stark gestreute Struktur ihrer Siedlungseinheiten aufweisen. Dabei wird u.a. die Problematik aufgegriffen, welche sich aus der post-kolonialen Neustrukturierung der dörflichen Verwaltungsgrenzen ergibt. Das dritte Beispiel, Watumaeta, ein Dorf im Norden des Kecamatan Lore Utara, steht für ein unter kolonialer Ägide gegründetes Dorf, bei welchem besonders dem Wüst-

\section{Pazifik Forum Bd. 12}


fallen ursprünglicher Siedlungsgebiete und der Ausdehnung des in Kultur genommenen Dorfterritoriums ein forschungsthematischer Platz eingeräumt wird.

\subsubsection{Fallbeispiel Maranatha}

Die lokale Transmigrationssiedlung Maranatha steht beispielhaft für eine geplante Siedlung. Maranatha ist das Ergebnis einer ab 1969 von staatlichen Stellen geleiteten und mit Unterstützung der Heilsarmee durchgeführten lokalen Umsiedlung der Bevölkerung mehrerer Siedlungen in den westlichen Randgebirgen des Palu-Tals (vgl. Kap. 3.4.1). Maranatha besteht aus sechs Dorfteilen, in welchen die Bewohner jeweils eines oder mehrerer der ehemaligen Herkunftsdörfer leben (vgl. Abb. 36). Ein Blick auf die ursprüngliche Planungskarte Maranathas zeigt die deutliche Planstruktur des Dorfes auf (vgl. Abb. 15). Noch deutlicher wird die Reissbrettstruktur derartiger lokaler Transmigrationssiedlungen am Beispiel des Ende der 1970er Jahre entstandenen Dorfes Kamarora in Palolo (vgl. Abb. 28).

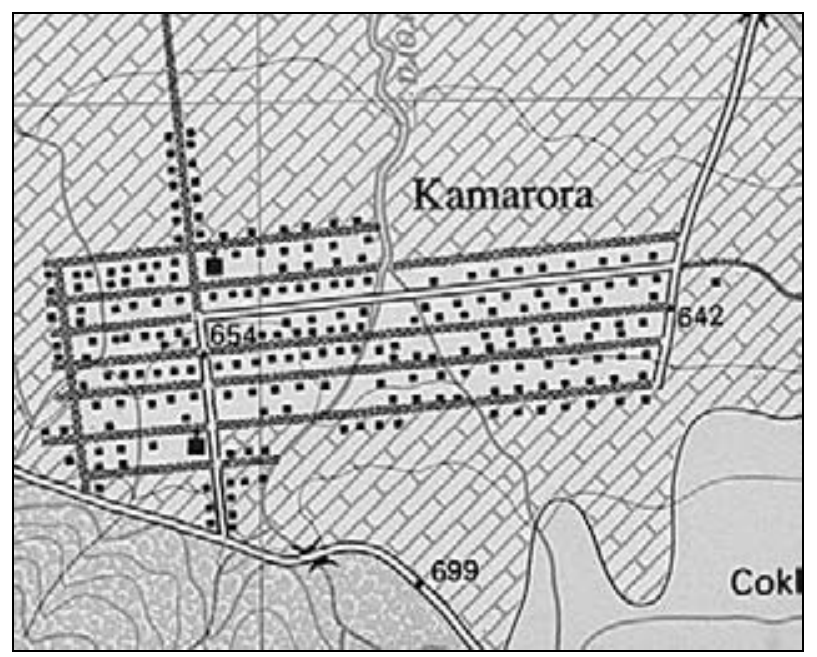

Abb. 28 Die Transmigrationssiedlung Kamarora

(Quelle: Auschnitt aus dem Kartenblatt 2114-43 der Peta

Rupabumi (TK 50), hrsg. von BAKOSURTANAL, Bogor)

Maranatha entstand in einem Waldgebiet südöstlich von Bora. Da das Dorf bei seiner Gründung fernab der Strassen lag, bot es optimale Voraussetzungen für eine planmäßige Anlage des Siedlungsgebietes. Nachdem eine Verbindungsstrasse zur Hauptstrasse Palu-Kulawi fertiggestellt worden war, wurden die Bewohner Maranathas, die anfangs in einfachen Baracken wohnten, aufgefordert sich entlang der neuen Dorfstrasse anzusiedeln. Das Zentrum von Maranatha bildet der Marktplatz, welcher für die gesamte Region v.a. durch den angegliederten Viehmarkt eine große 
Bedeutung erhält. Von dort aus erreicht man die verschiedenen Dorfteile entlang der beiden in Nord-Süd- und West-Ost-Richtung gelegenen Hauptstrassen des Dorfes. Aus der Planstruktur fällt allein der Dusun 6 (Lampio) heraus, welcher sich in ca. $2 \mathrm{~km}$ Entfernung von der Hauptsiedlung befindet. Maranatha unterscheidet sich neben der planmäßigen Anlage des Ortes auch von den in der Untersuchungsregion mehrheitlich vorhandenen Siedlungen mit Straßendorfcharakter.

\subsubsection{Fallbeispiele Lempelero und Bolapapu}

Nicht alle der in der Untersuchungsregion vorhandenen Dörfer weisen ein in sich geschlossenes Siedlungsbild auf. Das Dorf Lempelero, welches auf eine bewegte Siedlungsgeschichte zurückblicken kann, ist ein Beispiel dafür (vgl. Abb. 29). Folgt man der Hauptstrasse von Norden nach Süden durch das Kecamatan Kulawi, erreicht man als letzten Ort Lempelero. Nach dem Ortsschild befinden sich links und rechts entlang der Asphaltstrasse Häuser. Erst wenn man das Haus des Dorfbürgermeisters aufsucht, welches sich oberhalb der Strasse am Hang befindet und über einen unasphaltierten Weg zu erreichen ist, bemerkt man, dass sich das Wohngebiet Lempeleros entlang dieses Weges noch weiter den Hang hinauf erstreckt. Dort, nahe dem Fußballplatz des Ortes, befindet man sich in dem am frühesten besiedelten Gebiet Lemepleros. Die alteingesessenen Bewohner des Dorfes hatten sich im Jahr 1972 hier niedergelassen, nachdem ein verheerender Erdrutsch ihr ehemaliges Dorf Tompi Bangka zerstört hatte. Nahezu alle Gebäude des Ortes waren dabei in den nahen Lariang-Fluss gerissen worden.

Ihren Ursprung hatte die Bevölkerung von Tompi Bangka in dem neun Kilometer weiter westlich gelegenen Ort Tompi, das 1911 Kaudern (1925b: 38) zufolge 20 Häuser zählte. Um Verwechslungen mit dem Dorf Tompi Bugis, welches von der Lokalbevölkerung meist auch nur Tompi genannt wird, zu vermeiden, wird der Ort ihrer Vorfahren von der Lokalbevölkerung Lempeleros als Tompi Tua (altes Tompi) bezeichnet. Bis 1969 siedelten die Bewohner von Tompi Tua nach Tompi Bangka um, nachdem sich in dem alten Dorf ein schwerer Erdrutsch ereignet hatte. Im Jahr 1960 hielten sich in Tompi Bangka ca. 60 Familien auf.

Nach dem Erdrutsch von 1972 bot die Regierung den Bewohnern von Tompi Bangka an, sich an dem Polara genannten Ort der heutigen Hauptsiedlung Lempeleros niederzulassen. Ursprünglich war kaum jemand aus Tompi Bangka gewillt, nach Polara zu ziehen. Nur die Drohung des angesehenen Bürgermeisters, andernfalls sein Amt niederzulegen, veranlasste schließlich ca. 80 Haushalte, das Pipikoro-Gebiet zu verlassen und sich im Gimpu-Tal anzusiedeln. Nicht alle Dorfbewohner folgten diesen Vorstellungen der Regierung und siedelte sich statt dessen in Wua und Wangka an, deren Territorien auch zum Adat-Land von Tompi Tua gehörten und wo sich bereits Ackerfelder dieser Menschen befanden. Die Siedlung Wua, die Tompi Bangka räumlich am nächsten liegt, ist von der Ortschaft Kaha an der breiten Schotterstrasse, die von Tompi Bugis aus in Richtung Pipikoro führt, über einen 
kleinen, ca. zwei Kilometer langen Weg erreichbar. Etwa 25 Familien entschieden sich nach dem Erdrutsch für Wua als neuen Wohnort. Muu, welches ebenfalls dem Adat-Territorium von Tompi Tua zugerechnet wird, wurde von den Bewohnern Tompi Bangkas zuerst nicht als Zielort gewählt, da seine Lage direkt am Lariang die von dem Erdrutsch traumatisierten Menschen davon abhielt. Erst in den 1990er Jahren erfolgte die Migration eines Teils der Bevölkerung nach Muu, das durch die LKW-taugliche Schotterstrasse eine günstigere Verkehrs- und dadurch bessere Marktanbindung versprach.

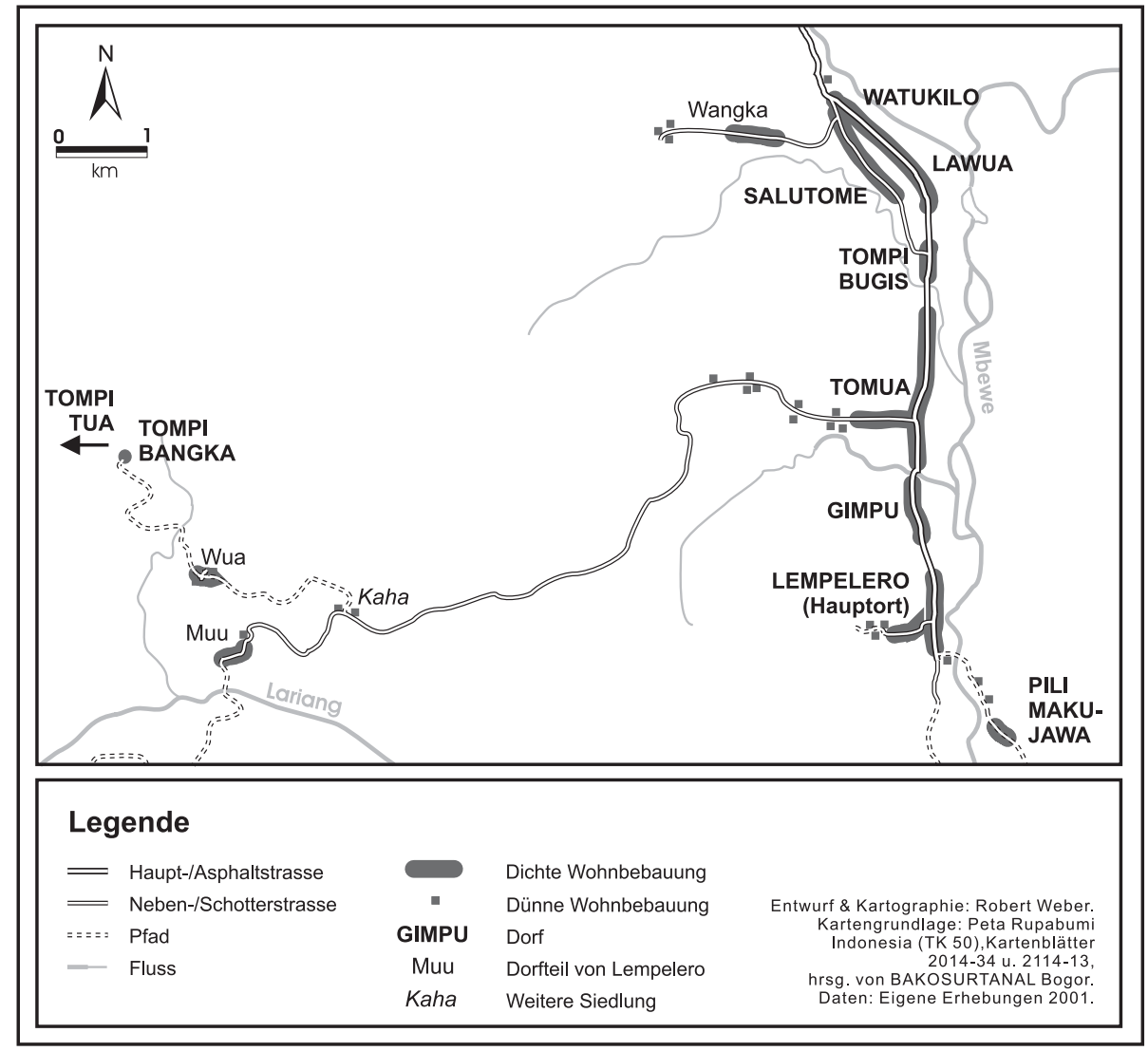

Abb. 29 Räumliche Verteilung der einzelnen Dorfteile von Lempelero sowie der Dörfer Tompi Tua und Tompi Bangka 
Die Siedlung Wangka befindet sich am Hang westlich des Dorfes Salutome ${ }^{101}$, in einer Entfernung von ca. $6 \mathrm{~km}$ zur Hauptsiedlung Lempeleros, und ist von der Asphaltstrasse Palu-Kulawi über einen kleinen, per Auto befahrbaren Weg erreichbar. Der Ort ist verwaltungstechnisch zweigeteilt. Dem einen Teil steht der Kepala Dusun (Dorfteilchef) von Lempelero vor, dem anderen der von Mataue, einem Nachbardorf von Bolapapu. Zu keinem der Mutterdörfer besteht eine räumliche Nähe, doch steuertechnisch wird die Bevölkerung entweder Lempelero oder Mataue zugerechnet. Anfangs befanden sich in Wangka Familien aus Mataue und Bolapapu, welche im Rahmen eines lokalen Umsiedlungsprojekts angesiedelt wurden. Bis heute bestehen unter der Bevölkerung sowie von Seiten der Lokal- und Regionalpolitiker unterschiedliche Planungen bzw. Wünsche, wie mit dieser verwaltungstechnischen Konstellation umgegangen werden soll. Die vier Hauptlösungsansätze schlagen die komplette Zuordnung Wangkas zu Lempelero, zu Mataue oder zu Salutome bzw. die Gründung eines eigenständigen Dorfes Wangka vor. Unter der Bevölkerung, welche sich Lempelero zugehörig empfindet, wird v.a. der erste Ansatz favorisiert. Als Argumentation wird dabei sehr deutlich die traditionelle Verbindung zu den gemeinsamen Vorfahren mit der lokalen Bevölkerung Lempeleros hervorgehoben.

Im Hauptort Lempeleros siedelte die Bevölkerung anfangs am Hang in der Nähe des heutigen Fußballplatzes. Nach dem Ausbau der Hauptstrasse von einem Pferdepfad auf Straßenbreite, die das Befahren mit dem Auto ermöglichte, verlegten im Jahr 1979 die ersten beiden Familien ihre Häuser an diese Strasse. Der Ausbau wurde v.a. von einem Rattanhändler aus Bolapapu vorangetrieben, um den Abtransport dieses Waldprodukts zu erleichtern. Bis 1982 folgten alle anderen Haushalte. Erst 1990, als das Gebiet entlang der Strasse kaum noch Platz bot, wurden wieder erste Häuser am ursprünglichen Siedlungsort errichtet.

Mit Bolapapu, dem Hauptort des Kec. Kulawi, kann ein weiteres Beispiel dafür aufgezeigt werden, dass nicht alle Dörfer der Untersuchungsregion über ein geschlossenes Siedlungsbild verfügen. Übersetzt bedeutet Bolapapu „verbranntes Dorf“. Ältere Bewohner des Ortes berichten, dass die Bezeichnung des Ortes, welcher zuvor den Namen „Bola“ (Dorf) trug, von einer Auseinandersetzung mit Bewohnern des Lindu Sees zu vorkolonialer Zeit herrühren. Ein Bewohner von Bola soll eine Lindu-Frau entführt haben. Daraufhin zogen Mitglieder des Clans der Frau in die Kulawi-Ebene und brannten die Häuser des Ortes Bola nieder (vgl. auch

101 Salutome, wie auch das Dorf Watukilo, wurde in der Folge des DI/TII-Konflikts gegründet, als zahlreiche Rampi- und Seko-Familien aus Südsulawesi nach Kulawi geflüchtet waren. Salutome existierte zuvor bereits als Ort an der Hauptstrasse Palu-Kulawi auf der Höhe von Toro. Die Regierung ordnete allerdings die Umsiedlung an die heutige Stelle an, da im Gebiet des urspünglichen Ortes nicht ausreichend flaches Land für die Nassreisbewirtschaftung zur Vefügung stand. 
Kaudern 1941: 106f, der das Ereignis auf die 1850er Jahre datiert). ${ }^{102}$ Das Dorf besteht aus fünf Dorfteilen (vgl. Abb. 30). ${ }^{103}$ Dusun 1 und 2 bilden den Hauptort des Dorfes und befinden sich in dem, im Vergleich zu Palolo, Napu oder auch Gimpu, relativ kleinen Kulawi-Tal. Dusun 1 bildet das politische, wirtschaftliche (Handel) und soziale (Wohngebiet der Nachkommen der „Aristrokatenschicht") Zentrum von Bolapapu. Ursprünglich siedelten seine lokalen Bewohner an den strategischen Berghängen, die heute im Süden an das Dorf Mataue angrenzen. Dort existierten von Norden nach Süden die Siedlungsgebiete Liohi (auf Höhe des heutigen Kantor Camat104), Lemo (im Bereich des heutigen staatlichen Gästehauses; Pasanggrahan), Panapa, Bola(papu) (dort befand sich das $l o b 0^{105}$ ) und Panua. In Leo, Bola(papu) und Panua leben auch heute noch einige Menschen, während in den 1960er Jahren die letzten Bewohner Panapa verließen. Heute findet man dort nur noch landwirtschaftliche Felder. Grubauer erwähnt in seinen Aufzeichnungen über eine von ihm im Jahr 1911 durchgeführten Reise Lemo als den Hauptort des KulawiTals:

„Nachdem wir so ziemlich die ganze Talbreite hinter uns hatten, sahen wir auch das Hauptdorf Lemo hoch auf einem Hügel vor uns liegen und dicht dabei, auf glücklich gewählter Stelle mit freiem Ausblick auf das ganze Talgebiet das Gouvernements-Unterkunftshaus. In nicht gerade präsentabler Toilette, aber in bester Laune bielt ich meinen Einzug in das freundliche Logis. Auch meine Soldaten waren in gehobener Stimmung; befand sich doch in Lemo ein ständiges Kommando von 20 Mann, dessen Kaserne in geringer Entfernung im Tale am O-Flusse lag." (1913: 550).

Das Zentrum von Dusun 1 bildet die Hauptstraße, an der sich ab den 1920er Jahren erst chinesische, später auch arabische und Bugis-Händler angesiedelt haben. Westlich der Straße befanden sich bis in die 1970er Jahre noch ausgedehnte Nassreisfelder. Doch nach einer verheerenden Flut wurden die Felder aufgegeben. Nach und nach wurden dort Wohnhäuser errichtet. Vor der Grenze zwischen Dusun 1 und 2 befinden sich noch die Gebiete Kamunji und Pobia.

102 Im Gegensatz dazu berichten Kruyt (1938, Bd. 1: 185f) und Aragon (2000b: 93), dass Krieger der Tobaku-Region (Pipikoro) für den Überfall auf Bola verantwortlich gewesen seien. Acciaioli (2002) spricht in bezug auf den Brand von Bola von einer der berühmtesten Legenden der Region, zu welcher vermutlich noch zahlreiche weitere Versionen in der lokalen Narrative existieren.

103 Im Jahr 2003 wurden die Dusun 4 und 5 zu einem eigenständigen Dorf mit Namen Namo zusammengeführt.

104 Das Kantor Camat ist das Gebäude der Landkreisverwaltung.

105 Traditionelles Versammlungshaus, in dem sich maradika und totua ngata zur Beratung und Rechtsprechung trafen. In Toro befand sich bis zum Beginn des 21. Jahrhunderts das einzige im alten Stil erhaltene lobo Kulawis. Seit 2003 werden auch in anderen Dörfern Kulawis mit Unterstützung von CSIADCP (Central Sulawesi Integrated Area Development and Conservation Project) die traditionellen Gebäude wiederaufgebaut. 


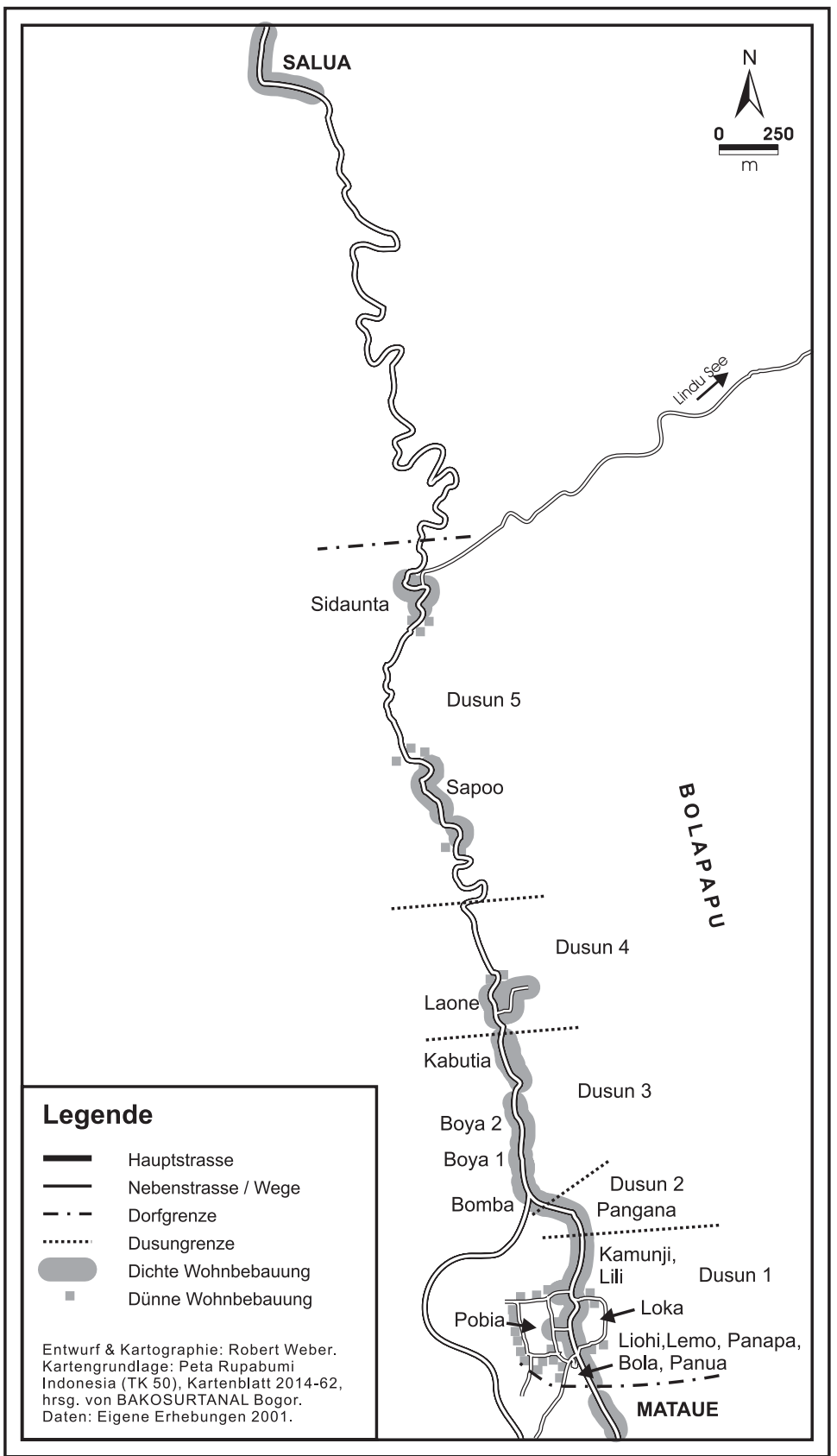

Abb. 30 Das Dorf Bolapapu und die räumliche Verteilung seiner Dorfteile 2001 
Das Wohngebiet von Dusun 1 entlang der Dorfhauptstrasse geht ohne Unterbrechung in das des Dusun 2 über. Auch hier befinden sich in den Gebieten Lili und Pangana zahlreichen Nachfahren der ehemaligen „aristokratischen“ Klassen der maradika und totua ngata (vgl. den nachfolgenden Exkurs zur soziopolitischen Gesellschaftsstratifikation in Kulawi).

Je weiter man sich über Bomba nach Boya 1, Boya 2 und Kabutia (bilden zusammen den Dusun 3) nach Laone (Dusun 4), Sapoo und Sidaunta (beide Dusun 5) entlang der Hauptstrasse Palu-Kulawi nach Norden bewegt, desto größer werden die Abstände zwischen den einzelnen bebauten Gebieten. Sidaunta ist ca. acht Kilometer vom Zentrum von Dusun 1 entfernt.

\section{Exkurs: Traditionelle soziopolitische Stratifikation der Gesellschaft in Kulawi}

Die traditionelle Gesellschaft in der Region Kulawi kennt drei Hauptklassen. Dies sind die „Adeligen“, 106 die Freien und die Sklaven (vgl. Abb. 31). Die Gruppe der „Adeligen“ setzt sich aus den maradika, totua ngata und tadulako (Kriegshelden) zusammen. Letztere sind die Ratgeber des bzw. der raja, welcher oder welche aus der Gruppe der maradika oder totua ngata bzw. tadulako entstammt. ${ }^{107}$ Die größte Bevölkerungsgruppe stellen die todea, die sog. Freien. Auf der untersten Ebene der Gesellschaft stehen die batua, ${ }^{108}$ die Unfreien bzw. Sklaven. Diese sind in der Regel Kriegsgefangene und den Familien der „Adels"schicht unterworfen. Der jeweilige Status in der Gesellschaftsordnung wurde in erster Linie vererbt. Es gab zwei Möglichkeiten, seinen Gruppenstatus zu verändern. Erstens konnte man unfreiwillig durch die bereits erwähnte Gefangennahme während kriegerischer Auseinandersetzungen auf den Sklavenstatus absinken. Zweitens konnte man durch Heirat in eine höhere Klasse aufsteigen. Die Heirat zwischen Sklaven und „Adeligen“ war aber ausgeschlossen. Auch Ehen zwischen todea und batua kamen kaum vor, da die Person aus der Gruppe der Unfreien mit der Heirat automatisch in die Gruppe der Freien aufgestiegen wäre, was einen unzulässigen Aufstieg bedeutet hätte. Generell kam es bis zur offiziellen Abschaffung dieses Klassensystems im Jahr 1962 nur sehr selten zu Eheschließungen zwischen Mitgliedern unterschiedlicher sozialer Statusgruppen. Der Mann einer höheren Statusgruppe konnte problemloser eine Frau niederen Status heiraten. Einem todea-Mann gelang es allerdings nicht, eine ,adelige“ Frau zu ehelichen. Die niederländischen Kolonialherren ließen das traditionelle soziopolitische Gesellschaftssystem weitgehend unangetastet (vgl. Abb. 32).

106 Mit „Adelige“ bzw. „Aristokraten“ seien die Personen bezeichnet, welche traditionell der obersten sozialen Schicht einer Dorfgemeinschaft bzw. einer ethnischen Gruppe, den sog. maradika, angehören. Sie werden als die Gründer bzw. deren Nachkommen der Gemeinschaft angesehen. Nach Kruyt (1938, Bd. 1: 500) wurde maradika durch die Bugis-Sprache vom Sanskrit-Begriff maharaddhika abgeleitet, welcher sich auf Personen bezieht, die von bestimmten sozialen Lasten befreit sind.

107 Sowohl in Kulawi als auch in Lore gab es auch weibliche raja. Kruyt (1908: 1311) verweist auf den großen Einfluss, den Frauen im Napu-Tal (besonders im Bereich der Landnutzung) traditionell hatten, wodurch es auch nicht selten war, dass Frauen hohe soziopolitische Positionen bekleideten.

108 In Toro ist die Bezeichnung budak für Sklaven verbreitet. 


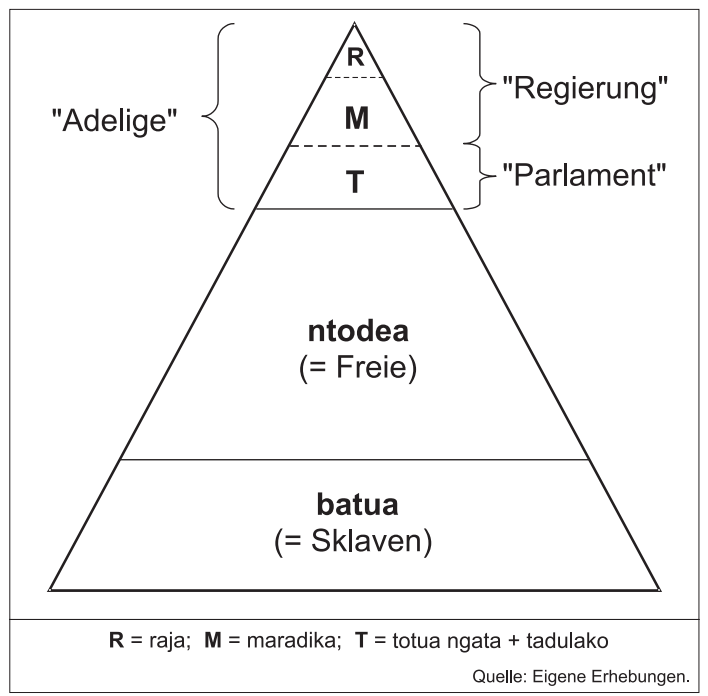

Abb. 31 Soziopolitische Stratifikation der Gesellschaft im präkolonialen Kulawi

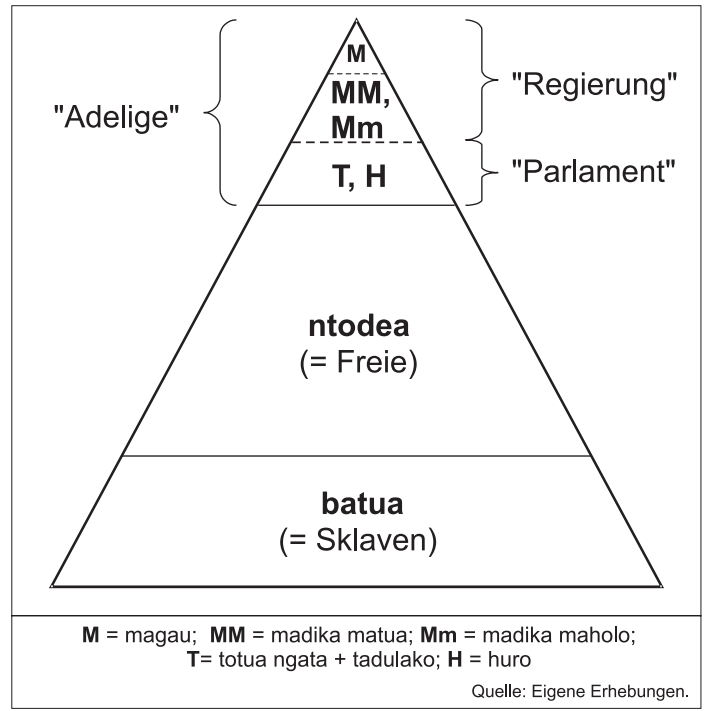

Abb. 32 Soziopolitische Stratifikation der Gesellschaft im kolonialen Kulawi 
Sie änderten v.a. die Bezeichnung raja in magau um. Eine weitere Modifikation war die Einführung der Positionen madika matua und madika mabolo. Während erstere Person das nördliche Kulawi regierte, besaß die zweite Macht über das südliche Kulawi, einschließlich der Pipikoro-Region. ${ }^{109}$ Obwohl in den 1960er Jahren offiziell abgeschafft, besteht die Gesellschaftsordnung in Kulawi bis heute latent weiter fort. Alle Mitglieder einer lokalen Dorfgemeinschaft kennen ihren eigenen traditionellen Status und den ihrer Mitbewohner. Die Felduntersuchungen haben jedoch ergeben, dass nur sehr wenige Menschen bereit sind, über dieses Thema offen zu sprechen. Selbst lokale Mitglieder einer Dorfgemeinschaft stoßen bei Nachforschungen in diesem Bereich meist auf eine Mauer des Schweigens, wie Pak Jakob für das Dorf Bolapapu bestätigt, der sich selbst seit einigen Jahren mit der Lokalgeschichte und insbesondere mit der traditionellen Sozialstruktur beschäftigt.

Das Dorf Bolapapu mit seinen fünf Dorfteilen stellt ein Verwaltungskonstrukt dar und kann nicht auf gemeinsame historische Wurzeln zurückgeführt werden. Bis 1979 zählte sogar das heutige Dorf Salua, welches sich ca. 4 km nördlich von Sidaunta befindet, als Dusun 6 noch zum Dorf Bolapapu. Im Rahmen der administrativen Neuregelungen von 1979 erhielt Salua den Status eines eigenständigen Dorfes. Auch in bezug auf die Religionszugehörigkeit der Bevölkerung stellt Bolapapu keine Einheit dar. Während vor allem die Bevölkerung von Dusun 1 und 2 mehrheitlich christlich missioniert wurde, sind die Bewohner von Laone, Sapoo und Sidaunta stark muslimisch geprägt. In Laone machen Muslime 95\% der Gesamtbevölkerung aus. Der Hintergrund hierfür ist die Missionstätigkeit eines arabischen Händlers, der während der Kolonialzeit nach Laone kam. Unter der Herrschaft der Niederländer wurden die Bewohner der Dusun 3 bis 5 aus den höher gelegenen Bergregionen in das sich nach Norden hin verjüngende Tal umgesiedelt. Die Vorfahren der heutigen lokalen Bevölkerung von Dusun 4 und 5 lebten ursprünglich in Namo, einem Siedlungsgebiet etwa $1 \mathrm{~km}$ westlich von Laone. Die Tatsache, dass seit 2003 diese beiden Dusun wieder den Dorfnamen Namo tragen, bestätigt das Konstrukt des Dorfes Bolapapu.

Im Fall von Laone zwangen die Niederländer die Bewohner des Siedlungsgebiets Namo sich im Tal niederzulassen, wo sich bald der Ort Tolumanu gründete. ${ }^{110}$ (vgl. Abb. 33) Tolumanu bedeutet „Hühnerei“, wodurch der Siedlung eine prosperierende Zukunft bescheinigt werden sollte. Etwa 30 Familien bewohnten dieses Gebiet östlich des heutigen Laone zu jener Zeit.

109 In der kolonialzeitlichen Region Lore im Osten des Untersuchungsgebiets baute sich die Gesellschaft folgendermaßen auf: magau, biti magau (Stellvertreter des magau), galara (Pendant zu totua ngata), kepala kampung (Dorfchef), masyarakat (einfache Bevölkerung), budak (Sklaven). Es gab drei biti magau, von welchen je einer über die Gebiete Bada, Besoa und Napu herrschte.

110 Neben Tolumanu wurde die Bevölkerung von Namo auf Veranlassung der Kolonialherren auch nach Sapoo und Sidaunta umgesiedelt. Namo war ursprünglich ein Schutzposten der tadulako, welche von dort aus Kulawi nach Norden gegen feindliche Eindringlinge absicherten. 


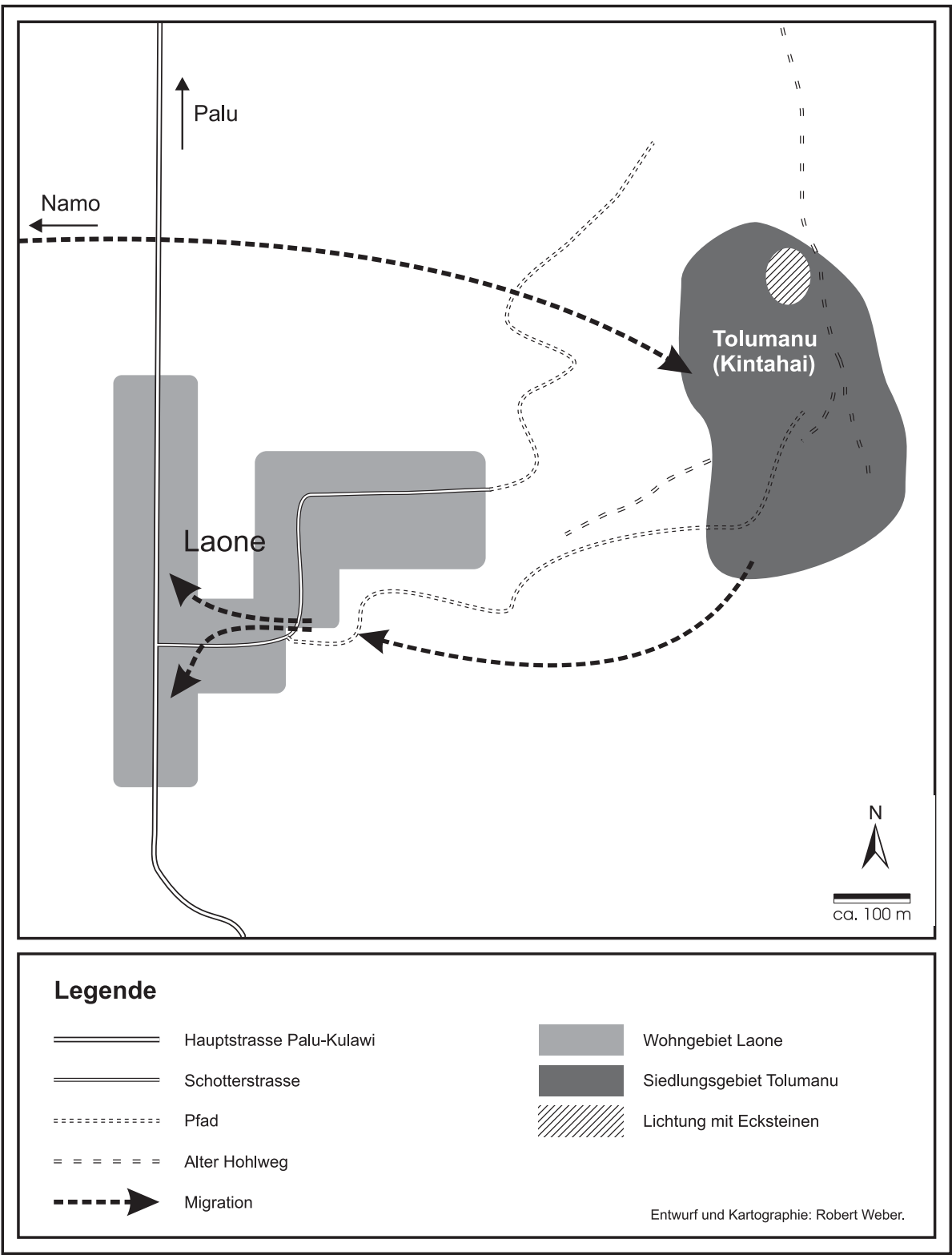

Abb. 33 Kartenskizze von Laone und seiner Besiedlung 
Noch heute zeugen einige Ecksteine, ${ }^{111}$ auf welchen die Tragebalken der Häuser lagerten, und Reste von Hohlwegen von der damaligen Siedlung. ${ }^{112}$ Etwa 20 Jahre, nachdem die ersten Familien nach Tolumanu gekommen waren, verlagerte sich das Wohngebiet näher in Richtung Hauptstrasse. Anfangs bildete die 1959 gebaute Grundschule das neue Zentrum des Ortes, der bereits vor den kolonialen Umsiedlungsmaßnahmen Laone genannt wurde. Aus Laone, das mit „Gras“ zu übersetzen ist, beschafften die Bewohner des alten Namo bereits Gras als Futter für ihre Büffel und Kühe. Nachdem die Menschen Tolumanu verlassen hatten, gaben sie dem Ort den Namen Kintahai, ,altes Dorf“" (kinta = „Dorf“, bai = „alt, ehemalig“). Mit dem Ausbau der Hauptstrasse Palu-Kulawi und der zunehmenden Bedeutung des Handels von landwirtschaftlichen Exportgütern wie Kaffee und Kakao verlagerten mehr und mehr Bewohner ihre Häuser an diese Strasse.

Die Beispiele Lempelero und Bolapapu stellen siedlungsgeographische Ausnahmen in der Lore-Lindu-Region vor. Sie zeigen, dass sich traditionelle Gruppenbezüge heute nicht generell in einem geschlossenen Siedlungsbild wiederspiegeln müssen (Bsp. Lempelero) und dass die Lokalbevölkerung der von den übergeordneten Regierungsebenen definierten Dörfer sich nicht zwingend auf gemeinsame Wurzeln zurückführen lässt (Bsp. Bolapapu).

\subsubsection{Fallbeispiel Watumaeta}

Watumaeta zählte im Jahr 2001 insgesamt 1.449 Einwohner in 362 Haushalten $(\mathrm{HH})$. In Dusun 1 befanden sich 337 Personen $(82 \mathrm{HH})$, in Dusun 2.664 Personen (173 HH) und in Dusun 3.449 Personen (107 HH) (eigene Erhebungen). Ab 1929 wurde das Territorium des heutigen Watumaeta von Kulawi-Familien und lokalen Familien besiedelt. Die lokalen Familien stammten ursprünglich aus den Nachbardörfern Wuasa und Alitupu sowie aus dem ehemaligen Siedlungsgebiet Powanuanga. Bis zu jener Zeit diente das Gebiet als Jagdgrund für die Bevölkerung von Wuasa und Tawaelia (heute: Sedoa). Im Jahr 1938 wurde das Dorf Watumaeta gegründet. Der erste Wohnkomplex mit sechs Häusern entstand Anfang der 1930er Jahre östlich des ehemaligen Verbindungsweges nach Wuasa (vgl. Abb. 34).

In Watumaeta hatten sich bereits während der ersten Jahre des Bestehens Bugis und Toraja als regionale Migranten niedergelassen, welche in Nachbarschaft zur lokalen Dorfbevölkerung wohnten.

111 Diese Konstruktionsweise soll sich besonders in Hinblick auf die in Zentralsulawesi häufig vorkommenden, schwachen Erdstöße als erdbebensicher erwiesen haben.

112 Einer dieser Wege führt weiter bis zum Lindu See. Er wurde auf Verlangen der Niederländer angelegt. 


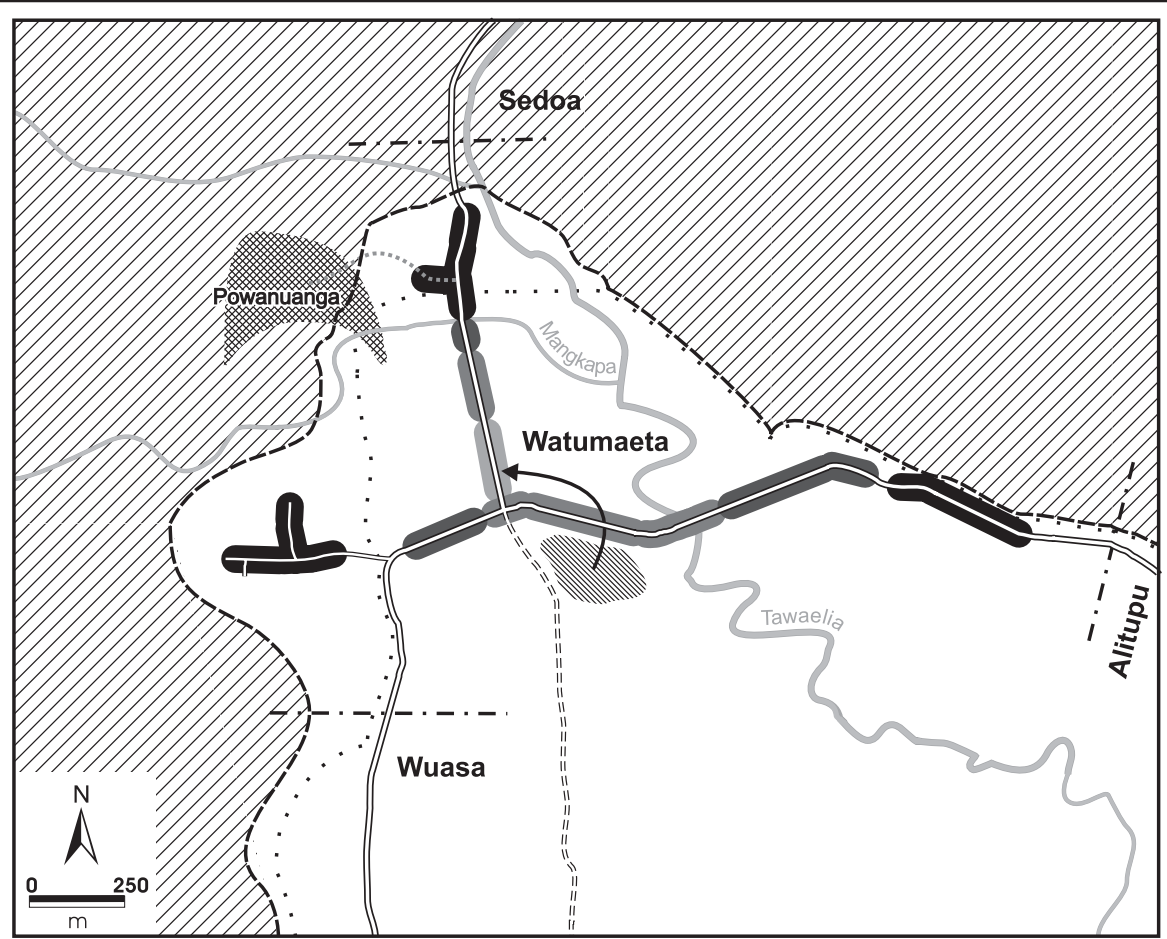

\section{Legende}

$=$ Asphaltstrasse

Unbefestigte Strasse

$====$ Ehem. Weg von Watumaeta nach Wuasa

nn: Pfad

- - Dorfgrenze

Fluss

. . Ungefähre Waldgrenze 1990

- - Ungefähre Waldgrenze 2000

H//, Ungefähres Waldgebiet 2000
Räumliche Ausbreitung der Wohngebiete:

Erste Häuser (ca. 1930)

ca. $1938-1942$

ca. $1950-1990$ (Verdichtung ab ca. 1980)

ca. 1991-1995

ca. ab 1996

$\longrightarrow \quad$ Verlagerung des Wohngebiets (ab 1938)

Entwurf \& Kartographie: Robert Weber Kartengrundlage: Peta Rupabumi Indonesia (TK 50), Kartenblatt 2014-62, hrsg. von BAKOSURTANAL Bogor. Daten: Eigene Erhebungen 2001.

Abb. 34 Raum-zeitliche Ausdehnung der Wohnareale in Watumaeta 
Während die Bugis dem Damar-Handel nachgingen ${ }^{113}$, waren die Toraja die ersten, die in Watumaeta sawab-Felder anlegten und zusätzlich die lokale Bevölkerung im Tausch gegen Wasserbüffel in der Verwendung von Sägen zum Fällen von Bäumen unterrichteten. Beide Gruppen verließen Watumaeta in den 1950er Jahren. Für die Bugis lag der Grund ihrer Abwanderung, nach Poso bzw. zurück nach Südsulawesi, im Rückgang des Damar-Handels. Für die Toraja kann Abdulkadir-Sunito (2002) zufolge angenommen werden, dass der Aufenthalt in Watumaeta allein der Kapitalakkumulation in Form der für die Toraja sehr bedeutenden Wasserbüffel diente.

Ab 1940 verlagerte die lokale Bevölkerung ihre Häuser an den Weg, der in Richtung Norden aus Watumaeta herausführt. Westlich davon befanden sich die ersten Nassreisfelder des Dorfes, während die mit Reis, Mais und Cassava bestellten Trockenfelder sich nördlich und südlich des ersten Wohnkomplexes befanden. Mit zunehmender Entfernung von der Weggabelung in Richtung Mangkapa-Fluss vergrößerte sich auch der Abstand zwischen den Häusern, da die Menschen dort noch in Hütten auf ihren sawah-Feldern wohnten. Ein Teil der Familien siedete ab 1940 zudem in die Nähe des Tawaelia-Flusses um. Im Laufe der Jahre näherten sich die 1940 entstandenen Wohnbereiche räumlich an, bis schließlich ein geschlossener Wohnkomplex in L-Form mit einer Nord- und einer Ostachse entstand. Erst nach dem Umbau der Straßenverbindung zwischen Watumaeta und Wuasa im Jahr 1982 erfuhr das Dorf schließlich eine Ausdehnung des Wohngebiets nach Westen hin. Die neu geschaffene Strasse sollte die im Bereich der geradlinigen Wegverbindung nach Wuasa befindlichen sumpfigen Böden umgehen. Während sich im Norden der neuen Strasse bereits sawah- und im Süden Trockenfelder befanden, wurde 1994 entlang der Strasse das erste Haus errichtet, dem bald weitere folgten. Im Jahr 1984 wurde die Straßenverbindung nach Alitupu im Osten verbessert, woraufhin ab 1992 das erste Haus östlich des Tawaelia-Flusses von Bugis-Migranten gebaut wurde.

Der Ausbau der Verbindungsstrasse nach Palolo im Jahr 1982 war, wie bereits erwähnt, eine bedeutende Ursache für die Migration von Bugis und anderen Gruppen in das Kecamatan Lore Utara. Der Kakaoboom der 1990er Jahre führte schließlich zur Zuwanderung einer großen Zahl von Kakaobauern aus Südsulawesi. Die Folgen dieser Migration zeigen sich in der Ausdehnung der Wohnbezirke Watumaetas nach Osten hin, wo sich heute in erster Linie die Häuser von Migranten aus Südsulawesi und Java befinden. Nachdem sich im Laufe der 1990er Jahre das Wohnareal entlang der Strasse nach Alitupu mehr und mehr verdichtete und das Angebot an freien Landflächen abnahm, bildete sich westlich der Strasse nach Wuasa gegen Ende der 1990er Jahre ein weiteres Siedlungsgebiet, das von Bugis be-

113 Das Waldprodukt wurde von ihnen an chinesische Händler in Poso verkauft. 
wohnt und bewirtschaftet wird. Dieses Gebiet erstreckt sich mittlerweile hinter die offizielle Grenze des Lore-Lindu-Nationalparks.

Auch Powanuanga, das ehemalige Siedlungsgebiet eines Teils der Dorfgründer, liegt weit hinter der Nationalparksgrenze. Das Gebiet befindet sich am Hang der das Tal nach Westen hin begrenzenden Berge. Wie in Laone (Dusun 4 des Dorfes Bolapapu; vgl. Kap. 4.2.2) lassen sich auch in Powanuanga noch Relikte der ehemaligen Siedlungsaktivität erkennen (vgl. Abb. 35). Der Hang weist in diesem Bereich eine deutlich sichtbare Terrassierung auf, die zur Vereinfachung der Feldbestellung durchgeführt wurde. Ebenso befindet sich dort ein eingewachsener sog. lesung batu, ein Steinbottich, in dem der Spelz vom Reiskorn getrennt wird (vgl. Kap. 5.1). Seit den Jahren 2000/2001 wird das Gebiet zwischen Dorfstrasse und Powanuanga, auch über die Nationalparksgrenze hinweg landwirtschaftlich in Kultur genommen.

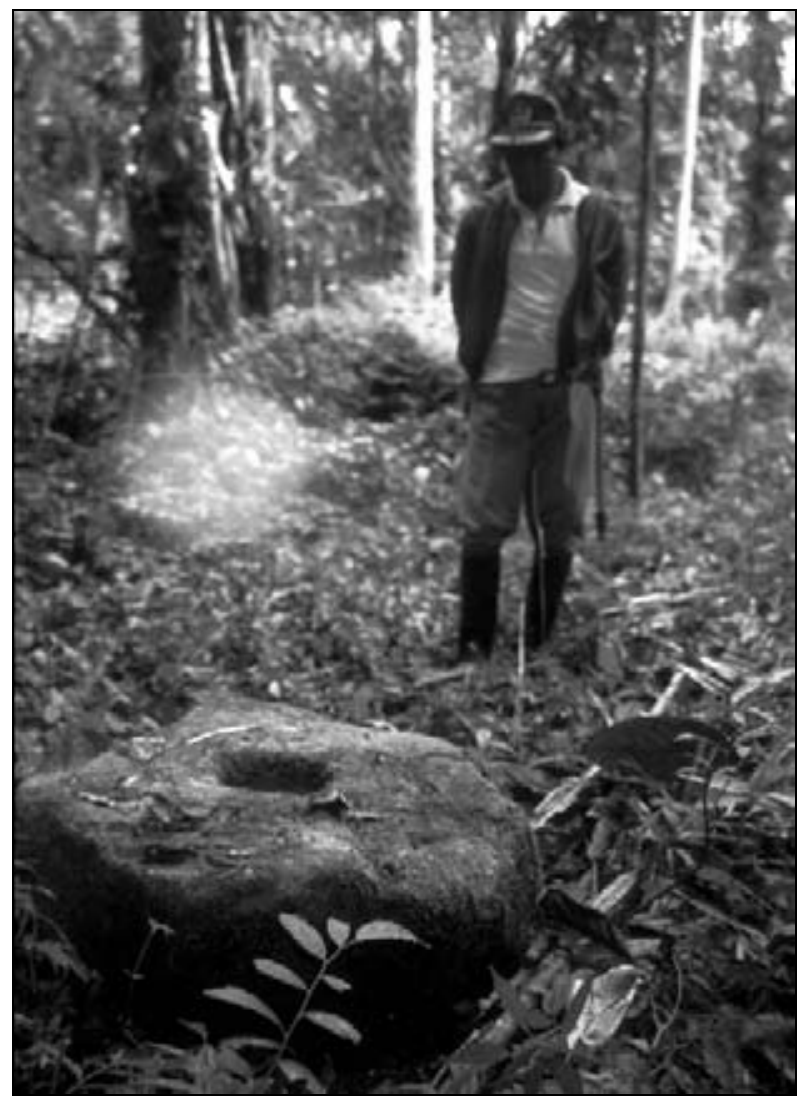

Abb. 35 Ein alter lesung batu in Powanuanga als Hinweis auf die ehemalige anthropogene Nutzung dieses Gebiets

(Quelle: Eigene Aufnahme, 2002) 


\subsection{Sozialräumliche Segregation}

Es sind i.d.R. Stadtlandschaften, auf die sich Forschungsarbeiten zur sozialräumlichen Segregation in Industrie- oder „Entwicklungsländern“ beziehen (vgl. u.a. Darden 1972; Dittmann 2004; Fischer \& Parnreiter 2002; Grunsven 1992; Harth 1997; Hershkovitz 1981; Schwedler 1985; Thong 1976). Bezogen auf Indonesien finden sich neben Arbeiten zur urbanen Segregation (vgl. z.B. Firman 2004) auch Untersuchungen zu Segregationsprozessen im ländlichen Raum. Diese stehen meist in Zusammenhang mit der indonesischen transmigrasi-Politik (vgl. Bubandt 1998; Hoey 2003; Hoppe \& Faust 2004).

Die vorliegende Arbeit untersucht hingegen die sozialräumlichen Segregationsprozesse auch unter Einbeziehung spontaner Migration auf der Ebene von Dörfern im ländlichen Raum der Lore-Lindu-Region. Die städtische Segregation in Palu, welche u.a. in bezug auf die Land-Stadt-Migration ein durchaus interessantes Forschungsfeld darstellt, wird hierbei nicht mit eingeschlossen.

\subsubsection{Allgemeine Überlegungen zum Verhaltensmuster sozialer Abgrenzung}

Segregation wird nach Leser definiert als „Prozeß der räumlichen Trennung und Abgrenzung von sozialen Gruppen gegeneinander, insbesondere innerbalb einer Siedlungseinheit, sowie der dadurch hervorgerufene Zustand." (1997: 769).114 Die Segregation beruht dabei auf gemeinsamen Merkmalen der segregierten Gruppe, welche zugleich die Unterscheidungsmerkmale zur übrigen Bevölkerung bilden (ebd.).

In der Lore-Lindu-Region ist Segregation besonders mit ethnischer und religiöser Zugehörigkeit der Bevölkerung verbunden. Während eines Aufenthalts in der Untersuchungsregion wird dies im Kontakt mit der Bevölkerung schnell deutlich. Bei einem Besuch in einer Familie kommt es kaum vor, dass die eben genannten Kategorien nicht angesprochen werden. Den Einstieg in diese Thematik soll das nachfolgende Beispielgespräch des Verfassers (V) und dessen indonesischen Assistenten (A) bei der ersten Kontaktaufnahme mit dem Oberhaupt (O) einer Gastfamilie erleichtern: 115

V/A: Guten Morgen, der Herr!

114 Bezüglich des Terminus „Segregation“ zeigt sich in der Literatur, ähnlich wie bei der Verwendung anderer Begriffe, dass er zwar oft Verwendung findet, jedoch nur selten klar definiert wird.

115 Zum besseren Verständnis sei erklärt, dass Kaili eine ethnische Gruppe sowie eine Sprache in der Untersuchungsregion ist. Diese lässt sich in mehrere dialektale Untergruppen teilen: z.B. Kaili-Da'a, Kaili-Ija oder die in diesem Beispiel erwähnte Gruppe Kaili-Ledo. Das Gespräch wurde vom Verfasser kurz danach in Form eines Gedächtnisprotokolls niedergeschrieben. Der indonesische Assistent steuerte die in der Lokalsprache Kaili-Ledo geführten Gesprächsteile bei. 
V: Wir wollen in diesem Dorf Forschung machen. Der Bürgermeister hat uns gesagt, dass wir bei Ihnen während dieser Zeit übernachten können.

O: $\quad$ Guten Morgen! Oh ja, kommen Sie doch herein! kommen Sie, kommen Sie!

( $V$ und $A$ treten ein.)

O: Nehmen Sie doch Platz!

(V und A nebmen Platz)

A/V: Danke!

O: $\quad$ Woher sind Sie? (an $V$ gerichtet)

V: $\quad$ Ich komme aus Deutschland.

O: Ah, Deutschland. Beckenbauer, Schumacher.

$\mathrm{V}: \quad J a$, genau.

O: $\quad$ Gut! Sind Sie Protestant?

V: $\quad$ Nein, ich bin Katholik.

O: Ah, Katholik. Ich bin Protestant, BK, Heilsarmee. Aber wir sind beide Christen. (lächelt und dentet auf ein Jesusbild an der Wand) Hier sind die meisten Christen. Und Sie? (an A gerichtet)

A: Ich bin Muslim.

O: Ah, Muslim. Ja, davon gibt es hier auch ein paar, Bugis und Javaner. Aber die meisten sind Christen. Sind sie Bugis?

A: Nein, ich bin Kaili.

O: Ah, wirklich? (blickt skeptisch) Ich dachte, Sie wären Bugis. Sehen aus wie ein Bugis. (lacht)

A: $\quad$ Nein, nein, ich bin Kaili.

O: Ja? Ich dachte, Sie wären Bugis.

A: (wechselt in die Kaili-Sprache) Nein, ich bin Kaili. Kaili-Ledo.

O: $\quad$ (wechselt auch in die Kaili-Sprache) Ah, Ledo. Sehr gut! Ich bin auch Kaili-Ledo. Woher kommen Sie?

A: Ich komme aus Boyaoge.

O: Oh, Boyaoge. Da wohnt ein Onkel von mir. Gayus. Kennen Sie den?

A: $\quad$ Ähm, nein, den kenne ich nicht. Ich war aber auch einige Jahre zum Studieren auf Java, in Bandung.

O: Ah ja, der wohnt da aber schon lange. Aber Sie sind in Boyaoge geboren? 
A: Ja, bin ich, war aber eben eine Zeit lang in Bandung.

O: Ja, ja. Sehr gut!

Ethnizität und Religionszugehörigkeit spielen in diesem Beispiel eine zentrale Rolle. Während der Forscher das Weltbild des Gastgebers vom christlichen Deutschen bestätigt, bedarf es in bezug auf den indonesischen Assistenten der gemeinsamen Lokalsprache, um das Wohlwollen des Gastgebers zu erhalten. Die Herkunft wird skeptisch hinterfragt und durch die Erwähnung des Onkels getestet. Bei diesen Mustern handelt es sich jedoch weder um Einzigartigkeiten der Lore-Lindu-Region, noch um eine Besonderheit der indonesischen Gesellschaft. Überträgt man dieses Gesprächsexempel beispielsweise auf Deutschland, kommt man schnell zu dem Schluss, dass es sich hierbei um universelle, gesellschaftliche Grundzüge handelt, welche je nach Prägung der Gesprächspartner unterschiedlich stark zur Geltung kommen. Diese beinhalten die Bildung von Wir-Gruppen und den Umgang mit Stereotypen. Eine Wir-Gruppe kann dabei durch ethnische (in übersteigerter Form auch rassistische) Klassifikationen definiert werden, ebenso auch durch religiöse, politische, ökonomische oder soziale Zuschreibungen. Mit Hilfe von Stereotypen werden Andere von Mitgliedern einer Wir-Gruppe zugeordnet. Diese Verhaltensweisen sind einerseits abhängig von der kulturellen Prägung einer Gesellschaft, andererseits aber - und das ist besonders entscheidend - auch von der jeweiligen Sozialisierung des einzelnen Individuums.

Es drängt sich nun die Frage auf, warum das Verhaltensmuster in dieser Arbeit dann überhaupt so ausführlich erwähnt wird. Die Antwort liegt in der Tatsache, dass es hier nicht darum geht, auf kulturrassistische Art das „,typisch Andere“ einer Region im Vergleich zu der eigenen Herkunftsregion zu ergründen. Bei tiefergehender Betrachtung wird nämlich deutlich, dass sich eine Vielzahl vermeintlich regionaloder gruppenspezifischer Verhaltensweisen über die jeweiligen räumlichen oder sozialen Grenzen hinweg sehr stark ähneln.116 Vielmehr sollen Ursachen und Gründe dafür gefunden werden, warum sich bestimmte Aspekte in einer Region so darstellen, wie sie es tun, und ob sich innerhalb der untersuchten Region Unterschiede diesbezüglich feststellen lassen.

116 Der Verfasser machte selbst die Erfahrung, dass er zu Beginn seines Feldaufenthalts in Zentralsulawesi viele Verhaltensweisen der lokalen Bevölkerung voreilig als „typisch indonesisch“ klassifizierte. Nach genauerer Reflexion kam er jedoch zu dem Schluss, dass sich oft die gleichen, oder zumindest sehr ähnliche Verhaltensmuster ebenso z.B. unter der deutschen Bevölkerung finden lassen. 


\subsubsection{Zur Entwicklung multi-ethnischer Dorfgemeinschaften in der Lore- Lindu-Region}

Mit der Zuwanderung in die Untersuchungsregion erhöhte sich auch die Vielfalt ethnischer Gruppen in den einzelnen Dörfern. Im Zuge staatlich geplanter oder spontaner, lokaler Migration erhöhte sich die Vielfalt lokaler ethnischer Gruppen in den Siedlungen. Neben den lokalen Gruppen wie Kaili, Kulawi und Napu, sowie deren Untergruppen, leben in der Lore-Lindu-Region gegenwärtig auch ethnische Gruppen aus anderen Regionen Sulawesis, z.B. Minahasa und Manado aus Nordsulawesi, Bugis, Toraja, Rampi oder Makassar aus Südsulawesi, sowie Poso, Pamona oder Luwuk aus Zentralsulawesi. Von anderen indonesischen Inseln kamen v.a. Javaner, Sundanesen und Balinesen in die Untersuchungsregion.

In der Lore-Lindu-Region lassen sich Dörfer mit weitgehend homogener ethnischer Bevölkerungsstruktur von jenen mit heterogener Struktur unterschiedlichen Grades unterscheiden. Generell kann man die Aussage treffen, dass mit der Verschlechterung des Zugangs zu den wirtschaftlichen Absatzmärkten die ethnische Homogenität der Dörfer zunimmt. Diese ökonomische Erklärung lässt sich im großen Maßstab in der Untersuchungsregion wiederfinden und hat angesichts der zunehmenden Bedeutung von cash crops in der Region auch eine gewisse Berechtigung. Die Untersuchungsdörfer Lawe und Lempelero im Kec. Kulawi weisen eine relativ hohe Bevölkerungshomogenität auf. Gleichzeitig befindet sich Lawe in der verkehrstechnisch gering erschlossenen Pipikoro-Region und Lempelero am südlichen Ende der Hauptstrasse von Palu nach Kulawi, deren baulicher Zustand sich in Richtung Lempelero zunehmend verschlechtert. Ein weitverbreitetes Argument für die Abwanderung aus Lawe und anderen Dörfern der Pipikoro-Region in die Nähe der Hauptstrasse Palu-Kulawi ist die unbefriedigende Transportsituation für marktfähige Agrarprodukte einerseits und für den Erwerb von in der Region nicht verfügbaren Gütern. Das ursprünglich als Untersuchungsdorf vorgesehene Rompo im Süden von Lore Utara zeichnet sich ebenfalls durch eine schlechte Verkehrsanbindung an die Marktzentren aus. Auch in diesem Dorf besteht nach AbdulkadirSunito (2002) nur eine geringe ethnische Heterogenität der Dorfbevölkerung. Das Beispiel des Dorfes Maranatha (Kec. Sigi-Biromaru), welches sehr gut an das Verkehrsnetz angeschlossen ist und nur ca. $20 \mathrm{~km}$ von der Provinzhauptstadt Palu entfernt liegt, zeichnet sich hingegen durch eine relativ geringe ethnische Heterogenität aus. Zwar ist in der Verteilung der Wohngebiete Maranathas deutlich zu erkennen, dass die Herkunftsdörfer der in dieses Dorf umgesiedelten Bevölkerung einen Einfluss auf die räumliche Vetreilung ihrer Wohnareale im Dorf hatte (vgl. Abb. 36). Alle diese Personen gehören jedoch der gleichen ethnischen Gruppe der Kaili an. Es passt somit nicht in das o.g. Erklärungsschema. Umgekehrt verhält es sich mit dem Vergleich der beiden Dörfer Sintuwu und Berdikari (jeweils im Kec. Palolo). Obwohl Berdikari deutlich bessere verkehrstechnische Voraussetzungen vorweisen kann als Sintuwu, findet man in beiden Dörfern nicht nur eine ethnisch heterogene Bevölkerungsstruktur vor, sondern zudem einen insgesamt hohen Anteil an 
Migranten. Bereits hier zeigt sich, dass allgemeingültige Erklärungsansätze nur bedingt geeignet sind, die Entwicklung multi-ethnischer Dorfgemeinschaften in der Lore-Lindu-Region zu erklären, auch wenn die Marktökonomie einen durchaus bedeutenden Einfluss hat. Je nach Migrationsgeschichte der Bewohner und den ökonomischen sowie soziopolitischen Rahmenbedingungen eines Dorfes entwickelten sich im Laufe der Zeit unterschiedliche Konstellationen der ethnischen Zusammensetzung von Dorfgemeinschaften.

Abhängig von der Größe, vom ökonomischen Status, von den sozialen und politischen Netzwerken sowie von den kulturellen Besonderheiten der ethnischen Gruppen entwickelte sich ferner in jeder Dorfgesellschaft eine besondere Ausprägung des community building und der Segregation. Im Folgenden werden diese Ausprägungen anhand der Untersuchungsdörfer analysiert. Erklärungsansätze für die individuelle Entwicklung multi-ethnischer Dorfgemeinschaften, welche über die rein ökonomische Betrachtungsweise hinausgehen, werden ebenfalls erst in dem folgenden Kapitel gegeben, da sie vielfach auch für die Segregationsentwicklung bedeutend sind.

\subsubsection{Segregationsmuster im Vergleich}

Vergleicht man die Untersuchungsdörfer, so fällt auf, dass eine mehr oder weniger stark ausgeprägte räumliche Segregation der Wohnbereiche einzelner ethnischer Gruppen sehr häufig ist. In Maranatha ist zwar keine ethnische, aber zumindest eine deutliche Segregation nach Herkunftsorten innerhalb der Gruppe der Kaili festzustellen (vgl. Abb. Abb. 36). Der Grund hierfür ist jedoch der Plancharakter dieses lokalen Umsiedlungsdorfes. Hier war es von der Regierung durchaus gewollt, dass die ehemaligen Sozialstrukturen der Dorfverbände beibehalten werden, um der umgesiedelten Bevölkerung die Eingewöhnung zu erleichtern (Lagarens 2002). Daneben hatten aber auch die Menschen selbst ein Interesse, in der Nähe der ihnen aus dem gemeinsamen Herkunftsort vertrauten Menschen zu wohnen. Die relativ wenigen regionalen und nationalen Migranten, v.a. Javaner, Toraja und Bugis, siedelten sich im Zentrum Maranathas an. Hierfür war die Nähe zum Marktplatz ein ausschlaggebender Grund. Neben dem Dorf Pandere ist die räumliche Segregation besonders in den Untersuchungsdörfern in Palolo und Lore Utara augenfällig. Doch auch in Bolapapu oder Lempelero ist eine, wenn auch nicht so ausgeprägte, Segregation erkennbar.

Grundsätzlich könnte man davon ausgehen, dass sich die lokalen Bevölkerungsgruppen, welche sich als erste in einem Dorf angesiedelt haben - die tuhan kampung, die "Herren des Dorfes", wie sie oft genannt werden - , im Zentrum des jeweiligen Dorfes befinden, während die zugewanderten Familien sukzessive die Dorfränder besiedeln. Dies setzt aber voraus, dass die Zuwanderung erst spät erfolgt und/oder das zentrale Territorium für die lokale Bevölkerung reserviert bleibt. Dieser Umstand trifft sehr deutlich auf Watumaeta zu, daneben auch auf Berdikari und 
Pandere. Nach der frühen Zuwanderung von Toraja und Bugis nach Watumaeta in den 1930er Jahren, welche sich im damaligen Dorfzentrum niedergelassen, in den 1950er Jahren den Ort jedoch wieder verlassen hatten (vgl. Kap. 4.2.3), konnte sich die lokale Bevölkerung bis in die 1980er Jahre im Zentrum Watumaetas ausbreiten.

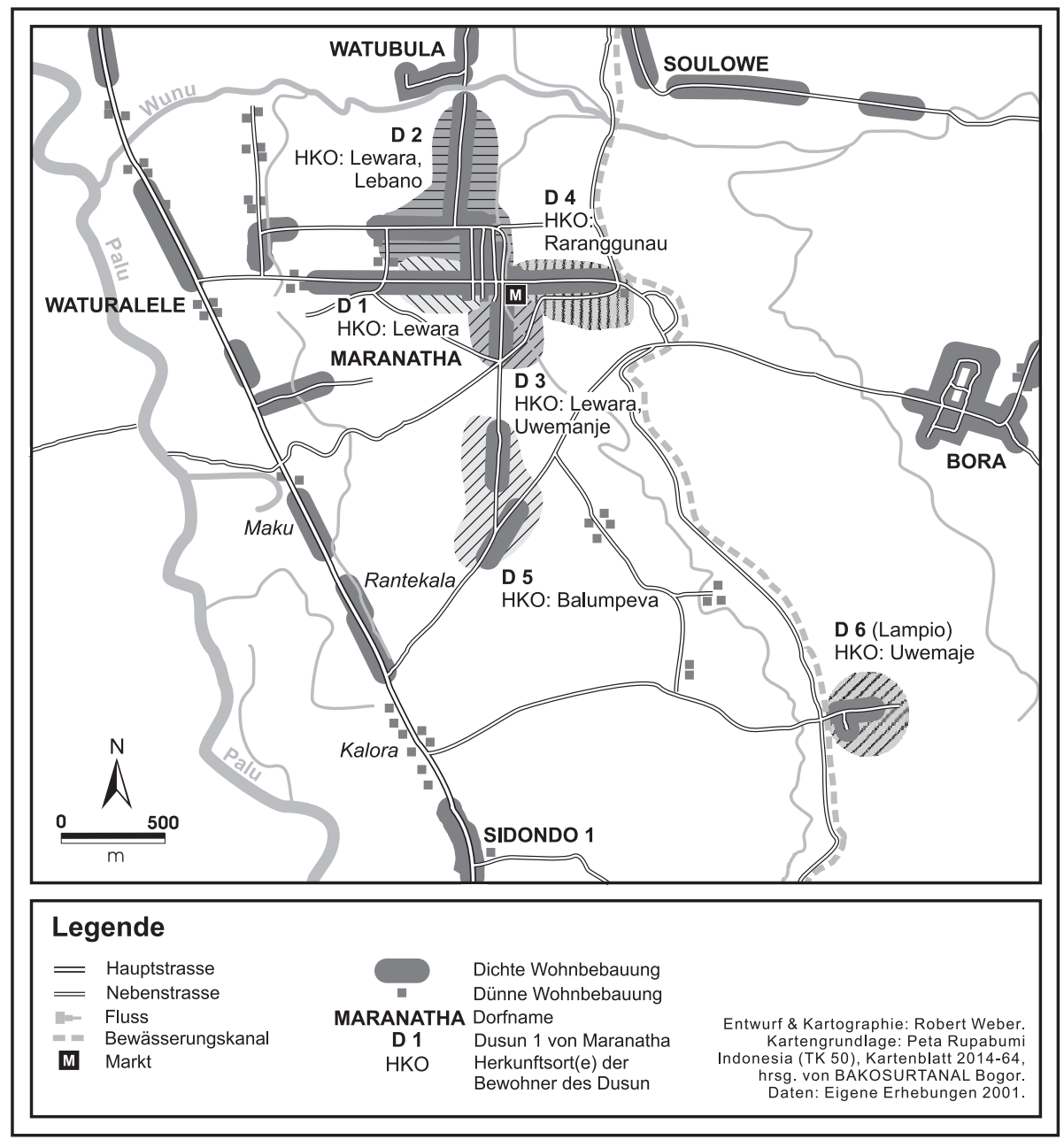

Abb. 36 Räumliche Segregation der Bevölkerung von Maranatha nach Herkunftsorten

Die Migranten aus Südsulawesi, welche ab den späten 1980er Jahren, in besonders großem Umfang aber ab Mitte der 1990er Jahre im Zuge des Kakaobooms nach Watumaeta zogen, befinden sich heute am westlichen und östlichen Rand des Dorfes. Im Osten dehnten sie das Siedlungsgebiet bis an die Grenze zum benachbarten Alitupu aus. Dort leben neben einer Bugis-Mehrheit auch einige 
Javaner, die Gemüseanbau betreiben. Im Westen siedeln sie an der Waldrandgrenze zum Lore-Lindu Nationalpark, welche sich in diesem Bereich in den letzten Jahren deutlich nach hinten verlagert hat. Der Landerwerb der Migranten ist zu einen großen Teil mit den illegalen Verkaufspraktiken des Dorfchefs verbunden gewesen. Im Dorfzentrum befinden sich weiterhin die alteingesessenen Familien, welche die politische Macht im Dorf innehaben.

Auch in Berdikari hatten sich in den Anfangsjahren des Dorfes (Ende der 1950er Jahre) einige Toraja angesiedelt, die am östlichen Rand des heutigen Zentrums (Dusun 1) wohnten und angeblich wegen Adat-Differenzen den Ort bald wieder verließen. Somit blieben die Dorfgründer aus dem Pipikoro-Gebiet Kulawis und die in der Folge zugewanderten Kulawi-Familien als tuhan kampung unter sich und erweiterten im Laufe der Zeit das Siedlungsgebiet nach Osten. ${ }^{117}$ Nachdem Mitte der 1970er Jahre der Weiterbau der Hauptstrasse des Palolo-Tals bis zum östlichen Talende erfolgt war, um später die Verbindung in das Napu-Tal (Lore Utara) herzustellen, siedelten sich viele Toraja im heutigen Dusun 3 am östlichen Ende Berdikaris an, wo ihnen vom Dorfchef Land zur Kultivierung zur Verfügung gestellt wurde. Sie bilden heute die größte Migrantengruppe in Berdikari. Ferner zogen auch Bugis nach Berdikari, die heute zum größten Teil in Dusun 3, aber auch in Dusun 2 leben. Die Anzahl der Kulawi-Familien nimmt von Dusun 1 bis Dusun 3 stetig ab. Die relativ wenigen Migranten in Dusun 1 sind v.a. Inhaber von Läden oder Besitzer der vier in Berdikari existierenden Reismühlen. Als die Zuwanderung in die Dusun 2 und 3 einsetzte, besaßen viele Kulawi-Familien mit Wohnsitz im Dusun 1 dort einen Teil ihrer Felder. Viele von ihnen verkauften diese Felder an Migranten und erwarben im Gegenzug Land in der Nähe des Dusun 1. Während die Kepala Dusun (Dorfteilvorsteher) 1 und 2 von der Gruppe der Kulawi gestellt werden, hat dieses Amt in Dusun 3 ein Bugis inne.

Das Dorf Pandere ist in drei Dusun gegliedert. Die lokale Bevölkerung der Kaili ist hauptsächlich in den Dusun 1 und 3 angesiedelt, während Dusun 2 von Migranten aus Nord- (Manado) und Südsulawesi (Bugis) geprägt ist. Der Siedlungskomplex in Dusun 1 entwickelte sich im Rahmen der 1927 von den Niederländern durchgeführten lokalen Umsiedlung der Bewohner des östlichen Gebirges. Heute bilden die ab Mitte der 1960er Jahre zugezogenen Bugis die größte Migrantengruppe im Zentrum von Pandere (Dusun 1). Auf dem seinerzeit noch unkultivierten Land im Norden von Pandere ließen sich ab 1929 die ersten Manado-Familien nieder. Die Landverfügbarkeit sowie die Nachbarschaft zum nördlich anschließenden christlich-javanischen Transmigrationsdorf Kalawara waren für ihre Ansiedlung besonders ausschlaggebend. Ab den 1980er Jahren, als in Dusun 1 kein freies Land mehr zur Ver-

117 Obwohl es sich bei den Pipikoro-Familien streng genommen auch um (lokale) Migranten handelt, werden sie in bezug auf die Thematik dieses Kapitels als Nicht-Migranten eingestuft, da sie die alteingesessene Bevölkerungsgruppe von Berdikari darstellen. 
fügung stand, siedelten sich Bugis auch in Dusun 2 an. Das dritte Dusun umfasst einen Großteil der ursprünglichen Siedlungsgebiete der Lokalbevölkerung. Die Kaili, welche sich entweder der kolonialen Umsiedlung an die Hauptstrasse widersetzten oder nach einiger Zeit wieder in die Berge zurückkehrten, besiedelten bis in die 1980er Jahre die Orte Saluponi, Salupongara und Bualo. Mit der Entwicklung des Dusun 2 zogen einige ihrer Bewohner näher an die Hauptstrasse, die mit dem neuen Wohngebiet durch einen schlechten Schotterweg verbunden ist. Heute lebt etwa ein Viertel der Bevölkerung von Dusun 3 noch in den genannten drei Orten. Es drängt sich die Frage auf, ob im Fall Pandere die Religion einen gewissen Einfluss auf die Segregation im Dorf hat. Einen Hinweis dafür liefert der Umstand, dass es sich bei der lokalen Kaili-Bevölkerung wie bei den Bugis um Muslime handelt. Obwohl die ersten Manado-Migranten bereits vor den Bugis nach Pandere kamen, wurde ihnen Land im späteren Dusun 2 zugeteilt, während die Bugis Felder in Dusun 1 erhielten. Diese Erklärung berücksichtigt jedoch nicht die Tatsache, dass die Bugis größenteils Land von der lokalen Bevölkerung kauften und dadurch Zugang zum Dusun 1 erlangten. Den ersten Bugis-Migranten wurde zudem ein dezentrales Sumpfgebiet in Dusun 1, fernab der Hauptstrasse, zugewiesen, und sie mussten ab der siebenten Ernte einen Teil des Ertrags an die lokale Bevölkerung abtreten. Die Religion kann daher eher als sekundärer Faktor oder, standorttheoretisch gesehen, als weicher Standortfaktor betrachtet werden.

Die Segregationsentwicklung in Sintuwu (Palolo) kommt bei einem Vergleich mit den drei soeben untersuchten Beispieldörfern Watumaeta am nächsten. Auch hier spielte der Dorfchef eine wichtige Schlüsselrolle in der Verteilung der Landressourcen. Allerdings sind es hier gerade die Migranten, welche sich, wenn auch keineswegs ausschließlich, im Dorfzentrum befinden. Sintuwu ist eines jener Dörfer in Palolo, welche ab den 1960er Jahren im Zuge der Bestrebungen der Regierung, das gesamten Palolo-Tal zu besiedeln und zu kultivieren, komplett neu gegründet wurden. Sintuwu selbst wurde 1961 gegründet. Die ersten Bewohner des Ortes waren Kaili aus Bakubakulu, einem Ort im Übergangsbereich vom Palu-Tal in das Palolo-Tal. Sie wurden von der Regierung zur Umsiedlung in das seinerzeit noch bewaldete Gebiet des späteren Sintuwu gezwungen, als die Regierung einerseits den Wanderfeldbau stoppen und andererseits die Ausdehnung von Nassreisfeldern vorantreiben wollte. Neben Bakubakulu wurden auch Familien aus Bunga zur Ansiedlung in Sintuwu gezwungen. Diese ersten Siedler rodeten in Gruppenarbeit den Wald im Bereich zwischen dem Gumbasa-Fluss und der heutigen Dorfstrasse in Dusun 1 und 2. Bis zur Ankunft der ersten Migranten, die nicht aus der Palolo-Region stammten, hatte jede Familie etwa 3 ha Land gerodet. Ab den späten 1960er Jahren ließen sich die ersten Bugis in Sintuwu nieder. Die Beschäftigungsoption als Holzfäller für das Unternehmen Kebun Sari zu arbeiten, ließ weitere Bugis in das Dorf zuwandern. Ein unzureichendes Bewässerungssystem einerseits und die Zuwanderung finanzkräftiger Migranten aus Südsulawesi andererseits führten ab den 
1980er Jahren dazu, dass die lokalen Kaili zunehmend ihre ehemaligen Nassreisfelder an Bugis verkauften, welche die Reis- in Kakaofelder umwandelten.

Ebenfalls ab den späten 1960er Jahren siedelten sich Familien aus dem Pipikoro-Gebiet Kulawis in Sintuwu an. Die ersten von ihnen arbeiteten als Rattansammler. Sie erhielten vom Bürgermeister Sintuwus ein Stück Land in der Nähe der südlichen Berghänge zur Bewirtschaftung zur Verfügung gestellt. Als das Holzunternehmen Kebun Sari 1977 die sog. Jalan Jepang entlang der südlichen Berghänge bauen ließ, bestand noch keine Straßenverbindung zum Siedlungsgebiet Sintuwus. Diese wurde zwei Jahre später errichtet.

Im Laufe der Jahrzehnte haben sich in Sintuwu verschiedene Wohnareale herausgebildet, in welchen jeweils eine dieser drei ethnischen Gruppen nahezu ausschließlich angesiedelt ist (vgl. Abb. 37). Diese Verteilung ist zum einen den sozialen Netzwerken der Migrantengruppen, zum anderen dem Handeln des von 1969 bis 2002 amtierenden Bürgermeister von Sintuwu geschuldet. Während die Kaili-Bevölkerung in ihrem ursprünglichen Wohngebiet verblieb, errichteten die Kulawi-Familien ihre Häuser nach dem Ende der Aktivitäten der Firma Kebun Sari entlang des westlichen Teils der Jalan Jepang. Die ersten Migranten aus Kulawi hatten bereits Land in der Nähe der Strasse vom Bürgermeister zugewiesen bekommen. Weiteres Land an der Strasse wurde ihnen schließlich von diesem verkauft. Zwar hatten auch die Kaili seit der Anbindung Sintuwus an diese Strasse ein Interesse, sich dort anzusiedeln. Der Bürgermeister verweigerte ihnen dies jedoch mit dem Hinweis, dass das Land bereits verkauft sei. Die ersten Bugis-Familien konnten sich nur allmählich größere Landareale aneignen. Die ab den 1980er Jahren zugewanderten wohlhabenderen Migranten aus Südsulawesi begannen hingegen sofort mit dem Ankauf von Land in Sintuwu. Auch hier spielte der damalige Bürgermeister eine zentrale Rolle. Er verkaufte sowohl Land im heutigen Zentrum des Ortes, als auch entlang des östlichen Teils der Jalan Jepang (vgl. auch Kap. 5.4 und 6.4).

Der räumlichen Dominanz der Bugis-Bevölkerung im Zentrum des Dorfes, sowie deren ökonomischer Dominanz als Großgrundbesitzer von Kakaofeldern begegnen die Kaili eher mit Neid als mit Ablehnung. Den Kaili ist bewusst, dass die heutige Verteilung im Dorf maßgeblich auf das Verhalten des ehemaligen Bürgermeisters zurückzuführen ist. Viele von ihnen zeigen sich aber durchaus enttäuscht darüber, dass nicht sie, als alteingesessene Bevölkerung, das Dorfzentrum dominieren. Diese Enttäuschung versuchen sie zu kompensieren, indem sie in den vergangenen Jahren damit begonnen haben, Land jenseits der Jalan Jepang und innerhalb des Lore-Lindu Nationalparks zu roden und danach an Bugis-Migranten zu verkaufen. Sie hoffen, sich dadurch zumindest in ökonomischer Hinsicht an die erfolgreichen Migranten annähern zu können. Interessant ist dabei wiederum die Rolle des neuen Dorfchefs, der die Eingriffe in den Nationalpark aktiv unterstützt. 


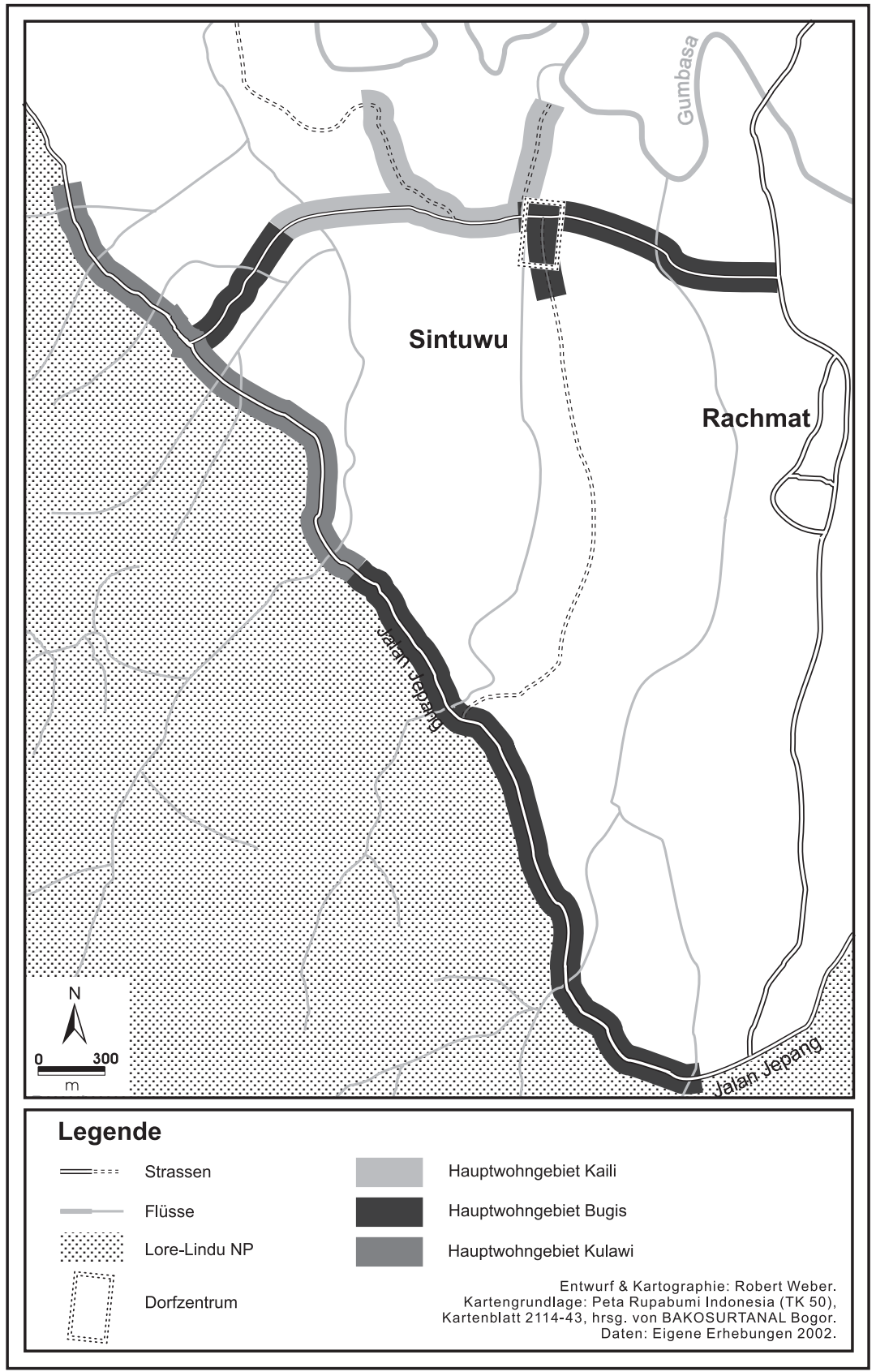

Abb. 37 Sozial-räumliche Segregation in Sintuwu 
Er knüpft damit an die dorfpolitische Funktion des ersten Dorfchefs als Verantwortlicher für die Verteilung von Landressourcen im Interesse der KailiBevölkerung an, während sein direkter Vorgänger v.a. seine persönlichen wirtschaftlichen Interessen und die der Migranten bediente (vgl. auch Sitorus 2002: 14ff).

Das Dorf Wanga (Kec. Lore Utara) nimmt in bezug auf die Segregation unter den Untersuchungsdörfern eine gewisse Sonderrolle ein. Unter dem Gesichtspunkt räumlicher Segregation ist der Ort deutlich zweigeteilt. Dusun 2, das entgegen der sonst in der Region üblichen Regel das Dorfzentrum bildet, ist fast ausschließlich von der Lokalbevölkerung besiedelt. ${ }^{118}$ Lediglich einige Bugis und Toraja wohnen ebenfalls in diesem Gebiet. Das Dusun 1 war bis Anfang der 1990er Jahre nahezu unbewohnt. Im Jahr 1991 migrierten 15 Kulawi-Familien von Kamarora (Kec. Palolo) nach Wanga. Die aus der Pipikoro-Region stammenden Familien hatten sich in den 1980er Jahren im Rahmen eines lokalen Transmigrationsprojekts in Kamarora angesiedelt. ${ }^{119} \mathrm{Da}$ sie das in Kamarora zur Verfügung gestellte Land als zu klein ansahen, um auch ihren Kindern eine günstige Zukunft zu sichern, entschlossen sich die Kulawi-Leute, dem Hinweis zu folgen, dass in Wanga noch ausreichend freies Land vorhanden sei. Von der Dorfregierung wurde den Familien im Dusun 1 Land geliehen. Eine entsprechende Regelung in Wanga sieht vor, dass Neuankömmlinge erst eine Bewährungsphase durchlaufen müssen, bevor sie als vollständige Dorfmitglieder anerkannt werden. Zudem soll kein Verkauf von Land an Migranten stattfinden. Die Kulawi-Familien, welchen in der Folgezeit noch einige weitere Familien aus dem Pipikoro-Gebiet folgten, bauen hauptsächlich Mais an, da der Nassreisanbau aufgrund von Vogelplagen nur von geringem Erfolg ist. Nach Informationen der Dorfbewohner ist die Bedrohung durch Vögel in Dusun 2 geringer, da dort die sawah-Felder dicht beieinander liegen und somit leichter überwacht werden können. Die Kulawi-Bevölkerung, die bis heute weitgehend nur über geliehenes Land verfügt, stellt eine ökonomisch relativ schwache Gruppe im Dorf dar. Das gleiche gilt für die aus Flores im Jahr 1999 über Poso nach Wanga migrierten Menschen, die ihr Herkunftsgebiet aufgrund eines verheerenden Erdbebens verlassen mussten. Sie arbeiteten anfangs auf einem Fünf-Hektar-Areal, das einem Chinesen gehörte, welcher Wanga aber schon bald wieder verlassen hatte. Seitdem sind sie

118 In Wanga verlief die Dorfteilgrenze anfangs parallel zur Hauptstrasse. Erst mit der zunehmenden Besiedlung wurde die Grenze entlang des Wanga-Flusses gezogen, welcher in etwa im rechten Winkel zur Strasse verläuft.

119 Der Hauptgrund für diese Migration war der Wunsch der Menschen, Nassreis zu bewirtschaften, was ihnen aufgrund des steilen Reliefs ihres Herkunftsgebiets dort nicht möglich war. Einige der Familien hatten sich zuvor bereits am Lindu See angesiedelt, wo ihnen Land für den sawah-Anbau zur Verfügung gestellt wurde. Nachdem das Transmigrationsprojekt angelaufen und Kamarora errichtet worden war, migrierten sie dorthin. 
auf Lohnarbeit angewiesen. Trotz der räumlichen Segregation offenbart sich das Verhältnis zwischen lokaler Bevölkerung und Migranten als relativ harmonisch, was durch die Integration der Migranten z.B. bei lokalen Hochzeitsfeiern belegt wird. Zwar werden auch in den anderen Untersuchungsdörfern die Beziehungen zwischen Lokalen und Migranten - zumindest vordergründig - als gut dargestellt. Zwischen den Zeilen und aus informellen Gesprächen lesen sich in manchen der Dörfer jedoch durchaus Spannungen heraus. Diese können für Wanga nicht bestätigt werden. Der Grund dafür scheint in der Tatsache zu liegen, dass in Wanga die lokale Bevölkerung nicht nur die soziopolitische, sondern auch die ökonomische Macht behalten hat. Dies kann neben der vergleichsweise peripheren Lage des Dorfes v.a. durch die Dorfregelungen bzgl. des Landverkaufs erklärt werden. Wanga zählt daher auch zu jenen Dörfern, deren Bewohner trotz der Nähe zum Nationalpark selbst keine nennenswerten Eingriffe in die unter Schutz gestellten Waldgebiete unternehmen. ${ }^{120}$

120 Statt dessen werden die Bewohner des nördlichen Nachbardorfes Dodolo (ursprünglicher Gebietsname: Toe) beschuldigt, die Nationalparkgrenzen zu verletzen. 


\section{Der Wandel von Landnutzung und Landbesitz}

Zu Beginn des 21. Jh. stellen Nassreis und Mais unter den einjährigen Kulturen sowie Kakao und Kaffee unter den mehrjährigen Kulturen die vier in der LoreLindu-Region wichtigsten ladwirtschaftlichen Anbauprodukte dar (vgl. Abb. 38). ${ }^{121}$ Das mit Abstand größte Einkommen wird dabei aus dem Verkauf von Nassreis und Kakao generiert.

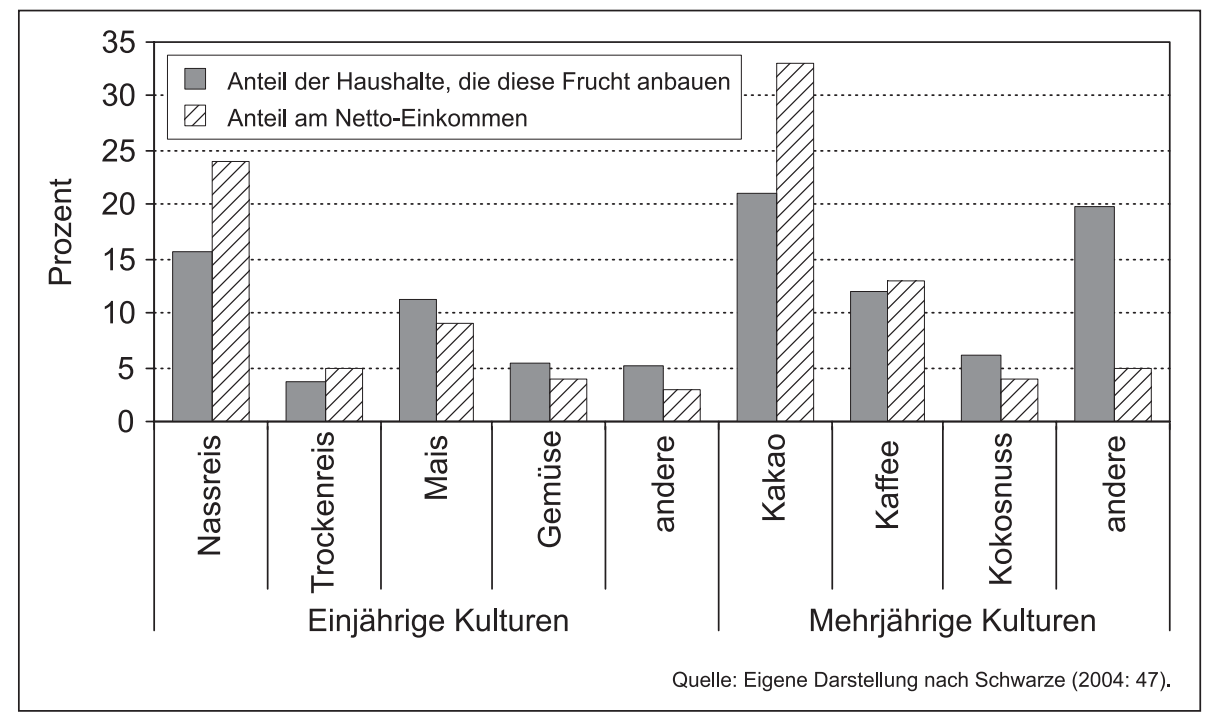

Abb. 38 Anteile der 2001 in der Lore-Lindu-Region angebauten Feldfrüchte und ihre Relevanz für das Haushalteinkommen der Landwirte

Vergleicht man nun die Einkommensrelevanz der Anbauprodukte in den einzelnen Kecamatan der Untersuchungsregion, ergibt sich jedoch ein deutlich differenzierteres Bild (vgl. Abb. 39). Die Nassreisproduktion hat die mit Abstand größte ökonomische Bedeutung im Palu-Tal (Sigi-Biromaru), während Kulawi eine gewisse Bedeutung als einzigem Marktproduzenten von Trockenreis zukommt. Neben Palolo ist v.a. in Lore Utara der Maisanbau für die Bevölkerung von ökonomischer

121 Die Daten basieren auf einem Haushaltsample des STORMA-Teilprojekts A4 $(\mathrm{n}=272)$. 
Relevanz. Unter den mehrjährigen Kulturen hat der Kaffee für die Menschen in Kulawi einen enormen wirtschaftlichen Stellenwert. In Palu wird dieser Kaffee unter

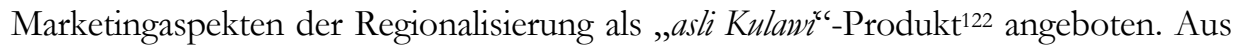
dem cash crop Kakao wird in Palolo über ein Viertel des Gesamteinkommens aus landwirtschaftlicher Produktion in der Untersuchungsregion generiert. Der geringe Stellenwert in Lore Utara lässt sich damit erklären, dass der Kakao dort erst gegen Ende der 1990er Jahre eine rasante Verbreitung erfuhr und viele der Felder daher noch keine großen Verkaufserträge erzeugen.

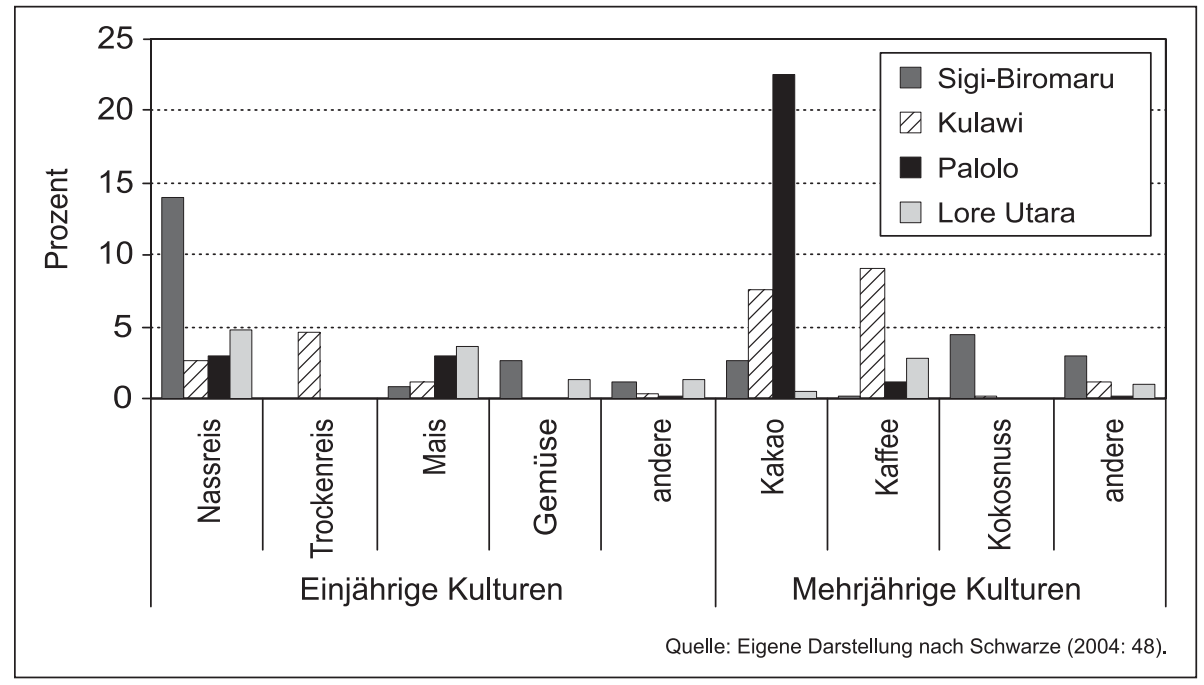

Abb. 39 Relevanz verschiedener Anbauprodukte für die Haushaltseinkommen der Landwirte 2001 nach Kecamatan

\subsection{Traditionelle Landnutzung}

Sucht man in der Lore-Lindu-Region nach Relikten der traditionellen Landwirtschaft, wird man vor allem im Pipikoro-Gebiet im westlichen Kulawi fündig. Fernab der asphaltierten Strassen und nur zu Fuß, auf dem Pferd oder (teilweise) mit dem Motorrad erreichbar, kann man in den Dörfern noch erahnen, auf welche Weise im Zentralsulawesi des frühen 20. Jh. Landwirtschaft betrieben wurde. Die sich deutlich vom dichten Wald abhebenden Brachefelder zeugen davon, dass in diesem Gebiet

122 Zu übersetzen als: "Original aus Kulawi”. 
noch heute Brandrodungswanderfeldbau betrieben wird. Zwar bestehen in einem Dorf wie Lawe heutzutage feste Siedlungsstrukturen und hat die Einführung von Kaffee und Kakao zur Anlegung permanente Felder geführt, der Anbau von saisonalen Produkten wie Mais, Trockenfeldreis oder Cassava erfolgt jedoch weiterhin in Feldrotation. Der Mechanisierungsgard in der Landwirtschaft liegt weit unter dem Durchschnitt der gesamten Lore-Lindu-Region. Handtraktoren sind nicht nur aufwendig in der Anschaffung, da die schlechte Verkehrsinfrastruktur des Pipikoro-Gebiets sowohl den Transport erschwert als auch die Transportkosten erhöht. Die Geräte wären bei Feldern mit einer Hangneigung von bis zu 80 Grad auch nur bedingt einsatzfähig (vgl. Abb. 40).

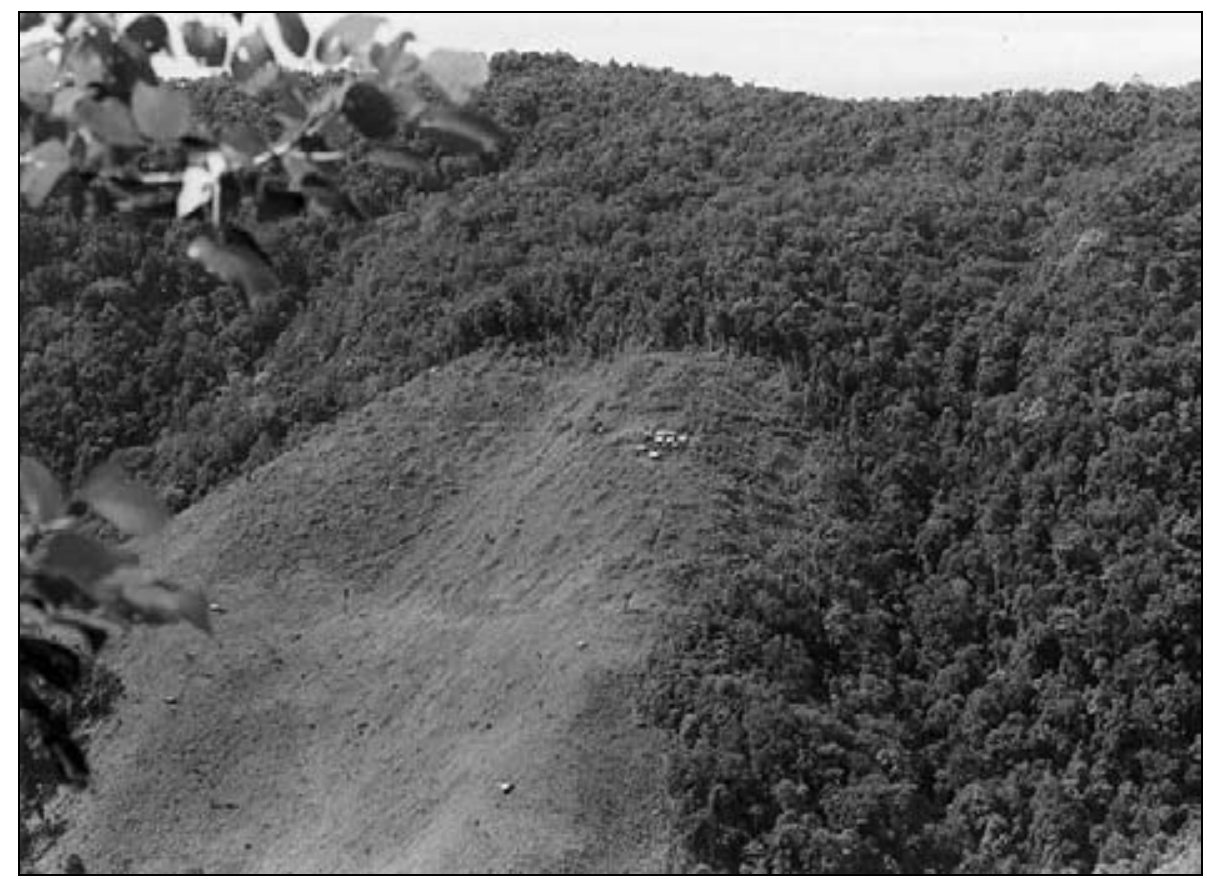

Abb. 40 Brachefeld in der Nähe von Lawe

(Quelle: Eigene Aufnahme, 2001)

Die Weiterverarbeitung des geernteten Reises erfolgt noch immer traditionell mit Hilfe eines lesung batu oder lesung kayu, einem Trog aus Stein bzw. Holz. Der getrocknete Reis wird dabei in den Trog gefüllt, welcher in der Mitte eine runde Vertiefung aufweist. Mit einer hölzernen Stange wird dann in monotonem Rhythmus senkrecht in die Vertiefung geschlagen, wobei sich der Spelz von den Reiskörnern trennt. Bereits Burkhard (2002a: 7ff) verweist darauf, dass die von Dove (1983) als Trugschlüsse identifizierten Mythen über den Wanderfeldbau auch für die Untersuchungsregion zutreffen. So kann weder behauptet werden, dass sich das kultivierte 
Land in kommunalem Besitz befindet, noch trifft es zu, dass die Flächen von einer ganzen Gemeinde bestellt werden. ${ }^{123}$ Die Rodung des Waldes für die neuen Agrarflächen erfolgte (und erfolgt noch immer, z.B. im Pipikoro-Gebiet) in Arbeitsgruppen, die im Napu-Tal z.B. horobo genannt werden (Burkhard 2002a: 7). Während früher vorwiegend Mitglieder einer extended kin group eine Arbeitsgruppe bildeten, wird nun, wie etwa aus dem Dorf Lawe zu erfahren ist, im gesamten Dorf nachgefragt, wer sich an einer solchen Arbeitsgemeinschaft beteiligen möchte. Nachdem für jedes Mitglied der Gruppe eine Fläche gerodet worden war, markierte jeder Bauer sein Feld, wodurch es von da an als Besitz seiner Familie ,registriert“ war. Meist wurde die paruja, das Durchstampfen des Bodens mit Hilfe einer größeren Anzahl an Büffeln (je nach Feldgröße bis zu 50 Tiere), ebenfalls in Gemeinschaftsarbeit dieser Gruppe nacheinander für jedes Feld durchgeführt. Die weitere Bestellung des Feldes wurde häufiger von der Familie durchgeführt, welche das Feld besaß, als in Gruppenarbeit. Der Ertrag der Felder war keinem System kommunaler Redistribution unterworfen. Vielmehr gehörten die Feldfrüchte dem jeweiligen Besitzer des Feldes. Man könnte verleitet sein, dem Einfluss der niederländischen Kolonialherren einen entsprechenden Wandel von Gemeinde- zu Privatbesitz zuzuschreiben. Die Niederländer stärkten die formale Betonung der Kernfamilie, indem sie durch die Veränderung der Siedlungsstruktur (permanente Dörfer, permanente Häuser) im Rahmen der Besteuerung von landwirtschaftlichen Erträgen nicht das gesamte Dorf, sondern jede einzelne Familie in Form von Kopfsteuern fokussierten (vgl. Logeman 1922: 131f; Voorn 1925: 15). ${ }^{124}$

Auf den gerodeten Flächen wurden Trockenfeldreis (padi ladang), Mais, Cassava und Gemüse angebaut. Während der Kolonialzeit kamen teilweise noch Kaffeefelder hinzu. Reis wurde entweder im Wechsel mit den anderen Produkten (außer Kaffee) oder gemischt angebaut, wobei der Reis meist in der Mitte und die anderen Früchte an den Rändern gepflanzt wurden. Bevor die ersten Händler in die Hoch-

123 Henley (2002: 193) kommt, bezugnehmend auf Adriani \& Kruyt (1950-1951, Bd. 3: 26f), zu dem Schluss: „In sparsely populated Central Sulawesi, by contrast, all of the fallow land belonging to a single village was held in common and reallocated each year by an unpredictable process of consultation and concensus". Den in dem zitierten Satz genannten Kontrast zeichnet er in Rückgriff auf die Arbeiten von Wilken (1873) und Graafland (1898) am Beispiel der im Vergleich zu Zentralsulawesi dichter bevölketen Region der Minahasa nach, wo eng definierte Verwandschaftsgruppen ein System dauerhafter Landrechte selbst für die Dauer der Brachezeit pflegten (Henley 2002: 193). Allerdings ist hierbei anzumerken, dass sich das Werk von Adriani \& Kruyt nicht mit dem westlichen Teil Zentralsulawesis befasst, welchem die Lore-Lindu-Region zuzurechnen ist, sondern mit dem östlichen Teil. Somit stehen die Ausführungen weniger im Widerspruch zu den Ergebnissen des Verfassers sowie von Burkhard (2002a), sondern deuten vielmehr auf die heterogene Ausgestaltung von Landbesitzrechten in unterschiedlichen Regionen Zentralsulawesis hin.

124 Auf landwirtschaftliche Produkte, sog. buah tanah, wurde eine Steuer von fünf Prozent des Ertrags erhoben. Den jeweiligen Dorfchefs wurden acht Prozent der eingenommenen Steuern als Lohn zur Verfügung gestellt (Logeman 1922: 132). 
landregionen (z.B. Kulawi) kamen, wurden Güter wie Salz im Tiefland (z.B. im Palu-Tal) im Austausch mit Reis ${ }^{125}$, Gold ${ }^{126}$ und anderen Produkten erworben. Wie in Kulawi wurde auch im Napu-Tal bereits zu vorkolonialen Zeiten Gold geschürft. Dieses wurde anfangs hauptsächlich für Tributgaben an den raja von Sigi benutzt. Nachdem sich die ersten chinesischen Händler in Poso angesiedelt hatten, wurde das Gold dort gegen Salz und andere Luxusgüter jener Zeit getauscht (vgl. auch Kruyt \& Kruyt 1921: 407).

Der Brandrodungswanderfeldbau war zur Zeit der Ankunft der Niederländer in der Lore-Lindu-Region weit verbreitet. Gleiches gilt aber auch für den Nassreisanbau oder zumindest für das Wissen darüber. Allerdings waren es nicht die Kolonialherren, die den Nassreisanbau im Untersuchungsgebiet erstmals eingeführt hatten. Dies belegen zeitgenössische Quellen und jüngere Untersuchungen. Das Vorkommen von Nassreisfeldern im Palu-Tal wird im 18. Jh. bereits von Valentijn (1724: 74f) erwähnt. Auch Hissink (1909: 62f) fielen aufgelassene sawab-Felder in dieser Region auf, wofür er kriegerische Auseinandersetzungen vor der Ankunft der Niederländer und die Entwaldung der das Tal säumenden Berghänge verantwortlich macht. Metzner (1981: 47) fand heraus, dass sich im Palu-Tal bereits zu Zeiten von Rumphius (1628-1702) ausgedehnte Nassreisareale befunden haben sollen. Dabei stellt Metzner die Hypothese auf, dass die sawah-Technik in diesem Gebiet den Bugis zu verdanken sei, welche seit Jahrhunderten die Küsten um Palu aufsuchten und sich dort nach der niederländischen Einnahme der südsulawesischen Stadt Makassar als Fischer und Händler niederließen (ebd.). Diese Hypothese eines frühen Einflusses durch Menschen von außerhalb Zentralsulawesis wird auch von den zeitgenössischen Aufzeichnungen Grubauers (1923) bestätigt. Er weißt nicht nur darauf hin, dass sich bei einer Fahrt von Sidondo nach Palu noch alte Spuren von sawahFeldern erkennen ließen (Grubauer 1923: 151), sondern führt weiter aus: „Da die Kunst Sawahs anzulegen, wie sie in höchster Entwicklung in Java existiert, den Kajeli [Kaili; Anm. d. Verf.] unbekannt ist, so darf hiernach auf sehr frühe Einwanderung fremder Elemente geschlossen werden." (ebd.). Die Berichte der niederländischen Kolonialverwalter in Zentralsulawesi verweisen ebenfalls auf die Existenz vorkolonialer Nassreisfelder im Palu-Tal. Van Hengel (1910: 24) bezieht sich beispielsweise auf alte Bewässerungsgräben, welche unter kolonialer Führung verbessert bzw. verlängert wurden. Von ehemals ausgedehnten sawah-Gebieten, die sich durch die Trockenheit im Palu-Tal in Kakteenwälder verwandelt hätten, geht auch Tideman (1926: 3) in seinem Report aus. Sarasin \& Sarasin fassen die Landschaft südlich des Dorfes Sibalaya (Palu-Tal) in ihren Reiseaufzeichnungen folgendermaßen zusammen:

125 Im Tauschhandel zwischen Kulawi und dem Palu-Tal wurde in den 1930er Jahren z.B. ein Liter Salz mit einem Liter Reis getauscht.

126 Das Gold wurde aus den Flüssen gewaschen. Es handelte sich dabei jedoch nur um geringe Mengen. 
„Weiter zunächst durch wohl bevölkertes Land; Kulturpflanzungen wechseln mit Strichen niederen Waldes, in denen elegante Pandanus-Bäumchen und Pbilodendron-Lianen auffallen; dann wieder Reisfelder, oft durch gut angelegte Kanäle bewässert und Grasebenen, mit Büffelherden bevölkert. Die Kokospalmen, welche die Dörfer umschatten, fallen durch besondere Höhe auf." (1905, Bd. 2: 19).

Das Gebiet südlich von Pakuli in Richtung Kulawi wird von den beiden Naturforschern so wahrgenommen: „Die Gegend blieb zunächst wohl bebaut, Pflanzung folgte auf Pflanzung; nach einiger Zeit jedoch wurden die Kulturflecke durch immer ausgedehntere Stücke Waldes unterbrochen, worauf dieser zuletzt Oberhand gewann." (ebd.: 21). Kruyt \& Kruyt (1921: 407) vermerken in ihren Aufzeichnungen, dass es auch im Napu-Tal bereits vorkoloniale Nassreisfelder gegeben hatte. Bei ihrem Besuch des Tals fallen ihnen alte Erddämme auf, die wie Begrenzungsdämme von sawah-Feldern aussehen. Nach Kruyt (1908: 1313f) ist die Verwahrlosung ehemaliger Nassreisfelder im Napu-Tal auf den Umstand zurückzuführen, dass die Bevölkerung den scheinbar einfacheren Weg der Nahrungsgewinnung mittels kriegerischer Überfälle auf die Bevölkerung des Poso-Tieflands präferierten. Eine zeitliche Einordnung des Nassreisanbaus in den Gebieten außerhalb des Palu-Tals anhand des empirischen Datenmaterials ist nur mäßig aufschlussreich. Zum einen lässt die lange Zeitspanne von einem Jahrhundert nicht zu, entsprechende Zeitzeugen zu finden. Zum anderen widersprechen sich die Aussagen der Kindergeneration zu sehr. Manche behaupten, dass die Niederländer für die Einführung des Nassreisanbaus verantwortlich sind. Andere schließen diese Option völlig aus. Die Ursachensuche für diese große Differenz in den Angaben lässt jedoch Spielraum für vielfältige Spekulationen. ${ }^{127}$ Verlässlicher erscheinen hier die Quellen zeitgenössischer Reiseaufzeichnungen. So lässt sich die vorkoloniale Nassreisbewirtschaftung anhand der Augenzeugen Sarasin \& Sarasin auch für das Kulawi-Tal belegen:

„Von dem Hügel herab, auf dem wir uns befanden, siebt man südwärts auf einen mitten im Gebirge sich ausdehnenden Kessel, welcher gegen Westen seinen Ausgang hat, sonst aber amphitheatralisch ringsum ansteigt, wobei die stufenweise angelegten Reisfelder gewissermaßen die Sitzreiben darstellen, (...) das schöne Gebirgskulturland von Kuláwi."(1905, Bd. 2: 27).

Die traditionelle Landnutzung war in erster Linie Subsistenzwirtschaft und diente daher der Erzeugung von Grundnahrungsmitteln wie Reis, Mais (s.u.), Cassava und Gemüsesorten. Die Feldrotationsdauer von 7-10 Jahren, welche den von Rasul \&

127 Beide Behauptungen können beispielsweise durch eine antikoloniale Grundhaltung der Interviewten gefärbt sein. Die Aussage, dass sawah-Felder bereits vor der Kolonialherrschaft existierten, könnte dabei dadurch begründet sein, dass man seine Vorfahren vor der Vermutung in Schutz nehmen will, sie seinen völlig primitiv gewesen. Den Niederländern die Einführung des Nassreisanbaus zuzuschreiben, könnte auf dem Denkmuster basieren, dass die Kolonialherren alles - und zwar ohne Rücksicht auf die lokale Bevölkerung - verändert haben, so auch den Reisanbau. 
Thapa (2003: 496) für Südostasien genannten Durchschnitt von 15 bis 20 Jahren deutlich unterschritt, erlaubte den bewirtschafteten Böden die erforderliche Neuanreicherung mit Mineralstoffen, um die Fruchtbarkeit zu gewährleisten. Noch heute kann in Dörfern wie Lawe weitgehend auf den Einsatz von Düngemitteln verzichtet werden, da die Böden nach der Brachezeit wieder ausreichend fruchtbar sind. Die niedrige Bevölkerungsdichte der in kleinen Gruppen siedelnden Familienverbände (vgl. hierzu Kap. 4) ermöglichte die Einhaltung der Rotationsdauer der Felder. In manchen Bergregionen stellte Reis durchaus ein Luxusprodukt dar. Dort ernährten sich die Menschen vorwiegend von Knollenfrüchten, wie z.B. Cassava. Bei Nahrungsmangel ging diese Bevölkerung in den Wald, um nach Sago zu suchen (Hissink 1909: 121; Vorstman 1935: 19f).

Nassreisfelder wurden traditionell mit Hilfe von Wasserbüffeln bestellt. Bei dieser als paruja bezeichneten Bewirtschaftungsweise wurde eine Herde von Büffeln über das Feld getrieben, um den Boden umzugraben. Büffel wurden traditionell zur Verhinderung ihrer völligen Verwilderung für einige Zeit des Jahres eingefangen. Die restliche Zeit verbrachten die Tiere in freier Wildbahn (vgl. auch Kap. 5.2.1). Durch das Sammeln der zahlreichen Büffel zum Weiden auf gerodeten Waldflächen wurde eine Wiederbewaldung verhindert, wodurch sich neben den natürlich entstandenen auch anthropogen beeinflusste Grasflächen entwickelten. Die verstärkte Sesshaftmachung der Bevölkerung während der Periode des kolonialen Siedlungsausbaus dürfte eine nicht unbedeutende Rolle bei der Zunahme der Grasflächen gespielt haben. Henley ist davon überzeugt, ,that the great majority of large grasslands ultimately owed their existence to the deliberate creation of pasture for grazing. "(2004: 313).

Befragt man ältere Menschen in der Lore-Lindu-Region nach dem Sortiment der von ihren Eltern angebauten Agrarprodukte, so wird gelegentlich auch der Mais erwähnt. Diese Pflanze wurde nach Aragon (1996d: 57) im westlichen Zentralsulawesi erst während der niederländischen Kolonialherrschaft eingeführt. Die Kolonialverwaltung propagierte den Anbau von Mais auf den abgeernteten Reisflächen, um den Ertrag der Felder zu steigern (Ansingh 1937: 8). Die Aussagen der befragten Bauern verwundern allerdings nicht, wenn man bedenkt, dass laut Aragon (1996a: 57) der gemeinsame Anbau von Reis und Mais schon bald als traditionell im Gewohnheitsrecht (adat) verankert galt. Im Napu-Tal taucht die vorkoloniale Kenntnis der Maispflanze in einer Volkslegende auf. Demnach brachten Bewohner von Gowa ${ }^{128}$ (Südsulawesi) Mais nach Napu. Sie übergaben den Menschen einige Körner, doch diese dachten, es handle sich um Gift, und warfen die Körner weg. Drei Monate später entdeckten die Menschen die herangewachsenen Maispflanzen. In der Lokalsprache Napus heißt Mais gogoa. Da es bereits vor dem 20. Jh. vereinzelte Kontakte zwischen den Bevölkerungsgruppen Zentral- und Südsulawesis gab,

128 Der Respondent nennt dieses Gebiet nur „Goa“. 
ist es durchaus möglich, dass in diesem Teil des Untersuchungsgebiets bereits vor der Einflussnahme der Niederländer Mais angebaut wurde. Kruyt (1908: 1313) untermauert diese Vermutung, indem er Cassava, Mais und Bananen als Hauptbestandteil des Speiseplans des Großteils der Bevölkerung im Napu-Tal zu vorkolonialer Zeit beschreibt. Reis wurde hingegen vornehmlich von der ,adeligen“ Bevölkerungsschicht verzehrt.

\subsection{Von der Subsistenzwirtschaft in die Weltmarktproduktion}

Bereits unter den Niederländern wurde in der Lore-Lindu-Region mit der Einrichtung eines Exportsektors in der Landnutzung begonnen. Dies betraf weniger die von den Niederländern in der Region eingeführte Kaffeepflanze, welche dort zu jener Zeit nur in geringen Mengen angebaut wurde. „A greater expansion of smallholding coffee also occurred, particularly in the West Coast region of Sumatra, and enormous further areas of coconut were planted in Celebes (Sulawesi)", betont Barlow (1989: 246). Schon im Jahr 1910 berichtet Van Hengel (1910: 26) von der Ausbreitung der Kokoskulturen in $\operatorname{der}$ Afdeeling Donggala. Kokospalmen fand man, bezogen auf die Untersuchungsregion, v.a. im Palu-Tal vor (Hissink 1909: 121). In den Hochlandregionen, z.B. in Kulawi, wurden hingegen nur wenige Kokospalmen gepflanzt (Vorstman 1935: 24).

Die Einflussnahme der Kolonialadministration auf die Landnutzung der Bevölkerung stellte sich bei der Kopraproduktion - ,[...] copra is een voornaam artikel van witvoer [...] " (ebd.: 122) -, mehr noch als bei Reis, besonders als im Eigeninteresse begründet dar. Pro Haushalt waren mindestens 50 Kokospalmen zu pflanzen. Bei jeder Vergrößerung der Familie mussten 10 weitere Palmen gepflanzt werden. Da es sich um ein Exportprodukt handelte, wurde die Einhaltung dieser Auflagen viel strenger eingefordert als in anderen landwirtschaftlichen Bereichen. Es erging der Befehl, einen geregelten Kokospalmenanbau zu unterhalten und vertrocknete Blätter sofort zu verbrennen, um Heuschrecken- und Raupenplagen zu verhindern (Van Hengel 1910:26). Der von der Kolonialregierung forcierte Kokospalmenanbau hatte Metzner (1981: 49) zufolge im Zusammenhang mit der Ausdehnung der Bewässerungswirtschaft weitreichende ökologische und wirtschaftliche Folgen. Als mit der Ausbreitung der sawab-Flächen unter niederländischer Ägide Kokospalmen plötzlich und in großem Maße von der Schildmotte befallen wurden, ${ }^{129}$ habe man erkannt, dass nicht komplette Kokosgärten, sondern einzeln in den Nassreisfeldern stehende Palmen bevorzugte Ausbreitungszentren dieser Schädlinge waren. Von Anfang an wurde von der Kolonialverwaltung überwacht, dass keine unreifen Ko-

129 Nach Metzner (1981: 49) fielen im Jahr 193344.000 Palmen der Schildmotte zum Opfer, zwei Jahre später sogar 100.000 . 
kosnüsse gepflückt wurden, die eigentlich für die Kopra-Erzeugung bestimmt waren. (Hissink 1909: 121). Die positive Quotierung des Kokospalmenproduktes Kopra lies die Kokoskultur in den darauffolgenden Jahren einen enormen Zuwachs verzeichnen (Logeman 1922: 66). Die Ausdehnung des Anbaus von Kokospalmen im Zuge des Preisbooms für Kopra während der 1930er Jahre bewirkte streckenweise eine Vernachlässigung der Nassreisbewirtschaftung (Van Rhijn 1941: 43). Doch spätestens die negative Veränderung der Weltmarktpreise als Folge des Beginns des 2. Weltkriegs löste dieses Problem von selbst.

\subsubsection{Vom Wasserbüffel zum Handtraktor - Ausbau und Technisierung des Nassreisanbaus}

War die Technik des Nassreisanbaus nicht von den Niederländern eingeführt worden, so waren diese immerhin für die Expansion der sawah-Anbauflächen verantwortlich. Mit der lokalen Umsiedlung der Bergbevölkerung in die Talebenen der Lore-Lindu-Region wurde auch die landwirtschaftliche Inwertsetzung der Täler vorangetrieben (Logemann 1922: 63). In Regionen wie Pipikoro oder Palolo wurde jedoch weiterhin vorwiegend Trockenreisanbau betrieben, was v.a. durch die physisch-geographischen Voraussetzungen begründet ist. Auch die in anderen Bergregionen verbliebene oder nach erfolgloser Umsiedlung ${ }^{130}$ dorthin zurückgekehrte Bevölkerung setzte die Trockenfeldbewirtschaftung fort.

Während die hohen Niederschlagsmengen in Kulawi oder Napu (Lore Utara) ausreichten, um die sawah-Felder mit genügend Wasser zu versorgen, erforderte das von Metzner (1981) ausführlich dargestellte Phänomen des Trockentals südlich von Palu eine technische Lösung. Die bedeutendste Veränderung geht dabei auf den Bewässerungsplan des ehemaligen Directeur van landschapswerken, Ingenieur S. Fenger Petersen, zurück (Ansingh 1937: 32f). Im Jahr 1931 entstand somit das GumbasaBewässerungssystem. Ein für die lokalen Verhältnisse gewaltiges Wehr südlich des Dorfes Pandere kanalisierte von da ab das Wasser des Gumbasa-Flusses und führte es den unterschiedlichen Dörfern zu. Ferner wurden auch die nördlicher gelegenen Flüsse Wunu und Paneki in den Bewässerungsplan mit einbezogen. In Abb. 41 sind die Gumbasa-, Wunu- und Paneki-Bewässerungskanäle deutlich als gestrichelte Liniensignatur erkennbar. Nach Norden hin schließt die Karte mit Palu (Paloe) und dem Beginn der Palu Bucht ab.

130 Vgl. hierzu die Ausführungen zur Ethischen Politik der Niederländer in Kapiteln 2.3.3, 3.3.1 und 6.1. 


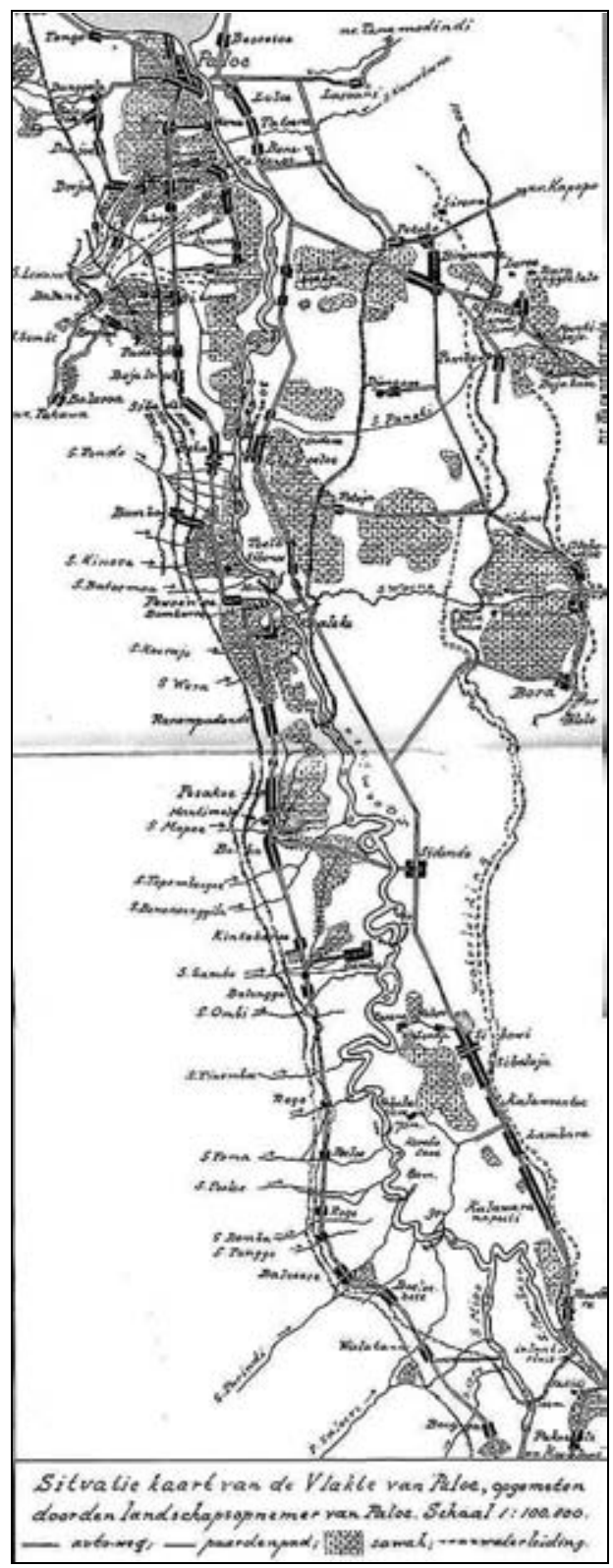

Abb. 41 Nassreisflächen im Palu-Tal 1938

(Quelle: Kruyt 1938: Anhang)

Von Pandere im rechten unteren Bereich der Karte erstreckt sich der GumbasaBewässerungskanal nach Norden. Der Fenger-Petersen-Plan hatte zum Ziel, die 
gesamte Osthälfte des Palu-Tals für den Nassreisanbau nutzbar zu machen. Ursprünglich sollten dabei 24.000 bou⿰ ${ }^{131}$ (ca. $170 \mathrm{~km}^{2}$ ) bewässerungsfähige Agrarfläche geschaffen werden. Allerdings konnte das Projekt aus Kostengründen nur in kleineren Dimensionen verwirklicht werden.

Mit Hilfe des sog. Sombe-Lewara-Plans (Vorstman 1935: 20) wurden die sawabFelder des westlichen Palu-Tals vor Überflutungen und Verschlammung geschützt. Die beiden Flüsse, welchen der Plan seinen Namen verdankt, führten von den unbewaldeten Hängen Erdmaterial und Steine mit sich, welche sich am Fuß der westlichen Bergkette des Palu-Tals in großen Mengen ablagerten. In Folge starker Niederschläge konnten diese Ablagerungen vielerorts durchbrochen werden und die dahinter liegenden Nassreisfelder vernichten. Der Sombe-Lewara-Plan konnte diesen negativen Effekt durch Verbesserungen an den Flussbettungen verringern.

Neben der Ausdehnung der Nassreisproduktion und der Errichtung von moderneren Bewässerungssystemen hatte die niederländische Kolonialverwaltung auch einen Einfluss auf die Technisierung der Feldbewirtschaftung. Das traditionelle paruja-System sollte der Verwendung des Pfluges weichen. Zwar erkannten die Kolonialherren, dass die herkömmliche Methode durchaus ihren Zweck erfüllte (Logeman 1922: 64), dennoch hielten sie an einer Propaganda für den Pflug als fortschrittliches Werkzeug fest, da dessen Einsatz zeitsparender wäre (Van Rhijn 1941: 82). ${ }^{132}$ Ab dem Jahre 1937 wurden im Archipel hergestellte, einfache und billige Pflüge zum Selbstkostenpreis in der Untersuchungsregion verkauft, um der Bevölkerung demonstrieren zu können, wie gut das Pflügen auch ohne Büffel funktioniert (Ansingh 1937: 9). ${ }^{133}$ Da sich aber die Mehrzahl der lokalen Bauern den Kauf eines eigenen Pflugs nicht leisten konnten, wurde von der Kolonialregierung zuvor bereits angeregt, Pflüge dorfweise zur Verfügung zu stellen, die sich die Bewohner dann teilen könnten (Logeman 1922: 64). Verbreitete sich der Gebrauch des Pfluges während der Kolonialzeit nur zögerlich, führte spätestens der Rückgang der Anzahl an Büffeln in der Lore-Lindu-Region zu einem flächendeckenden Übergang von der parija- zur Pflugbewirtschaftung (vgl. Abb. 42).

Die Beziehung von Übergang zur Pflugwirtschaft und Abnahme der Büffelmenge ist als sich gegenseitig bedingendes System zu verstehen. Auf der einen Seite wurden mit der Technisierung in der Landwirtschaft weniger dieser Tiere benötigt, auf der anderen Seite zwang der Rückgang der Büffelzahl die Bevölkerung, $\mathrm{zu}$ anderen Anbaumethoden überzugehen. Der Rückgang der Büffelpopulation ist auf mehrere Faktoren zurückzuführen. Bereits für das Ende der 1950er Jahre wird

131 Ein bouw entspricht $7.096 \mathrm{~m}^{2}$.

132 Für einen Hektar sawah-Boden fielen nach Ansingh (1937: 9) bei einem Einsatz von 10 bis 15 Büffeln etwa 140 parija-Stunden an.

133 Nach Auskunft eines älteren Informanten wurde der Pflug im Palu-Tal zum ersten Mal im Jahr 1920 eingeführt. 
z.B. in Lore Utara eine Verringerung des Büffelbestandes mit dem Einfluss der sog. Permesta-Bewegung in Verbindung gebracht. Bewohner des Dorfes Watumaeta (Kec. Lore Utara) berichten, dass sie anfangs die Rebellen, später die sie verfolgende Nationalarmee mit Nahrungsmitteln versorgen mussten. Hierfür wurden u.a. zahlreiche Büffel geschlachtet, um den Essensbedarf decken zu können. Im Vergleich dazu hatten die Veränderungen im Siedlungswesen und Tierepidemien einen bedeutend größeren Einfluss auf die Büffelpopulation.

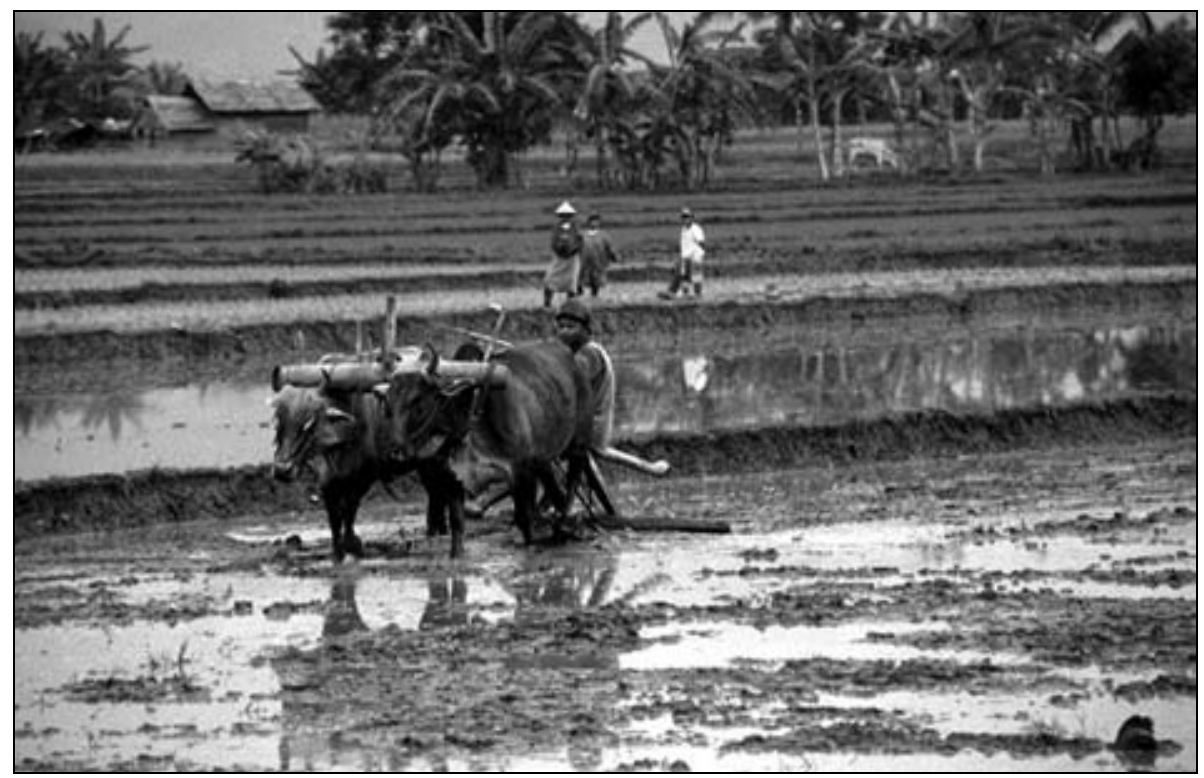

Abb. 42 Pflugbewirtschaftung eines Nassreisfeldes im Palu-Tal (Quelle: Eigene Aufnahme, 2000)

Traditionell wurden Wasserbüffel die meiste Zeit frei gehalten, d.h. sie hielten sich in nicht umzäunten Arealen im Wald oder auf Grassflächen auf. Nur für die paruja wurden die Tieren eingefangen und zu den Feldern geführt. Der Ortsname Gimpu in Südkulawi, welcher mit „Büffelranch“ übersetzt werden kann, legt die Vermutung nahe, dass sich diese Tiere auf umzäuntem Gelände befunden haben. Aus dem Interviewmaterial ergibt sich, dass Büffel jedoch nur in gewissen Abständen eingefangen wurden, um eine komplette Verwilderung der Tiere zu vermeiden. Ein Nebeneffekt war zudem die Demonstration von sozialem Status innerhalb der lokalen Gesellschaft durch die Ansammlung der wertvollen Büffel, die zum größten Teil dem bzw. der raja und seinen/ihren Angehörigen gehörten. Für das Napu-Tal beschreibt Sunito (2004: 83) zudem die aus der hierarchischen Besitzverteilung von Wasserbüffeln entstandenen Schuldner-Beziehungen der gewöhnlichen Bevölkerung zu der Schicht der „Adeligen“. Die Büffel spielten in der traditionellen Gesellschaft neben der Verwendung in der Landwirtschaft auch eine wichtige soziokultu-

\section{Pazifik Forum Bd. 12}


relle Rolle (u.a. für Adat-Rituale; s.u.). Somit mussten diejenigen, die einen Büffel für derartige Anlässe benötigten, in die Schuld der Büffel besitzenden „Adeligen“ eintreten, wodurch die sozial-hierarchischen Gesellschaftsstrukturen gefestigt wurden. Der Büffelbestand während der ersten Hälfte des 20. Jh. kann in Ermangelung statistischer Erfassung nur anhand von Zeitzeugenaussagen und wenigen zeitgenössischen Quellen rekonstruiert werden. Augenzeugen berichten, dass sich in den Wäldern und im Grasland von Lore Utara während der ersten Hälfte des 20. Jh. Büffel und andere Tiere in unvorstellbar großer Zahl aufgehalten hatten. Dies wird auch für die Region Kulawi bestätigt (vgl. auch Boonstra van Heerdt 1914: 644; Kruyt 1909: 358). Mit der Ausweitung der kultivierten Siedlungsgebiete verringerten sich sukzessive die Weideflächen für Büffel. Zudem haben Krankheiten zu der Dezimierung des Viehbestands beigetragen. Daneben spielen auch Adat-Zeremonien und die Funktion von Büffeln als veräußerbares Kapital eine gewisse Rolle für den Rückgang der Büffelmenge.

In der bis heute noch größtenteils mündlich weitergetragenen AdatGesetzgebung stellt der Büffel eine zentrale Maßeinheit dar. Vergehen wie z.B. außerehelicher Sexualkontakt werden mit der Opferung eines Büffels geahndet. Vor dem Einfluss der christlichen Missionierung, die Menschopfer untersagte, wurden die Schuldiggesprochenen noch durch den Tod bestraft. Denn in der traditionellen Vorstellung konnte nur das Blut die Sünde reinwaschen. Nach dem kirchlichen und staatlichen Verbot solcher Praktiken, wurden die zu opfernden Menschen durch Büffel ersetzt. ${ }^{134}$ Selbst in heutiger Zeit wird das Strafmaß der Adat-Gesetzgebung noch in Büffeln berechnet, auch wenn diese aufgrund der Bestandsdezimierung letztendlich immer häufiger durch andere Tiere, z.B. Kühe, Ziegen, Schweine oder Hühner, ersetzt werden können. ${ }^{135}$ Neben Adat-Strafen waren auch andere AdatZeremonien, wie z.B. Bestattungsfeiern, für den Rückgang der Büffelzahlen verantwortlich. ${ }^{136}$ Waren es traditionell nur ,aristokratische“ Familien, die für ihre Verstorbenen Büffel opferten und als Gastmahl anboten, änderte sich dies mit dem offiziellen Ende der Exklusivität der Noblen. Zunehmend konnten auch andere Familien der lokalen Dorfgesellschaft aufwendigere Adat-Beerdigungen inszenieren,

134 Im Fall des koloa-Rituals kam es vor dessen Verbot zu Tötungen von Sklaven durch Aufschlitzen mit der Machete. Nach dem Verbot wurde die Person durch eine Bananenstaude ersetzt.

135 Die Handhabung differiert zwischen den einzelnen Adat-Einzugsgebieten. In manchen Gemeinden und Fällen wird vom lembaga adat (Adat-Rat) noch heute auf der Bezahlung einer Strafe in Form von Büffeln bestanden. Die gesamte, weitverzweigte Familienverwandtschaft wird in die Pflicht genommen, die Strafe zu begleichen. Teilweise wird hierfür ein Teil des Landbesitzes verkauft, um einen Büffel erwerben zu können. Schweine werden nur in christlichen Gemeinden als Äquivalent benannt, während Ziegen vornehmlich in Adat-Prozessen muslimischer Gemeinden vorkommen.

136 Kruyt (1908: 1296) berichtet von Büffelschlachtungen im Napu-Tal in Folge eines schweren Erdbebens am 13. Januar 1902. An mehreren Orten wurden jeweils zehn Büffel geschlachtet, um damit die Erdgeister zu besänftigen. 
wodurch reiche Dorfbewohner ihren sozialen Status in der Gemeinschaft steigern konnten. In manchen Fällen wurden Büffel zur Bezahlung der Schulausbildung für die Kinder verkauft. Die Käufer waren v.a. Händler aus Palu.

Der Bürgermeister von Wanga (Kec. Lore Utara) weist auf einen weiteren $\mathrm{Zu}$ sammenhang zwischen Büffeln und Landwirtschaft hin. Von Mitte der 1940er bis Mitte der 1950er Jahre wurde durchgehend mehr sawah als Trockenfeldreis angebaut. Zwischen ca. 1957 und 1970 veränderte sich dieses Verhältnis. Der Grund hierfür liegt in der Domestizierung der Büffel. Da die frei laufenden Büffel besonders nachts eine große Gefahr für die Menschen darstellten, drängte die indonesische Regierung ab Mitte der 1950er Jahre darauf, Büffel in umzäunten Gehegen auf den Grasflächen zu halten, um die Entwicklung der Siedlungen nach den Vorstellungen der Regierung zu gewährleisten (Sunito 2004: 78). In Wanga gab es, so der Dorfbürgermeister, einen Büffelhirten. Als dieser unerwartet starb, begannen die Büffel wieder zu verwildern. Niemand im Dorf war mehr in der Lage, die Büffel einzufangen und auf die jeweiligen Nassreisfelder zu schicken.

Henley (2004: 311) führt den Ausbau der Verkehrsinfrastruktur als einen weiteren Grund für den Rückgang der Büffelpopulationen an. Er verweist dabei auf den fast vollständigen Austausch von Büffeln durch Kühe in Gorontalo zwischen 1860 und 1930. Diese Entwicklung, die nach Merkensen (1927: 118ff, zit. in Henley (2004: 311)) zu jener Zeit auch in anderen Regionen des Archipels vorkam, könnte dazu geführt haben, dass der Büffel als Transportmittel für unwegsames Terrain zunehmend an Bedeutung verlor. Einen weiteren Einfluss der Erweiterung des Strassen- und Wegenetzes während der ersten Dekaden nach der Unabhängigkeitserklärung Indonesiens sieht Sunito (2004: 79) in bezug auf den Verkauf von getrocknetem Büffelfleisch. Mit dem leichteren Zugang zu den Hochlandregionen des Untersuchungsgebiets stieg auch die Nachfrage nach diesem Fleisch und somit dessen Verkaufspreis. Die lokale Bevölkerung erkannte schnell den wirtschaftlichen Vorteil. Zudem begannen die lokalen Eliten, ihre Jagdkenntnisse wohlhabenden Besuchern aus Palu oder Poso zur Verfügung zu stellen und Jagdtouren für dieses Klientel zu organisieren. Die Dezimierung der Büffelpopulation in der Lore-LinduRegion wirft besonders in Hinblick auf die soziokulturelle Rolle des Wasserbüffels die Frage auf, ob dadurch neben den landwirtschaftlichen Auswirkungen auch die soziale Stratifizierung der lokalen Gesellschaften nachhaltig umstrukturiert wurde. Schließlich haben die Schuldnerbeziehungen zwischen „Freien“ und „Adeligen“ von der Präsenz der Büffel gelebt. Wie in Kap. 6.2 noch ausgeführt werden wird, bestehen die sozialen Hierarchien latent bis heute fort. Zwar wurden die direkten Abhängigkeitsverhältnisse im Laufe der Zeit aufgeweicht, dennoch sind sich die Menschen ihrem traditionellen sozialen Status in der Gesellschaft bewusst. Dieses Bewusstsein ist bis in die Gegenwart in vielen Fällen unterschwellig verhaltensbestimmend.

Nach Angaben der regionalen Statistikbehörden sank die Anzahl der Büffel im Kab. Donggala bis zum Jahr 2000 auf nur noch $32 \%$ des Bestands von 1970, wobei das Kec. Kulawi mit über $50 \%$ der Gesamtmenge einen deutlichen Spitzenplatz 
einnimmt (BPS 1971: 51; BPS 2002: 216). Im Kab. Poso erfolgte eine Reduzierung des Büffelbestandes im gleichen Zeitraum sogar auf gerade noch $14 \%$ der Anzahl von 1970 (BPS 1971: 51; BPS 2001: 182). ${ }^{137}$ Dieser enorme Rückgang ist neben den anderen genannten Gründen mit der Technisierung in der Landwirtschaft zu erklären. Die unter der niederländischen Herrschaft beginnende Verbreitung des Pfluges, dehnte sich während der Unabhängigkeit Indonesiens weiter aus und verdrängte den Büffel als landwirtschaftliches Arbeitstier zugunsten der Kühe.

Im Jahr 1979 erfolgte mit der Einführung des ersten Handtraktors in der Untersuchungsregion ein weiterer maßgeblicher Technisierungsschritt in der Landwirtschaft. Nach Angaben der regionalen Landwirtschaftsbehörde (Dinas Pertanian) in Palu führt der Einsatz von Handtraktoren gegenüber der traditionellen paruja-Methode zu einer Halbierung des Arbeitsaufwands bei der Bestellung von Nassreisfeldern. Nach Maertens (2003: 44) verfügen 81 Prozent der Dörfer mit sawah-Bewirtschaftung über mindestens einen Handtraktor. In den meisten Untersuchungsdörfern der vorliegenden Arbeit sind die Traktoren in Privatbesitz und können von anderen Dorfbewohnern gemietet werden. Entsprechende Aussagen von Interviewpartnern legen den Schluss nahe, dass dies auch für zahlreiche andere Dörfer der Lore-Lindu-Region zutrifft. Die technische Effizienz im Nassreisanbau wurde neben der Einführung von Handtraktoren auch durch die sog. Grüne Revolution erhöht. Ab den 1970er Jahren verbreitete sich der Gebrauch chemischer Pflanzenschutzmittel in der Lore-Lindu-Region, begleitet von der Einführung neuer Reissorten, die höhere Erträge versprachen. Allerdings waren bessere Ernteergebnisse dabei abhängig von einem kostenintensiven Einsatz von Dünger und Pestiziden.

Die Neuerungen zeigten durchaus Erfolge. Rangierte Indonesien während der 1960er und 1970er Jahre noch auf Platz eins der Länder mit der weltweit höchsten Rate an der Reiseinfuhr, so konnte das Land 1984 dank verschiedener Programme zur Intensivierung der Reisproduktion schließlich den Selbstversorgungsstatus für dieses Grundnahrungsmittel erreichen (Ambler 1989: 56). Aus ihrer Untersuchung in 80 Dörfern der Untersuchungsregion zieht Maertens (2003: 45) den Schluss, dass solche Programme nicht alle Gebiete gleichermaßen begünstigten. Zwar fanden in drei Vierteln der 80 Dörfer entsprechende Projekte von Seiten der regionalen Landwirtschaftsinstitutionen statt, es hatten jedoch v.a. Regionen mit höherer Bevölkerungsdichte davon profitiert. Zudem war die Verbreitung des Gebrauchs von Dünger und Pestiziden abhängig von den finanziellen Kapazitäten und dem entsprechenden Know how der Bauern. Es verwundert daher nicht, dass es v.a. die wohlhabenden Kakaobauern aus Südsulawesi sind, die gegenwärtig mit einem hohen

137 Aufgrund der fehlenden Aufschlüsselung der Daten von 1970 nach Kecamatan ist ein Vergleich mit den Werten von 2000 auf der Basis der untersuchten Kecamatan leider nicht möglich. Entsprechendes gilt für die Angaben zu Lore Utara und Lore Selatan im Kab. Poso. 
Einsatz dieser Zusatzstoffe arbeiten. Sie verfügen nicht nur über das notwendige Kapital, sondern konnten - zumindest ein Teil von ihnen - bereits in den 1970er Jahren Erfahrungen im Bereich moderner Landwirtschaft während ihres Aufenthalts auf Kakaoplantagen in Malaysia sammeln (vgl. das folgende Kapitel).

\subsubsection{Der Kakaoboom - Wohlstandsmotor auf tönernen Füßen}

Kakao wird in Südostasien seit dem 17. Jh. angebaut (Burger \& Smit 2001: 41). Auf Java wurde die Kakaopflanze bereits im frühen 18. Jh. eingeführt, wo sie v.a. im Ostteil der Insel in großen, von niederländischen Firmen errichteten Plantagen angebaut wurde und 1930 eine Produktionsmenge von 1.500 Tonnen erreichte (Akiyama et al. 1996: 2). Eine Kaffeemissernte auf der Insel im Jahr 1880 ermutigte zahlreiche Bauern, auf den Kakaoanbau umzusteigen (International Cocoa Organisation 1998). Der Anteil der kleinbäuerlichen Produktion von Kakaobohnen, welcher in bezug auf die Landbesitzstrukturen in der Lore-Lindu-Region für diese Arbeit von besonderem Interesse ist, blieb bis in die 1980er Jahre marginal. Zwar wurde in diesen Produktionsgrößen im Laufe der Zeit vor allem auch auf den Molukken, Sumatra und Sulawesi Kakao angebaut, die kleinbäuerliche Gesamtproduktion von Kakao überschritt 1980 jedoch nur knapp die 1.000-Tonnen-Marke (Akiyama et al. 1996: 2). Ab Ende der 1980er Jahre erfuhr die kleinbäuerliche Produktion v.a. auf Sulawesi einen gewaltigen Auftrieb und nahm von ca. 17.000 Tonnen im Jahr 1989 auf über 151.000 Tonnen im Jahr 1994 zu (vgl. Abb. 43). Erreichte der Anteil Indonesiens an der Weltproduktion von Kakao im Jahr 1985 nur zwei Prozent, waren es 2000 bereits $11 \%$, mit zunehmender Tendenz (Burger \& Smit 2001: 41). Die Anzahl kleinbäuerlicher Kakaoproduzenten in Indonesien nahm 1999 den weltweit dritthöchsten Wert nach Elfenbeinküste und Ghana ein (vgl. Abb. 44).

Bereits in dem Zeitraum 1820 bis 1880 wurde Kakao auf Sulawesi eingeführt (Li 2002c: 419). In Zentralsulawesi kam die Kakaopflanze, dessen Bepflanzung als Zweitgewächs unter der Kokospalme vorgenommen werden konnte, schon während der niederländischen Kolonialzeit vor. Sie ging aber durch den Befall von Phytopthora und Kakaomotte zugrunde (Van Rhijn 1941: 50; vgl. auch Burger \& Smit 2001: 41). Seinen Aufschwung erfuhr diese Anbaufrucht in erster Linie durch Bugis aus Südsulawesi, welchen Pelras (1998: 27) „a tradition specifically open to change" zuspricht. Als der Weltmarktpreis für Kakao in den späten 1970er Jahren einen rasanten Aufschwung erfuhr (vgl. Abb. 45), fand eine große Anzahl von Männern aus Südsulawesi Arbeit auf den Kakaoplantagen des malaysischen Sabah. Dort eigneten sie sich nicht nur das nötige Wissen über den Kakaoanbau an, sondern konnten auch eine gewisse Menge an Kapital anhäufen (Akiyama et al. 1996: 4). 


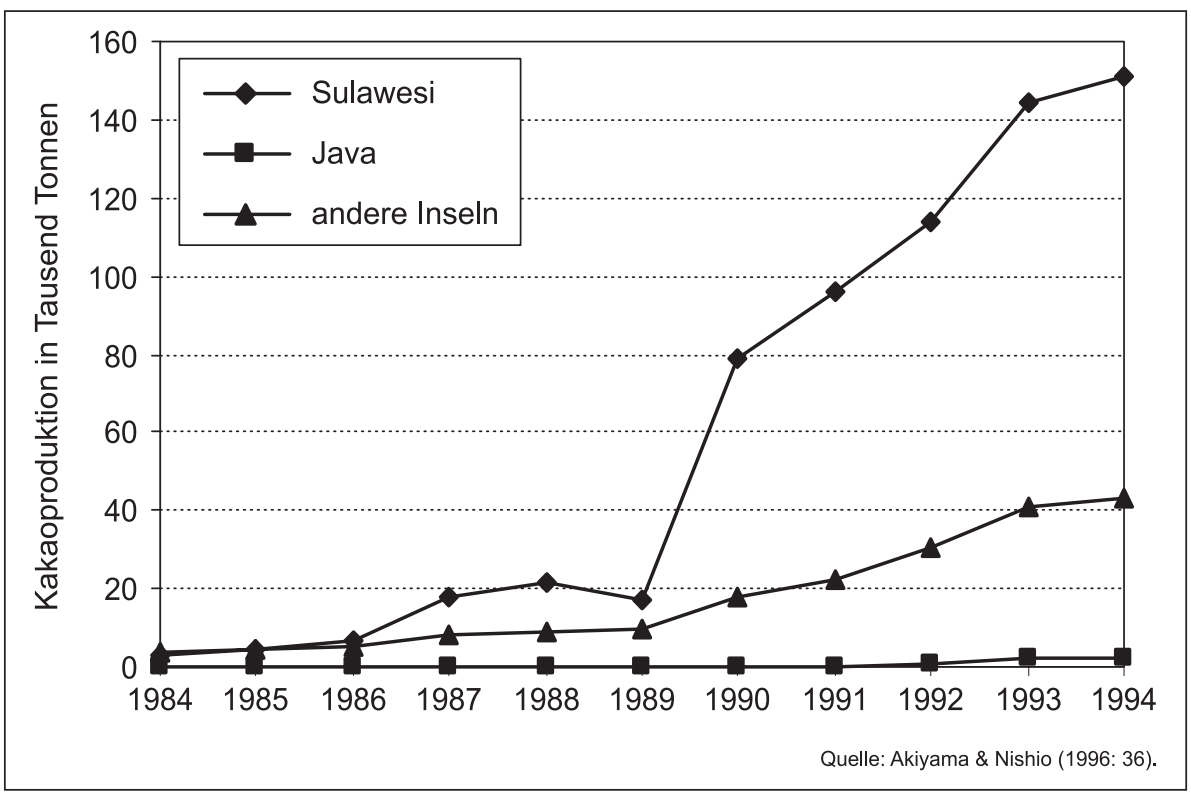

Abb. 43 Kakaoproduktion von Kleinbauern in Indonesien 1984 - 1994

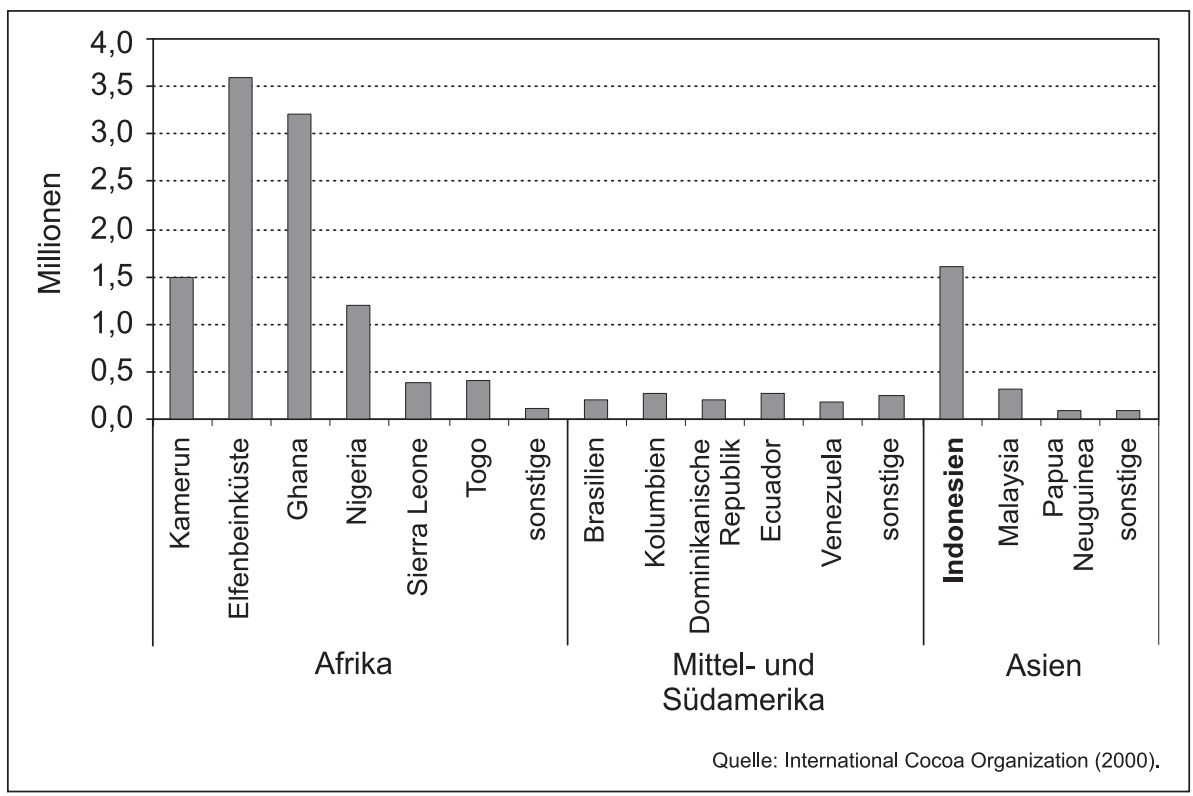

Abb. 44 Anzahl der Kakao produzierenden Kleinbauern im Ländervergleich 1999 
Die Grundlage für diese Arbeitsmigration wurde jedoch schon ab der Mitte des 20. Jh. geschaffen. In den 1950er Jahren führten die beginnende Landverknappung, der Rückgang von Erwerbsmöglichkeiten im nicht-landwirtschaftlichen Sektor und die beginnende DI/TII (Darul Islam/Tentara Islam Indonesia)-Rebellion in Südsulawesi als Push-Faktoren zu einer Abwanderung von Bugis in andere Regionen Indonesiens und nach Malaysia (Röll 1979: 27ff).

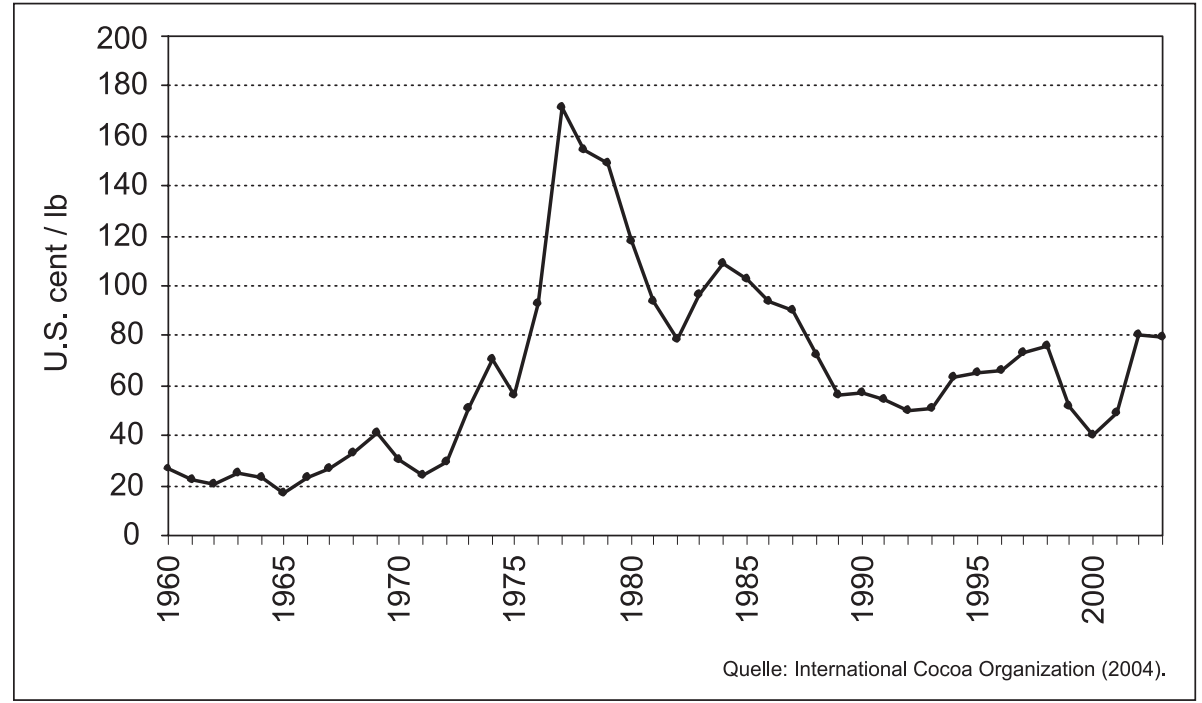

Abb. 45 Durchschnittlicher Jahresweltmarktpreis für Kakao 1960 - 2003

Ein geschichtlicher Vergleich der Kakaoproduktion in anderen Regionen (vgl. Ruf 1995) zeigt, dass die meisten Kakao-Booms mit enormen Migrationsbewegungen in Verbindung stehen. Das Zusammenspiel der Faktoren Preiszuwachs, Verfügbarkeit von Land und Migration im Zusammenhang mit Kakaoboom wurde erstmals in Ghana während der 1950er Jahre beobachtet (vgl. Hill 1956, 1963). In anderen Ländern konnten später ähnliche Prozesse festgestellt werden (vgl. Ruf \& Ehret 1993; Jamal \& Pomp 1976).

Nach Ruf \& Yoddang (2001: 99ff) beförderten in Sulawesi neben Landreserven und einem großen Arbeitskräftereservoir sechs weitere Faktoren den Boom des Kakaoanbaus. Als erster Faktor sind hohe Niederschläge v.a. in den Bergregionen, zu nennen. Zweitens führte die DI/TII-Bewegung der 1950er und 1960er Jahre zu einer positiven Kakaoentwicklung. Bei Vorstößen der DI/TII-Mitglieder in die Waldregionen konnten diese einen Überblick über verfügbare Landflächen gewinnen. Nach dem Ende der Bewegung migrierten sie dann zu den Orten, die sie sich gemerkt hatten, und pflanzten dort erst Tabak und Gewürznelken, später Kakao an. Drittens stellte die malaysische Provinz Sabah (Nordborneo), welche die DI/TII-Bewegung unterstützte, Informationen über den intensiven Kakaoanbau 
zur Verfügung. Sabah war später auch eines der Zielgebiete für buginesische Arbeitsmigranten, die dort weitere Informationen sammeln konnten. Zwar kehrten nur wenige der Plantagenarbeiter aus Sabah wieder nach Südsulawesi zurück, deren Know-how und finanzieller Aufschwung hatten jedoch einen bedeutenden Einfluss auf die Entwicklung des Kakaoanbaus auf Sulawesi (Röll 1979: 6). Viertens profitierten die Bauern Südsulawesis auch von dem Innovationssprung durch die sog. Grüne Revolution im Reisanbau. Mit diesem Wissen wanderten die BugisBauern in andere Gebiet, um dort ihr Wissen umzusetzen. Ebenso veranlassten die Produkte Gewürznelke und Tabak Migrationen in Gebiete, die auch für den Kakaoanbau geeignet sind. Zusätzlich setzten neue Technologien in der Nassreisbewirtschaftung wie Handtraktoren oder Herbizide Arbeitskräfte im Reisanbau frei, wodurch die Abwanderung in Waldrandgebiete anderer Regionen der Insel verstärkt wurde. Fünftens sind Bugis für ihre Fähigkeiten im Handel bekannt. ${ }^{138}$ Der Kakao bot ihnen die Möglichkeit, ihre Geschäftstätigkeit auf dieses neue Feld auszudehnen. Sechstens gibt es bei den Bugis z.B. das traditionelle gadaiSystem (Pfandsystem). Dabei übernimmt ein Geldverleiher ein Stück Farmland für bis zu drei Jahre. Er zahlt dafür einen Pfandbetrag an den Landbesitzer. Nach der Rückgabe des Landes erhält der Besitzer den Pfandbetrag zurück, während der Geldverleiher das innerhalb der drei Jahre aus dem Land erwirtschaftete Einkommen behalten darf. Manche Eltern ermöglichen somit ihren Kindern, mit Hilfe des Pfandbetrags in andere Gebiete, z.B. in die Lore-Lindu-Region, auszuwandern und dort Land zu kaufen.

In der Untersuchungsregion boten v.a. die Kecamatan Palolo und Lore Utara optimale Voraussetzungen für die Ansiedlung der neuen Kakaobauern und der Entwicklung eines Kakaobooms. Noch war in diesen beiden Gebieten landwirtschaftlich nutzbares Land vorhanden. Die hohen Niederschlagsmengen waren zudem eine ideale Voraussetzung für einen ertragreichen Kakaoanbau (vgl. auch Ruf 1997; Li 2002c). Mit der Einführung ${ }^{139}$ des Kakao wird von Zeitzeugen in der gesamten Untersuchungsregion durchgehend der Name Latif in Verbindung gebracht. Dieser kam Anfang der 1980er Jahre als buginesischer Händler in die einzelnen Dörfer und überließ den Einwohnern Kakaosamen mit dem Hinweis, dass deren Anpflanzung in Zukunft zu hohen Einnahmen führen würde. Erst die Ansiedlung von Kakaobauern aus Südsulawesi in der Region führte jedoch dazu, dass auch lokale Bauern dieser Frucht ernsthaft Beachtung schenkten.

138 Damit soll jedoch keine pauschale, deterministische Zuschreibung getroffen werden.

139 Wie oben bereits erwähnt existierten Kakaopflanzen in Zentralsulawesi Anfang des 20. Jahrhunderts bereits. Es ließen sich jedoch keine gesichterten Quellen dafür finden, dass auch in der LoreLindu-Region, speziell in den Gebieten Palolo und Napu (Lore Utara), der Kakao zu jener Zeit schon bekannt war. Aus dem vorhandenen Interviewmaterial lässt sich eher das Gegenteil vermuten. 
Die Ansiedlung der Bugis in den einzelnen Dörfern Palolos und Lore Utaras erfolgte nicht wahllos, sondern, nach einer anfänglichen Pionierphase, hauptsächlich in Form von Kettenmigration basierend auf einem Informationsnetzwerk zwischen Herkunfts- und Zielregion (vgl. Kap. 3.6). Mit zunehmender Verschlechterung der Landverfügbarkeit und Beschäftigung im nicht-landwirtschaftlichen Sektor Südsulawesis bei gleichzeitiger Verbesserung der für den Kakaoabsatz wichtigen Verkehrsinfrastruktur im Ostteil der Lore-Lindu-Region erhöhte sich auch der Anteil der Bugis an der dortigen Bevölkerung. Analog zur Migrationsentwicklung war der Anteil des Kakao an der landwirtschaftlichen Produktion in Palolo während der 1980er Jahre um ein Vielfaches höher als in Lore Utara. Erst nachdem sich das verfügbare Agrarland in Palolo verknappte, begann der Kakaoboom auch im südlichen Nachbarkecamatan. Das im Vergleich zur Lokalbevölkerung viel höhere Kapitalvermögen vieler der Migranten erlaubte diesen den Ankauf von Land, welches danach in der Regel mit Kakao in Monokultur bepflanzt wurde. Die steigenden Kakaopreise erlaubte es den Migranten ihr Vermögen weiter zu steigern.

Der Zunahme des wirtschaftlichen Wohlstands zahlreicher Bugis wurde nur von einem Teil dieser in Form äußerlicher Veränderungen des Hauses, und somit des Siedlungsbildes, zur Schau gestellt. Innerhalb relativ kurzer Zeit wurden Holzhäuser z.B. durch Ziegelhäuser ersetzt, deren Fußböden und Wände mit Fließen versehen wurden. Mobile Statusobjekte wie Fernsehgeräte, Stereoanlagen, VideoCD-Player, teure Möbel, Motorräder oder gar Autos ließen zumindest auf den zweiten Blick erkennen, dass sich eine neue wirtschaftliche Schicht in den Dörfern etabliert hatte, welche ihren Wohlstand offenkundig dem Kakaoanbau verdankte. Diese neue Disparität führte bei der lokalen Bevölkerung einerseits zu Neid gegenüber den wohlhabenden Migranten, andererseits zu dem Versuch, durch eigenen Kakaoanbau ebenfalls eine prosperierende Zukunft zu erlangen. Allerdings bestand für viele der lokalen „Kakaopioniere“ das Problem, dass ihnen die für eine hohe Produktivität erforderliche Startkapital fehlte. Aufgrund des großen Inputs, welches während der Anfangsjahre der Bewirtschaftung bis zur ersten Ernte geleistet werden muss, stellt der erste Anbau von mehrjährigen Pflanzen, wie z.B. Kakao, ein gewisses Risiko dar (vgl. Eijkemans 1995: 164).

Das Wissen über den Kakaoanbau schauten sich die Lokalen bei den Migranten ab. Bei einer geringen Zahl der Befragungen gaben die Landwirte an, sich bei den Zugewanderten aktiv, im Gespräch mit den Bugis, über die Anbaumethoden informiert zu haben. Dieses mehrheitlich passive Verhalten zeugt zum einen von einer geringen sozialen Interaktion zwischen ansässiger und zugewanderter Bevölkerung (vgl. Kap. 4.3). Zum anderen kann es als eine Erklärungsmöglichkeit dafür herangezogen werden, warum die Produktivität der Kakaofelder der Migranten mehrheitlich höher ist als jene der lokalen Bevölkerung. In vielen Untersuchungsdörfern kann man beobachten, dass der Boden unter den Kakaobäumen auf Feldern lokaler Bauern weniger stark von Laub freigehalten wird, wodurch sich die Gefahr von Pflanzenkrankheiten und Schädlingsbefall erhöht. Ebenso werden die reifen Früchte 
unregelmäßiger abgeerntet. Neben dem geringen Informationsaustausch ist jedoch auch die unterschiedliche Landnutzungsstruktur zwischen der Mehrzahl der lokalen und zugewanderten Bauern für die voneinander abweichende Pflege der Felder und die daraus resultierende Produktivität verantwortlich. Besitzen sehr viele BugisHaushalte fast ausschließlich Kakaofelder, so bewirtschaften lokale Haushalte meist noch mindestens ein weiteres Feld mit Reis. Oft kommt noch ein drittes Feld mit paruja-Anbau hinzu. Der lokale Bauer kann folglich viel weniger Arbeitszeit auf die Kakaoproduktion verwenden als der buginesische Kakaobauer. Das Thema Arbeitszeit führt dabei zu einem weiteren Punkt, der die Ungleichheit ausmacht. Wie bereits erwähnt, etablierten sich viele der Kakaobauern, die aus Südsulawesi in die Lore-Lindu-Region kamen, relativ bald zu einer wohlhabenden Schicht. Ihnen war es daher möglich, nicht nur Dünger und Pflanzenschutzmittel zur Ertragssteigerung zu kaufen, sondern auch Lohnarbeiter auf ihren Feldern zu beschäftigen. Sie stellten dabei entweder Mitglieder ihrer eigenen ethnischen Gruppe ein, die noch nicht über die finanziellen Möglichkeiten für den Ankauf eigener Felder verfügten. In zahlreichen der beobachteten Fälle waren dies die Neffen reicherer Kakaobauern. Oder es wurden Mitglieder der lokalen ethnischen Gruppe(n) engagiert. Diese Entwicklung der zunehmenden abhängigen Lohnarbeit im ländlichen Raum stellt einen entscheidenden Faktor für eine ganze Reihe von Veränderungsprozessen in der Kulturlandschaft der Lore-Lindu-Region dar.

Ab der Mitte der 1990er Jahre kam es in der Region zu einem regelrechten Kakaoboom. Die Asienkrise von 1997 führte durch die enorme Entwertung der indonesischen Rupiah bei gleichzeitig leicht ansteigenden Weltmarktpreisen für Kakao (vgl. Abb. 45) zu einer steilen Aufwärtsbewegung der Abnehmerpreise für Kakao. Die ein Jahr später folgende politische Instabilität des Landes ${ }^{140}$, welche mit dem Rücktritt von Präsident Suharto im Jahr 1998 ein vorläufiges Ende fand, hielt die nationalen Kakaopreise weiterhin auf hohem Niveau. Für die Lore-Lindu-Region hatte dies eine gewaltige Zunahme der Kakaoanbauflächen zur Folge. Sie erhöhte sich zwischen 1990 und 2001 um etwa das 4,5-fache (Maertens 2003: 37), so deutlich wie kein anderes Anbauprodukt in der Region. Dies hatte seine Ursache in der steigenden Zuwanderung einerseits und in dem vermehrten Anbau von Kakao durch die Lokalbevölkerung andererseits. Letzterer wurde auch durch Maßnahmen

140 Indonesien wurde von der Asienkrise vergleichsweise hart getroffen. Die ökonomischen Auswirkungen, wie Preissteigerungen und die Gefährdung von Arbeitsplätzen, waren der Auslöser für die politischen Unruhen von 1998. Die gewaltsamen Ausschreitungen richteten sich neben US-amerikanischen Unternehmen wie McDonald's v.a. gegen die chinesische Bevölkerungsminderheit und wurden von einer strukturell antisemitischen Stimmungsmache begleitet - zum Teil initiiert oder unterstützt von Militär und politischen Gruppen. Während dieser Unruhen kam es zu über Tausend Toten, unzähligen Verletzten, vergewaltigten Frauen und zerstörten Gebäuden (vgl. u.a. Dieter 1999; Zha 2000; Chua-Franz 2002). 
regionaler Landwirtschaftsbehörden, durch die Vergabe von Kakaosamen und die Bereitstellung von Anbauinformationen unterstützt.

Bis jetzt scheint sich der Kakao zu einem wahren Wohlstandsmotor der LoreLindu-Region entwickelt zu haben. Eine einseitige Ausrichtung der Landwirtschaft auf dieses Exportprodukt, für welches Abnehmer wie das Süßwarenunternehmen Mars gute Absatzmöglichkeiten bieten, kann jedoch bei sinkenden Kakaopreisen zu gravierenden sozioökonomischen Folgen führen. Eine im Rahmen dieser Arbeit durchgeführte Risikountersuchung weißt dabei ein diverses Bild auf. Die Interviewten wurden mit dem Zukunftsszenario konfrontiert, dass der Kakaopreis innerhalb kurzer Zeit rapide absinken und der Preis für ein anderes Verkaufsprodukt, z.B. Kaffee, gleichzeitig stark zunehmen würde. Anschließend wurde ihnen die Frage gestellt, wie sie sich in einer solchen Situation in bezug auf ihre Kakaofelder verhalten würden. Als ein generelles Ergebnis ist festzuhalten, dass sich die überwiegende Mehrheit der befragten Bauern bislang noch keine Gedanken für den Fall dieses Szenarios gemacht haben. Einige wenige gaben an, dass sie das Kakaofeld in ein Kaffeefeld umwandeln würden. Eine größere Anzahl der Befragten schloss diese Option mit großer Wahrscheinlichkeit aus. Oft wurde dabei auf die Entwicklung von der Kaffee- zur Kakaoproduktion verwiesen. Als der Kaffeepreis seinerzeit absank und zugleich der Kakaopreis anstieg, wurden vielerorts die Kaffeebäume gefällt und an deren Stelle Kakaosetzlinge gepflanzt. Andere verfuhren dieser Art mit ihren ehemaligen Gewürznelkenfeldern. Nachdem die Gewürznelkenernte mit dem Sturz Suhartos und dem Ende des von dessen Sohn beherrschten nationalen Handelsmonopols nur noch geringe Erträge einbrachte, wurde in zahlreichen Fällen der Gewürznelkenanbau durch den Kakaoanbau ausgetauscht. Diese Gruppe der Befragten würde entweder, sofern dies möglich ist, die neue Anbaufrucht zwischen die Kakaobäume bzw. die Pflanzen eines anderen Feldes, z.B. eines palawija-Feldes, setzen, oder ein zusätzlich erschlossenes/erworbenes Feld mit der neuen Frucht bepflanzen. Sie hätten dadurch die Möglichkeit, so die Aussagen, die Entwicklung des Kakaopreises abzuwarten, und könnten je nach Preisentwicklung zumindest die Inputkosten für die Kakaobewirtschaftung ausgleichen. Die Befragten geben zudem zu Bedenken, dass ein radikaler Wechsel von Kakao zu Kaffee mehrere Jahre einer negativen Haushaltsbilanz bedeuten würde, da der frisch gepflanzte Kaffee nicht sofort Erträge abwerfen würde.

Ruf \& Yoddang (1999: 9) haben ein ähnliches, Kakao bewahrendes Verhalten von Bauern in anderen Frontierzonen Sulawesis mittels einer Gegenüberstellung von Kakao und Nassreis ausgemacht. Solange der Kakaopreis über dem für Nassreis liegt, würden die Landwirte ihre Kakaoanpflanzungen nicht antasten. Als Hauptgrund wird das Verhältnis von Arbeit und Ertrag angeführt, da Reis nach jeder Ernte neu ausgesät werden muss, während man im Fall der Kakaobäume nach 
einem einmaligen Aufwand etwa 20 bis 25 Jahre lang automatisch ${ }^{141}$ Ernteerträge erhält. Besonders auf der Ostseite der Lore-Lindu-Region, in Palolo und Lore Utara, verweisen in bezug auf die Neuerschließung von landwirtschaftlichen Landflächen zahlreiche lokale Bauern auf das vorhandene Adat-Land, den Boden ihrer Vorfahren. Dieses befindet sich in den Dörfern des südlichen Palolo und des nördlichen Lore Utara zu einem Großteil auf dem Gebiet des Lore-Lindu Nationalparks. Hieran zeigt sich auch die ökologische Dimension des angesprochenen Zukunftsszenarios (vgl. u.a. auch Kap. 6.3). ${ }^{142}$ Der Finanzrahmen der Mehrzahl der lokalen Bauern ist allerdings so gering, dass sie durch das starke Absinken des Kakaopreises in ihren Wohlstandsaussichten auf jeden Fall wieder weit zurückgeworfen würden. Seine Reisfelder würde deshalb auch kaum einer der Befragten antasten, da sie die existentielle Ernährungsgrundlage dieser Menschen darstellen.

Interessant ist auch die Überlegung, wie sich ein solches Zukunftsszenario auf die Entscheidungen jener Haushalte auswirken würde, welche ausschließlich Kakao anbauen, also auf die Haushalte der Kakaomigranten. Diese würden sich in der Mehrheit abwartend verhalten, ihren landwirtschaftlichen Bestand gegebenenfalls auch durch zusätzliche Anbauprodukte mit aktuell hohen Verkaufspreisen zwischenpflanzen. Sie gehen meist nicht davon aus, dass sich eine Krise des Kakaopreises lange Zeit halten würde und spekulieren darauf, dass sie diese Phase dank ihrer finanziellen Rücklagen überbrücken könnten.

Die Ergebnisse zeigen, dass, besonders bei der lokalen Bevölkerung, ein Zukunftsdenken nur schwach ausgeprägt ist. Vielmehr sind den Menschen aber die Erfahrungen aus der Vergangenheit mit der Krise des Kaffee- oder Gewürznelkenpreises bewusst. Ebenso ist ihnen klar, welch wichtige existenzsichernde Funktion der Besitz eines Reisfeldes besitzt. Es kann daher zwar davon ausgegangen werden,

141 Natürlich müssen Kakaofelder ständig gepflegt werden, sollen sie gute Erträge liefern. Allerdings bestätigen die meisten befragten Bauern der Untersuchungsregion, dass der Aufwand für ein Kakaofeld deutlich geringer ist, als der für ein sawah-Feld. In dem Dorf Maranatha häuften sich bei den Interviews und in informellen Gesprächen hingegen die positiven Aussagen über den Nassreisanbau. Zwar gebe es eine arbeitsintensive Phase der Anpflanzung und der Ernte, während der Monate des Pflanzenwachstums bliebe jedoch genügend Zeit für andere Aktivitäten, wie etwa einer Beschäftigung im Dienstleistungssektor (z.B. Motorradtaxi-Fahrer). Zur Relativierung sei angefügt, dass sich Maranatha, inmitten der Ebene des Palu-Tals, nicht in einer für die Kakaoproduktion günstigen geographischen Lage befindet und nur über einen geringen Anteil an Kakaofeldern verfügt. In den 1980er und 1990er Jahren sind einige Familien nach Palolo oder Lore Utara gezogen, um dort Kakao anzubauen. Der größte Teil der Dorfbevölkerung lebt weiterhin vom Nassreisanbau und unterhält höchstens einige Kakaobäume direkt vor oder hinter dem Wohngebäude.

142 Ruf \& Yoddang (2001: 106) verweisen daneben auf die enormen potentiellen Umweltschäden durch den Kakaoboom: In Regionen, in welchen die Kakaobäume bereits über 20 Jahre alt sind, zeigen sich meist deutliche Erosions- und Degradationserscheinungen in den Talbereichen. Neben dem Kakaoanbau trägt jedoch die Holzwirtschaft durch Kahlschlagverfahren eine maßgebliche Mitschuld an diesen Umweltveränderungen. 
dass das Gros der Bauern im Fall des genannten Szenarios je nach dessen Ausprägung den erhofften Wohlstand nicht würde erlangen können. Es würde jedoch maximal auf den Status quo vor Beginn des Kakaobooms zurückgeworfen. Da zudem das Kreditwesen in der Lore-Lindu-Region nur gering ausgeprägt ist, müsste auch nicht mit einer signifikanten Verschuldungsentwicklung gerechnet werden, ausgelöst durch unvorsichtige Anschaffungen von Statussymbolen in dem Glauben an eine stetig steigende Einkommensentwicklung. Der Wohlstandsmotor Kakao steht zwar gegenwärtig auf tönernen Füssen, das Zerbrechen der Füße, um diese Metapher weiter zu bemühen, führt jedoch in der Regel nicht zu einem freien Fall an die Tiefe.

\subsubsection{Vom nachbarschaftlichen Arbeitskräftetausch zur Lohnarbeit}

Die Veränderungen in der Landnutzung der Lore-Lindu-Region erwirkten auch einen gravierenden Wandel in bezug auf die sozioökonomischen Arbeitsbeziehungen. Grund dafür ist in erster Linie die Kapitalisierung aller Lebensbereiche menschlicher Interaktion. Vor dem Einfluss der Niederländer bestanden Tauschbeziehungen, auf Makroebene zwischen den Königreichen (kerajaan), auf Mikroebene unter Mitgliedern einer Verwandtschaftsgruppe (kin group). Die Makrobeziehungen lassen sich für das Untersuchungsgebiet vor allem zwischen dem kerajaan Sigi und dem kerajaan Kulawi belegen. Sigi hatte in vorkolonialer Zeit als größtes Königreich Zentralsulawesis nach Ausfechtung zahlreicher Kriege einen Dominanzstatus gegenüber Kulawi erworben. Die Eintracht zwischen Hoch- und Tieflandgruppen wurde in Form von Geschenkgaben aufrecht erhalten (Aragon 1996d: 48ff), welche im Abstand von mehreren Jahren erfolgten (Henley 2004: 8). Auf der Mikroebene bestand ein System des verwandtschaftlichen Arbeitskräftetauschs auf der Basis genereller Reziprozität. Diese hebt sich von der direkten Reziprozität dadurch ab, dass eine erbrachte Leistung der einen Seite der Tauschbeziehung keine direkte Gegenleistung erwarten lässt. Statt dessen geht dieses System davon aus, dass sich die Leistungen beider Seiten auf lange Sicht gesehen ausgleichen werden. Falls nicht auf beiden Seiten der Tauschbeziehung eine moralische Grundverankerung gegeben ist, welche die Voraussetzung für eine Nivellierung der jeweils geleisteten Dienste oder Güter bildet, kippt das System und es entsteht eine negative Reziprozität. ${ }^{143}$ Der verwandtschaftliche Austausch von Arbeitskräften für die Feldbestellung, welchen Schrauwers als Teil einer „moral economy“ (1999: 114) identifiziert, entwickelte

143 Ein klassisches Beispiel hierfür ist das kapitalistische Lohnarbeitssystem, welches der Arbeitskraft als Leistung der einen Seite die Lohnzahlung als Leistung der anderen Seite gegenüberstellt. Die Ungleichheit erfolgt dabei durch die Einbehaltung des Mehrwerts der Ware, der durch die Arbeitskraft produziert wurde. 
sich aus dem traditionellen Modus des Wanderfeldbaus. Waldflächen für neue Anbaufelder wurden von den Familien eines Clans gemeinschaftlich gerodet. Dabei fällten alle Mitglieder erst die Fläche für das Feld der einen Familien. Danach rodeten alle die Fläche für das Feld der nächsten Familie. Erst nachdem alle Flächen gerodet waren, begannen die einzelnen Familien mit der Feldbestellung. In dem Dorf Lawe (Pipikoro-Region) hat sich diese Methode bis heute erhalten. Für andere Gemeinden, welche gegenwärtig noch Wanderfeldbau betreiben (in erster Linie handelt es sich dabei um die Pipikoro-Region), wird diese Kontinuität ebenfalls berichtet. Im Laufe der Zeit hat dieses Prinzip in weiten Teilen der Lore-LinduRegion an Bedeutung verloren (vgl. auch Burkhard 2002b: 33). ${ }^{144}$

Die Landbesitzverhältnisse in den Dörfern der Lore-Lindu-Region änderten sich maßgeblich durch den Zuzug von Migranten aus Südsulawesi. Jene Migranten, die bereits während der 1960er und 1970er Jahre in die Region kamen, erhielten meist freies Waldland von den Dorfchefs zur Verfügung gestellt. In Berdikari (Kec. Palolo) zählten Menschen aus Tanah Toraja zu den ersten Zugewanderten, abgesehen von den Kulawi-Leuten, die das Dorf gegründet hatten. Die in den 1960er Jahren zugewanderten Toraja erhielten ein Waldstück zugeteilt, das sie roden und anschließend bewirtschaften konnten. In den 1980er Jahren zugewanderte Familien mussten bereits größtenteils Land kaufen. Während wohlhabende Migrantenfamilien in der Lage waren Land zu kaufen, konnten ärmere Migranten auf die sozialen Netzwerke innerhalb ihrer ethnischen Gruppe zurückgreifen. Oft wird ein Verwandter, z.B. ein Onkel, angegeben, der in dem Ort bereits ansässig war und seine Verwandten aufgefordert hatte, ebenfalls dorthin zu ziehen. Die Neuankömmlinge erhielten dann in der Regel ein kleines Stück Land geliehen, durch dessen Wertschöpfung sie später in der Lage waren, selbst Land zu kaufen. Manche verdingten sich zuerst solange als Lohnarbeiter, z.B. in einer Reismühle oder als Rattansammler, bis sie sich den Kauf eines Stückes Land leisten konnten.

Speziell in den Dörfern, deren verfügbare Landfläche durch die Einrichtung des Lore-Lindu-Nationalparks verkleinert wurde und in welche eine große Anzahl von kaufkräftigen Migranten zugewandert war, nahmen die Lohnarbeitsverhältnisse v.a. während des letzten Jahrzehnts des 20. Jh. besonders zu. ${ }^{145}$ Zuvor ergaben sich Beschäftigungsmöglichkeiten im Lohnarbeitsbereich hauptsächlich außerhalb des eigenen Dorfes, z.B. in Palu oder Poso, durch die Ausdehnung des sekundären und

144 Für seine Untersuchungsdörfer in Palolo und Lore Utara kommt Burkard (2002b: 33f) sogar zu dem Ergebnis, dass eine Hilfe selbst von Verwandten nicht mit Sicherheit erwartet werden könne. Vielmehr würden sich Neid und Missgunst unter der lokalen Bevölkerung ausbreiten, welche sogar so weit gehen würden, dass andere beschuldigt würden, faul zu sein. Letzteres wird aus den eigenen Befragungen v.a. von jenen Migranten gegenüber der lokalen Bevölkerung geäußert, die sich selber eine hohe tägliche Arbeitsintensität bescheinigen.

145 Nach Schwarze (2004: 54) ist der Anteil der in der Landwirtschaft beschäftigten Lohnarbeiter im regionalen Vergleich innerhalb der Untersuchungsregion in Palolo am höchsten. 
tertiären Wirtschaftssektors. Die Veränderung der Landbesitzverhältnisse in den Dörfern schaffte schließlich auch dort einen größer werdenden Arbeitsmarkt. Viele lokale Bauern bestellten vor dem Einfluss insbesondere der Bugis aus Südsulawesi vergleichsweise kleine Landparzellen. Aufgrund der wachsenden Nachfrage nach Land durch die Migranten verkauften sie diesen Teile ihres brachliegenden Landes, um entweder die Schulausbildung ihrer Kinder finanzieren oder ihren Lebensstandard demjenigen anpassen zu können, der sich schon bald bei vielen der zugewanderten Familien offenbarte. Zwar wussten die lokalen Bauern, dass Erbteilung und Mitgift ihre eigenen Landressourcen angreifen würden. Sie hatten jedoch die Sicherheit der noch vorhandenen Landareale an den Hängen der Berge. Doch diese Areale wurden mit der Einrichtung des Nationalparks größtenteils unter staatlichen Schutz gestellt. Gleichzeitig stieg bei den Großgrundbesitzern unter den Migranten der Bedarf an Arbeitskräften, da sie nicht in der Lage waren, das gesamte Land selbst zu bestellen, oder weil sie selbst vorwiegend Handel betrieben und gleichzeitig über das nötige Kapital verfügten, Lohnarbeiter auf ihren Feldern anzustellen. Der Bedarf an Arbeitskräften wurde zudem durch Landbesitzer erhöht, die in Palu wohnten und im Zuge des Kakaobooms Land in den einzelnen Dörfern erworben hatten. Bei steigender Landverknappung kann davon ausgegangen werden, dass die Nachfrage nach Lohnarbeitsbeschäftigungen und die dadurch entstehenden Abhängigkeitsverhältnisse in der Lore-Lindu-Region in Zukunft weiter zunehmen werden.

\subsection{Landkonflikte - ein Problem der Zuwanderung?}

Nach Sunderlin \& Resosudarmo (1999) wird Bevölkerungswachstum oft zu voreilig als einzige Ursache für die fortschreitenden Entwaldungsprozesse in Indonesien angesehen. Auch wenn dabei dem Faktor Migration tatsächlich ein hoher Stellenwert zugemessen werden kann, ,it should be seen as an intermediate variable, and not as an independent variable. " (ebd.: 152). In der Lore-Lindu-Region ist der Zuzug von regionalen Migranten neben den Auswirkungen auf die Entwaldungsprozesse an den Grenzen des Lore-Lindu Nationalparks (vgl. Kap. 6.4) auch in bezug auf Landkonflikte nicht als eine unabhängige Einflussgröße zu werten. In der Untersuchungsregion lassen sich zwei Bereiche ausmachen, welche zu Landkonflikten führen. Der eine hängt mit staatlich geplanten Umsiedlungen zusammen, der andere mit Landverkäufen und der Zertifizierung von Land.

Ein Beispiel für den ersten Fall stellt der Landkonflikt zwischen Siliwanga, einer Transmigrationssiedlung und (ehemaligen) Bewohnern des benachbarten Dorfes Wanga (Kec. Lore Utara) dar (vgl. Abb. 16). Nachdem Anfang der 1990er Jahre Balinesen im Rahmen eines transmigrasi-Projekts auf einem Teil des Dorfterritoriums von Wanga angesiedelt worden waren, forderte die Regierung einige Familien aus Wanga auf, in Form einer transmigrasi sisipan nach Siliwanga umzusiedeln. Durch diese spezielle Form der Transmigration sollten sich die Familien aus Wanga zwischen den Familien aus Bali ansiedeln (sisipan), um eine engere Verbindung zwischen 
lokaler und zugewanderter Bevölkerung zu schaffen und einen Wissenstransfer über Technologien im Nassreisanbau von den Migranten zur lokalen Bevölkerung zu befördern. Dass sich schließlich Konflikte um das den jeweiligen Familien zur Verfügung gestellte Land aufkamen, kann nicht singulär auf die Tatsache zurückgeführt werden, dass eine Zuwanderung stattgefunden hat. Vielmehr liegt der Grund in einer ungenauen Landvergabe durch die Regierung, wodurch in einigen Fällen ein Stück Land doppelt vergeben wurde.

Im zweiten Fall, dem Komplex der Landverkäufe und -zertifizierungen, stellt die Zuwanderung ebenfalls nur eine ,intermediate variable" (Sunderlin \& Resosudarmo 1999: 152) dar. Anhand der geführten Interviews wird deutlich, dass die lokale Bevölkerung in der Lore-Lindu-Region lange Zeit über keinerlei staatlich zertifiziertes Land verfügte, obwohl die gesetzlichen Grundlagen nach Pamungkas (1995) bereits seit der Verabschiedung des Landwirtschaftsgesetzes (UU Pokok Agraria) von 1960 geschaffen waren. Landbesitzangelegenheiten waren - und sie sind es überwiegend noch heute - durch das Adat geregelt. Gerodetes Land wurde entsprechend markiert und dem Dorfrat mündlich angegeben, und war somit dauerhafter Besitz der jeweiligen Familie (vgl. Kap. 5.2.3). Es waren schließlich Migranten, v.a. aus Südsulawesi, die Land von der lokalen Bevölkerung aufkauften und dieses danach zum Teil staatlich zertifizieren ließen. In Dörfern wie Sintuwu (Palolo) oder Watumaeta (Lore Utara) kam es daraufhin teilweise zu Landkonflikten, welche ihre Ursache jedoch vornehmlich in den illegalen Verkaufspraktiken der jeweiligen Dorfchefs hatten. Diese verkauften Land der lokalen Bevölkerung - in manchen Fällen handelte es sich sogar um Grundstücke, die sich auf dem Territorium des Nachbardorfes befanden - ohne deren Wissen an kaufkräftige Migranten. Hatten die jeweiligen Migranten ihr erworbenes Land bereits mit einem Besitzzertifikat auszeichnen lassen, fühlten sich die betroffenen ehemaligen Besitzer doppelt betrogen. Zum einen wurde ihr Land unrechtmäßig verkauft, zum anderen konnten sie gegen den Verkauf nicht weiter vorgehen, da sie selbst nicht einmal über ein Schriftstück verfügten, das ihnen hätte bescheinigen können, dieses Stück Land jemals besessen zu haben. Da die Migranten beim Kauf nicht darüber informiert waren, dass es sich um eine illegale Transaktion handelte, kann ihnen nicht der Vorwurf gemacht werden, wissentlich einen Landkonflikt provoziert zu haben.

Die beiden genannten Beispiele verdeutlichen, dass nicht die Migration an sich die Gefahr von Landkonflikten erhöht, sondern dass vielmehr die politisch-administrativen Rahmenbedingungen (z.B. staatliche vs. gewohnheitsrechtliche Landzertifizierung) und die Handlungsweisen einzelner Akteure (Regierung, Dorfchefs) ausschlaggebend sind. 


\section{$6 \quad$ Politische Einflüsse auf die Veränderungen der Kulturlandschaft}

\subsection{Die Ethische Politik der Niederländer}

\subsubsection{Der kolonialpolitische Entstehungsprozess der Ethischen Politik}

Während der Hochphase der sog. Befriedungspolitik in Aceh und anderen Gebieten der äußeren Inseln setzte in den Niederlanden ein Umdenken bzgl. der Kolonialpolitik ein. Der Journalist J. P. Brooshooft ${ }^{146}$ veröffentlichte 1901 eine Broschüre mit dem Titel „De ethische koers in de koloniale politiek“, in der er ein größeres Verantwortungsgefühl für die Einheimischen in der Kolonie einforderte und dabei auf „de edelmoedige drang van den sterkere om de zwakkere rechtvaardig te behandeln" (LocherScholten 1981: 177) verwies. Bereits zwei Jahre vor Brooshooft hatte C. Th. Van Deventer ${ }^{147}$ in seinem Artikel „Een eereschuld“ die Ausbeutung der Kolonie angeprangert. Neben einer finanziellen Wiedergutmachung dieser Schuld ${ }^{148}$ forderte Van Deventer ${ }^{149}$ auch einen moralischen Ausgleich ein: „Rechtschaffenheit, Redlichkeit und Pflichtbewnßtsein sollten künftige Leitgedanken der Kolonialpolitik bilden. "(Wedema 1998: 1). Nachdem auch die niederländische Königin in ihrer Thronrede im Jahr 1901 eine moralische Berufung aussprach, unterstützte das Parlament in der Mehrheit diese Ethische Politik (Prince 1996: 35).

Im Jahr 1849 wurde die Frage des Ministers der Kolonien, ob, unter Berücksichtigung des Schutzes der einheimischen Bevölkerung, dessen Gewährung erste Pflicht der Regierung wäre, noch Inseln im indischen Archipel mit Aussicht auf eine erfolgreiche europäische Kolonisation ausgewiesen werden könnten, nach diversen Untersuchungen noch verneint (Metzger 1892: 19f). Mit dem Aufkommen der Mo-

146 J. P. Brooshooft war Chefredakteur der in Semarang ansässigen Tageszeitung De Locomotief.

147 Conrad Theodor van Deventer wurde 1857 in den Niederlanden geboren. Er studierte ab 1875 in Leiden Jura und promoviete 1879 zum Thema „Zijn naar de Grondwet onze koloniën deelen des rijks?“" 1880 ging er nach Niederländisch-Indien und kehrte 1897 wieder nach Europa zurück. Zwei Jahre später ließ er seine Eindrücke in den Artikel „Een eereschuld“ in der Zeitschrift De Gids einfließen (Encyclopaedie 1921, Bd. 1: 594).

148 Zwischen 1867 und 1878 gingen 187 Mio. Gulden als Transfer aus der Kolonie in die Niederlande (Cribb 1993: 226). Mit Beginn der Ethischen Politik wurden 30 Mio. Gulden für die Wohlfahrt in Niederländisch-Indien bereitgestellt (ebd.: 234).

149 Neben Van Deventer waren auch Snouck Hurgronje, Van Vollenhoven, Van Kol u.a. Verfechter der Ethischen Politik (Middendorp 1929: 45). 
ral in der Kolonialpolitik wandelte sich diese Sichtweise. Gerade um die Bevölkerung zu schützen, musste nach der Logik der Niederländer diesen Menschen eine mit „westlichen Werten“ erfüllte „Entwicklung“ zuteil werden, die jedoch erst nach der „Befriedung“ der jeweiligen Gebiete und der Installation einer niederländischen Verwaltung in Gang kommen konnte.

Beflügelt wurde die Entwicklung einer „ethischen“ Politik ${ }^{150}$, wie bereits zuvor die liberale Phase, u.a. durch den von Douwes Dekker unter dem Pseudonym „Multatuli“ veröffentlichten Roman „Max Havelaar“, der als Insiderbericht eines Beamten die Situation in den Kolonien anprangerte. Von einem akademischen Zirkel in Leiden, welcher die Ethische Politik thematisiert und in die Kolonialpolitik gehoben hatte, wurde dieser Roman mit Vorliebe rezitiert. Nach Van Niel wurde dieser Roman fälschlicherweise als Anklage gegen das Zwangsanbausystem und gegen den Kolonialismus an sich gedeutet. Aber:

"If there is one thing that Multatuli was not, it was an anti-colonialist. Quite to the contrary he believed, along with the Europeans of his day, that colonial rule was essentially good for native peoples. What he wanted to see was a colonialism that applied rules of justice and equality and did not exploit the population, especially the defenceless little man. "(Van Niel 1991: 22).

Zwar beriefen sich die Gegner des cultuurstelsel 151 auf diesen Roman, doch, so Van Niel, ,the little man was no better off under the new governing system; in fact, many would argue that he came to be noticeably worse off." (ebd.). Auch unter dem Banner der Ethischen Politik taten sich nach Otterspeer (1989:204) große Unterschiede auf zwischen dem, was die Professoren in Leiden mit dieser moralischen Neuausrichtung verbanden, und der Umsetzung in der Kolonialpolitik. Bereits 1879 hatte Abraham Kruyper, der Gründer der calvinistischen Anti-revolutionaire Partij, welche 1898 die Laissezfaire-Politik der wirtschaftsliberalen Ära ablöste, im damaligen Parteiprogramm von „ethischem“ Kolonialismus gesprochen. Kruyper ging von einer moralischen Ver-

150 In dieser Arbeit werden die Schreibweisen „Ethische Politik“ und ,'ethische’ Politik“ synonym verwendet. Die Verwendung der Anführungszeichen bei der Kleinschreibung von ,ethische“ soll die Tatsache unterstreichen, dass die Bedeutung dieses Begriffs bei der praktischen Umsetzung dieser Politik nur bedingt zutraf.

151 Der Übergang vom „Kaufmanns“kolonialismus zum Staatskolonialismus wurde vornehmlich durch die Einführung des sog. cultuurstelsel, eines Systems des staatlich gelenkten Zwangsanbaus, geprägt. Begründet hatte dieses System der Niederländer Johannes van den Bosch, welcher 1830 in Niederländisch-Indien tätig war und von 1834 bis 1839 die Leitung des Kolonialministeriums übernahm (Encyclopaedie 1921, Bd. 1: 382ff; Frank 1911: 22; Ricklefs 1981: 114). Durch einen Regierungsbeschluss von 1830 waren alle Wälder und alle noch nicht in Besitz genommenen Ländereien zu Staatseigentum erklärt worden. Ein Fünftel der bewirtschafteten Fläche musste von nun an mit Exportprodukten wie Kaffee, Tee, Indigo, Zucker und Tabak bepflanzt werden. Die Kolonialregierung nahm diese Kontingente zu einem festgesetzten Preis ab. Die Arbeitskräfte für die Plantagen wurden durch sog. heerendiensten, Zwangsarbeitsdienste, oder gegen einen Minimallohn über die lokalen Fürsten rekrutiert (Frank 1911: 24; Kötter et al. 1979: 86). 
pflichtung aus, die Kontrolle über immer größere Gebiete der Kolonie zu erstrecken. Seine Partei forderte zudem eine stärkere Missionstätigkeit unter der einheimischen Bevölkerung. Einer seiner Schüler, A. W. F. Idenburg, wurde bald nach seinem Erscheinen auf der politischen Bühne Kolonialminister und war von 1909 bis 1916 Gouverneur-Generaal von Niederländisch-Indien (Schöffer 1978: 87; Wedema 1998: 17).

Doch was war nun der Kern der Ethischen Politik? Der Sozialist Henri H. Kol fasste diesen folgendermaßen zusammen:

„Das Wobl der Eingeborenen, ibre physische, mentale und moralische Entwicklung muss das böchste Ziel unsrer Kolonialpolitik sein. Die ökonomische Evolution zu befördern, mit der größtmöglichen Minimierung der unvermeidlichen Belastung der Übergangszeit, ist unsere vornehmste Aufgabe. Die unzü̈htige Beherrschung und die kapitalistische Ausbeutung unserer Kolonien muss einer Staatskunde gegenseitiger Verantwortlichkeit platzmachen. "(1901: 7). ${ }^{152}$

Boomgaard (1993: 248) stimmt Cribb (1993) zu, dass keine Zielvorgaben bzgl. nationalem Pro-Kopf-Einkommens, Reisertrags pro Hektar oder Kindersterblichkeit gesetzt wurden, sondern es wurde von den Politikern der vage Begriff der volksverheffing (Heraushebung der Bevölkerung aus seiner Situation) propagiert. Die Entwicklung des Anbaus ausreichender Nahrungsmittel war nach Prince (1995: 35) eines der zentralen Aufgabenfelder dieser Wohlfahrtspolitik: „One did hope to achieve one's aims by agricultural research, by agricultural education and by agricultural extension. "Die Emanzipation ${ }^{153}$ der einheimischen Bevölkerung war ein weiterer zentraler Punkt jener Politik. Diese sollte nicht nur in wirtschaftlicher, sondern auch in politischer Sicht die lokale Elite aus ihrer Passivität holen und am politischen Leben teilhaben lassen. Dabei ergab sich jedoch das Dilemma, dass diese Emanzipation auf keinen Fall die niederländische Herrschaft in Frage stellen durfte (Wedema 1998: 2).

Nach Dahm (1990: 69) waren diese Bildungsmaßnahmen die Voraussetzung für einen modernen indonesischen Nationalismus, wie er sich im 20. Jh. herausbildete. Sich entwickelnde Forderungen nach Mitsprache wurden von den Niederländern durch die Einrichtung von Gremien wie dem Volksraad, ${ }^{154}$ welcher keine politischen

152 Zitat im Original: „Het welzijn der inboorlingen, hun physieke, verstandlijke en moreele ontwikkeling moet het hoogste doel zijn onzer koloniale politiek. De economische evolutie te bevorderen, met verzachting zooveel moglijk van het onvermijdelijk overgangstijdperk, is onze voornamste taak. Het baatzuchtig beheer en de kapitalistische exploitie onzer koloniën moet plaats maken voor een staatkunde van zedelijke verantwoordelijkheid.“"

153 In Anlehnung an Dahm (1974) versteht der Verfasser der vorliegenden Arbeit unter Emanzipation den Bewusstwerdungsprozess der kolonisierten Bevölkerung, der durch westliche Bildung und soziale Besserstellung v.a. bei deren Eliten zu einem Einheitsbewusstsein führte. Die eigentliche Erlangung der Unabhängigkeit im anti-kolonialen Sinn ist davon zu trennen.

154 Der Volkssaad, welcher am 18. Mai 1918 zum ersten Mal zusammentrat, hatte 39 Sitze. 19 Mitglieder (davon zehn einheimische) wurden gewählt, 19 (davon fünf einheimische) wurden nominiert. Der Vorsitzende wurde von der niederländischen Krone ernannt (Palmier 1962: 17). 
Vollmachten besaß, befriedigt. 155 Die Ethische Politik sollte eine „Entwicklungshilfe" ${ }^{156}$ für die einheimische Bevölkerung darstellen. Das sog. Gidslandsgedachte, das Konzept einer Führungsnation, trat mit dem Sendungsbewusstsein auf, den Niederlanden einen europäischen Vorbildstatus in Sachen „ethischer“ Politik einzuräumen (Wedema 1998: 18). Mit Van Vollenhoven, welcher nach Kuitenbrouwer (1991: 322) das militärische Vorgehen der Kolonialmacht in den sog. Außenbesitzungen mit der Bezeichnung seines Landes als der "Jeanne d'Arc unter den Nationen“ zu legitimieren versuchte, zeichnete sich eine Instrumentalisierung der Ethischen Politik zur Imagepflege der niederländischen Kolonialpolitik ab.

Laut Kuitenbrouwer (1991: 18) stellten Nationalismus, Gidslandsgedachte und die Ethische Politik die drei Säulen der Propagierung eines ,non-imperialistic image "dar, an dem den Niederlanden um die Wende zum 20. Jahrhundert gelegen war. Coté (1996: 89) sieht die Ethische Politik als kulturelle Transformation mit dem Anliegen, die ökonomische Basis der Kolonialmacht neu zu formulieren. Die Ethische Politik verfehlte seiner Meinung nach sein Ziel, und die Idee eines idealistischen europäischen Nationalismus wurde zur Praxis eines neuen Imperialismus. „Colonialism and this particular phase in colonialist expansion was central in the reconstitution of nationalism in Dutch society in metropolitan Europe as it was in the case of every other colonial power. "(ebd.).

Nach Coté war die Politik von Beginn an weder emanzipatorisch noch humanitär, sondern allein für staatsbildende, herrschaftsfestigende Absichten ausgelegt worden. Humanismus war nur ein Alibi, um mit der Überzeugung von einer Überlegenheit westlicher, technisch-rationaler Kultur in indigene Gesellschaften eingreifen zu dürfen. „The white man's burden“ und andere Prädikate dienten den neuen Imperialisten als Banner für ihre Politik (ebd.: 89f).157

155 Doch selbst da hielt sich die Kolonialmacht selten an die Gesetze. „Noch weniger Federlesens freilich machte die Kolonialregierung mit den sogenannten „Extremisten', Nationalisten, die es wagten, außerhalb der auf regionaler und kommunaler Ebene geschaffenen Räte für die Unabhängigkeit zu werben und die Bevölkerung gegen die Kolonialmacht aufzuwiegeln. Gegen diese „undankbaren Gesellen', die sich weigerten, auf den für die Nationalisten geschaffenen „Spielwiesen $^{6}$ zu verbleiben und die statt dessen die offene Konfrontation suchten, gab es genügend Paragraphen in den Strafgesetz-Bestimmungen, und in Zeiten von wirtschaftlicher Notlage wurde hart durchgegriffen." (Dahm 1990: 69).

156 Mit dem Begriff der sog. Entwicklungshilfe wird bis in die heutige Zeit meist ein Überlegenheitsdenken transportiert, dem die Argumentation zugrunde liegt, die Empfängerseite dieser Hilfe sei unterentwickelt. Die Definitionsmacht über die Bedeutung dessen, was unter Entwicklung und der daraus gefolgerten Unterentwicklung zu verstehen ist, behält sich i.d.R. die Geberseite vor.

157 Nach Cotés (1996: 89f) Ansicht waren auch das Streben der einheimischen Bevölkerung nach mehr Mitsprache und die Gründungen politischer Organisationen, welche eine Unabhängigkeit von der Kolonialmacht einforderten, nicht durch die Ethische Politik entstanden. Vielmehr beflügelte ein Gefühl moralischer Überlegenheit nationalistischer Prägung in der niederländischen Kolonialpolitik den entstehenden indonesischen Nationalismus. Dahm (1990: 69) hingegen ist der Überzeugung, dass erst die im 20. Jahrhundert einsetzenden Bildungsmaßnahmen der Kolonialregierung die Voraussetzung für einen modernen indonesischen Nationalismus waren. 
Locher-Scholten (1981) grenzt sich von Coté ab. Ihr ist bewusst, dass das „ethische“ Konzept auch expansionistische und herrschaftssichernde Elemente in sich barg. Daneben sieht sie aber auch den Entwicklungscharakter darin enthalten. Die einheimische Bevölkerung sollte in Richtung einer niederländisch geführten und nach westlichem Vorbild funktionierenden Selbstverwaltung „entwickelt“ werden (Locher-Scholten 1981: 201). Ethische Politik definiert sie als ,[...] darauf gerichtet, den gesamten indonesischen Archipel unter die reale Herrschaft der Niederlande zu bringen, und auf die Entwicklung von Land und Volk dieses Gebiets in die Richtung von Selbstverwaltung unter niederländischer Leitung und nach westlichem Modell." (ebd.). ${ }^{158}$ Unter der Doppelzielsetzung „Land und Volk“ sei einerseits (Land) die Ausbreitung von landwirtschaftlichen Betrieben und Verbesserungen der Infrastruktur zu sehen, andererseits (Volk) der Ausbau von Bildung und Gesundheitswesen sowie die Beteiligung der einheimischen Bevölkerung an der Verwaltung zu verstehen. Das westliche Modell beinhalte u.a. auch die in den 1920er Jahren einsetzende Vereinheitlichung des Rechts für alle Einwohner der Kolonie. Der Begriff der Selbstverwaltung (zelfbestuur) ist als indirekte Herrschaft mittels lokaler Fürsten und deren „Beamten“ zu sehen. 159 Er hat nichts mit dem emanzipatorischen Gedanken von Selbstverwaltung zu tun oder mit jener Forderung, die indonesische Nationalisten zur Durchsetzung der Unabhängigkeit ausgaben.

Ferner sieht Locher-Scholten die Ethische Politik als Einheit und Entgegenstellung, wie sie sich in den beiden Blöcken von Vaderlands Club ${ }^{160}$ und N.S.B. ${ }^{161}$ einerseits sowie der Stuw-Gruppe ${ }^{162}$ andererseits wiederfinden (vgl. Abb. 46). Erstere plädierten für die Reichseinheit, einem bleibenden Band mit den Niederlanden. Ihnen standen die Verfechter eines Indië voor Indië gegenüber, die ontvoogding (wörtlich übersetzt: „Entvormundung ${ }^{6}$ ), also eine politische und verwaltungsmäßige Emanzi-

158 Zitat im Original: ,[...] gericht op het onder reël Nederlands gezag brengen van de gehele Indonesische archipel èn op de ontwikkkeling van land en volk van dit gebied in de richting van zelfbestuur onder Nederlandse leiding en naar westers model."

159 Auf die Einrichtung der Selbstverwaltungen mittels sog. Korte Verklaringen (Kurzdeklarationen) wurde bereits in Kap. 2.3.3 genauer eingegangen.

160 Der 1929 gegründete Vaderlandse Club sprach mit seinem rechtsnationalistischen und wirtschaftsfreundlichen Programm v.a. die niederländischen Siedler in der Kolonie an (Wedema 1998: 11).

161 Nationaal-sozialistische Beweging. Diese faschistische Bewegung ging aus einer Vielzahl kleiner Organisationen hervor. Ab 1933 war sie auch in Niederländisch-Indien aktiv. Nach anfänglichen Erfolgen hatte die Bewegung mit Ausbruch des 2. Weltkriegs fast alle Einheimische und Europäer im Archipel gegen sich (Blok et al. 1978: 397).

162 Die Stuw-Gruppe setzte sich aus 13 Personen zusammen, die im politischen Leben Batavias nicht unbekannt waren. Durch ihre Positionen hatten sie meist mehr Kontakt mit der einheimischen Bevölkerung als ihre Amtskollegen in den Niederlanden. Parteipolitisch lagen sie nicht auf einer Linie, können aber grob unter der Bezeichnung , liberal“ zusammengefasst werden. Unter dem Einfluss der Ideen von Snouck Hurgronje und Van Vollenhoven wurde dieser Gruppe bewusst, dass eine Entwicklung auf der Adat-Kultur basieren müsste. Ihre Zeitschrift De Stuw erschien von 1930 bis 1934 in Batavia als Gegenstimme zum Vaderlandse Club. (Locher-Scholten 1981: 119 u. 211). 
pation forderten. Beide Seiten plädierten auf ihre Weise für Verbesserung des Status quo im Sinne einer Wohlfahrtspolitik, beide bezogen sich auch auf die indonesische Gemeinschaft. Sie unterschieden sich aber in ihren Urteilen über staatskundliche, bildungspolitische und wirtschaftliche Ideen. „Man kann sagen, dass die Anti-Ethici sich im bewahrenden Sinn mehr an die Masse richten, während die Neo-Ethici die Entwicklung der Elite verfolgten."(Locher-Scholten 1981: 54). ${ }^{163}$

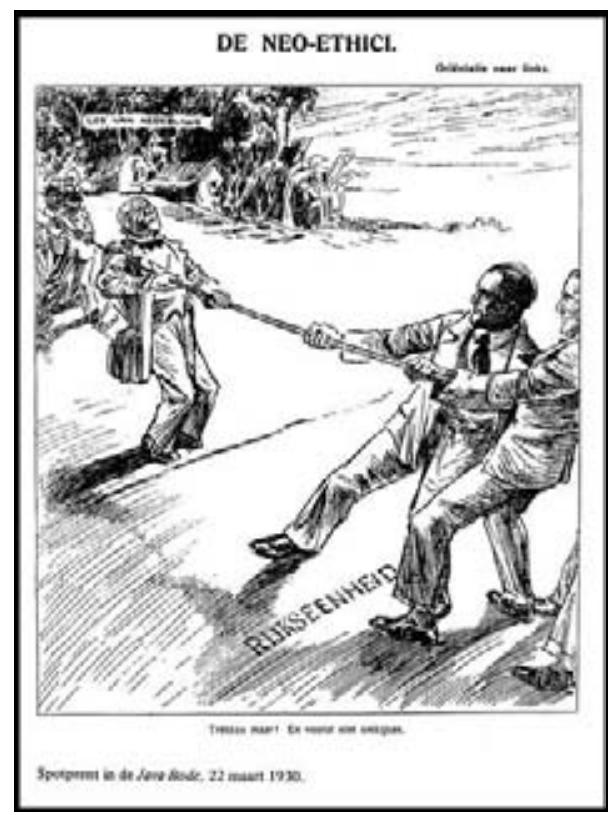

Abb. 46 Karikatur über den politischen Kampf zwischen der Stuw-Gruppe und dem Reichseinheitsblock: „Zieht doch! Und vor allem nicht umschauen.“

(Quelle: Locher-Scholten 1981: nach 72)

Nach Wedema (1998: 7) betonen Locher-Scholten wie Kuitenbrouwer das angeblich moralisch überlegene Nationalismusverständnis der Niederlande, die im Gegensatz zum Imperialismus Großbritanniens oder Frankreichs keinem zynischen Profit- und Machtstreben in den Kolonien erlegen seien. Die Niederlande vermieden es also lange Zeit im Interesse eines ,non-imperialistic image" (Kuitenbrouwer 1991: 18), ihre Kolonialpolitik der Expansion auf die bis dahin kaum erschlossenen Außenbesitzungen als imperialistisch zu bezeichnen und bemühten dazu die Ab-

163 Zitat im Original: „Men kann zeggen, dat de anti-ethici zich in konserverende zin meer op de massa richtten, de neo-ethici de ontwikkeling van de elite volgden." 
grenzung von der Politik ihrer europäischen Rivalen. ${ }^{164}$ Vielmehr war ihnen daran gelegen, ihr Land als ein Musterland der „ethischen Entwicklungshilfe“ zu präsentieren. Caldwell \& Utrecht kommen zu dem Ergebnis, dass die „Entwicklungspoli-

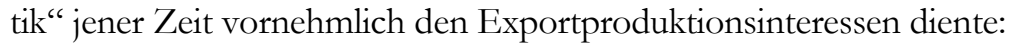

„In fact, the expenditures undertaken were all of most benefit to the foreign companies and the colonial administration, in providing improved roads, ports and other facilities, and in providing healthier and harder working labourers and slightly better educated but low level clerical employees. "(1979: 33).

Sie legen damit die Ethische Politik als reines Mittel zum Zweck bar jeder moralischen Einsicht bloß. Ähnlich äußert sich Semaoen bereits 1927: „Mit der Absicht, eine Monopolstellung zu erobern, kam Holland zu der Politik der ,Einmischung' in indonesische Angelegenheiten. "(Semaoen 1927: 12).

Die Anfangsjahre des 20. Jh. waren geprägt von einer boomenden Zucker- und Kopraproduktion und ermöglichten es somit, Bestandteile der Ethischen Politik umzusetzen. Die Situation verschlechterte sich für den Kolonialstaat nach der ersten Dekade aber zunehmend. „Colonial Indonesia was bound to the Netherlands by burden of its debt.", fasst Prince (1996: 67) die Situation zu Beginn der 1930er Jahre zusammen, die Niederländisch-Indiens Waren auf dem Weltmarkt unattraktiv, weil zu teuer, machte. Der Archipel versuchte sich von seinem Mutterland zu lösen, doch erst 1936 gelang es den Politikern in Batavia, sich gegen die Torpedierungen seitens des Kolonialministeriums in Den Haag und der Bank der Niederlande zu behaupten und den Gulden abzuwerten. ${ }^{165}$ Da sich die Niederlande aber weiterhin ihre Position im Kolonialhandel sichern konnten, veränderte sich für Niederländisch-Indien nur wenig (ebd.). Durch seine einseitige Ausrichtung auf den Export von Agrarprodukten und Bodenschätzen sowie seine Situation als Schuldnerland traf die Wirtschaftskrise von 1929 die Kolonie besonders schwer. Die Exportpreise fielen stärker als die des Imports und bescherten Niederländisch-Indien eine fortschreitende Verschlechterung seiner Terms of trade. Panikkar umschreibt die beiden Jahrzehnte um die Weltwirtschaftskrise folgendermaßen:

„Auch in Indonesien und Indochina merkten die Kolonialbehörden, daß der Kolonialimperialismus im Rückgang war. Wie in Indien ist auch hier das Buch der Geschichte für jene Epoche voll von Konflikten, ausgedehnten nationalen Erbebungen, brutalen Unterdrückungen durch Kolonialregierungen und zwischendurch schwächlichen Versuchen, einen ,goldenen Mittelweg' zu finden,

164 Wedema beschreibt in diesem Zusammenhang eine Facette der politischen Auseinandersetzung: „Als die niederländische Linke auf die Parallele zwischen britischem Imperialismus und dem niederländischen Verhalten in Atjeh verwiesen, reagierten ibre politischen Gegner mit einer nationalistisch motivierten Rechtfertigung: Im Gegensatz zum unzivilisierten "Piratennest' Atjeh bandele es sich bei den Burenrepubliken um Gebiete mit Bevölkerungen niederländischer Abstammung." (1998: 17).

165 Seit 1854 war der niederländisch-indische Gulden an den niederländischen Gulden gekoppelt (Prince 1995: 28). 
auf dem sich die nationalen Bestrebungen mit den materiellen Interessen der ,Mutterländer'vereinbaren ließen."(1955: 282).

Ähnlich wie über dessen wahres Wesen besteht auch über das Ende der Ära der Ethischen Politik in der Wissenschaft keine Einigkeit. Cribb (1990: 8) sieht ein vorläufiges Ende 1920 erreicht, als erkannt wurde, dass das Ziel, die Kolonie als sich selbst tragende Einheit aufzubauen, nicht erreicht werden konnte. Kipp (1990: 33) zieht das Aufkommen von nach Unabhängigkeit strebenden und sich öffentlich artikulierenden Gruppen aus den Reihen der einheimischen Bevölkerung heran, wonach spätestens 1913 ein Ende zu setzen wäre. Locher-Scholten (1981: 206 u. 213) teilt die Zeit in drei Perioden auf. Von 1894 bis 1905 wurden die letzten Gebiete der äußeren Inseln „befriedet“ und auf ein „Entwicklungsprogramm“ vorbereitet, bis 1920 wurde diese „Entwicklung“ der einheimischen Bevölkerung nach „ethischen“ Grundsätzen umgesetzt, während die Jahre bis 1942 von einer Konsolidierungsphase bestimmt waren. Letztere Phase setzt bei Wedema 1927 an, dem Jahr, „in welchem die Kolonialmacht nach der erfolgreichen Niederschlagung einer angeblich von Kommunisten angezettelten Aufstandsbewegung zu einer deutlich repressiveren Politik gegenüber einheimischen Organisationen überging. "(1998: 3).

\subsubsection{Die Bedeutung der Ethischen Politik für die Lore-Lindu-Region}

Aus den kolonialen Quellen über die Aktivitäten der Niederländer in der LoreLindu-Region lassen sich Elemente dieser Ethischen Politik finden. Neben der Förderung des Anbaus von Exportprodukten wie etwa Kopra (vgl. Henley 2002), wurde ein besonderer Wert auf die ausreichende Versorgung der Bevölkerung mit Grundnahrungsmitteln gelegt. Hierzu wurden der Nassreisanbau enorm ausgedehnt und - hauptsächlich, aber nicht ausschließlich, im Palu-Tal - großflächige Bewässerungssysteme installiert (vgl. Hengel 1910: 24 u. 74; Hirschmann 1934: 31; Logeman 1922: 63f; Van Tideman 1926: 5 u. 44; Vorstman 1935: 20). Im Zusammenhang mit den lokalen Zwangsumsiedlungen von den Bergen in die Täler tauchen in den Berichten der örtlichen Kolonialbeamten einige Stellen auf, welche den Einfluss der Ethischen Politik auf das koloniale Handeln vermuten lassen. Vorstman hält zum Beispiel angesichts der offenbaren Unzufriedenheit der umgesiedelten Bevölkerung mit ihrer neuen Wohnumgebung in seinem Bericht an das Kolonialministerium im Mutterland fest: „Man sollte diese Menschen dann auch einfach in ihre Berge zurückkehren lassen. "(1935: 13). ${ }^{166}$

Allerdings zeigen sich auch Interessenkonflikte der Kolonialherren zwischen dem Wohl der Bevölkerung einerseits und den Wünschen der Kolonialverwaltung

166 Zitat im Original: „Men zou deze zielige menschen dan ook graag naar hun bergen laten terugkeeren." 
nach wirtschaftlicher Erschließung der Region und Kontrolle der Bewohner andererseits. Ein Beispiel hierfür liefert die Argumentation bezüglich des Baus eines Weges in die westlich des Palu-Tals gelegene Pekawa-Bergregion:

\begin{abstract}
„Das sog. Pekawa-Bergland ist durch diesen Weg aus seiner Isolierung entrückt. Ich will nicht behaupten, dass die Bergbevölkerung selbst dies sehr schätzt, doch vom Standpunkt der Verwaltung aus ist der Weg von großer Bedeutung, und die Ebenenbevölkerung soll hier eifrig mit dem Anbau von Gemüse, Kaffee und anderer Hochlandkulturen beginnen." (Vorstman 1935: 8$) \cdot{ }^{167}$
\end{abstract}

Als ein Entgegenkommen gegenüber der lokalen Bevölkerung verweist Vorstman im Zusammenhang mit dem Steuerrückstand dieser Menschen auf den Dienst im Straßenbau und argumentiert, dass ,,[...] hierdurch der nichts besitzenden Bergbevölkerung die Gelegenheit gegeben wird, ihre ausstehenden Steuerzahlungen (fast drei und mehr Jahre lang wurde nichts bezablt) durch Arbeit in nicht allzu großem Abstand von ibrem Wobnort und in geeignetem Klima zu begleichen. "(ebd.). ${ }^{168}$ Aus der Sichtweise der Niederländer setzten mit der Erschließung der sog. Äußeren Inseln des Archipels Fürsorge für die und Entwicklung der lokalen Bevölkerung nach westlichem Modell als Element der Ethischen Politik ein. Diese neue Politik war jedoch im Grunde nur ein probates Mittel zur Umsetzung der kolonialen Interessen. Die angestrebte sog. Indisierung der Verwaltung erforderte z.B. die Einrichtung eines Schulsystems. Dieses stellte gleichzeitig einen Beleg für die „Entwicklungshilfe“ der Niederlande als Bestandteil der Ethischen Politik dar. Nach Aussagen von Zeitzeugen war es jedoch zunächst nur den traditionellen Eliten gestattet, die Schulen zu besuchen. Bildungseinrichtungen höheren Grades waren ausschließlich dieser Bevölkerungsgruppe vorbehalten. Für die einfache Bevölkerung reichte es den Kolonialherren aus, wenn diesen der Umgang mit Geld für eine ordnungsgemäße Steuerentrichtung gelehrt wurde.

Mittels der Ethischen Politik wurde die Kulturlandschaft der Lore-Lindu-Region weiterhin orientiert an den Interessen der Kolonialmacht verändert, wobei dieser Wandel von den Niederländern jedoch im Vergleich zur Kolonialpolitik des 19. Jh. als grundsätzlich zum Wohle der Lokalbevölkerung begriffen wurde. Die Errichtung eines Straßen- und Wegenetzes diente zwar in erster Linie der Schaffung einer Infrastruktur für den Absatz von landwirtschaftlichen (Export-)Produkten, sie sollte die Bewohner entlegener Gebiete aber zugleich aus ihrer Isolation befreien. Die lokalen Zwangsumsiedlungen wurden mit dem Zweck besserer Kontrolle und

167 Zitat im Original: „Het z.g. Pekawa bergland is door deze weg uit zijn isolement verlost. Ik will niet bewerden dat de bergbevolking zelf hierop erg gesteld zal zijn, maar uit bestuursoogpunt is de weg van veel belang en de vlaktebevolking zal hier graag groenten, koffie en andere bergcultures beginnen."

168 Zitat im Original: ,,[...] hierdoor de niets-bezittende bergbevolking in de gelegenheid wird gesteld haar achterstallige belastingen (vaak 3 en meer jaren was niets betaald) in arbeid te voldoen op niet to groote afstand van hun woonplaats in een voor hen passend klimaat." 
Eintreibung der Steuern begründet, sollte den Betroffenen aber auch einen größeren Infrastrukturzugang ermöglichen. Zudem wurden diese Umsiedlungsprojekte, wie bereits erwähnt, nicht so rigide umgesetzt wie in anderen Regionen zuvor. Die Niederländer ermöglichten eine Schulausbildung, auch wenn diese vornehmlich der Ausbildung von Befehlsempfängern für die Kolonialverwaltung diente und das niederländische Personal entlasten sollte, und die höheren Bildungsebenen nur den einheimischen Eliten vorbehalten wurde. Daneben beeinflussten die Niederländer auch das Gesundheitswesen, einem nach Locher-Scholten (1981:201) weiteren Kernbereich der Umsetzung ,ethischer“ Politik. Als häufigste Krankheiten im Untersuchungsgebiet wurden von den Kolonialbeamten Malaria, Influenza, Frambösie, ${ }^{169}$ Ruhr und Lepra ausgemacht. Die Konzentration der Bevölkerung in den neu gegründeten Siedlungen (vgl. Kap. 4) erhöhte die Gefahr der Entstehung und Ausbreitung von Krankheiten. So brach im Jahr 1918 in Zentralsulawesi eine InfluenzaEpidemie aus, die u.a. durch die vorherrschende Malaria noch verstärkt wurde (Logeman 1922: 74f). 1934 wurde die Malaria v.a. am Lindu See als so schlimm eingeschätzt, dass die gesamte Bevölkerung dieses Gebietes einer viertägigen Tabletten-Kur unterzogen wurde (Vorstman 1935: 29). Um Epidemien vorzubeugen, ordneten die Kolonialverwalter schon frühzeitig an, dass die Behausungen, Wege und Plätze in den Dörfern sauber zu halten seien. Bereits 1913 erwähnt Grubauer (1913: 557f) diese Verordnung und die Furcht der Bewohner vor Überprüfungen der Sauberkeit in den Dörfern durch Kolonialbeamten. ${ }^{170}$

\subsection{Bildungspolitik und die Bedeutung von Bildung für die ökonomische Entwicklung}

Zu vorkolonialer Zeit war die (Aus-)Bildung auf den familiären Bereich beschränkt und betraf einerseits die Weitergabe landwirtschaftlicher und gewohnheitsrechtlicher Kenntnisse. Erst mit der niederländischen Eroberung wurde in der Lore-LinduRegion unter dem Einfluss der sog. Ethischen Politik ein staatliches Schulsystem eingeführt (vgl. Kap. 6.1). Im Jahr 1910 existierten bereits Schulen in Biromaru, Sidondo (beide heute im Kec. Sigi-Biromaru) und in Lemo (späteres Bolapapu; Kec.

169 Tropische Infektionskrankheit mit himbeerähnlichen Hautwucherungen.

170 Beim Erscheinen Grubauers im Dorf Búro (das heutige Puro'o) am Lindu See begannen die Frauen des Dorfes hastig damit, die Umgebung der Hütten sauber zu fegen. Der Forschungsreisende hält weiter fest: „Diese ungewöbnliche Wirkung meines Erscheinens hing mit einer Gowvernements-Verfügung zusammen, die es den an Reinlichkeit nicht gewöhnten Eingeborenen zur Pflicht machte, Wobnungen und Dorplatz stets in Stand zu balten. Die Befolgung dieser zur Besserung der sanitären Verbältnisse gerade am LinduSee sebr notwendigen Maßregel wird von Zeit zu Zeit durch den in Lemo stationierten Inlandsassistenten nachgeprïft, und wer die Leute auch nur flüchtig kennengelernt hat, versteht, daß sie einen heiligen Respekt vor solchen Visiten baben."(Grubauer 1913: 557f). 
Kulawi) (Van Hengel 1910: 40). Ab 1933 wurde von der Kolonialregierung das Schulgeld eingeführt, welches zu zahlreichen Absenzen, v.a. in Kulawi, führte und daher in dieser Teilregion bald wieder eingestellt wurde (Vorstman 1935: 30). In Wanga (Kec. Lore Utara), das seinerzeit als Sitz des raja noch der Hauptort des Napu-Tals war, wurde 1931 eine Sekolah Rakyat (SR; Volksschule) errichtet.

Von großer Bedeutung für die Entwicklung des Schulsystems in den HochlandRegionen war die christliche Missionierungstätigkeit. Die Missionare waren gleichzeitig Priester und Lehrer. Die errichteten Kirchengebäude - anfangs nur einfache Häuser - dienten beiden Zwecken. Während anfangs besonderer Wert auf eine schulische Ausbildung der höchsten Schicht der lokalen Gesellschaftsstratifikation, also der „Adeligen“, gelegt wurde, um das für eine erfolgreiche Verwaltung ihrer Einflussgebiete durch die Niederländer (z.B. im Bereich der Steuerangelegenheiten) notwendige Grundwissen zu erlangen, wurden im Rahmen der fortschreitenden Missionierung auch die anderen gesellschaftlichen Schichten in den Schulunterricht mit einbezogen. Um die Bevölkerung sowohl für die religiöse, als auch für die schulische Ausbildung zu gewinnen, arbeiteten die Missionare mit Hilfe von Geschenken wie Süßigkeiten, Seife etc. (vgl. auch Aragon 1992, 1996d). Die gewöhnliche Bevölkerung erhielt während der Kolonialzeit i.d.R. eine dreijährige Grundschulausbildung. Der Besuch höherer, administrativer Schuleinrichtungen war weiterhin der lokalen Führungsschicht vorbehalten.

Besonders während der Ära der Orde Baru unter Präsident Suharto wurde das Schulsystem im Rahmen der Fünf-Jahres-Pläne (repelita) für die Entwicklung des Archipels ausgedehnt. Eine Vielzahl an staatlichen Schulen, sog. Sekolah Dasar Instruksi Presiden (SD Inpres; Grundschulen auf Instruktion des Presidenten) wurden auch in der Lore-Lindu-Region errichtet, und es wurde eine sechsjährige Schulpflicht gesetzlich beschlossen. ${ }^{171}$ Daneben entstand eine Reihe von weiterführenden Schulen (SMP, SMA, SMK etc.). Während die höheren Schulen anfangs nur in den Städten Palu und Poso existierten, wurde dieses Angebot sukzessive auch auf die politischen und ökonomischen Zentren der einzelnen Kecamatan ausgedehnt. Dennoch war - und ist bis heute - der Zugang selbst zu Grundschulen von der finanziellen Situation der einzelnen Familien abhängig.

Das Fallbeispiel des Dorfes Pandere (Kec. Sigi-Biromaru) weist eine deutliche Differenzierung des Grads der Schulausbildung zwischen lokaler Bevölkerung und den aus Manado (Nordsulawesi) zugewanderten Familien auf. In diesem Dorf stellt sich dieser Unterschied sogar räumlich dar, da die Migranten aus Nordsulawesi die Bevölkerungsmehrheit in Dusun 2 ausmachen, während sich die lokale Bevölkerung auf die Dorfteile 1 und 3 konzentrieren. Die Befragungen haben ergeben, dass die lokalen Kaili im Vergleich zu den Manado-Familien der Schulausbildung ihrer Kin-

171 In Indonesien dauert die Grundschulausbildung sechs Jahre. 
der nur einen sehr geringen Stellenwert beimessen. So erklärten mehrere Respondenten in Dusun 1, dass es ihnen ausreichte, wenn ihre Kinder die ersten Jahre der Grundschule absolvierten. Die Kinder müssten doch nur rechnen, schreiben und lesen können, so die Meinung, und wären danach als Arbeitskraft in der familiären Landwirtschaft viel wichtiger. Allein anhand der Ausstattung der Wohnhäuser in den drei Dusun ist eine deutliche Unterscheidung der finanziellen Verhältnisse der beiden ethnischen Gruppen zu erkennen. Es ist daher zu vermuten, dass die finanziellen Möglichkeiten eine, wenn auch kaum offen ausgesprochene, dennoch vorhandene Basis für diese ablehnende Haltung der lokalen Bevölkerung darstellen. Da Manado bereits im 19. Jh. von den Niederländern erobert worden war, genoss die dortige lokale Bevölkerung vergleichsweise früh bereits eine schulische Ausbildung. Dadurch hatten die ab 1929 nach Pandere zugewanderten Familien einen deutlichen Bildungsvorsprung, welchen sie entsprechend frühzeitig in Form ökonomischer Wertschöpfung umsetzen konnten. Daneben spielt aber auch die Religion eine gewisse Bedeutung. Die christlich missionierte Manado-Bevölkerung Panderes kann von der Nähe zu den im Nachbarort Kalawara ${ }^{172}$ vorhandenen Missionsschulen profitieren, während den muslimischen Kaili-Familien nur die staatliche Grundschule zur Verfügung steht.

Die empirischen Untersuchungen in der gesamten Untersuchungsregion haben allerdings ergeben, dass die Bereitschaft bzw. der Wunsch der Eltern, ihren Kindern eine höhere Schulausbildung zu ermöglichen, nicht allein davon abhängig ist, ob die Eltern selbst bereits einen höheren Schulabschluss haben oder ob sie über die ausreichenden finanziellen Möglichkeiten dafür verfügen. Viele ärmere und geringer gebildete Eltern geben an, dass sie sich wünschen, dass ihre Kinder jene Ausbildungsmöglichkeiten nutzen können, die ihnen selbst nicht möglich waren. Dazu sind sie auch bereit, das benötigte Kapital zu akquirieren. Die Tatsache, dass viele Familien Land verkaufen, um die schulische Ausbildung ihrer Kinder zu finanzieren, untermauert diese Selbsteinschätzungen.

Es lässt sich somit sagen, dass sich in weiten Teilen der Bevölkerung im Laufe der Zeit ein gewisses Bewusstsein über die Bedeutung schulischer Ausbildung für die ökonomischen Zukunftschancen entwickelt hat. Mit der ab Mitte der 1990er Jahren begonnenen Ausdehnung des lukrativen Kakaoanbaus auf den Feldern der lokale Bevölkerung und der Vergrößerung des schulischen Angebots auch in Regionen, die sich in größerer Distanz zur Provinzhauptstadt Palu befinden, kann angenommen werden, dass der Anteil der lokalen Bevölkerungsgruppen am tertiären Wirtschaftssektor deutlich zunehmen wird. Abhängig ist dies, wie auch schon oben angesprochen, jedoch nicht unwesentlich von der individuellen Setzung von Präferenzen. So kann z.B. unter der Gruppe der zugewanderten, wohlhabenden Bugis

172 Das Dorf Kalawara wurde 1906 als Transmigrationsdorf für christliche Javaner gegründet (vgl. Kap. 3.4.2). 
beobachtet werden, dass sich wirtschaftliche Prosperität oft stärker in der Anschaffung von Statussymbolen (Kraftfahrzeuge, Fernseher etc.) äußert als in dem Grad der Schulausbildung der Kinder.

\subsection{Kontinuitäten und Brüche traditioneller Herrschaftsstrukturen}

Mit der Verabschiedung des Regionalen Verwaltungsgesetzes (Gesetz Nr. 1/1957) wurden bereits während der Herrschaftsperiode von Präsident Sukarno sämtliche autonomen Verwaltungseinheiten abgeschafft, welche bis zu diesem Zeitpunkt noch von Sultanen oder traditionellen Führern regiert worden waren. Dennoch dauerte es in Zentralsulawesi beispielsweise noch bis in die aktuelle Reform-Ära (reformasi), bis das Amt des Provinzgouverneurs nicht mehr entlang der Verwandtschaftslinie der vorkolonialen Herrscherdynastie weitergegeben wurde. Kontinuitäten alter Herrschaftsstrukturen zeigen sich auch in der Lore-Lindu-Region. Bis 1998 stellten die Nachkommen des traditionellen Fürstengeschlechts Lamakarate den Camat von Sigi-Biromaru. Der Ehemann der Tochter der letzten Königin (raja) von Lore ${ }^{173}$ war noch Anfang des 21. Jh. Bürgermeister des Dorfes Wanga in Lore Utara.

In Bolapapu (Kec. Kulawi) gibt der frühere Mitarbeiter und spätere Leiter der lokalen Schul- und Erziehungsbehörde und gleichzeitiger Nachfahre einer der traditionellen „Aristokraten“familien, Jore Pamei, zwei Beispiele für die latent noch immer vorhandenen Strukturen der vorkolonialen Klassen- und Schichtengesellschaft in Kulawi. Zum einen berichtet er von einer Begebenheit in seinem Haus: Ein Mitglied der traditionellen Schicht der Sklaven sucht das Haus des Erzählers auf und möchte mit dessen Ehefrau sprechen. Pamei bittet die Person ins Haus mit der Bemerkung, seine Frau halte sich im Moment in der Küche im hinteren Bereich des Gebäudes auf. Der Gast wiederholt seine Bitte und bleibt weiterhin an der Haustür stehen, da er weiß, dass es sich bei den Gastgebern um ,aristokratische“ Nachfahren handelt. Die klassische Gesellschaftsordnung untersagt den Angehörigen der Sklavenschicht, das Haus von „Adeligen“ zu betreten. Pamei schließt seine Erzählung mit der Anmerkung, dass es einer mehrmaligen Aufforderung bedurfte, bis der Gast schließlich in tief gebeugter Haltung am Hausherren vorbei zur Küche ging. Die andere berichtete Gegebenheit spielt sich innerhalb des Dienstverhältnisses in der Schul- und Erziehungsbehörde ab. Pamei erklärt, dass die traditionelle Gesellschaftsordnung im Dienst keine Beachtung mehr findet. Ist sein Vorgesetzter Angehöriger einer traditionell niedrigeren Klasse/Schicht, so führt Pak Jore selbstver-

173 Die Gebiete Tawaeli (heute: Sedoa, nördlich des Napu-Tals gelegen), Napu (Lore Utara), Besoa (heute Teil des neu gegründeten Kecamatan Lore Tengah) und Bada (Lore Selatan) sind seit 1916 unter dem Kollektivbegriff Lore bekannt (Koloniaal Verslag 1917: 35). 
ständlich dessen Anweisungen aus. Außerhalb des Dienstverhältnisses kommt es jedoch durchaus vor, dass sich sein Vorgesetzter ihm gegenüber entsprechend der traditionellen Ordnung verhält.

Diese Beispiele weisen auf die selbst rund 50 Jahre nach der offiziellen Abschaffung traditioneller Klassen- und Schichtstrukturen bestehenden Kontinuitäten hin. Sie zeigen auch auf, welche Bedeutung die Regelungen im Gewohnheitsrecht (adat) auch heute noch haben. Die traditionelle soziale Stratifikation der ethnischen Gruppe ist zwar offiziell abgeschafft, dennoch spielen sie unterschwellig eine wichtige Rolle im alltäglichen Zusammenleben. Gegen Ende der 1980er Jahre wurde für die Dorfebene per Gesetzentscheid ein entscheidender Schritt hin zur Demokratisierung vormals eher monarchischer Vergabemuster für politische Ämter vollzogen. Das Gesetz von 1979 zur Neustrukturierung der indonesischen Verwaltungseinheiten legte auch die Direktwahl des Bürgermeisters durch die Dorfbevölkerung fest. Somit waren es nicht mehr allein die Familien eines Dorfes, welche traditionell die soziopolitische Autorität darstellten, die über die Ernennung eines ihrer Mitglieder zum Dorfoberhaupt bestimmten. Trotz dieser Veränderungen zeigen die oben genannten Beispiele, dass sich die traditionellen Machtverhältnisse bis in das 21. Jh. gehalten haben.

Die Ära der Orde Baru während der Regierungszeit von Präsidenten Suharto zementierte eine politische Gesellschaftsstruktur, deren Facetten einen einschneidenden Einfluss auf die Ausprägung der Kulturlandschaft der Lore-Lindu-Region hatten. Zwei der Untersuchungsdörfer, Sintuwu und Watumaeta, stehen beispielhaft für die Auswirkungen der Jahrzehnte langen nationalen Politik autoritär-hierarchischer politischer Strukturen unter der Suharto-Regierung, die bis auf die Dorfebene reichten. Ein streng hierarchischer Aufbau der Gesellschaft war vor der Regierungsübernahme Suhartos in Indonesien nicht unbekannt. Die oben erwähnte vorkoloniale soziale Stratifizierung war ebenso hierarchisch aufgebaut und wurde auch unter der Kolonialherrschaft in ihrem Kern nicht angetastet. Auch nach der Unabhängigkeit Indonesiens führte allein schon die zentralistische Führung des Landes zu einer grundsätzlichen Beibehaltung der Top-down-Struktur. Es drängt sich folglich die Frage auf, warum nun gerade das Suharto-Regime hervorzuheben ist. Die Herrschaftsordnung, welche die traditionellen Fürsten als Regenten relativ autonomer Verwaltungseinheiten vorsah, wurde 1957 unter der Präsidentschaft von Suhartos Vorgänger, Sukarno, abgeschafft. Zehn Jahre später kam es zu einer Vermischung von traditionellen Rangordnungen in den Dörfern einerseits und der allgegenwärtigen Regierungspartei GOLKAR andererseits. Wie sich in Watumaeta sehr deutlich erkennen lässt, wird dort die Dorfregierung von Mitgliedern der alteingesessenen Dorffamilien gestellt. Diese genießt allein schon deswegen hohen Respekt bei der übrigen Dorfbevölkerung. Die gleichzeitige Mitgliedschaft in der Partei GOLKAR stattet den Bürgermeister zudem mit den Insignien absoluter Autorität aus.

Es ist gerade die Wahrnehmung der Dorfautoritäten als verlängerter Arm des autoritär-repressiven Systems des Suharto-Regimes, welche es z.B. Dorfbürgermei- 
stern während jener Zeit ermöglichte, ihr Amt zu Zwecken der persönlicher Bereicherung zu missbrauchen. In den Dörfern Sintuwu und Watumaeta nutzten die Dorfchefs diesen Machtstatus aus, um ohne öffentlich geäußerter Kritik illegale Landverkäufe durchzuführen (vgl. Kap. 5.4 und 6.4.2). In Watumaeta sollen zudem staatliche Zuwendungen in Form von Pestizidsprühgeräten, Saaten und ähnlichem nicht gleichmäßig an die Dorfbevölkerung, sondern nur innerhalb des Clans der traditionellen Herrschaftsfamilien verteilt worden sein. Erleichtert wird solches Handeln lokaler Autoritäten neben dem generellen Machtstatus durch eine, im Vergleich zu anderen Provinzen Sulawesis (Akib 2002; Aragon 2002) oder zu Java (Burkard 2002), hohe Intransparenz lokalpolitischer Entscheidungen und Belange. So ist es in der Lore-Lindu-Region nicht üblich, dass z.B. die Art und der Umfang staatlicher Zuwendungen in den Dörfern öffentlich gemacht werden (z.B. durch Aushänge).

Der Niedergang des Suharto-Regimes und die beginnenden Demokratisierungsbestrebungen des Landes ab 1998 führten im Fall des Dorfes Sintuwu zu offen geäußertem Protest der Lokalbevölkerung gegen das Agieren ihres Bürgermeisters und mündeten schließlich in dessen Amtsenthebung. Das neu eingesetzte Dorfoberhaupt agiert nun stärker im Interesse der lokalen Bevölkerung und versteht es dabei, durch diese Strategie eine lokalpolitischen Machtstatus, wenn auch in anderer Form, aufrecht zu erhalten. Das folgende Kapitel (6.4) wird u.a. aufzeigen, wie sich diese Strategie darstellt und welchen Einfluss sie auf den aktuellen Kulturlandschaftswandel hat.

\subsection{Das Spannungsfeld Nationalpark}

\subsubsection{Historische Entwicklung der Mensch-Wald-Interaktion in der Untersuchungsregion}

In Indonesien sind nach Lynch \& Talbott (1995: 22) zwischen 80 und 95 Mio. Menschen von der Ressource Wald abhängig. Der Bedeutung von Wald als existenzsichernde Grundlage ist sich auch die Bevölkerung in der Lore-Lindu-Region nicht generell unbewusst. Bereits vor hundert Jahren bemühten sich die Bewohner des waldarmen Südens des Napu-Tals intensiv darum, die wenigen jungen Baumtriebe zu schützen, um die Versorgung mit Brenn- und Bauholz zu gewährleisten (Kruyt 1908: 1285). Das lokale Gewohnheitsrecht beinhaltete Regelungen zum Umgang mit den Waldressourcen. Große Bedeutung kam hierbei der animistischen Glaubensvorstellung zu. Bevor das Christentum im Zuge der niederländischen Eroberung in den Bergregionen eingeführt worden war, glaubte die Lokalbevölkerung an von Geistern beseelte Bäume und Steine. In der Umgebung solcher Bäume durfte kein Holz geschlagen werden. Da die christlichen Missionare jedoch den animistischen Glauben verboten hatten, mag es nicht verwundern, wenn damit auch der Respekt der Bevölkerung vor der Ressource Wald nachließ. Ferner existieren bis 
heute Adat-Regeln, die den Kahlschlag an Uferböschungen verbietet, damit der Flusserosion entgegengewirkt werden kann. Vielerorts wurde diese Regel jedoch schon verletzt. Die unausweichliche Folge sind Überschwemmungen der dicht an den Flüssen angelegten Felder.

Diese Beispiele sollen nicht zu einem falschen, allzu romantisierenden Bild von der Mensch-Wald-Beziehung in der Lore-Lindu-Region verleiten. Auch wenn man die Einschätzung von Donner, ,that the local people on this island [Sulawesi; Anm. d. Verf.] do not understand or even care about ecological hazards" (1987: 178), für überzogen halten mag, darf die Realität ökologischer Waldschäden nicht übersehen werden. In der bereits zu vorkolonialer Zeit am dichtesten besiedelten Subregion, dem Palu-Tal, wurden schon in früher Zeit die unbewaldeten westlichen Berghänge erwähnt (Adriani \& Kruyt 1898: 467). Aufgrund der höheren Bevölkerungsdichte als in anderen Teilregionen konnten nur kurze Rotationszeiten für den Brandrodungswanderfeldbau eingehalten werden. Das trockene Klima des Palu-Tals beschleunigte die Degradierung der Hänge, was Verhoef (1937: 22) zu seiner Bezeichnung der PaluRegion als „stervend land" (sterbendes Land) veranlasste. Die Entwaldung dieser Berge stellte zu Beginn des 20. Jh. ein so großes Problem dar, dass der Kolonialverwalter Voorn (1925: 17f) dem Schutz der noch bestehenden Waldgebiete sowie der Wiederaufforstung die gleich hohe Dringlichkeit beimaß wie dem Ausbau der Bewässerungsanlagen für den Nassreisanbau. Neben der Einrichtung einer Waldschutzgrenze um das Palu-Tal, oberhalb derer keine Landwirtschaft mehr betrieben werden durfte, wurde unter niederländischer Führung auch die Wiederaufforstung mit Pinien durchgeführt (Vorstman 1935: 15). Ein noch heute sichtbares Beispiel hierfür sind die Pinienwälder nahe dem Ort Matantimali. ${ }^{174}$ Im Gegensatz zum PaluTal bestand beispielsweise in der Region Kulawi während der Kolonialzeit keine Notwendigkeit, Maßnahmen für den Schutz der Ressource Wald durchzuführen (ebd.). Allerdings haben Respondenten in Bolapapu (Kec. Kulawi) ausgesagt, dass bereits zur Zeit der niederländischen Kolonialherrschaft etwa $2 \mathrm{~km}$ östlich der Strasse Palu-Kulawi eine Waldgrenze eingerichtet wurde. Jenseits dieser Linie durften keine Felder angelegt werden.

Die von mehreren älteren Interviewpartnern bestätigte intensive landwirtschaftliche Tätigkeit an den östlichen Hängen des Kulawi-Tals (heute: vorwiegend vom Lore-Lindu Nationalpark eingenommen) während der ersten Hälfte des 20. Jh., wird auch von einer Fotographie Grubauers (1913) aus dem Jahre 1911 bestätigt. Das Foto (vgl. Abb. 47) zeigt die Berghänge hinter dem Dorf Lemo, die

174 Im Schatten dieser Pinien ließen die Niederländer auch eine sog. hill station errichten. Die Tochter des letzten raja von Palu erinnert sich noch daran, wie sie dort seinerzeit als Köchin eingesetzt wurde, während die Kolonialherren das kühle Klima und das Panorama auf die Palu Bucht, das Palu-Tal und bis in das Palolo-Tal genossen hatten. Am Rande sei noch angemerkt, dass die dort lebende Kaili-Da’a-Bevölkerung heute zu einer der ärmsten der Lore-Lindu-Region zählt. 
größtenteils bis hinauf zu den Bergkämmen gerodet und landwirtschaftlich genutzt wurden. In der Gegenüberstellung mit einem im Jahr 2001 aufgenommenen Foto (vgl. Abb. 48) wird der Einfluss des Verbots von Brandrodungsfeldbau und der Etablierung des Nationalparks besonders deutlich.

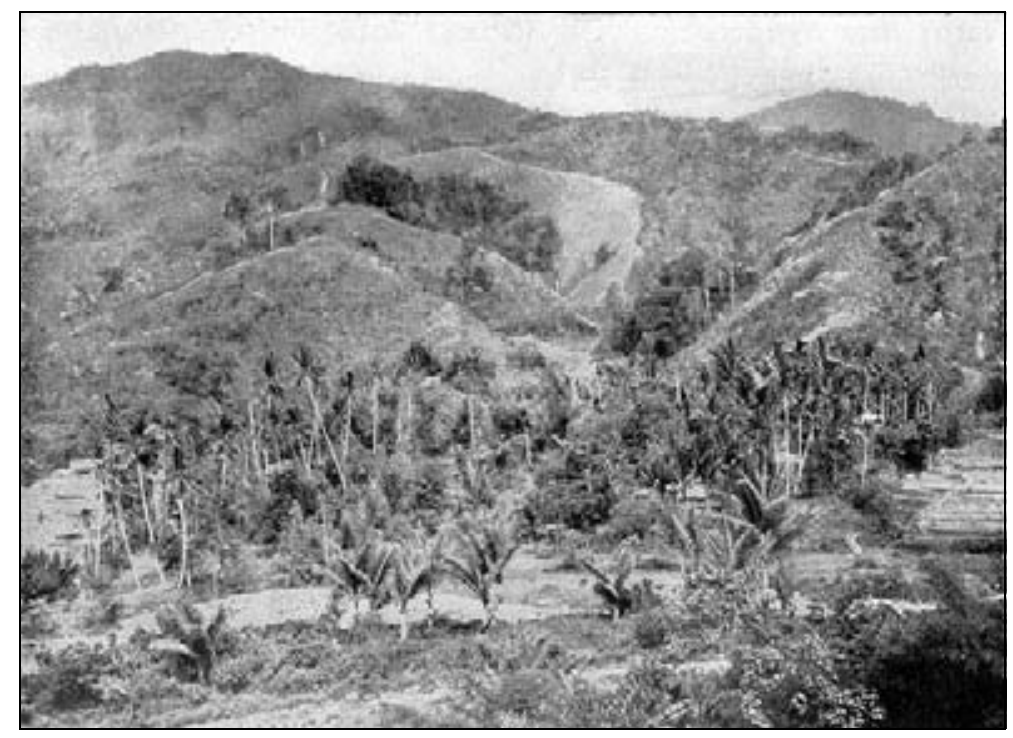

Abb. 47 Blick von Westen aus auf den Ort Lemo und die entwaldeten Hänge des heutigen Lore-Lindu Nationalparks 1911

(Quelle: Grubauer 1913: 551)

Vor dem Einfluss des Staates auf die Nutzung der Ressource Wald war ein offener Zugang zu Waldflächen gewährleistet. Für die lokale Bevölkerung stellte die Umwandlung von Wald in landwirtschaftliche Felder ein "quasi natural right" (Fremerey 2002: 7) dar, welches nur von einigen Adat-Regelungen, wie sie oben bereits erwähnt wurden, beschnitten war. Wie Burkhard (2002b: 15) deutlich macht, stellt die Landreserve Wald in erster Linie eine Grundlage für soziale Sicherungssysteme der Bevölkerung dar. Den von Burkhard (ebd.) insbesondere für die Regionen Palolo und Napu belegten Hauptbeweggrund für das Roden von Waldflächen, nämlich die Akquirierung von Ackerland für die nachfolgende Generation, bestätigen auch die Aussagen von Befragten aus dem westlichen Teil der Untersuchungsregion.

Bedingt durch den bereits während der Kolonialzeit begonnen Wechsel von der Wanderfeldwirtschaft zur Kultivierung permanenter Felder, insbesondere der Nassreisfelder, wurde in weiten Teilen der Lore-Lindu-Region der Druck von den Waldressourcen genommen. Zwar wirkten natürliche und durch Migration induzierte Bevölkerungszuwächse dieser Druckabnahme entgegen. Die Waldkonversion beschränkte sich jedoch vornehmlich noch auf den Waldbestand in den Tallagen (z.B. 
des Palolo-Tals). Bei zunehmender Bevölkerungsdichte wurde der Trockenanbau von Reis in den 1970er Jahren in vielen Teilregionen untersagt. Dies verstärkte die Inwertsetzung von Wald- und Grasflächen in den Talbereichen zusätzlich. Heute findet man diese Anbauweise mit Feldrotation nur noch in den entlegenen Gebieten Pipikoro (Kulawi) und südliche Lore-Region vor. Unter der Regierung Suharto wurden in einigen Gegenden Konzessionen an Holzfirmen vergeben. Im Palolo-Tal nahm z.B. die Firma PT Kebun Sari die Extraktion von Exporthölzern vor. Die sog. Jalan Jepang (japanische Strasse), benannt nach einem japanischen Ingenieur, welcher den Bau der Strasse leitete, entlang der Hänge des südlichen Talabschnitts wurde ausschließlich für diese Aktivitäten errichtet. Die Bevölkerung bewirtschaftete zwar Kaffee- und Gewürznelkenfelder an den Waldrändern und innerhalb des Waldes, niedrige Marktpreise dieser Produkte verhinderten aber eine großflächige Ausdehnung dieser Areale. Teilweise resultieren die geringen Ertragsaussichten sogar darin, dass manche Felder nicht mehr regelmäßig gepflegt oder komplett aufgelassen wurden.

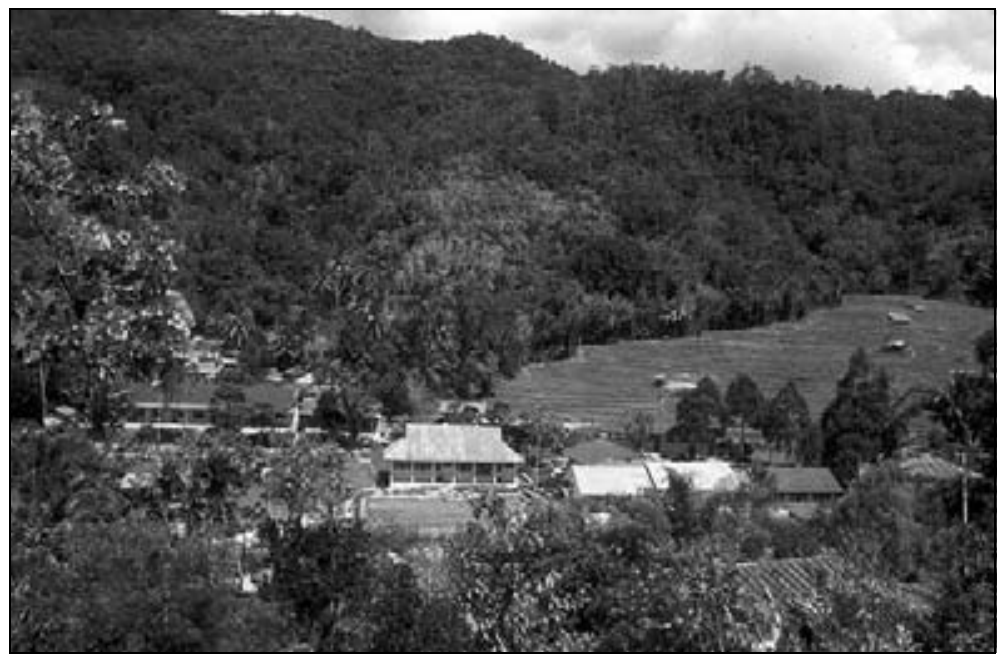

Abb. 48 Blick vom Regierungs-Gästehaus (ehem. Lemo) von Bolapapu aus nach Osten auf die Hänge des Lore-Lindu Nationalparks

(Quelle: Eigene Aufnahme, 2001)

Ab den 1980er Jahren führten zwei Ereignisse zu einem einschneidenden Wandel in der Mensch-Wald-Interaktion. Bugis-Migranten machten die Kakaopflanze in der Lore-Lindu-Region bekannt und kauften Land von der lokalen Bevölkerung. Im Jahr 1982 wurden zwei seit den 1970er Jahren bestehende Waldreservate, das Lore Kalamanta Wildlifereservat im Kabupaten Poso und das Lore Lindu Wildlifereservat im Kab. Donggala, zum Lore-Lindu Nationalpark zusammengefasst und vom Ministerium für Landwirtschaft eingerichtet. Der 231.000 Hektar umfassende Na- 
tionalpark wurde 1993 schließlich vom Forstministerium anerkannt (Anzdec 1997: 10). In vielen Bereichen des Nationalparks weist der Bestand von Sekundärwald auf die frühere landwirtschaftliche Nutzung des Areals hin (Schulze 2002). Im Zuge des Damar-Booms vor Beginn der Unabhängigkeit Indonesiens suchte die lokale Bevölkerung laut World Wild Life Found (1981:11) nahezu das gesamte Gebiet innerhalb der heutigen Parkgrenzen auf. Auch die ältere Generation der lokalen Bewohner der Untersuchungsregion weiß dies zu bestätigen. Mit dem Rückgang des Marktpreises für Damar und der mit der Zunahme der Nassreisbewirtschaftung einhergehenden, verstärkten Sesshaftigkeit der Bevölkerung ging die aufreibende Extraktion dieses Waldprodukts nach und nach zurück. Ab den 1960er Jahren waren nur noch sehr wenige Menschen im Damar-Geschäft aktiv. Zudem wurde das Sammeln von Damar schließlich von der Nationalparkverwaltung endgültig untersagt. Auch die Extraktion anderer Forstprodukte, wie etwa Rattan, wurde verboten. Bereits angelegte Kaffeefelder durften zwar weiterhin abgeerntet werden. Das Erweitern bestehender Felder oder gar das Anlegen neuer Agrarflächen war jedoch untersagt (ebd.). Das staatliche Verbot der Rattan-Extraktion bedeutet allerdings nicht, dass allein dadurch weniger Menschen dieses Waldprodukt sammelten. Erstens findet das Rattangeschäft in der Region weiterhin statt. Ein Teil dieses Rattan stammt aus den geschützten Zonen des Nationalparks. Aufgrund der Brisanz dieses Themas ist es jedoch kaum möglich, verlässliche Informationen, z.B. bzgl. des Umfangs der Rattanextraktion, zu erhalten. Dafür sind zu viele Interessengruppen (u.a. auch staatliche Stellen) im Rattanhandel involviert. Zweitens wird z.B. in Watumaeta und Wanga (Lore Utara) berichtet, dass Rattan ab den 1980er Jahren an Attraktivität verloren hat, weil die Menschen der vergleichsweise unbeschwerlicheren Bewirtschaftung von Feldern mit Kakao und anderen cash crops den Vorzug gaben. Zudem muss man in vielen Gebieten mittlerweile sehr tief in den Wald vordringen, um noch lohnenswerte Mengen an Rattan vorzufinden.

Der Wandel der Mensch-Wald-Interaktion in der Lore-Lindu-Region ist in den sozioökonomischen und soziokulturellen Veränderungen begründet. Vor dem Beginn der christlichen Missionierung hatte die Allbeseelung noch einen starken Einfluss auf die Nutzung der Waldressourcen. Mit dem nachlassenden Interesse v.a. der jüngeren Generation an den gewohnheitsrechtlichen Regelungen werden dem Wald zunehmend andere Attribute begemessen. Die wachsende Bedeutung von Landbesitztiteln und Agroforstproduktion zur Gewinnung von Marktprodukten wie Kakao zeigen die Ökonomisierung der Umwelt auf. Diese wird nun zur Ware, die zur Befriedigung neuer Bedürfnisse der Moderne verkauft werden kann. Wird die Verfügbarkeit der Ressource Land durch die staatliche Einrichtung von Waldschutzgebieten wie z.B. dem Lore-Lindu Nationalpark verknappt, erhält der Wald für die Bevölkerung einen bis dahin nicht wahrgenommenen wirtschaftlichen Wert und kann sich zum Fokus politisch-ökonomischer Konflikte entwickeln. 


\subsubsection{Der Dongi-Dongi-Konflikt und seine Folgen}

Die Nationalparkgrenze wurde nach der Parkgründung als zunächst noch vorläufig deklariert. Erst nach der Durchführung verschiedener Surveys zur Zonierung des Parkareals sollten die entgültigen Grenzen markiert werden. Nach Aussage der Vertreter mehrerer Dörfer, deren Dorfterritorium in den Nationalpark hineinreicht, fand die definitive Bestimmung der Parkgrenze nicht im Konsens mit der jeweiligen Dorfbevölkerung statt. Es gab beispielsweise keine Dorfversammlungen, in denen die Grenzziehung mit den staatlichen Vertretern diskutiert werden konnte. Dass die lokale Bevölkerung sich selbst nur bedingt aktiv für eine entsprechende Kommunikation einsetzte, dürfte der allgemeinen politischen-gesellschaftlichen Struktur während der Suharto-Ära geschuldet sein. In dieser Struktur herrschten klare Topdown-Entscheidungsprozesse vor. Kritik aus der Bevölkerung an staatlichen Vorhaben war nicht erwünscht und wurde repressiv unterbunden. Am Beispiel des Dorfes Watumaeta (Kec. Lore Utara) zeigen sich die Kommunikationsmängel bei der Festlegung der Parkgrenze. Seinerzeit hätten die Beamten der Nationalparkverwaltung ein Mitglied des Ältestenrates (lembaga adat) von Watumaeta, einen alten, gebrechlichen Mann, ausgewählt. Dieser sollte die Beamten in den Wald begleiten und zeigen, wo das tanah adat, das gewohnheitsrechtlich definierte Land der lokalen Dorfbevölkerung endet. Da der Mann aufgrund seiner physischen Konstitution keine weiten Strecken mehr zurücklegen konnte, sei die Grenze an dem Punkt definiert worden, bis zu dem der alte Mann zu gehen in der Lage war. Dieser Punkt befände sich jedoch noch weit vor dem Ende des $A$ dat-Gebiets.

NGOs unterschiedlicher Couleur versuchten, auf die Entscheidungsprozesse in bezug auf den Nationalpark einzuwirken. Als zwei Beispiele für die entgegengesetzten Positionen können die lokale NGO Yayasan Tanaha Merdeka (YTM; Organisation für ein freies Land) und die US-amerikanische NGO The Nature Conservancy (TNC) genannt werden. Erstere setzt sich vor allem für die Belange der lokalen Bevölkerung ein, während sich letztere explizit dem Naturschutz widmet. Die YTM konnte bereits im Zuge der Auseinandersetzungen um das staatliche Vorhaben einer Umsiedlung der Bevölkerung des Dorfes Katu im südlichen Lore Utara Erfolge verbuchen. Das Dorf bildet eine Enklave innerhalb des Nationalparks. Die Verhandlungen der NGO mit den staatlichen Behörden führten dazu, dass nur ein Teil der Bevölkerung, die des Dorfteils Dodolo, das Territorium des Nationalparks 1989 verlassen musste. Die Menschen aus Dodolo wurden nach Toe, einem ursprünglichen Teilgebiet des Dorfes Wanga (Kec. Lore Utara) umgesiedelt. Da sie den Namen ihres Herkunftsgebietes behalten durften, erhielt das Dorf im Zielgebiet (Toe) den Namen Dodolo.

Abgesehen von solchen punktuellen Auseinandersetzungen wurde die Existenz des Lore-Lindu Nationalparks von der Bevölkerung bis in die Mitte der 1990er Jahre weitgehend hingenommen. Maßgeblich dafür verantwortlich war einmal mehr das autoritär-repressive Regime unter Suharto. Mit dem Regierungswechsel von 1998, welcher dem Sturz Suhartos folgte, ging der Versuch einher, eine Demokrati- 
sierung Indonesiens herbeizuführen. Dezentralisierung und Regionale Autonomie ${ }^{175}$ waren neben demokerasi (Demokratie) fortan die Schlagworte, welche die Reformpolitik der späten 1990er Jahre begleiteten. Wie im gesamten Archipel wurde dieser Politikwechsel auch von der lokalen Bevölkerung der Lore-Lindu-Region als Chance begriffen, nun ihre Rechte und Ansprüche durchsetzen zu können. Vielerorts resultierte dies in blutigen Regionalkonflikten zwischen lokalen und zugewanderten Bevölkerungsgruppen, wie etwa in Zentralkalimantan, auf den Molukken, auf Flores oder in der Poso-Region Zentralsulawesis (vgl. u.a. Aragon 2000a, 2001; Freischlad 2002). Eine andere Ausprägung dieses politischen Wandels zeigte sich in der Untersuchungsregion in Form des sog. Dongi-Dongi-Konflikts.

Eine der Ursachen des Dongi-Dongi-Konflikts reicht bis in die 1970er Jahre zurück. Im Jahr 1979 wurde am südöstlichen Ende des Palolo-Tals das Dorf Kamarora gegründet, dessen Bevölkerung sich aus Migranten eines staatlich geplanten, lokalen Umsiedlungsprojekts zusammensetzte. ${ }^{176}$ Bereits fünf Jahre davor wurden Familien aus den Bergregionen des Kab. Donggala in das neu gegründete Dorf Rachmat umgesiedelt. Wie bereits in Kap. 3.4.1 erwähnt, wurden bei solchen Projekten von der Regierung in der Regel zwei Hektar Land pro umgesiedelter Familie versprochen. Oft wurden diese Vorgaben jedoch nicht eingehalten. Auch in Kamarora und Rachmat erhielten die Familien effektiv meist nur maximal einen Hektar Land, teilweise noch weniger. Während der repressiven Suharto-Regierung wurde dieser Tatbestand von der Bevölkerung anfangs weitgehend widerstandslos hingenommen. In den Jahren 1996 und 1998 erbat die Dorfbevölkerung von Kamarora eine Ausweitung der Landfläche, da die Einrichtung des Lore-Lindu Nationalparks das nutzbare Territorium des Dorfes enorm verringert hatte. Diese Bitte blieb jedoch von der Regierung unbeantwortet. Neben Kamarora und Rachmat waren auch andere Dörfer ${ }^{177}$ am südlichen Rand des Palolo-Tals, welcher an den Nationalpark grenzt, von der Beschneidung ihrer Dorfareale betroffen. Noch während der Suharto-Ära unternahm die lokale Bevölkerung Versuche, Teile des Dongi-DongiGebiets, welches sich entlang der Hauptstrasse von Palolo nach Lore Utara erstreckt und innerhalb der Grenzen des Nationalparks liegt, zu besetzen. Die Forstpolizei ging jedoch sofort gegen die Betreffenden vor und beschuldigte sie des illegalen Holzeinschlags. Ermutigt von der nationalen Reformpolitik und unterstützt von NGOs und Holzfirmen begannen im Juni 2001 mehrere Hundert Menschen, v.a. aus den Dörfern Kamarora und Rachmat, einen flächenhaften Kahlschlag des Waldes entlang der genannten Hauptstrasse. Innerhalb weniger Wochen wurden ca.

175 Das Gesetz 22/1999 regelt die Umsetzung der Regionalen Autonomie.

176 Es handelte sich dabei um Da'a- und Kulawi-Familien. Sie mussten ihre Herkunftsgebiete aufgrund eines vom Departmen Sosial und von der Forstbehörde geplanten Schutzareals für ein Wassereinzugsgebiet verlassen.

177 Hierbei handelt es sich um die Dörfer Bakubakulu, Bobo, Kadidia, Sigimpu, Sintuwu und Tongoa. 
1.500 ha Wald des Lore-Lindu Nationalparks gerodet und die Zufahrt in das Gebiet blockiert. Bis heute wurde keine Lösung für den Dongi-Dongi-Konflikt gefunden. Statt dessen siedelten sich im Laufe der Monate immer mehr Familien in dem Gebiet an. Mittlerweile existieren dort zwei Siedlungen mit Häusern, Feldern, Kirchen und kleinen Läden.

Doch die Rodungen im Dongi-Dongi-Gebiet und die Wahrnehmung einer nicht intervenierenden Staatsgewalt waren nur der Auslöser für eine räumlich viel weiter reichende Reaktion der lokalen Bevölkerung. Das Spannungsfeld Nationalpark und die Auseinandersetzung mit dem Problem verknappender Landressourcen dehnte sich entlang der Ostseite des geschützten Waldgebiets aus. In verschiedenen Dörfern von Palolo und Lore Utara begannen Mitglieder der lokalen Bevölkerungsgruppen damit, den Wald innerhalb der Nationalparksgrenze zu roden und Felder anzulegen oder dieses neu erschlossene Land an Migranten in ihren Dörfern zu verkaufen. Bemerkenswert ist hierbei, mit welcher Offenheit viele Dorfbewohner ihr Handeln erzählen. Auf die Frage, warum sie nun in den Nationalpark eindringen, erhält man den Fall Dongi-Dongi als Grund genannt. Wenn die anderen Leute dort die Parkgrenzen verletzen und den unter Schutz gestellten Wald roden können, ohne dafür vom Staat belangt zu werden, so die Argumentation, hätte man selbst erst recht die Freiheit, ebenso zu handeln. Dabei wird der Unterschied zwischen der eigenen lokalen Dorf- und der Dongi-Dongi-Bevölkerung in bezug auf Territorialansprüche angeführt, welche vom Gewohnheitsrecht abgeleitet werden. Das Dorfterritorium innerhalb des Nationalparks stellt das von den Vorfahren vermachte Adat-Land (tanah adat) dar. Somit hätte die Lokalbevölkerung einen traditionellen Anspruch auf dieses Land, welches vom Staat beschlagnahmt wurde.

\subsubsection{Räumliche Differenzierung von Wahrnehmung und Handeln in bezug auf den Lore-Lindu-Nationalpark}

Diese für die gesamte Region entlang der östlichen Nationalparkgrenze gezeichnete Wahrnehmung in bezug auf Dongi-Dongi und den Lore-Lindu Nationalpark (s.o.) übersieht jedoch deren unterschiedliche Ausprägungen von Dorf zu Dorf. Wie auch Ebersberger et al. (2002) aufzeigen, ist der Grad der Verletzung der Parkgrenze in den jeweiligen Dörfern abhängig von verschiedenen Faktoren. Allein bei einer vergleichenden Gegenüberstellung der drei für diese Thematik relevanten Untersuchungsdörfer der vorliegenden Arbeit zeigen sich deutliche Unterschiede. In den beiden Dörfern Sintuwu (Kec. Palolo) und Watumaeta (Kec. Lore Utara) sind die Rodungen innerhalb des Nationalparks bereits deutlich sichtbar (vgl. Abb. 49 u. Abb. 50).

Auf dem Gebiet des Dorfes Wanga (Kec. Lore Utara) erscheint der Wald jenseits der Grenze als noch relativ intakt. Dies deckt sich mit der Beurteilung der Landreserven und -verteilung der drei Dörfer. Während in den Dörfern Sintuwu und Watumaeta ein hoher Anteil von kaufkräftigen Migranten, v.a. aus Südsulawesi, 
eine steigende Verknappung der Ressource Land auf Seiten der Lokalbevölkerung bewirkte, setzt sich die Gruppe der Migranten in dem Dorf Wanga aus mehrheitlich finanzschwachen Familien zusammen. Deren Land ist meist nur geliehen, womit der Landbesitz in Wanga von der lokalen Bevölkerungsgruppe dominiert ist. Wanga verfügt im Vergleich zu den anderen beiden zudem noch über einen höheren Anteil an kultivierbarem Land außerhalb des Parkterritoriums. Auf Adat-Land innerhalb des Nationalparks wird sowohl von der lokalen Bevölkerung in Watumaeta als auch in Wanga Bezug genommen, während dies in Sintuwu kaum der Fall ist. Als einziges der drei Dörfer verfügt Wanga durch den Fischfang im Rano See über eine bedeutende Einkommensquelle außerhalb des Ackerbaus.

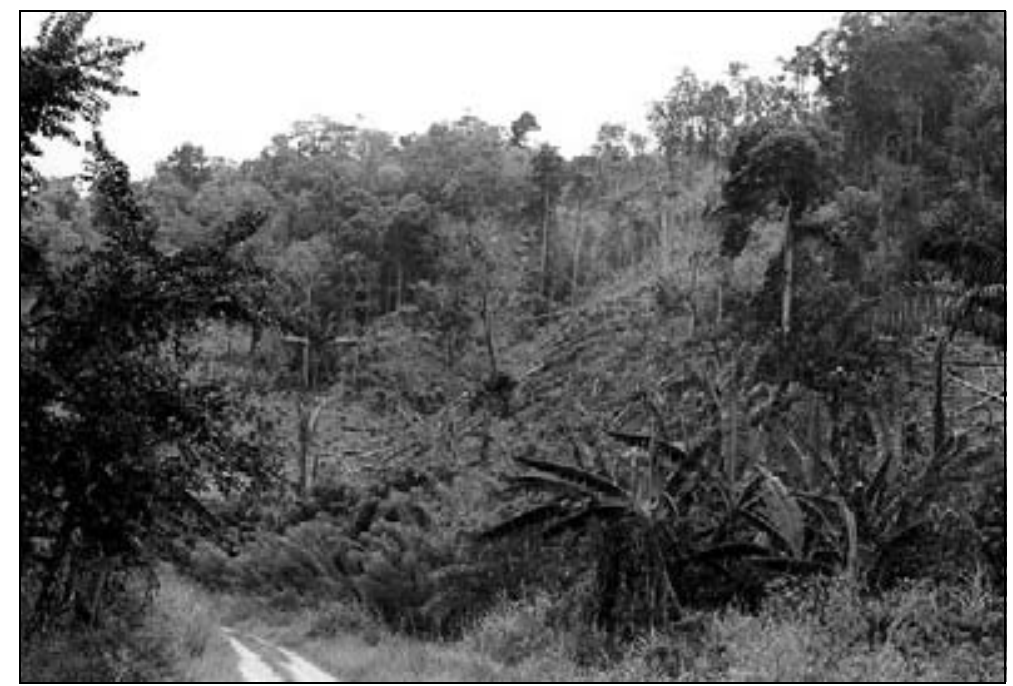

Abb. 49 Rodung des Waldes in Sintuwu entlang der sog. Jalan Jepang (Quelle: Eigene Aufnahme, 2002)

Fasst man diese verschiedenen Faktoren zusammen, zeigt sich, dass allein das Vorhandensein von traditionellem Ahnenland innerhalb des Nationalparks zwar im Rahmen der Rechtfertigung für das Eindringen in den Park eine Rolle spielt. Die Existenz dieses Adat-Landes ist jedoch, wie die Beispiele Wanga und Sintuwu belegen, kein zwingender Indikator dafür, dass die Bevölkerung des entsprechenden Dorfes die Waldschutzgrenze verletzt. Auch der Faktor Migration reicht hierfür allein nicht aus, denn das Dorf Wanga hatte ebenfalls eine Zuwanderung erfahren. Es handelt sich dabei allerdings um weniger finanzstarke Migranten, welche nicht in der Lage sind, in großen Mengen Land von der Lokalbevölkerung anzukaufen, um somit zu einer Abnahme der Landressourcen der lokalen Bevölkerung beizutragen. Zudem hat sich bei den Befragungen herausgestellt, dass die jeweilige Dorfpolitik einen zentralen Einfluss auf die Mensch-Wald-Interaktion der Dorfbewohner ausübt. Im Fall Wanga ist die Dorfführung zum einen auf eine gelenkte Zuwanderung 
bedacht. Neuankömmlinge erhalten zuerst einen Interimsstatus. Sie müssen sich über einen gewissen Zeitraum hinweg bewähren und erhalten ein Stück Land als Leihe. Der Verkauf von Land ist nur innerhalb der lokalen Bevölkerungsgruppe erlaubt und muss in jedem Fall der Dorfverwaltung gemeldet werden. Zudem erkennt die Regierung von Wanga die Grenzen des Lore-Lindu Nationalparks an.

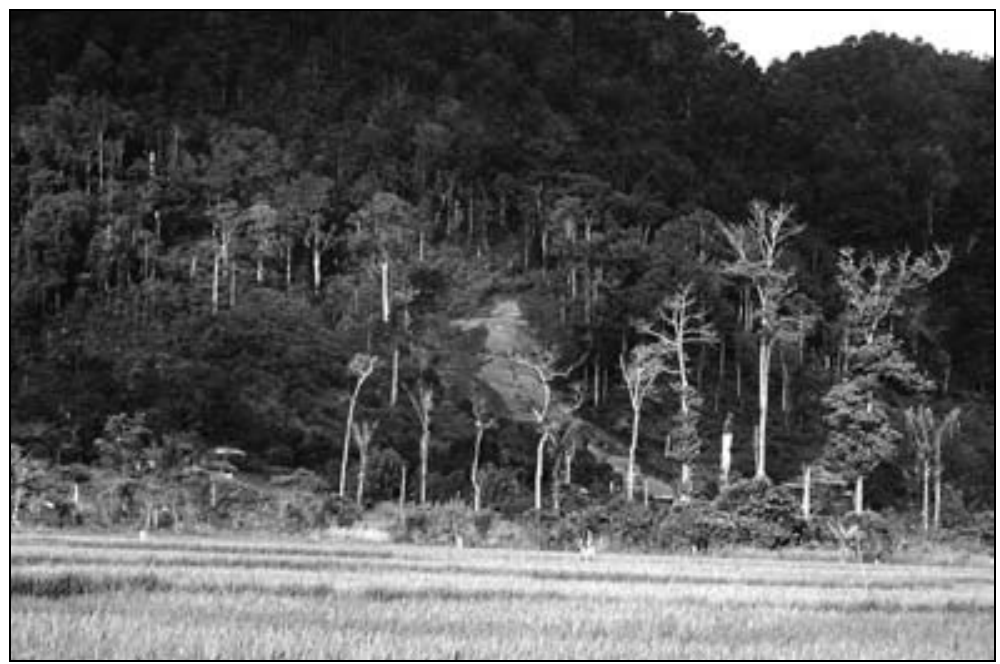

Abb. 50 Hangerosion in Sintuwu

(Quelle: Eigene Aufnahme, 2002)

In Watumaeta kamen hingegen zahlreiche Fälle von illegalen Landverkäufen durch die Dorfführung vor. Hierbei wurde Land, welches sich im Besitz anderer Dorfbewohner befand, an zahlungskräftige Migranten veräußert. Um den Unmut unter der betroffenen Bevölkerung zu mindern, verhielt sich die Dorfregierung in bezug auf anschließende Rodungen von Wald im Nationalpark passiv bis unterstützend.

Auch in Sintuwu kam es während der 1990er Jahre zu einem großflächigen Verkauf von Land, welches lokale Dorfbewohner besaßen, durch den Dorfbürgermeister, der Angehöriger der Bugis-Gruppe im Dorf war. Nachdem dieser auf das gewaltsame Drängen der lokalen Bevölkerung hin vom Camat abgesetzt worden war, wurde dem neuen Anwärter auf den Bürgermeisterposten, diesmal einem Mitglied der lokalen Kaili-Bevölkerung, von der Lokalbevölkerung klar zu verstehen gegeben, dass er sich anders als sein Vorgänger verhalten sollte. Dieser vertrat nun im besonderen Maße die Interessen der finanzschwachen und landarmen lokalen Bevölkerung und stellte sich an die Spitze derjenigen, die Forstranger aus dem Dorf vertrieben, um ungestört in den Nationalpark eindringen zu können. Beachtlich ist die Freimütigkeit, mit welcher der mittlerweile als Dorfbürgermeister vereidigte Anwärter sein Handel ungefragt am Ende eines informellen Interviews erwähnte. Er verlangte auch nicht, dass diese Angaben vertraulich behandelt werden, was das 
hohe Maß seiner Überzeugung von der Rechtmäßigkeit seines Handelns und dem der lokalen Dorfbevölkerung besonders deutlich werden lässt.

Die Reaktion der lokalen Bevölkerung wurde bisher v.a. für den östlichen Teil des Untersuchungsgebiets (Kec. Palolo, Kec. Lore Utara) behandelt. Doch wie hat sich die Einrichtung des Lore-Lindu-Nationalparks auf das Handeln der Menschen auf der Westseite des Parks ausgewirkt? Hierbei kommt besonders die Region Kulawi in Betracht, die im westlichen Untersuchungsgebiet den größten Teil der Nationalparksgrenze einnimmt. Zu behaupten, die Westgrenze des Parks werde strikt eingehalten, würde die Realität verzerren. Selbst in dem Dorf Toro, dessen Repräsentanten großen Wert darauf legen, dass die Dorfgemeinde die Grenzen des Nationalparks respektiert und Verletzungen der Auflagen durch die Nationalparksbehörde mittels revitalisierter Adat-Gesetze konsequent verfolgt werden, liegen Anzeichen dafür vor, dass Eingriffe in den Park vorgenommen und nicht geahndet werden (Burkhard 2004; Lorenz 2004).

Allerdings vermitteln die empirischen Daten aus den Untersuchungsdörfern in Sigi-Biromaru und Kulawi den Eindruck, dass diese Gebiete in bezug auf die Verletzung der Nationalparksgrenze erheblich stabiler sind als jene im östlichen Untersuchungsgebiet. Hierfür lassen sich eine Reihe von Erklärungen heranziehen. Viele der Dörfer, v.a. in Kulawi, welche an den Nationalpark grenzen, weisen noch relativ große, landwirtschaftlich nutzbare Landflächen auf, die sich von den Siedlungen aus nach Westen hin erstrecken. Im Gimpu-Tal des südlichen Kulawi verläuft die Hauptstrasse und somit der Kernbereich der Siedlungsgebiete auf der Westseite des Tals. Dadurch ist die Entfernung zwischen Dorfkern und potenziellen Feldern innerhalb des Nationalparks deutlich größer als z.B. im südlichen Palolo oder nördlichen Lore Utara, wo sich die Siedlungen nahe an der Nationalparksgrenze befinden und mittlerweile erhebliche Eingriffe in die Randzone des Parks vorgenommen wurden. Ein weiterer erklärender Faktor ist die im Vergleich zur Ostseite der Lore-Lindu-Region deutlich weniger stark ausgeprägte $\mathrm{Zu}$ wanderung wohlhabender Kakaobauern. Entsprechend geringer sind auch die Landverkäufe lokaler Bauern und der damit einhergehende Bedarf an zusätzlichem Agrarland. Auch die bereits erwähnten illegalen Landverkaufspraktiken von Dorfautoritäten konnten für das westliche Untersuchungsgebiet nicht nachgewiesen werden. Zudem hat sich der sog. Dongi-Dongi-Konflikt auf der Ostseite ereignet und dürfte durch die größere Distanz einen geringeren Einfluss auf das Verhalten der Bevölkerung von Kulawi und des südlichen Sigi-Biromaru haben. 


\section{Zusammenfassende Schlussbetrachtung}

Der Ausgangspunkt dieser Arbeit war es, den Kulturlandschaftswandel in der LoreLindu-Region während des 20. Jh. historisch-geographisch zu untersuchen, um damit eine Lücke in der Grundlagenforschung zur Region Zentralsulawesi zu schließen (vgl. Kap. 1.3) und einen Informationspool für die Erklärung aktueller Probleme in der Region zur Verfügung zu stellen (vgl. Kap. 1.1). Das Hauptanliegen der Arbeit lag hierbei in der Rekonstruktion der kulturlandschaftlichen Veränderungsprozesse und ihrer Einflussfaktoren. Bei der chronologischen Erfassung des Kulturlandschaftswandels stand die in der Haupthypothese formulierte Frage im Mittelpunkt, in wieweit dieser Wandel einer räumlichen Heterogenität unterliegt und wodurch diese potenzielle Heterogenität verursacht ist (vgl. Kap. 1.3).

Zur Beantwortung dieser Frage wurden neben Archivquellen, Sekundärliteratur, statistischen Datenbeständen, Karten und Fotographien insbesondere die vom Verfasser in neun ausgewählten Dörfern der Lore-Lindu-Region geführten qualitativen Interviews sowie aus der teilnehmenden Beobachtung gewonnene Informationen herangezogen. Die große Bedeutung dieser empirischen Daten liegt darin, dass sie es ermöglichen, zum einen das bereits vorhandene Datenmaterial zu ergänzen und zu überprüfen, und zum anderen jene Hintergrundinformationen und Erklärungsansätze zu liefern, die zur Analyse von Kontinuitäten und Wandel der Kulturlandschaft(en) erforderlich sind.

Die erarbeiteten Ergebnisse belegen, dass während des 20. Jh. sowohl die gesamte Untersuchungsregion gleichermaßen betreffende Veränderungen stattgefunden haben als auch räumlich unterschiedliche. Die leitende Hypothese kann somit positiv und die darin aufgeworfene Frage mit einem Ja beantwortet werden. Besondere Beachtung ist dabei der Tatsache zu schenken, dass sich die Heterogenität des Kulturlandschaftswandels auf verschiedenen räumlichen Ebenen abspielt. Manche der Veränderungen sind nur auf ein oder mehrere Kecamatan bezogen, andere sind auf Teilregionen eines oder mehrerer Kecamatan begrenzt. Darüber hinaus lassen sich selbst Unterschiede von Dorf zu Dorf offenlegen.

Die Lore-Lindu-Region war bereits in vorkolonialer Ära nicht gänzlich von ihrer Außenwelt isoliert. Das Palu-Tal wurde zu jener Zeit z.B. bereits durch den von Händlern eingeführten Islam beeinflusst. Auch innerhalb der Region bestanden zwar voneinander abgegrenzte Herrschaftsbereiche, die jedoch Kontakte zueinander auf der Basis kriegerischer Auseinandersetzung hatten und in der Folge in ein Vasallenverhältnis der Hochland-,,Fürstentümer" zu dem an Macht gewinnenden SigiReich im Tiefland mündeten. Dennoch war es die konkrete Unterwerfung der Re- 
gion unter die niederländische Krone zwischen 1904 und 1908, welche zu einem ersten massiven Kulturlandschaftswandel führte.

Bezogen auf die Bevölkerungsverteilung in der Untersuchungsregion erzwangen die Niederländer mit Hilfe von lokalen Zwangsumsiedlungen der Bergbevölkerung die räumliche Konzentration eines Großteils der Menschen in den jeweiligen Tallagen ihres Herkunftsgebiets. Damit verbunden war die Errichtung einer Vielzahl von Dörfern entlang der auf Befehl der Niederländer angelegten Strassen und Wege, welche die traditionell weit verbreiteten, verstreuten und durch verwandtschaftliche Zugehörigkeit definierten Siedlungsstrukturen ablösten. Der Grund für diese Neuordnung der Siedlungsgebiete war neben einer leichteren Kontrolle der Bevölkerung die bessere Handhabung des von den Niederländern eingeführten Steuer- und Abgabensystems.

Im Bereich der Landnutzung trieben die Kolonialherren die Wiederbewirtschaftung aufgelassener Nassreisfelder und deren flächenmäßige Ausdehnung voran. Einer der Gründe hierfür waren die bereits zu Beginn des 20. Jh. deutlich sichtbare, durch den bis dato praktizierten Wanderfeldbau hervorgerufene Entwaldung der westlichen und östlichen Randgebirge des nördlichen Palu-Tals, welche die Niederländer veranlasste, eine Waldschutzgrenze einzurichten. Unterstützt wurde dieses Vorhaben durch ehrgeizige Bewässerungsprogramme im trockenen Palu-Tal, während in den regenreicheren Hochtälern (Napu, Kulawi, Gimpu) die Regen- oder Flussbewässerung beibehalten wurde.

Neben der Einführung der Kaffeepflanze, deren Erträge während der niederländischen Herrschaft aufgrund mangelnder Kenntnisse nur in geringem Umfang verkauft wurden, legte der Vorstoß von buginesischen, chinesischen und arabischen Händlern in die ,,befriedeten“ Hochlandregionen und die beginnende Monetarisierung die ersten Grundsteine für einen Wandel von der Subsistenz- zur Marktwirtschaft. Es ist allerdings zu betonen, dass die Lore-Lindu-Region zu jener Zeit in weitaus geringerem Umfang in die Weltwirtschaft eingebunden war, als es z.B. auf Java, Sumatra oder den Molukken der Fall war. Ebenso blieben die traditionellen Gesellschaftsstrukturen von der Kolonialpolitik weitgehend unangetastet. Diese Strukturen sind trotz ihrer offiziellen Abschaffung bis heute latent im Bewusstsein der Bevölkerung verankert und weisen deutliche Kontinuitäten bzgl. der Besetzung politischer Ämter auf.

Neben der seit der niederländischen Unterwerfung einsetzenden christlichen Missionierung der noch animistischen Teile der lokalen Bevölkerung (v.a. in den Hochlandregionen) erstreckten sich die kolonialen Einflüsse mit dem Beginn der dreijährigen japanischen Besatzung (1942-1945) außerdem auf die Zwangskultivierung von Baumwolle in der gesamten Untersuchungsregion sowie auf den kriegsbedeutenden Erzabbau in der Pipikoro-Region Kulawis. Trotz dieser zahlreichen Facetten des Kulturlandschaftswandels während der ersten Hälfte des 20. Jh., betrafen diese Veränderung meist die gesamte Lore-Lindu-Region. Erst in der zweiten Hälfte des Jahrhunderts zeichnete sich eine stärkere räumliche Ausdifferenzierung des 
Kulturlandschaftswandels ab. Hierbei lassen sich drei Hauptursachen für die heterogene Entwicklung ausmachen: die Migrationsprozesse, der Boom des cash crop Kakao und die Einrichtung des Lore-Lindu Nationalparks.

Die lokalen Umsiedlungsprogramme der Niederländer wurden nach der Unabhängigkeit und besonders ab der Suharto-Ära fortgesetzt. Befanden sich während der Kolonialzeit Herkunfts- und Zielort der Umsiedlungen jedoch noch im gleichen Sub-Distrikt, ${ }^{178}$ so wurden im unabhängigen Indonesien auch Umsiedlungen zwischen diesen Gebieten durchgeführt. Die Folge war eine durch spontane lokale Migration hervorgerufene Entstehung multi-ethnischer Dorfgemeinschaften. Dabei können die Bergregionen westlich und östlich des Palu-Tals sowie die PipikoroRegion in Kulawi als wichtigste Abwanderungsgebiete zusammengefasst werden, während v.a. das bis in die 1960er Jahre noch wenig erschlossene Palolo das Hauptzielgebiet darstellte. Die im Rahmen des indonesischen transmigrasi-Programms durchgeführten nationalen Umsiedlungen von Javanern, Sundanesen und Balinesen hatten ebenfalls Palolo (Javaner, 1965) und in noch stärkerem Umfang das Kec. Lore Utara (alle drei Gruppen, 1990-1992) als Zielgebiete. Die größte Bedeutung für die Bildung multi-ethnischer Gemeinden hatte jedoch die spontane, regionale $\mathrm{Zu}$ wanderung v.a. aus Südsulawesi.

Die spontane Migration in der Lore-Lindu-Region ist von einer Reihe von Push- und Pullfaktoren geleitet, welche teilweise die verschiedenen ethnischen Gruppen übergreifend sind, teilweise auf bestimmte Gruppen begrenzt sind. Die für den Kulturlandschaftswandel bedeutendste ethnische Migrantengruppe sind die Bugis aus Südsulawesi. Ein Teil von ihnen hatte, wie auch Chinesen und Araber, bereits während der Kolonialzeit ihre Handelstätigkeit und -interessen als Hauptmotiv für ihre Zuwanderung in das Untersuchungsgebiet. Den größten Teil der heute in der Region lebenden Bugis bewegte jedoch die zunehmende Bedeutung des Exportprodukts Kakao und die Landverknappung in Südsulawesi einerseits sowie die Verfügbarkeit von anbautechnisch günstigem Land in Zentralsulawesi andererseits zur Migration in die Untersuchungsregion. Die Ansiedlung dieser Bugis erfolgte in besonderem Maße in Palolo und Lore Utara. Der Grund hierfür war die junge Erschließung Palolos und der Bau der Straßenverbindung zwischen Palolo und Lore Utara im Jahr 1982. Hierdurch war nicht nur eine verbesserte Erreichbarkeit des Napu-Tals für Migranten geschaffen, sondern auch die Marktanbindung an die Provinzhauptstadt Palu verbessert worden. Die Bugis-Familien, welche den Kakao in der Lore-Lindu-Region bekannt machten, verfügten zu einem großen Teil über das nötige Kapital, um Land für den großflächigen Kakaoanbau von der Lokalbevölkerung aufzukaufen. Die Lokalbevölkerung verkaufte bereitwillig Teile ihres Landbesitzes, da sie sich einerseits von dem Vorbild der prosperierenden Bugis zum

178 Gemeint sind damit die heutigen administrativen Einheiten der Kecamatan, welche sich in etwa mit den kolonialen Landschappen decken. 
Kauf von Statusgütern anregen ließen und andererseits auf ihre durch das traditionelle Adat-Recht gewährleitsteten Landbesitzreserven an den Berghängen vertrauten. Allerdings fanden in einigen Dörfern auch illegale Landverkäufe im großen Umfang durch Dorfrepräsentanten an die Migranten statt. Die Ermöglichung dieses Handelns ist durch die individuelle, von Dorf zu Dorf unterschiedliche Ausnutzung des durch das autoritär-repressive Suharto-Regime formierten Machtgefügen auf Dorfebene zu erklären. Die definitive Grenzziehung des Lore-Lindu Nationalparks Anfang der 1990er Jahre, welche die Regierung der lokalen Bevölkerung gegenüber als Provisorium erklärt hatte, bedeute jedoch nicht nur ein vorübergehendes, sondern ein permanentes Konversionsverbot der geschützten Waldgebiete in Agrarland. Die durch Erbteilung schrumpfenden, nicht verkauften Landgrundstücke der Lokalbevölkerung führten einen Teil der Menschen in die Abhängigkeitsverhältnisse der Lohnarbeit. Diese nahm mit der Zuwanderung wohlhabender Migranten und deren Arbeitskräftebedarf zur Bewirtschaftung ihrer im Vergleich zu den Verhältnissen der Lokalbevölkerung größeren Agrarflächen stetig zu. Eine weitere Auswirkung der Landverknappung der lokalen Bevölkerung sind die in jüngster Vergangenheit einsetzenden massiven Eingriffe in den Lore-Lindu Nationalpark. Die Rodung der geschützten Waldflächen betrifft v.a. die Ostseite der Untersuchungsregion, in der das Landproblem zwischen Lokalen und Migranten am größten ist. Zudem hat der maßgeblich durch die nationale Politik der Dezentralisierung und Demokratisierung geförderte und zum Teil auf der Landproblematik in lokalen Umsiedlungsdörfern basierende sog. Dongi-Dongi-Fall zwischen Palolo und Lore Utara in besonderer Weise dazu beigetragen, dass in den beiden Kecamatan die lokale Bevölkerung die Parkgrenze verletzten. Allerdings muss auch hier je nach Dorf unterschieden werden.

Neben dem Eintritt in Lohnarbeitsverhältnisse und dem Eingriff in den Nationalpark verursachte das Landproblem auch eine weitere Migration von Teilen der lokalen Bevölkerung. Diesem Migrationsmotiv gingen während des 20. Jh. bereits eine Reihe weiterer Motive für die Zu- und Abwanderung der lokalen Bevölkerung in der Region voraus. Waren es während der ersten Hälfte des Jahrhunderts v.a. Heiratsbeziehungen, welche zu einer Migration in ein anderes Dorf führten, kamen etwa ab den 1970er Jahren die steigende Nachfrage im sekundären und tertiären Wirtschaftssektor, der Ausbau des Bildungssystems und die Errichtung höherer Schulen, die Suche nach einem für den Anbau von Kakao günstigen Land, sowie die zum Teil durch mediale Einflüsse erzeugte Bewusstwerdung der persönlichen Unzufriedenheit in der jeweiligen Herkunftsregion als Wanderungsmotive hinzu. Die genannte Nachfrage nach Arbeitskräften hatte v.a. Migrationen in die Zentren des jeweiligen Sub-Distrikts, mehr noch aber in die Provinzhauptstadt zur Folge. Ebenso verhielt es sich mit den Wanderungsbewegungen zu Ausbildungszwecken. Zum Teil führten die Beschäftigungsangebote die Migranten auch aus der LoreLindu-Region und aus Zentralsulawesi hinaus. Zielregionen für jene Migranten, die sich dem Boom des Kakaoanbaus anschließen wollten, waren in erster Linie Palolo 
und Lore Utara, zu einem weitaus geringerem Teil das Kec. Kulawi. Von letzterem ist jedoch das verkehrsinfrastrukturell rückständige Pipikoro-Gebiet auszuschließen. Der Großteil dieser Migranten hatte seinen Ursprung im Palu-Tal, welches aufgrund der hohen Bevölkerungsdichte und der vergleichsweise ungünstigeren klimatischen Bedingungen nur ein geringes Ausbaupotential für den Kakaoanbau bot. Ferner zählt Pipikoro aus dem o.g. Grund dazu.

Sowohl für lokale, als auch für regionale Migranten nimmt die Existenz ethnischer und familiärer sozialer Netzwerke einen herausragenden Stellenwert im Entscheidungsprozess zur Migration ein. Dieser Aspekt trug neben anderen Faktoren dazu bei, dass sich in jedem Dorf eine nach Quantität und Qualität unterschiedliche ethnische Zusammensetzung der Bevölkerung entwickelte. Die jeweilige Komposition schlug sich auch in einer mehr oder wenig stark ausgeprägten sozialräumlichen Segregation innerhalb der Dörfer nieder. Es konnte aufgezeigt werden, dass verschiedene Faktoren in von Dorf zu Dorf unterschiedlicher Intensität an der Entstehung und Ausformung der jeweiligen Segregationsmuster beteiligt waren: der ökonomische und politische Status der jeweiligen Bevölkerungsgruppen, die Landvergabepolitik der Dorfadministration, die soziale Interaktion zwischen den Gruppen und die Religionszugehörigkeit.

Zudem unterscheiden sich die Dörfer der Lore-Lindu-Region hinsichtlich der Siedlungsform. Während die relative Gleichzeitigkeit von Verkehrswegeausbau und Dorfgründungen in der überwiegenden Zahl der Fälle eine Ausformung der Dörfer als Straßensiedlungen bewirkte, weichen einige Dörfer von dieser Form ab. Dies betrifft z.B. die in Folge lokaler oder nationaler Umsiedlungsmaßnahmen neu geschaffenen Dörfer mit planmäßiger Struktur (z.B. Maranatha, Kamarora, Siliwanga). Aber auch das Dorf Sintuwu erfüllt nur bedingt die Kriterien eines Straßendorfes. Ebenso weichen v.a. Dörfer in verkehrsinfrastrukturell schlechter erschlossenen Regionen (z.B. Lawe oder Lonebasa in der Pipikoro-Region, Rompo oder Watutau in Lore Utara) von dieser Form ab. Ferner weisen nicht alle Dörfer der Region ein kompaktes Siedlungsgebiet (wie z.B. Lawe) auf, sondern sind besonders im Zusammenhang mit der Orientierung entlang der Hauptstrassen oft in mehrere, mehr oder weniger abgeschlossene Einzelsiedlungen unterteilt. Das Dorf Lempelero steht hierbei für einen Extremfall mit drei Dusun, die, durch dazwischenliegende andere Dörfer getrennt, in einer mehr als $10 \mathrm{~km}$ großen Entfernung zur Hauptsiedlung des Ortes liegen. Die einzelnen Siedlungsteile, die i.d.R. jeweils ein eigenes Dusun der administrativen Dorfeinheit umfassen, beruhen auf den kolonialzeitlich gegründeten und post-kolonial zusammengeführten Siedlungen zu größeren Verwaltungseinheiten.

$\mathrm{Zu}$ den Veränderungsprozessen in der Landnutzung ist neben den bereits genannten Entwicklungen die Technisierung der Landwirtschaft, insbesondere des Nassreisanbaus, und die weitgehende Abschaffung des Brandrodungswanderfeldbaus hervorzuheben. Die Technisierung im Bereich der Landnutzung hatte seine Anfänge bereits während der niederländischen Kolonialzeit mit dem Bau von Be- 
wässerungsanlagen und der Einführung des Pfluges. Der Pflug, welcher erst nach der Unabhängigkeit Indonesiens eine weite Verbreitung in der Region fand, löste zunehmend das traditionelle paruja-System ab, welches das Pflügen mit Hilfe von Wasserbüffeln darstellte. Der Rückgang der Büffelpopulationen, für den in dieser Arbeit eine Vielzahl von Einflussfaktoren genannt wurden, verstärkte den Einsatz von Pflügen, die ab den 1980er Jahren teilweise motorisierte Handtraktoren ersetzt wurden. Der Übergang von der Wanderfeld- zur permanenten Feldbewirtschaftung machte schließlich den Einsatz von Düngern erforderlich, während die zunehmende monokulturelle Landnutzung (v.a. Nassreis und Kaffee bzw. Kakao) den Bedarf an Pestiziden erhöhte. Da der Einsatz dieser Zusatzstoffe sowohl von dem Know how ihrer Anwendung als auch von der finanziellen Situation eines Haushalts abhängig ist, sind es zu einem großen Teil die wohlhabenden Kakaobauern aus Südsulawesi, die diesbezüglich neben dem notwendigen Kapital auch auf ihre frühzeitigen, in Malaysia gesammelten Erfahrungen zurückgreifen können.

Während Mais und Cassava als Subsistenzprodukte (Mais wird z.T. auch nach Kalimantan exportiert) für die meisten der lokalen Familien bis heute eine große Rolle spielen, wurden die cash crops Kaffee und Gewürznelken mittlerweile weitgehend vom Kakao abgelöst. Kaffee ist v.a. noch in Kulawi ein wichtiges Marktprodukt, welches, mit einem regionalen Marketinglabel versehen, z.B. in Palu angeboten wird. Zahlreiche Familien haben jedoch ab den 1990er Jahren ihre Kaffeeund/oder Gewürznelkenfelder durch Kakaofelder ersetzt oder bewirtschaften diese in Mischkultur. Die empirischen Untersuchungen haben ergeben, dass sich bei vielen Menschen in der Lore-Lindu-Region ein stärker vorausschauendes Handlungsverhalten etabliert hat. Nur noch wenige der Befragten, die sich in den 1990er Jahren für einen radikalen Wechsel des Anbauprodukts entschieden hatten, würden bei einem gravierenden Verfall des Kakaopreises wieder in gleicher Weise verfahren.

Die Zusammenfassung der einzelnen Aspekte des Kulturlandschaftswandels in der Lore-Lindu-Region während des 20. Jh. zeigt, dass sich dort über ein Jahrhundert hinweg eine Vielzahl von Veränderungsprozessen im ökonomischen, sozialen, kulturellen und politischen Bereich vollzogen haben. Darüber hinaus wurde aufgezeigt, dass dieser Wandel räumlich differenziert zu betrachten und zu bewerten ist. Die physisch-realen und soziokulturell konstruierten Kulturlandschaften dieser Region Zentralsulawesis sind durch regional und lokal unterschiedliche Kontinuitäten und Dynamiken bestimmt. 


\section{Quellen- und Literaturverzeichnis}

\section{Quellen aus dem Nationaalarchief in Den Haag}

Ansingh, W. (1937): Nota inzake het landschap Sigi-Dolo. [Nationaalarchief, KIT 1206]

Hirschmann, F. Ch. H. (1934): Vervolg-memorie (op. M. Junius) inzake de afdeeling Donggala. [Nationaalarchief, KIT 1202, 49 S. + Anhang]

Logeman, F. H. W. J. R. (1922): Memories van overgave van de residentie Manado. [Nationaalarchief, MMK 304, 182 S. + Anhang]

Ministerie van Kolonien: Politieke Verslagen Buitengewesten 1898-1940, Celebes, Kaarten en Tekeningen. [Micro-Fiche Nr. 722 A]

Tideman, J. (1926): Memories van overgave van de residentie Manado.

[Nationaalarchief, MMK 305, 198 S. + Anhang]

Van Hengel, J. (1910): Memories van overgave van de residentie Manado.

[Nationaalarchief, MMK 303, 78 S.]

Van Rhijn, M. (1941): Memories van overgave van de residentie Manado.

[Nationaalarchief, MMK 308, 288 S.]

Voorn, M. C. (1925): Aanvullende memories inzake de onderafdeeling Palu.

[Nationaalarchief, KIT 1198, 25 S. + Anhang]

Vorstman, J. A. (1935): Aanvullende Memories van overgave van de onderafdeeling Paloe. [Nationaalarchief, KIT 1203, 39 S. + Anhang]

\section{Literaturverzeichnis}

Abdulkadir-Sunito, M. (2004): Orang kampung and pendatang: analysis of demographic structures and migration in two forest-margin villages, Central Sulawesi. In: Gerold, G., Fremerey, M. \& E. Guhardja (Hrsg.): Land use, nature conservation and the stability of rainforest margins in Southeast Asia. Berlin. S. 89-104.

Acciaioli, G. (1981): Knowing what you are doing. A review of Pierre Bourdieu's Outline of a Theory of Practice. In: Canberra Anthropology, Bd. 4, H. 1, S. 23-51.

- (1985): Culture as art: from practice to spectacle in Indonesia. In: Canberra Anthropology, Bd. 8, H. 1+2, S. 148-172.

- (1987): Networks and nets: principles and processes in Bugis migration strategies to Lake Lindu, Central Sulawesi. (= International Workshop on Indonesian Studies, No.2)

- (1989): Searching for good fortune. The making of a Bugis shore community at Lake Lindu, Central Sulawesi. The Australian National University, Canberra. 
- (1999): Principles and strategies of Bugis migration: some contextual factors relating to ethnic conflict. In: Masyarakat Indonesia, Bd. 25, H. 2, S. 239-269.

- (2000): Kinship and debt. The social organization of Bugis migration and fish marketing at Lake Lindu, Central Sulawesi. In: Tol, R., Van Dijk, K. \& G. Acciaioli (Hrsg.): Authority and enterprise among the peoples of South Sulawesi. Leiden.

- (2001): Grounds of conflict, idioms of harmony : custom, religion, and nationalism in violence avoidance at the Lindu Plain, Central Sulawesi. In: Indonesia, Bd. 72, S. 81-114.

Adriani, N. (1913): De reis van den heer W. J. M. Michielsen naar het Posso-Meer, 12-17 Juli 1869. In: De Indische Gids, Bd. 35, H. 2, S. 1612-1618.

- (1915): Maatschappelijke, speciaal economische verandering der bevolking van Midden-Celebes, sedert de invoering van het nederlandsch gezag aldaar. In: Tijdschrift van het Koninklijk Nederlandsch Aardrijkskundig Genootschap, tweede serie deel XXXII, S. 457-475.

Adriani, N. \& A. C. Kruyt (1898): Von Poso naar Parigi, Sigi en Lindoe. In: Tijdschrift voor Zendingswetenschap, Bd. 42, S. 369-535.

- (1912-1914): De Bare'e-sprekende Toraja's van Midden-Celebes. Batavia. 3 Bde.

- (1950-1951): De Bare'e-sprekende Toradjas van Midden-Celebes (de OostToradjas). Amsterdam. 3 Bde.

Akita, T. \& J. J. K. Szeto (2000): Inpres Desa Tertinggal (IDT) program and indonesian regional inequality. In: Asian Economic Journal, Bd. 14, Nr. 2, S. 167-186.

Akiyama, T. \& A. Nishio (1996): Indonesia's cocoa boom: hands-off policy encourages smallholder dynamism. Washington D.C. (= Policy research working papers, no. 1580, The World Bank)

Alene, A., Zeller, M., Schwarze, S. \& N. Nuryartono (2005): The extent and determinants of production efficiency of farmers in the rainforest margins in Central Sulawesi, Indonesia: Implications for land use and support services. Wird publiziert in: Quarterly Journal of International Agriculture.

Ambler, J. S. (1989): Adat and aid : management of small scale irrigation in West Sumatra. New York.

Anderson, B. (1983): Imagined communities. London, New York.

Anderson, K. \& F. Gale (1992): Introduction. In: Anderson, K. \& F. Gale (Hrsg.): Inventing places: studies in cultural geography. Melbourne. S. 1-14.

ANZDEC (1997): Report on the Central Sulawesi integrated area development and conservation project. Palu, Sulawesi.

Aragon, L. V. (1992): Divine justice: cosmology, ritual and protestant mission in Central Sulawesi, Indonesia. Urabana.

- (1996a): 'Japanese time' and the mica mine: experiences of the occupation in the western Central Sulawesi highlands. In: Journal of Southeast Asian Studies, Bd. 27, H. 1, S. 49-63.

- (1996b): Reorganizing the cosmology: The reinterpretation of deities and religious practice by protestants in Central Sulawesi. In: Journal of Southeast Asian Studies, Bd. 27, H. 2, S. 350-373. 
- (1996c): Suppressed and revised performances: Raego' songs of Central Sulawesi, Indonesia. In: Ethnomusicology, Bd. 40, H. 3, S. 413-439.

- (1996d): Twisting the Gift: Translating Precolonial into Colonial Exchanges in Central Sulawesi, Indonesia. In: American Ethnologist, Bd. 23, H. 1, S. 43-60.

- (2000a): Can Central Sulawesi Christians and Muslims get along?: An analysis of Indonesian regional conflict. In: Antropologi Indonesia, Bd. 24, H. 63, S. 54-64.

- (2000b): Fields of the Lord: animism, Christian minorities, and state development in Indonesia. Honolulu.

- (2001): Communal violence in Central Sulawesi: where people eat fish and fish eat people. In: Indonesia, Bd. 72, S. 45-79.

- (2002): Waiting for Peace in Poso. In: Inside Indonesia, Nr. 70, S. 11-12.

- (2003): Missions and omissions of the supernatural: indigenous cosmologies and the legitimisation of 'religion' in Indonesia. In: Anthropological Forum, Bd. 13, H. 2, S. 131-140.

Babcock, T. G. \& F. H. Cummings (1984): Land settlement in Sulawesi, Indonesia. In: Malaysian journal of tropical geography, Bd. 10, S. 12-25.

Balibar, E. \& I. Wallerstein (1992): Rasse, Klasse, Nation. Ambivalente Identitäten. Hamburg.

Banks, M. (1996): Ethnicity: Anthropological Constructions. London, New York.

Barnett, C. (1998): The cultural turn: fashion or progress in human geography? In: Antipde, Bd. 30, H. 4, S. 379-394.

Barth, F. [Hrsg.] (1969): Ethnic groups and boundaries. The social organization of cultural difference. London, Oslo.

Bastian, A. (1889): Indonesien oder die Inseln des malayischen Archipels. Borneo und Celebes. Reiseergebnisse und Studien. Berlin.

Behrendt, R. F. (1965): Soziale Strategie für Entwicklungländer. Entwurf einer Entwicklungssoziologie. Frankfurt am Main.

Bigalke, T. (1981): A social history of “Tana Toraja” 1870-1965. Diss., University of Wiskonsin, Madison.

Birner, R. \& M. Mappatoba (2002): Community agreements on conservation in Central Sulawesi: a coase solution to externalities or a case of empowered deliberative democracy? Bogor, Göttingen, Kassel. (= STORMA Discussion Paper Series Sub-Program A, Nr. 3)

Bleeker, P. (1856): Reis door de Minahassa en de Moluksche Archipel, gedaan in de maanden September en October 1855. Batavia.

Blok, D. P. \& W. Prevenier [Hrsg.] (1978): Algemene Geschiedenis der Nederlande, Bd. 14. Haarlem.

Bodman, A. (1991): Weavers of influence: the structure of contemporary geographic research. In: Transactions of the Institute of British Geographers, Bd. 16, S. 321-337.

Bodman, A. (1995): Binding ties or scattered fragments?: the structure of contemporary geographic research. In: Transactions of the Institute of British Geographers, Bd. 24, S. 34-53. 
Boomgaard, P. (1986): The welfare service in Indonesia, 1900-1942. In: Itinerario, Bd. 10, S. 57-82.

Boomgard, P. \& A. J. Gooszen (1991): Population Trends 1795-1942. (=Changing Economy in Indonesia, Bd. 11)

Boomgard, P. (1993): Upliftment down the drain? Effects of welfare measures in late colonial Indonesia. In: Dirkse, J.-P. (Hrsg.): Development and social welfare: Indonesia's experiences under the New Order. Leiden. S. 247-253.

Boonstra van Heerdt, R. (1914): De berglandschappen behoorende tot de onderafdeeling Paloe van Midden-Celebes. In: Tijdschrift van het Koninklijk Nederlandsch Aardrijkskundig Genootschap (2nd series), Bd. 31, S. 618-644.

Bourdieu, P. (1976): Entwurf einer Theorie der Praxis auf der ethnologischen Grundlage der kabylischen Gesellschaft. Frankfurt am Main.

BPS (1971): Sulawesi Tengah dalam angka tahun 1970. Palu.

- (1981): Registrasi penduduk propinsi Sulawesi Tengah akhir tahun 1980. Palu.

- (1991): Sulawesi Tengah dalam angka tahun 1990. Palu.

- (2001): Kabupaten Poso dalam angka 2000. Poso.

- (2002): Kabupaten Donggala dalam angka. Donggala regency in figures, 2001. Palu.

BPS Jakarta (2001): Statistics Indonesia. http:/ /www.bps.go.id (Zugriff: Februar 2004).

Bubandt, N. (1998): Timber plantations in Indonesia: approaching the predicaments of modern utopia. Canberra. (= Resource Management in Asia-Pacific Working Paper, Nr. 15)

Burger, K. \& H. P. Smit (2001): International market responses to the Asian Crisis for rubber, cocoa and coffee. In: Gerard, F. \& F. Ruf (Hrsg.): Agriculture in crisis: people, commodities and natural resources in Indonesia, 1996-2000. Montpellier, Richmond. S. 31-48.

Burkard, G. (2002a): Natural resource management in Central Sulawesi: Past experiences and future prospects. Bogor, Göttingen, Kassel. (= STORMA Discussion Paper Series Sub-Program A, Nr. 8)

- (2002b): Stability or sustainability? Dimensions of socio-economic security in a rainforest margin. Bogor, Göttingen, Kassel. (= STORMA Discussion Paper Series Sub-Program A, Nr. 7)

Caldwell, M. \& E. Utrecht (1979): Indonesia. An alternative history. Sydney.

Chua-Franz, C. (2002): Indonesiens Chinesen. Konstruktion und Instrumentalisierung einer ethnischen Minderheit. Hamburg. (= Mitteilungen für Asienkunde, Bd. 361)

Coates, B., Bishop, K. D. \& D. Gardner (1997): A guide to the birds of Wallacea: Sulawesi, the Moluccas and Lesser Sunda Islands, Indonesia. Alderly, Queensland.

Conservation International (2002): Biodiversity hotspots - Wallacea. http://www.biodiversityhotspots.org (Zugriff: Februar 2004).

Cosgrove, D. (1984): Social formation and symbolic landscape. London.

Cosgrove, D. \& P. Jackson (1987): New directions in cultural geography. In: Area, Bd. 19, S. 95-101. 
Coté, J. (1979): The colonization and schooling of the To Pamona of Central Sulawesi, 1894 to 1924. Melbourne.

- (1996): Colonising Central Sulawesi: the 'ethical policy' and imperalist expansion 1890-1910. In: Itinerario, Bd. 20, S. 87-107.

Crang, M. (1998): Cultural geography. London, New York.

Cribb, R. (1993): Development Policy in the early 20 th century. In: Dierkse, J.-P. (Hrsg.): Development and social welfare: Indonesia's experiences under the New Order. S. 225-245.

- (1994): Introduction. The late colonial state in Indonesia. In: Cribb, R. (Hrsg.): The late colonial state in Indonesia: political and environmental foundations of the Netherlands Indies, 1880-1942. Leiden, S. 1-9.

Cribb, R. \& C. Brown (1995): Modern Indonesia: a history since 1945. Singapore.

Cronon, W. [Hrsg.] (1996): Uncommon ground: rethinking the human place in nature. New York.

Dahm, B. (1990): Der Dekolonisationsprozeß Indonesiens. Endogene und exogene Faktoren. In: Mommsen, W. J. (Hrsg.): Das Ende der Kolonialreiche Dekolonisierung und die Politik der Großmächte. Frankfurt am Main. S. 67-88.

Darden, J. T. (1972): Spatial dynamics of residential segregation of Afro-Americans in Pittsburgh. Pittsburgh.

Dechert, G. (2003): Nutrient dynamics and their control in land use systems of forest margins in Central Sulawesi, Indonesia. Diss., Georg-August-Universität Göttingen.

Departmen Pendidikan dan Kebudayaan [Hrsg.] (1996/1997): Sejarah daerah Sulawesi Tengah. Palu.

Dieter, H. (1999): Die Asienkrise. Ursachen, Konsequenzen und die Rolle des Internationalen Währungsfonds. Marburg.

Dittmann, A. (2004): Segregation und Migration in städtischen Zentren zwischen Hindukusch und Himalaya. In: 54. Deutscher Geographentag. Bern. S. 515-522.

Donner, W. (1987): Land use and environment in Indonesia. London.

Dove, M. R. (1983): Theories of swidden culture and the political economy of ignorance. In: Agroforestry Systems, Bd. 1, S. 85-99.

Ebersberger, S., Weber, R \& M. Hoppe (2002): Perceptions of protected forest areas: decisive facotrs and impacts on people's attitudes. The case of 7 communities in the vicinity of Lore Lindu National Park, Central Sulawesi, Indonesia. Poster, präsentiert auf dem International Symposium "Land Use, Nature Conservation, and the Stability of Rainforest Margins in Southeast Asia", Bogor, 29.9.-3.10.2002.

Eijkemans, C. H. (1995): Profitability or security. Decision-making on land use among Toba Batak peasants in North Sumatra, Indonesia. Nijmegen.

Emmer, P. C. \& E. Schmitt [Hrsg.] (1988): Dokumente zur Geschichte der europäischen Expansion, 7 Bde.

Encyclopaedie (1921): Encyclopaedie van Nederlandsch-Indie. 4 Bde., Den Haag, Leiden. 
Erasmi, S., Twele, A., Ardiansyah, M., Malik, A. \& M. Kappas (2004): Mapping deforestation and land cover conversion at the rainforest margins in Central Sulawesi, Indonesia. In: EARSeL eProceedings, Bd. 3, H. 3, S. 288-297.

Evernden, N. (1992): The social creation of nature. Baltimore.

Ewald, K. C. (1996): Traditionelle Kulturlandschaften. Elemente und Bedeutung. In: Konold, W. (Hrsg.): Naturlandschaft - Kulturlandschaft. Die Veränderung der Landschaften nach der Nutzbarmachung durch den Menschen. Landsberg. S.99-119.

Fasbender, K. \& S. Erbe (1990): Towards a new home: Indonesian managed mass migration. Hamburg.

Faust, H. (im Druck): Vergleichende Kulturgeographie - Eine empirische Untersuchung der Integrationsprozesse in Agrarkolonisationsräumen Boliviens, der Elfenbeinküste und Indonesiens. Göttingen. (= Göttinger Geographische Abhandlungen, H. 115, 2006).

Faust, H. \& M. Chairil (2002): Social conflicts about nature conservation. International seminar on "Sustainable development: socio-economic \& environment problems in Indonesia”. Göttingen. (= Seminar Proceeding Paper)

Faust, H., Maertens, M., Weber, R., Nuriatono, N., Van Rheenen, T. \& R. Birner (2003): Does migration lead to destabilization of forest margins? - Evidence from an interdisciplinary field study in Central Sulawesi. Bogor, Göttingen, Kassel. (= STORMA Discussion Paper Series Sub-Program A, Nr. 11)

FAO (2001): FRA 2000 main report. Rom. (= FAO Forestry Paper 140) http://www.fao.org/forestry/site/7949/en (Zugriff: Oktober 2003)

- (2003): Statistical database FAOSTAT. http://www.fao.org (Zugriff: Oktober und November 2003)

Fearnside, P.M. (1997): Transmigration in Indonesia: lessons from its environmental and social impacts. In: Environmental Management, Bd. 21, Nr. 4. S. 553-570.

Firman, T. (2004): New town development in Jakarta Metropolian Region: a perspective of spatial segregation. In: Habitat International, Bd. 28, S. 349-368.

Fischer, K. \& Parnreiter, C. (2002): Transformation und neue Formen der Segregation in den Städten Lateinamerikas. In: Geographical Helvetica, Bd. 57, H. 4, S. 245-252.

Frank, G. (1911): Niederländisch-Indien. Eine Finanzquelle für das Mutterland. Würzburg.

Freischlad, J. (2002): Binnenflüchtlinge in Indonesien. Berlin. (= IndonesienInformation, Nr. 3) http://home.snafu.de/watchin/II_3_02/II_3_02.htm (Zugriff: März 2004)

Fremerey, M. (2002): Local communities as learning organisations: the case of the village of Toro, Central Sulawesi, Indonesia. Bogor, Göttingen, Kassel. (= STORMA

Discussion Paper Series Sub-Program A, Nr. 6)

Glaser, B. G. \& A. L. Strauss (1967): The discovery of grounded theory. Chicago.

Gooszen, H. (1999): A demographic history of the Indonesian archipelago, 1880-1942. Leiden. (= Verhandelingen van het Koninklijk Instituut voor Taal-, Land- en Volkenkunde, Bd. 183)

\section{Pazifik Forum Bd. 12}


Graafland, N. (1898): De Minahassa: Haar verleden en haar tegenwoordige toestand. Batavia. 2 Bde.

Greider, T. \& L. Garkovich (1994): Landscapes: the social construction of nature and the environment. In: Rural Sociology, Bd. 59, H. 1, S. 1-24.

Grubauer, A. (1913): Unter Kopfjägern in Central-Celebes. Ethnologische Streifzüge in Südost- und Central-Celebes. Leipzig.

- (1923): Celebes: Ethnologische Streifzüge in Südost- und Central-Celebes. Hagen i. W., Darmstadt.

Grunsven, L. (1992): Integration versus segregation: ethnic minorities and urban politics in Singapore. In: Tijdschrift voor Economische en Sociale Geografie, Bd. 83, H. 3, S. 196-215.

Guiness, P. (1977): Changing focus of the transmigration program. Yogyakarta.

Hardjono, J. M. (1977): Transmigration in Indonesia. Melbourne.

Hart, J. C. van der (1853): Reize rondom het eiland Celebes en naar eenige der Moluksche Eilanden. s'Gravenhage.

Harth, A. (1997): Soziale Ausdifferenzierung und räumliche Segregation in den Städten der Neuen Bundesländer: allgemeine Befunde und eine Fallstudie in Halle/Saale. In: Schäfer, U. (Hrsg.): Städtische Strukturen im Wandel. Opladen. S. 251-365.

Head, L. (2000): Cultural landscape and environmental change. London.

Hefner, R. W. (1990): The political economy of mountain Jva: An interpretative history. Berkley, Los Angeles.

Henley, D. (1989): The idea of Celebes in history. Clayton, Victorya. (=Centre of Southeastasian Studies, Working Paper Nr. 59)

- (2002): Population, economy and environment in island Southeast Asia: an historical view with special reference to Northern Sulawesi. In: Singapore Journal of Tropical Geography, Bd. 23, H. 2, S. 167-206.

- (2004): Fertility, food and fever: population and environment in North and Central Sulawesi before 1930. Leiden.

Hershkovitz, S. (1981): Spatial segregation trends in cities. Monticello. (= Public administration series, $\mathrm{Nr} .812$ )

Herskovits, M. J. (1958): Acculturation. The study of culture contact. Gloucester.

- (1967): Cultural dynamics. New York.

Hill, P. (1956): The Gold Coast farmer. Oxford.

- (1963): Migrant cocoa farmers of Southern Ghana, a study in rural capitalism. Cambridge.

Hissink (1912): Nota van toelichting, betreffende de zelfbesturende landschappen Paloe, Dolo, Sigi en Beromaroe. In: Tijdschrift voor Indische Taal-, Land- en Volkenkunde, Bd. 54, S. 58-129.

Hoey, B. A. (2003): Nationalism in Indonesia: building imagined and intentional communities through transmigration. In: Ethnology, Bd. 42, H. 2, S. 109-126.

Holmgren, R. J. \& A. Spertus (1989): Early Indonesian textiles from three island cultures. New York. 
Hoppe, M. (2003): Auswirkungen der Transmigration auf Kulturlandschaft und soziokulturelles Zusammenleben im Naputal, Zentral-Sulawesi - eine kulturgeographische Untersuchung in drei Siedlungen am Rande des Lore Lindu Nationalparks. Unveröffentlichte Diplomarbeit, Georg-August-Universität Göttingen.

Hoppe, M. \& H. Faust (2004): Transmigration and integration in Indonesia - impacts on resource use in the Napu Valley, Central Sulawesi. Bogor, Göttingen, Kassel. (= STORMA Discussion Paper Series Sub-Program A, Nr. 13)

Houben, V. J. H. (1994): Profits versus ethics. Government enterprises in the late colonial state. In: Cribb, R. (Hrsg.): The late colonial state in Indonesia: political and environmental foundations of the Netherlands Indies, 1880-1942. Leiden, S. 193221.

Hubbard, P., Kitchin, R., Bartley, B. \& D. Fuller (2002): Thinking geographically. Space, theory and contemporary human geography. London, New York.

Hugo, G. J. (1980): Population movements in Indonesia during the colonial period. In: Fox, J. J. (Hrsg.): Indonesia: Australian perspectives, Bd. 1: Indonesia, the making of a culture. Canberra. S. 95-134.

Ingold, T. (1993): The temporality of landscape. In: World Archaeology, Bd. 25, S. 152-174.

International Cocoa Organisation (1998): Information on the origin/history of cocoa and its spread around the world. http:/ /www.icco.org (Zugriff: Oktober 2004)

- (2000): How many smallholders are there worldwide producing cocoa? http:/ / www.icco.org (Zugriff: Oktober 2004)

- (2004): ICCO monthly and annual averages of daily prices of cocoa beans, 1960 2003. http://www.icco.org (Zugriff: Februar 2005)

Jackson, P. (1989): Maps of meaning. London, New York.

Jamal, S. \& M. Pomp (1976): Smallholder adoption of tree crops: A case study of cacao in Sulawesi. In: Bulletin of Indonesian Economic Studies, Bd. 29, H. 3, S. 69-94.

Jones, M. \& K. Daugstad (1997): Usage of the 'cultural landscape' concept in Norwegian and Nordic landscape administration. In: Landscape research, Bd. 23, H. 3, S. $267-$ 281.

Jorgensen, D. (1989): Participant observation: a methodology for human studies. Newbury Park. (= Applied Social Research Methods Series, Bd. 15)

Kahn, J. (1993): Constituting the Minangkabau: peasants, culture and modernity in colonial Indonesia. Providence.

- (1999): Culturalising the Indonesian uplands. In: Li, T. M. (Hrsg.): Transforming the Indonesian uplands: marginality, power and production. London. S. 79-101.

Kaschuba, W. (1999): Konstruktionen: Identität und Ethnizität. In: Kaschuba, W. (Hrsg.): Einführung in die europäische Ethnologie. München. S. 132-146.

Kaudern, W. (1925a): Migrations of the Toradja in Central Celebes. Results of the author's expedition to Celebes 1917-1920. Göteborg. (= Ethnographical studies in Celebes, Bd. 2) 
- (1925b): Structures and settlements in Central Celebes. Results of the author's expedition to Celebes 1917-1920. Göteborg. (= Ethnographical studies in Celebes, Bd. 1)

- (1941): The noble families or maradika of Kulawi, Central Celebes. In: Ethnologiska Studier, Bd. 11 (1940), S. 29-117.

Kebschull, D. (1984): Transmigration - Indonesiens organisierte Völkerwanderung. Bonn.

- (1986): Transmigration in Indonesia. Hamburg.

- (1987): Transmigration - the Indonesian resettlement programme. Bielefeld.

Kebschull, D. \& K. Fasbender (1987): Transmigranten in Indonesien - Ihre Motive und Erfahrungen. In: Internationales Asienforum. Bd. 18, Nr. 3/4, S. 279-290.

Keil, A., Kleinhans, A., Schwarze, S., Birner, R., Gerold, G. \& S. Lipu (2003): Forest conservation, water availability and water use in Central Sulawesi, Indonesia. In: Die Erde, Bd. 134, H. 4, S. 147-164.

Kelle, U. (1994): Empirisch begründete Theoriebildung. Zur Logik und Methodologie interpretativer Sozialforschung. Weinheim.

Kelle, U. \& S. Kluge (1999): Vom Einzelfall zum Typus. Fallvergleich und Fallkontrastierung in der qualitativen Sozialforschung. Opladen.

Kerjasama Badan Perencanaan Pembangunan Nasional Yayasan Agro Ekonomika (1995): IDT terhadap biaya transport pemasaran yang mahal: kasus Desa Lawe, Kec. Kulawi. Penelitian data dasar untuk pengembangan sosial ekonomi masyarakat desa tertinggal parah di propinsi Sulawesi Tengah, Maluku, dan Irian Jaya. o. O.

Kipp, R. S. (1990): The early years of a Dutch clonial mission. The Karo field. Michigan.

Kohl, K.-H. (1998): Ethnizität und Tradition aus ethnologischer Sicht. In: Assman, A. \& H. Friese (Hrsg.): Identitäten. Frankfurt am Main. (= Erinnerung, Geschichte, Identitäten, Bd. 3), S. 269-287.

Kol, H. H. (1901): Onterwerp-program voor de Nederlandsch koloniale politiek. Te behandelen op het congres der Socialdemokratische arbeiderspartij te Utrecht 1901. Amsterdam.

Koloniaal Verslag (1917): Koloniaal Verslag. s'Gravenhage.

Koninklijk Nederlandsch Aardrijkskundig Genootschap [Hrsg.] (1938): Atlas van tropisch Nederland. Batavia.

Kornrumpf, Martin (1935): Mensch und Landschaft auf Celebes. Breslau.

Kößler, R. (1994): Postkoloniale Staaten. Elemente eines Bezugsrahmens. Hamburg.

Kötter, H., Roeder, R. O. G. \& K. H. Junghans [Hrsg.] (1979): Indonesien. Geographie, Geschichte, Religion, Staat, Gesellschaft, Bildungswesen, Politik, Wirtschaft. Tübingen, Basel. (= Buchreihe Ländermonographien, Bd. 11)

Kotilainen, E.-M. (1992): "When the bones are left". A study of Material Culture of Central Sulawesi. Helsinki. (= Transactions of The Finnish Anthropological Society, No. 31) 
Kreisel, W., Weber, R. \& H. Faust (2004): Historical impacts on use and management of natural resources in the rainforest margins of Central Sulawesi. In: Gerold, G., Fremerey, M. \& E. Guhardja (Hrsg.): Land use, nature conservation and the stability of rainforest margins in Southeast Asia. Berlin. S. 39-65.

Kruyt, A. C. (1903a): Beobachtungen an Leben und Tod, Ehe und Familie in Zentralcelebes. In: Zeitschrift für Sozialwissenschaft, Bd. 6, S. 707-714.

- (1903b): Gegevens voor het bevolkingsvraagstuk van een gedeelte van MiddenCelebes. In: Tijdschrift van het Koninklijk Nederlandsch Aardrijkskundig Genootschap, tweede serie deel XX, S. 190-205.

- (1906): Het animisme in den Indischen Archipel. 's-Gravenhage.

- (1908): De berglandschappen Napoe en Besoa in Midden-Celebes. Tijdschrift van het Koninklijk Nederlandsch Aardrijkskundig Genootschap (2nd series), Bd. 25, S. 1271-1344.

- (1909): Het landschap Bada in Midden-Celebes. In: Tijdschrift van het Koninklijk Nederlandsch Aardrijkskundig Genootschap (2nd series), Bd. 26, S. 349-380.

- (1926): Pakawa, een landstreek in de onderafdeeling Paloe. In: Tijdschrift Koninklijk Nederlandsch Aardrijkskundig Genootschap, Bd. 43, H. 4, S. 526-544.

- (1929): The influence of Western civilisation on the inhabitants of Poso (Central Celebes). In: Schierke, B. (Hrsg.): The effect of western influence on native civilisations in the Malay Archipelago. Batavia.

- (1935): De Bewoners van Midden-Celebes, en de culturen, die invloed op hen hebben uitgeoefend. o.O.

- (1938): De West-Toradjas op Midden-Celebes. 4 Bde. Amsterdam.

(= Verhandelingen der Koninklijke Nederlandsche Akademie van Wetenschapen te Amsterdam, Afdeeling Letterkunde, Nieuwe Reeks, Deel XL)

Kruyt, A. C. \& J. Kruyt (1921): Verslaag van een reis nar het landschap Napoe in de Onderafdeeling Posso (Celebes). In: Tijdschrift van het Koninklijk Nederlandsch Aardrijkskundig Genootschap (2nd series), H. 38, S. 400-414.

Konold, W. (1996a): Von der Dynamik einer Kulturlandschaft. Das Allgäu als Beispiel. In: Konold, W. (Hrsg.): Naturlandschaft - Kulturlandschaft. Die Veränderung der Landschaften nach der Nutzbarmachung durch den Menschen. Landsberg. S.121-136.

Kuitenbrouwer, M. (1991): The Netherlands and the rise of modern imperialism: colonies and foreign policy, 1870-1902. New York.

Lamnek, S. (1993): Qualitative Sozialforschung. Bd. 1: Methodologie. Weinheim.

Lebar, F. M. [Hrsg.] (1972): Ethnic groups of insular Southeast Asia, Vol. 1: Indonesia, Andaman Islands, and Madagaskar. New Haven.

Leirissa, R. Z. (1996): 'Copracontracten': an indication of economic development in Minahasa. In: Lindblad, T. (Hrsg.): Historical foundations of a national economy in Indonesia, 1880s-1990s. Amsterdam u.a.. S. 265-277.

Leser, H. [Hrsg.] (1997): DIERCKE-Wörterbuch Allgemeine Geographie. München, Braunschweig. 
Le Vine, V. T. (1997): Conceptualizing ethnicity and ethnic conflict. A controversy revisted. In: Studies in comparative international development, Nr.2, S. 45-75.

Li, T. M. [Hrsg.] (1999): Transforming the Indonesian uplands: marginality, power and production. London.

- (2000): Articulation indigenious identity in Indonesia. Resource politics and the tribal slot. Berkeley. (= Berkeley Workshop on Environmental Politics, working paper n. 00-7)

- (2001a): Masyarakat adat, difference, and the limits of recognition in Indonesia's forest zone. In: Modern Asian Studies, Bd. 35, H. 3, S. 645-676.

- (2001b): Relational histories and the production of difference on Sulawesi's upland frontier. In: Journal of Asian Studies, Bd. 60, H. 1., S. 41-66.

- (2002a): Engaging simplifications: community based resource management, market processes and state agendas in upland Southeast Asia. In: World Development, Bd. 30, H. 2, S. 265-283.

- (2002b): Ethnic cleansing, recursive knowledge, and the dilemmas of sedentarism. In: International Journal of Social Science, Bd. 173, S. 361-371.

- (2002c): Local histories, global markets: cocoa and class in upland Sulawesi. In: Development and Change, Bd. 33, H. 3, S. 415-437.

Liefer, M. (1996): Dictionary of the modern politics of Southeast Asia. London.

Lineton, J. A. (1975): An Indonesian society and its universe. A study of the Bugis of South Sulawesi (Celebes) and their role within the wider social and economic system. Diss., University of London.

Locher-Scholten, E. (1981): Ethiek in fragmenten. Vijf studies over koloniaal denken en doen van Nederlanders in de Indonesische Archipel 1877-1942. Zeist.

Locher-Scholten, E. (1994): Dutch expansion in the Indonesian Archipelago around 1900 and the imperialism debate. In: Journal of Southeast Asian Studies, Bd. 25, H. 1, S. 91-111.

Loze, T. H. M. (1929): De indische zelfbesturende landschappen in het nieuwe staatsbestel. 's-Gravenhage.

Lynch, O. J. \& K. Talbott (1995): Balancing acts: community-based forest management and national law in Asia and the pacific. Washington.

Maertens, M. (2003): Economic modeling of agricultural land use patterns in forest frontier areas: theory, empirical assesment and policy implications for Central Sulawesi, Indonesia. Berlin.

Maertens, M., Zeller, M. \& R. Birner (2002): Explaining agricultural land use in villages surrounding the Lore Lindu National Park in Central Sulawesi, Indonesia. Indonesia. Bogor, Göttingen, Kassel. (= STORMA Discussion Paper Series Sub-Program A, Nr. 4)

- (2004): Does technical progress in agriculture have a forest saving or a forest clearing effect? Theory and evidence from Central Sulawesi. In: Gerold, G., Fremerey, M. \& E. Guhardja (Hrsg.): Land use, nature conservation and the stability of rainforest margins in Southeast Asia. Berlin. S. 179-197. 
Mattulada (1990): Penduduk terasing. Suata kajian etnologi nusantara. In: Mamar, S. \& H. Sulaiman (Hrsg.): Suku terasing di Sulawesi Tengah. Palu. S. 1-6.

- (1991): Manuisa dan kebudayaan Kaili di Sulawesi Tengah. In: Antropologi Indonesia, Bd. 48, H. 15, S. 110-182.

Mappatoba, M. (2004): Co-management of protected areas: the case of community agreements on conservation in the Lore Lindu National Park, Central Sulawesi, Indonesia. Göttingen.

McDonald, P. (1980): An historic perspective to population growth in Indonesia. In: Fox, J. J. (Hrsg.): Indonesia: Australian perspectives, Bd. 1: Indonesia, the making of a culture. Canberra. S. 81-94.

Merchant, C. (1980): The death of nature: women ecology and the scientific revolution. San Francisco.

Merkens, J. (1927): Bijdrage tot de kennis van den kerbouw en de kerbouwenteelt in Nederlandsch Oost-Indië. Diss., Rijksuniversiteit Utrecht.

Metzger, E. (1892): Europäische Ansiedler in Niederländisch Ost-Indien. Hamburg.

Metzner, Joachim (1981): Palu (Sulawesi):Problematik der Landnutzung in einem klimatischen Trockental am Äquator. In: Erdkunde, Bd. 35, S. 42-54.

Middendorp, W. (1929): The administration of the outer provinces of the Netherlands Indies. In: Schierke, B. (Hrsg.): The effect of western influence on native civilisations in the Malay Archipelago. Batavia. S. 34-70.

Mitchell, D. (2000): Cultural geography - a critical introduction. Oxford.

Muir, R. (1998): Landscape a wasted legacy. In: Area, Bd. 30, S. 263-271.

Myers, N., Mittermeier, R. A., Mittermeier, C. G., da Foncesca, G. A. B. \& J. Kent (2000): Biodiversity hotspots for conservation priority. In: Nature, Bd. 403, S. 853-858.

Naylor, L. L. (1996): Culture and change. An introduction. Westport.

Nirboyo, K. (1990): Perubahan sosial masyarakat terasing. Kasus desa resettlement Maranata Kabupaten Donggala. In: Mamar, S. \& H. Sulaiman (Hrsg.): Suku terasing di Sulawesi Tengah. Palu. S. 64-107.

Nooy-Palm, C. H. M. (1975): Introduction to the Sa'dan Toraja people and their country. In: Archipel, Bd. 10, S. 53-92.

Norton, W. (1989): Explorations in the understanding of landscape. A cultural geography. New York, Westport, London. (= Contributions in Sociology, Nr. 77)

- (2000): Cultural geography: themes, concepts, analyses. Toronto.

o.A. (1912): Nota van toelichting over de Berglandschappen boven het Paloedal. In: Tijdschrift voor Indische Taal-, Land- en Volkenkunde (1912), S. 1-26.

Olive, C. A. (1998): Land use change and sustainable development in Segara Anakan, Java, Indonesia: interactions among society, environment and development. Waterloo. (=Department of Geography Publication Series, University of Waterloo, Nr. 51)

Oswald, W. \& M. Schöpfle (1987): Systematic fieldwork: ethnographic analysis and data management. Newbury.

\section{Pazifik Forum Bd. 12}


Otterspeer, W. (1989): The ethical imperativ. In: Otterspeer, W. (Hrsg.): Leiden oriental connections: 1850-1940. Leiden. S. 204-230.

Palmier, L. H. (1962): Indonesia and the Dutch. London.

Pamei, J. (1997): Perang bulu Momi: perlawangan Towoalangi raja Kulawi terhadap Belanda. Paper, präsentiert auf einem Seminar über die Schlacht am Berg Momi. Palu. 8. November 1997.

Pamungkas, S.-B. (1995): Die 10 größten Probleme der indonesischen Wirtschaft. In: Indonesien-Information, Nr. 3, o.S.

http://home.snafu.de/watchin/II_3_95/zehn_groessten_probleme.htm (Zugriff: Oktober 2004)

Peet, R. (1998): Modern geographic thoughts. Oxford.

Pelras, C. (1998): Bugis culture: a tradition of modernity. In: Robinson, K. \& M. Paeni (Hrsg.): Living through histories: culture, history and social life in South Sulawesi. Canberra. S. 19-28.

Popper, K. R. (1984) [1934]: Logik der Forschung. Tübingen.

Prince, G. H. A. (1995): Dutch Policy in Colonial Indonesia 1900-1942. In: Jahrbuch für Wirtschaftsgeschichte, Bd. 1995, H. 1, S. 23-44.

- (1996): Monetary policy in colonial Indonesia and the position of the Java bank. In: Lindblad, T. (Hrsg.): Historical foundations of a national economy in Indonesia, 1880s-1990s. Amsterdam u.a.. S. 55-70.

Rasul, G. \& G. B. Thapa (2003): Shifting cultivation in the mountains of South and Southeast Asia: regional patterns and factors influencing the change. In: Land Degradation \& Development, Bd. 14, S. 495-508.

Reid, A. (1988-1993): Southeast Asia in the age of commerce, 1450-1680. New Haven.

- (1997): Inside out. The colonial displacement of Sumatra's population. In: Boomgard, P., Colombijn, F. \& D. Henley (Hrsg.): Paper Landscapes. Explorations in the environmental history of Indonesia. Leiden.

Ricklefs, M. C. (1981): A history of modern Indonesia: c. 1300 to the present. London.

Robinson, I.. \& P. Richards (2003): Introduction. In: Robertson, I. \& P. Richards (Hrsg.): Studiying cultural landscapes. London. S. 1-18.

Röll, W. (1979): Indonesien. Entwicklungsprobleme einer tropischen Inselwelt. Stuttgart.

Ruf, F. (1995): Booms et Crises du Cacao. Les Vertiges de l'or Brun. Karthala, Paris.

- (1997): From Tree-Crop Planting to Replanting: 1997: A new Turning-Point for the Sulawesi Cocoa Boom. In: Cirad-Askindo (Hrsg.): Workshop on the Future of Indonesian Cacao through Replanting and Pest Disease Control. Jakarta. S.. 13-46.

Ruf, F. \& P. Ehret (1993): Compétitivité et cycles du cacao. Vrais et faux problèmes sous éclairage Indonésien. In: Etienne, G., Griffon, M. \& P. Guillaumont (Hrsg.): Afrique-Asie: Performances agricoles comparées. Paris, S. 255-301.

Ruf, F. \& Yoddang (2001): Cocoa migrants from boom to bust. In: Gerard, F. \& F. Ruf (Hrsg.): Agriculture in crisis: people, commodities and natural resources in Indonesia, 1996-2000. Montpellier, Richmond. S. 97-156. 
Sauer, C. O. (1925): The Morphology of landscape. In: University of California Publications in Geography 2, S. 19-54. Nachgedruckt in Leighly, J. [Hrsg.] (1963): Land and life: a selection from the writings of Carl Ortwin Sauer. Berkeley. S. 351-379.

Sarasin, P. \& F. Sarasin (1901): Entwurf einer geographisch-geologischen Beschreibung der Insel Celebes. Wiesbaden. (= Materialien zur Naturgeschichte der Insel Celebes, Bd. 4)

- (1905): Reisen in Celebes ausgeführt in den Jahren 1893-1896 und 1902-1903. Wiesbaden. 2 Bde.

Savitri, L. A. (2004): Agrarian change and gendered local knowledge: the shift of domination. A case study of lokal knowledge of Kulawi community in Bolapapu village, Kulawi sub-district, Donggala regency, Central Sulawesi. Unveröffentlichte M.Sc. thesis, Institut Pertanian Bogor.

Schaafhausen-Betz, S. (1998): Auswirkungen spontaner Landnahme in Ostkalimantan. Berlin. (= Berliner geographische Schriften, Bd. 27)

Scherer-Hall, R. (1996): Kleines Lexikon der historischen Kulturlandschaft und ihrer Elemente. Mit tabellarischer Übersicht von historischen Kulturlandschaftselementen. Köln.

Schöffer, I. (1978): Dutch "Expansion" and Indonesian reactions: some dilemmas of modern colonial rule (1900-1942). In: Wesseling, H. L. (Hrsg.): Expansion and reaction. Leiden. S. 78-100.

Schlüter, O. (1906): Die Ziele der Geographie des Menschen. München u.a.

- (1928): Die analytische Geographie der Kulturlandschaft erläutert am Beispiel der Brücken. In: Zeitschrift der Gesellschaft für Erdkunde, Sonderband, S. 388-411.

Scholz, U. (1992): Transmigrasi - ein Desaster? Probleme und Chancen des indonesischen Umsiedlungsprogramms. In: Geographische Rundschau, Bd. 44, H. 1, S. 33-39.

Schrauwers, A. (1998): "Let's party": State Intervention, Discursive Traditionalism and the Labour Prcess of Highland Rice Cultivators in Central Sulawesi, Indonesia. In: Journal of peasant studies, Bd. 25, H. 3, S. 112-130.

- (1999): "It's not economical": the market roots of a moral economy in highland Sulawesi, Indonesia. In: Li, T. M. (Hrsg.): Transforming the Indonesian uplands: marginality, power and production. London. S. 105-129.

- (1999): Negotiating parentage: the political economy of "kinship" in Central Sulawesi, Indonesia. In: American Ethnologist, Bd. 26, H. 2, S. 310-323.

- (2000a): Colonial 'Reformation' in the Highlands of Central Sulawesi, Indonesia, 1892-1995. Toronto, Buffalo, London.

- (2000b): Three weddings and a performance: marriage, household, and development in the highlands of Central Sulawesi, Indonesia. In: American Ethnologist, Bd. 27, H. 4, S. 855-876.

Schulze, C., Steffan-Dewentert, I. \& T. Tscharntke (2004): Effects of land use on butterfly communities at the rain forest margin: a case study from Central Sulawesi. In: Gerold, G., Fremerey, M. \& E. Guhardja (Hrsg.): Land use, nature conservation and the stability of rainforest margins in Southeast Asia. Berlin. S. 282-297. 
Schurmer-Smith, P. [Hrsg.] (2002): Doing cultural geography. London u.a.

Schuyt, P. (1911): Van dag tot dag op reis naar de landschapen Napoe, Besoa en Bada. In: Mededeelingen van wege het Nederlandsch Zendelinggenootschap, Bd. 55, S. $1-26$.

Schwarze, S. (2004): Determinants of income generating activities of rural households. a quantitative study in the vicinity of the Lore-Lindu National Park in Central Sulawesi/Indonesia. Diss. Georg-August-Universität Göttingen.

Schwarze, S. \& M. Zeller (2005): Income diversification of rural households in Central Sulawesi, Indonesia. In: Quarterly Journal of International Agriculture, Bd. 44, S. 61-73.

Schwedler, H.-U. (1985): Arbeitsmigration und urbaner Wandel: eine Studie über Arbeitskräftewanderung und räumliche Segregation in orientalischen Städten am Beispiel Kuweits. Berlin.

Semaoen (1927): Indonesien hat das Wort. Der Niedergang des holländischen Imperialismus. Hamburg, Berlin.

Seymour, S. (2000): Historical geographies of landscape. In: Graham, B. \& C. Nash (Hrsg.): Modern historical Geographies. London. S. 193-217.

Simmons, I. G. (1993): Interpreting nature: cultural constructions of the environment. London.

Sitorus, F. (2002a): Land, ethnicity and the competing power: agrarian dynamics in forest margin communities in Central Celebes, Indonesia. Bogor, Göttingen, Kassel. (= STORMA Discussion Paper Series Sub-Program A, Nr. 5)

- (2002b): "Revolusi chokelat": social formation, agrarian structure, and forest margins in upland Sulawesi, Indonesia. In: Gerold, G., Fremerey, M. \& E. Guhardja (Hrsg.): Land use, nature conservation and the stability of rainforest margins in Southeast Asia. Berlin. S. 105-118.

Spit, H. J. (1911): De indische zelfbesturende landschappen. Hun mate van zelfstandigheid. 's-Gravenhage.

Soemardjan, S. \& K. Breazeale (1993): Cultural change in Indonesia. Impact of village development. Surakarta.

Stattersfield, A. J., Crosby, N. J., Long, A. G. \& D. C. Wage (1998): Endemic bird areas of the world: Priorities for bird conservation. Cambridge.

Steinke, I. (1999): Kriterien qualitativer Forschung. Ansätze zur Bewertung qualitativempirischer Sozialforschung. Weinheim, München.

Steward, J. H. (1955): Theory of culture change: the methodology of multilinear evolution. Urbana.

Sunderlin, W. \& I. A. P. Resosudarmo (1999): The effect of population and migration on forest cover in Indonesia. In: Journal of Environment and Development, Bd. 8, H. 2, S. 152-169.

Sunito, S. (2004): Robo and the water buffalo: the lost souls of the pekurehua of the Napu Valley. In: Gerold, G., Fremerey, M. \& E. Guhardja (Hrsg.): Land use, nature conservation and the stability of rainforest margins in Southeast Asia. Berlin. S. 67-88. 
Sutarto, E. (2003): The 'cultural core' in multi ethnic communities and its impact on agrarian resource management. Bogor, Göttingen, Kassel. (= STORMA Discussion Paper Series Sub-Program A, Nr. 10)

Terkenli, T. S. (2005): New landscape spatialities: the changing scales of function and symbolism. In: Landscape and Urban Planning, Bd. 70, S. 165-176.

Thong, L. (1976): Patterns of urban residential segregation. The case of Kuala Lumpur. In: Tropical Geography, Bd. 43, S. 41-48.

Thorbecke, E. \& T. van de Pluijm (1993): Rural Indonesia: socio-economic development in a changing environment. New York. (= IFAD studies in rural poverty, no. 3)

Tilley, V. (1997): The terms of the debate. Untangeling language about ethnicity and ethnic movement. In: Ethnic and radical studies, Nr. 3, S. 497-522.

Tsing, A. L. (1993): In the realm of the diamond queen. Princeton.

Uhlig, H. (1984): Spontaneous and planned agricultural settlement. A general view of the present clearing-colonization in the ASEAN countries of South East Asia. Hamburg. (= Gießener Geographische Schriften, Bd. 58)

Valentijn, F. (1724-1726): Oud en Nieuwe Oost-Indiën, Vervattende een Naaukerige en uitvoerige Verhandelinge van Nederlands Mogentheyd in die Gewesten, benevens eene wydlustige Beschryvinge der Moluccos, Ambonia, Banda, Timor, en Solor, Java, en alle de Eylanden onder de zelve Landbestieringen behoorende; het Comptoir op Suratte, en de Levens der Groote Mogols. Dordrecht, Amsteram. 5 Bde.

Van Niel, R. (1991): A perception of a perception of a perception: Multatuli's view of Java in the 1850s. In: Canadian Journal of Netherlandic Studies, Bd. 12, S. 21-29.

Van Rheenen, T., Elbel, C., Schwarze, S., Nuryatono, N., Zeller, M. \& B. Sanim (2004): Encroachments on primary forests: are they really driven by dispair? In: Gerold, G., Fremerey, M. \& E. Guhardja (Hrsg.): Land use, nature conservation and the stability of rainforest margins in Southeast Asia. Berlin. S. 199-213.

Vatikiotis, M. R. J. (1993): Indonesian politics under Suharto. Order, development and pressure for change. London, New York.

Velthoen, E. (2002): Mapping Sulawesi in the 1950s. In: Nordholt, H. S. \& G. Asnan (Hrsg.): Proceedings Workshop Indonesia in Transition: Work in Progesss, Padang, 21 - 25 August 2002., o.S. http://www.knaw.nl/indonesia/transition/workshop/work_in_progress06.pdf (Zugriff: März 2004)

Verhoef, L. (1937): Typen van stervend land in den Nederlandsch Indischen Archipel: Het Paloedal (Midden Celebes). In: Tectona, Bd. 30, S. 220-222.

Vrocklage, B. A. G. (1936): Borneo, Celebes und Molukken. Münster/Westfalen. (= Die sozialen Verhältnisse Indonesiens. Eine kulturgeschichtliche Untersuchung, Bd. 1)

Wander, H. (1965): Die Beziehungen zwischen Bevölkerungs- und

Wirtschaftsentwicklung, dargestellt am Beispiel Indonesiens. Tübingen. (= Kieler Studien, Bd. 70) 
Waltert., M., Langkau, M., Maertens, M., Härtel, M.., Erasmi, S. \& M. Mühlenberg (2004): Predicting the loss of bird species from deforestation in Central Sulawesi. In: Gerold, G., Fremerey, M. \& E. Guhardja (Hrsg.): Land use, nature conservation and the stability of rainforest margins in Southeast Asia. Berlin. S. 327-349.

Weber, R. (2000a): “Ethische” Kolonialherrschaft in Zentralsulawesi.

Kulturgeographische Analyse der memories van overgave. In: Pacific News, Nr. 15, S. 4-5.

- (2000b): Die Kolonialzeit des heutigen Indonesien und ihre Auswirkungen auf Bevölkerung und Kulturlandschaft von Zentral-Sulawesi. Unveröffentlichte Diplomarbeit, Georg-August-Universität Göttingen.

- (2004): Terrorismus, Regionalkonflikte und ihre Auswirkungen auf den Tourismus in Indonesien. In: Faust, H., Reeh, T., Gee, K. (Hrsg.): Freizeit und Tourismus. Konzeptionelle und regionale Studien aus kulturgeographischer Perspektive. Göttingen. (= ZELTForum - Göttinger Schriften zu Landschaftsinterpretation und Tourismus, Bd. 2)

Weber, R., Kreisel, W. \& H. Faust (2003): Colonial interventions on cultural landscape of Central Sulawesi by „Ethical Policy” - Impacts of the Dutch rule in Palu and Kulawi valley 1905-1942. In: Asian Journal of Social Science, Bd. 31, H. 3, S. 398-434.

Wedema, S. (1998): "Ethiek" und Macht: die niederländisch-indische Kolonialverwaltung und Indonesiens Emanzipationsbestrebungen 1901-1927. Stuttgart.

Welz, G. (1994): Die soziale Organisatiom kultureller Differenz. Zur Kritik des Ethnosnegriffes in der anglo-amerikanischen Diskussion. In: Berding, H. (Hrsg.): Nationales Bewußtsein und kollektive Identität. Studien zu der Entwicklung des kollektiven Bewußtseins in der Neuzeit. Frankfurt am Main. S. 66-81.

Werlen, B. (1993): Society, action and space: An alternative human geography. London.

Werlen, B. \& S. Wälty [Hrsg.] (1995): Kulturen und Raum - Theoretische Ansätze und empirische Kulturforschung in Indonesien. Chur, Zürich. (= Konkrete Fremde Studien zur Erforschung und Vermittlung anderer Kulturen, Bd. 10)

Wichmann, A. (1890): Bericht über eine im Jahre 1888-89 im Auftrage der Niederländischen Geogr. Gesellschaft ausgeführten Reise nach dem Indischen Archipel. In: Tijdschrift Koninklijk Nederlandsch Aardrijkskundig Genootschap, Bd. 7, S. 907-994.

Whitten, A. J., Mustafa, M. \& G. S. Henderson (1988): The ecology of Sulawesi. Yogyakarta.

Wilken, G. A. (1873): Het landbzit in de Minahasa. In: Mededeelingen van wege het Nederlandsch Zendelinggenootschap, Bd. 17, S. 107-137.

Women and Geography Study Group (1997): Feminist geographies: explorations in diversity and difference. Harlow.

World Wild Life Found [Hrsg.] (1981): Lore Lindu National Park management plan 1981 - 1986. Bogor.

Wsevolod, W. I. (1981): Definitions of Ethnicity. Ontario. (= Occasional papers in ethnic and immigration studies, Bd. 79, Nr. 6) 
Yin, R. (1984): Case study research: design and methods. Beverly Hills.

Zha, D. (2000): China and the May 1998 riots in Indonesia. Exploring the issues. In: The Pacific review, Bd. 14, H. 4, S. 557-576.

Zelinski, W. (1973): The cultural geographie of the United States. Englewood Cliffs.

Zeller, M., Schwarze, S. \& T. van Rheenen (2002): Statistic sampling frame and methods used for the selection of vilages and households in the scope of the research program on stability of rainforest margins in Indonesia. Bogor, Göttingen, Kassel. (= STORMA Discussion Paper Series Sub-Program A, Nr. 1)

\section{Experteninterview und persönliche Kommunikation}

Abdulkadir-Sunito, M. (2002): Persönliche Kommunikation. Palu

Acciaioli, G. L. (2002): Persönliche Kommunikation. Bogor.

Akib, M. (2001): Persönliche Kommunikation. Palu.

Aragon, L. (2002): Persönliche Kommunikation. Palu.

Burkard, G. (2002): Persönliche Kommunikation. Palu.

- (2004): Persönliche Kommunikation. Palu.

Henley, D. (2000): Persönliche Kommunikation. Leiden.

Lagarens, A. (2002): Experteninterview im Departmen Sosial, Palu, am 17.6.2002.

Lorenz, W. (2004): Persönliche Kommunikation. Palu.

Schulze, C. H. (2002): Persönliche Kommunikation. Palu. 


\section{Anhang}

\section{Leitfragen/-themen für die Haushaltinterviews}

- Demographische Daten des Respondenten/der Respondentin und seiner/ ihrer Familie

- Landbesitz/-nutzung der Eltern

- Falls Migrant/-in: Migrationsdaten, Lebensbedingungen im Herkunftsort/gebiet

- Biographie des Respondenten/der Respondentin

- Bevölkerungs-, Siedlungs-, Landnutzungs-, ökonomische, soziokulturelle und Infrastruktur im Untersuchungsdorf zu verschiedenen Zeitpunkten

- Innovationen (landwirtschaftlich, soziokulturell, etc.) durch verschiedene ethnische Gruppen im Dorf

- Unterschiede zwischen lokalen und Migrantengruppen im Dorf

- Innovationen von staatlicher Seite

- Soziale Interaktion im Dorf

- Wahrnehmung des und Einstellung zum Lore-Lindu Nationalpark

- Einschätzung der persönlichen und dorfbezogenen zukünftigen Entwicklung durch den Respondenten/die Respondentin

\section{Leitfragen/-themen für die Interviews mit Repräsentanten im Untersuchungsdorf}

- Dorfbürgermeister (Kepala Desa): wie bei Haushaltsinterviews, zusätzliche Daten zur allgemeinen Dorfentwicklung, Problemfelder im Dorf.

- Dorfteilvorsteher (Kepala Dusun): wie bei Haushaltsinterviews, zusätzliche doftteilspezifische Daten, Problemfelder im Dorfteil.

- Vertreter des Adat-Rates: gewohnheitsrechtliche Regelungen im Dorf zur Landnutzung, Adat-Rituale, Adat-Sanktionen, historische Veränderungen des Adat, Vergleich des lokalen Adat mit dem Adat anderer Regionen. 


\section{Abstract}

\section{Change of cultural landscape in Central Sulawesi - Historical-geographical analysis of an Indonesian mountainous rainforest region}

The focus of this volume is on an empirical qualitative analysis of the historical changes in the (wo)man-environment interaction in an ecologically sensitive rainforest margin area of Indonesia. Along the four thematic scopes population, settlement structures, land property/land use, and political impacts the change of the cultural landscape in the Lore Lindu region is investigated for the first time for the whole twentieth century. The results support a better understanding of recent socio-economic and ecological issues in this region.

The study extends the classical geographical research on cultural landscape by the socio-cultural constructs of cultural landscape that are discussed in the course of the 'Cultural Turn' in human geography. The empirical data results from a field research in Central Sulawesi between February 2001 and September 2003. During this time period qualitative-biographical in-depth interviews were conducted with households, representatives, and key informants of nine selected villages. Furthermore, besides participant observation, secondary information sources (archival documents, statistics, maps, photographs, literature) were evaluated. Besides making available this knowledge base on the historical development the study emphasizes the great importance of qualitative research methods for revealing explanations of processes of change.

The results confirm the working hypothesis, which assumes that the change of the cultural landscape in the research area is subject to a time-spatial heterogeneity resulting from a multi-dimensional network of processes of change. Crucial foundations for the recent status of the cultural landscape in the Lore Lindu region are already based on the impacts of the Dutch colonial rule (1904 - 1942). This can be observed in almost all fields of reference. Extensive forced resettlements from the mountains down to the lowland and upland plains were accompagnied by the establishment of permanent settlements along new built roads. This allowed for an easier implementation of the colonial rulers' tax system, a better control of the local people and an effective recruitment of forced laborer for the construction of roads and irrigation channels. The Dutch promoted the expansion of paddy rice cultivation in the plains in stead of slash-and-burh shifting cultivation at the hills. The introduction of the plough and technical irrigation systems increased the agricultural mechanization while the introduction of the coffee plant and the beginning monetarization of trade heralded the transformation from subsistence to world market production. 
The traditional socio-political stratification of the indigenous societies was only slightly modified as the Dutch embarked on a strategy of indirect rule based on the local nobles as mediator of the colonial interests. The customary set of beliefs, in contrast, was superposed by the spread of the Christian religion. Although the Dutch missionaries put much effort into the Christianization of the people, former animist elements survived until today like it can be observed for all prominent religions in Indonesia. Closely linked to the mission was the establishment of a formal schooling system as the missionaries posed as teachers, too. The introduction of schools was one result of the so-called ethical policy, which influenced the Dutch colonial policy since the beginning of the $20^{\text {th }}$ century. This policy, however, did not fully meet its initial aim of an upliftment of the people in the colony.

Between 1942 and 1945 the Japanese replaced the Dutch rulers in Indonesia. Despite their relatively short period of presence the Japanese activities had major impacts on the cultural landscape of the study region, especially concerning agriculture. The local people were forced to plant cotton trees which made them neglecting the cultivation of food crops and thus resulted in cases of starvation. In the remote Pipikoro region of Kulawi sub-district the Japanese rulers forced the male villagers to exploit a mica mine. Besides, the Japanese language had to be learned and military exercises had to be done.

Although the colonial period was characterized by numerous facets of the cultural landscape's processes of change, most of them pertained to Lore Lindu region as a whole. Starting in the 1950s, multi-layered migration processes boosted an increasing differentiation of regional and local population, settlement, and land use patterns. While local resettlement programs beginning in the late 1960s and interisland transmigration schemes during the 1990s tied in with the Dutch colonial migration policy, spontaneous migration became a relevant phenomenon in the study area since the late 1950s. In this context, the biggest impact on the cultural landscape is connected to the members of the Bugis ethnic group immigrating from South Sulawesi. It was them who introduced the cacao plant around 1980, currently the most important cash crop in the region, and many of which were not only skilled in cacao cultivation but also had the financial means to purchase land. Especially since the mid 1990s, when the market prices for cacao boomed, the number of Bugis migrants in the Lore Lindu region increased considerably. An apparent difference of welfare between most Bugis migrants and locals emerged, which, among other reasons, made many local farmers shift their land use patterns from food crop dominated to cash crop dominated. Increasing land sales by the autochthonous villagers coming along with the implementation of the Lore Lindu national park as a restricted area resulted in a conflict situation when, in some sub-regions, the autochthonous farmers started to claim their customary land reserves, major parts of which then were located within the park territory. It has to be emphasized, however, that migrants in general and Bugis migrants in particular are not the direct causers of forest encroachments in the study region. 
Besides Bugis migrants, a large variety of different ethnic groups from within and outside the study region moved spontaneously and reshaped, in the course of the second half of the $20^{\text {th }}$ century, the formerly comparatively homogeneous ethnic composition of the local communities. Transmigrants, most of which originated in Java or Bali, and locally resettled migrants contribute to the current patchwork of numerous different ethnic groups living in the Lore Lindu region. Multi-ethnic villages of different formation, social interaction and distrubtion of political and economic power developed depending on ethnic networks, local politics and infrastructural settings. In many cases the major ethnic groups are concentrated in spatially different residential areas within the village. As main reasons for these sociospatial segregation patterns shape up ethnic networking and political decisions of the village's administration.

Village policies do not only play a role in residential segregation processes but also in the allocation of land. Informal continuities of the hierachical society structures as remnants of the traditional kingdoms and the implementation of the authoritarian Orde Baru system at the village level do have effects on land transactions (loan or sale) witihin a village. Here again however, it depends on the individual behavior of the village leaders whether and how land is allocated. Besides, the respective village policies influence spontaneous migrants' selection of their destination, which is communicated within the migrant groups by means of intra-ethnic networking. Influenced by the improvement of road infrastructure and thus better market access the eastern parts of the study region became, since the early 1980s, the most favored destination for migrants interested in the cultivation of perennial cash crops. This development resulted in a high dynamism of the cultural landscape's change in this area, even though not showing the same intensity in every sub-region and village.

The agricultural structure of the local farmers was characterized for decades by a land use mix of rice, cassava, vegetables and fruits. This system was extended by the additional cultivation of coffee starting during the Dutch colonial period and of clove since the 1970s. In most cases however, the fodd crops held the highest share within this agricultural pattern. Since the 1990s, cacao became the most important cash crop in the study region. Decreasing market prices for coffee and clove fueld this development making many local farmers converting fields cultivated with these crops into cacao fields. In some cases, even paddy rice fields got converted in recent years representing a shift from a food crop first strategy to a cash crop first strategy. This significant agricultural change is likely to hold economic risks as the households' income then strongly depends on the market prices of just one single crop.

The findings reveal that recent problems in the study area cannot be explained without the knowledge about the differentiated historical regional and village developments. Furthermore, the study demonstrates that the socio-cultural constructs of cultural landscape, which are created and developed in the perceptions of the respective local and migrant groups, play a decisive role for the formation of spatially different processes of change in this region. 


\section{Ringkasan}

\section{Perubahan pemandangan budaya di Sulawesi Tengah - Analisis bersejarah-geografis di suatu daerah hutan hujan Indonesia}

Buku ini mengandung analisis pembangungan pemandangan budaya (cultural landscape) di daerah Lore Lindu, Sulawesi Tengah, semasa abad ke-20. Penulis menyelidiki perubahan-perubahan di bidang sosial, budaya, demografi, ekonomi, infrastruktur dan politik semasa waktu dari awal zaman Belanda di daerah tersebut sampai masa sekarang.

Setelah orang Belanda masuk dearah Lore Lindu pada tahun-tahun 1904-1907 mereka memaksa orang-orang lokal pindah rumah dari gunung-gunung ke lembahlembah. Atas peraturan Belanda orang asli terpaksa tinggal di desa yang baru didirikan dekat jalan yang dibangungkan pakai kerja paksa. Orang lokal harus mulai menanam pohon kelapa dan memperluaskan lahan-lahan padi sawah. Selain itu orang Belanda memperkenalkan agama kristen, tanaman kopi, irigasi teknis, uang sebagai alat pembayaran dan sistem sekolah. Zaman Jepang (1942-1945) berkaitan dengan peraturan menanam pohon kapas, ini bahkan di petak-petak yang sebelumnya ditanami dengan tanaman makanan pokok. Salah satu akibat perintah itu adalah kasus-kasus kelaparan.

Sistem resettlemen lokal dilanjutkan oleh pemerintah Indonesia mulai pada tahun 1960-an. Pada awal tahun 1990-an beberapa program transmigrasi berlangsung. Selain jenis migrasi tersebut migrasi spontan adalah jenis yang paling penting. Kelompok etnis yang paling besar di antara migran-migran itu adalah kelompok orang Bugis yang berasal dari Sulawesi Selatan. Mereka memperkenalkan tanaman coklat di daerah Lore Lindu dan banyak dari mereka mempunyai cukup banyak modal untuk membeli lahan dari orang-orang lokal. Banyak keluarga-keluarga lokal yang menjual bagian lahan mereka berpikir bahwa nanti mereka masih bisa menggunakan tanah adat mereka sebagai warisan anak cucu mereka. Namun setelah batas taman nasional Lore Lindu diimplementasikan dengan larangan menebang pohon di dalam taman nasional mulai ada masalah kekurangan lahan untuk orang-orang lokal. Oleh karena itu di beberapa desa sudah ada kasus-kasus konflik antara masyarakat lokal dan pegawai kehutanan. Tidak hanya orang-orang Bugis pindah ke desa-desa di daerah Lore Lindu tetapi orang-orang dari banyak kelompok etnis yang berasal dari di dalam daerahnya dan dari daerah dan propinsi lain pindah di/ke daerah Lore Lindu mulai pada tahun 1950an. Salah satu hasil migrasi itu adalah pembentukan pola masyarakat multi-etnik yang berbeda dari desa ke desa. Perbedaan ini berkaitan dengan beberapa faktor, yaitu komunikasi dalam jaring-jaring etnis, politik pemerintah desa, dll.

Ini hanya beberapa contoh pembangungan pemandangan budaya di daerah Lore Lindu yang berdiskusi dalam buku ini. Analisis ini membuktikan pembangunan-pembangunan sama dan beda antara wilayah-wilayah dan antara desa-desa. 



\section{Pazifik Forum}

\section{Bisher erschienene Bände der Reihe Pazifik Forum:}

WAIBEL, MICHAEL/JORDAN, ROLF/SCHNEIDER, HELMUT (Hrsg.) (2006): Krisenregion Südostasien - Alte Konflikte und neue Kriege. Schriftenreihe PAZIFIK FORUM der Arbeitsgemeinschaft für Pazifische Studien e.V.; Band 11, Horlemannverlag, Bad Honnef 2006. 172 S. ISBN 3-89502-217-9.

WAIBEL, MICHAEL/KREISEL, WERNER (Ed.) (2005): The Pacific Challenge - Development Trends in the $21^{\text {st }}$ century. Schriftenreihe PAZIFIK FORUM der Arbeitsgemeinschaft für Pazifische Studien e.V.; Volume 10, Universitätsverlag Göttingen, Göttingen 2005. 176 S. ISBN 3-938616-10-5.

WAIBEL, MICHAEL/THIMM, TANJA/KREISEL, WERNER (Hrsg.) (2005): Fragile Inselwelten. Tourismus, Umwelt und indigene Kulturen. Schriftenreihe PAZIFIK FORUM der Arbeitsgemeinschaft für Pazifische Studien e.V.; Band 9, Horlemannverlag, Bad Honnef 2005. 256 S. ISBN 3-89502-204-7.

KREISEL, WERNER/MARSDEN, PETER H./WAIBEL, MICHAEL (Hrsg.) (2003): Wandel, Werte und Wirtschaft im pazifischen Raum. In: Schriftenreihe PAZIFIK FORUM der Arbeitsgemeinschaft für Pazifische Studien e.V.; Band 8, Göttingen. ISBN 3-89744-226-4.

Sonderpreis bei Bestellung über die APSA: 14 Euro

THIMM, TANJA (2002): Kulturwandel und Tourismus in Französisch-Polynesien. In: Schriftenreihe PAZIFIK FORUM der Arbeitsgemeinschaft für Pazifische Studien e.V. Hrsg:: KREISEL, W., WAIBEL, M.); Band 7, Göttingen. ISBN 389744-191-8.

Sonderpreis bei Bestellung über die APSA: 14 Euro

FAUST, H. (1996): Verstädterung in Fiii - Besonderheiten in den Stadt-Land-Beziehungen eines insularen pazifischen Entwicklungslandes. Aachen 1996. In: Schriftenreihe PAZIFIK FORUM der Arbeitsgemeinschaft Pazifische Studien Aachen e.V.; (Hrsg.: KREISEL, W., FENSTERSEIFER, C., DICKMANN, F., VOSSEN, J.); Band 6, Aachen. Sonderpreis bei Bestellung über die APSA: 20 Euro

KREISEL, W./MARSDEN P. H. (Hrsg.) (1995): "First Peoples, First Voices": Indigene Völker zwischen Fremdbestimmung und Selbstbehauptung. In: Schriftenreihe PAZIFIK FORUM der Arbeitsgemeinschaft für Pazifische Studien Aachen e.V.; Band 5. Aachen.

Sonderpreis bei Bestellung über die APSA: 10 Euro

KREISEL, W./VOSSEN, J./DICKMANN, F. (Hrsg.) (1995): Wirtschaft und Umwelt im asiatisch-pazifischen Raum. In: Schriftenreihe PAZIFIK FORUM der Arbeitsgemeinschaft für Pazifische Studien Aachen e.V.; Band 4. Aachen. Sonderpreis bei Bestellung über die APSA: 10 Euro

KREISEL, W./MARSDEN P. H./DAVIS, G. V./JANSEN, J. (Hrsg.) (1992): Neuseeland im Pazifischen Raum. In: Schriftenreihe PAZIFIK FORUM der Arbeitsgemeinschaft für Pazifische Studien Aachen e.V.; Band 3. Aachen.

KREISEL, W./VOSSEN, J./DICKMANN, F. (Hrsg.): Der Tourismus in der pazifischen Inselwelt. In: Schriftenreihe PAZIFIK FORUM der Arbeitsgemeinschaft für Pazifische Studien Aachen e.V.; Band 2. Aachen 1991.

KREISEL W./VOSSEN, J./DICKMANN, F. (Hrsg.): Entwicklungstendenzen und Entwicklungsstrategien im pazifischen Inselraum. In: Schriftenreihe PAZIFIK FORUM der Arbeitsgemeinschaft für Pazifische Studien Aachen e.V.; Band 1. Aachen 1990.

\section{IHR ANSPRECHPARTNER FÜR BESTELLUNGEN:}

Dr. Michael Waibel; Geographisches Institut der Universität Göttingen

Goldschmidtstr. 5; 37077 Göttingen

Email: mwaibel@gwdg.de

Tel.: +49-551-39.80.55 



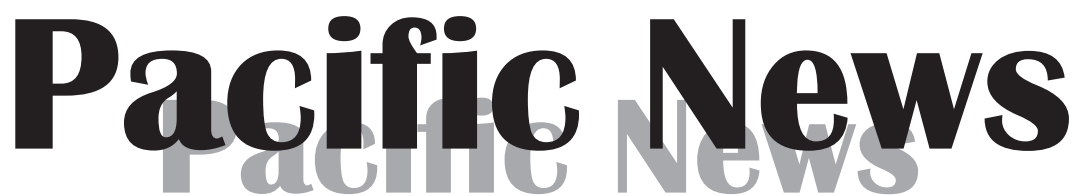

Das Magazin für Nachrichten - Mitteilungen - Hintergründe aus dem asiatisch-pazifischen Raum

Die PACIFIC NEWS ist das periodisch erscheinende Informationsmedium der Arbeitsgemeinschaft für Pazifische Studien e.V. (APSA) und wird in Zusammenarbeit mit der Abt. Kultur- und Sozialgeographie des Geographischen Instituts der Universität Göttingen erstellt.

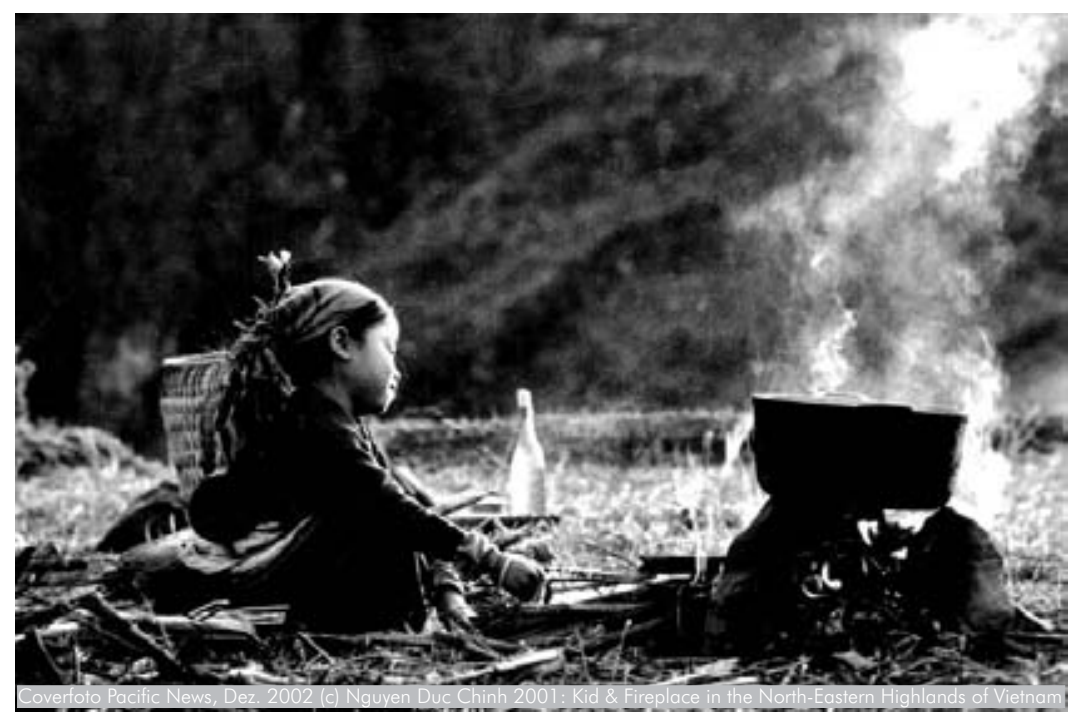

In der PACIFIC NEWS werden aktuelle Projekte und Forschungsarbeiten präsentiert sowie neueste Entwicklungen im asiatisch-pazifischen Raum vorgestellt. Besonderer Wert wird auf Interdisziplinarität gelegt. Die Redaktion freut sich über Artikel aus allen Bereichen der Kultur- und Gesellschafts- sowie Wirtschaftswissenschaften. Ferner ist es ein Bestreben, engagierten Nachwuchswissenschaftlern eine seriöse Publikationsplattform zu bieten.

Die PACIFIC NEWS erscheint halbjährlich in Göttingen. Interessierte Autoren möchten sich an die Redaktion (redaktion@pacific-news.de; Geographisches Inst. der Universität Göttingen, Goldschmidtstr. 5, 37077 Göttingen) wenden. Bitte beachten Sie unsere Hinweise und Richtlinien für Beiträge im Internet. 